CIVIL ENGINEERING STUDIES

Illinois Center for Transportation Series No. 19-012

UILU-ENG-2019-2012

ISSN: 0197-9191

\title{
Development of Long-Term Aging Protocol for Implementation of the Illinois Flexibility Index Test (I-FIT)
}

\author{
Prepared by \\ Imad L. Al-Qadi \\ Hasan Ozer \\ Zehui Zhu \\ Punit Singhvi \\ Uthman Mohamed Ali \\ Mohammed Sawalha \\ Arturo Francisco Espinoza Luque \\ Javier J. García Mainieri \\ University of Illinois at Urbana-Champaign \\ Thomas G. Zehr \\ Illinois Department of Transportation
}

Research Report No. FHWA-ICT-19-009

A report of the findings of

ICT PROJECT R27-175

Development of Long-Term Aging Protocol for Implementation of the Illinois Flexibility Index Test (I-FIT)

https://doi.org/10.36501/0197-9191/19-012

Illinois Center for Transportation

August 2019 

TECHNICAL REPORT DOCUMENTATION PAGE

\begin{tabular}{|l|l|}
$\begin{array}{l}\text { 1. Report No. } \\
\text { FHWA-ICT-19-009 }\end{array}$ & $\begin{array}{l}\text { 2. Government Accession } \\
\text { N/A }\end{array}$ \\
\hline $\begin{array}{l}\text { 4. Title and Subtitle } \\
\text { Development of Long-Term Aging Protocol for Implementation of the } \\
\text { Illinois Flexibility Index Test (I-FIT) }\end{array}$
\end{tabular}

\section{Authors}

Imad L. Al-Qadi, Hasan Ozer, Zehui Zhu, Punit Singhvi, Uthman Mohamed Ali, Mohammed Sawalha, Arturo Francisco Espinoza Luque, Javier J. García Mainieri, and Thomas G. Zehr

\section{Performing Organization Name and Address \\ Illinois Center for Transportation \\ Department of Civil and Environmental Engineering \\ University of Illinois at Urbana-Champaign \\ 205 North Mathews Avenue, MC-250 \\ Urbana, IL 61801}

\section{Sponsoring Agency Name and Address}

Illinois Department of Transportation (SPR)

Bureau of Research

126 East Ash Street

Springfield, IL 62704

\section{Recipient's Catalog No.}

N/A

\section{Report Date}

August 2019

6. Performing Organization Code N/A

\section{Performing Organization Report No.}

ICT-19-012

UILU-ENG-2019-2012

\section{Work Unit No.}

N/A

11. Contract or Grant No.

R27-175

13. Type of Report and Period Covered

Final Report 1/1/2017-8/31/2019

14. Sponsoring Agency Code

\section{Supplementary Notes}

Conducted in cooperation with the U.S. Department of Transportation, Federal Highway Administration.

https://doi.org/10.36501/0197-9191/19-012

\section{Abstract}

The objective of this study was to evaluate long-term aging effects on asphalt mixtures using the Illinois Flexibility Index Test (I-FIT) and to develop a corresponding long-term aging protocol. The study recommended the use of the forced-draft oven as the aging equipment because of its availability, feasibility, practicability, capacity, and acceptable variability. A fully prepared semicircular I-FIT specimen was chosen as the state of material during aging because it is practical, has limited operational variability, and its integrity is maintained during aging. The aging of compacted specimens for three days at $203^{\circ} \mathrm{F}\left(95^{\circ} \mathrm{C}\right)$ was found to be similar to aging for five days at $185^{\circ} \mathrm{F}\left(85^{\circ} \mathrm{C}\right)$ according to AASHTO R30, which is believed to be able to simulate up to 10 years of field aging. Hence, the $3 \mathrm{D} / 95 \mathrm{C}$ aging method was chosen as the key component of the long-term aging protocol. Based on statistical analysis and oven-aging specimens immersed in argon, it was shown that the trends of aging after $1 \mathrm{D} / 95 \mathrm{C}$ were similar to that observed after 3D/95C and 5D/85C. Hence, 1D/95C may be used as an indicator of 3D/95C aging for quality control purposes. Aging protocols were developed for laboratory-produced laboratory-compacted (LPLC) and plant-produced laboratorycompacted (PPLC) specimens. For LPLC, I-FIT on unaged and 3D/95C aged specimens should be conducted in all cases. A specific mix must have a mean flexibility index (FI) of unaged and 3D/95C aged specimens greater than 8.0 and 5.0 , respectively, to be accepted. For PPLC, I-FIT procedure should be conducted on both unaged and 3D/95C aged specimens in all cases, while 1D/95C may be used by contractors to screen problematic mixes. Flexibility index higher than 8.0 and 4.0 for unaged and 3D/95C aged specimens, respectively, must be satisfied.

\section{Key Words}

Asphalt Mixture, Fracture, Illinois Flexibility Index Test (I-FIT), Long-term Aging, Flexibility Index, Aging Rate

19. Security Classif. (of this report)

Unclassified

20. Security Classif. (of this page)
Unclassified

\section{Distribution Statement}

No restrictions. This document is available through the National Technical Information Service, Springfield, VA 22161.

Reproduction of completed page authorized
21. No. of Pages

$80+$ appendices
22. Price N/A 



\section{ACKNOWLEDGMENT, DISCLAIMER, MANUFACTURERS' NAMES}

This publication is based on the results of "ICT-R27-175: Development of Long-Term Aging Protocol for Implementation of the Illinois Flexibility Index Test (I-FIT)." ICT-R27-175 was conducted in cooperation with the Illinois Center for Transportation; the Illinois Department of Transportation; and the U.S. Department of Transportation, Federal Highway Administration.

Members of the Technical Review Panel (TRP) were the following:

- Tom Zehr (TRP Chair), Illinois Department of Transportation

- Jim Trepanier, Illinois Department of Transportation

- Dennis Bachman, Federal Highway Administration

- Ron Price, Illinois Department of Transportation

- Brian Pfeifer, Illinois Department of Transportation

- George Houston, Illinois Department of Transportation

- Brian Hill, Illinois Department of Transportation

- Kevin Burke, Illinois Asphalt Pavement Association

- Jeff Kern, Open Road Paving Company, LLC

- Megan Swanson, Illinois Department of Transportation

- Steve Robinson, Illinois Department of Transportation

- Matt Mueller, Illinois Department of Transportation - Retired

The contents of this report reflect the view of the authors, who are responsible for the facts and the accuracy of the data presented herein. The contents do not necessarily reflect the official views or policies of the Illinois Center for Transportation, the Illinois Department of Transportation, or the Federal Highway Administration. This report does not constitute a standard, specification, or regulation.

Trademark or manufacturers' names appear in this report only because they are considered essential to the object of this document and do not constitute an endorsement of product by the Federal Highway Administration, the Illinois Department of Transportation, or the Illinois Center for Transportation. 


\section{EXECUTIVE SUMMARY}

As a result of the Illinois Center for Transportation (ICT) project "R27-128: Testing Protocols to Ensure Performance of High Asphalt Binder Replacement Mixes Using RAP and RAS," a practical and reliable test method, the Illinois Flexibility Index Test (I-FIT), was developed to screen asphalt concrete (AC) cracking potential. The test method evaluates $A C$ mixes at $77^{\circ} \mathrm{F}\left(25^{\circ} \mathrm{C}\right)$ and at a loading head displacement rate of $1.97 \mathrm{in} / \mathrm{min}(50 \mathrm{~mm} / \mathrm{min})$. The flexibility index (FI), derived from I-FIT results, is a simple index parameter correlated to fundamental crack growth mechanisms in the fracture process zone. The parameter has the ability to distinguish AC mixes with varying characteristics that may result in different cracking potential. The integration of the I-FIT method into the Illinois Department of Transportation's (IDOT) hot-mix asphalt (HMA) design specifications is underway. Several steps are required to complete the implementation, including field validation, industry acceptance, and development of a long-term aging protocol. Therefore, this project was identified to develop protocols and propose thresholds for long-term aged plant- and laboratory-produced surface mixtures.

This report presents the outcomes from ICT R27-175 project "Development of Long-Term Aging Protocol for Implementation of the Illinois Flexibility Index Test (I-FIT)," which may be used by IDOT to fine-tune I-FIT AC surface mixture specifications for acceptance procedures and criteria. To accomplish the objectives of this study, flexibility characterization of a wide range of plant- and labproduced AC surface mixtures using various aging techniques such as a forced-draft oven, vacuum oven, and pressure aging vessel under different conditions were investigated. A suitable long-term aging protocol was developed. Thresholds were then developed for FI based on the testing results and discussion amongst the research team and the project Technical Review Panel (TRP) members. Additionally, field cores that experienced up to five years of field aging from both R27-175 and R27161 projects were evaluated to validate the proposed protocol and thresholds.

The FI decreases consistently after long-term aging, and this effect is primarily due to changes in the post-peak slope. The impact of aging varies with respect to different $A C$ mixes and is affected by voids in mineral aggregate (VMA), low-temperature PG grade, mix type, aggregate blend water absorption, and effective asphalt content.

The development of a long-term aging protocol for I-FIT consists of selection of equipment, state of material during aging, temperature, and aging time. The forced-draft oven has been selected as the aging equipment because of availability, feasibility, practicability, capacity, and acceptable variability. A fully prepared semi-circular I-FIT specimen has been chosen as the state of material during aging due to its high practicability and limited operational variability while maintaining integrity. $203^{\circ} \mathrm{F}$ $\left(95^{\circ} \mathrm{C}\right)$ is the optimal set-up temperature for forced-draft oven aging considering efficiency without changing the aging mechanism. Three days at $203^{\circ} \mathrm{F}\left(95^{\circ} \mathrm{C}\right)$ was shown to be able to reach an aging extent similar to that of 5D/85C, which is reported to simulate up to 10 years of field aging. However, both statistical analysis and a limited argon gas study suggested that $1 \mathrm{D} / 95 \mathrm{C}$ has the same aging mechanism as 3D/95C (5D/85C) and can distinguish AC mixes' susceptibility to aging. Consequently, 1D/95C may be used by contractors as a first-step indicator for 3D/95C behavior. 
As for long-term aging thresholds for the lab mix design process of new mixes, I-FIT on unaged and 3D/95C aged specimens should be conducted in all cases. FI thresholds of 8.0 and 5.0 have been proposed for unaged and aged I-FIT specimens, respectively.

As for the plant production process, I-FIT should be conducted for both unaged and 3D/95C aged specimens in all cases. FI thresholds of 8.0 and 4.0 are proposed for unaged and aged I-FIT specimens, respectively, based on test results of this project. However, 1D/95C may be used by contractors as an option to screen problematic mixes at an earlier stage. A correction must be applied to the thresholds for plant-produced mixes if they experience more than one month of summer season (June to September in Illinois) shelf aging in non-climate-controlled storage. 


\section{TABLE OF CONTENTS}

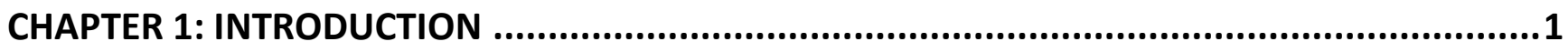

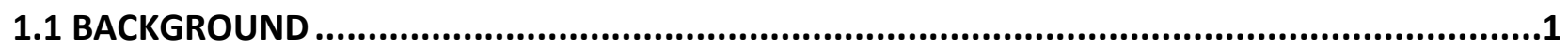

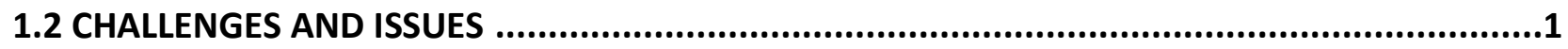

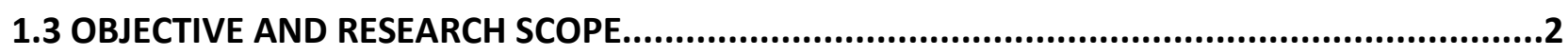

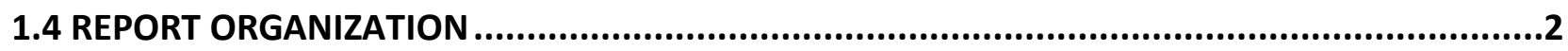

CHAPTER 2: SUMMARY OF LITERATURE ON LONG-TERM AGING OF ASPHALT CONCRETE

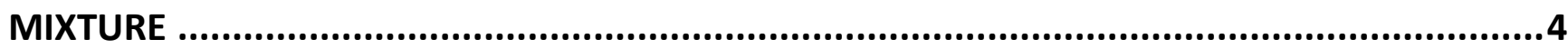

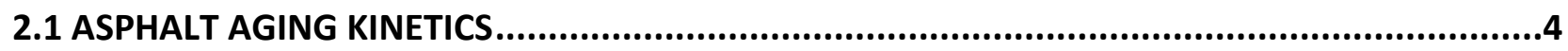

2.2 LABORATORY SIMULATION OF ASPHALT CONCRETE AGING ...........................................6

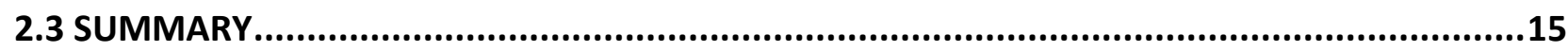

CHAPTER 3: MATERIAL SAMPLING, INVENTORY, AND EXPERIMENTAL METHODS...............19

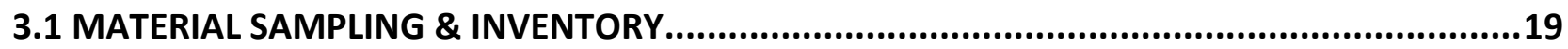

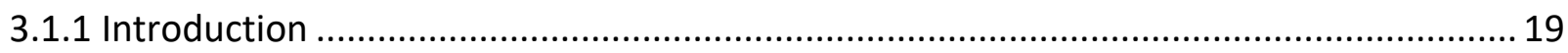

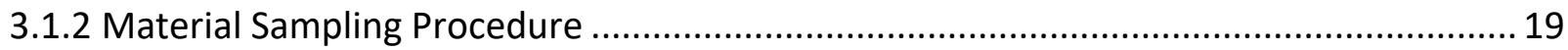

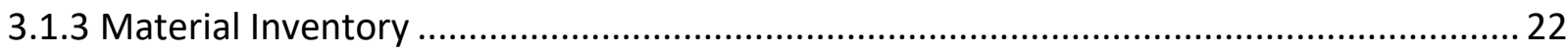

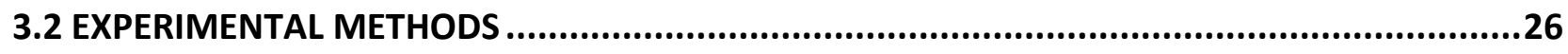

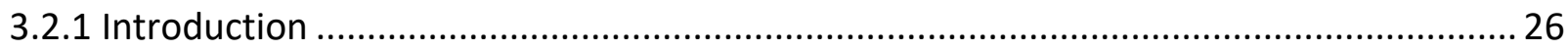

3.2.2 Selection of State of Material during Aging ................................................................ 27

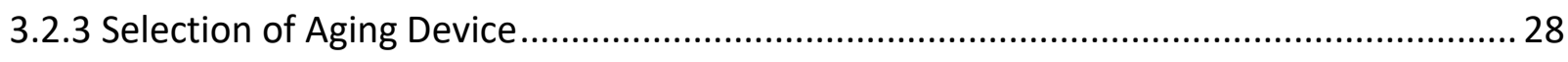

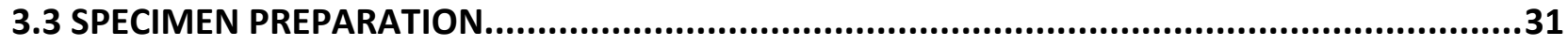

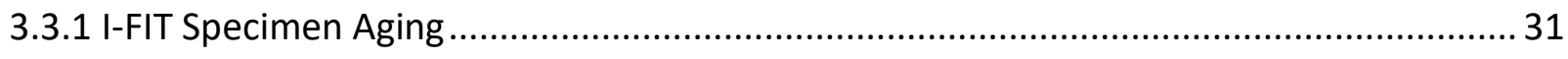

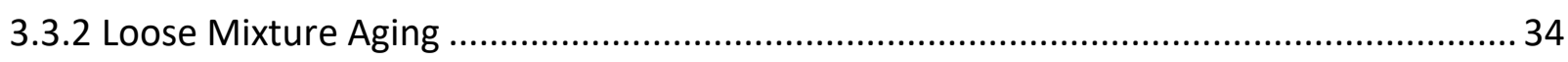

3.4 BINDER TESTING RESULTS SUMMARY ............................................................................34

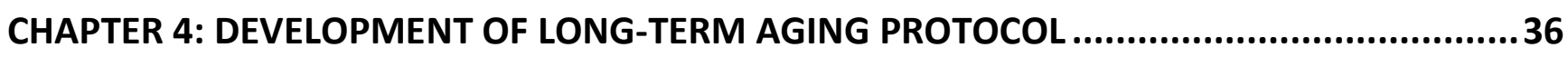

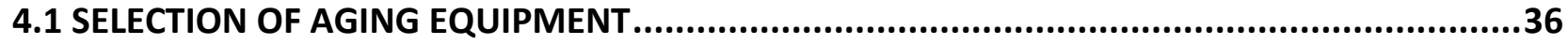

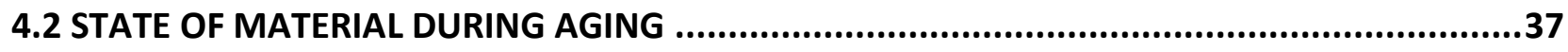

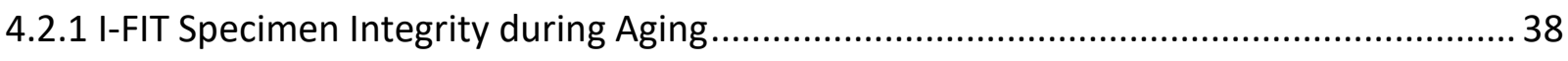

4.2.2 I-FIT Results for Compacted and Loose Mix Aged Specimens ........................................ 40 
4.3 I-FIT RESULTS OF LONG-TERM AGED AC ...................................................................43

4.4 SELECTION OF AC AGING TEMPERATURE...........................................................52

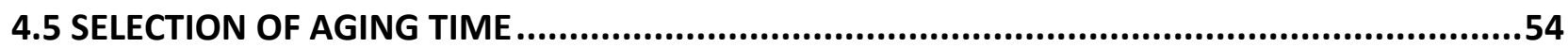

4.5.1 Statistical Analysis of Mix Design Parameters' Effect on Aging Rate ............................. 54

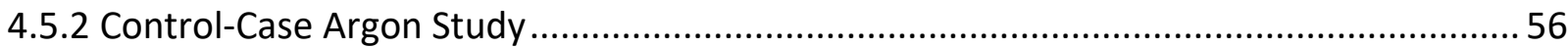

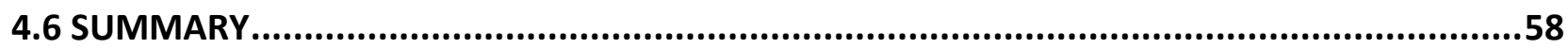

CHAPTER 5: DEVELOPMENT AND VALIDATION OF LONG-TERM AGING THRESHOLDS AND

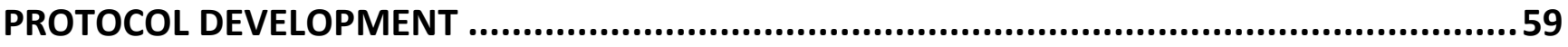

5.1 LABORATORY-PRODUCED AND LABORATORY-COMPACTED SPECIMENS.........................59

5.2 PLANT-PRODUCED AND LABORATORY-COMPACTED SPECIMENS...................................60

5.2.1 Development of Plant Production Process Thresholds .............................................. 60

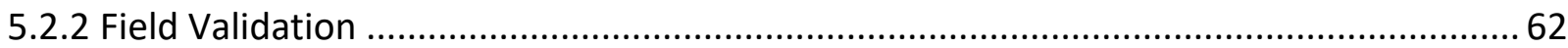

5.3 SHELF-AGED PLANT-PRODUCED MIXES THRESHOLDS .................................................67

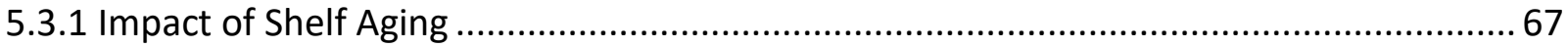

5.3.2 Development of Thresholds for Shelf-Aged Plant-Produced Mixtures .......................... 71

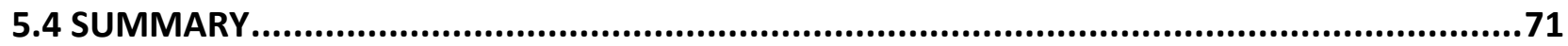

CHAPTER 6: SUMMARY, FINDINGS, AND RECOMMENDATIONS .................................73

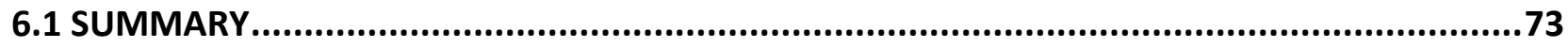

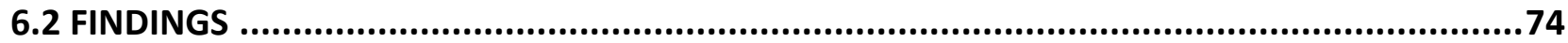

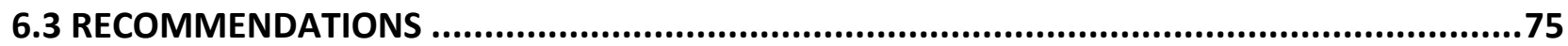

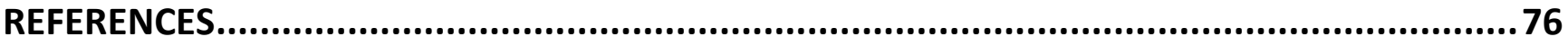

APPENDIX A: JOB MIX FORMULAS OF PLANT-PRODUCED MIXES ..................................81

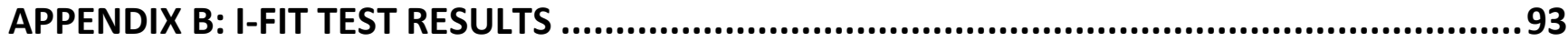

APPENDIX C: R27-175 MIXES FIELD CORES I-FIT RESULTS .........................................117

APPENDIX D: SHELF AGING I-FIT RESULTS......................................................... 123

APPENDIX E: R27-161 MIXES FIELD CORE I-FIT RESULTS .......................................... 130

APPENDIX F: COMPARISON BETWEEN AASHTO R30 AND PROPOSED PROTOCOL ............ 134

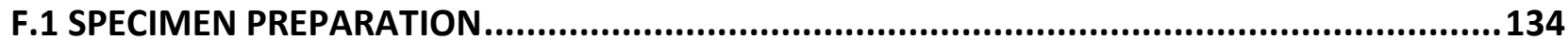




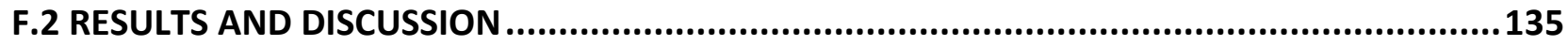

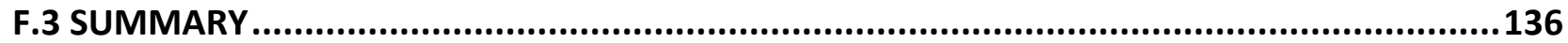

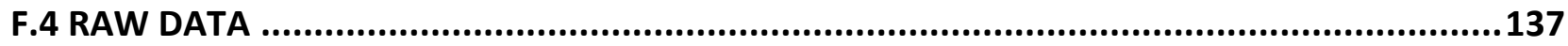




\section{LIST OF FIGURES}

Figure 2.1. Carbonyl $(C=O)$ and sulfoxide $(S=O)$ species appeared after aging (Lu et al. 2008).

Figure 2.2. Illustration showing speed of changes of a rheological property (viscosity) over aging time for SHRP asphalts. Test temperature and pressure were $60^{\circ} \mathrm{C}$ and 2 atmospheric pressure, respectively....

Figure 2.3. Oxidative-aging system without a high-pressure vessel. The air is passed into the compacted sample encased by a rubber membrane (Khalid 2002)

Figure 2.4. Effect of different aging regimes and moisture conditioning on mix stiffness (Airey et al. 2003).

Figure 2.5. Effect of aggregate types on aging (Airey et al. 2003).

Figure 2.6. Asphalt-aging system combining oxidation, heating, and moisture (Collop et al. 2007). ... 10

Figure 2.7. Weather-Ometer used to simulate aging of asphalt mixture or compacted sample (Hagos, 2008).

Figure 2.8. Schematic of UV-aging system utilized in the study and key features of the system on the right side (Xiao et al. 2013)

Figure 2.9. The fracture-energy value for non-aged, regularly aged (AASHTO R30), and UV-aged samples (data from Xiao et al., 2013).

Figure 3.1. Asphalt sample collection from the plant in a loader.

Figure 3.2. Placement of the sample on a flat surface. 20

Figure 3.3. Spread the sample to flatten conical heap.

Figure 3.4. Sampling from different locations.

Figure 3.5. Collected samples ready for transport.

Figure 3.6. Gradations for all mixes used in this project. 25

Figure 3.7. Test matrix.

Figure 3.8. Loose mixture aging.

Figure 3.9. I-FIT specimen aging: (a) specimens ready for aging; (b/c) aged specimen. 28

Figure 3.10. I-FIT specimen after PAV aging. 29

Figure 3.11. Vacuum oven. 30

Figure 3.12. Forced-draft oven. 30

Figure 3.13. Material splitting and batched samples. 31

Figure 3.14. Conditioning of batched samples before compaction. 
Figure 3.15. Extruded SGC pills with $180 \mathrm{~mm}$ height.

Figure 3.16. Specimen preparation steps from left to right: slicing, halving, and notching.

Figure 3.17. Trays containing specimens ready for aging process.

Figure 4.1. I-FIT specimen deformed after PAV aging.

Figure 4.2. Most used forced-draft ovens (Lemke et al. 2018).

Figure 4.3. Air voids comparison before and after aging. 38

Figure 4.4. Dimension changes after aging: (a) 1D/95C; (b) 3D/95C; (c) 5D/85C. 40

Figure 4.5. Plant Mix 1, I-FIT results comparison. 41

Figure 4.6. Plant Mix 2, I-FIT results comparison. 41

Figure 4.7. Plant Mix 3, I-FIT results comparison. 41

Figure 4.8. Plant Mix 5 I-FIT results comparison. 42

Figure 4.9. Plant Mix 6, I-FIT results comparison. 42

Figure 4.10. Lab Mix 1, I-FIT results comparison. 42

Figure 4.11. Loading vs. displacement curves of unaged and aged PM2 specimens for various periods at: (a) $75^{\circ} \mathrm{C}$, (b) $85^{\circ} \mathrm{C}$, and (c) $95^{\circ} \mathrm{C}$.

Figure 4.12. Unaged and Aged I-FIT results for all AC mixes. 51

Figure 4.13. Correlation between $\mathrm{Fl}$ and binder m-value. 52

Figure 4.14. Distorted I-FIT specimen (SMA) after 1D/110C aging. 53

Figure 4.15. Temperature variability of probes at different specified locations in forced-draft oven (Lemke et al. 2018). 53

Figure 4.16. Preparation process for argon aging. 57

Figure 4.17. Flexibility index comparison of aging in air vs. in argon. 57

Figure 5.1. Long-term aging protocol and thresholds for lab mix design process using I-FIT. 59

Figure 5.2. Long-term aging using I-FIT (a) protocol and thresholds for plant production, and (b) optional approach.

Figure 5.3. Progression of field cores FI from the R27-175 project: (a) uncorrected FI; (b) corrected FI for both thickness and air void. 65

Figure 5.4. Progression of field cores FI from the R27-161 project: (a) uncorrected FI; (b) corrected FI for both thickness and air void. 65

Figure 5.5. Progression of field cores FI from the 2016 IDOT Pilot Projects: (a) uncorrected FI; (b) corrected FI for both thickness and air voids. 
Figure 5.6. Field correlation between transverse cracking length FI for different rounds (Al-Qadi et al.

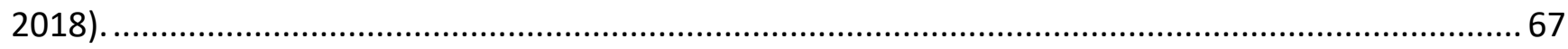

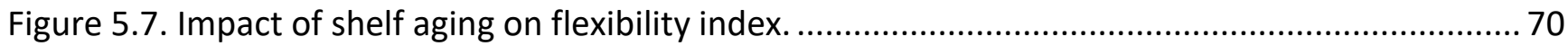

Figure 6.1. Long-term aging protocol for the Illinois Flexibility Index Test (I-FIT)............................ 74

Figure F.1. Compacted $180 \mathrm{~mm}$ pills aging in a forced-draft oven. ............................................... 134

Figure F.2. Comparison of FI for AASHTO R30 and proposed protocol using aged specimens. ..........135 


\section{LIST OF TABLES}

Table 2.1. Comparison between Loose Mix and Compacted Specimens in the Aging Procedure (Kim et al. 2018)

Table 2.2. Comparison between Oven and PAV Aging Methods (Kim et al. 2018).....

Table 2.3. Summary of Long-Term Aging Protocols Used/Recommended in Previous Studies

Table 3.1. Grading and Source of Asphalt Binders Used in the Study.... 23

Table 3.2. Details of PPLC Mixtures 24

Table 3.3. Design Details for PPLC Mixtures 24

Table 3.4. Aggregate Information 24

Table 3.5. Details of LPLC Mixtures 25

Table 3.6. Hamburg Wheel Test Information for LPLC Mixes 25

Table 3.7. Details of Field Core Samples. 26

Table 3.8. Detailed Information of the Forced-draft Oven Used in This Study. 30

Table 3.9. Test Data for Performance Graded Asphalt Binders Collected for the R27-175 Project ...... 35

Table 4.1. Comparison of Aging Equipment 36

Table 4.2. Pros and Cons of Aged Compacted and Loose AC Mixture 43

Table 4.3. Linear Regression Analysis Results for 1D/95C and 5D/85C Aging 55

Table 4.4. Summary of Mix Design Parameters' Effect on Aging Rate. 56

Table 5.1. LPLC Specimens Long-term Aging Flexibility Checking Results 60

Table 5.2. PPLC Specimens Long-term Aging Flexibility Checking Results 62

Table 5.3. Field Core Inventory Based on Dates of Coring .63

Table 5.4. I-FIT Summary of Additional Field Cores from Illinois Districts Provided by IDOT 64

Table A.1. PM1 81

Table A.2. PM2 .82

Table A.3. PM3 83

Table A.4. PM5 .84

Table A.5. PM6 85

Table A.6. PM7 86

Table A.7. PM8 87 


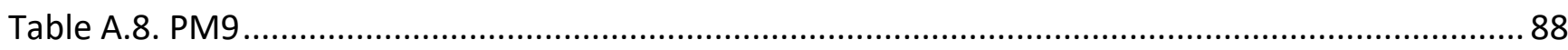

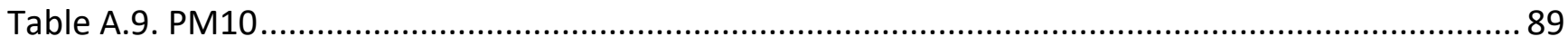

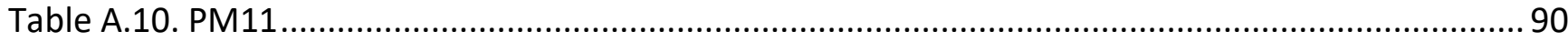

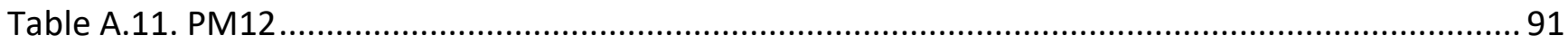

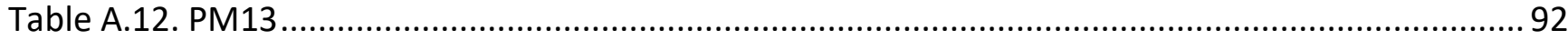

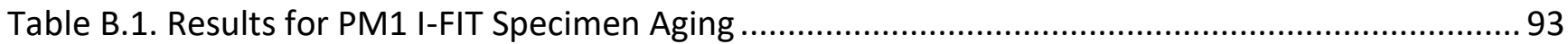

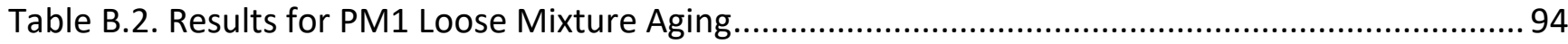

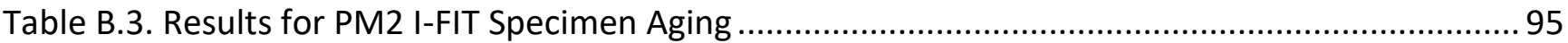

Table B.4. Results for PM2 Loose Mixture Aging................................................................................ 96

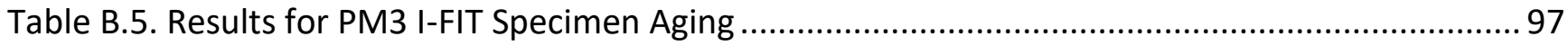

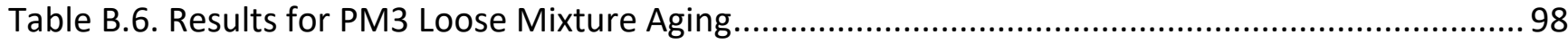

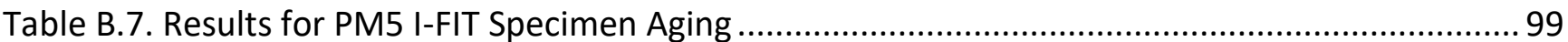

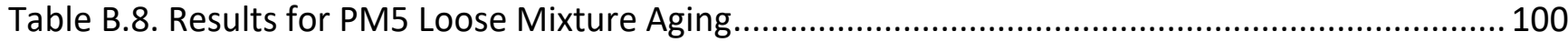

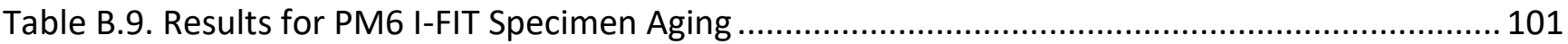

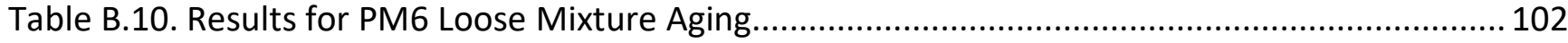

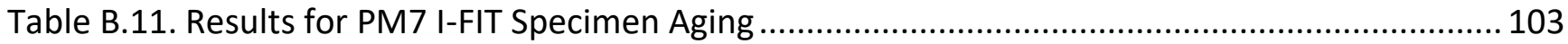

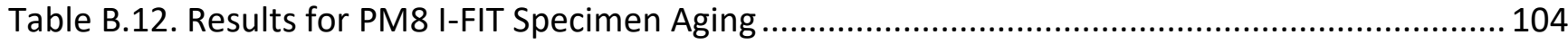

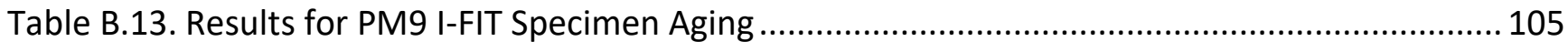

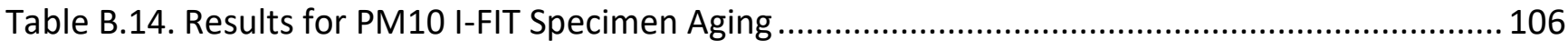

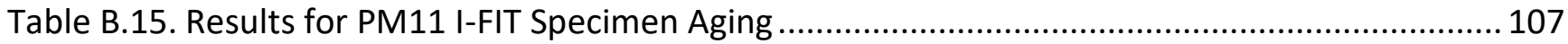

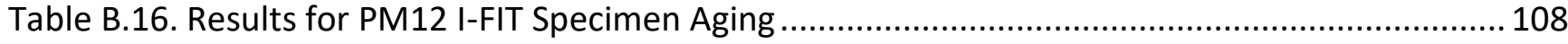

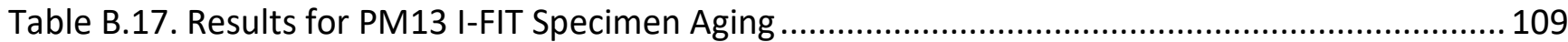

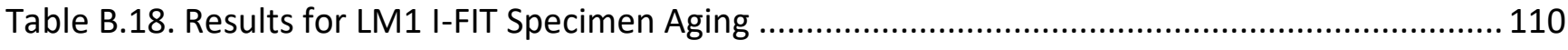

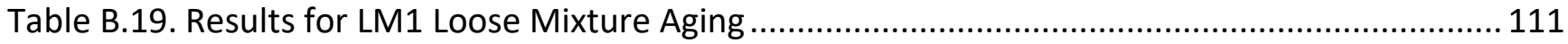

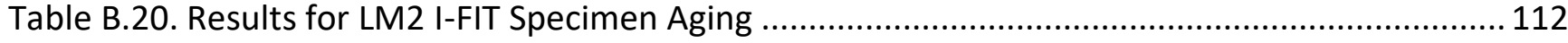

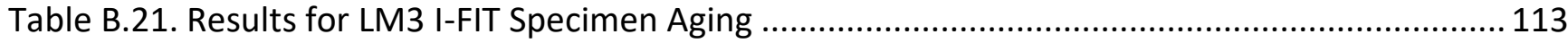

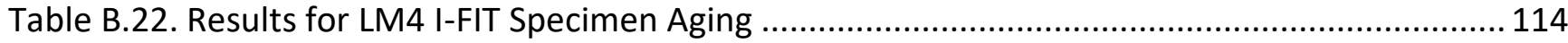

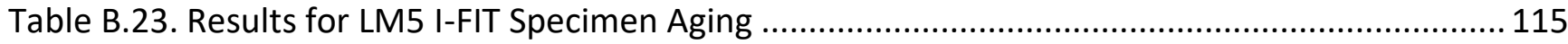

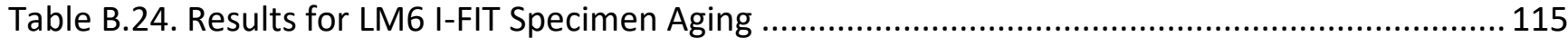

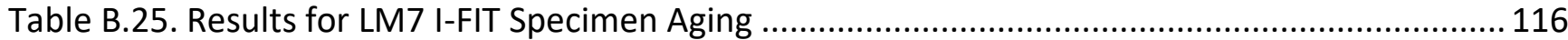


Table C.1. Results for PM1 Field Cores

Table C.2. Results for PM2 Field Cores

Table C.3. Results for PM3 Field Cores

Table C.4. Results for PM5 Field Cores

Table C.5. Results for PM6 Field Cores

Table C.6. Results for PM7 Field Cores

Table C.7. Results for PM12 Field Cores

Table C.8. Results for PM13 Field Cores

Table C.9. Six Months Field Cores Results for PM1

Table C.10. Six Months Field Cores Results for PM2

Table C.11. Six Months Field Cores Results for PM3 120

Table C.12. Six Months Field Cores Results for PM5

Table C.13. Six Months Field Cores Results for PM6

Table C.14. Six Months Field Cores Results for PM7

Table C.15. Six Months Field Cores Results for PM12

Table C.16. Six Months Field Cores Results for PM13

Table C.17. Twelve Months Field Cores Results for PM1

Table C.18. Twelve Months Field Cores Results for PM2

Table C.19. Twelve Months Field Cores Results for PM3

Table D.1. Six Months Shelf Aging for PM2

Table D.2. Six Months Shelf Aging for PM3

Table D.3. Six Months Shelf Aging for PM5

Table D.4. Six Months Shelf Aging for PM6

Table D.5. Six Months Shelf Aging for PM8

Table D.6. Six Months Shelf Aging for PM9

Table D.7. Six Months Shelf Aging for PM10

Table D.8. Six Months Shelf Aging for PM11

Table D.9. Six Months Shelf Aging for PM12

Table D.10. Six Months Shelf Aging for PM13. 
Table D.12. Twelve Months Shelf Aging for PM2 ................................................................ 128

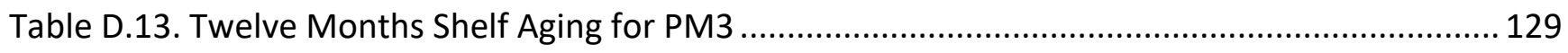

Table E.1. Field Core Results for R27-161 Project ................................................................ 130

Table E.2. One Year Field Core Results for R27-161 Project ...................................................... 130

Table E.3. Two Year Field Core Results for R27-161 Project .................................................... 131

Table E.4. Three Year Field Core Results for R27-161 Project................................................... 132

Table E.5. Four Year Field Core Results for Project R27-161 ................................................. 133

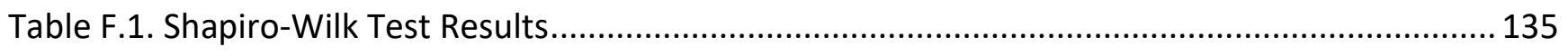

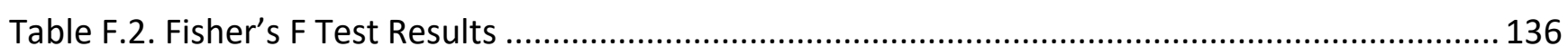

Table F.3. Independent Two-Sample T Test Results................................................................. 136

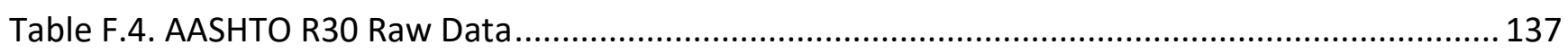





\section{CHAPTER 1: INTRODUCTION}

\subsection{BACKGROUND}

The increased use of recycled materials and asphalt binder modification affects overall asphalt concrete (AC) pavement performance. High use of recycled asphalt material content adversely affects the cracking performance of AC mixtures. The Illinois Center for Transportation (ICT) project "R27128: Testing Protocols to Ensure Performance of High Asphalt Binder Replacement Mixes Using RAP and RAS" resulted in a practical and reliable test method, the Illinois Flexibility Index Test (I-FIT), which was developed to screen the $A C$ mixes with cracking potential. The test method evaluates $A C$ mixes at $77^{\circ} \mathrm{F}\left(25^{\circ} \mathrm{C}\right)$ and at a loading head displacement rate of $1.97 \mathrm{in} / \mathrm{min}(50 \mathrm{~mm} / \mathrm{min})$. The flexibility index $(\mathrm{FI})$, derived from I-FIT results, is a simple index parameter correlated to fundamental crack growth mechanisms in the fracture process zone. The parameter can identify mixes with varying characteristics that may result in different cracking potential (Al-Qadi et al. 2015).

Asphalt concrete cracking is a common distress type found in Illinois pavements. The cracking results from several factors, which include using recycled asphalt materials like recycled asphalt shingles (RAS) and recycled asphalt pavement (RAP), quality and content of binder, poor aggregate structure, external loads, pavement structure, and environmental impacts. Asphalt concrete mixture aging is one of the natural phenomena that affects overall pavement cracking performance. Aging causes binder stiffening and results in higher potential of cracking in AC. However, the stiffening of AC mixtures has positive effect on rutting performance due to increased resistance to permanent deformation.

The aging of $A C$ mixtures is a highly complex chemical phenomenon. Apart from material properties, the degree of aging in $A C$ in the real world also depends on the pavement temperature, the presence of moisture, oxygen, and the extent of ultraviolet exposure. In addition, the change in material characteristics over time is directly related to these environmental changes. Hence, AC mixtures placed in various regions experience variable deterioration over time, which leads to differences in pavement performance.

Therefore, a distinct need exists for a comprehensive study to assess the aging of AC mixtures and the long-term impacts on overall pavement cracking performance in Illinois. In addition, the development of a practical long-term aging protocol complementing the current cracking performance test, I-FIT, is necessary to ensure pavements meet projected service lives.

\subsection{CHALLENGES AND ISSUES}

Cracking of AC pavements in Illinois is a prominent concern. The I-FIT test developed as per ICT project R27-128 filled the gap of a required performance test to evaluate the cracking potential in AC mixtures. However, the method that was developed does not incorporate the long-term effects due to aging on the cracking performance of AC mixtures. In addition, with increased use of recycling, modification of RAS and RAP binder by adding softer binders has become more common. 
Additionally, additives used to soften binders may impact AC long-term aging, hence, affecting pavement performance.

Several studies on AC mixture aging were conducted to investigate the possibility of implementing a laboratory aging methodology to predict the long-term behavior of pavement performance. These studies are discussed in Chapter 2 as part of the literature review. The current state-of-the-art literature characterizes laboratory aging of AC mixture as per AASHTO R30, which has its own challenges. For a performance test, a five-day aging period is not practical. Hence, there is a need for a rapid, easy, and reliable aging protocol suitable for industry. In addition, AASHTO R30 has not been field validated in Illinois. As stated earlier, long-term aging effects are a function of location with changing environmental patterns. There is currently no major study that specifically addresses determining the future performance of present AC mixtures. Therefore, there is a gap in the literature to guide the asphalt industry to reliably and cost effectively quantify the long-term performance of Illinois pavements.

\subsection{OBJECTIVE AND RESEARCH SCOPE}

The objective of this study was to evaluate the long-term aging effects on AC mixtures using I-FIT. This study aimed to develop a long-term aging protocol with specifications developed for I-FIT. Thresholds were to be developed for plant- and laboratory-produced AC mixtures as part of the study.

In addition, the study addressed the following research and practical concerns related to AC mixture aging:

- Effect of AC mixture aging on FI values.

- Effect of aging method in the presence of an inert gas environment.

- Effect of storage time and duration of shelf aging of the plant-produced mixtures on FI values.

- Binder source effects on Fl with aging.

- Field validation of the proposed aging protocol.

To achieve the objectives, a detailed experimental program was developed with a variety of plantand laboratory-produced mixtures representative of those used in Illinois. Laboratory mixes were especially designed to understand the effects of binder source on mixture aging potential in the presence of recycled material, RAP. The combination of these mixtures was used to develop the protocol for aging and corresponding thresholds.

\subsection{REPORT ORGANIZATION}

The report is organized in six chapters.

Chapter 1 presents an introduction, major challenges and issues, research objective, and scope of the work. 
Chapter 2 discusses the recent state-of-the-art literature on the aging of AC mixtures, related mechanisms, and existing laboratory aging protocols.

Chapter 3 presents material sampling procedures and inventory, experimental methods, specimen preparation, binder testing results, and details of testing materials.

Chapter 4 illustrates the comparison of various aging equipment and different states of material during aging. Aging temperature and duration were selected along with the discussion of asphalt concrete aging characterization using I-FIT. A long-term aging protocol is proposed.

Chapter 5 discusses the development of long-term aging thresholds for different scenarios: lab mix design process, plant production process, and shelf-aged plant-produced mixtures. The finalized aging protocol, along with associated thresholds, are presented.

Chapter 6 presents the summary and key findings of this project as well as the study recommendations. 


\section{CHAPTER 2: SUMMARY OF LITERATURE ON LONG-TERM AGING OF ASPHALT CONCRETE MIXTURE}

This chapter summarizes and discusses the literature compiled from previous studies conducted to simulate AC long-term aging in a lab. Asphalt aging kinetics was discussed first, followed by a detailed discussion of asphalt mixtures long-term aging methods.

\subsection{ASPHALT AGING KINETICS}

When asphalt (an organic hydrocarbon) reacts with atmospheric oxygen under different thermal forces, such as heat or ultraviolet radiation, oxidized products are created. Therefore, the concentration of oxidative products and the speed at which they are produced is a function of time, temperature, oxygen, diffusion flux, and physiochemical characteristics of the chemical species present (Peterson 2009). For instance, a common oxidative species (i.e., also called chemical functional group in asphalt), carbonyl $(\mathrm{C}=\mathrm{O})$, has a relatively lower initial production rate than that of the sulfoxide species $(\mathrm{S}=\mathrm{O})$, which is another oxidative species occurring during aging. However, the production rate of sulfoxide is significantly reduced, and this rate becomes almost constant after the first five hours of oxidative aging. As these oxidative species are produced, the molecular association between the core asphalt fractions changes. As a result, the physical performance properties of asphalt are also altered. As indicated by previous research, Fourier Transform Infrared Spectroscopy (FTIR) can be utilized to obtain information about the presence of carbonyl and sulfoxide in oxidized asphalt. In the FTIR library, an infrared-light absorption at $1700 \mathrm{~cm}^{-1}$ represents carbonyl formation $(C=O)$ and at $1030 \mathrm{~cm}^{-1}$ represents sulfoxide formation $(\mathrm{S}=0)$, as determined by the chemical-bond energy of these chemical species formed during the oxidation process. Studies have reported that they appeared to have a higher peak intensity in aged samples than unaged samples, as shown in Figure 2.1 (Hagos 2008; Yehualaeset 2010).

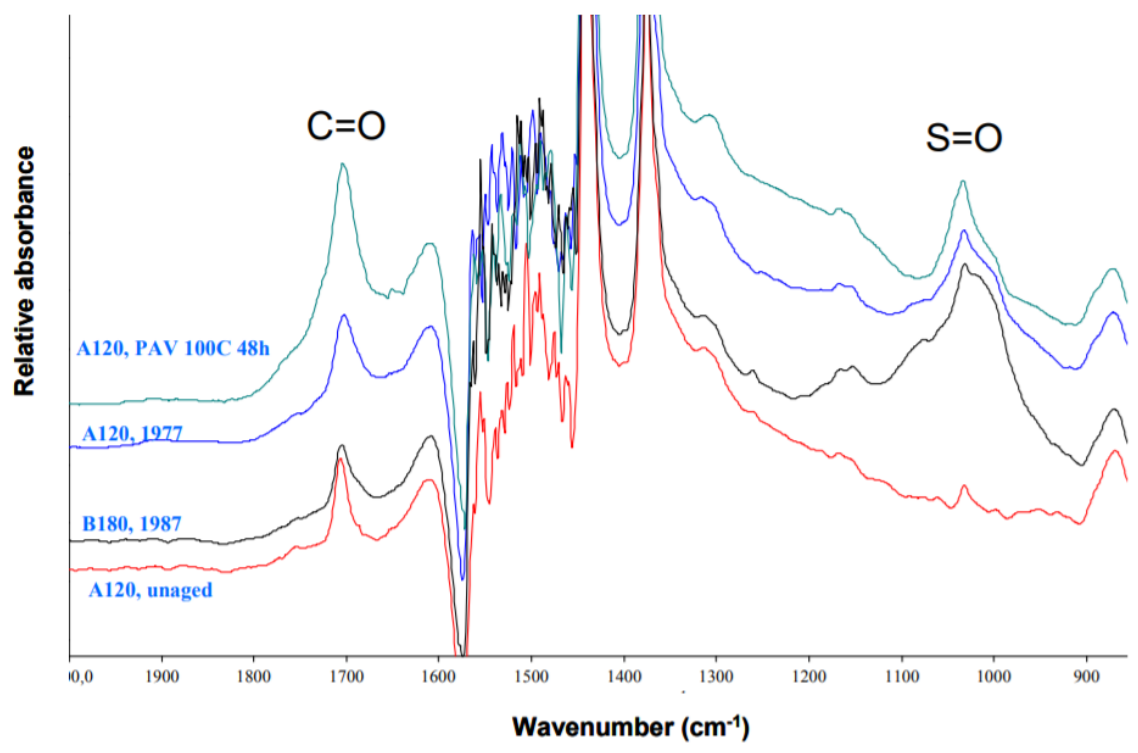

Figure 2.1. Carbonyl $(C=0)$ and sulfoxide $(S=0)$ species appeared after aging (Lu et al. 2008). 
Figure 2.2 shows the aging kinetics of three SHRP (Strategic Highway Research Program) asphalt samples that demonstrates how a critical physical property (i.e., viscosity) changes over time as asphalt ages. Two clear aging regimes exist: at the early stage, aging occurs at a fast rate; later, aging progresses at a slower rate. To be specific, after the first 50 hours of PAV aging, the aging effect (the rate of change viscosity increase) is significantly reduced. This trend can be noticed regardless of the types of asphalt tested in the study. Therefore, Herrington et al. (1994) hypothesized that the increase in the viscosity of asphalt is a hyperbolic function, a supposition that was then supported by many other studies, e.g., Peterson (2009).

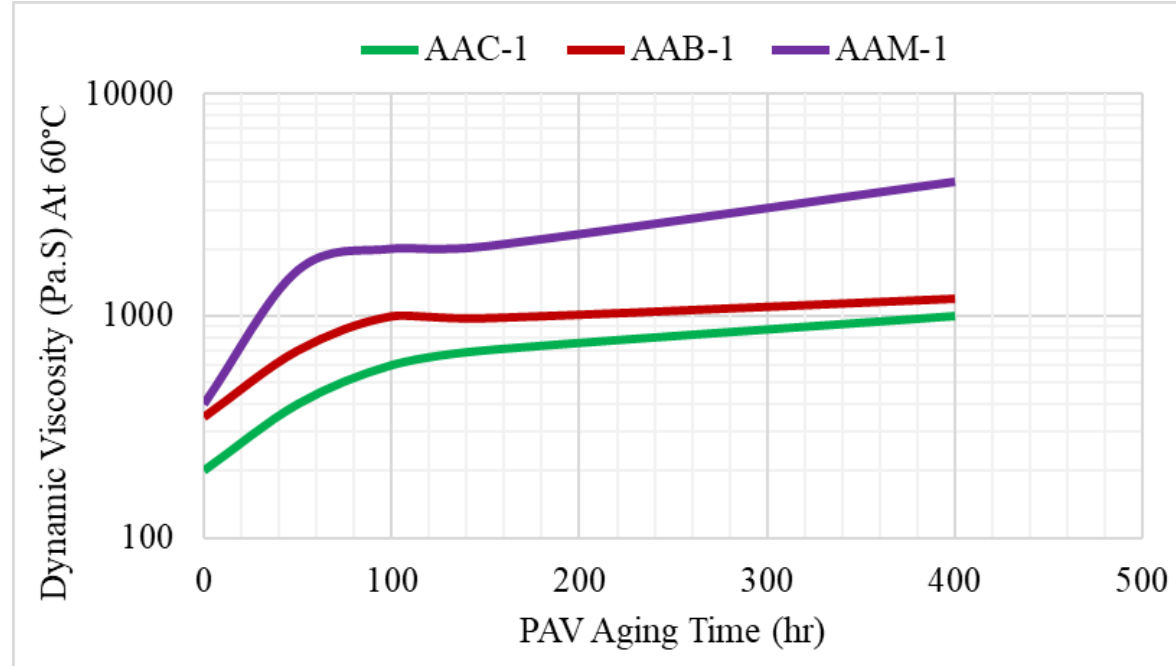

Figure 2.2. Illustration showing speed of changes of a rheological property (viscosity) over aging time for SHRP asphalts. Test temperature and pressure were $60^{\circ} \mathrm{C}$ and 2 atmospheric pressure, respectively.

From a UK study, Khalid (2002) reported on the stiffening modulus of asphalt binder. As expected, like viscosity, the stiffness modulus increased as asphalt aged over time; the rate of stiffening was also reported to change over time. The stiffening rate exhibited two slightly different regimes; the rate of increase in stiffness initially was high and decreased as time elapsed. In this study, another interesting observation was made: the volumetric flow rate of air can have a significant impact on the stiffening of asphalt. The impacts air (oxidation) can have on asphalt chemo-engineering performance are discussed in detail later in this chapter. To understand the time-temperature sensitivity, many studies-e.g., Bell et al. (1994) - reported that the speed of aging can be accelerated with an increase in temperature and as elapsed time increases.

In another UK study, Wu (2009) investigated in his doctoral thesis how aggregate petrography, i.e., physiochemical properties of aggregates, influences the aging of asphalt binder. The study found that the charged and polarized aggregate surface can have both accelerating and decelerating impacts on aging as a result of both adsorption and absorption within an asphalt-aggregate mixture system. Adsorption is an adhesion concept that theorizes that the molecular/atomic forces occur between the surfaces as the free electrons move onto the surfaces. Absorption is a similar idea to adsorption, but action is through fluid permeating into a solid. While the desired decelerative effect is the result 
of the absorption of asphalt polar components, accelerated aging can occur from the mineral components of aggregate that provide the potential for oxidative aging.

\subsection{LABORATORY SIMULATION OF ASPHALT CONCRETE AGING}

Many attempts have been made to simulate the aging of asphalt materials, at both the binder and mixture levels. Most of them targeted simulating and testing asphalt binder aging. Therefore, there is a wealth of data on asphalt binder aging, which sheds little light on asphalt-mixture aging.

Furthermore, few studies exist that have shown correlation between laboratory-aging data with field aging.

As indicated in the previous section, aging occurs in two regimes: short term and long term. Shortterm aging happens during the mixing, storage, transporting, and laydown processes at the construction site. Long-term aging, on the other hand, occurs during the service life of pavement as asphalt interacts with environmental and mechanical factors such as oxygen, heat, UV irradiation, moisture, and traffic action (Bell 1989; Fernández-Gómez et al. 2016; Peterson 2009; Hagos 2008; Wu et al. 2010; Baek et al. 2012; Anderson et al. 2012; Canestrari et al. 2013; Xiao et al. 2013; Hachiya 2003). This section presents the summary of long-term and short-term aging procedures attempted in research. Only literature from recent years (particularly after the development of the SHRP SuperPave program) has been reviewed and discussed chronologically.

Through a SHRP research study in the late 1980s, Bell first systematically compiled the research on the topic of asphalt aging and presented a critical review and summary of the research gap for the pavement community (Bell 1989). The study reported: "Compared to research on asphalt cement, there has been little research on the aging of asphalt mixtures, and, to date, there is no standard test. Pavement engineers understand the need to model the effects of short- and long-term aging of asphalt-aggregate mixtures in structural design procedures, and while some research has addressed this need, as of yet no standard procedure has emerged to address it."

With the results on asphalt-mixture aging, the AASHTO R30 procedure was developed to simulate short-term and long-term aging of asphalt mixture (Wu 2009; Bell et al. 1994; AASHTO R 30; Monismith et al. 1994; Kliewer et al. 1995). In the AASHTO R30 method, loose mixture with a thickness ranging from 0.98 to 1.97 in ( 25 to $50 \mathrm{~mm}$ ) is placed in a pan and aged for four hours at $275^{\circ} \mathrm{F}\left(135^{\circ} \mathrm{C}\right)$ in a forced-draft oven for short-term aging, with the mixture stirred every hour to maximize uniform aging. To simulate long-term aging, the short-term-aged mixture is then compacted. The pills are then aged for 120 hours at $185^{\circ} \mathrm{F}\left(85^{\circ} \mathrm{C}\right)$ to simulate long-term aging. Bell (1994) reported that the short-term procedure (four hours at $275^{\circ} \mathrm{F}\left[135^{\circ} \mathrm{C}\right]$ ) is adequate to simulate aging during mixing, transportation, and compaction. The study also reported that this aging procedure is enough to simulate aging up to two to three years for some climatic regions. In addition, the study cautiously mentioned that the long-term procedure may simulate up to 10 years of field aging, although the conclusion was made with a very limited number and highly skewed data collected from several specific climatic regions.

Houston et al. (2007) reported that temperature across the United States varies and the AASHTO R30 procedure employs only one temperature in aging. Therefore, the long-term aging procedure might 
not produce reasonable aging results for all regions and that the prediction of field aging for more than 10 years may be unrealistic. Furthermore, this standard is not based on a study that considers the effects of the air-void content of the mixture, a factor that intuitively would seem to have a significant effect on aging. The ambient and entrapped oxygen chemically reacts with asphalt and, ultimately, changes the chemical composition of binder and affects its engineering properties.

Through an NCHRP project on the asphalt-aggregate mixture analysis system, Von Quintus (1992) also attempted to simulate long-term aging using a forced-draft oven, in which compacted asphaltmixture specimens were first aged for two days at $140^{\circ} \mathrm{F}\left(60^{\circ} \mathrm{C}\right)$. Then, the specimens were rotated to maximize uniformity and kept in the oven for an additional five days at $224.6^{\circ} \mathrm{F}\left(107^{\circ} \mathrm{C}\right)$. Mechanical tests such as resilient modulus, indirect tensile test (IDT), strain at failure, and indirect tensile creep at $41^{\circ} \mathrm{F}\left(5^{\circ} \mathrm{C}\right)$ were used to examine the aging effect. No correlation was made with field-aging data. In terms of aging at a high temperature (e.g., at $224.6^{\circ} \mathrm{F}\left[107^{\circ} \mathrm{C}\right]$ ), later studies-e.g., Bell et al. (1994) and Reed (2010) - reported that the elevated temperature may damage the integrity of the specimen due to slumping from self-weight, particularly for high-void content (porous asphalt) and softer grade asphalt mixtures (Nicholls et al. 2007).

To accelerate oxidative aging, Bell et al. employed high-pressure aging equipment and reported that the utilization of high-pressure oxidative equipment in an aging procedure can damage the integrity of the specimen (reducing air-void content by slumping and producing changes in shape). Bell (1994) found a significant increase in resilient modulus (the performance parameter employed in the evaluation) due to aging. To avoid risks related to compromising the integrity of the specimens and those associated with high-pressure aging equipment, Bell (1994) developed another long-term aging protocol, called low-pressure oxidative aging, that employs passing oxygen through the sample at a temperature of $140^{\circ} \mathrm{F}\left(60^{\circ} \mathrm{C}\right)$ or $185^{\circ} \mathrm{F}\left(85^{\circ} \mathrm{C}\right)$ and a pressure $(100 \mathrm{psi})$ relatively lower than that of the high-pressure oxidation system.

It is to be noted that a similar issue was reported by previous studies that also utilized comparatively higher pressure (145 psi) for oxidative aging of compacted specimens (Kim et al. 1986; Von Quintus et al. 1989). Another study found that rapid increase in the resilient modulus occurred in the first few days of aging (Li and Nazarian 1995), suggesting that a long-term aging protocol can be developed, yet with an aging period of shorter duration.

To improve the pressure/oxidative-aging procedure, while reducing high pressure and temperature as discussed in the preceding paragraph, Khalid (2002) developed a system that involves encasing the sample in a sealed system that ensures passing of air through mixture samples without the use of a pressure vessel, as presented in Figure 2.3. Another motivation of this development was that the effect of aging at a high temperature does not correlate well with field-aging data. The study recommended an aging temperature of $140^{\circ} \mathrm{F}\left(60^{\circ} \mathrm{C}\right)$ and air pressure of 2 to $5 \mathrm{Lt} / \mathrm{min}$ (less than 10 psi). Note that this system took 21 days to simulate the semi-field-aging condition of about a year for a porous-grade asphalt mix that has a very high air-void content. The duration would be significantly longer for a dense-graded mix. 

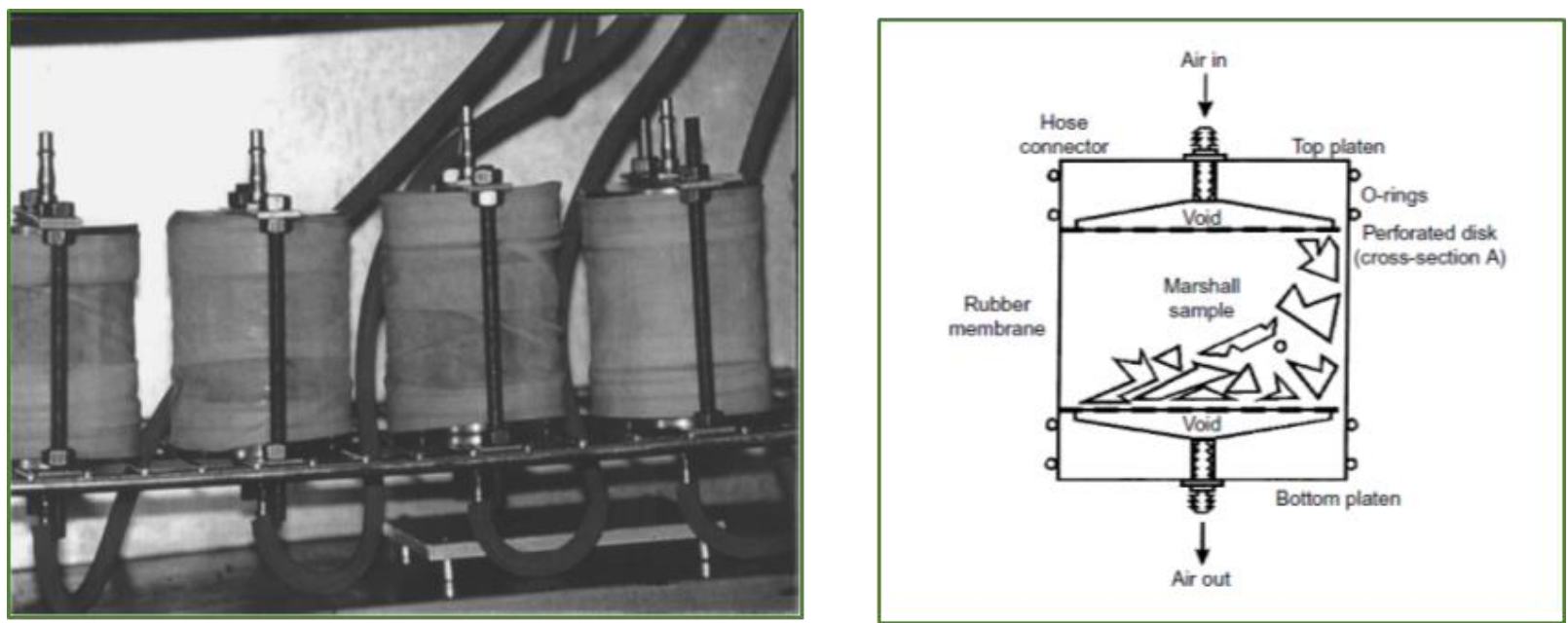

Figure 2.3. Oxidative-aging system without a high-pressure vessel. The air is passed into the compacted sample encased by a rubber membrane (Khalid 2002).

In 1995 at Nottingham University, UK, Scholz (1995) investigated the interaction effect of asphalt, aggregate, and moisture on asphalt pavement durability. He investigated the effect of different mineral aggregates on asphalt binder aging, which involved two hours of conditioning at mix-design temperature, and reported that aging was observed (viscosity increased, phase angle decreased). However, a meaningful difference was not observed in the aging effect between the aggregate types tested. A similar observation was also made in another British study, Wu (2009). However, opposite conclusions were also made in the literature. Monismith et al. (1994) stated: "It should be noted that the aging of asphalt-aggregate mixes is influenced by both the asphalt and aggregate. Aging of the asphalt alone, and subsequent testing, does not appear to be an adequate means of predicting mix performance because of the apparent mitigating effect aggregate has on aging. Moreover, the aging of certain asphalts is strongly mitigated by some aggregates but not by others. This appears to be related to the strength of the chemical bonding (adhesion) between the asphalt and aggregate." Therefore, it is the opinion of the authors that further research might be needed to address this issue; and aggregate specifications can be developed to account for the aging effect, if needed.

At the 2003 RILEM Conference in Zurich, Airey et al. (2003) presented an aging protocol that enables the researchers to examine oxidative, heat, and moisture effects. In this protocol, six regimes were tried to condition a 3.94 in $(100 \mathrm{~mm})$ (diameter) by 2.56 in $(65 \mathrm{~mm})$ (height), disk-shaped, compacted sample: (1) No-aging; (2) AASHTO R30; (3) AASHTO R30 plus partially saturated oven; (4) Lowpressure oxidation; (5) Pressure aging vessel (PAV); and (6) PAV plus partially saturated, pressureaged. Figure 2.4 shows the effect of the aging on compacted-mix performance measured by indirect tensile stiffness modulus. Each procedure tried increased mixture modulus. However, with moisture conditioning, the modulus value decreased, as expected, due to weakening of the adhesion between binder and aggregate in the presence of moisture. Ma et al. (2011) also reported that moisture can accelerate aging and further negatively affect performance of asphalt material. 


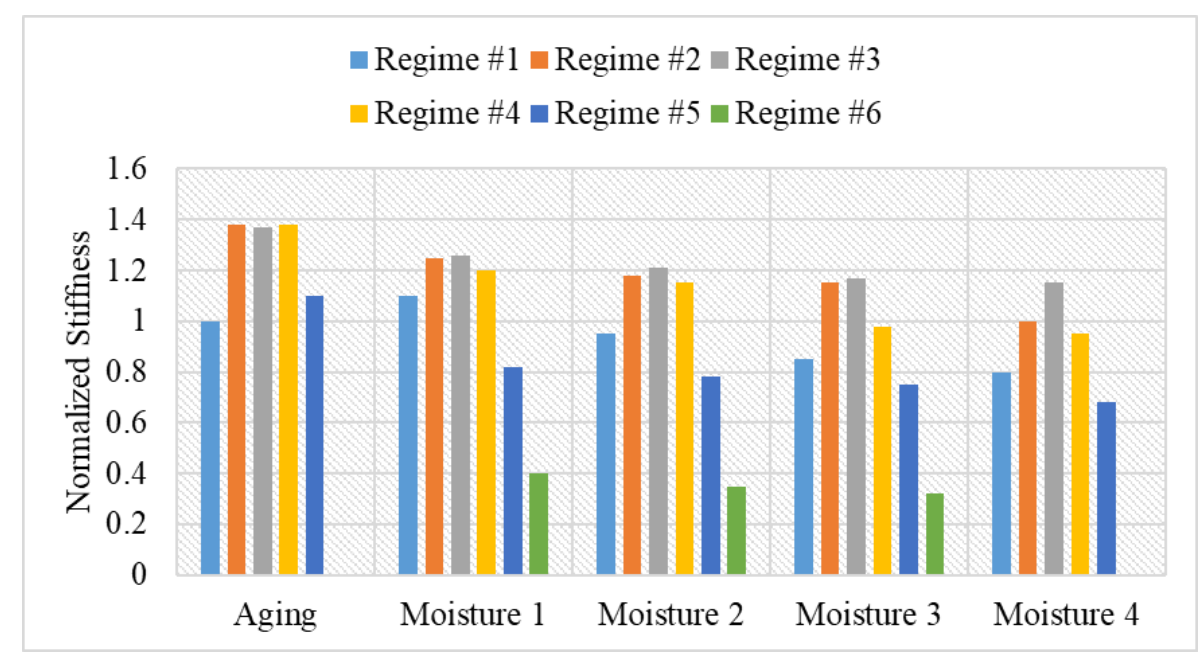

Figure 2.4. Effect of different aging regimes and moisture conditioning on mix stiffness (Airey et al. 2003).

Note that under aging Regime 5 (PAV), an attempt was also made to understand the interaction effect of aggregate and binder on aging. For this purpose, three 15-penetration-grade and one 50 penetration-grade asphalt binders, along with two types of coarse aggregates (dolomite and granite), were used. The study reported that dolomite aggregate outperformed the granite aggregate under all scenarios tested, as presented in Figure 2.5.

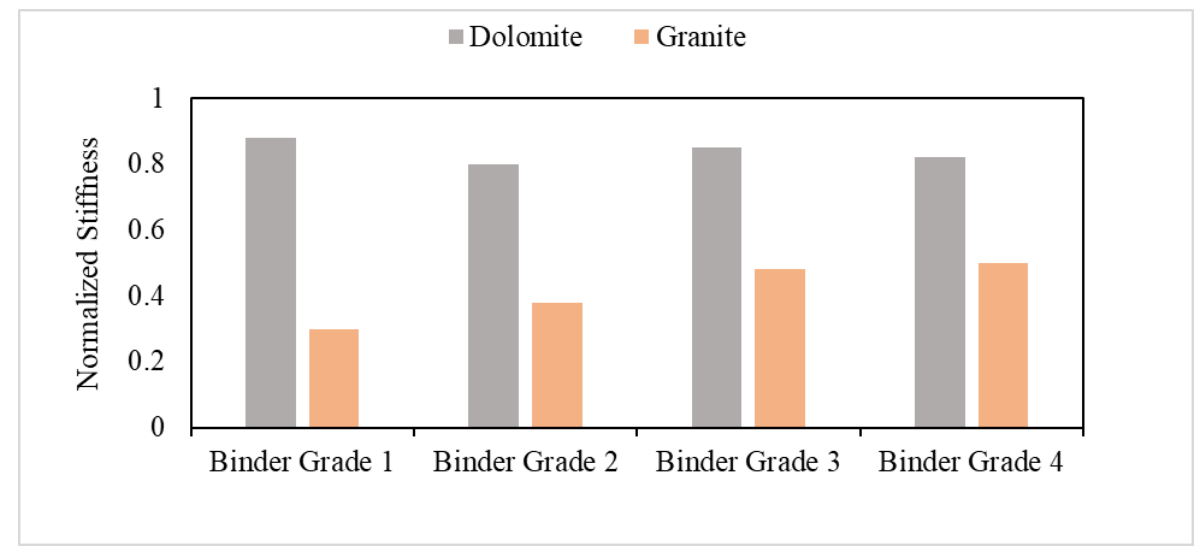

Figure 2.5. Effect of aggregate types on aging (Airey et al. 2003).

Collop et al. (2007) modified the PAV system so that the pressure vessel is partially filled with distilled water; and then water is temperature-conditioned at $185^{\circ} \mathrm{F}\left(85^{\circ} \mathrm{C}\right)$ for at least two hours (Figure 2.6). In a simultaneous action, compacted specimens to be tested are also pre-saturated and then placed in the preconditioned PAV system with water and pressured at $304.58 \mathrm{psi}(2.1 \mathrm{MPa})$ for 65 hours. The final conditioned specimens are tested for indirect tensile stiffness modulus to obtain the combined aging effect of oxygen, heat, and moisture on the compact asphalt concrete mixture. 

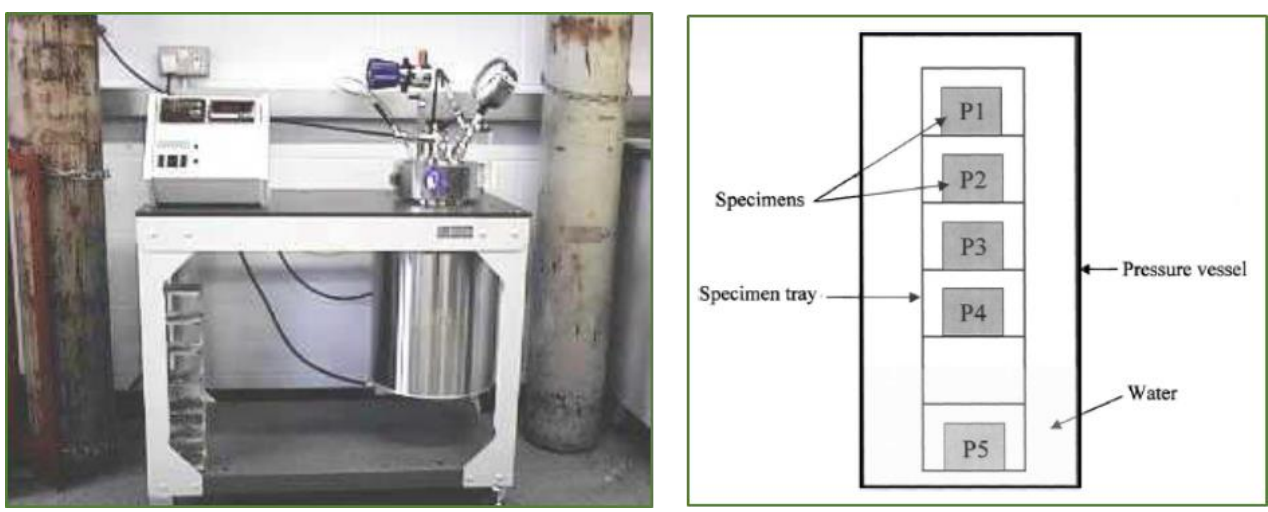

Figure 2.6. Asphalt-aging system combining oxidation, heating, and moisture (Collop et al. 2007).

In his doctoral work at Delft University in the Netherlands, Hagos (2008) conducted a comprehensive study to examine the aging effect on porous-grade asphalt mixture. This study also combined heating, oxidation, and moisture, along with a new variable-UV radiation. To simulate the different aging conditions involving these variables, the study employed a weather chamber (also called WeatherOmeter, equipment popular in the material science discipline), shown in Figure 2.7. Various aging protocols were tested. For the long-term condition, the study used $194^{\circ} \mathrm{F}\left(90^{\circ} \mathrm{C}\right)$ for a duration of 185 hours. For other experiment parameters (e.g., UV radiation, humidity, rain) and for detailed information, it is worthwhile to consult Hagos (2008). Some of the significant conclusions made from analysis of binder extracted from aged mix include that the lab aging method did not exhibit as severe of aging as that in the field.

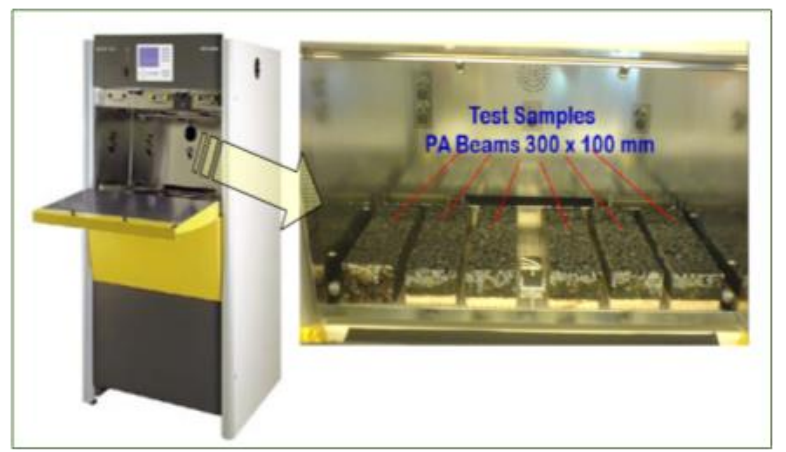
Key features:
- Rain and humidity simulator
- Broad range of UV radiation
- Sophisticated heating and cooling svstem

Figure 2.7. Weather-Ometer used to simulate aging of asphalt mixture or compacted sample (Hagos, 2008).

In another study at Delft University, Yehualaeset (2010) aged mortar, comprises of binder, filler, and fine aggregate less than 0.02 in, instead of binder or mixture and found significant correlation with field-aging data. The aging procedure involved heating mortar in an oven for two hours at $329^{\circ} \mathrm{F}$ $\left(165^{\circ} \mathrm{C}\right)$ and then placing the specimen in a PAV system for seven days at $194^{\circ} \mathrm{F}\left(90^{\circ} \mathrm{C}\right)$ with an air pressure of $304.58 \mathrm{psi}(2.1 \mathrm{MPa}$ ). Comparing chemical and rheological tests, the study concluded that this procedure can simulate 10 years of field aging. It also found satisfactory aging results with loose mix for porous-grade asphalt. For an aging-gradient effect, this study showed aging intensity declined as the depth of pavement increased. 
Based on a comparative study of different long-term aging protocols-Belgian method (conditioned at $140^{\circ} \mathrm{F}\left[60^{\circ} \mathrm{C}\right]$ ), RILEM method (conditioned at $185^{\circ} \mathrm{F}\left[85^{\circ} \mathrm{C}\right]$ ), PAV, and UV aging-Mollenhauer et al. (2012) reported that the aging of a loose mixture in a forced-draft oven is a more feasible option than a PAV system. Following the RILEM protocol (similar to AASHTO R30), the study found that aging of asphalt after nine days at $185^{\circ} \mathrm{F}\left(85^{\circ} \mathrm{C}\right)$ is more severe than aging for 14 days at $140^{\circ} \mathrm{F}\left(60^{\circ} \mathrm{C}\right)$, which is in line with the aging-kinetics discussion presented in the introductory section of this chapter. Contrary to many studies, this study, however, did not observe a UV radiation effect (i.e., photooxidation) in aging. The study also tried aging a loose mixture in the PAV and found that aging at $194^{\circ} \mathrm{F}\left(90^{\circ} \mathrm{C}\right)$ for 20 hours can produce results comparable to the RILEM protocol or AASHTO R30. Note that this option is unreasonable from a practical viewpoint, as the PAV aging system does not have enough capacity to allow preparing test specimens for regular mechanical testing.

Baek et al. (2012) conducted an experimental study on a control mix with varying aging durations of four hours at $135^{\circ} \mathrm{C}$ and two, four, and eight days at $185^{\circ} \mathrm{F}\left(85^{\circ} \mathrm{C}\right)$ for loose-mix samples and examined the performance for dynamic modulus. The study reported that the dynamic modulus value increased with aging duration and found that under all dynamic modulus testing temperatures $\left(14^{\circ} \mathrm{F}\left[-10^{\circ} \mathrm{C}\right]\right.$, $\left.41^{\circ} \mathrm{F}\left[5^{\circ} \mathrm{C}\right], 68^{\circ} \mathrm{F}\left[20^{\circ} \mathrm{C}\right], 104^{\circ} \mathrm{F}\left[40^{\circ} \mathrm{C}\right], 129.2^{\circ} \mathrm{F}\left[54^{\circ} \mathrm{C}\right]\right)$, the protocols resulted in significantly different modulus, except between the conventional short-term-aged specimens (aging at $275^{\circ} \mathrm{F}\left[135^{\circ} \mathrm{C}\right]$ for four hours) and two-day-aged specimens for a few instances. Also, the different aging protocols clearly differentiated the damage characteristic curves of the aged mixtures. Xiao et al. (2013) conducted an investigation examining thermal- and UV-aging effects on HMA and WMA with an airvoid content of 7, 4, and 2\%-mimicking short-, medium-, and long-term air-void content in realworld pavement. Various performance measures such as rutting, flow, indirect tensile strength, and elastic and fracture energy were considered in the aging-effect comparison. For aging compacted specimens, the study utilized the AASHTO protocol; and for UV aging, the study developed a custombuilt oven with UV lamps, as can be seen in Figure 2.8.

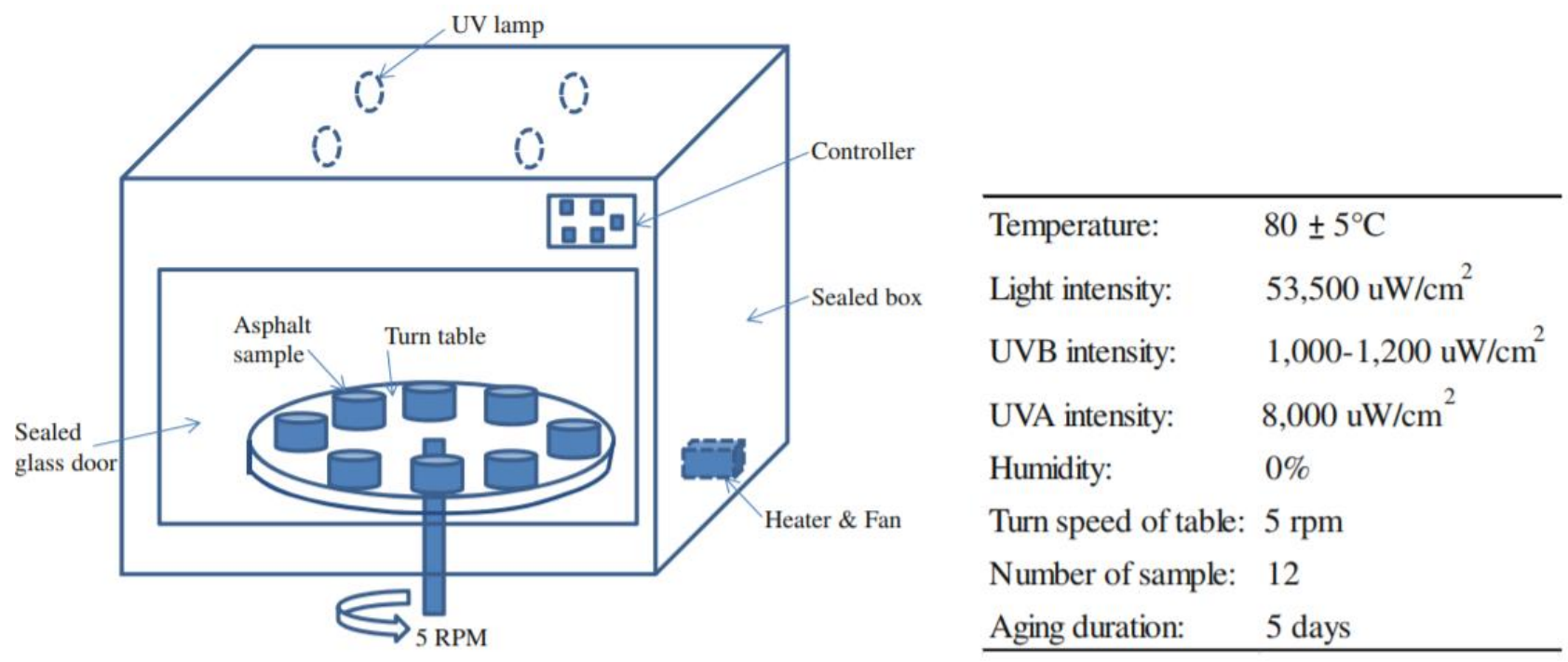

Figure 2.8. Schematic of UV-aging system utilized in the study and key features of the system on the right side (Xiao et al. 2013). 
Figure 2.9 shows that the mixtures with the most air voids store the least fracture energy regardless of aging type, binder type, and aggregate source. The unaged mixture exhibits just a marginally higher fracture energy than the thermally aged mixture, followed by the UV-aged mixture. Figure 2.9 also shows that the UV-aging procedure is generally more prone to reducing the fracture resistance of an asphalt mixture than is the standard thermal-aging procedure. In another study, based on data collected from retrieved binder from the mix with two aging treatments (UV and thermal), Mouillet et al. (2014) found that UV aging is dominant over thermal aging in producing some chemical carbonyl functional groups $(\mathrm{C}=\mathrm{O})$, which ultimately changes asphalt chemical and rheological properties. Zeng et al. (2015) found that, for a given UV-aging system, ambient temperature under $122^{\circ} \mathrm{F}\left(50^{\circ} \mathrm{C}\right)$ has less influence on the rheological performance of the asphalt binder.

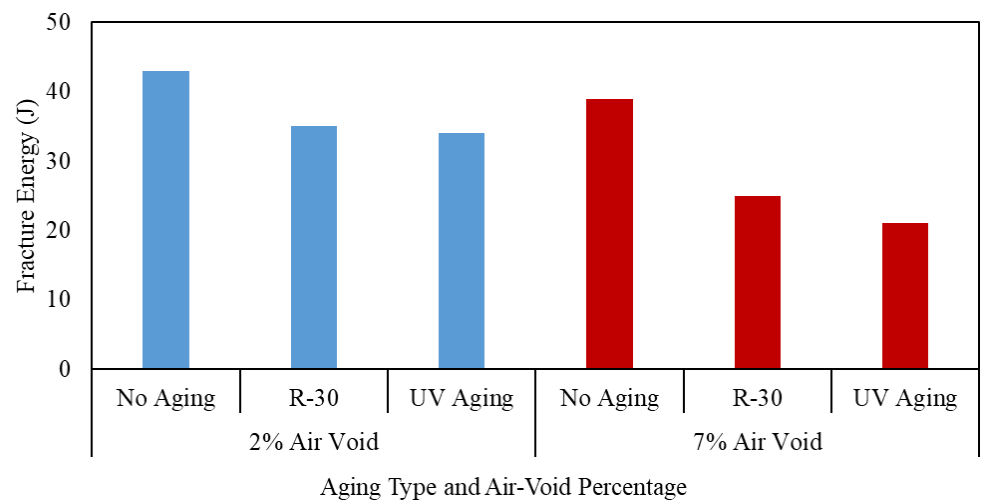

Figure 2.9. The fracture-energy value for non-aged, regularly aged (AASHTO R30), and UV-aged samples (data from Xiao et al., 2013).

Like all other laboratory tests, lab-aging procedures also have limitations to appropriately simulate field conditions. Therefore, a reliable field-performance prediction cannot be produced from using these procedures. To mitigate this problem, NCHRP has recently taken some steps to correlate the results between lab aging, field aging, and other environmental data. For instance, through NCHRP Project 9-52, Texas A\&M University has conducted a comprehensive study. In this research, a wide variety of mixes, aging protocols (short-term aging plus five-day vs. two weeks at $185^{\circ} \mathrm{F}\left[85^{\circ} \mathrm{C}\right]$ ), lab, plant, and field environmental factors were considered in the experimental matrix to examine how these factors affect aging at the binder and mixture levels. The mix-level performance was examined by conventional mechanical tests data such as resilient and complex modulus and rut depth - while binder aging was examined by rheological and chemical properties obtained by rheological test equipment and FTIR, respectively (Yin et al. 2017; Newcomb et al. 2015).

The study suggested that consideration of cumulative degree days, or CDD (a concept utilized in climatic science discipline, estimated as a total sum of time and temperature of each day for the entire year), as a variable in aging prediction yields better results than treating time and temperature separately. The study found that the aging effect on an asphalt sample with a CDD value of 17,500 was equivalent to 12 months in service in warmer climates and 23 months in service in colder climates. Further, no statistically significant difference was noticed in terms of the aging effect between the mixes manufactured in plant, made in the lab, or sampled at the construction site. 
Surprisingly, the study also did not find a noticeable difference between the aging effects for five days and two weeks of aging conditioning. Based on the limited data, the research also developed the following model (Equation 1) that can be used to predict the modulus value for the field-aged sample.

$M_{R}$ Ratio $=1+2.73 * 10^{5} * \exp ^{\left\{-\left(\frac{1.24 * 10^{21}}{C D D}\right)\right\}}$

where $M_{R}$ Ratio stands for resilient modulus ratio between long-term-aged (either five days or two weeks, both at $185^{\circ} \mathrm{F}\left[85^{\circ} \mathrm{C}\right]$ ) and short-term-aged (two hours at $275^{\circ} \mathrm{F}$ [ $\left.135^{\circ} \mathrm{C}\right]$ ) specimen.

Similarly, in a recently published study by Chen et al. (2018) at the National Center for Asphalt Technology (NCAT), researchers tried to select a laboratory loose-mix aging protocol for NCAT's topdown cracking experiment. Existing pavements show that top-down cracking typically initiated after 70,000 CDD, which was selected as the target of the aging process. Then, several predesigned loosemix aging protocols were evaluated by laboratory experiments. Based on rheological and oxidation results obtained from the dynamic shear rheometer (DSR), bending beam rheometer (BBR), and (Fourier Transform Infrared Spectroscopy) FTIR tests; the $24-\mathrm{hr}, 275^{\circ} \mathrm{F}\left(135^{\circ} \mathrm{C}\right)$ protocol yielded the most significant level of asphalt aging, followed by the $12-\mathrm{hr}, 275^{\circ} \mathrm{F}\left(135^{\circ} \mathrm{C}\right)$ protocol, five-day, $203^{\circ} \mathrm{F}$ $\left(95^{\circ} \mathrm{C}\right)$, protocol, and six-hour, $275^{\circ} \mathrm{F}\left(135^{\circ} \mathrm{C}\right)$ protocol, respectively. These aging protocols represent greater than $235,000 \mathrm{CDD}, 80,000$ to $157,000 \mathrm{CDD}, 48,000$ to $80,000 \mathrm{CDD}$, and approximately 48,000 $\mathrm{CDD}$, respectively. Among these four proposed protocols, 5 -day $/ 95^{\circ} \mathrm{C}$ protocol was the most representative one. However, due to practical implementation challenges, researchers tried to find an alternative protocol with shorter aging duration in this study. They claimed that no significant difference in the oxidation-hardening relationship of asphalt binders were observed for mixes aged at $203^{\circ} \mathrm{F}\left(95^{\circ} \mathrm{C}\right)$ versus $275^{\circ} \mathrm{F}\left(135^{\circ} \mathrm{C}\right)$. In addition, DSR and FTIR results indicated that loose mix aging for eight hours at $275^{\circ} \mathrm{F}\left(135^{\circ} \mathrm{C}\right)$ and five days at $203^{\circ} \mathrm{F}\left(95^{\circ} \mathrm{C}\right)$ were likely to achieve an equivalent aging level. Finally, $8-\mathrm{hr} / 135^{\circ} \mathrm{C}$ loose mix aging was suggested. One important point that needs to be emphasized here is that the $8-\mathrm{hr} / 135^{\circ} \mathrm{C}$ aging protocol was proposed by using nonlinear regression based on $24-\mathrm{hr} / 135^{\circ} \mathrm{C}, 12-\mathrm{hr} / 135^{\circ} \mathrm{C}$, and $6-\mathrm{hr} / 135^{\circ} \mathrm{C}$ data. However, the 8 -hr $/ 135^{\circ} \mathrm{C}$ aging could not be validated and achieve idealized CDD.

In a recently completed five-year NCHRP project 09-54, Kim et al. (2018) developed a procedure calibrated and validated with field data to simulate long-term aging of asphalt mixtures for performance testing and prediction. Researchers classified the asphalt mixture laboratory aging procedures that have been tried based on three main concerns: 1) the state of the material during aging (compacted specimen vs. loose mix); 2) the pressure level (oven aging vs. pressurized aging); and 3 ) the aging temperature.

Regarding compacted specimen and loose mix aging, Table 2.1 was presented to illustrate the pros and cons of these two methods. 
Table 2.1. Comparison between Loose Mix and Compacted Specimens in the Aging Procedure (Kim et al. 2018)

\begin{tabular}{|l|l|l|}
\hline \multirow{5}{*}{ Loose Mix } & Pros & $\begin{array}{l}\text { a) Homogenous aging in the mixture } \\
\text { b) Higher oxidation rate than compacted mix } \\
\text { c) Maintaining specimen integrity a non-issue }\end{array}$ \\
\cline { 2 - 3 } Compacted & Cons & $\begin{array}{l}\text { a) Difficulties associated with compaction of aged loose mix, which limits its use for } \\
\text { producing specimens for performance testing } \\
\text { b) Limited amount of materials can be aged in standard PAV chamber }\end{array}$ \\
\cline { 2 - 3 } & Pros & $\begin{array}{l}\text { a) Can produce aged sample for performance tests if slumping is minimized through } \\
\text { use of wire mesh }\end{array}$ \\
\cline { 2 - 3 } & Cons & $\begin{array}{l}\text { a) Slower oxidation rate than loose mix } \\
\text { b) Integrity of the specimens is compromised at high temperatures and pressures due } \\
\text { to slump, cracking upon pressure release, and differences in the coefficient of thermal } \\
\text { expansion between binder and aggregate } \\
\text { c) Oxidation gradients exist radially and throughout height of the specimen }\end{array}$ \\
\hline
\end{tabular}

Similarly, Table 2.2 listed pros and cons within two aging equipment: traditional oven and PAV.

Table 2.2. Comparison between Oven and PAV Aging Methods (Kim et al. 2018)

\begin{tabular}{|l|l|l|}
\hline \multirow{5}{*}{ Oven Aging } & Pros & $\begin{array}{l}\text { a) Available and easy to perform and control } \\
\text { b) Large amount of material can be aged }\end{array}$ \\
\cline { 2 - 3 } Pressure Aging & Cons & $\begin{array}{l}\text { a) High variability among ovens, especially in terms of air drafting } \\
\text { b) More time needed to age materials in the oven than in the PAV } \\
\text { c) Maintaining compacted specimen integrity is required, especially at high } \\
\text { temperatures }\end{array}$ \\
\cline { 2 - 3 } & Pros & $\begin{array}{l}\text { a) Pressure can expedite the aging process } \\
\text { b) More reliable than oven aging due to less equipment variability between laboratories }\end{array}$ \\
\hline & $\begin{array}{l}\text { a) Due to limited capacity of the vessel, less material can be aged in each aging cycle } \\
\text { unless new device is developed } \\
\text { b) Integrity of compacted samples during and after testing is a major concern }\end{array}$ \\
\hline
\end{tabular}

Regarding aging temperature, the researchers suggested using less than $212^{\circ} \mathrm{F}\left(100^{\circ} \mathrm{C}\right)$ for two reasons. Firstly, the disruption of polar molecular associations and sulfoxide decomposition become critical at temperatures that exceed $212^{\circ} \mathrm{F}\left(100^{\circ} \mathrm{C}\right)$, which are inaccessible at lower temperatures. Secondly, aging temperature over $212^{\circ} \mathrm{F}\left(100^{\circ} \mathrm{C}\right)$ leads to asphalt mastic drain-down because of the low viscosity of asphalt binder at elevated temperatures.

Prior to the experimental study, two important sub-investigations were conducted to support that. The first one is a sensitivity study, which can help researchers to understand the significance of observed differences in asphalt binders AIPs (Aging Index Properties) in terms of asphalt mixture performance. The second is to select proper and efficient chemical and rheological AIPs. Carbonyl + sulfoxide peak $\left(\mathrm{C}+\mathrm{S}\right.$ peak) and $\mathrm{G}^{*}$ at $147.2^{\circ} \mathrm{F}\left(64^{\circ} \mathrm{C}\right)$ and $10 \mathrm{rad} / \mathrm{s}$ were selected as the chemical and rheological AIP, respectively (Rad et al. 2018). 
A comprehensive experimental program was then conducted to select the most appropriate longterm aging method and the abovementioned three factors were evaluated, respectively. The integrity of the specimens following aging, the rate of oxidation quantified using the AIPs of the extracted binder, versatility, and the cost of the various procedures were compared in order to select the most promising aging procedure. The key findings were listed as below.

- Loose mix aging was found to have further oxidation than compacted specimen aging under the same conditions.

- Long-term loose mix aging was determined to have no significant effect on compaction.

- The current AASHTO R30 aging procedure can lead to an oxidation gradient from the periphery to the center of the specimen, which violates the fundamental integrity requirement of a performance test.

- Standard PAV cannot generate enough aged loose materials for performance testing.

- Performance test results showed that compacted specimens were damaged during the PAV aging process.

- The kinetics and mechanisms of oxidation were believed to change with an increase of aging temperature from $203^{\circ} \mathrm{F}\left(95^{\circ} \mathrm{C}\right)$ to $275^{\circ} \mathrm{F}\left(135^{\circ} \mathrm{C}\right)$ since the relationship between binder rheology and chemistry changed significantly.

- Aging temperature did not affect the relationship between binder rheology and chemistry if it is at or under $203^{\circ} \mathrm{F}\left(95^{\circ} \mathrm{C}\right)$, which implies that the oxidation mechanism did not change.

- The rate of oxidation increased with an increase in temperature.

Based on the above claims, researchers recommended using loose mix aging at $203^{\circ} \mathrm{F}\left(95^{\circ} \mathrm{C}\right)$ for longterm aging.

Another important component of an aging protocol is the laboratory aging durations. In this project, researchers developed nation-wide aging duration maps to match the AIPs of field cores at varying depths. Since loose-mixture oven aging leads to a kinetics-controlled reaction, the kinetics model can be applied to loose-mix aging without considering diffusion. A kinetic model was developed and validated in this project and can be calibrated using AIP measurements obtained from isothermal aging at a single temperature. Finally, CAI (climate aging index), developed by simplifying the kinetics model, was used to get laboratory aging durations to match a given field condition using hourly pavement temperature histories at depths of $0.24 \mathrm{in}(6 \mathrm{~mm}), 0.79 \mathrm{in}(20 \mathrm{~mm})$, and $1.97 \mathrm{in}(50 \mathrm{~mm})$. All maps are available in their final research report (Kim et al. 2018).

\subsection{SUMMARY}

The mechanisms of aging and effect of aging on the characteristics and field performance of AC mixtures were documented from studies available in the literature. Short- and long-term aging was simulated in the lab environment primarily to explore the following aspects. Aging resistance of mixes were characterized for use in mechanistic simulations. The aging resistance of different mixtures (HMA, WMA) was characterized. The effect of neat and modified binders was explored. Different 
aging factors including heating, oxidation, UV, and moisture were determined. Table 2.3 provides a brief summary of the main factors employed in some previous studies that addressed long-term aging.

In addition, the following conclusions can be made from previous studies:

- Age-hardening occurs only in the viscoelastic component of an asphalt-aggregate mixture system, i.e., the asphalt binder. During the age-hardening process, properties of the chemical constituents of asphalt change as asphalt reacts with atmospheric oxygen and the process is influenced by oxygen-diffusion flux and various thermal forces (e.g., heat, UV radiation) of the natural environment. The changes in chemical constituents and in molecular groups are reflected in chemical and rheological performance properties of the asphalt binder. In particular, as asphalt ages, it loses its adhesive property, becomes stiffer and embrittled, and exhibits reduced fatigue endurance.

- Asphalt-aggregate mix design parameters such as air-void ratio and aggregate petrography (porosity, chemical compositions, morphology) can either accelerate or decelerate aging. Therefore, in asphalt-aging research design or to predict mix performance, full consideration should be given to all mix-related parameters to account for how these parameters affect aging and to obtain their quantitative information.

- Two regimes are believed to exist in the aging of asphalt mixtures. The first one happens during the mixing, transportation, and construction stages; while the second one occurs during its service life. The rate of aging is found out to be much higher in the first regime.

- Various environmental and design factors can contribute to the age-hardening of asphalt. These factors include atmospheric oxygen, dissolved oxygen in moisture, moisture, ambient and pavement-layer temperature, UV irradiation, aggregate petrography, mix-design parameters, and binder chemical compositions. However, few studies have attempted to quantify the effect of these factors sufficiently.

- To simulate the short- and long-term aging of asphalt mixture, AASHTO R30 is followed throughout the United States. For long-term aging, loose mixture is first short-term aged with a spread thickness of 25 to $50 \mathrm{~mm}$ in a pan at $135^{\circ} \mathrm{C}$ for four hours in a forced-draft oven, with the mixture stirred every hour to maximize uniformity in aging. The mixture is then compacted and placed in the oven at $85^{\circ} \mathrm{C}$ for five days. It is arguably believed that AASHTO R30 is safe to predict long-term aging up to 10 years.

- Other procedures than AASHTO R30 are presented Table 2.3. However, there was a need to develop a procedure that is efficient and practical and maintains the integrity of the material.

- Studies reported that when a loose mix is aged for a long period, the coated binder on each particle is extremely oxidized, whereas this is not the case when a compacted specimen is aged. In addition, $\mathrm{G}_{\mathrm{mm}}$ increased in the long-term aging process. However, an aging gradient is observed in the compacted specimen, unlike the loose mix (which can be stirred). Note that an aging gradient is also observed in field-aged cores. 
- Above $100^{\circ} \mathrm{C}$, the binder's chemical and rheological properties are significantly affected. Hence, $95^{\circ} \mathrm{C}$ is the highest aging temperature that can be safely utilized. Four to eight days is a common duration for examining long-term aging characteristics of asphalt mixes.

- Modulus value is a very common performance metric utilized for gauging the aging effect. Other mechanistic performance metrics (e.g., permanent deformation, fracture energy) have also been used.

- A standard lab aging protocol should be developed identifying the sample size, oven type, aging temperature and duration, etc.

Table 2.3. Summary of Long-Term Aging Protocols Used/Recommended in Previous Studies

\begin{tabular}{|c|c|c|c|c|c|}
\hline Time & Test method & $\begin{array}{l}\text { Temp } \\
\left({ }^{\circ} \mathrm{C}\right)\end{array}$ & Duration & $\begin{array}{l}\text { Sample } \\
\text { state }\end{array}$ & $\begin{array}{l}\text { New observation(s) or previous issue(s) } \\
\text { addressed }\end{array}$ \\
\hline $\begin{array}{l}\text { Since } \\
\text { adoption }\end{array}$ & AASHTO R30 & 85 & 5 days & Compacted & \\
\hline 2018 & $\begin{array}{l}\text { Rahbar- } \\
\text { Rastegar et } \\
\text { al. (2018) }\end{array}$ & $\begin{array}{l}85,95 \\
135\end{array}$ & $\begin{array}{l}1,5,12 \\
\operatorname{day}(s)\end{array}$ & $\begin{array}{l}\text { Compacted } \\
\text { Loose }\end{array}$ & \\
\hline 2018 & $\begin{array}{l}\text { Chen et al. } \\
\text { (2018) }\end{array}$ & 135 & 8 hours & Loose & Use CDD concept to generate aging protocol. \\
\hline 2018 & $\begin{array}{l}\text { Kim et al. } \\
(2018)\end{array}$ & 95 & $\begin{array}{l}\text { Depends on } \\
\text { time, } \\
\text { depth, } \\
\text { location }\end{array}$ & Loose & $\begin{array}{l}\text { (1) Nation-wide aging duration maps were } \\
\text { generated; } \\
\text { (2) Sensitivity study on the significance of } \\
\text { observed differences in asphalt binders AIPs in } \\
\text { terms of asphalt mixture performance; } \\
\text { (3) C+S peak and G* at } 64^{\circ} \mathrm{C} \text { and } 10 \mathrm{rad} / \mathrm{s} \text { can } \\
\text { efficiently represent chemical \& rheological AIP, } \\
\text { respectively; } \\
\text { (4) Modified ME design with pavement aging } \\
\text { model. }\end{array}$ \\
\hline 2017 & $\begin{array}{l}\text { Elwardany } \\
\text { et al. (2017) }\end{array}$ & 70-95 & $1-35$ days & $\begin{array}{l}\text { Compacted } \\
\text { Loose }\end{array}$ & $\begin{array}{l}\text { Small specimens can be used to minimize } \\
\text { slumping issue for complex modulus test. }\end{array}$ \\
\hline 2015 & $\begin{array}{l}\text { Newcomb } \\
\text { et al. (2015) }\end{array}$ & 85 & $\begin{array}{l}5 \text { days or } 2 \\
\text { weeks }\end{array}$ & Compacted & $\begin{array}{l}\text { Cumulative degree days (CDD) had significant } \\
\text { effect on aging. }\end{array}$ \\
\hline 2014 & $\begin{array}{l}\text { Tarbox et al. } \\
\text { (2012) }\end{array}$ & 85 & $2,4,8$ days & Compacted & \\
\hline
\end{tabular}




\begin{tabular}{|c|c|c|c|c|c|}
\hline Time & Test method & $\begin{array}{l}\text { Temp } \\
\left({ }^{\circ} \mathrm{C}\right)\end{array}$ & Duration & $\begin{array}{l}\text { Sample } \\
\text { state }\end{array}$ & $\begin{array}{l}\text { New observation(s) or previous issue(s) } \\
\text { addressed }\end{array}$ \\
\hline 2012 & $\begin{array}{l}\text { Baek et al. } \\
\text { (2012) }\end{array}$ & 85 & $2,4,8$ days & Compacted & \\
\hline 2013 & $\begin{array}{l}\text { Azari et al. } \\
\text { (2013) }\end{array}$ & 85 & $2,5,9$ days & Compacted & $\begin{array}{l}\text { Aging resulted in increase in permanent } \\
\text { deformation resistance. }\end{array}$ \\
\hline 2011 & $\begin{array}{l}\text { Morian et al. } \\
\text { (2011) }\end{array}$ & 60 & $\begin{array}{l}3,6,9 \\
\text { months }\end{array}$ & Compacted & $\begin{array}{l}\text { Binder source had a significant effect. Aggregate } \\
\text { source had no effect. }\end{array}$ \\
\hline 2010 & Reed (2010) & 85 & 5,14 days & $\begin{array}{l}\text { Compacted } \\
\text { Loose }\end{array}$ & $\begin{array}{l}\text { Loose mix worked like an extremely oxidized RAP. } \\
\text { Compaction job was tough. Modulus value } \\
\text { decreased for loose-mix compacted samples. } \\
\text { Compacted specimen's integrity degraded, as } \\
\text { measured by before-and-after AV ratio. }\end{array}$ \\
\hline 2008 & Hagos (2008) & 95 & 185 hours & Compacted & $\begin{array}{l}\text { Utilized a Weather-Ometer to simulate UV } \\
\text { radiation, rain, humidity }\end{array}$ \\
\hline 2007 & $\begin{array}{l}\text { Collop et al. } \\
(2007)\end{array}$ & 85 & 65 hours & Compacted & $\begin{array}{l}\text { Modified PAV system to incorporate water bath } \\
\text { for simulating heat, oxidation, moisture aging }\end{array}$ \\
\hline 2005 & $\begin{array}{l}\text { Houston et } \\
\text { al. (2005) }\end{array}$ & $\begin{array}{l}80,85 \\
90\end{array}$ & 5 days & Compacted & $\begin{array}{l}\text { Air void had a significant effect in field-aging. } \\
\text { Aging at } 85^{\circ} \mathrm{C} \text { for } 5 \text { days, compared with } 7-10 \\
\text { years of field-aging data; lab specimen aged more } \\
\text { than field-aged sample when air voids were less } \\
\text { than } 8 \% .\end{array}$ \\
\hline 2003 & $\begin{array}{l}\text { Airey et al. } \\
(2003)\end{array}$ & 85 & 5 days & Compacted & $\begin{array}{l}\text { Involved testing partially saturated specimens in } \\
\text { PAV system }\end{array}$ \\
\hline 2000 & Khalid (2000) & 60 & 1-21 days & Compacted & Air flow 3 liter/min \\
\hline 1995 & $\begin{array}{l}\text { Li et al. } \\
(1995)\end{array}$ & 85 & 5 days & Compacted & $\begin{array}{l}\text { Fast increment in modulus value occurs in first } \\
\text { few days due to aging. }\end{array}$ \\
\hline 1995 & Scholz (1995) & 85 & 4 days & Compacted & $\begin{array}{l}\text { Aging resulted in effect in U.K. that was equivalent } \\
\text { to field-aging of } 15 \text { years in U.S. }\end{array}$ \\
\hline 1994 & $\begin{array}{l}\text { Bell et al. } \\
\text { (1994) }\end{array}$ & 60,85 & 5 days & Compacted & Air pressure $100+$ psi, specimens damaged \\
\hline
\end{tabular}




\section{CHAPTER 3: MATERIAL SAMPLING, INVENTORY, AND EXPERIMENTAL METHODS}

\subsection{MATERIAL SAMPLING \& INVENTORY}

\subsubsection{Introduction}

Asphalt concrete surface mixtures used in this study covered a wide spectrum of mixture types that were commonly used at the time of the study. Mixes varied based on production technique, $\mathrm{N}$ design, mixture type (dense-graded and SMA), amount of recycled content, type of binder, and binder content. The following AC mixtures were collected and prepared for testing as part of the experimental program:

- Twelve plant-produced and lab-compacted (PPLC) mixtures sampled from various plants in Illinois.

- Seven lab-produced and lab-compacted (LPLC) mixtures designed as part of the study.

- Field core samples corresponding to the plant mixtures were also collected at various intervals of pavement life (at placement, after six months, and after 12 months).

In addition, a total of 17 different binders used in the PPLC and LPLC specimens were collected and verified for SuperPave performance grading.

\subsubsection{Material Sampling Procedure}

This part describes in detail the sampling procedure implemented for collecting materials for the R27175 project. The materials collected for this project included binder, mineral filler, aggregates, RAP, RAS, and plant-produced mixtures.

\subsubsection{Asphalt Binder}

Binders were sampled by AC producers as plant personnel deemed appropriate, for safety considerations. For each source, at least 15 gallons (56.78 liters) of binder were collected in galvanized steel cans of one-gallon capacity.

\subsubsection{Aggregate, RAP \& RAS}

Aggregates were collected as per IDOT procedure (Aggregate Technician Course Manual). Virgin aggregates, RAP, and RAS were collected for mixes used in the project.

\subsubsection{Mineral Filler}

Two five-gallon buckets of fine materials were collected for each mix investigated. No specific sampling procedure was implemented. Respirators were used to avoid inhaling particles while filling buckets with mineral filler. 


\subsubsection{Plant Mixtures}

Different mixture sampling procedures were investigated in the ICT project R27-8 (Elseifi 2007). It was recommended that the behind the paver sampling method is the optimal procedure. However, samples were collected from the plant due to large quantities of AC mixture needed in this study. The sampling procedure used to collect plant samples is summarized as follows:

1. Filled the front wheel loader with 3.3-4.4 US tons (3-4 tons) of asphalt mixture. This might be completed in one or more drops, depending upon the plant and loader that are used (Figure 3.1).
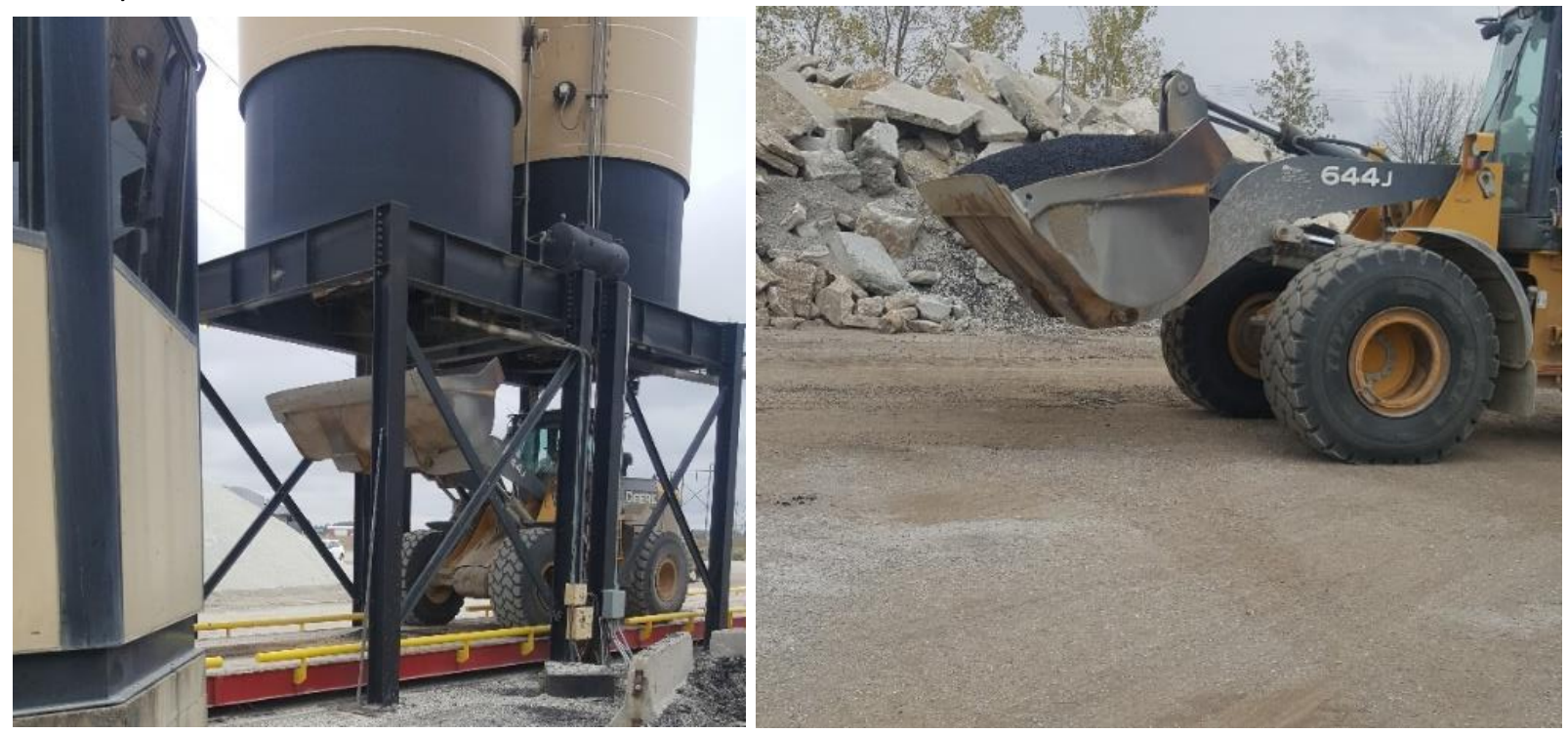

Figure 3.1. Asphalt sample collection from the plant in a loader.

2. Dumped the material into a pile on the ground. Then, mixed the pile by scooping material and dumping it back on the top of the pile several times at right angles to previous scoops (Figure 3.2). This was done three times. Special attention must be given to not dig into the underlying material, which would contaminate the pile.

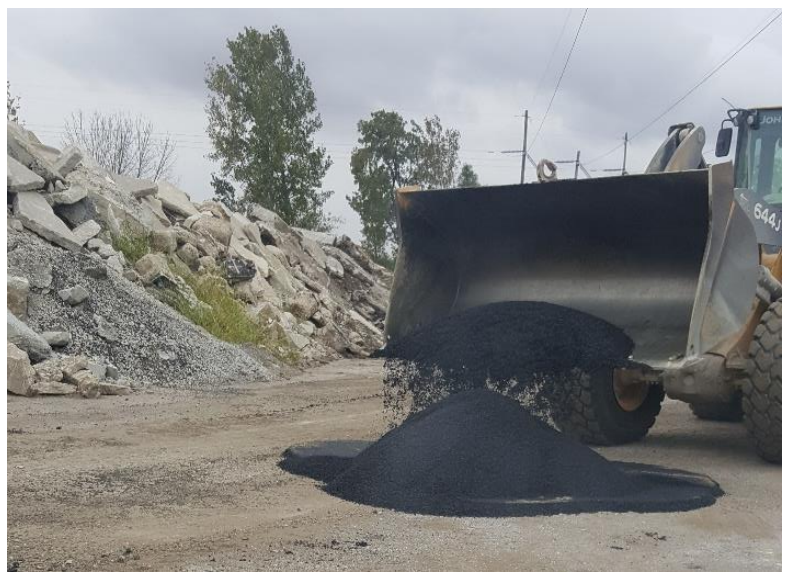

Figure 3.2. Placement of the sample on a flat surface. 
3. Back dragged the pile to provide a flat sample pad (Figure 3.3).
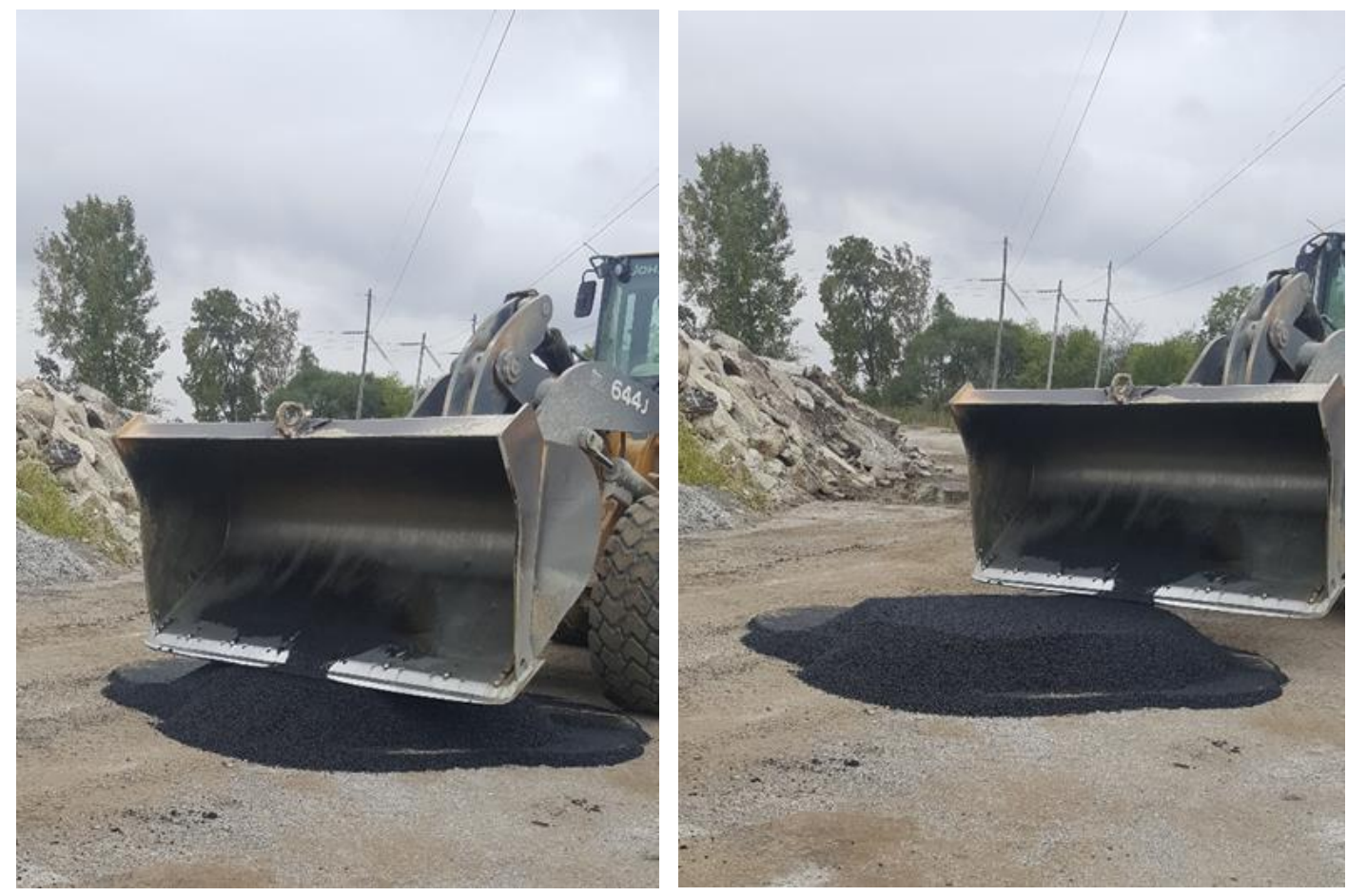

Figure 3.3. Spread the sample to flatten conical heap.

4. Sampled material from multiple locations on the pad for each bag (Figure 3.4).
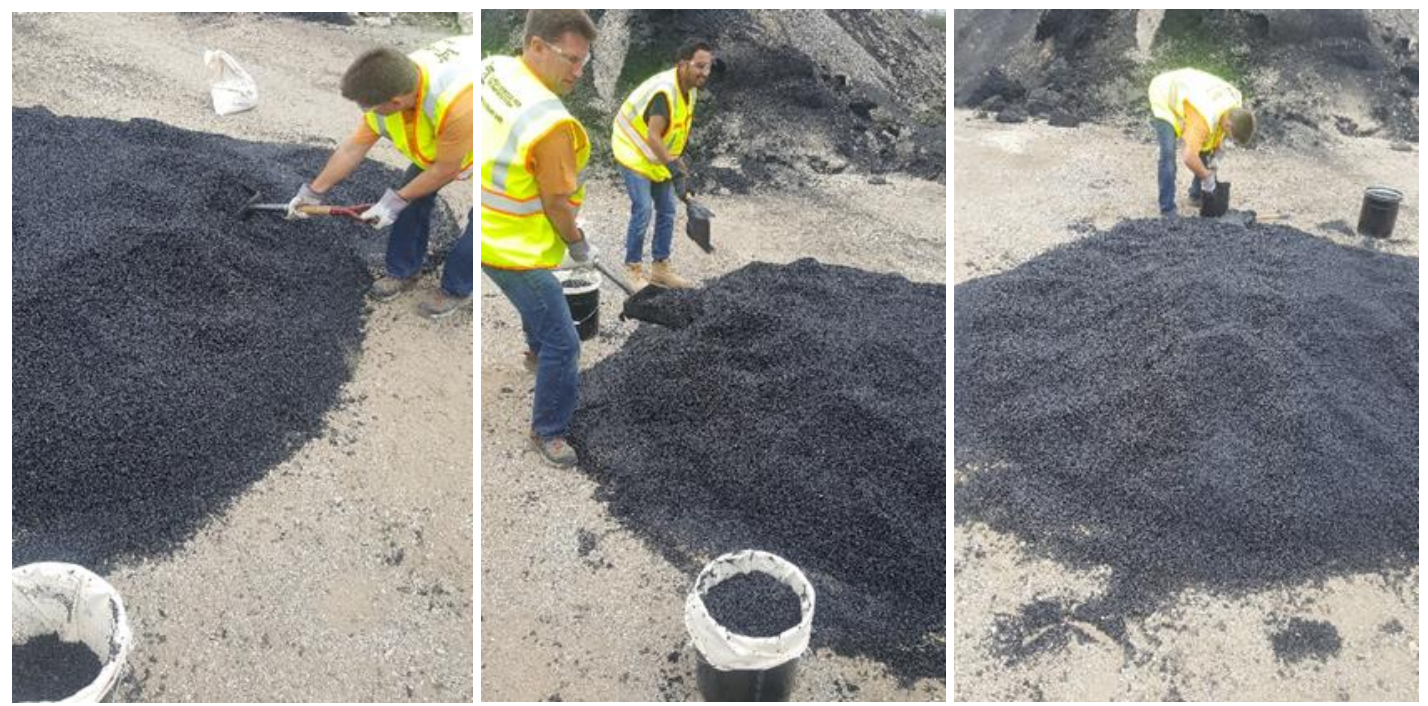

Figure 3.4. Sampling from different locations. 
5. Finally, sealed the bags and loaded them into the trailer to be stored and tested at the Advanced Transportation Research and Engineering Laboratory (ATREL). Each bag was labeled adequately to uniquely identify the material (date, project, material designation, etc.) (Figure 3.5).
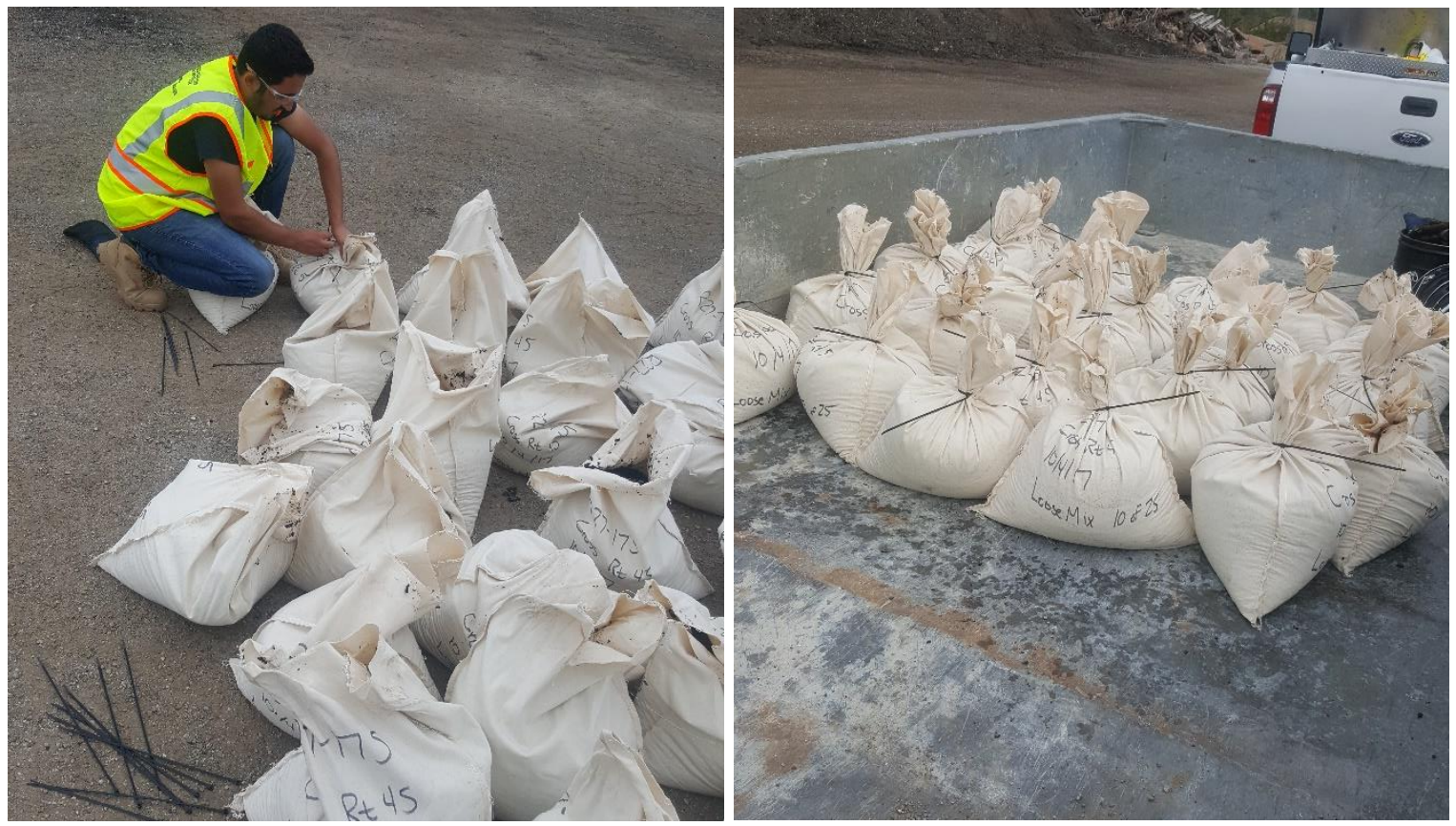

Figure 3.5. Collected samples ready for transport.

\subsubsection{Material Inventory}

Twelve AC surface mixtures were collected from the plants as well as component materials used in the production of those mixes. In addition, seven laboratory-designed mixtures were also developed in the laboratory for the evaluation of the binder source effect on long-term aging. The details of the mixtures collected are presented below.

Asphalt mixtures used in this study were classified into types based on their production technique. Mixture types include plant-produced, laboratory-compacted (PPLC) mixtures and laboratoryproduced, laboratory-compacted (LPLC) mixtures. They are mostly dense-graded, and two SMA mixes are also included. The $\mathrm{N}$-design of the mixes ranged from N50 to N90. The mixes obtained in the study from the asphalt plants were used in pavements with different traffic levels: interstate, state highways, and low-volume roads. Field core samples were obtained shortly after placement and at approximately six-month and 12-month ages. In addition to the plant mixtures, laboratory mixtures were designed to understand the impact of aging with a change in binder source and added recycled content.

\subsubsection{Asphalt Binders}

Asphalt binders were collected from each of the asphalt concrete plants from which the plantproduced mixtures were collected. In addition, binders with similar performance grade, but with 
different sources, were also gathered to understand the effect of source variability on the cracking potential of asphalt mixtures. Binder samples were also obtained which were modified with recycled engine oil bottoms (ReOB) at $9 \%$ by weight of the binder. The effect of source variability and presence of ReOB on Flexibility Index (FI) was studied for laboratory-designed mixtures consisting of $0 \%, 20 \%$, and $26 \%$ ABR (asphalt binder replacement) mixtures at various aging conditions. The matrix also included polymer modified binders. Table 3.1 shows the details of the binders used in the study.

\subsubsection{Plant-Produced, Laboratory-Compacted (PPLC) Mixtures}

Table 3.2 summarizes the list of mixtures used in the study. A wide variety of surface mixes were used in the study with $\mathrm{N}$-design ranging from 50 to 90 and with modified and neat binders. Two SMA mixes were added to the experimental plan as their aging resistance may potentially be different than that of dense-graded mixes. It should be noted that Mix 4 was removed from the test matrix for evaluation due to quality issues at the time of sampling. Table 3.3 presents the mix design details of PPLC mixtures.

\subsubsection{Laboratory-Produced, Laboratory-Compacted (LPLC) Mixtures}

These mixtures were designed to study the effect of RAP, binder type, and binder source. The RAP was sampled from contractor stockpile and was characterized based upon binder content and extracted gradation. The aggregates used to design the LPLC mixtures were the same as for Mix 2 with details provided in Table 3.4. The details of the mix designs are presented in Table 3.5. Hamburg wheel test information is provided in Table 3.6, which shows that all mixes passed IDOT specification.

Table 3.1. Grading and Source of Asphalt Binders Used in the Study

\begin{tabular}{|c|c|c|c|}
\hline $\begin{array}{c}\text { Binder } \\
\text { ID }\end{array}$ & $\begin{array}{c}\text { Binder } \\
\text { Grade }\end{array}$ & $\begin{array}{c}\text { Binder } \\
\text { Modification }\end{array}$ & $\begin{array}{c}\text { Associated } \\
\text { Mix }\end{array}$ \\
\hline S1 & PG 64-22 & N/A & PM1, LM1 \\
\hline S2 & PG 70-22 & SBS & PM2 \\
\hline S3 & PG 70-22 & SBS & PM3 \\
\hline S4 & PG 58-28 & N/A & PM4, LM4 \\
\hline S5 & PG 58-28 & N/A & PM5 \\
\hline S6 & PG 64-28 & SBS & PM6 \\
\hline S7 & PG 70-28 & SBS & PM7 \\
\hline S8 & PG 70-22 & SBS & PM8 \\
\hline S9 & PG 76-28 & SBS & PM9 \\
\hline S10 & PG 76-22 & SBS & PM10 \\
\hline S11 & PG 58-28 & N/A & PM11 \\
\hline S12 & PG 70-28 & SBS & PM12 \\
\hline S13 & PG 70-28 & SBS & PM13 \\
\hline S14 & PG 58-28 & N/A & LM3, LM6 \\
\hline S15 & PG 64-22 & N/A & LM2 \\
\hline S16 & PG 58-28 & N/A & LM5 \\
\hline S17 & PG 58-28 & 9\% ReOB & LM7 \\
\hline
\end{tabular}


Table 3.2. Details of PPLC Mixtures

\begin{tabular}{|c|c|c|c|c|}
\hline \multirow{2}{*}{$\begin{array}{c}\text { Mix } \\
\text { Designation }\end{array}$} & $\begin{array}{c}\text { Sample Date } \\
\text { (same as } \\
\text { paving date) }\end{array}$ & Mix Type & \multicolumn{2}{|c|}{ Hamburg Wheel Information } \\
\cline { 4 - 5 } & $6 / 6 / 2017$ & N70D & 7500 & 1.4 \\
\hline PM1 & $6 / 14 / 2017$ & N90D & 15000 & 4.8 \\
\hline PM2 & $6 / 16 / 2017$ & N90E & 15000 & 3.4 \\
\hline PM3 & $9 / 18 / 2017$ & N70D & 10000 & 5.0 \\
\hline PM5 & $10 / 4 / 2017$ & N70D & 10000 & 7.7 \\
\hline PM6 & $10 / 20 / 2017$ & 9.5 SMA & 20000 & 2.7 \\
\hline PM7 & $10 / 27 / 2017$ & N50D & 7500 & 7.6 \\
\hline PM8 & $11 / 1 / 2017$ & N70D & 20000 & 2.3 \\
\hline PM9 & $11 / 1 / 2017$ & N50E & 20000 & 4.1 \\
\hline PM10 & $11 / 2 / 2017$ & N50E & 7500 & 4.1 \\
\hline PM11 & $11 / 3 / 2017$ & N70E & 20000 & 4.4 \\
\hline PM12 & $11 / 14 / 2017$ & 12.5 SMA & 20000 & 2.4 \\
\hline PM13 & & &
\end{tabular}

Table 3.3. Design Details for PPLC Mixtures

\begin{tabular}{|c|c|c|c|c|c|c|c|c|c|}
\hline ID & N Design & NMAS $^{a}$ & VMA $^{b}$ & Asphalt Content (\%) & Binder Grade & $\mathrm{ABR}^{\mathrm{c}}$ & Mix Type & $A b^{d}$ & $\mathrm{Pbe}^{\mathrm{e}}$ \\
\hline PM1 $^{f}$ & 70 & 9.5 & 15.2 & 5.9 & $64-22$ & 20.7 & DG & 1.47 & 5.0 \\
\hline PM2 & 90 & 9.5 & 15.1 & 6.2 & $70-22$ & 9.2 & DG & 2.29 & 4.8 \\
\hline PM3 & 90 & 9.5 & 15.2 & 6.2 & $70-22$ & 9.6 & DG & 2.14 & 4.8 \\
\hline PM5 & 70 & 9.5 & 15.5 & 6.1 & $58-28$ & 20.3 & DG & 1.69 & 5.0 \\
\hline PM6 & 70 & 9.5 & 15.7 & 6.2 & $64-28$ & 7.9 & DG & 2.14 & 4.9 \\
\hline PM7 & 80 & 9.5 & 16.4 & 6.4 & $70-28$ & 30.2 & SMA & 0.85 & 5.7 \\
\hline PM8 & 50 & 9.5 & 15.0 & 6.0 & $70-22$ & 15.8 & DG & 1.83 & 4.8 \\
\hline PM9 & 70 & 9.5 & 15.0 & 5.7 & $76-28$ & 10.2 & DG & 1.15 & 4.8 \\
\hline PM10 & 50 & 9.5 & 15.2 & 6.0 & $76-22$ & 10.2 & DG & 2.04 & 4.9 \\
\hline PM11 & 50 & 9.5 & 15.4 & 6.0 & $58-28$ & 24.5 & DG & 1.86 & 4.9 \\
\hline PM12 & 70 & 9.5 & 15.0 & 6.0 & $70-28$ & 30.0 & DG & 1.69 & 4.7 \\
\hline PM13 & 80 & 12.5 & 17.3 & 6.3 & $70-28$ & 26.7 & SMA & 0.73 & 5.7 \\
\hline
\end{tabular}

${ }^{\text {a }}$ NMAS = Nominal Maximum Aggregate Size $(\mathrm{mm})$

b VMA = Voids in Mineral Aggregate (\%)

${ }^{\mathrm{c}} \mathrm{ABR}=$ Asphalt Binder Replacement (\%)

${ }^{\mathrm{d}} \mathrm{Ab}=$ Aggregate Blend Water Absorption (\%)

e Pbe $=$ Effective Asphalt Content Defined by Mass (\%)

${ }^{\mathrm{f}} \mathrm{PM}=$ Abbreviation of Plant Mixtures

Table 3.4. Aggregate Information

\begin{tabular}{|c|c|c|c|c|c|}
\hline Material ID & 022CM16 & 022CM16 & 028FM20 & $027 F M 01$ & $004 M F 01$ \\
\hline Type of Material & Dolomite & Limestone & Dolomite & Natural Sand & Mineral Filler \\
\hline
\end{tabular}


Table 3.5. Details of LPLC Mixtures

\begin{tabular}{|c|c|c|c|c|c|c|c|c|c|}
\hline ID & N Design & NMAS & VMA & Asphalt Content (\%) & Binder Grade & ABR & Mix Type & Ab & Pbe \\
\hline LM1 $^{\mathbf{a}}$ & 70 & 9.5 & 15.2 & 6.4 & $64-22$ & 0 & DG & 2.40 & 4.7 \\
\hline LM2 & 70 & 9.5 & 15.2 & 6.4 & $64-22$ & 0 & DG & 2.40 & 4.7 \\
\hline LM3 & 70 & 9.5 & 15.2 & 6.4 & $58-28$ & 20.0 & DG & 2.03 & 4.9 \\
\hline LM4 & 70 & 9.5 & 15.2 & 6.4 & $58-28$ & 20.0 & DG & 2.03 & 4.9 \\
\hline LM5 & 70 & 9.5 & 15.2 & 6.4 & $58-28$ & 20.0 & DG & 2.03 & 4.9 \\
\hline LM6 & 70 & 9.5 & 15.2 & 6.4 & $58-28$ & 26.0 & DG & 1.94 & 4.9 \\
\hline LM7 & 70 & 9.5 & 15.2 & 6.4 & $58-28($ ReOb) & 26.0 & DG & 1.94 & 4.9 \\
\hline
\end{tabular}

${ }^{a} \mathrm{LM}=$ Abbreviation of Lab Mixtures

Table 3.6. Hamburg Wheel Test Information for LPLC Mixes

\begin{tabular}{|c|c|c|}
\hline \multirow{2}{*}{ ID } & \multicolumn{2}{|c|}{ Hamburg Wheel Information } \\
\cline { 2 - 3 } & No. Passes & Depth (mm) \\
\hline LM1 & 7500 & 6.5 \\
\hline LM2 & 7500 & 3.8 \\
\hline LM3 & 5000 & 6.8 \\
\hline LM4 & 5000 & 8.2 \\
\hline LM5 & 5000 & 5.6 \\
\hline LM6 & 5000 & 5.6 \\
\hline LM7 & 5000 & 6.3 \\
\hline
\end{tabular}

All AC mixes excluding PM13 have a 9.5- $\mathrm{mm}$ nominal maximum aggregate size (NMAS). Aggregate gradations for each of these AC mixes are shown in Figure 3.6. All AC mixes excluding PM7 and PM13 are coarse dense-graded mixes, and their gradations are close to each other, excluding PM12, which still meets the requirements of IDOT. PM7 and PM13 are stone matrix asphalt (SMA) mixes with open aggregate gradation, as shown in Figure 3.6.

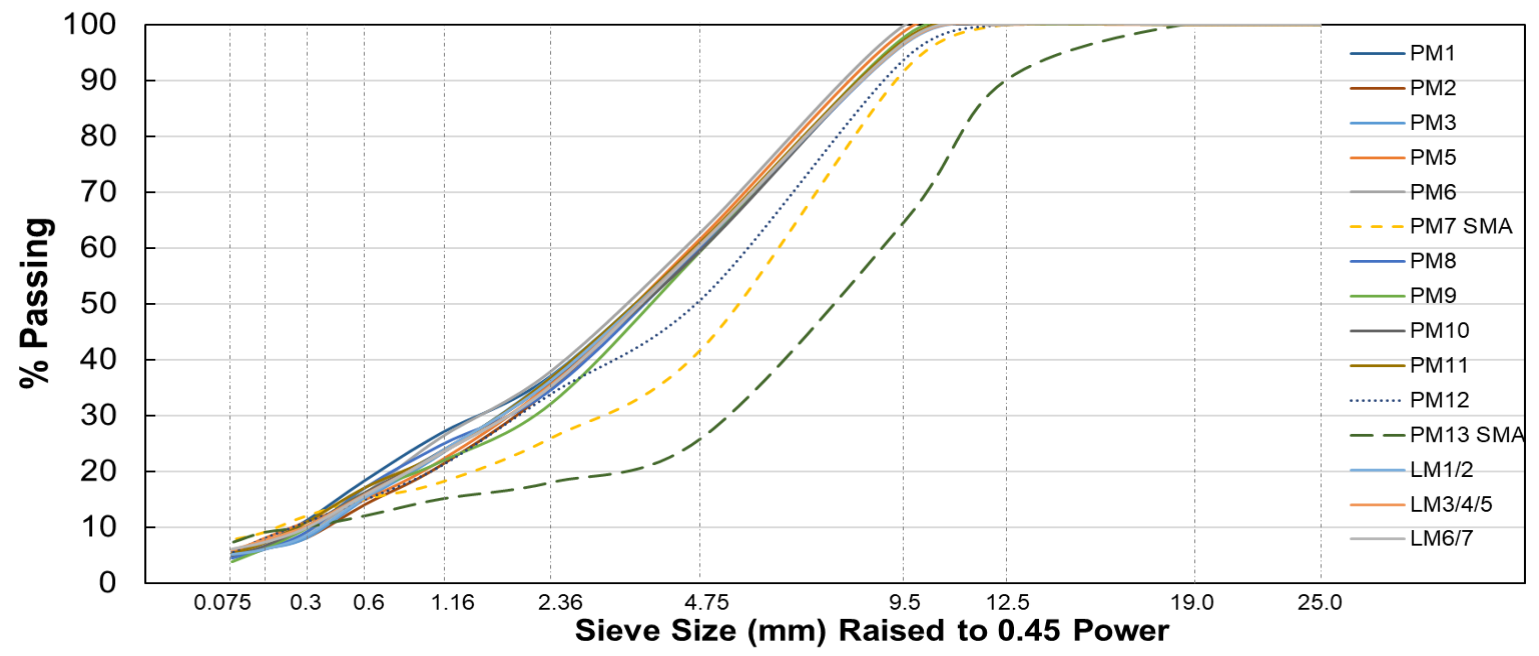

Figure 3.6. Gradations for all mixes used in this project. 


\subsubsection{Field Cores of Plant Mixes from R27-175 Project}

Field core samples for the current study were collected at various intervals from the locations where plant mixes (Table 3.7) were placed. These were collected for field validation of the developed protocol for long-term aging. The samples were collected at intervals of approximately six months from the time of construction. The summary of the field core samples collected is provided in Table 3.7.

Table 3.7. Details of Field Core Samples

\begin{tabular}{|c|c|c|c|c|}
\hline \multirow{2}{*}{$\begin{array}{c}\text { Mix } \\
\text { Designation }\end{array}$} & Mixtures & Field Core 1 & Field Core 2 & Field Core 3 \\
\cline { 3 - 5 } & Sampling Dates & \multicolumn{3}{|c|}{ Actual Sampling Dates } \\
\hline PM1 & $06 / 06 / 2017$ & $07 / 31 / 2017$ & $11 / 28 / 2017$ & $06 / 26 / 2018$ \\
\hline PM2 & $06 / 14 / 2017$ & $10 / 18 / 2017$ & $02 / 14 / 2018$ & $07 / 25 / 2018$ \\
\hline PM3 & $06 / 16 / 2017$ & $10 / 18 / 2017$ & $02 / 14 / 2018$ & $07 / 25 / 2018$ \\
\hline PM5 & $09 / 18 / 2017$ & $10 / 13 / 2017$ & $06 / 21 / 2018$ & $10 / 31 / 2018$ \\
\hline PM6 & $10 / 04 / 2017$ & $10 / 18 / 2017$ & $04 / 18 / 2018$ & $10 / 24 / 2018$ \\
\hline PM7 & $10 / 20 / 2017$ & $12 / 18 / 2017$ & $06 / 21 / 2018$ & $10 / 31 / 2018$ \\
\hline PM12 & $11 / 03 / 2017$ & $12 / 19 / 2017$ & $06 / 21 / 2018$ & $10 / 31 / 2018$ \\
\hline PM13 & $11 / 14 / 2017$ & $12 / 20 / 2017$ & $06 / 21 / 2018$ & $10 / 31 / 2018$ \\
\hline
\end{tabular}

\subsection{EXPERIMENTAL METHODS}

\subsubsection{Introduction}

To understand the effects of aging, it is important to develop a methodology to age the asphalt concrete (AC) samples in the laboratory, with the following objectives:

- Use of available aging devices which can be readily available for agency and contractors.

- Low-cost equipment with low-maintenance requirements.

- Enough capacity.

- Repeatable and reproducible across various labs.

- Good correlation to field aging and performance.

Various commonly used and readily available aging methods were investigated. This included PAV, vacuum oven, and conventional oven (forced-draft oven). In addition to the aging device, specific sample preparation methods were also investigated. The samples were aged both in a loose condition as well as in a fully prepared I-FIT specimen condition.

The simulated aging of AC in a laboratory environment can be achieved by oxidation and UV exposure in the presence of moisture. The scope of this study investigated effects caused primarily by oxidation. The extent of oxidation is a function of temperature, pressure, and the duration of the exposure. In order to understand the selected variables associated with the aging caused by oxidation, a test matrix was developed which is presented in Figure 3.7. The matrix included variables such as temperature, duration, pressure, and type of aging device used to determine the final protocol for aging AC mixtures. 


\subsubsection{Selection of State of Material during Aging}

Candidate states of material during aging include loose mixture and I-FIT specimen. The procedures followed for each of them are described as follows.

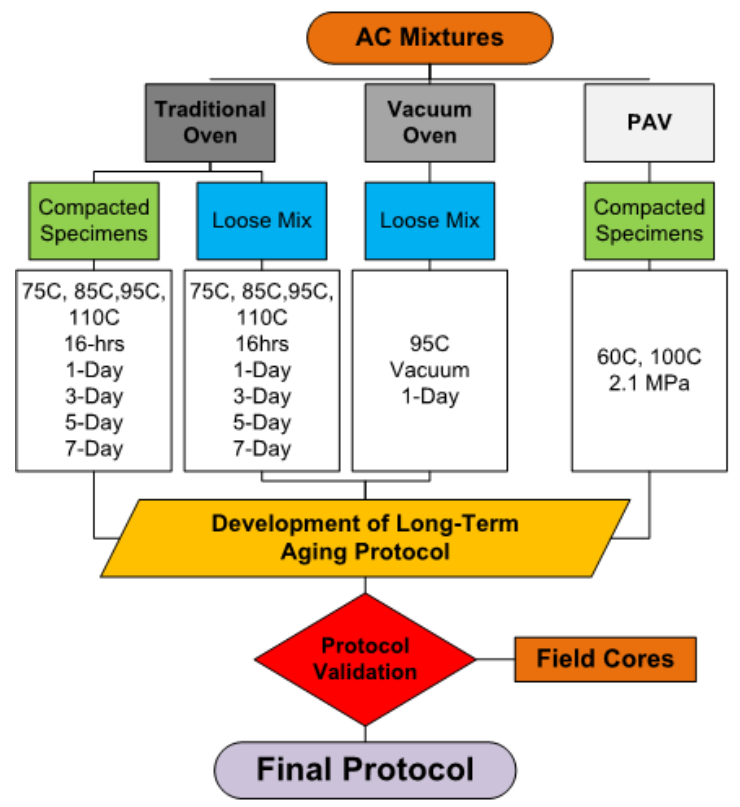

Figure 3.7. Test matrix.

\subsubsection{Loose Mixture Aging}

The material was aged in its loose state prior to compaction for the required aging time at a specific temperature followed by two hours of short-term aging at compaction temperature before being compacted. After the compaction, samples were fabricated and tested. Figure 3.8 shows the loose sample being aged. The same compaction temperature was used as recommended in the original mix design.

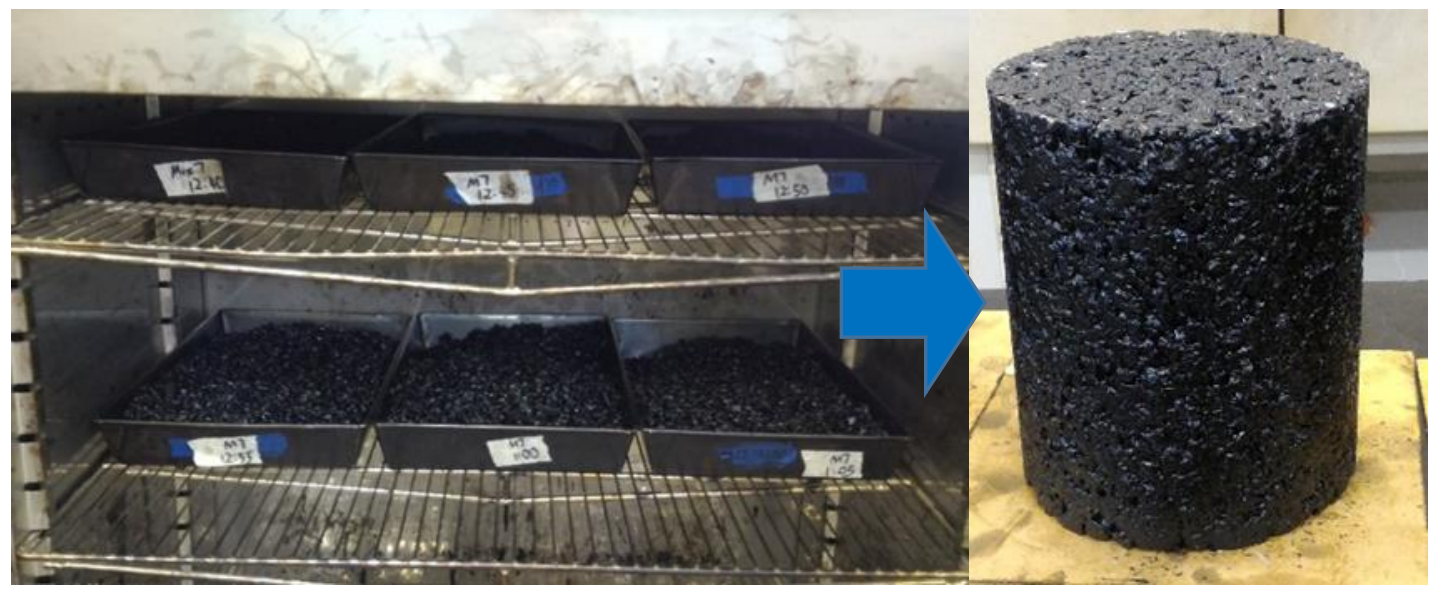

Figure 3.8. Loose mixture aging. 


\subsubsection{I-FIT Specimen Aging}

Samples were compacted following a short-term aging of two hours at the compaction temperature. The gyratory compacted cylinders (pills) were fabricated to the desired I-FIT geometry and were then aged for a specific time and duration. Figure 3.9 shows I-FIT specimens ready for aging and the aged specimens.

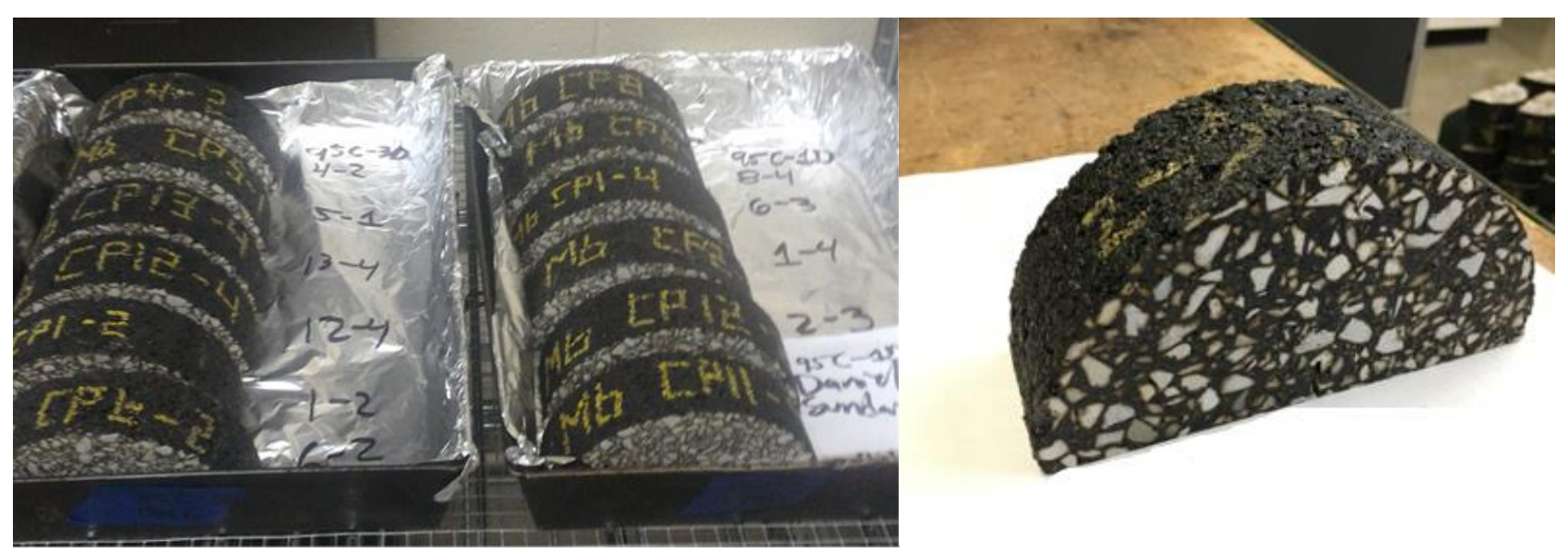

(a)

(b)

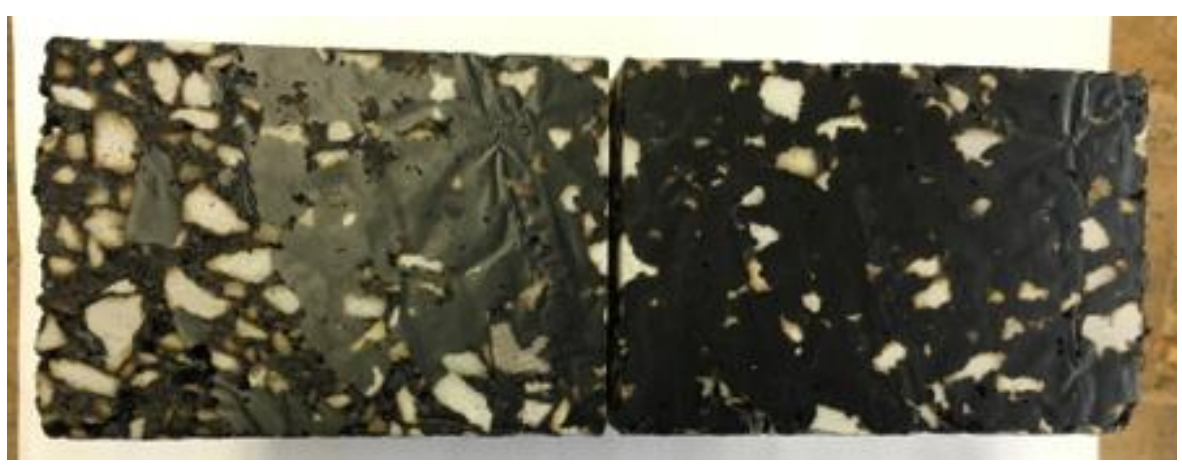

(c)

Figure 3.9. I-FIT specimen aging: (a) specimens ready for aging; (b/c) aged specimen.

\subsubsection{Selection of Aging Device}

Three aging devices were selected for the initial investigation including the pressure aging vessel (PAV) used in the SuperPave PG specification (AASHTO M320), vacuum oven used in sealant aging (AASHTO MP25), and a traditional forced-draft oven.

\subsubsection{Pressure Aging Vessel}

The same PAV device used in binder grading according to SuperPave specifications was used. Since the capacity of the PAV is very limited, only the fully prepared IFIT specimens were aged using this technique. Specimens were aged at $140^{\circ} \mathrm{F}\left(60^{\circ} \mathrm{C}\right)$ and $212^{\circ} \mathrm{F}\left(100^{\circ} \mathrm{C}\right)$ with the pressure of $304.58 \mathrm{psi}$ (2.1 MPa). Pressure was not used as a variable because it was found to be difficult and impractical to 
change the pressure. The settings needed to be adjusted by the equipment manufacturer. The current settings of temperature and pressure caused excessive deformations of specimens, as shown in Figure 3.10. Due to the mentioned practical drawbacks and potential damage to the specimens, this method was eliminated from further testing.

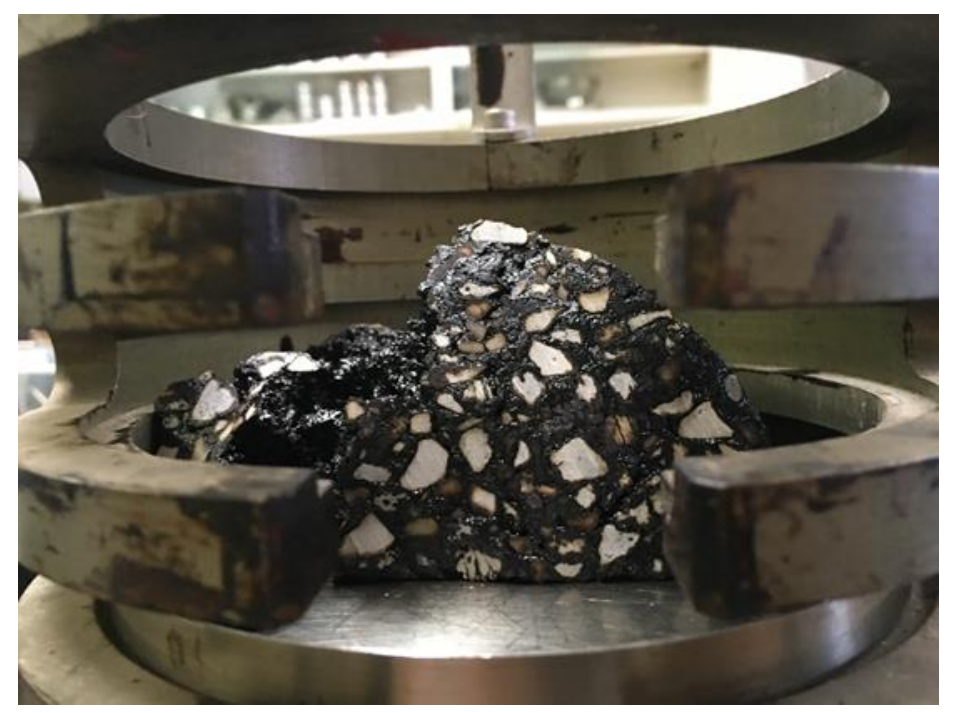

Figure 3.10. I-FIT specimen after PAV aging.

\subsubsection{Vacuum Oven Aging}

Vacuum oven aging is currently used in characterization and grading of the crack sealants. The device is used to age the sealant samples and is tested for low temperature performance. The device has comparatively more capacity to age the samples than that of the PAV device. The method was investigated to evaluate loose mix samples. The results were positive but posed a concern due to the practical issues. Firstly, this device is not readily available. This posed a challenge to continue using for further examination. Secondly, the capacity is limited to age materials that can prepare three 7.1 in (180 mm) gyratory-compacted cylinders (12 I-FIT samples). Thirdly, the vacuum created reduces the supply of oxygen. Figure 3.11 shows a typical vacuum oven used for aging AC mixtures in this project.

\subsubsection{Forced-draft Oven Aging}

This is the most commonly and widely available oven to contractors and agencies due to low initial and maintenance costs. It has much larger capacity than PAV and vacuum oven. Aging of both loose mix and I-FIT specimen was evaluated for a long-term aging protocol. The test matrix was applied extensively across various temperatures and durations in the study. The forced-draft oven used in this study is shown in Figure 3.12, and Table 3.8 provides detailed information. 


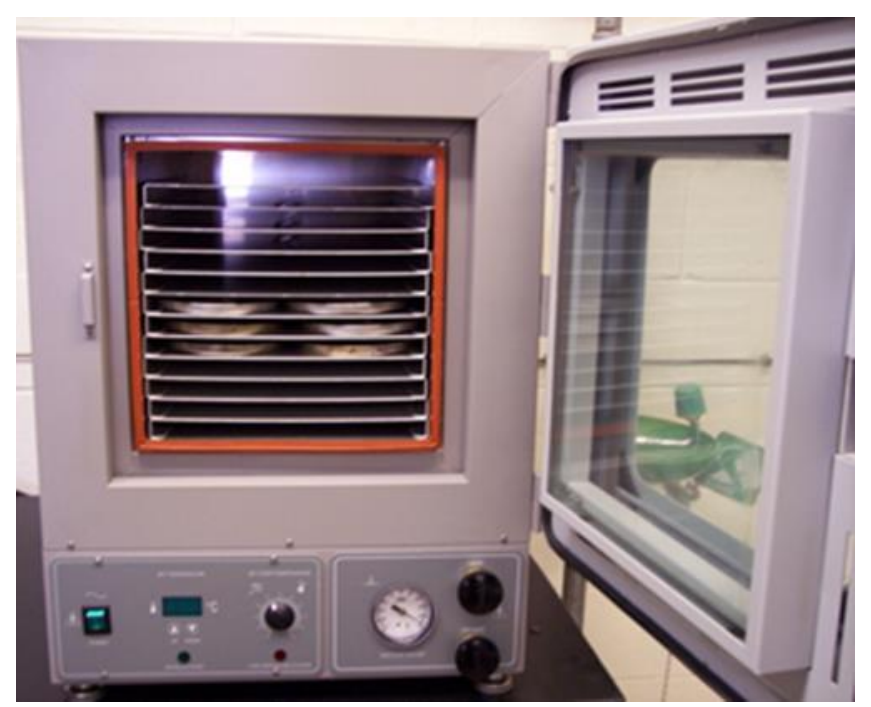

Figure 3.11. Vacuum oven.

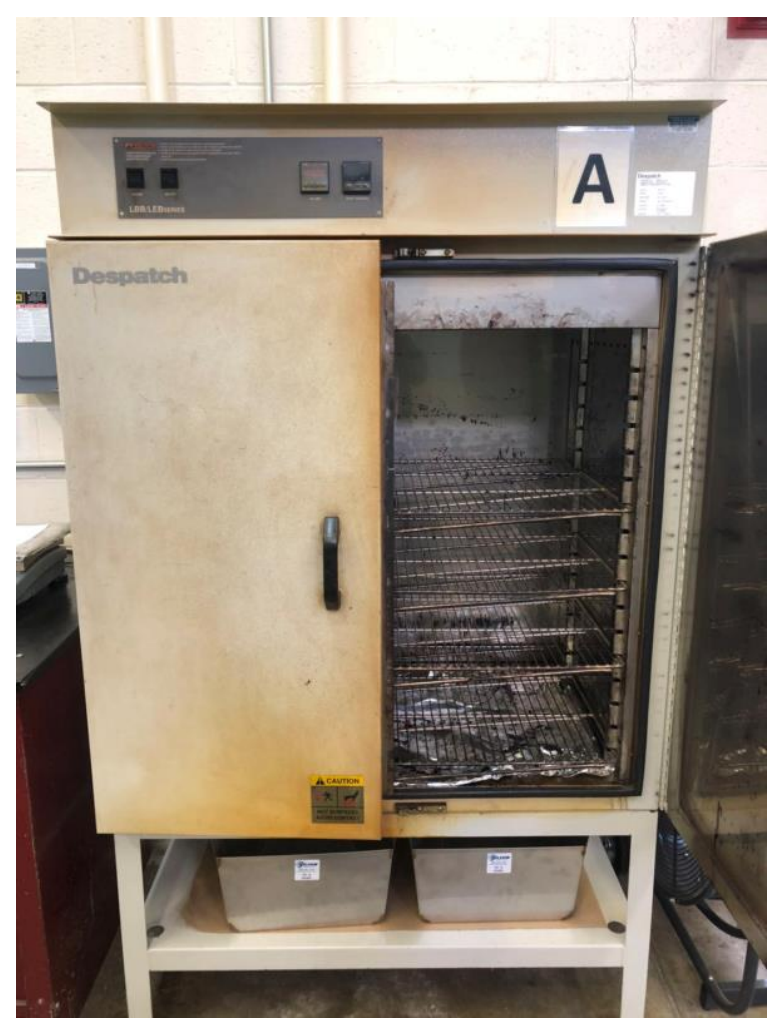

Figure 3.12. Forced-draft oven.

Table 3.8. Detailed Information of the Forced-draft Oven Used in This Study

\begin{tabular}{|c|c|c|c|c|c|}
\hline Model & Serial & Max Temp. & Power & Heater & Motor \\
\hline LBB2-18-1 & 182600 & $204^{\circ} \mathrm{C}\left(400^{\circ} \mathrm{F}\right)$ & $\begin{array}{c}\text { 240V-1PH-50/60 } \\
\mathrm{HZ}\end{array}$ & $\begin{array}{c}3600 \text { WATTS } 15.0 \\
\text { AMPS }\end{array}$ & 1/4HP 1.4AMPS \\
\hline
\end{tabular}


Out of the available aging devices, the most practical and suitable device selected for further evaluation was the forced-draft oven. The method addressed most of the requirements for an ideal aging protocol stated earlier in Section 3.2.1.

The preparation processes are discussed in Section 3.3. Loose mixture and I-FIT specimen aging, using the forced draft oven, is also presented. Appropriate procedures for specimen preparation and aging were determined and used for further testing.

\subsection{SPECIMEN PREPARATION}

\subsubsection{I-FIT Specimen Aging}

Specimen preparation of plant-mixed, lab-compacted (PPLC) mixes is comprised of all the steps required to fabricate a final I-FIT specimen from loose mix material. The first step was to heat up the bag with the sampled materials at a temperature of $275^{\circ} \mathrm{F}\left(135^{\circ} \mathrm{C}\right)$ for four hours. The materials were blended and split in accordance with AASHTO R47. The objective of splitting was to homogenize the sampled material and to prepare batches that contained the required amount of materials necessary for compaction (15.4 to $17.6 \mathrm{lbs}$ [7.0 to $8.0 \mathrm{kgs}$ )). The final material batches were poured into metal trays. Figure 3.13 shows an example of the splitting process and the final batched materials to the exact weight of the 7.1-in (180-mm) gyratory compacted pills.
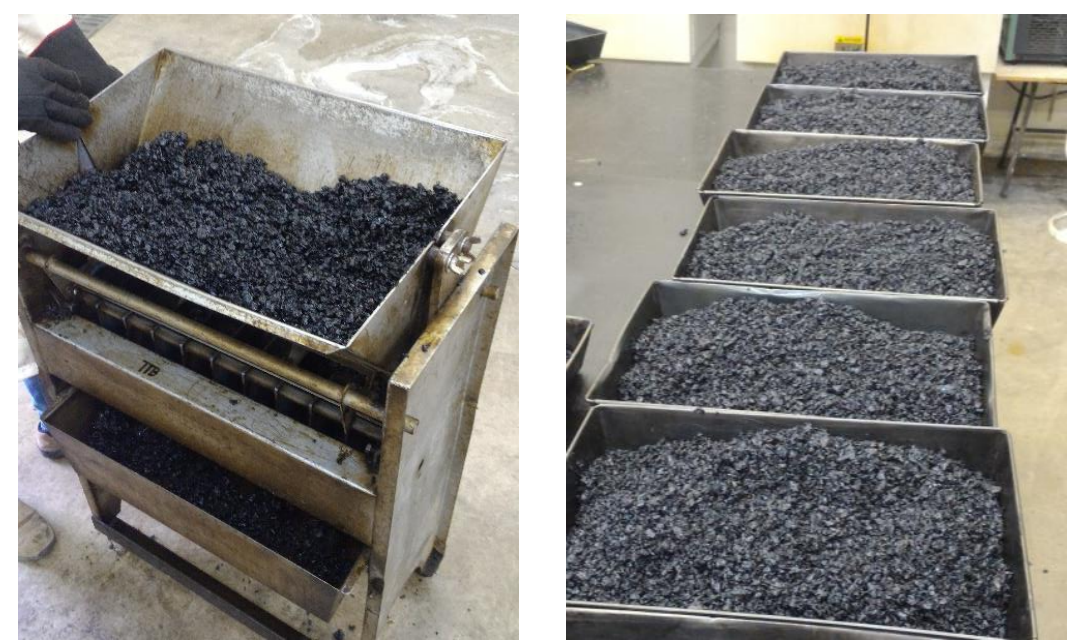

Figure 3.13. Material splitting and batched samples.

The next step was to subject the batched samples to a two-hour conditioning cycle so that the material could reach its required compaction temperature. This process was completed by using the forced-draft oven. To ensure that each sample was subjected to only two hours of heating before compaction, the trays were introduced into the oven with a five-minute lag between each other; Figure 3.14 shows an example of this set-up. After 60 minutes of conditioning, the materials in each tray were stirred to maintain uniform heating. 


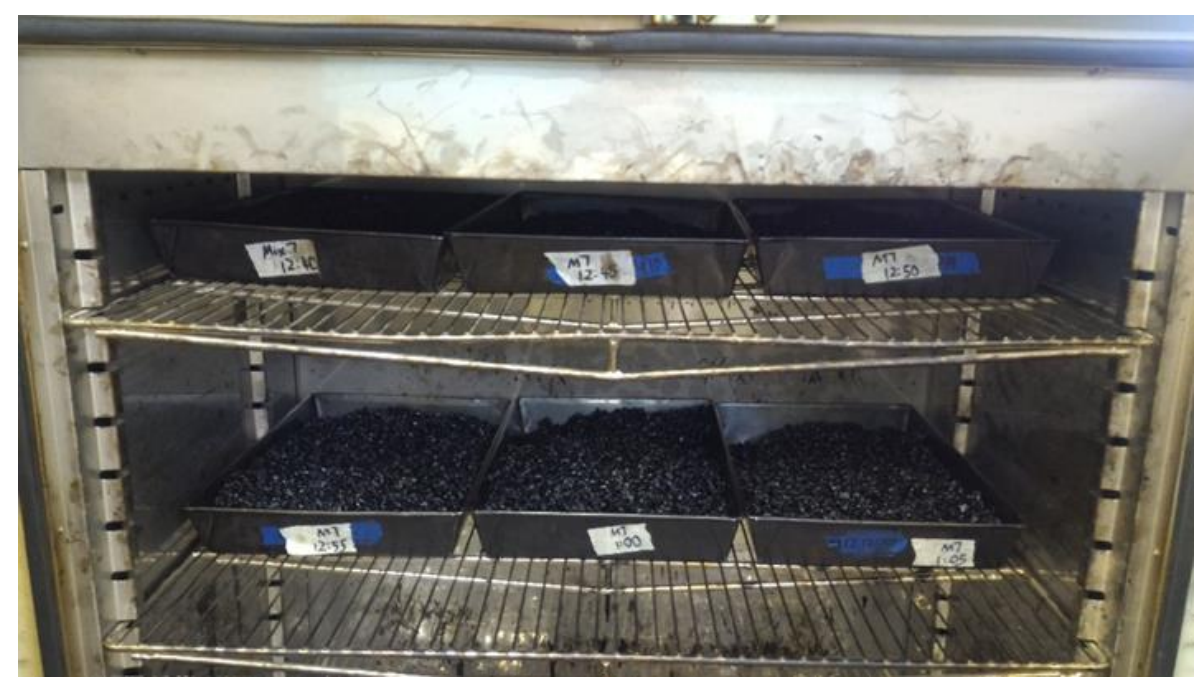

Figure 3.14. Conditioning of batched samples before compaction.

After the two-hour conditioning cycle, the materials were compacted. AASHTO TP124 was followed to fabricate 7.09 in (180 mm) high gyratory-compacted cylinders (also called pills). During the compaction process, the materials of each tray were transferred to the compaction mold using a chute to avoid any mix segregation. After compaction was completed, the materials were left in the mold for approximately five minutes before it was extruded. Once the pills were extruded, they were left undisturbed overnight at room temperature to allow proper cooling. The amount of mass used for the compaction of each mix was adjusted so that the air voids in the final semi-circular specimens were in the range of $7.0 \% \pm 0.5 \%$ for dense-graded mixes, and $6.0 \% \pm 0.5 \%$ for SMA mixes. Figure 3.15 shows an example of extruded pills cooling overnight.

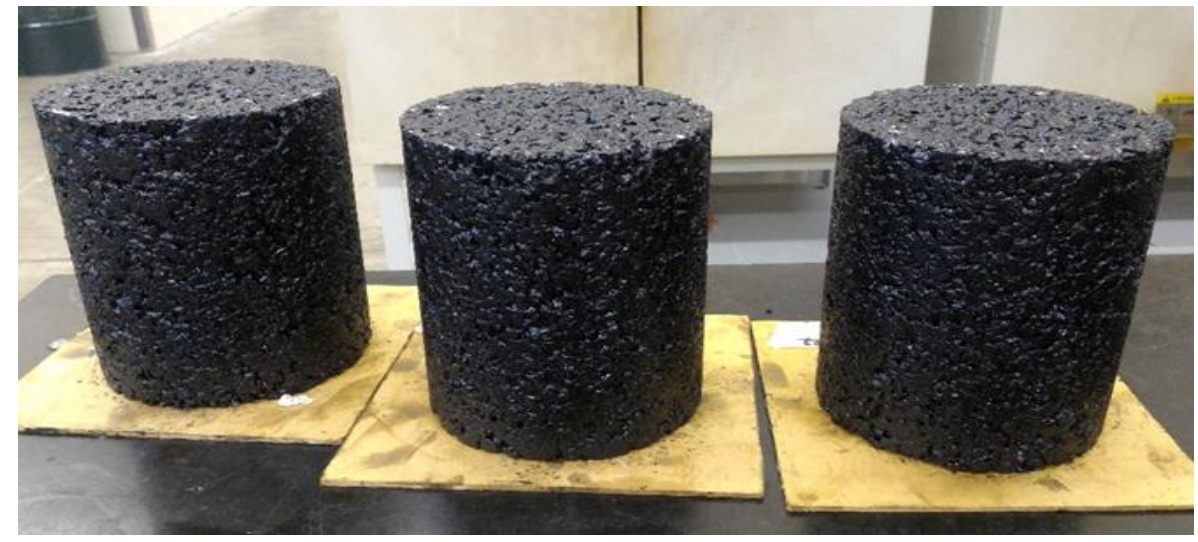

Figure 3.15. Extruded SGC pills with $180 \mathrm{~mm}$ height.

The final step was to process the SGC pills to obtain the final specimen geometry using masonry saws. Two circular slices of 1.97 in $(50 \mathrm{~mm})$ thick were cut from the center of the pills. The slices were later split in half to obtain a semi-circular geometry. Finally, a central notch was cut at the base of each specimen. Figure 3.16 shows the different processing stages. 

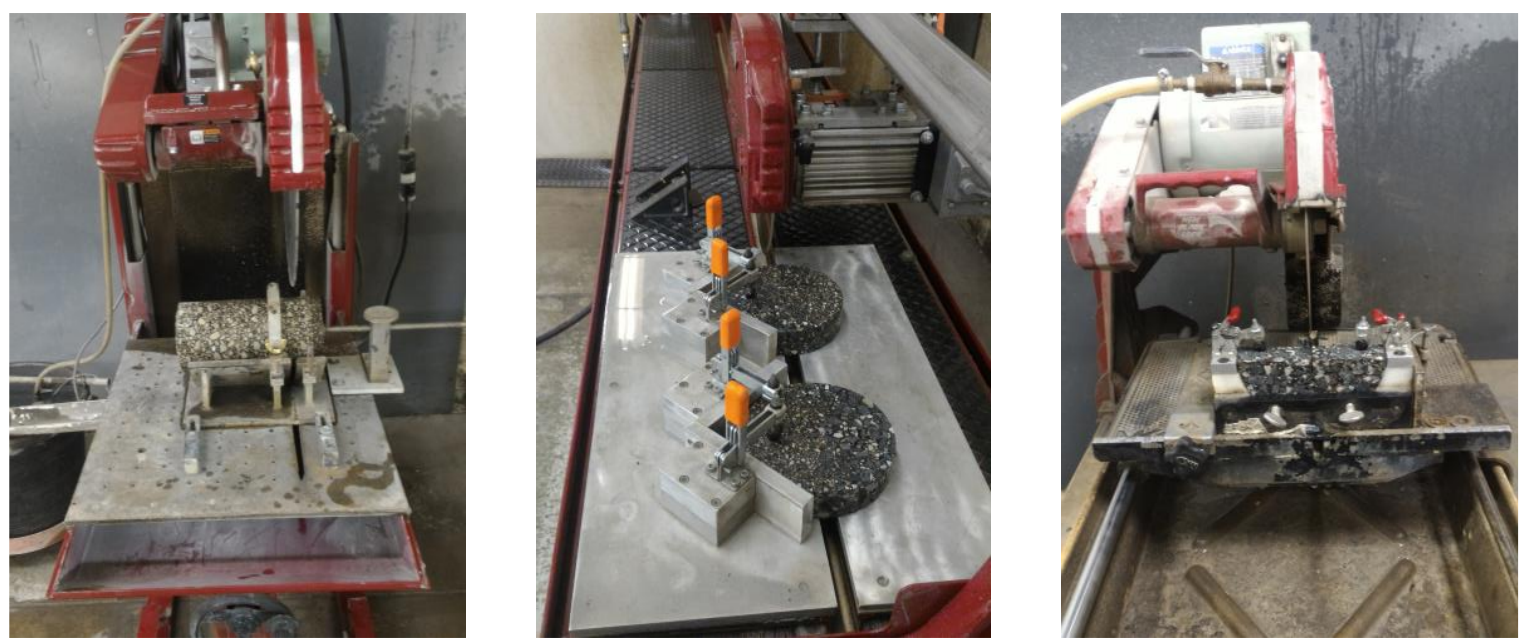

Figure 3.16. Specimen preparation steps from left to right: slicing, halving, and notching.

Air voids calculation was performed on the final notched semi-circular specimens using the actual measured theoretical maximum specific gravity $\left(\mathrm{G}_{\mathrm{mm}}\right)$ determined from the blended and split sample. Those specimens, compliant with the required air voids, were subjected to various long-term aging protocols included in the experimental plan. The procedure of preparing specimens for aging included organizing the selected specimens on aluminum foil upon metal trays and putting them in a forced-draft oven at the temperature and duration according to the aging cycle selected. Figure 3.17 shows an example of how the samples were set-up before being introduced to the oven.

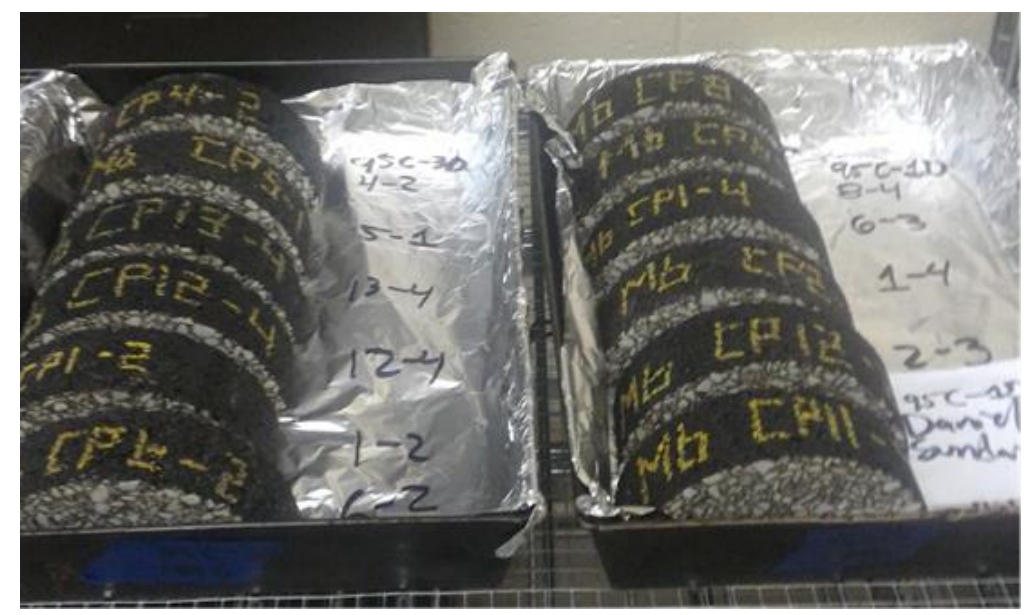

Figure 3.17. Trays containing specimens ready for aging process.

After the corresponding aging cycle was finished, the samples were taken from the oven and were cooled for a minimum of four hours in front of a fan. Once the samples cooled down, measurements of thickness, notch length, and ligament were taken to ensure that they were within the limits specified by I-FIT standard AASHTO TP124. Those specimens with the acceptable dimensions were conditioned in a water bath at $77^{\circ} \mathrm{F}\left(25^{\circ} \mathrm{C}\right)$ for two hours and then tested according to the AASHTO TP124. 
The preparation of laboratory-mixed, lab-compacted (LPLC) specimens was similar to the above procedures. The only exception was that instead of heating and splitting the sampled bags, AC raw materials (binder and aggregates) were first heated to the required mixing temperature, and then prepared as a loose AC mix using a mechanical stirrer. No four-hour reheating cycle was needed for LPLC.

\subsubsection{Loose Mixture Aging}

The same procedures of sample splitting were followed to split plant samples into the batches with an exact weight of a sample for compaction. The next step was to subject the batched samples to the aging condition required (example: 1-Day, 3-Day, and 7-Day at $85^{\circ} \mathrm{C}, 95^{\circ} \mathrm{C}$, and likewise) as shown in Figure 3.14. After aging was completed, the material was put into a two-hour conditioning cycle at $302^{\circ} \mathrm{F}\left(150^{\circ} \mathrm{C}\right)$ so that the material could reach its required compaction temperature as well as the aging extent being controlled. This process was done by using the forced-draft oven. The same procedures were followed in preparing the final I-FIT specimens after the completion of aging and compaction.

\subsection{BINDER TESTING RESULTS SUMMARY}

A total of 17 binders were collected and tested from various HMA plants and asphalt binder suppliers across Illinois. Test results are presented in this section.

Binder testing was conducted following AASHTO M320, AASHTO R29, AASHTO T316, AASHTO T240, AASHTO R28, AASHTO T315, and AASHTO T313 specifications. SuperPave binder grade and $\Delta T_{c}$ (difference in temperature passing m-value and stiffness criteria per ASTM D7643) were reported. $\Delta T_{c}$ was calculated after a single and double PAV. This parameter was found to be useful in correlating with the brittleness of AC after long-term aging (Sharma et al. 2017). Table 3.9 shows the testing results.

Out of the 17 binders tested, S1 and S5 failed the low temperature SuperPave criteria. Binder S11 failed the SuperPave fatigue criteria. However, one of the binders, S8, which was reported to be a PG70-22, was a PG 70-28 instead. All other SuperPave criteria were satisfied for all the binders tested.

The empirical threshold suggested for $\Delta \mathrm{T}_{\mathrm{c}}$ is $-5^{\circ} \mathrm{C}$. After $1 \mathrm{PAV}, \Delta \mathrm{T}_{\mathrm{c}}$ of most of the binders was greater than $-5^{\circ} \mathrm{C}$. Only three of the binders had $\Delta \mathrm{T}_{\mathrm{c}}$ less than $-5^{\circ} \mathrm{C}(\mathrm{S} 1, \mathrm{~S} 5$, and $\mathrm{S} 13)$. However, after the second PAV, only four (S10, S11, S14, and S16) of the binders remained greater than the $-5^{\circ} \mathrm{C}$ threshold. Four of the binder samples had the lowest $\Delta \mathrm{T}_{\mathrm{c}}$ values less than $-10^{\circ} \mathrm{C}(\mathrm{S} 1, \mathrm{~S} 2, \mathrm{~S} 9$, and $\mathrm{S} 13)$. 
Table 3.9. Test Data for Performance Graded Asphalt Binders Collected for the R27-175 Project

\begin{tabular}{|c|c|c|c|c|c|c|}
\hline \multirow{2}{*}{ Binder ID } & \multirow{2}{*}{ Provided PG Grade } & \multirow{2}{*}{ Actual PG Grade } & \multirow{2}{*}{ True Grade } & \multirow{2}{*}{ m-value } & \multicolumn{2}{|c|}{$\Delta \mathbf{T}_{\mathbf{c}}$} \\
\cline { 5 - 7 } & & & & PAV $^{\mathrm{b}}$ & 2-PAV \\
\hline S1 & PG 64-22 & PG 64-16 & PG 68.5-21.6 & $\mathbf{0 . 2 9 8}$ & -8.7 & -14.3 \\
\hline S2 & PG 70-22 & PG 70-22 & PG 72.1-23.1 & 0.307 & -2.9 & -10.2 \\
\hline S3 & PG 70-22 & PG 70-22 & PG 72.8-24.6 & 0.323 & -4.5 & -9.8 \\
\hline S4 & PG 58-28 & PG 58-28 & PG 59.0-28.3 & 0.303 & -1.5 & -7.5 \\
\hline S5 & PG 58-28 & PG 58-22 & PG 59.7-26.4 & $\mathbf{0 . 2 8 2}$ & -5.6 & -6.2 \\
\hline S6 & PG 64-28 & PG 64-28 & PG 66.2-28.7 & 0.307 & -4.2 & -8.7 \\
\hline S7 & PG 70-28 & PG 70-28 & PG 71.6-29.3 & 0.311 & -1.4 & -6.7 \\
\hline S8 & PG 70-22 & PG 70-28 & PG 74.7-31.4 & 0.322 & -1.8 & -8.3 \\
\hline S9 & PG 76-28 & PG 76-28 & PG 77.6-28.7 & 0.304 & -3.4 & -11.5 \\
\hline S10 & PG 76-22 & PG 76-22 & PG 77.9-25.3 & 0.328 & -0.5 & -1.6 \\
\hline S11 & PG 58-28 & PG 58-22 & PG 61.4-28.8 & 0.311 & 0.3 & -2.6 \\
\hline S12 & PG 70-28 & PG 70-28 & PG 71.2-29.5 & 0.312 & -1.7 & -6.6 \\
\hline S13 & PG70-28 & PG 70-28 & PG 74.7-31.2 & 0.316 & -5.9 & -16.6 \\
\hline S14 & PG 58-28 & PG 58-28 & PG 59.9-29.6 & 0.318 & 0.0 & -4.8 \\
\hline S15 & PG 64-22 & PG 64-22 & PG 66.1-22.3 & 0.304 & -3.1 & -7.4 \\
\hline S16 & PG 58-28 & PG 58-28 & PG 60.1-28.7 & 0.321 & 1.4 & -1.4 \\
\hline S17 & PG 58-28 & PG 58-28 & PG 58.5-32.4 & 0.329 & 4.3 & -9.2 \\
\hline
\end{tabular}

a 11 failed the fatigue criterion.

b PAV equals 20 hours in a pressure aging vessel according to AASHTO R28.

c 2 -PAV equals 40 hours in a pressure aging vessel. 


\section{CHAPTER 4: DEVELOPMENT OF LONG-TERM AGING PROTOCOL}

After the collection of the samples for testing, a testing plan was prepared to achieve the following goals: 1 ) identify candidate aging procedures, including testing equipment, specimen type, duration of aging, and temperature, and 2) test selected mixes using varying aging equipment and aging conditions. After an aging device was selected and testing parameters were set, an optimal aging duration and temperature were recommended.

\subsection{SELECTION OF AGING EQUIPMENT}

There are three types of AC laboratory aging equipment that are currently available: forced-draft oven, pressure aging vessel (PAV), and ultraviolet device. The forced-draft oven is the most available aging equipment, and it has received the most attention in other research studies. A pressure aging vessel introduces high pressure during the aging process, which can accelerate aging significantly. An ultraviolet device considers the UV effect on AC mixture's aging to simulate real-life aging. Table 4.1 compares the equipment in detail, including their pros and cons.

Table 4.1. Comparison of Aging Equipment

\begin{tabular}{|l|l|l|}
\hline \multirow{2}{*}{$\begin{array}{l}\text { Forced-Draft } \\
\text { Oven }\end{array}$} & Pros & $\begin{array}{l}\text { a) Availability, feasibility, and practicability } \\
\text { b) High capacity allows it to accommodate a large amount of material for aging }\end{array}$ \\
\cline { 2 - 3 } & Cons & $\begin{array}{l}\text { a) Variability among ovens, especially in terms of air drafting } \\
\text { b) Lower aging rate compared to PAV }\end{array}$ \\
\hline \multirow{3}{*}{ PAV } & Pros & $\begin{array}{l}\text { a) High pressure can shorten the aging time } \\
\text { b) Limited equipment variability }\end{array}$ \\
\cline { 2 - 3 } & Cons & $\begin{array}{l}\text { a) Limited capacity } \\
\text { b) Integrity of compacted samples during and after aging (Figure 4.1) }\end{array}$ \\
\hline \multirow{3}{*}{$\begin{array}{l}\text { Ultraviolet } \\
\text { Device }\end{array}$} & $\begin{array}{l}\text { a) Most relevant to field aging } \\
\text { b) Aging time may be quantitatively calculated }\end{array}$ \\
\cline { 2 - 3 } & Cons & $\begin{array}{l}\text { a) Availability } \\
\text { b) Limited capacity } \\
\text { c) Slow aging rate and long aging periods }\end{array}$ \\
\hline
\end{tabular}

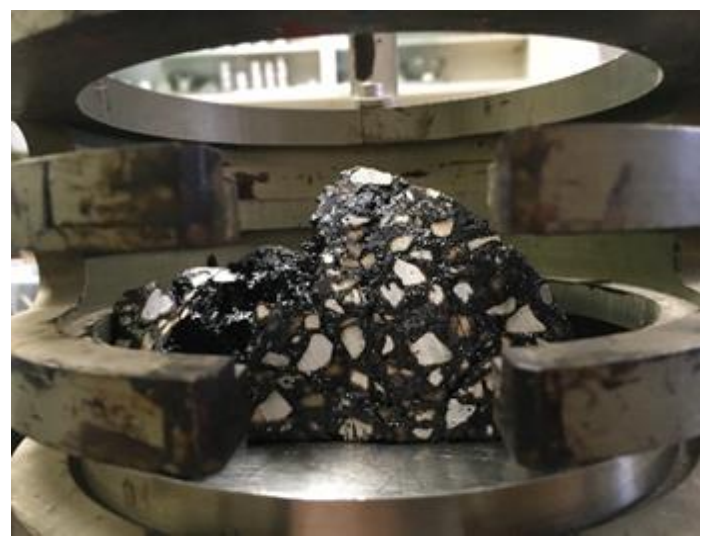

Figure 4.1. I-FIT specimen deformed after PAV aging. 
The goal of this project is to develop a practical aging protocol that can be readily implemented by IDOT and contractors. Therefore, using readily available equipment is a preferred option with an acceptable long-term field aging simulation capability. Therefore, the available equipment that allows technicians to age enough materials in a short period of time should be considered first. Moreover, since I-FIT is a performance test, maintaining specimen integrity throughout the aging process is important. In this case, the forced-draft oven was determined to be the most suitable equipment for a long-term aging protocol for I-FIT specimens. It was recognized that using a forced-draft oven may introduce additional variability due to air drafting. However, Lemke et al. (2018) investigated three most commonly used forced-draft ovens (Figure 4.2) and found that oven type did not have a significant effect on AC mixtures' I-FIT results after long-term aging.

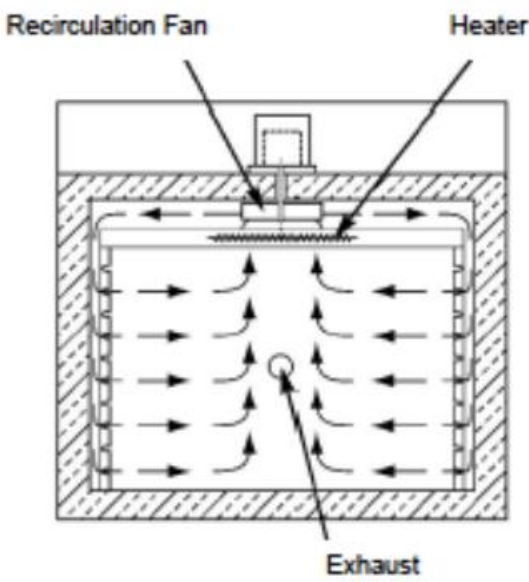

(a)

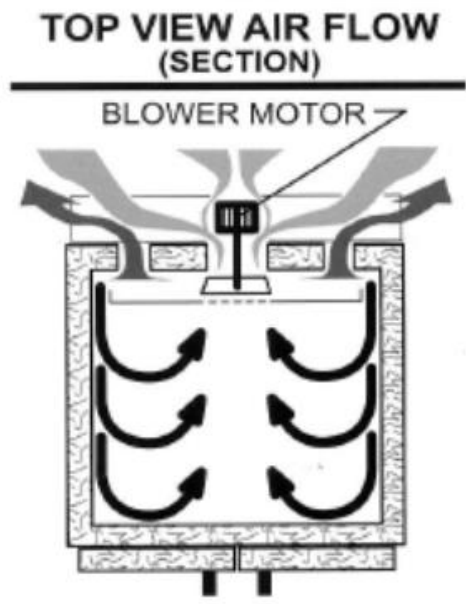

(b)

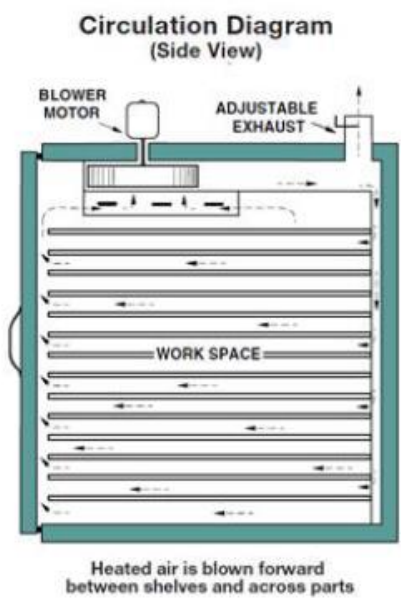

(c)

Figure 4.2. Most used forced-draft ovens (Lemke et al. 2018).

\subsection{STATE OF MATERIAL DURING AGING}

After a forced-draft oven has been selected as the aging equipment, the state of material during aging (I-FIT specimen vs. loose mixture) was determined. There are some advantages of aging compacted AC specimens as compared to aging loose $A C$ mix reported in the literature. The current standard long-term procedure defined in AASHTO R3O recommends the aging of gyratory compacted cylinders (pills). This method is practical, efficient, and operation variability is limited. However, specimen integrity (excessive distortions affecting geometry) and aging gradient (gradual reduction of aging from the surface of the specimen towards the center) are two issues with using compacted specimens for aging (Reed 2010; Houston et al. 2005). Distortion refers to the changes of air void contents and specimen dimensions after aging; while oxidation gradient leads to inconsistent properties throughout a specimen. These concerns were investigated in this study and the results are presented in Section 4.2.1.

Loose AC samples, on the other hand, were used in the development of long-term aging protocols in some of the recent studies, including the recently completed NCHRP 9-54 project (Partl et al. 2012; Van den Bergh 2011; Mollenhauer et al. 2011; Kim et al. 2018). Loose mixture aging was preferred in those studies for several reasons. Firstly, distortion is not a concern of loose AC mix aging; specimens 
are compacted after the aging process. Secondly, the aging gradient can be better controlled with stirring the $A C$ materials. Thirdly, loose $A C$ mix aging is believed to occur faster than that of compacted specimens because of larger surface area of binder film exposed to air. However, there are some drawbacks of loose mix aging, which may hinder the implementation of this approach. Binder becomes stiffer after aging, and hence, compaction becomes more difficult. The effect of higher gyratory effort and stirring may introduce additional variability to the test results. In addition, if loose mix is aged, then the air void content of the compacted and prepared test specimen may be outside the specification, which may require specimen re-fabrication. Using I-FIT specimens will save time and materials.

Both I-FIT specimen and loose AC mix aging methods were investigated in this study for selected plant mixes (PM1, 2, 3, 5, 6) and lab mix (LM1). A detailed comparison of the results is presented in Section 4.2.2.

\subsubsection{I-FIT Specimen Integrity during Aging}

Because of different thermal expansion coefficients for binder and aggregate at aging temperatures and creep due to specimen's own weight, the integrity of the specimens can be compromised under prolonged exposure to high temperatures. Hence, air voids and dimensions may change after aging. This problem becomes more significant for larger specimens and longer aging periods at higher temperatures. Kim et al. (2018) suggested using relatively small specimens to control this drawback. Distortion was checked by comparing the air voids and dimensions before and after aging. In addition, oxidation gradient is discussed in this section.

Two plant-produced AC mixtures (PM2 and PM3) and one lab-produced mix (LM1) were tested. The compacted specimens' air void contents were measured before and after one, three, five, and seven days of aging at three temperatures: $167,185,203^{\circ} \mathrm{F}\left(75,85,95^{\circ} \mathrm{C}\right)$; Figure 4.3 shows the results.

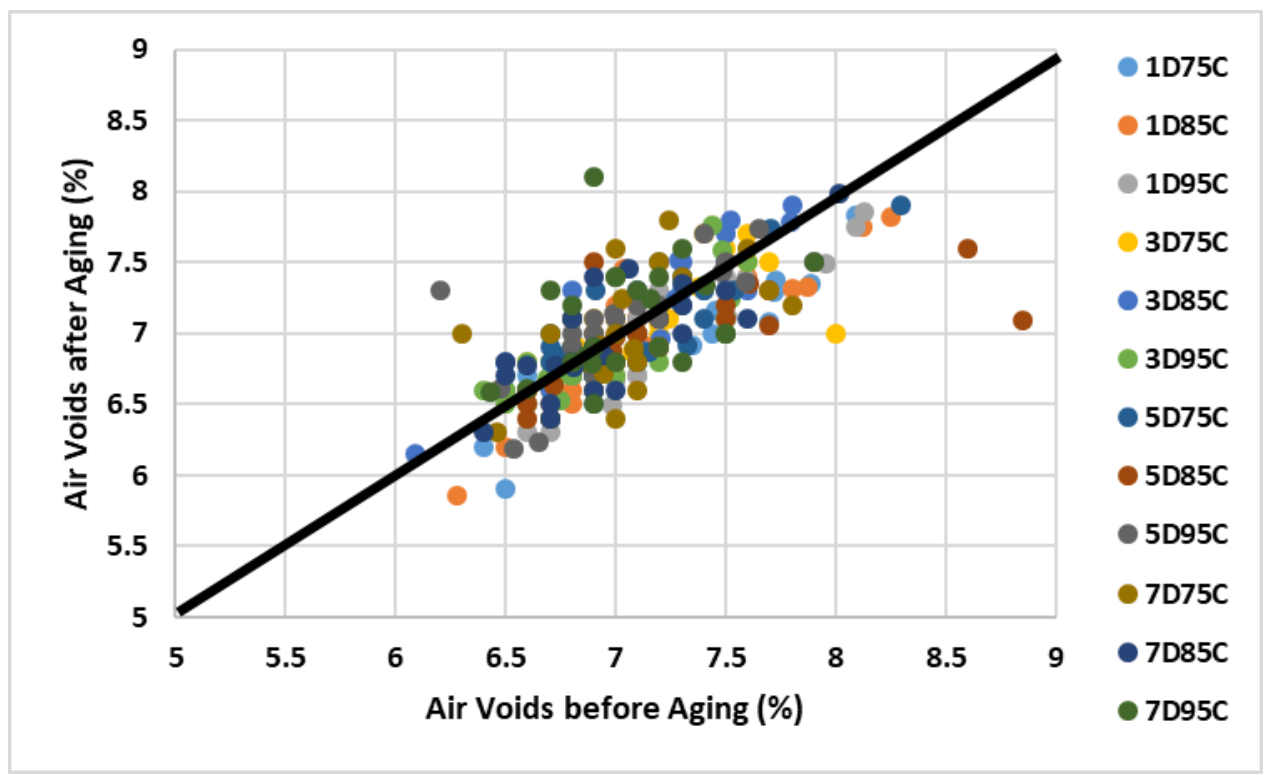

Figure 4.3. Air voids comparison before and after aging. 
Most of the data points lie on or near to the equality line in Figure 4.3, which suggests that air void changes before and after aging are limited. Also, various aging durations and temperatures do not show any specific trends, which implies that these changes are random.

Each of the specimen's dimensions, including thickness, ligament length, and notch length, were measured before and after the aging process for selected aging protocols: $1 D / 95 C, 3 D / 95 C$, and 5D/85C. Results are shown in Figure 4.4.

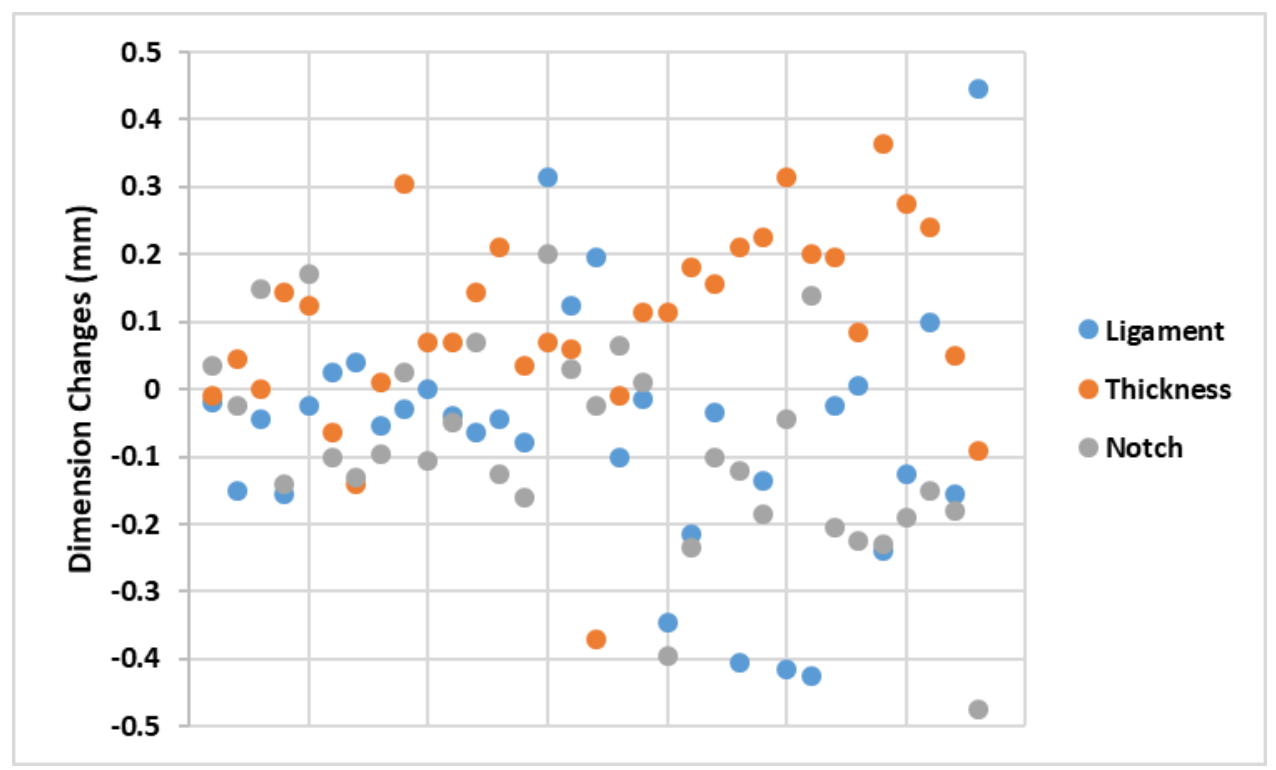

(a)

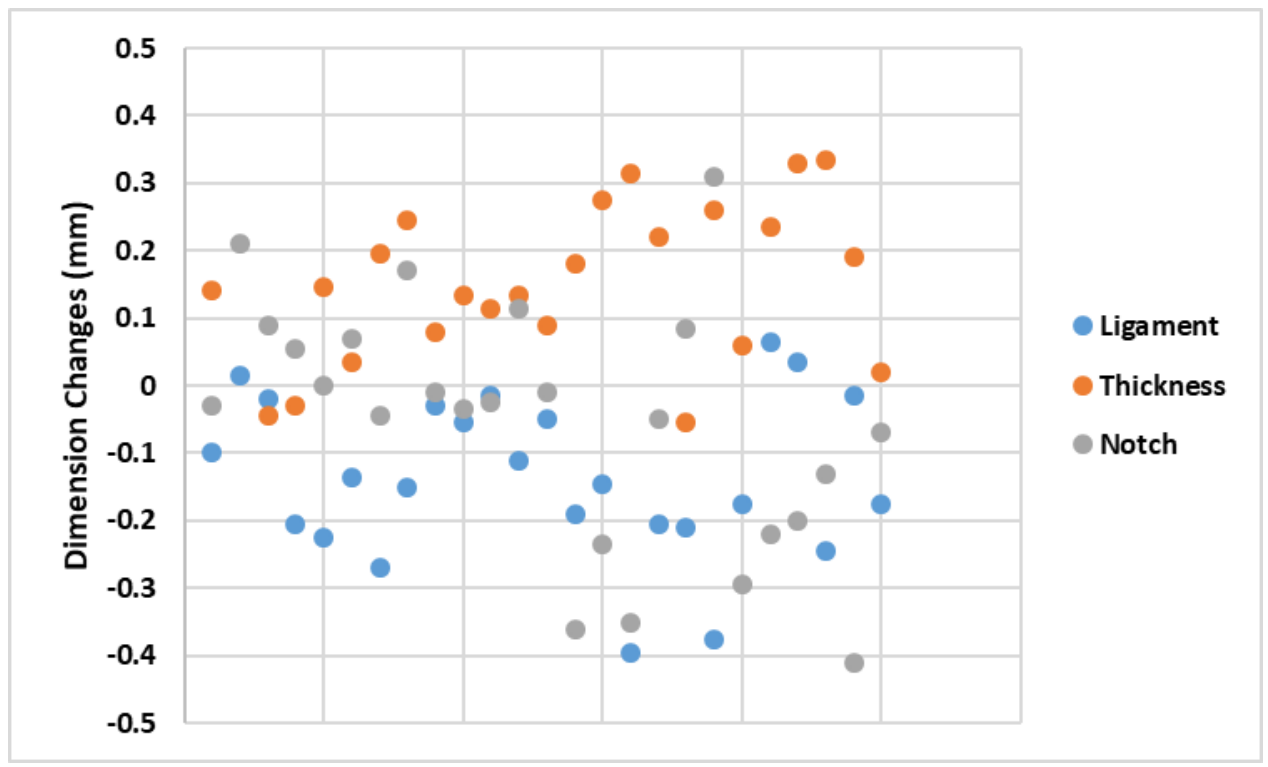

(b) 


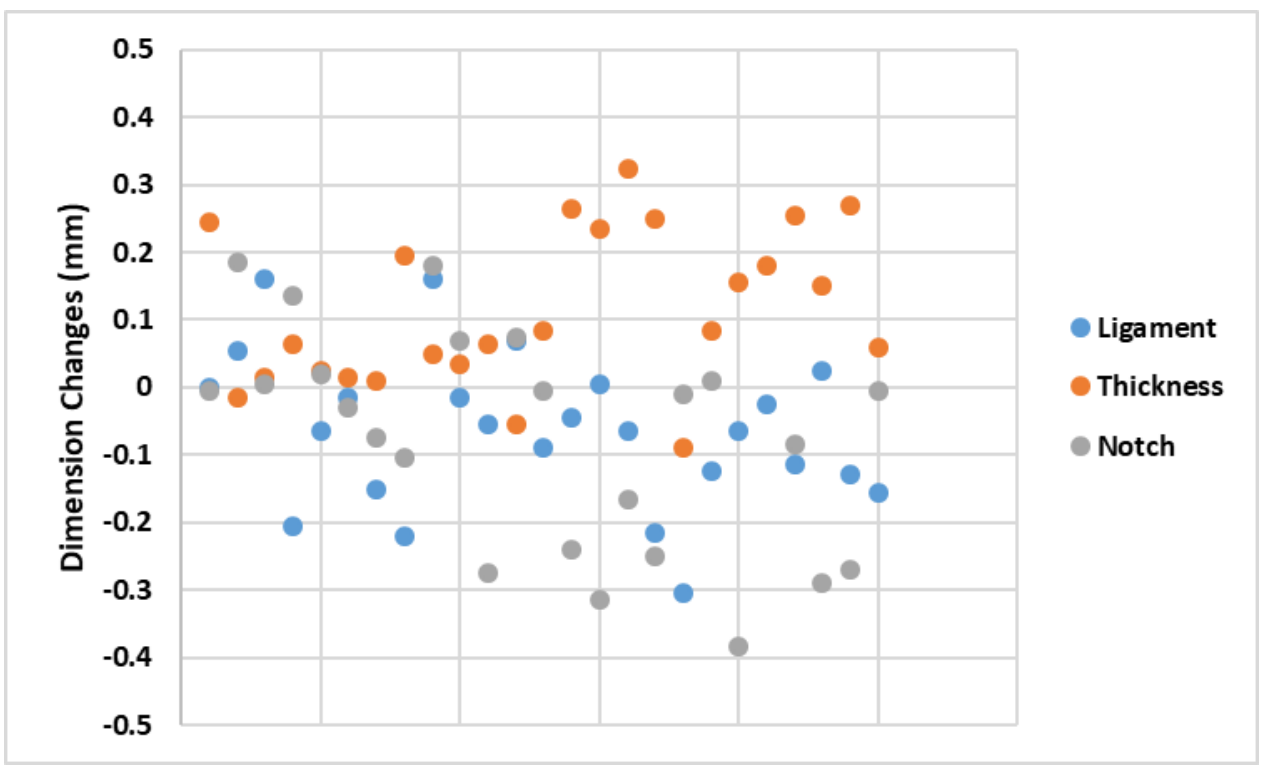

(c)

Figure 4.4. Dimension changes after aging: (a) 1D/95C; (b) 3D/95C; (c) 5D/85C.

As shown in Figure 4.4, most of the specimens had dimension changes smaller than $0.01 \mathrm{in}(0.3 \mathrm{~mm})$ and all of them were smaller than $0.02 \mathrm{in}(0.5 \mathrm{~mm})$. The changes are random and mainly resulted from operational variability. Hence, it is concluded that lab-simulated aging using forced-draft has limited impact on I-FIT specimen geometry.

\subsubsection{I-FIT Results for Compacted and Loose Mix Aged Specimens}

Both compacted and loose AC mix specimens were investigated for the effect of aging for selected plant mixes (PM1, PM2, PM3, PM5, and PM6) and lab-produced mix (LM1). The specimens were tested at varying aging durations and temperatures; one, three, five, and seven days of aging at 75, 85 , and $95^{\circ} \mathrm{C}$. A detailed comparison of the results is presented in Figure 4.5 through Figure 4.10. The right and left columns, in the histogram, for each aging condition represent aged I-FIT specimen and loose mixture results, respectively. Fracture energy and post-peak load slope are represented by bars in different colors, while $\mathrm{Fl}$ is shown in colored dots.

As shown in Figure 4.5 through Figure 4.10, aged loose AC mixture typically resulted in steeper postpeak slopes and lower FI than aged I-FIT specimens at the same temperature and aging duration. This suggests that aged loose AC mixture has a greater crack growth rate than aged I-FIT specimen. This could be related to faster aging. 


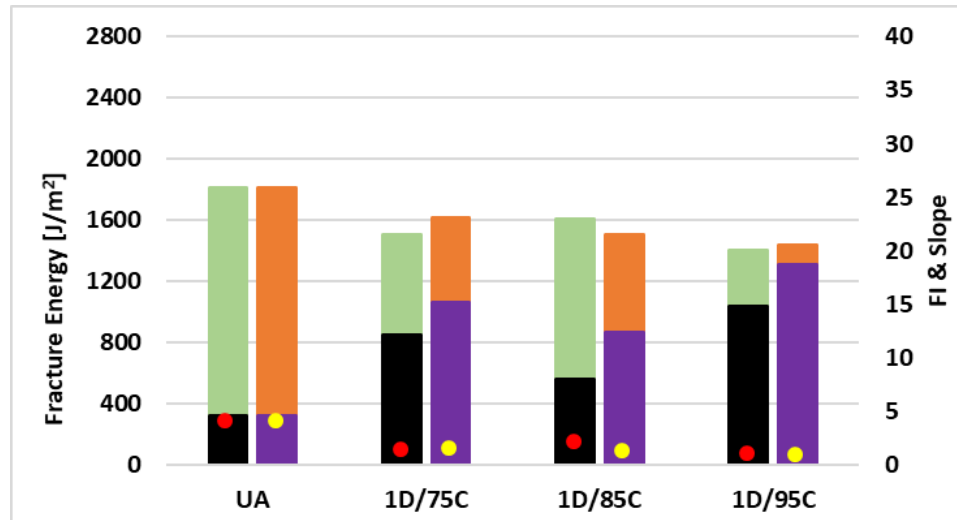

FE Comp. FE Loose $\square$ Slope Comp. ESlope Loose $\bullet$ FI Comp. FI Loose

Figure 4.5. Plant Mix 1, I-FIT results comparison.

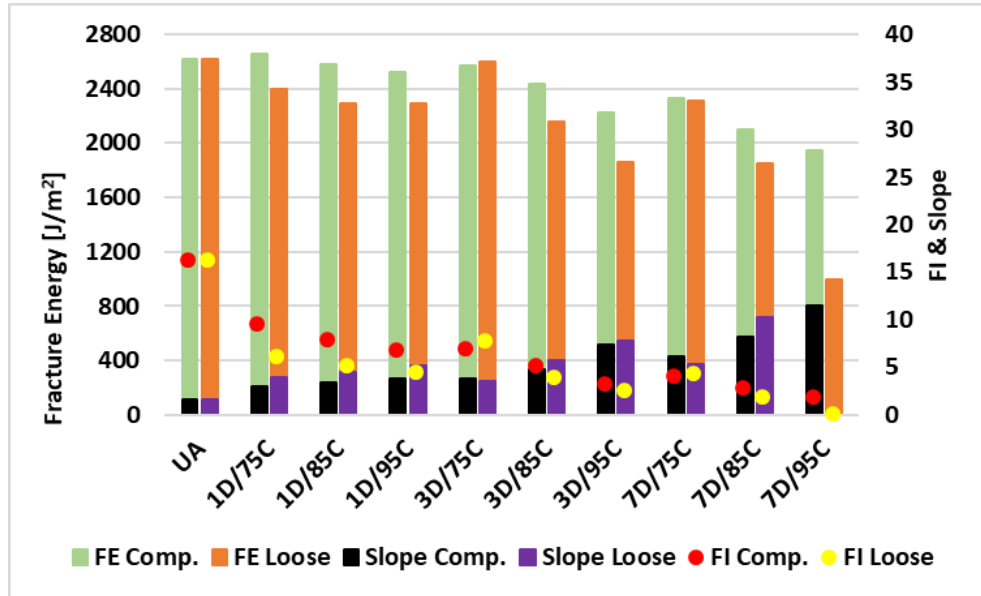

Figure 4.6. Plant Mix 2, I-FIT results comparison.

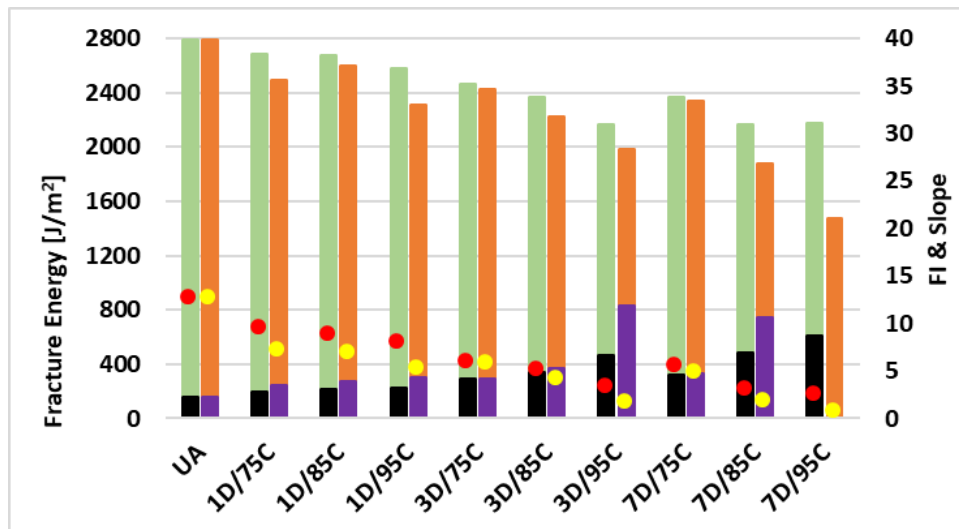

FE Comp. FE Loose $\square$ Slope Comp. I Slope Loose • FI Comp. FI Loose

Figure 4.7. Plant Mix 3, I-FIT results comparison. 


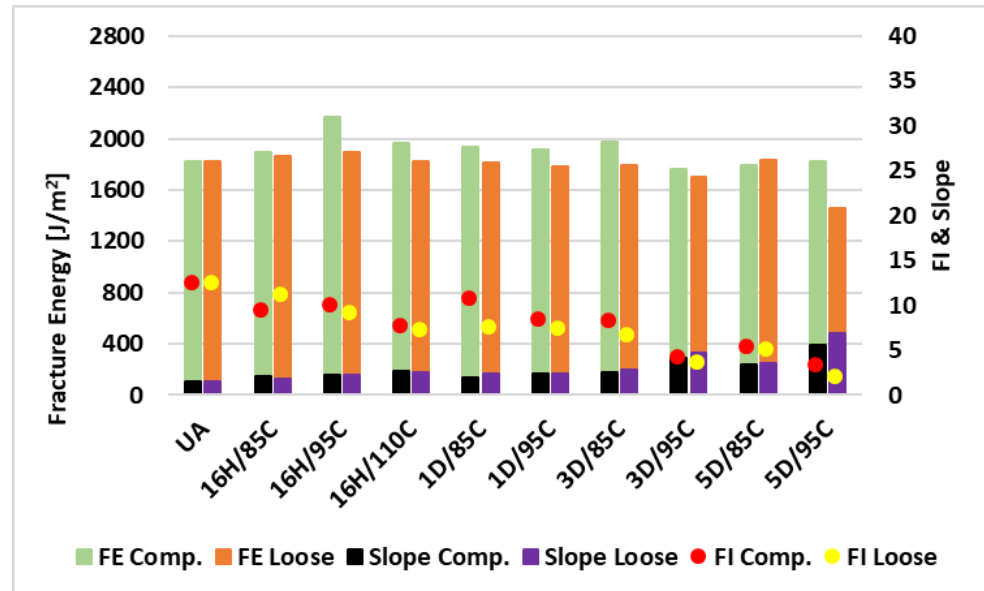

Figure 4.8. Plant Mix 5 I-FIT results comparison.

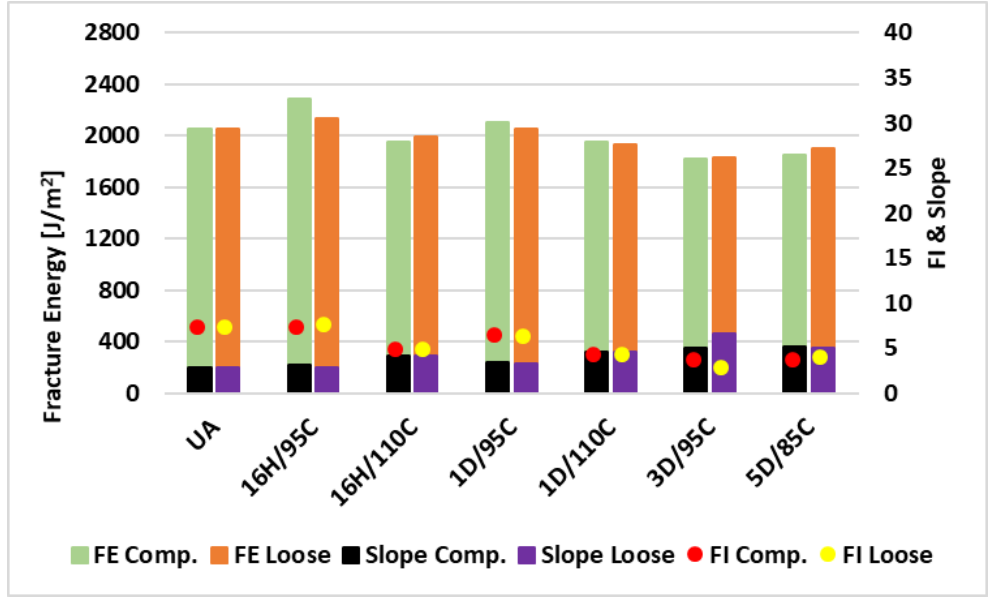

Figure 4.9. Plant Mix 6, I-FIT results comparison.

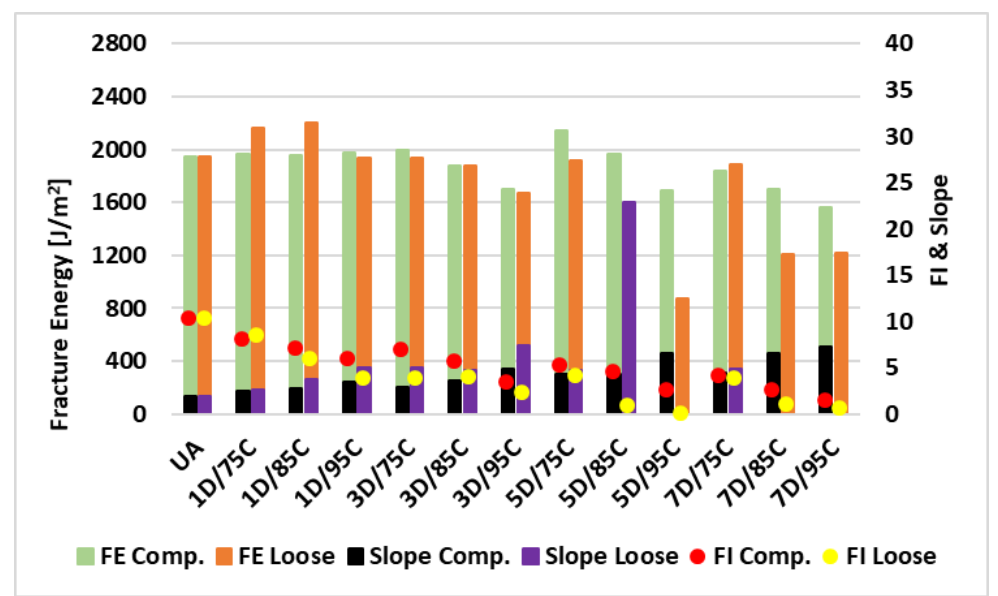

Figure 4.10. Lab Mix 1, I-FIT results comparison. 
The experimental data support the literature findings about the faster rate of aging of loose mixes compared to compacted specimens. However, the change in the rate of aging could also be AC mix dependent. Table 4.2 provides the pros and cons of the two aging methods with respect to I-FIT.

Table 4.2. Pros and Cons of Aged Compacted and Loose AC Mixture

\begin{tabular}{|l|l|l|}
\hline \multirow{2}{*}{ I-FIT Specimen Aging } & Pros & $\begin{array}{l}\text { a) Practicability } \\
\text { b) Limited operation variability }\end{array}$ \\
\hline & Cons & $\begin{array}{l}\text { a) Aging gradient } \\
\text { b) Slower aging rate }\end{array}$ \\
\hline \multirow{2}{*}{ Loose Mix Aging } & Pros & $\begin{array}{l}\text { a) Higher aging rate } \\
\text { b) Limited aging gradient } \\
\text { c) Specimen integrity }\end{array}$ \\
\cline { 2 - 3 } & Cons & $\begin{array}{l}\text { a) Stirring is needed during aging process } \\
\text { b) Operational variability } \\
\text { c) Compaction difficulty }\end{array}$ \\
\hline
\end{tabular}

Based on evaluation of both methods of aging using the forced-draft oven, this study concluded that compacted I-FIT specimen aging is the most appropriate method for the long-term AC aging protocol; although aged loose AC samples may be used for research.

\subsection{I-FIT RESULTS OF LONG-TERM AGED AC}

The next step was to evaluate the FI decay of various plant-produced and lab-produced specimens with temperature and aging duration. Figure 4.11 presents typical I-FIT load-displacement curves for PM2 specimens for one day, three days, five days, and seven days at various temperatures.

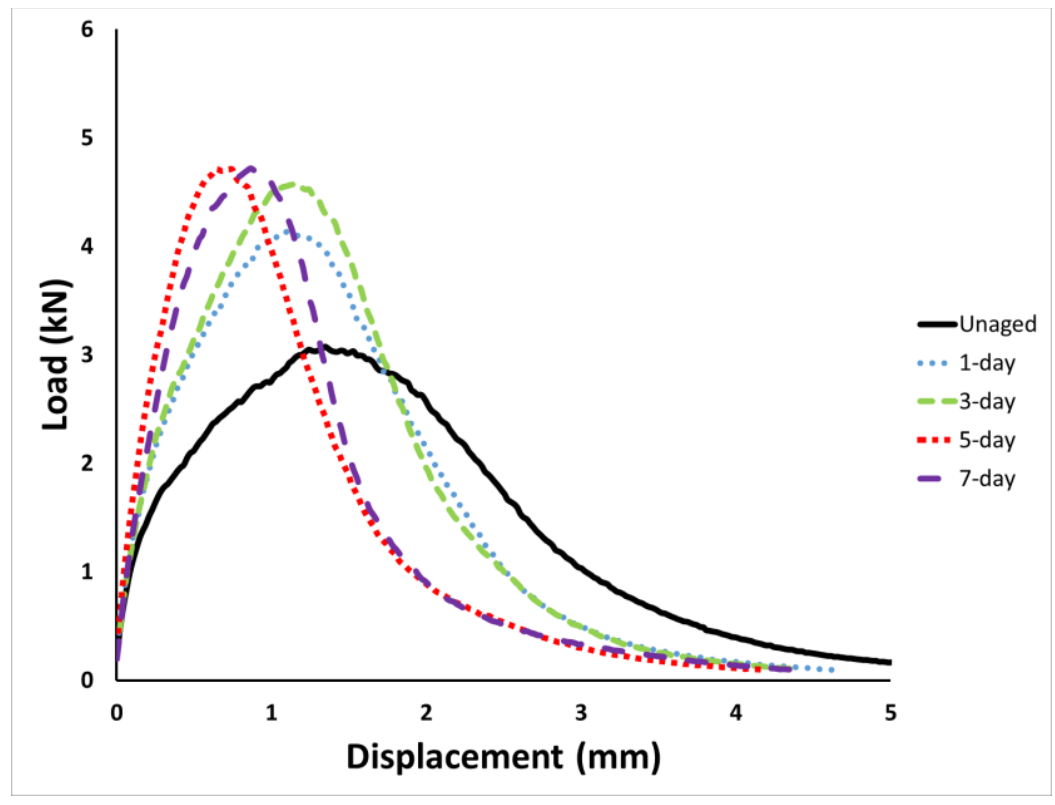

(a) 


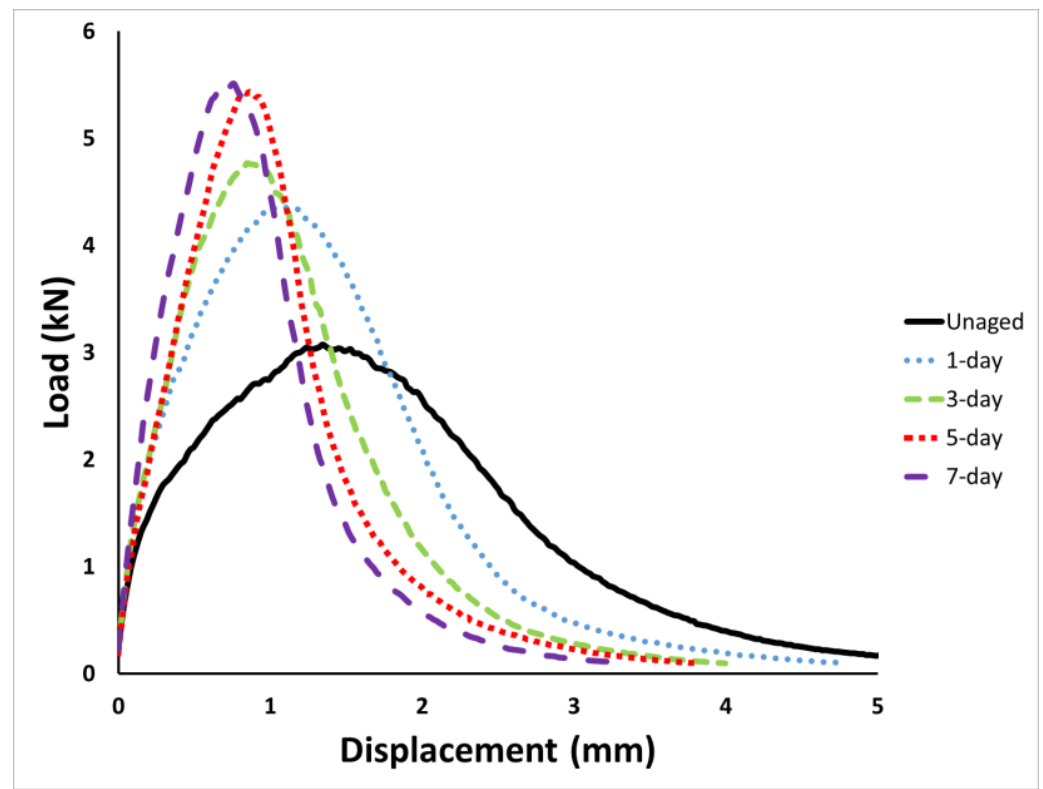

(b)

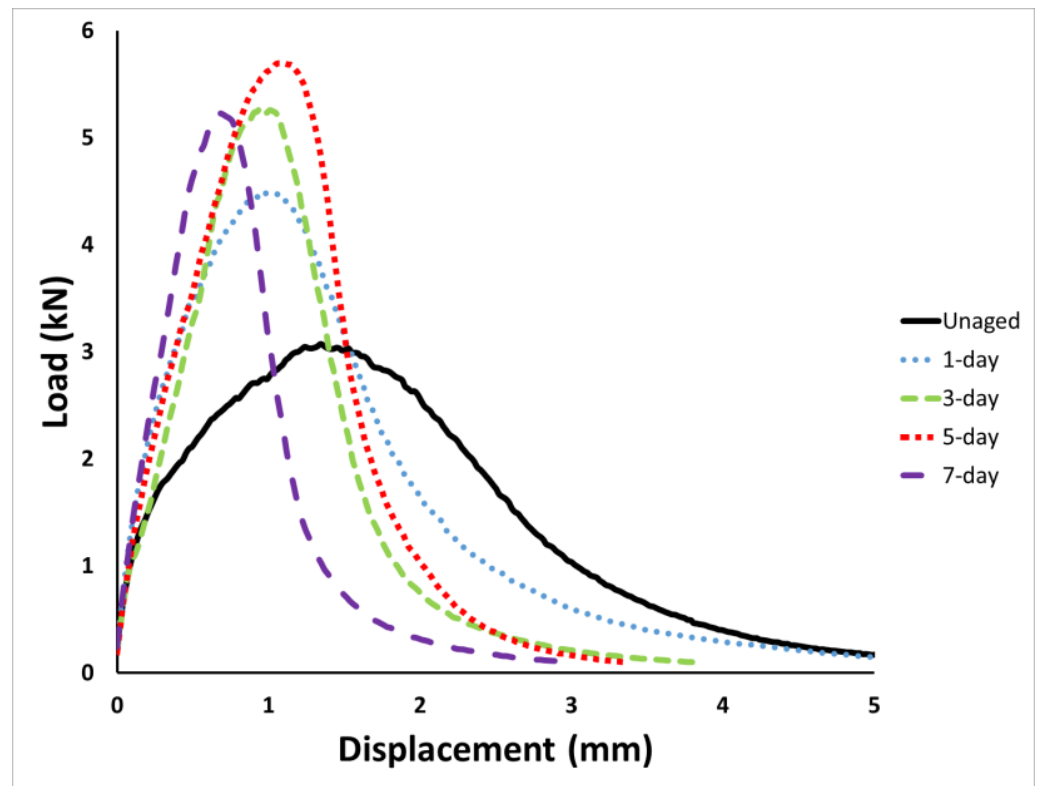

(c)

Figure 4.11. Loading vs. displacement curves of unaged and aged PM2 specimens for various periods at: (a) $75^{\circ} \mathrm{C}$, (b) $85^{\circ} \mathrm{C}$, and (c) $95^{\circ} \mathrm{C}$.

The change in the load-displacement curves after aging is evident. It was observed that the peak load and pre-peak slope (represents the rate of crack growth) significantly increased with any aging (both time and temperature). The post-peak slope became steeper with aging, indicating the rapid crack growth due to material brittleness increase. This also results in reducing the time to peak load with 
aging. The effects on pre-peak slope, peak load, and post-peak slope are most pronounced after one day of aging.

Fracture energy, slope, and FI were calculated for each AC mix at different aging conditions. Figure 4.12 illustrates I-FIT results for all the AC mixes under various aging conditions. The green bar represents FE. Post-peak load slope is represented by a black bar, while FI is represented by a red dot. Plant Mixture 1,1D/95C aged results are not completely shown; numerical issues were encountered in calculating the slope and FI because specimens were very brittle.

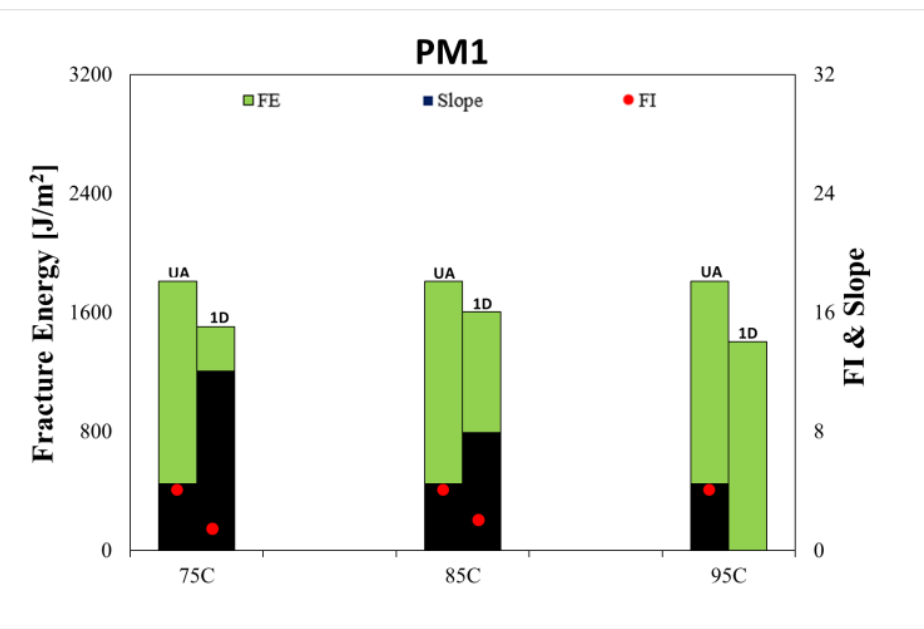

(a)

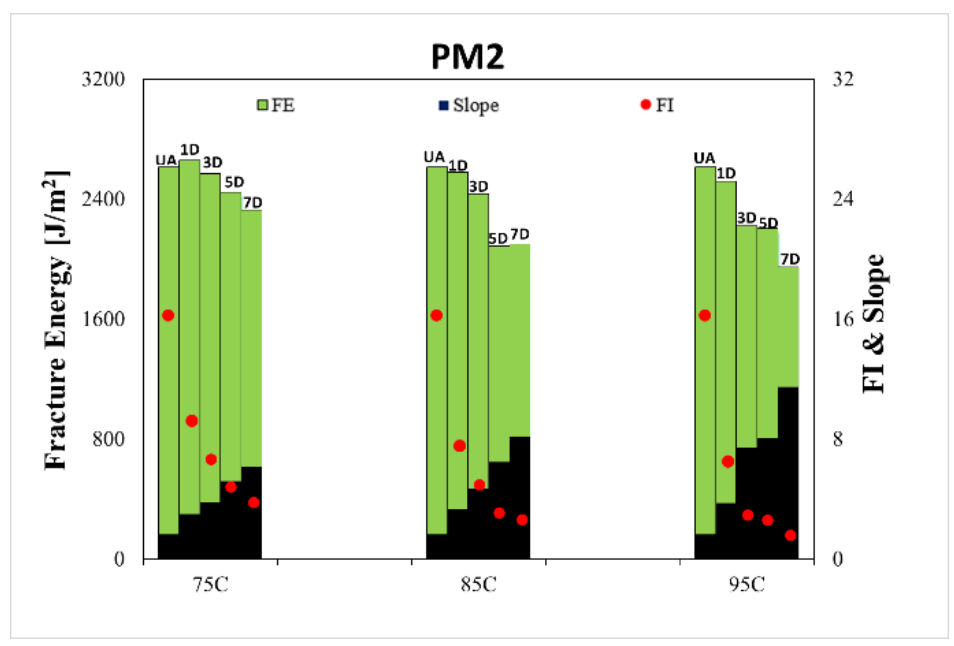

(b) 


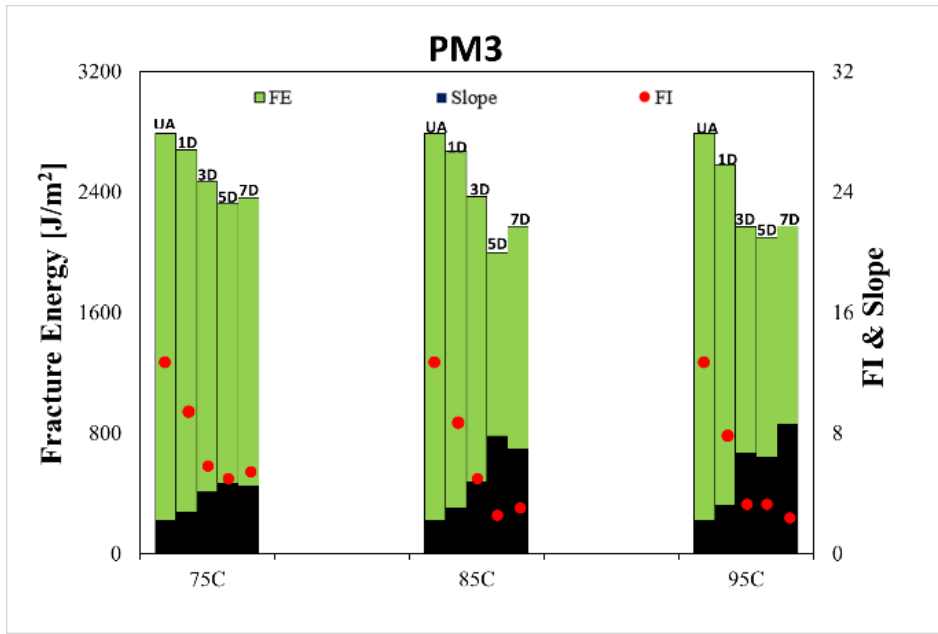

(c)

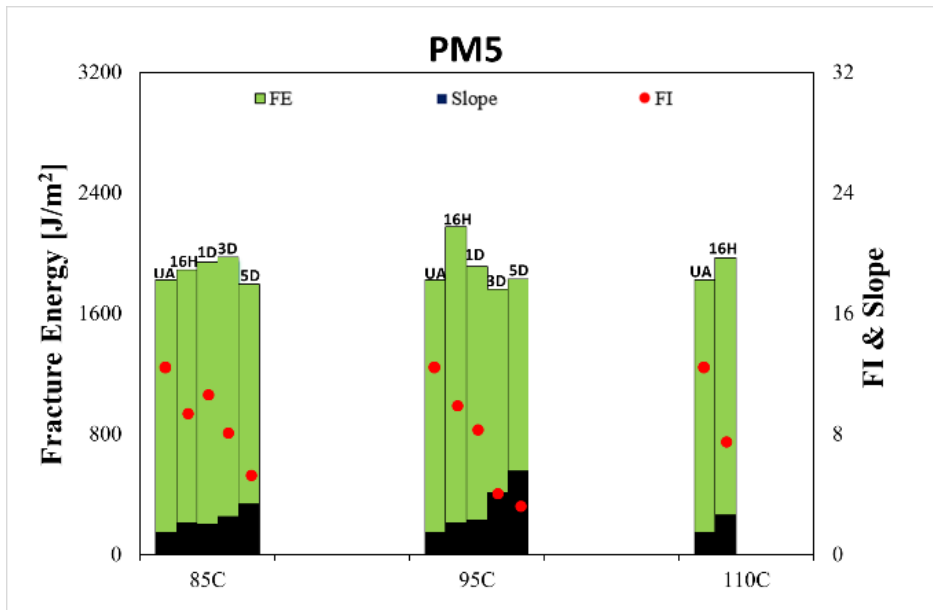

(d)

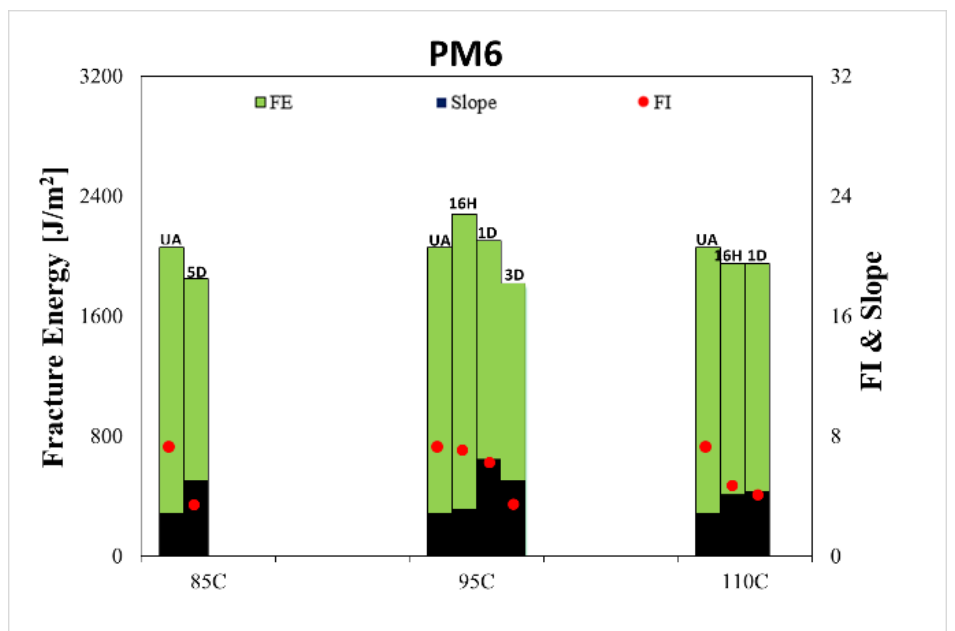

(e) 


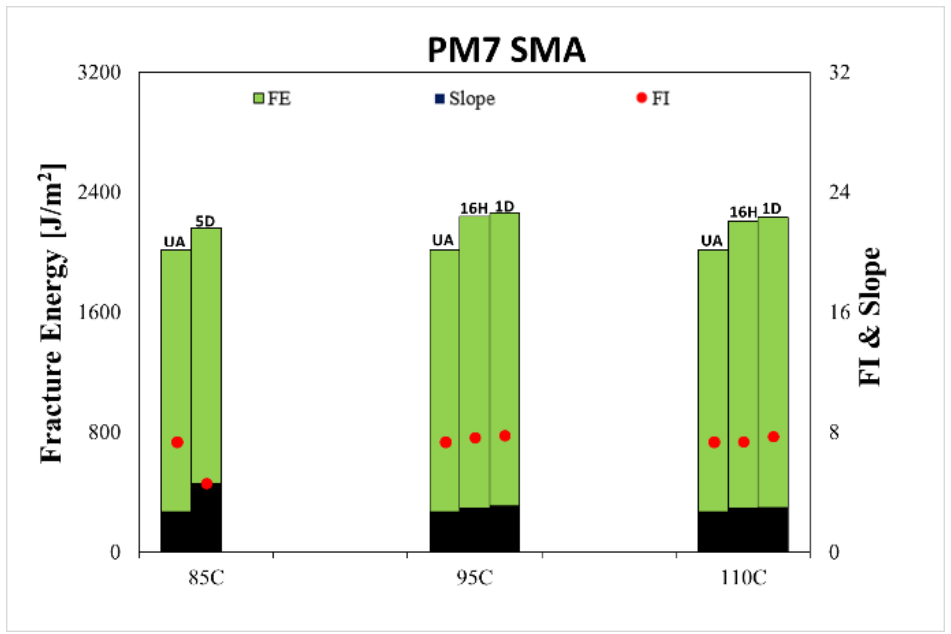

(f)

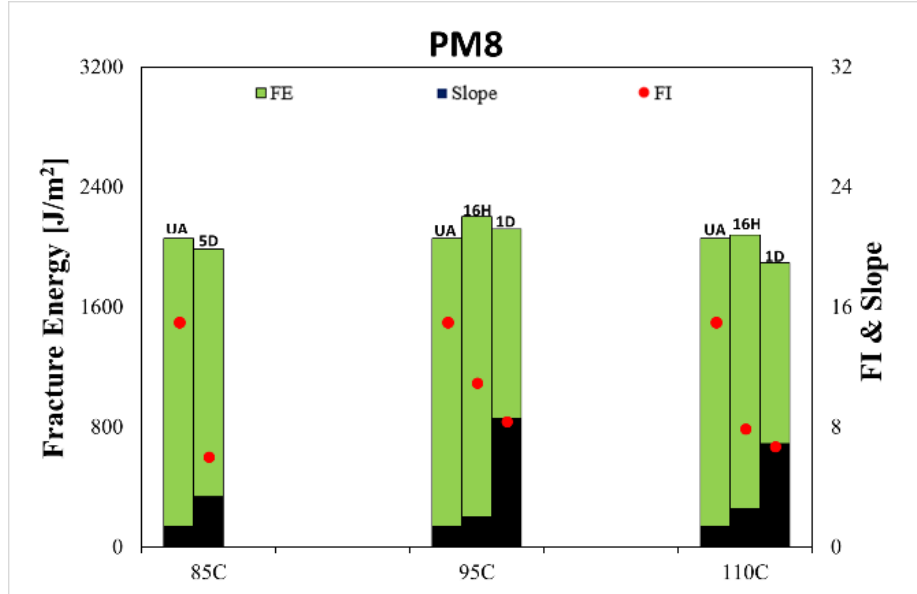

(g)

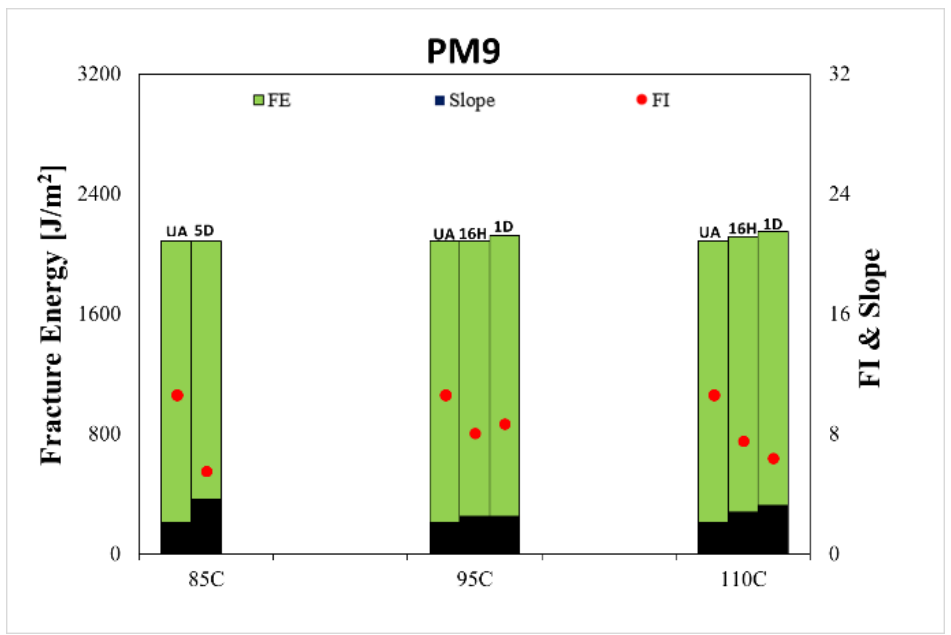

(h) 


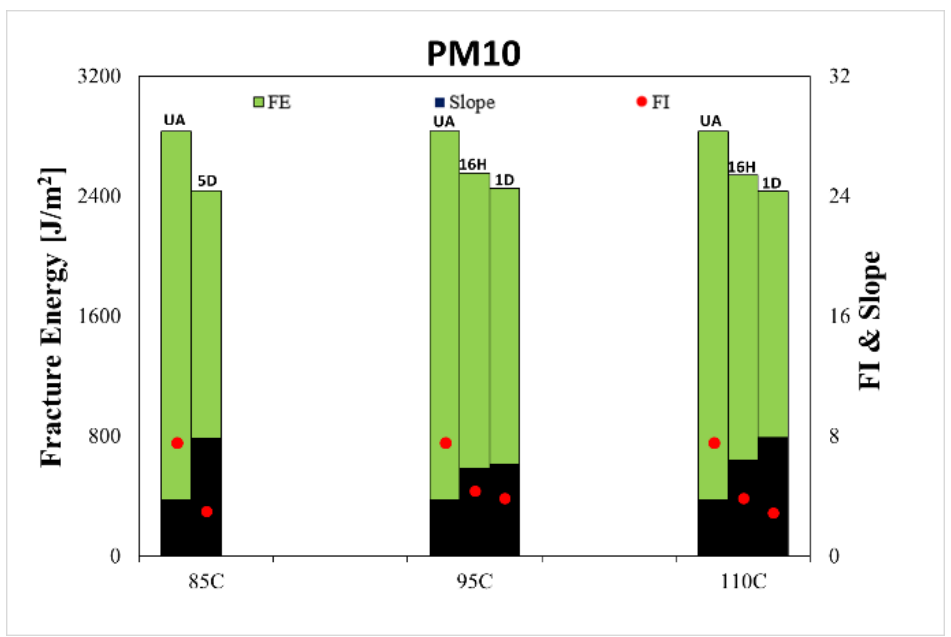

(i)

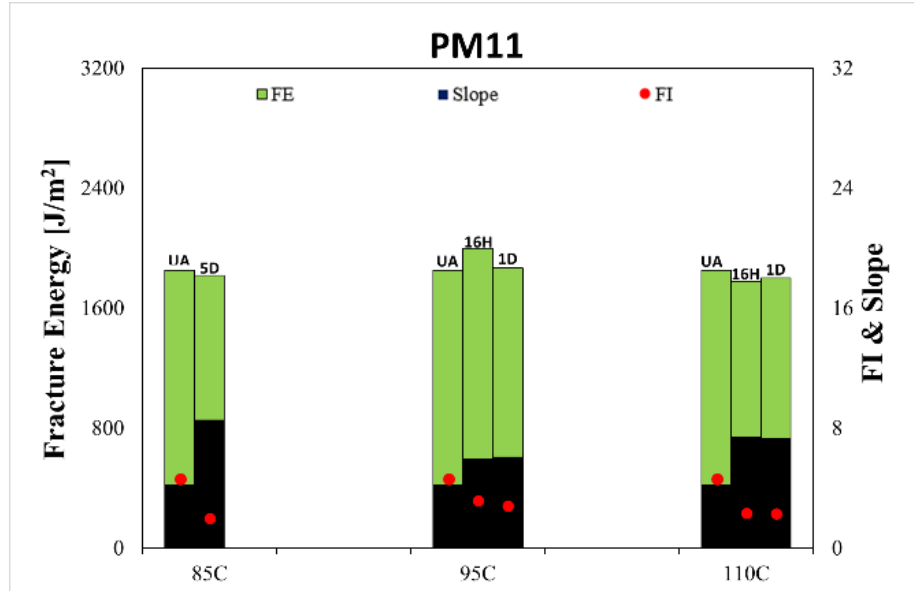

(j)

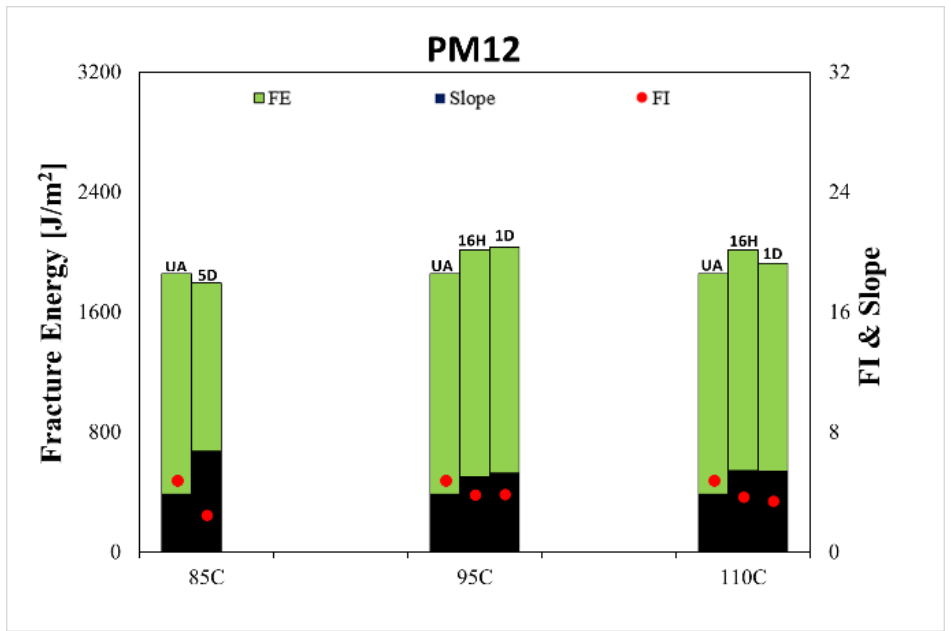

(k) 


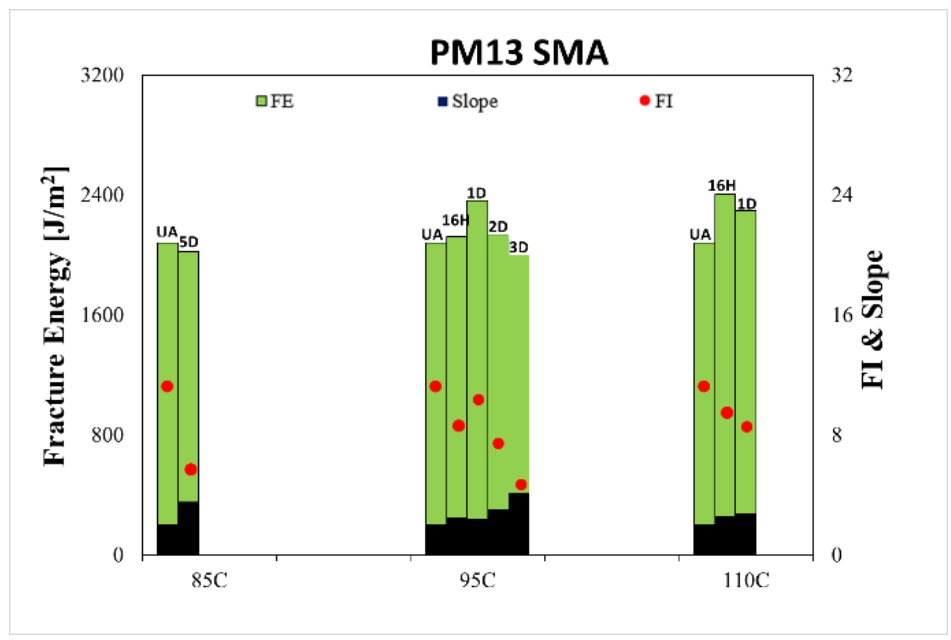

(I)

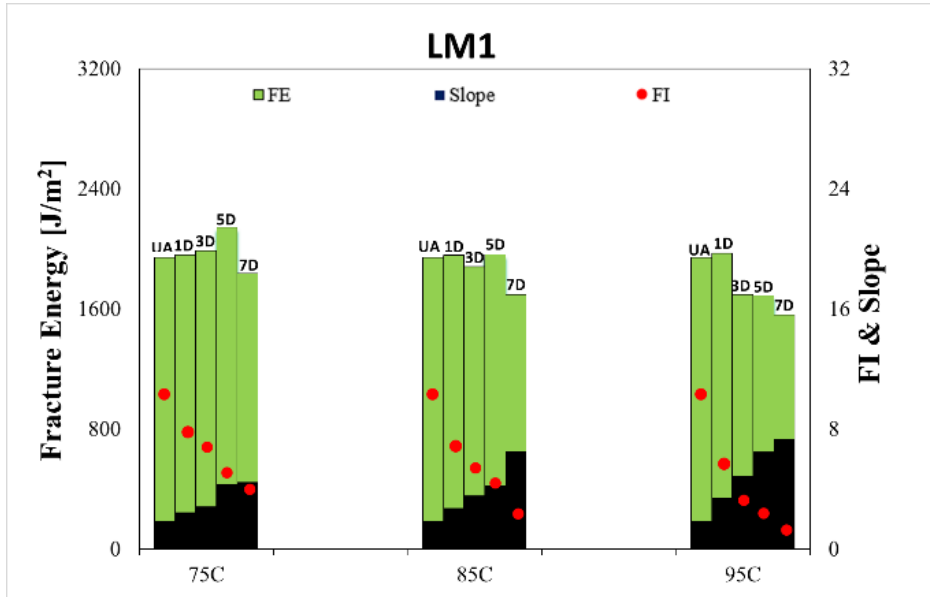

(m)

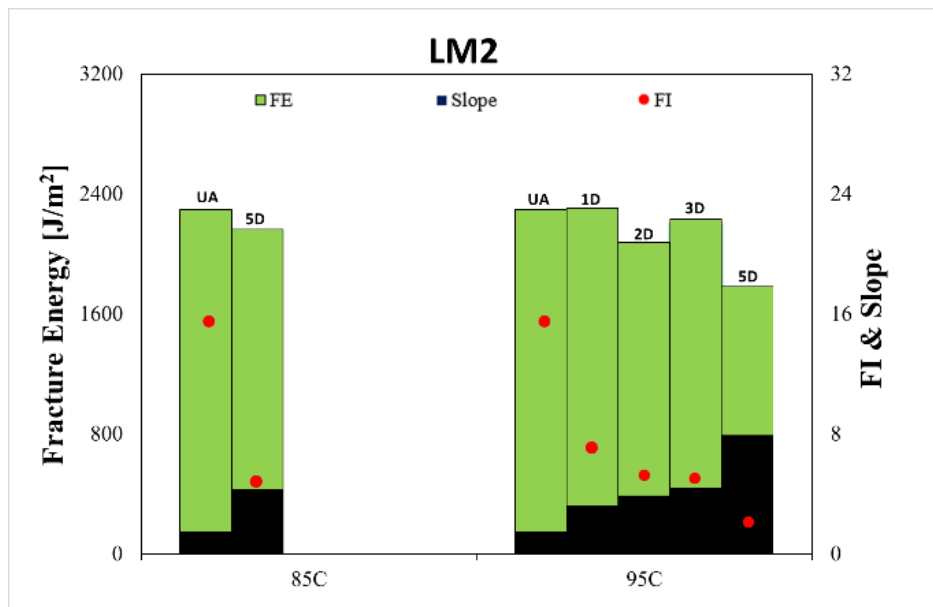

(n) 


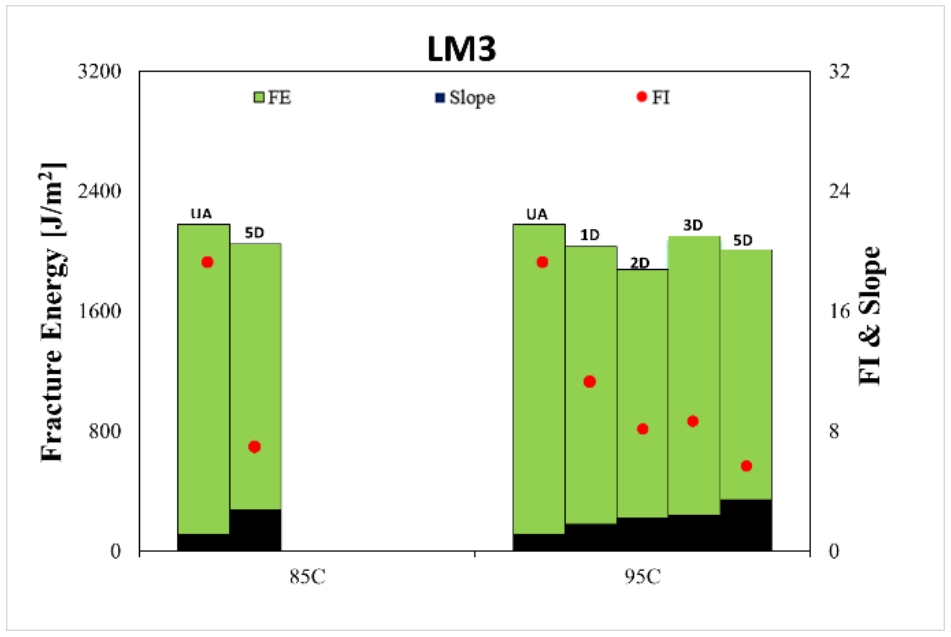

(o)

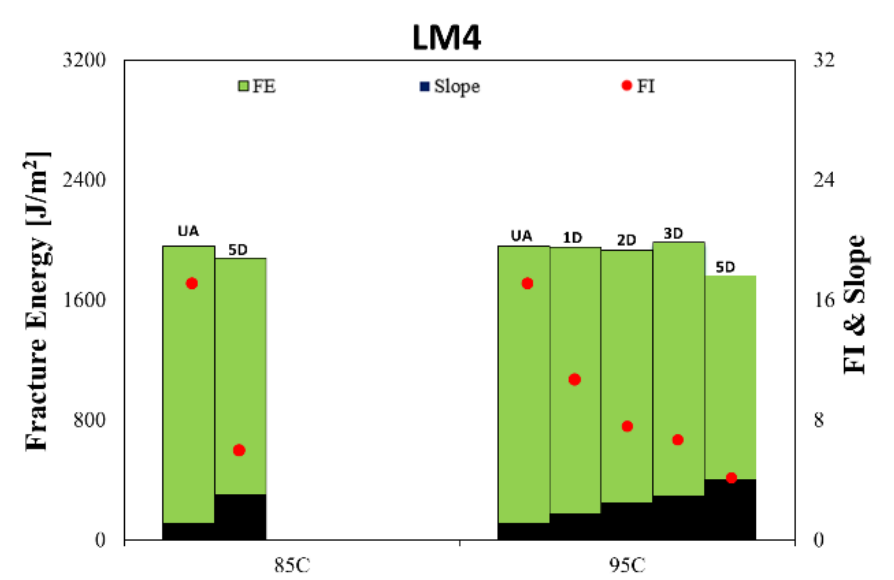

(p)

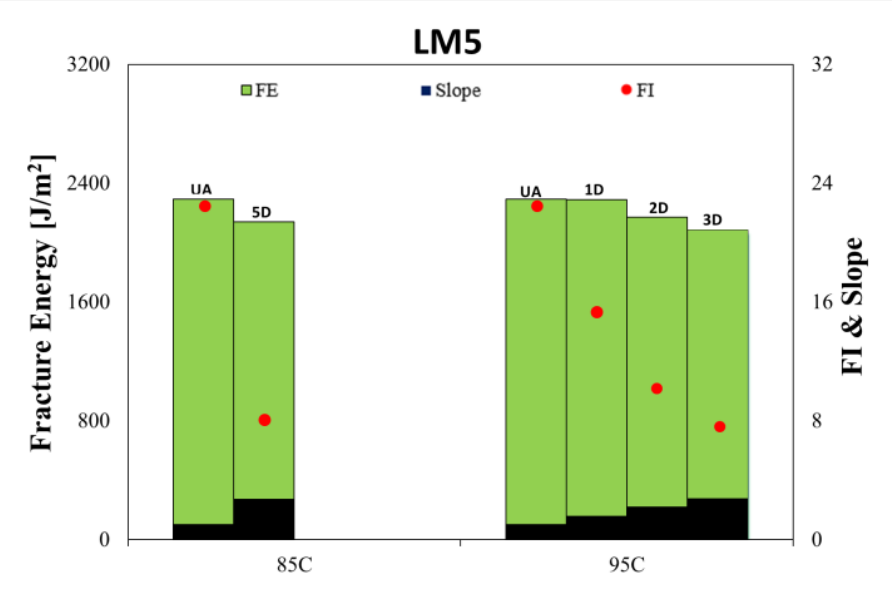

(q) 


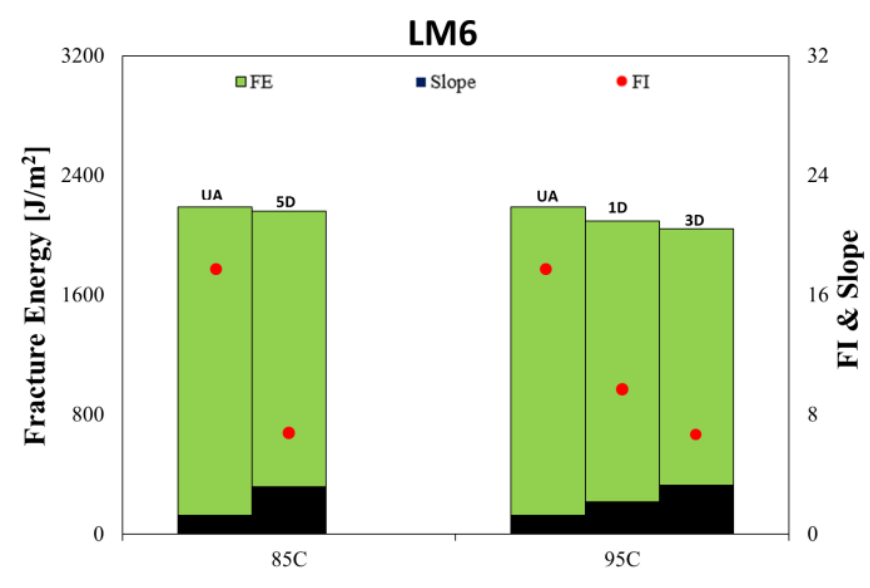

$(r)$

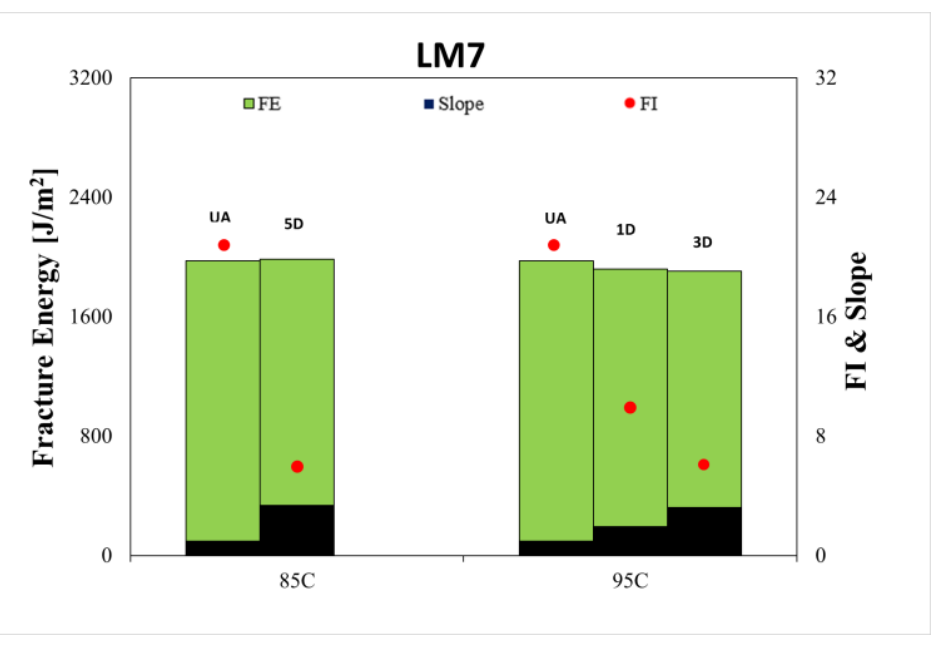

(s)

Figure 4.12. Unaged and Aged I-FIT results for all AC mixes.

As shown in Figure 4.12, the post-peak slopes, in general, increased with aging. It is interesting that the slope is positively and linearly correlated to aging time. However, there is no trend between fracture energy (FE) and aging; FE shows a drop if AC experienced severe aging.

Fl decreased with aging for all $\mathrm{AC}$ mixes under all aging conditions, excluding PM7 (SMA) under some early aging conditions (16H/95C, 1D/95C, 16H/110C, and 1D/110C). Fl decay curves with aging are convex functions, and they become flat when aging time increases significantly, especially after five days of aging. The rate of $\mathrm{FI}$ reduction (or aging rate) is dependent upon the AC mixture design and constituents. This is further addressed in Section 4.5.

Binder source plays an important role in aging development. LM1 and LM2 have the same mix design, but a different binder source. LM2 shows a higher FI under all aging conditions. Similarly, LM3, LM4 and LM5 have a different binder source, although all have the same mix design. The FI differences 
could be caused by different binder chemical compositions and/or refinery process. Each mix uses a binder with a different "true" PG: LM1 and LM2 use PG 68.9-21.6 and PG 66.7-22.3 binders; while LM3, LM4, and LM5 use PG 60.4-29.6, PG 59.3-28.3, and PG 60.7-28.7 binders, respectively.

Moreover, it can be noted that the m-value of binder significantly affects the flexibility index of AC. As shown in Figure 4.13, mixes that have the same design using binders with higher m-values resulted in a significant increase in FI under all conditions. LM6 and LM7 were excluded from the previous observation. LM7 used binder with $9 \%$ re-refined engine oil bottoms (ReOB), which induced a higher m-value and a higher unaged FI than LM6. However, it showed lower FI than LM6 after long-term aging. This shows that $\mathrm{ReOB}$ is an additive that has a negative impact on the long-term performance of AC and agrees with the findings of ICT project R27-SP28 (Ozer et al. 2016).

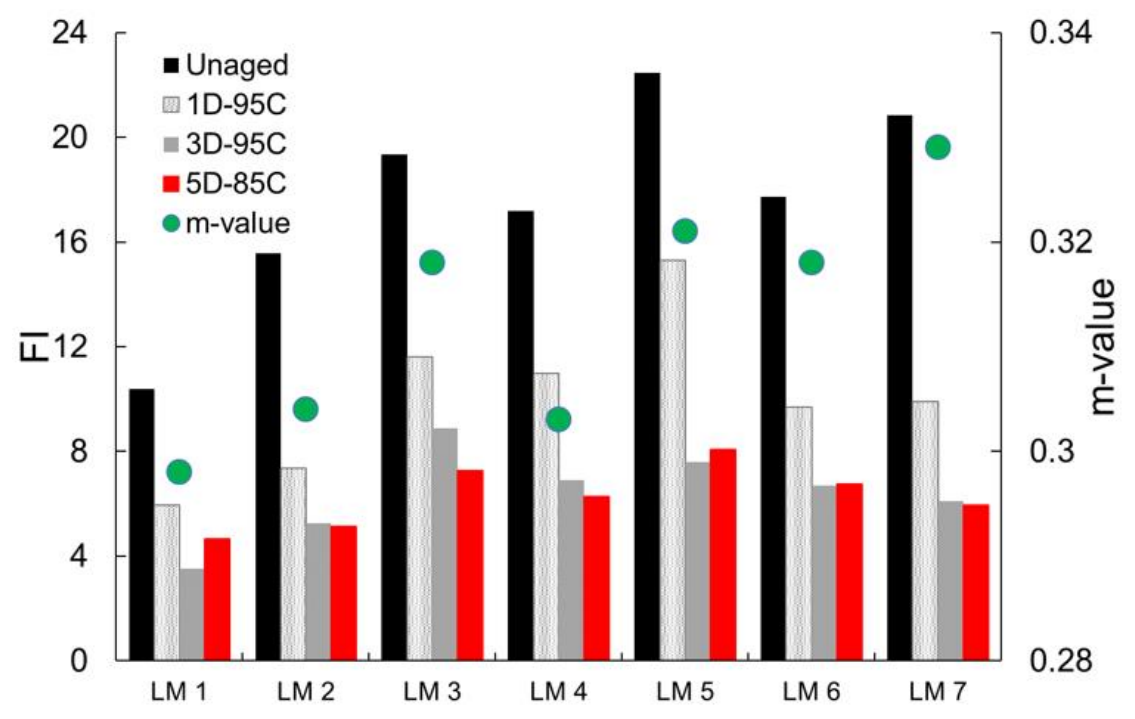

Figure 4.13. Correlation between $\mathrm{Fl}$ and binder $\mathrm{m}$-value.

In general, FI was able to capture the impact of various aging conditions. A major cause of the $\mathrm{FI}$ reduction is the increase of the post-peak slope. This finding suggests that aging plays a major role in $A C$ cracking potential. As shown, the decay in FI varied for different AC mixtures. Therefore, resistance to $A C$ crack propagation can be significantly affected by aging.

\subsection{SELECTION OF AC AGING TEMPERATURE}

A forced-draft oven and a I-FIT specimen have been selected as the aging equipment and state of material during the aging process, respectively. The next step is to identify the aging temperature. It is evident that as temperature increases, aging increases or the time to achieve the same aging can be reduced. However, higher temperature may change the aging mechanism, which introduces additional risk. Moreover, the I-FIT specimen is more likely to be distorted at relatively high temperatures (Figure 4.14). Hence, to maintain the practical and feasible aging process, a high temperature should be used as long as AC characteristics are not altered. 


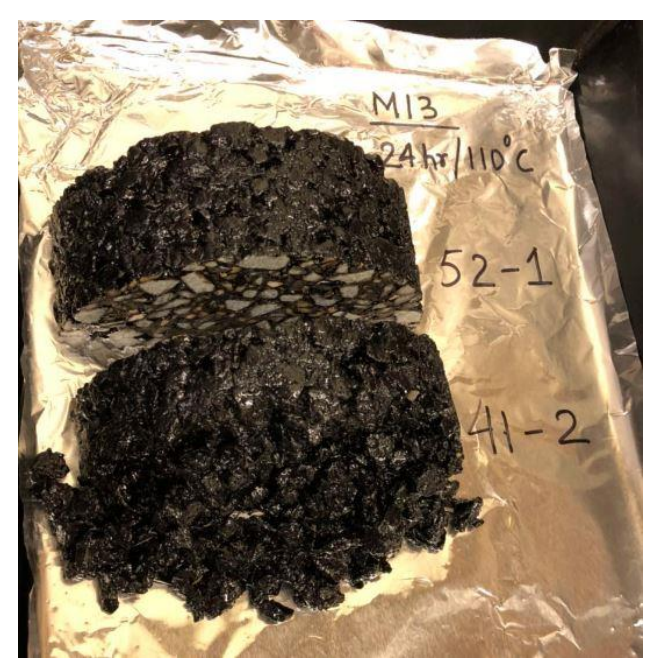

Figure 4.14. Distorted I-FIT specimen (SMA) after 1D/110C aging.

As shown in Table 2.1, previous studies employed temperatures ranging from $140^{\circ} \mathrm{F}\left(60^{\circ} \mathrm{C}\right)$ to $275^{\circ} \mathrm{F}$ $\left(135^{\circ} \mathrm{C}\right)$. Most of the studies were performed under $212^{\circ} \mathrm{F}\left(100^{\circ} \mathrm{C}\right)$ to avoid the disruption of polar molecular associations and sulfoxide decomposition and the drain-down of AC. Hence, I-FIT specimens should not be exposed to temperatures greater than $212^{\circ} \mathrm{F}\left(100^{\circ} \mathrm{C}\right)$. Specimens tested at temperatures below $212^{\circ} \mathrm{F}\left(100^{\circ} \mathrm{C}\right)$ in this study remained structurally intact. Hence, the aging temperature should be selected below $212^{\circ} \mathrm{F}\left(100^{\circ} \mathrm{C}\right)$.

As mentioned in Chapter 2, temperature variability inside the equipment is a limitation when using a forced-draft oven. In a recent study, temperature variability was investigated for samples located at different locations inside a frequently used forced-draft oven (Lemke et al. 2018). Figure 4.15 presents the results for an oven with a temperature set at $275^{\circ} \mathrm{F}\left(135^{\circ} \mathrm{C}\right)$. The difference in probe readings for the oven varied by as much as approximately $10^{\circ} \mathrm{F}\left(5.6^{\circ} \mathrm{C}\right)$ for different locations. Moreover, temperature varied by nearly $5^{\circ} \mathrm{F}\left(2.8^{\circ} \mathrm{C}\right)$ at the same location.

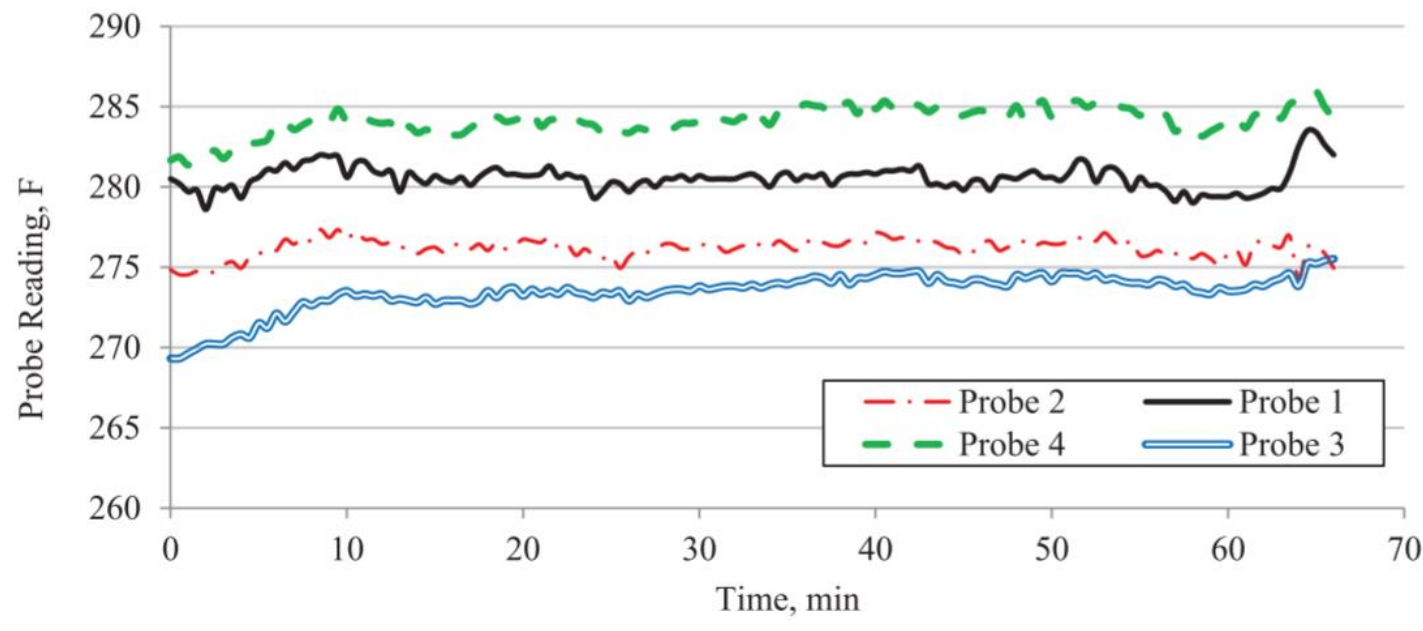

Figure 4.15. Temperature variability of probes at different specified locations in forced-draft oven (Lemke et al. 2018). 
The above facts suggest that the highest set-up temperature for forced-draft oven is $203^{\circ} \mathrm{F}\left(95^{\circ} \mathrm{C}\right)$, which can prevent specimens from experiencing any aging that exceeds $212^{\circ} \mathrm{F}\left(100^{\circ} \mathrm{C}\right)$.

\subsection{SELECTION OF AGING TIME}

The current long-term aging protocol recommended by AASHTO R30 is five days at $185^{\circ} \mathrm{F}\left(85^{\circ} \mathrm{C}\right)$ to simulate up to 10 years of field aging of $A C$. However, since the goal of this project is to develop a long-term aging protocol for I-FIT specimens that can be used for quality assurance during the production of AC mixtures, a shorter aging period is desired. Therefore, the feasibility of shorter aging durations was explored. In order to study the feasibility of aging for one day, a statistical analysis was implemented and a control case study using argon was conducted. The FI results after one and three days of aging at $203^{\circ} \mathrm{F}\left(95^{\circ} \mathrm{C}\right)$, which is higher than the AASHTO R30 recommended temperature, were shown in Figure 4.12. I-FIT specimens aged at 3D/95C typically resulted in similar FI values compared to those aged at 5D/85C. As would be expected, the impact on $\mathrm{Fl}$ after one day of aging is lower than three days of aging. However, since the option of one-day aging may be indicative of three-day aging and might be used in the protocols, a statistical analysis was performed. Details are provided in the following sub-sections.

\subsubsection{Statistical Analysis of Mix Design Parameters' Effect on Aging Rate}

As discussed in Section 4.3, different AC mixes have different FI reduction aging rates. Because of the limited available data for 3D/95C aged I-FIT specimens, linear regression was used to explore the effect of those parameters on the aging rate of $A C$ mixtures and to explore the relationship between $1 D / 95 C$ and $5 D / 85 C$ aging only. Inferences can be further made for 3D/95C since it resulted in similar aging extent as 5D/85C. Mix design parameters such as $\mathrm{N}$-design, voids in the mineral aggregate (VMA), asphalt content (AC \%), asphalt binder high temperature PG grade, low temperature PG grade, asphalt binder replacement (ABR), mix type (SMA or dense graded), and effective asphalt content $\left(P_{b e}\right)$ are considered in the analysis.

Simple and multiple linear regressions were implemented to explain the effect of various mix design parameters on the aging rate of $\mathrm{AC}$ mixtures under two different long-term lab simulated aging protocols: $1 \mathrm{D} / 95 \mathrm{C}$ and 5D/85C. The regression model has the following general form:

$y_{i}=\beta_{0}+\beta_{1} x_{1 i}+\beta_{2} x_{2 i}+\cdots+\beta_{p} x_{p i}+\epsilon_{i}$

where $y_{i}$ is the value of a continuous variable for observation $i$, and $x_{1 i}, x_{2 i}, \ldots, x_{p i}$ are the values of explanatory continuous variables. The term $\epsilon_{i}$ is the residual or error for individual $i . \beta_{0}, \beta_{1}, \beta_{2}, \ldots, \beta_{p}$ are the regression coefficients and are generally estimated by least-squares (Der et al. 2008).

Normality assumption of residual terms must be satisfied to correctly perform the linear regression analysis. A significance level $(\alpha)$ of 0.1 was used.

The FI for the unaged condition was referred to as $\mathrm{Fl}_{\text {unaged, }}$ while for a specific aging condition, it was referred to as $\mathrm{Fl}_{\text {aged. }}$. The effect of aging would result in a change in the $\mathrm{FI}$ and was quantified as aging rate. The aging rate was calculated using equation (3), and it was set up as the response variable $\left(y_{i}\right)$ in equation (2). 
Aging Rate $(\%)=\frac{F I_{\text {unaged }}-F I_{\text {aged }}}{F I_{\text {unaged }}} \times 100$

where $F I_{\text {unaged }}$ and $F I_{\text {aged }}$ represent the mean $\mathrm{FI}$ of unaged specimens and $1 \mathrm{D} / 95 \mathrm{C}$ or $5 \mathrm{D} / 85 \mathrm{C}$ aged specimens, respectively.

Simple linear regression was conducted first on each of these parameters individually to examine their statistical significance and their effect on the aging rate. The normality assumption was validated using a residual-quantile plot. The results for $1 \mathrm{D} / 95 \mathrm{C}$ and $5 \mathrm{D} / 85 \mathrm{C}$ aging are shown in Table 4.3. A p-value smaller than 0.1 indicates that the specific term is significantly affecting the aging rate. $\beta$ stands for parameter estimate, which implies aging rate change corresponding to a one-unit increase in the specific term. The lower and upper limits for parameter estimates using a $90 \%$ confidence interval are also helpful for identifying the significant terms qualitatively and quantitatively. If the $90 \%$ confidence interval includes 0 in between, the specific term will be concluded as not significant.

Table 4.3. Linear Regression Analysis Results for 1D/95C and 5D/85C Aging

\begin{tabular}{|c|c|c|c|c|c|c|c|c|c|}
\hline \multicolumn{10}{|c|}{ 1D/95C } \\
\hline & N Design & VMA & $A C \%$ & High Grade & Low Grade & ABR & Type $^{a}$ & $A b^{b}$ & Pbe \\
\hline p-value & 0.545 & 0.006 & 0.916 & 0.273 & 0.006 & 0.172 & 0.003 & 0.007 & 0.004 \\
\hline $\boldsymbol{\beta}$ & -0.265 & -21.493 & -2.379 & -0.817 & -4.054 & -0.691 & -40.472 & 24.925 & -38.140 \\
\hline 90\% Cl Lower & -1.011 & -33.261 & -40.992 & -2.073 & -6.279 & -1.534 & -60.690 & 10.800 & -58.068 \\
\hline 90\% Cl Upper & 0.482 & -9.724 & 36.234 & 0.438 & -1.830 & 0.151 & -20.254 & 39.050 & -18.212 \\
\hline \multicolumn{10}{|c|}{$5 \mathrm{D} / 85 \mathrm{C}$} \\
\hline p-value & 0.286 & 0.042 & 0.252 & 0.304 & 0.044 & 0.131 & 0.019 & 0.001 & 0.034 \\
\hline $\boldsymbol{\beta}$ & 0.273 & -9.780 & 15.848 & -0.455 & -1.921 & -0.450 & -19.674 & 17.214 & -17.423 \\
\hline 90\% Cl Lower & -0.158 & -17.515 & -7.454 & -1.201 & -3.455 & -0.943 & -32.859 & 9.724 & -30.561 \\
\hline 90\% Cl Upper & 0.704 & -2.046 & 39.150 & 0.292 & -0.387 & 0.043 & -6.489 & 27.705 & -4.286 \\
\hline
\end{tabular}

aType: stone matrix asphalt (SMA) and dense graded (DG) were coded as 1 and 0 , respectively, since linear regression does accept a categorical variable.

${ }^{\mathrm{b}} \mathrm{Ab}$ : aggregate blend water absorption (\%)

${ }^{c} \mathrm{P}_{\text {be }}$ : effective asphalt content by mass (\%)

From the analysis results in Table 4.3, VMA, low-temperature PG grade, mix type, aggregate blend water absorption, and effective asphalt content $\left(\mathrm{P}_{\text {be }}\right)$ are the significant terms affecting the aging rate for both $1 \mathrm{D} / 95 \mathrm{C}$ and $5 \mathrm{D} / 85 \mathrm{C}$ at a significance level of 0.1 . Table 4.4 summarizes these parameters' effect. 
Table 4.4. Summary of Mix Design Parameters' Effect on Aging Rate

\begin{tabular}{|c|c|c|c|c|}
\hline \multirow{2}{*}{ Mix Design Parameters } & Trend $^{\mathbf{b}}$ & Absolute Value $^{\mathbf{c}}$ & Trend & Absolute Value \\
\cline { 2 - 5 } & \multicolumn{2}{|c|}{ 1D/95C } & \multicolumn{2}{|c|}{ 5D/85C } \\
\hline VMA & Decrease & $21.49 \%$ & Decrease & $9.78 \%$ \\
\hline Low-Temperature PG Grade $^{*}$ & Increase & $4.05 \%$ & Increase & $1.92 \%$ \\
\hline Mix Type $^{\text {a }}$ & Decrease & $40.47 \%$ & Decrease & $19.67 \%$ \\
\hline Ab & Increase & $24.93 \%$ & Increase & $17.21 \%$ \\
\hline Pbe & Decrease & $38.14 \%$ & Decrease & $17.42 \%$ \\
\hline
\end{tabular}

a Type: stone matrix asphalt (SMA) and dense graded (DG) were coded as 1 and 0 , respectively, since linear regression does accept a categorical variable.

${ }^{b}$ Trend: aging rate increases or decreases when the specific mix design parameter increases.

${ }^{c}$ Absolute Value: change of aging rate (\%) when the specific mix design parameter increases by one unit.

As shown in Table 4.4, the significant AC mix design parameters and the trends of aging rate are the same for 1D/95C and 5D/85C. In addition, it appears that 1D/95C can distinguish AC mixes' susceptibility to aging. This fact implies that $1 \mathrm{D} / 95 \mathrm{C}$ can be an initial indicator of long-term aging to predict $A C$ 's behavior during its service life while being able to distinguish between AC mixes. However, 3D/95C method of aging should reflect the conditions of a long-term field aged material.

\subsubsection{Control-Case Argon Study}

The following test was performed with argon gas without oxygen. The goal was to check whether the FI decrease after one-day aging at high temperature is caused by oxidation or loss of volatiles.

Specimens were fabricated and exposed for $1 D / 95 \mathrm{C}$ and 3D/95C in air or argon, which is a chemically inert gas under most conditions and often used as an inert shielding gas in high-temperature industrial processes. Figure 4.16 showed the preparation process of specimens for aging.

A key step of the preparation process of argon aging is to purge as much air as possible in the container by replacing it with argon. Kinsley (2001) stated that when pumping in six times the volume of the container with argon, the container can have at least $99 \%$ of air removed. The plastic bag shown in Figure 4.16 contains at least nine gallons of gas, well in excess of the six times required to eliminate $99 \%$ of the air.

Figure 4.17 shows the results of $\mathrm{FI}$ changes due to aging in air vs. in argon under two aging protocols: $1 \mathrm{D} / 95 \mathrm{C}$ and $3 \mathrm{D} / 95 \mathrm{C}$. 

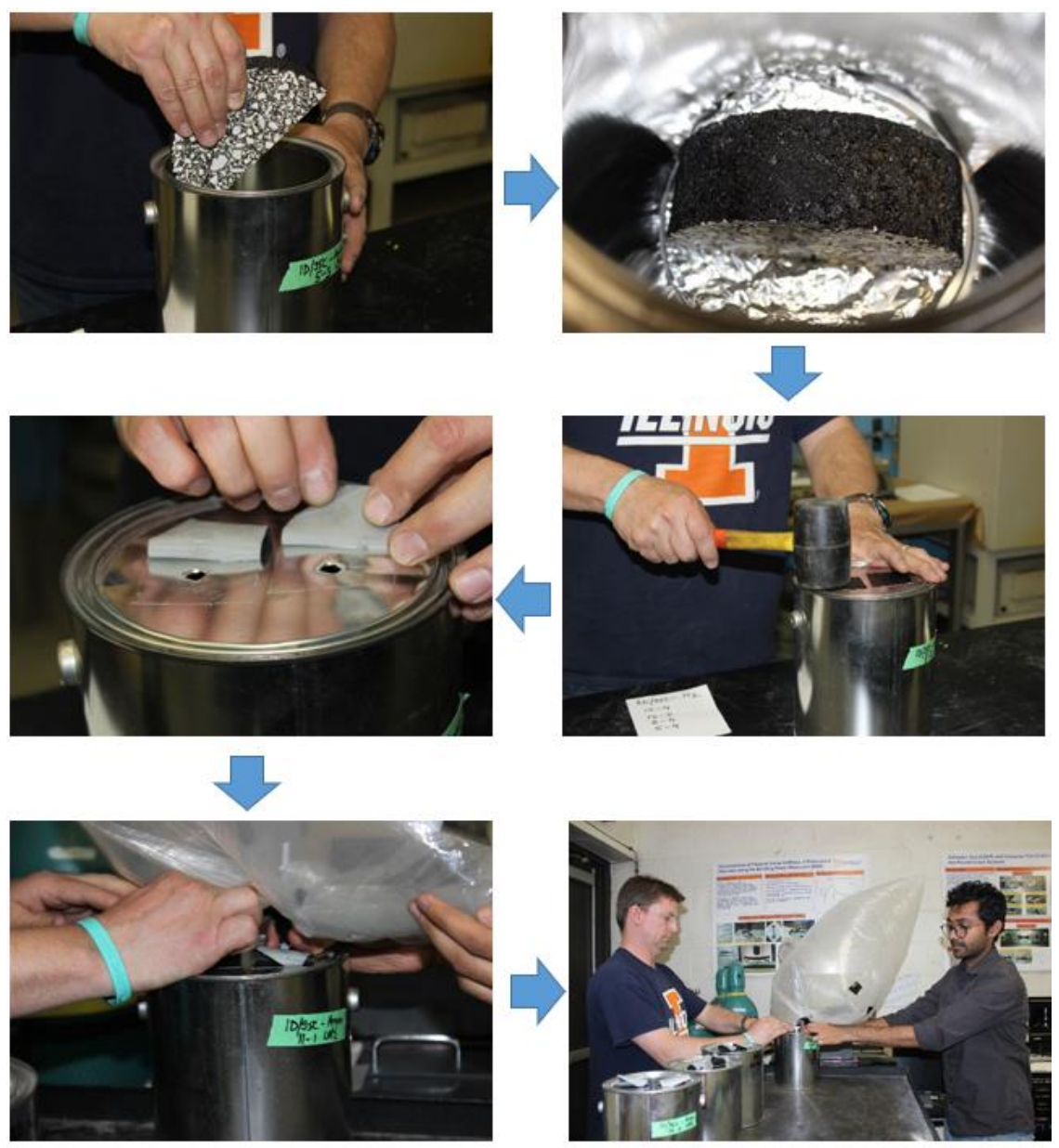

Figure 4.16. Preparation process for argon aging.

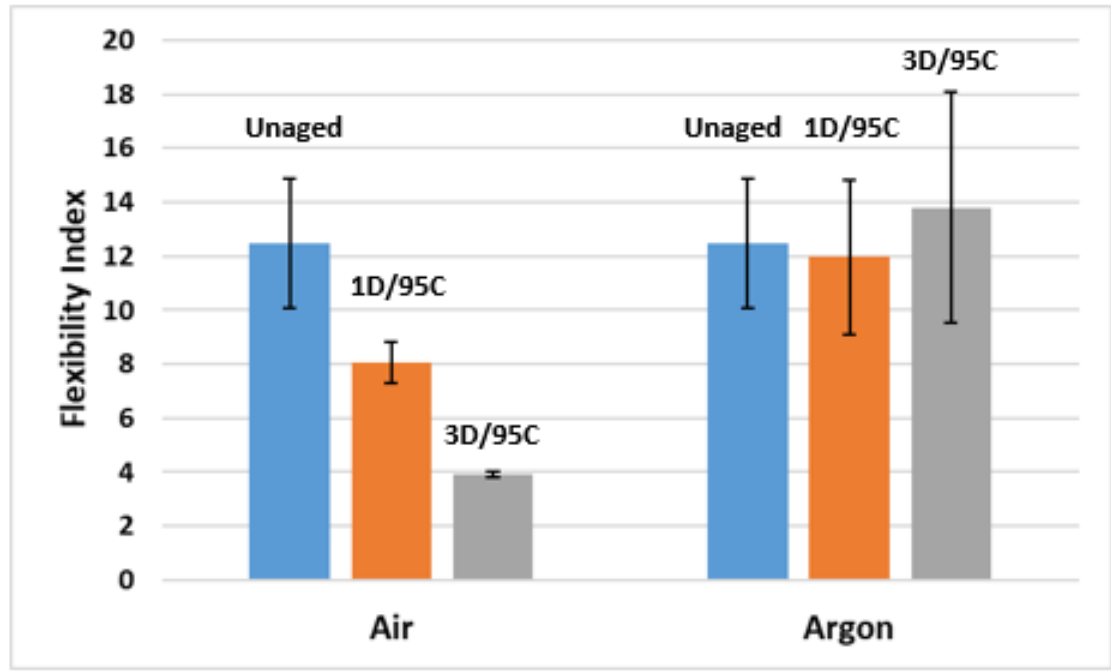

Figure 4.17. Flexibility index comparison of aging in air vs. in argon. 
As shown in Figure 4.17, FI decreased consistently for those specimens aged in air while it remained approximately the same under aging in argon. This demonstrates that oxidation is playing a leading role in the aging of I-FIT specimens under both aging protocols. Combining the results from the statistical analysis illustrated in Section 4.5.1 and the aging in argon, it is concluded that 1D/95C has the same aging mechanism as 3D/95C (5D/85C).

\subsection{SUMMARY}

The development of a long-term aging protocol for I-FIT comprises equipment selection, state of material during aging, and temperature and aging period. A forced-draft oven has been selected as the aging device due to its availability, feasibility, practicability, capacity, and acceptable variability. A fully prepared semi-circular I-FIT specimen has been chosen as the state of material during aging due to operation practicality. To maintain the aging mechanism, $203^{\circ} \mathrm{F}\left(95^{\circ} \mathrm{C}\right)$ is identified as the optimal set-up temperature for the forced-draft oven. Due to the good correlation between $\mathrm{FI}$ values of aged specimens after modified AASHTO R30's recommended 5D/85C method and 3D/95C aged samples, the method using 3D/95C aging was selected as the main method of aging. A detailed comparison between modified AASHTO R30, used in this project, and original method may be found in Appendix F.

A statistical analysis and argon study showed that 1D/95C and 3D/95C (5D/85C) have the same aging mechanism; while 1D/95C aging may differentiate $A C$ mixes' susceptibility to aging better than $3 \mathrm{D} / 95 \mathrm{C}(5 \mathrm{D} / 85 \mathrm{C})$ aging. As a result, the $1 \mathrm{D} / 95 \mathrm{C}$ aging can be used as an alternate for $3 \mathrm{D} / 95 \mathrm{C}$ in some scenarios.

The aging protocols and corresponding thresholds will be discussed further in Chapter 5. 


\section{CHAPTER 5: DEVELOPMENT AND VALIDATION OF LONG-TERM AGING THRESHOLDS AND PROTOCOL DEVELOPMENT}

In this chapter, long-term aging protocols and thresholds for lab-and plant-produced mixes have been developed.

\subsection{LABORATORY-PRODUCED AND LABORATORY-COMPACTED SPECIMENS}

A long-term aging protocol is proposed for laboratory-produced, laboratory-compacted (LPLC) specimens. This protocol may be used for mix design approval by IDOT prior to the mix being used in the field. The protocol requires I-FIT on unaged and 3D/95C aged specimens. A specific mix must have a mean $\mathrm{Fl}$ of unaged and 3D/95C aged specimens passing the proposed thresholds to be accepted. Figure 5.1 illustrates the proposed flowchart demonstrating the long-term aging protocol.

Preliminary thresholds are proposed after the interpretation of the FI results presented in Chapter 4 and with consideration of the following assumptions:

- The FI threshold for LPLC specimens after three-day aging at $203^{\circ} \mathrm{F}\left(95^{\circ} \mathrm{C}\right)$ is 5.0 , per the data analysis and Technical Review Panel (TRP) discussion. It is expected that LPLC specimens may have a higher FI threshold than that of PPLC specimens.

- A relatively strict criterion should be proposed for designing new mixes, which can provide contractors with enough tolerance during the production stage.

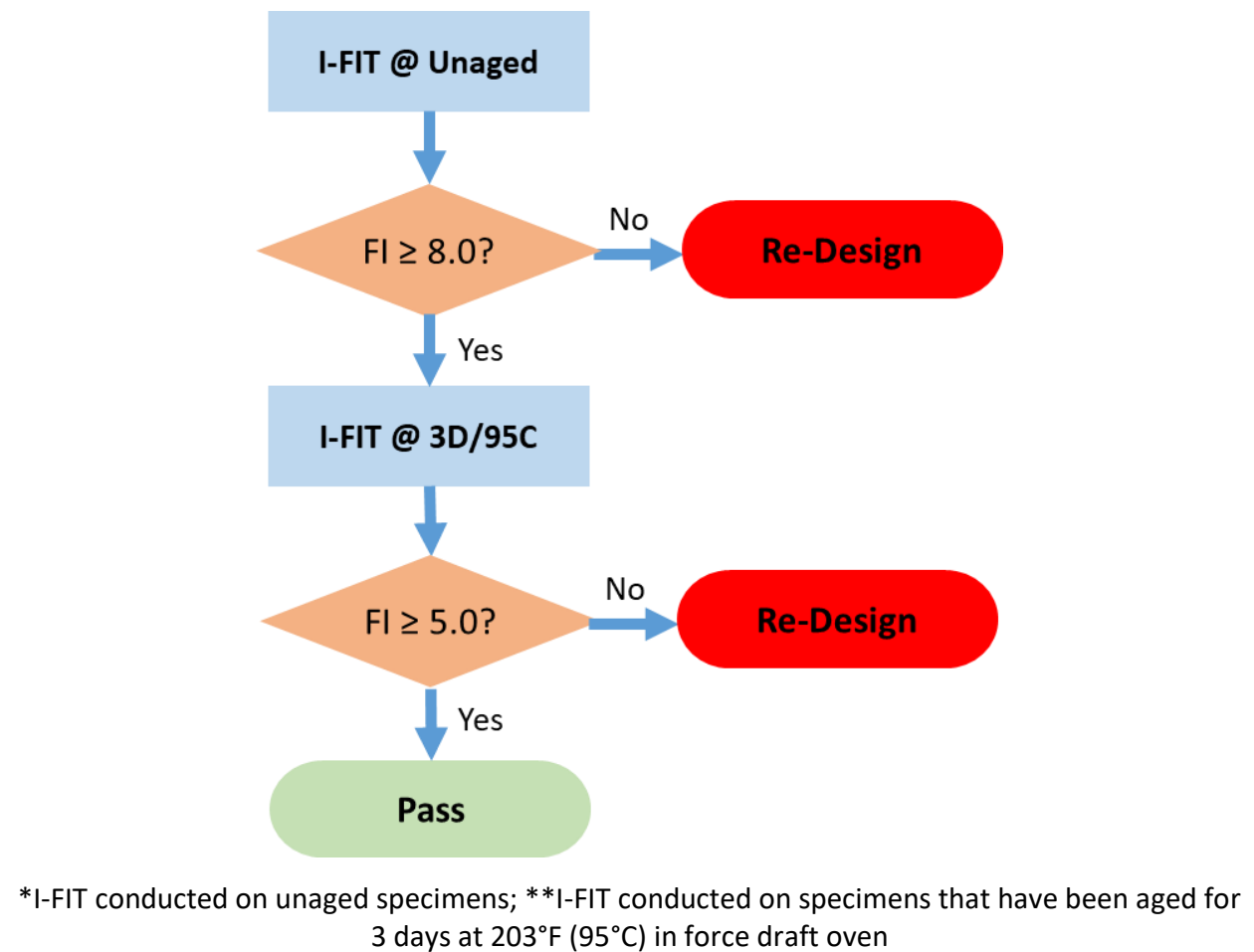

Figure 5.1. Long-term aging protocol and thresholds for lab mix design process using I-FIT. 
The protocol and thresholds were applied to the seven tested lab-produced mixes. Table 5.1 presents the results. One of the seven mixes failed the 3D/95C FI criterion.

Table 5.1. LPLC Specimens Long-term Aging Flexibility Checking Results

\begin{tabular}{|c|c|c|c|}
\hline Mix ID & Unaged FI & 3D/95C FI & Pass/Fail \\
\hline LM1 & 10.4 & 3.5 & Fail \\
\hline LM2 & 15.6 & 5.3 & Pass \\
\hline LM3 & 19.4 & 8.9 & Pass \\
\hline LM4 & 17.2 & 6.9 & Pass \\
\hline LM5 & 22.5 & 7.6 & Pass \\
\hline LM6 & 17.7 & 6.7 & Pass \\
\hline LM7 & 20.8 & 6.1 & Pass \\
\hline
\end{tabular}

\subsection{PLANT-PRODUCED AND LABORATORY-COMPACTED SPECIMENS}

\subsubsection{Development of Plant Production Process Thresholds}

A long-term aging protocol is proposed for plant-produced, laboratory-compacted (PPLC) specimens. I-FIT procedure should be conducted on both unaged and 3D/95C aged specimens in all cases, while $1 \mathrm{D} / 95 \mathrm{C}$ may be used by contractors to screen problematic mixes at an early stage. Figure 5.2 illustrates the proposed flowchart employed in the long-term aging protocol for PPLC specimens.

Preliminary thresholds are proposed after the interpretation of FI results presented in Chapter 4 and with consideration of the following assumptions:

- A dual criterion is used for $1 \mathrm{D} / 95 \mathrm{C}$ aged specimens. The mix fails if the $\mathrm{Fl}$ is less than 6.0 or the aging rate is greater than $45 \%$. This optional criterion would provide timely feedback for the contractor.

- The FI threshold for PPLC specimens after three-day aging at $203^{\circ} \mathrm{F}\left(95^{\circ} \mathrm{C}\right)$ is 4.0 based on limited mixes tested in this project. It should be noted that they had relatively low unaged $\mathrm{Fl}$. Of the 12 mixes tested, six failed the unaged FI criterion of 8.0. In addition, 10 out of 12 mixes contained binders that had $\Delta T_{C}$ less than $-5^{\circ} \mathrm{C}$. Hence, this suggests that a criterion of 4.0 , for $\mathrm{FI}$ threshold for PPLC specimens after three-day aging at $203^{\circ} \mathrm{F}\left(95^{\circ} \mathrm{C}\right)$, may be practical to achieve for mixes designed properly.

The FI threshold for PPLC is lower than that of LPLC specimens because PPLC is exposed to more aging than LPLC specimen during plant storage and reheating process of plant mixes. Also, this allows the contractor some additional margin to help ensure that a mix that passes during design will also meet the requirements during production. 


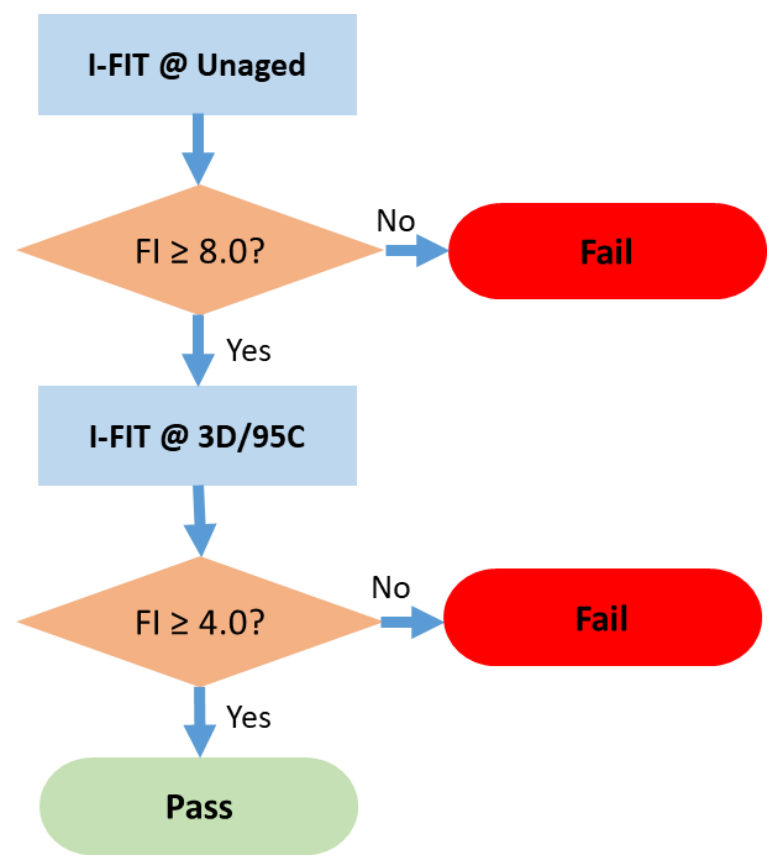

(a)

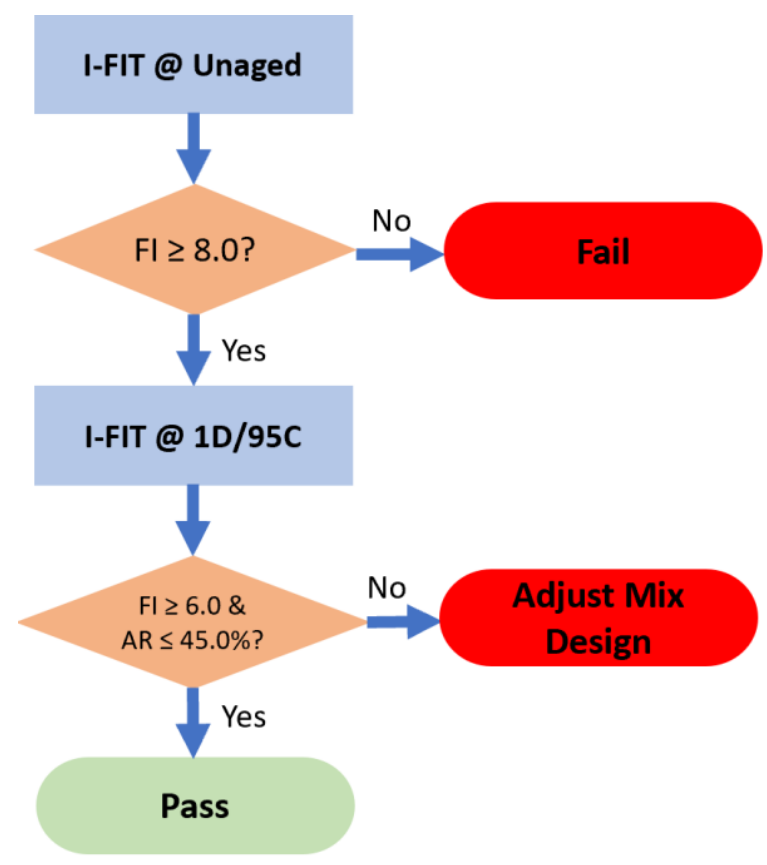

(b)*

*I-FIT conducted on specimens that have been aged for one-day at $203^{\circ} \mathrm{F}\left(95^{\circ} \mathrm{C}\right)$ in a forced-draft oven. This is an optional method for contractors to detect problematic mixes at an early stage.

Figure 5.2. Long-term aging using I-FIT (a) protocol and thresholds for plant production, and (b) optional approach

The protocol and thresholds were applied to the 12 plant-produced mixes in this project. The results are presented in Table 5.2. Although 3D/95C aging results were not available for all mixes, they can be estimated based on $5 \mathrm{D} / 85 \mathrm{C}$ aging results since a good correlation was established between $3 \mathrm{D} / 95 \mathrm{C}$ and $5 \mathrm{D} / 85 \mathrm{C}$ aging, as presented in Chapter 4 . Four out of 12 mixes passed the long-term aging flexibility check (see Table 5.2). PM7, which is an SMA, failed the unaged check only. Mixes PM1, PM6, PM10, PM11, and PM12 failed the unaged as well as the 3D/95C check. On the other hand, PM2 and PM3 failed the 3D/95C check.

If the optional 1D/95C check was applied, then the same mixes would have failed, excluding PM3. The $1 \mathrm{D} / 95 \mathrm{C}$ criterion nearly flagged all the mixes failed at $3 \mathrm{D} / 95 \mathrm{C}$. The analysis indicates that $1 \mathrm{D} / 95 \mathrm{C}$ is an efficient optional check that may be used by contractors to detect problematic mixes and to make adjustments at an early stage. 
Table 5.2. PPLC Specimens Long-term Aging Flexibility Checking Results

\begin{tabular}{|c|c|c|c|c|c|c|c|}
\hline Mix ID & Unaged FI & 1D/95C FI & 1D/95C Aging Rate & $3 \mathrm{D} / 95 \mathrm{C} \mathrm{FI}$ & $5 \mathrm{D} / 85 \mathrm{C} \mathrm{FI}$ & $\Delta T_{c} 2-P A V$ & Pass/Fail \\
\hline PM1 & 4.1 & 1.1 & 73.7 & $<1.0$ & $<1.0$ & -14.3 & Fail \\
\hline PM2 & 16.3 & 6.8 & 58.3 & 3.2 & 3.3 & -10.2 & Fail \\
\hline PM3 & 12.8 & 8.1 & 36.5 & 3.5 & 2.8 & -9.8 & Fail \\
\hline PM5 & 12.5 & 8.5 & 31.7 & 4.2 & 5.5 & -6.2 & Pass \\
\hline PM6 & 7.4 & 6.5 & 11.8 & 3.7 & 3.7 & -8.7 & Fail \\
\hline PM7 & 7.4 & 8.0 & -8.5 & N/A & 4.8 & -6.7 & Fail \\
\hline PM8 & 15.0 & 8.6 & 42.9 & N/A & 6.2 & -8.3 & Pass \\
\hline PM9 & 10.6 & 8.9 & 16.2 & N/A & 5.7 & -11.5 & Pass \\
\hline PM10 & 7.6 & 4.1 & 46.1 & N/A & 3.2 & -1.6 & Fail \\
\hline PM11 & 4.6 & 3.1 & 33.8 & N/A & 2.2 & -2.6 & Fail \\
\hline PM12 & 4.8 & 4.1 & 15.8 & N/A & 2.7 & -6.6 & Fail \\
\hline PM13 & 11.3 & 10.6 & 6.1 & 4.9 & 6.0 & -16.6 & Pass \\
\hline
\end{tabular}

PM1 and PM11 failed unaged FI check because of poor binder quality and relatively high ABR content. PM7 and PM12 showed marginal and low unaged FI respectively, mainly because of high ABR content (30\%) consisting of significant amount of RAS (4.0\% for PM7 and 3.5\% for PM12). However, PM7 performance was relatively better than PM12 due to two reasons: higher effective binder content and different AC mix design (PM7 - 5.7 \% effective AC, SMA mix; PM12 - 4.7\% effective AC, CG mix). PM6 and PM10 marginally failed unaged FI check and the reasons are unclear.

\subsubsection{Field Validation}

This section includes field cores tested from this project as well as from ICT project R27-161 "Construction and Performance Monitoring of Various Asphalt Mixes." The cores tested were as old as five years from the date of construction. The results are presented with and without specimen properties corrections. Equations (4) and (5) were used to correct FI for thickness and air void, respectively (Barry 2016; Rivera-Perez et al. 2017).

$F I_{\text {Thickness-Corrected }}=F I \times \frac{T}{50}$

where $F I$ stands for uncorrected $\mathrm{FI}$ and $T$ refers to specimen thickness in $\mathrm{mm}$.

$F I_{\text {Air Void-Corrected }}=F I \times \frac{0.0651}{A V-A V^{2}}$

where $F I$ stands for uncorrected $\mathrm{FI}$ and $A V$ refers to air void in percent. 
Eight field sections from project R27-175 and seven from project R27-161 were included for testing. The progression of FI from plant production to field performance over several years of monitoring is presented.

Field cores were collected at regular intervals after construction to keep track of the in-service $\mathrm{FI}$ change. Cores obtained in the R27-175 project are as old as one year, which is not sufficient to develop a direct correlation to the plant-produced mixtures tested after 3D/95C aging. However, the data from the R27-161 project field cores is more appropriate to use for field validation.

\subsubsection{Field Core Results Summary}

Table 5.3 shows the date of construction and dates of retrieval of the corresponding cores at regular intervals. Table 5.4 shows additional data on I-FIT of field cores collected and tested by IDOT approximately every year after construction from districts in Illinois for two years.

Table 5.3. Field Core Inventory Based on Dates of Coring

\begin{tabular}{|c|c|c|c|c|c|c|c|c|c|}
\hline Mix ID & $\begin{array}{c}\text { Date of } \\
\text { Construction }\end{array}$ & $\begin{array}{l}\text { 1st Core } \\
\text { Received }\end{array}$ & $\begin{array}{c}\text { Age } \\
\text { (years) } \\
1 s t \\
\text { Core } \\
\end{array}$ & $\begin{array}{l}\text { 2nd Core } \\
\text { Received }\end{array}$ & $\begin{array}{c}\text { Age } \\
\text { (years) } \\
\text { 2nd } \\
\text { Core } \\
\end{array}$ & $\begin{array}{l}\text { 3rd Core } \\
\text { Received }\end{array}$ & $\begin{array}{c}\text { Age } \\
\text { (years) } \\
\text { 3rd } \\
\text { Core }\end{array}$ & $\begin{array}{l}\text { 4th Core } \\
\text { Received }\end{array}$ & $\begin{array}{c}\text { Age } \\
\text { (years) } \\
4 \text { th } \\
\text { Core }\end{array}$ \\
\hline \multicolumn{10}{|c|}{ R27-175 } \\
\hline PM1 & $6 / 6 / 2017$ & $8 / 2 / 2017$ & 0.2 & $12 / 11 / 2017$ & 0.5 & $6 / 26 / 2018$ & 1.1 & & \\
\hline PM2 & $6 / 14 / 2017$ & $10 / 19 / 2017$ & 0.4 & $2 / 14 / 2018$ & 0.7 & $7 / 25 / 2018$ & 1.1 & & \\
\hline PM3 & $6 / 16 / 2017$ & $10 / 19 / 2017$ & 0.3 & $2 / 14 / 2018$ & 0.7 & $7 / 25 / 2018$ & 1.1 & & \\
\hline PM5 & 9/18/2017 & $10 / 27 / 2017$ & 0.1 & $6 / 21 / 2018$ & 0.8 & $10 / 31 / 2018$ & 1.1 & & \\
\hline PM6 & $10 / 4 / 2017$ & $10 / 19 / 2017$ & 0.0 & $4 / 18 / 2018$ & 0.5 & $10 / 24 / 2018$ & 1.1 & & \\
\hline PM7 & $10 / 20 / 2017$ & $12 / 21 / 2017$ & 0.2 & $6 / 21 / 2018$ & 0.7 & $10 / 31 / 2018$ & 1.0 & & \\
\hline PM12 & $11 / 3 / 2017$ & $12 / 21 / 2017$ & 0.1 & $6 / 21 / 2018$ & 0.6 & $10 / 31 / 2018$ & 1.0 & & \\
\hline PM13 & $11 / 14 / 2017$ & $12 / 21 / 2017$ & 0.1 & $6 / 21 / 2018$ & 0.6 & $10 / 31 / 2018$ & 1.0 & & \\
\hline \multicolumn{10}{|c|}{ R27-161 } \\
\hline $306 \mathrm{M} 30$ & $10 / 31 / 2013$ & $5 / 10 / 2015$ & 1.5 & $10 / 2 / 2016$ & 3.0 & $5 / 9 / 2017$ & 3.6 & $7 / 28 / 2018$ & 4.8 \\
\hline 156Y03 & $10 / 30 / 2014$ & $5 / 10 / 2015$ & 0.5 & $10 / 2 / 2016$ & 2.0 & $5 / 9 / 2017$ & 2.6 & $7 / 28 / 2018$ & 3.8 \\
\hline 157Y03 & $10 / 30 / 2014$ & $5 / 10 / 2015$ & 0.5 & $10 / 2 / 2016$ & 2.0 & $5 / 9 / 2017$ & 2.6 & $7 / 28 / 2018$ & 3.8 \\
\hline 159Y02 & $11 / 15 / 2014$ & $5 / 10 / 2015$ & 0.5 & $10 / 2 / 2016$ & 1.9 & $5 / 9 / 2017$ & 2.5 & $7 / 28 / 2018$ & 3.8 \\
\hline 140Y02 & $11 / 15 / 2014$ & $5 / 10 / 2015$ & 0.5 & $10 / 2 / 2016$ & 1.9 & $5 / 9 / 2017$ & 2.5 & $7 / 28 / 2018$ & 3.8 \\
\hline 177Y04 & $6 / 10 / 2015$ & $6 / 22 / 2015$ & 0.0 & $10 / 2 / 2016$ & 1.3 & $5 / 9 / 2017$ & 1.9 & $7 / 28 / 2018$ & 3.2 \\
\hline
\end{tabular}


Table 5.4. I-FIT Summary of Additional Field Cores from Illinois Districts Provided by IDOT

\begin{tabular}{|c|c|c|c|c|c|c|c|c|c|}
\hline \multirow[b]{2}{*}{ District } & \multicolumn{3}{|c|}{ Post Construction } & \multicolumn{3}{|c|}{ Year 1} & \multicolumn{3}{|c|}{ Year 2} \\
\hline & AV\% & $\begin{array}{c}\text { FI } \\
\text { Uncorr. }^{1}\end{array}$ & $\begin{array}{c}\text { FI } \\
\text { Corr. }^{2}\end{array}$ & AV\% & $\begin{array}{c}\text { FI } \\
\text { Uncorr. }\end{array}$ & $\begin{array}{c}\text { FI } \\
\text { Corr. }\end{array}$ & AV\% & $\begin{array}{c}\text { FI } \\
\text { Uncorr. }\end{array}$ & $\begin{array}{c}\text { FI } \\
\text { Corr. }\end{array}$ \\
\hline \multirow{2}{*}{1} & - & - & & 4.7 & 6.8 & 4.3 & 2.9 & 7.4 & 6.1 \\
\hline & - & - & - & 5.7 & 19.8 & 18.3 & 6.5 & 27.2 & 27.2 \\
\hline 2 & 1.7 & 31.9 & 22.5 & 1.0 & 18.4 & 12.9 & 1.1 & 15.1 & 10.3 \\
\hline \multirow{2}{*}{3} & 1.9 & 20.0 & 16.5 & 1.9 & 24.9 & 18.8 & 1.7 & 14.8 & 11.0 \\
\hline & 2.6 & 16.7 & 13.8 & 2.7 & 21.6 & 16.3 & 2.3 & 14.6 & 11.4 \\
\hline 4 & 8.6 & 46.6 & 35.4 & 6.3 & 13.4 & 9.1 & 3.0 & 9.3 & 6.4 \\
\hline \multirow{3}{*}{5} & 3.6 & 15.6 & 12.5 & 3.3 & 13.0 & 9.8 & 3.5 & 6.9 & 6.1 \\
\hline & 5.4 & 13.0 & 8.5 & 3.9 & 8.9 & 5.8 & 3.8 & 6.5 & 5.3 \\
\hline & 3.4 & 20.3 & 16.9 & 2.9 & 14.0 & 11.0 & 3.8 & 13.4 & 11.8 \\
\hline \multirow{2}{*}{6} & 5.0 & 32.8 & 31.1 & 4.4 & 10.7 & 7.7 & 2.9 & 10.6 & 7.9 \\
\hline & 6.2 & 60.2 & 45.5 & 1.7 & 17.3 & 15.0 & 2.7 & 18.9 & 15.5 \\
\hline 7 & 5.6 & 40.8 & 31.3 & 2.6 & 19.3 & 13.6 & 3.2 & 15.3 & 11.9 \\
\hline 8 & 5.7 & 11.8 & 11.7 & 4.7 & 7.5 & 5.3 & 3.7 & 3.0 & 2.4 \\
\hline 9 & - & - & - & 3.6 & 12.1 & 8.1 & 4.9 & 12.5 & 9.0 \\
\hline
\end{tabular}

${ }^{1} \mathrm{FI}$ Uncorr. refers to $\mathrm{FI}$ without any correction.

${ }^{2} \mathrm{FI}$ Corr. refers to FI corrected for thickness only of the tested core (equation [4]).

The progression of Fl obtained from field cores less than one and half years old is shown in Figure 5.3. No trend is noticed in the first six months for cores from this project. However, the reduction in $\mathrm{Fl}$ is evident after one year of service. Fl values were corrected for thickness and air void content. It is important to note that the corrected FI values show a consistent trend in FI with in-service aging compared to uncorrected FI. Figure 5.4 shows corrected and uncorrected FI from project R27-161. FI decay is evident with age of service in those cores after three years, while it is unclear in the early years of service (up to two years). In addition, the corrected FI values are more representative of performance reported in R27-161 (Al-Qadi et al. 2017). The field performance of R27-161 shows that the control mix $306 \mathrm{M} 30$ is the best-performing mix, which is supported by the corrected FI trends in Figure 5.4 . 


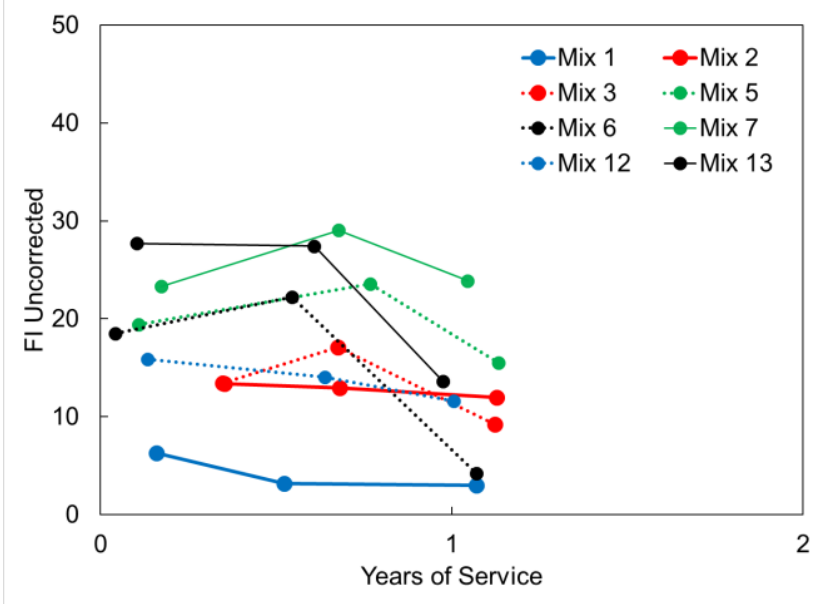

(a)

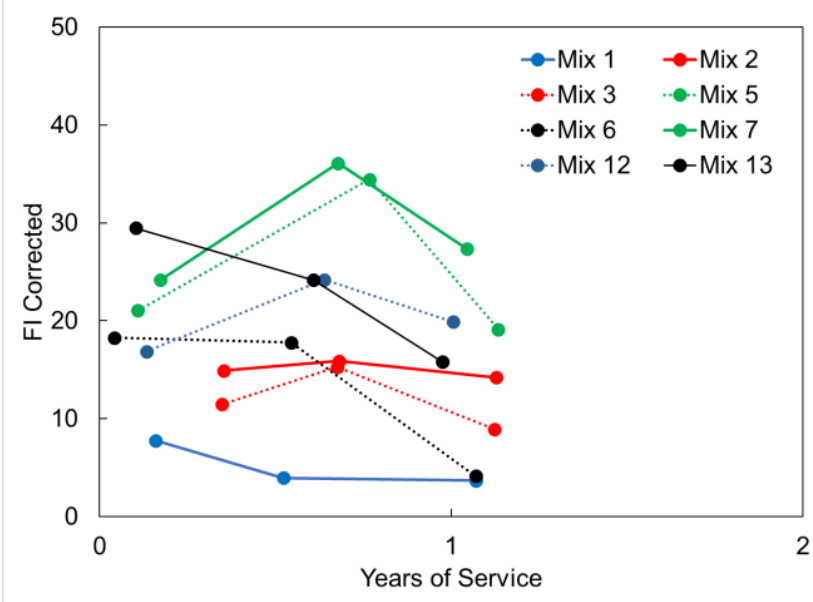

(b)

Figure 5.3. Progression of field cores FI from the R27-175 project: (a) uncorrected FI; (b) corrected FI for both thickness and air void.

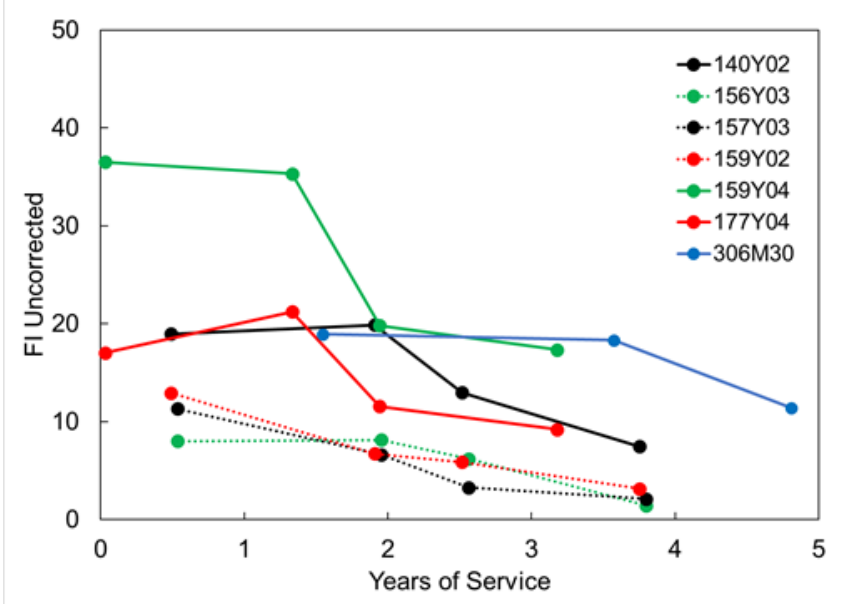

(a)

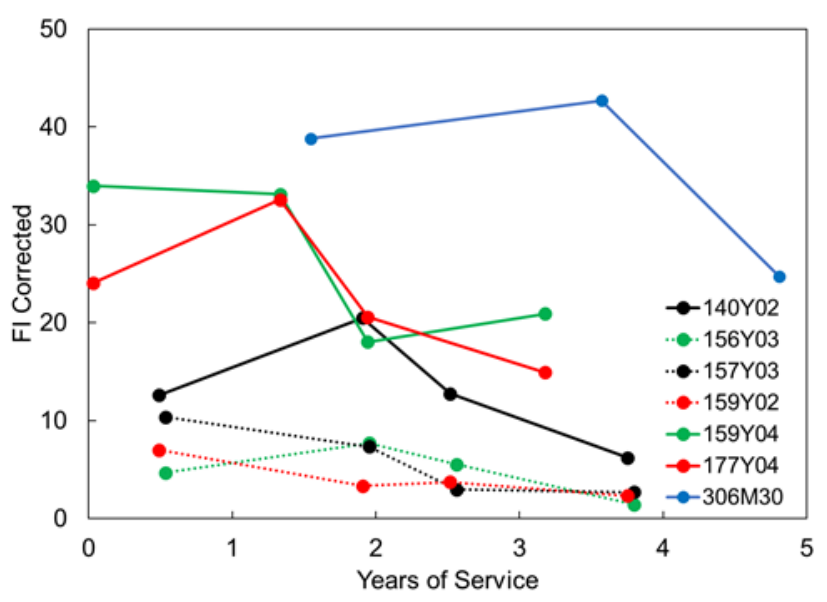

(b)

Figure 5.4. Progression of field cores FI from the R27-161 project: (a) uncorrected FI;

(b) corrected FI for both thickness and air void.

Additional field sections from varying districts in Illinois constructed in 2016 were evaluated using IFIT immediately after construction, followed by yearly testing through 2018 . The results for uncorrected and corrected $\mathrm{FI}$ are shown in Figure 5.5. Field cores that had air voids ranging from 3 to $8 \%$ were corrected for $\mathrm{Fl}$ using equation (5). In the first year of service, the corrected Fl decay is not evident. However, the results from the second year do reflect FI decay when compared to post construction. It is interesting to note that the corrected FI helps in distinguishing between the mixes more significantly than uncorrected FI. 


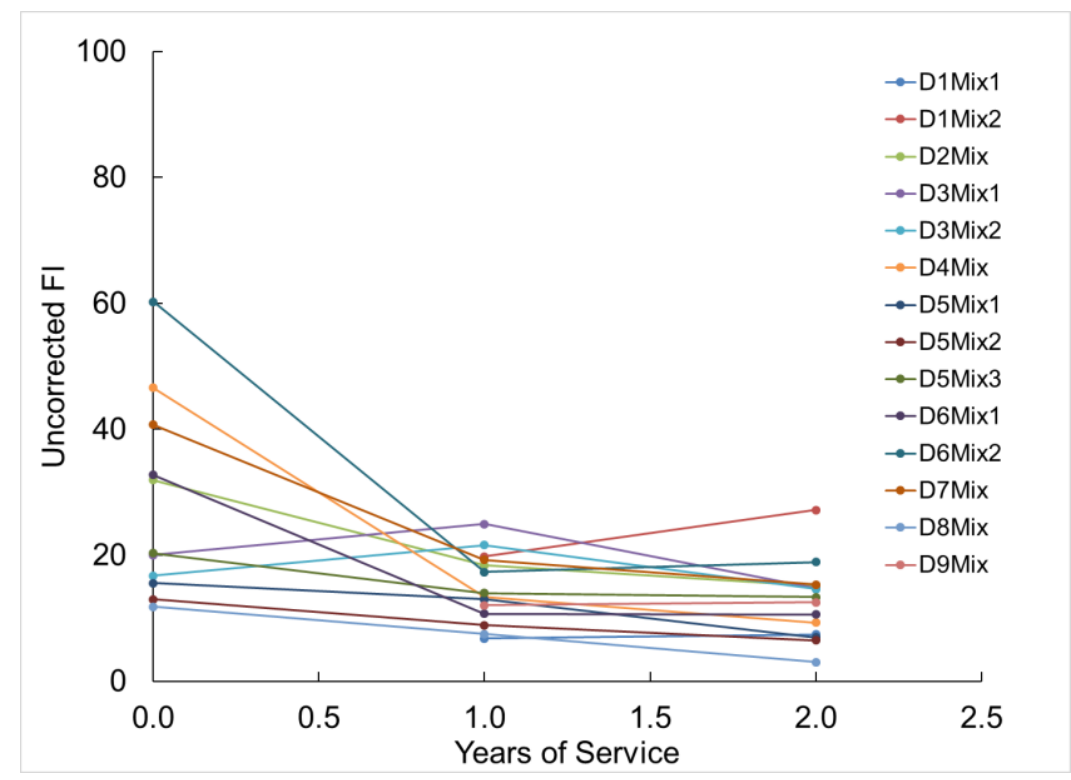

(a)

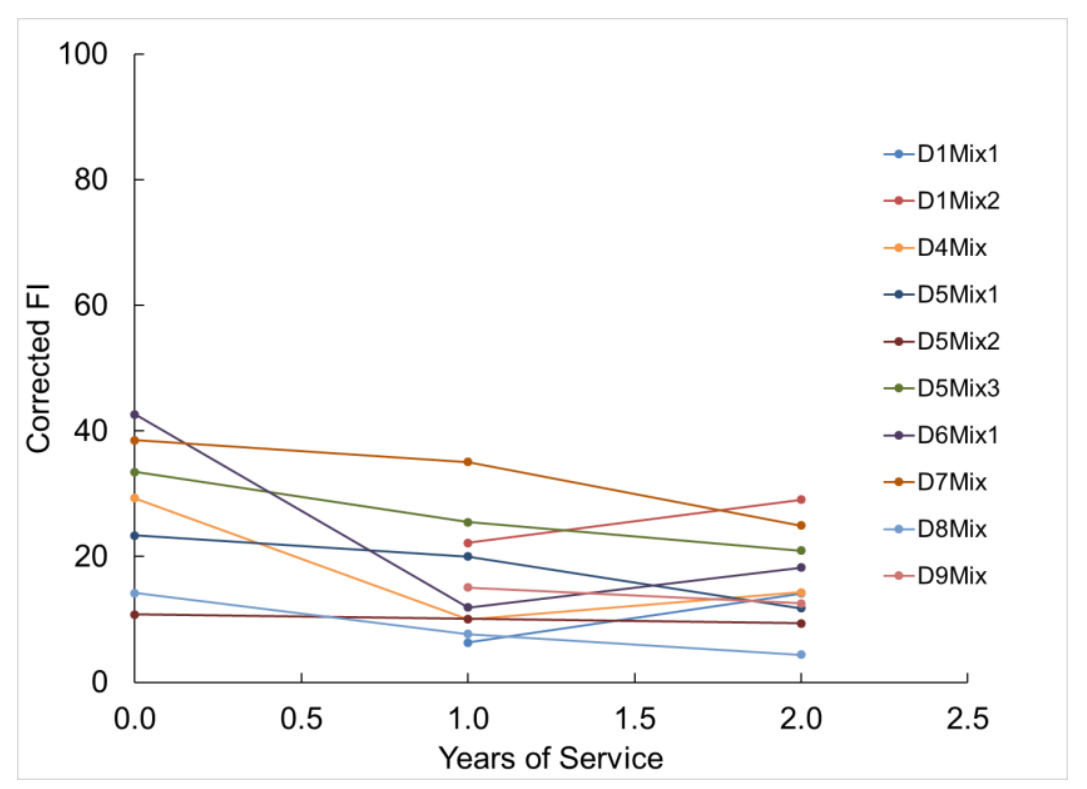

(b)

Figure 5.5. Progression of field cores FI from the 2016 IDOT Pilot Projects: (a) uncorrected Fl; (b) corrected FI for both thickness and air voids.

\subsubsection{Impact of Fl on Transverse Cracking}

Figure 5.6 shows the correlation of FI from field cores to transverse crack length for sections considered as part of project R27-161 collected over three years until 2017. In Figure 5.6, the trends of Fl show an inverse correlation to transverse crack length measured for the sections each year. In addition, the slope of the trends of FI vs. transverse cracking becomes steeper with age, indicating that the cracking increases significantly with the decrease in Fl. The inference from the field data 
support the notion that cracking increases with a decrease in $\mathrm{Fl}$ and an increase in the age of the AC mixtures. At this point, it is not possible to directly compare FI values from field cores and plantproduced or lab-produced mixes. This is due to validation purposes, as the sections presented in Figure 5.6 do not correspond to the AC mixes studied in the current project. Once new cores are obtained from the mixes used in the current project in the future, FI thresholds for aging can be better understood.

\subsection{SHELF-AGED PLANT-PRODUCED MIXES THRESHOLDS}

\subsubsection{Impact of Shelf Aging}

If $A C$ mixes are stored on a shelf in hot weather conditions without temperature control, they will experience aging until being tested. Therefore, the proposed protocols for the PMLC mixes cannot be applied to mixes with arbitrary shelving time in a relatively high-temperature environment. Because of the viscoelastic nature of the $A C$, high temperature would have significant impact. In order to account for the impact of shelf aging on $\mathrm{Fl}$, nine of the 12 PMLC mixes were tested after six months of shelf storage in canvas bags in a non-climate-controlled storehouse at the ATREL. In addition, two mixes, which experienced 12 months of shelf aging, were tested later. Figure 5.7 shows the results for progression of Fl after shelf aging.

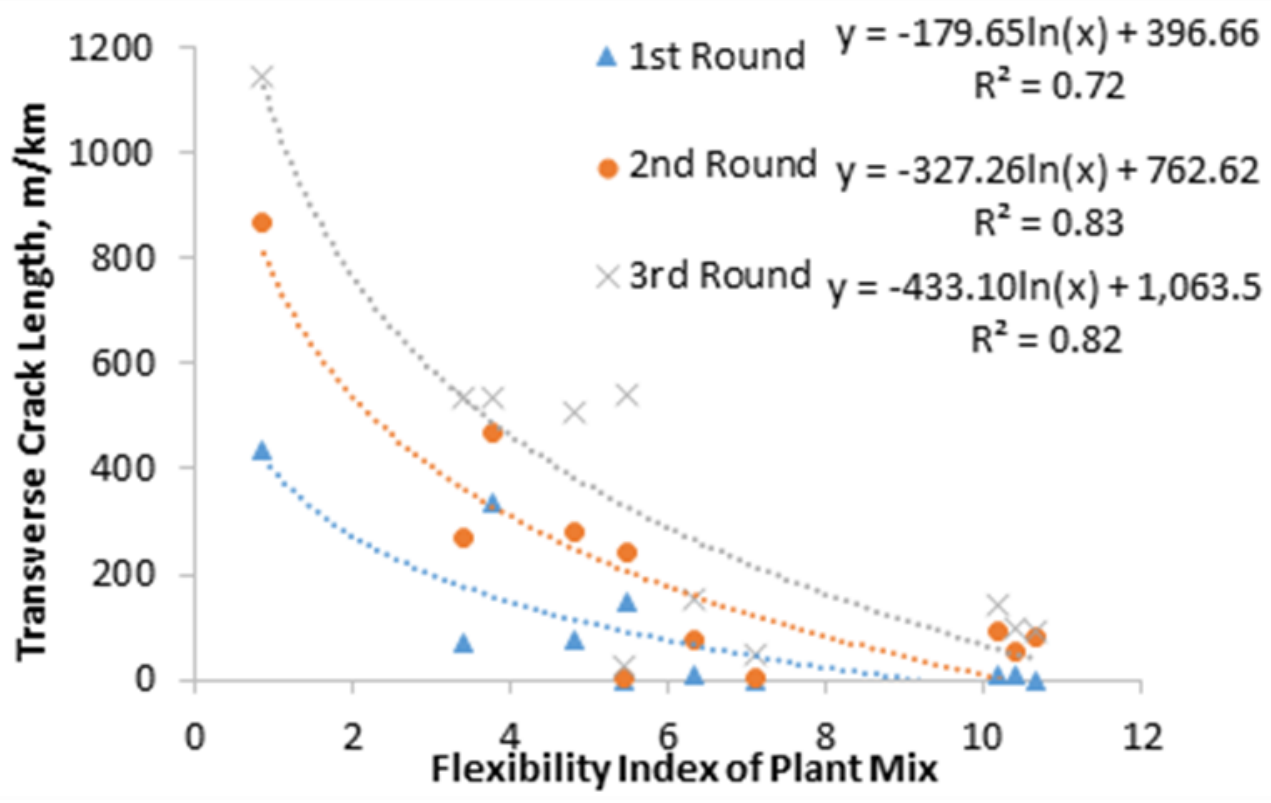

Figure 5.6. Field correlation between transverse cracking length FI for different rounds (Al-Qadi et al. 2018). 


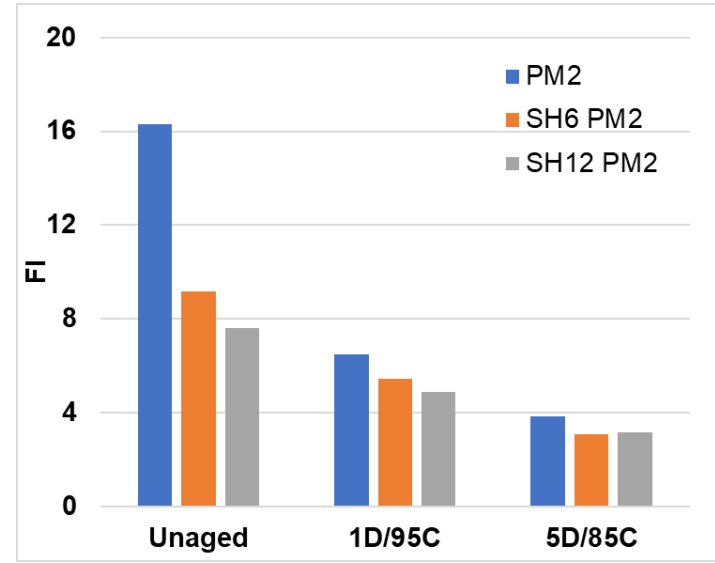

(a)

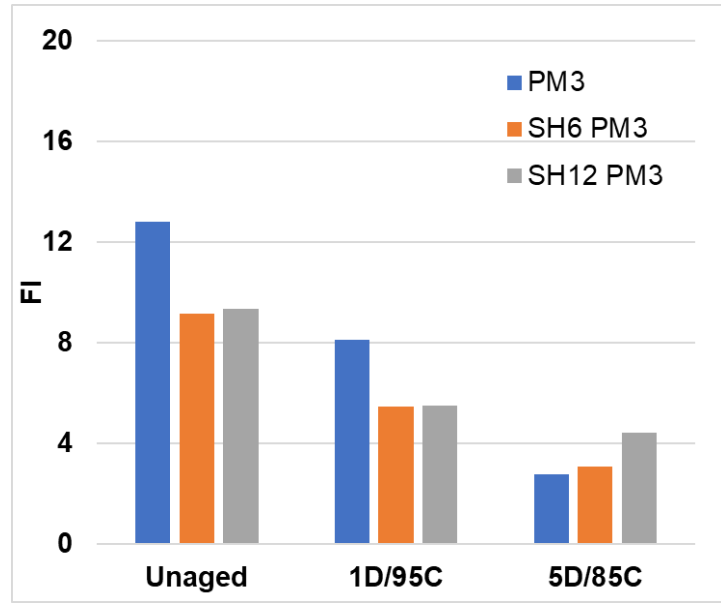

(b)

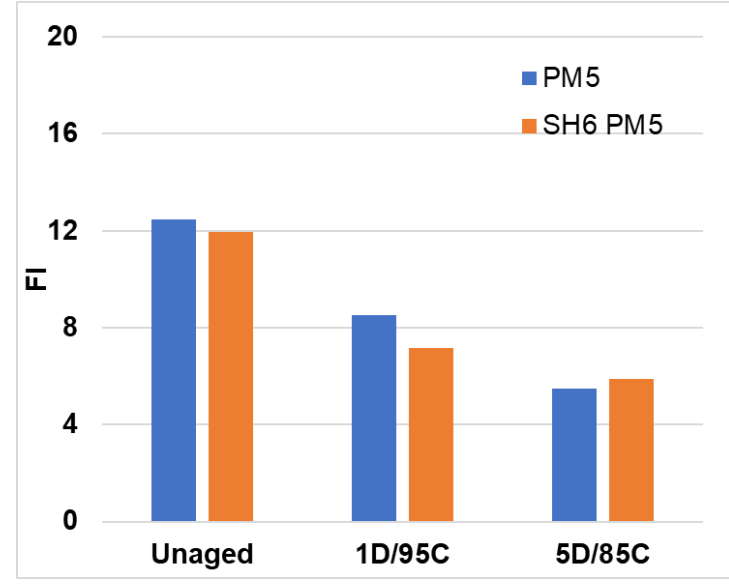

(c) 


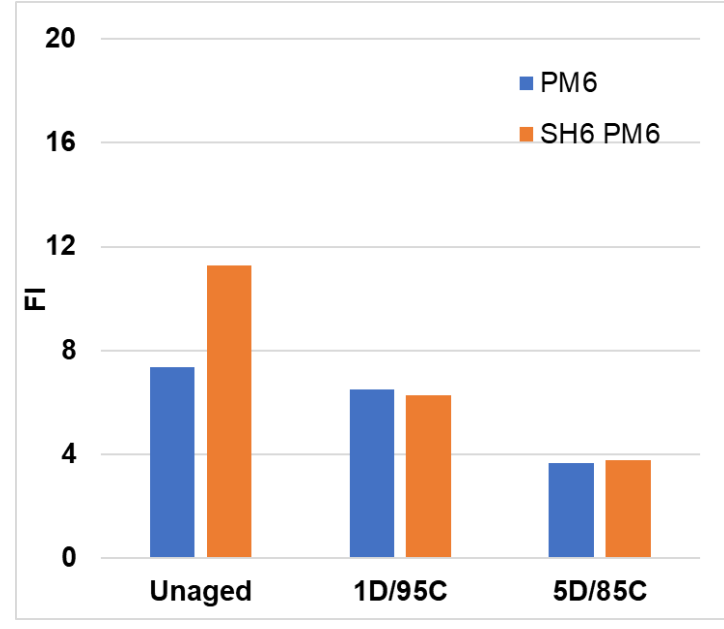

(d)

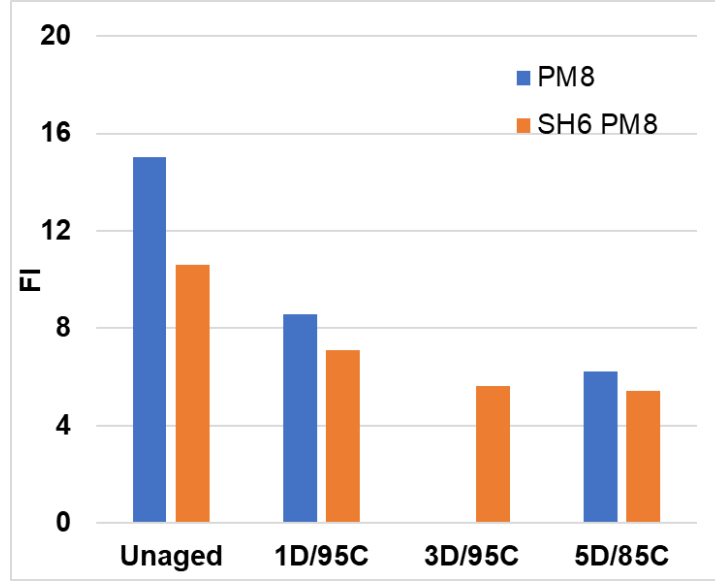

(e)

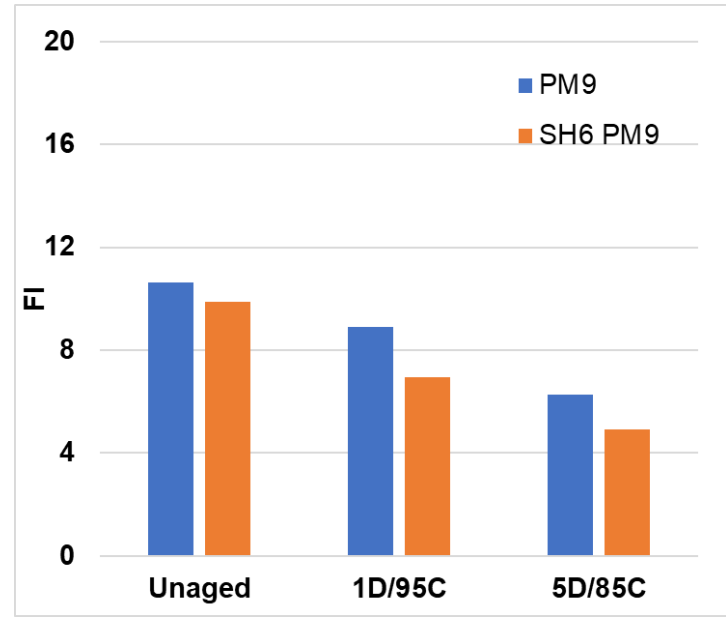

(f) 


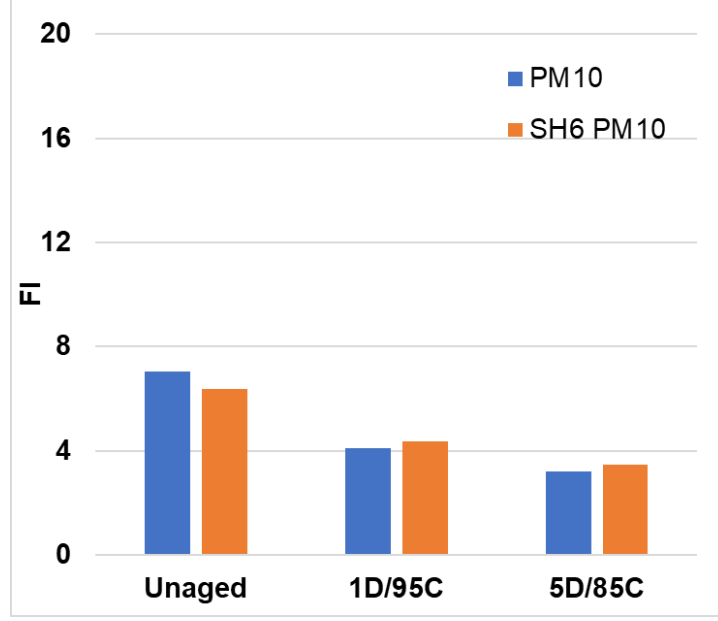

(g)

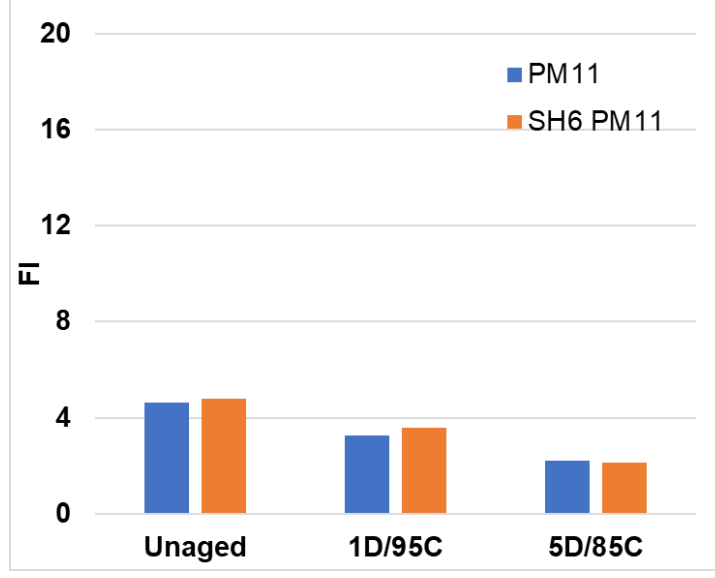

(h)

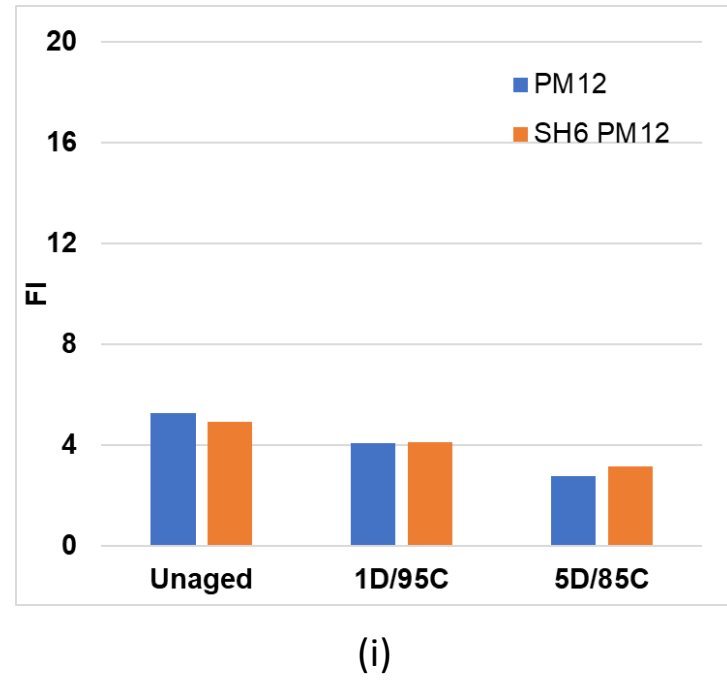

Figure 5.7. Impact of shelf aging on flexibility index. 
PM2 and PM3 were sampled in June 2017; tests after 6- and 12-month shelf aging were completed in December 2017 and June 2018, respectively. As can be seen in Figure 5.7, the first six months of shelf aging had a significant impact on the FI while the second six months had limited effect. At least half of the time in the first six months was in the summer season and most of the time in the second six months was in the winter season. Therefore, it could be concluded that three to five months of shelf aging in the summer season can significantly decrease the Fl, while the winter season nearly had no impact on flexibility of the mix.

PM5 was sampled in September 2017 and a six-month shelf aging process was completed in March 2018. This means the mix experienced around one month of shelf aging during the summer season. One month of summer shelf aging had nearly no impact on flexibility for this specific mix.

PM6 and PM8 were sampled in October 2017, and six-month shelf aging tests were completed in April 2017. This six-month shelf aging can be considered as the winter season. Although PM6 showed consistent results as expected, excluding unaged condition, PM8 showed decreasing $\mathrm{FI}$ for all conditions (unaged, 1D/95C, and 5D/85C) and the reason was unclear.

PM9, PM10, PM11, and PM12 were all sampled in November 2017 and six-month shelf aging tests were completed in May 2018. These four mixes can be considered to have been subjected to less than one month of summer season shelf aging and, as expected, the impact on flexibility was very limited.

Based on the above statements, the following conclusions can be made regarding the effect of shelf aging on the $\mathrm{FI}$ of $\mathrm{AC}$ :

- More than one month of summer season (June to September) shelf aging can significantly decrease the $\mathrm{FI}$ for $1 \mathrm{D} / 95 \mathrm{C}$ aging.

- Winter season shelf aging does not have significant impact on the Fl.

\subsubsection{Development of Thresholds for Shelf-Aged Plant-Produced Mixtures}

The same aging protocol proposed for PMLC mixes can be applied for shelf-aged mixes with some changes in the thresholds. I-FIT should be conducted on both unaged and 3D/95C aged specimens in all cases while $1 \mathrm{D} / 95 \mathrm{C}$ is only optional for contractors. Because IDOT requires all districts to test I-FIT specimens within three weeks upon sampling, a shelf-aging adjustment protocol is not included.

\subsection{SUMMARY}

Long-term aging protocols for I-FIT are proposed for plant-produced, laboratory-compacted (PPLC) and laboratory-produced, laboratory-compacted (LPLC) mixes. For LPLC mixes, the protocol requires I-FIT of unaged and 3D/95C aged specimens. The AC mix FI of unaged and 3D/95C aged specimens must be equal or greater than 8.0 and 5.0, respectively. For PPLC mixes, FI equal to or greater than 8.0 and 4.0 for unaged and 3D/95C aged specimens, respectively, must be satisfied. As an option for a quicker indication of the mix cracking potential, I-FIT procedure may be conducted on 1D/95C aged specimens. For $1 \mathrm{D} / 95 \mathrm{C}$, an $\mathrm{Fl}$ equal to or greater than 6.0 and an aging rate less than $45 \%$ may be considered by contractors as indicators of performance at three-day aging. For mixtures that are 
shelf-aged for more than one month in an uncontrolled environment during June to September, $\mathrm{FI}$ thresholds should be turned to consider shelf aging effect. Field cores from this study and the R27161 project were used to evaluate $\mathrm{Fl}$ progression in the field and its relationship to transverse cracking. It was shown that $\mathrm{Fl}$ and aging rate are correlated to transverse cracking progression. 


\section{CHAPTER 6: SUMMARY, FINDINGS, AND RECOMMENDATIONS}

\subsection{SUMMARY}

I-FIT procedure was developed as part of the ICT project R27-128 to allow practical evaluation of the cracking potential of AC mixtures. However, the method developed does not incorporate the longterm aging effect on cracking prediction. In addition, with increased use of recycled materials, binder softener use becomes common. There is a gap in the literature to guide the asphalt industry toward reliably and cost-effectively quantifying the long-term performance of Illinois asphalt pavements. The objective of this study was to evaluate long-term aging effects on asphalt mixtures using I-FIT and to develop a corresponding long-term aging protocol.

The development of a long-term aging protocol for I-FIT consists of the selection of equipment, state of material during aging, temperature, and aging duration. The forced-draft oven has been selected as the aging equipment due to its availability, feasibility, practicability, capacity, and acceptable variability. Semi-circular I-FIT specimens were chosen as the state of material during aging because it is practical, has limited operational variability, and maintains its integrity during aging. $203^{\circ} \mathrm{F}\left(95^{\circ} \mathrm{C}\right)$ is the optimal set-up temperature for forced-draft ovens, considering efficiency without altering the material due to changes other than the aging mechanism. The aging of I-FIT specimens for three days at $95^{\circ} \mathrm{C}$ was found to be similar to aging for five days at $85^{\circ} \mathrm{C}$, which is thought to simulate up to 10 years of field aging. Hence, the $3 \mathrm{D} / 95 \mathrm{C}$ aging method was chosen as the key component of the longterm aging protocol. Based on a statistical analysis and aging using argon gas, it was shown that the trends of aging after $1 \mathrm{D} / 95 \mathrm{C}$ are similar to those observed after $3 \mathrm{D} / 95 \mathrm{C}$ and $5 \mathrm{D} / 85 \mathrm{C}$. Therefore, $1 \mathrm{D} / 95 \mathrm{C}$ may be used to pre-screen and identify a non-borderline AC mixture as an alternate in some scenarios to shorten the time needed for the quality control process. Long-term aging protocols were then finalized for different scenarios as stated below.

Different protocols were developed for laboratory-produced, laboratory-compacted (LPLC) and plantproduced, laboratory-compacted (PPLC) I-FIT specimens. According to the protocol proposed for LPLC, I-FIT on unaged and 3D/95C aged specimens should be conducted in all cases. The FI for AC mixes must have an FI for unaged and 3D/95C aged specimens equal to or greater than 8.0 and 5.0, respectively.

For PPLC mixes, I-FIT procedure should be conducted on both unaged and 3D/95C aged specimens, while 1D/95C may be conducted by contractors to screen AC mixes for potential problems at an early stage. The required $\mathrm{FI}$ must be equal to or greater than 8.0 and 4.0 for unaged and 3D/95C aged specimens, respectively. An Fl equal to or greater than 6.0 and an aging rate equal to or less than $45 \%$ when conducted at 1D/95C may be considered by contractors as an optional criterion for an indication of three-day aging performance.

Figure 6.1 illustrates the aging protocols proposed for LPLC and PPLC AC mixes. 


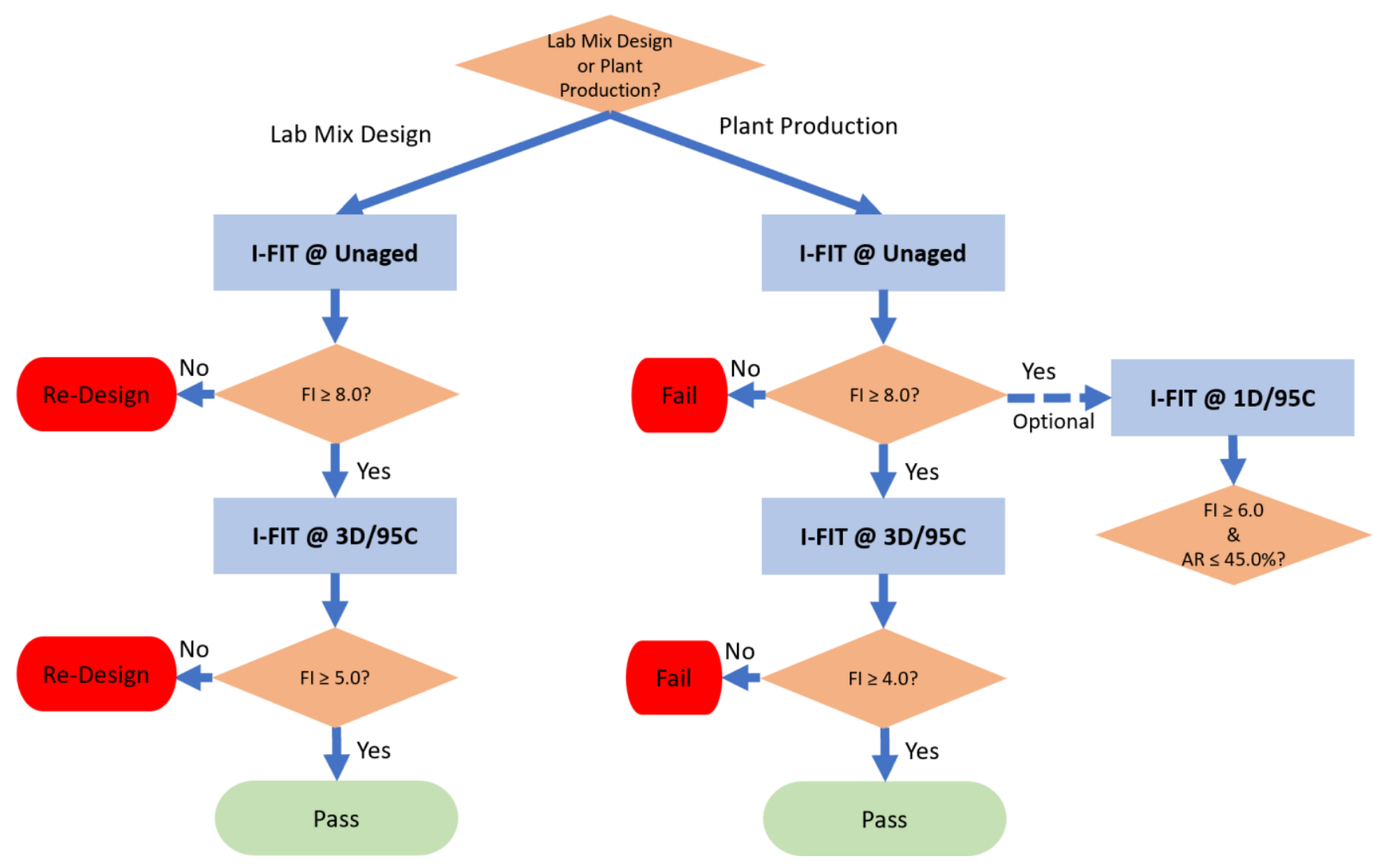

Figure 6.1. Long-term aging protocol for the Illinois Flexibility Index Test (I-FIT).

\subsection{FINDINGS}

The following findings can be drawn from this project:

- The Illinois Flexibility Index Test (I-FIT) is a valid and effective approach to capture the aging effect on AC mixtures.

- The forced-draft oven has been recognized as the most suitable aging equipment because of its availability, feasibility, practicability, capacity, and acceptable variability among ovens.

- Aging loose mix is faster than using compacted specimens, especially at high temperatures and long aging time. However, loose mix introduces operational variability.

- The compacted and fully prepared semi-circular I-FIT specimen has been chosen as the state of material during aging due to its high practicability, limited operational variability, and maintaining its integrity during aging.

- $203^{\circ} \mathrm{F}\left(95^{\circ} \mathrm{C}\right)$ is the optimal set-up temperature for the forced-draft oven considering efficiency while maintaining the aging mechanism.

- A three-day at $203^{\circ} \mathrm{F}\left(95^{\circ} \mathrm{C}\right)$ aging procedure is similar to aging for five days at $185^{\circ} \mathrm{F}\left(85^{\circ} \mathrm{C}\right)$, which is believed to simulate up to 10 years of field aging. 
- The decrease of Fl after aging, as indicated by a significant increase of the post-peak load slope, suggests that a mixture's flexibility is reduced and its potential for cracking is increased.

- Binder source and properties can significantly affect I-FIT results for both unaged and aged conditions.

- The impact of aging is mix dependent. However, aging generally reduces FI value. VMA, lowtemperature PG grade, mix type, aggregate blend water absorption, and effective asphalt content have a statistically significant impact on the aging rate of AC mixtures.

- The increase of each, or all of, effective asphalt content, and VMA, and a decrease of lowtemperature $P G$ grade, and aggregate blend water absorption induce a decrease in the aging rate of $A C$.

- Stone mastic asphalt (SMA) shows a significantly lower aging rate than dense-graded mixes.

- The FI decreases consistently when the AC specimens are aged in air while the FI remains the same under aging in argon. This suggests that oxidation is the main effect during the aging process.

- I-FIT performed on 1D/95C aged specimens is an optional test. Results may provide a reasonable indication of the aging behavior of AC during its service life and allows initial screening of $A C$ mixes at an earlier stage.

- More than one month of summer season (June to September) shelf aging can significantly decrease the AC Fl. On the other hand, winter season shelf aging does not have a significant impact on AC flexibility.

- The impact of shelf aging is significant on unaged specimens and is insignificant on 3D/95C.

\subsection{RECOMMENDATIONS}

- For long-term aging, it is recommended to age I-FIT specimens in a forced-draft oven at $203^{\circ} \mathrm{F}$ $\left(95^{\circ} \mathrm{C}\right)$. The $3 \mathrm{D} / 95 \mathrm{C}$ aging method was chosen as the key component of the long-term aging protocol.

- For laboratory-produced laboratory-compacted (LPLC) specimens, I-FIT procedure on unaged and $3 \mathrm{D} / 95 \mathrm{C}$ aged specimens should be conducted. The $\mathrm{Fl}$ criterion of at least 8.0 should be met for unaged specimens. The proposed FI threshold after 3D/95C long-term aging is 5.0.

- For plant-produced laboratory-compacted (PPLC) specimens, I-FIT procedure should be conducted on unaged specimens and meet an Fl criterion of at least 8.0 . The $3 \mathrm{D} / 95 \mathrm{C}$ longterm aging protocol should be used and the specimens should meet the Fl criterion of 4.0 or higher.

- The $1 \mathrm{D} / 95 \mathrm{C}$ protocol may be used as an optional test for informational purposes to provide an indication of the cracking potential of the $\mathrm{AC}$ mixture and/or to screen $\mathrm{AC}$ mixes. 


\section{REFERENCES}

AASHTO M 320-17. (2017). Standard Specification for Performance-Graded Asphalt Binder. Washington, DC: American Association of State Highways and Transportation Officials.

AASHTO MP 25-18. (2017). Standard Specification for Performance-Graded Hot-Poured Asphalt Crack Sealant. Washington, DC: American Association of State Highways and Transportation Officials.

AASHTO R 28-12. (2016). Standard Practice for Accelerated Aging of Asphalt Binder Using a Pressurized Aging Vessel. Washington, DC: American Association of State Highways and Transportation Officials.

AASHTO R 29-15. (2016). Standard Specification for Grading or Verifying the Performance Grade (PG) of an Asphalt Binder. Washington, DC: American Association of State Highways and Transportation Officials.

AASHTO R 30-02. (2016). Standard Practice for Mixture Conditioning of Hot Mix Asphalt (HMA). Washington, DC: American Association of State Highways and Transportation Officials.

AASHTO R 47-14. (2018). Standard Practice for Reducing Samples of Hot Mix Asphalt (HMA) to Testing Size. Washington, DC: American Association of State Highways and Transportation Officials.

AASHTO T 240-13. (2016). Standard Method of Test for Effect of Heat and Air on a Moving Film of Asphalt Binder (Rolling Thin-Film Oven Test). Washington, DC: American Association of State Highways and Transportation Officials.

AASHTO T 312-15. (2016). Standard Method of Test for Preparing and Determining the Density of Asphalt Mixture Specimens by Means of the SuperPave Gyratory Compactor. Washington, DC: American Association of State Highways and Transportation Officials.

AASHTO T 313-12. (2016). Standard Method of Test for Determining the Flexural Creep Stiffness of Asphalt Binder Using the Bending Beam Rheometer (BBR). Washington, DC: American Association of State Highways and Transportation Officials.

AASHTO T 315-12. (2016). Standard Method of Test for Determining the Rheological Properties of Asphalt Binder Using a Dynamic Shear Rheometer (DSR). Washington, DC: American Association of State Highways and Transportation Officials.

AASHTO T 316-13. (2016). Standard Method of Test for Viscosity Determination of Asphalt Binder Using Rotational Viscometer. Washington, DC: American Association of State Highways and Transportation Officials.

AASHTO TP 124-18. (2018). Standard Method of Test for Determining the Fracture Potential of Asphalt Mixtures Using the Flexibility Index Test (FIT). Washington, DC: American Association of State Highways and Transportation Officials.

Airey, G. D. (2003). State of the art report on ageing test methods for bituminous pavement materials. International Journal of Pavement Engineering, 4(3), 165-176.

Airey, G. D., Choi, Y. K., Collop, A. C., \& Elliott, R. C. (2003). Development of an accelerated durability assessment procedure for high modulus base (HMB) materials. Proceedings of the 6th 
International RILEM Symposium on Performance Testing and Evaluation of Bituminous Materials, PTEBM, 3, 160-166.

Al-Qadi, I. L., Lippert, D. L., Wu, S., Ozer, H., Renshaw, G., Murphy, T. R., ... \& Said, I. M. (2017). Utilizing lab tests to predict asphalt concrete overlay performance (Illinois Center for Transportation Series No. 17-026). Illinois Center for Transportation/Illinois Department of Transportation.

Al-Qadi, I. L., Ozer, H., Lambros, J., El Khatib, A., Singhvi, P., Khan, T., ... \& Doll, B. (2015). Testing protocols to ensure performance of high asphalt binder replacement mixes using RAP and RAS (Illinois Center for Transportation Series No. 15-017). Illinois Center for Transportation/Illinois Department of Transportation.

Al-Qadi, I. L., Wu, S., Lippert, D. L., Ozer, H., \& Safi, F. R. (2018). Field performance and materials characteristics evolution of asphalt pavements containing various recycled materials. Journal of Road Materials and Pavement Design (Accepted).

Anderson, D. A., \& Bonaquist, R. F. (2012). Investigation of short-term laboratory aging of neat and modified asphalt binders (Vol. 709). Transportation Research Board.

ASTM D7643-16. (2016). Standard Practice for Determining the Continuous Grading Temperature and Continuous Grades for PG Graded Asphalt Binders. West Conshohocken, PA: ASTM International.

Azari, H., \& Mohseni, A. (2013). Effect of short-term conditioning and long-term ageing on permanent deformation characteristics of asphalt mixtures. Road Materials and Pavement Design, 14(sup2), 79-91. https://doi.org/10.1080/14680629.2013.812833

Baek, C., Underwood, B., \& Kim, Y. (2012). Effects of oxidative aging on asphalt mixture properties. Transportation Research Record: Journal of the Transportation Research Board, 2296(1), 77-85. https://doi.org/10.3141/2296-08

Barry, M. K. (2016). An analysis of impact factors on the Illinois flexibility index test (Master Thesis, University of Illinois at Urbana-Champaign).

Bell, C. A. (1989). Summary report on aging of asphalt-aggregate systems (No. SHRP-A-305).

Bell, C. A., AbWahab, Y., Cristi, M. E., \& Sosnovske, D. (1994). Selection of laboratory aging procedures for asphalt-aggregate mixtures (No. SHRP-A-383). Strategic Highway Research Program.

Bell, C. A., Sosnovske, D., \& Wieder, J. A. (1994). Aging: Binder validation (No. SHRP-A-384). Washington, DC: Strategic Highway Research Program, National Research Council.

Bell, C. A., Wieder, A. J., \& Fellin, M. J. (1994). Laboratory aging of asphalt-aggregate mixtures: Field validation (No. SHRP-A-390).

Canestrari, F., Ferrotti, G., Lu, X., Millien, A., Partl, M. N., Petit, C., ... \& Raab, C. (2013). Mechanical testing of interlayer bonding in asphalt pavements. In Advances in interlaboratory testing and evaluation of bituminous materials (pp. 303-360). Springer, Dordrecht. https://doi.org/10.1007/978-94-007-5104-0_6

Chen, C., Yin, F., Turner, P., West, R. C., \& Tran, N. (2018). Selecting a Laboratory Loose Mix Aging Protocol for the NCAT Top-Down Cracking Experiment. Transportation Research Record, 
0361198118790639.

Collop, A. C., Choi, Y., \& Airey, G. D. (2007). Effects of pressure and aging in SATS test. Journal of Transportation Engineering, 133(11), 618-624. https://doi.org/10.1061/(ASCE)0733-

947X(2007)133:11(618)

Der, G., \& Everitt, B. S. (2008). A handbook of statistical analyses using SAS. Chapman and Hall/CRC.

Elseifi, M. (2007). Evaluation of Hot-Mix Asphalt Sampling Techniques.

Fernández-Gómez, W. D., Vides-Berdugo, A. C., Roncallo-Contreras, S. P., Bautista-Rondón, F., Rondón-Quintana, H. A., \& Reyes-Lizcano, F. A. (2016). Effects of environmental aging and ultra violet radiation on asphalt mixture dynamic modulus, permanent deformation and fatigue life. Revista Facultad de Ingeniería Universidad de Antioquia, (80), 89-96.

Hachiya, Y., Nomura, K., \& Shen, J. (2003). Accelerated aging tests for asphalt concretes. Sixth International RILEM Symposium on Performance Testing and Evaluation of Bituminous Materials (pp. 133-140). RILEM Publications SARL.

Hagos, E. T. (2008). The effect of aging on binder properties of porous asphalt concrete (Doctoral thesis, Delft University of Technology).

Herrington, P. R., Patrick, J. E., \& Ball, G. F. (1994). Oxidation of roading asphalts. Industrial \& Engineering Chemistry Research, 33(11), 2801-2809. https://doi.org/10.1021/ie00035a033

Houston, W. N., Mirza, M. W., Zapata, C. E., \& Raghavendra, S. (2007). Simulating the effects of hot mix asphalt aging for performance testing and pavement structural design. NCHR Research Results Digest, 324.

Houston, W. N., Mirza, M. W., Zapata, C. E., \& Raghavendra, S. (2005). Environmental effects in pavement mix and structural design systems. NCHRP, Project, 9-23.

Khalid, H. A. (2002, August). A new approach for the accelerated ageing of porous asphalt mixtures. Proceedings of the Institution of Civil Engineers-Transport, 153(3): 171-181. https://doi.org/10.1680/tran.2002.153.3.171

Kim, O. K., Montalvo, J., Bell, C. A., Hicks, R. G., Wilson, J. E., \& Boyle, G. E. (1986). Effect of moisture and aging on asphalt pavement life: Part 2-effect of aging (No. FHWA-OR-RD-86-01-2). Oregon. Dept. of Transportation. Research Unit.

Kim, Y. R., Castorena, C., Elwardany, M. D., Rad, F. Y., Underwood, S., Akshay. Gundla, ... \& Glaser, R. R. (2018). Long-term aging of asphalt mixtures for performance testing and prediction. Transportation Research Board.

Kinsley Jr, G. R. (2001). Properly purge and inert storage vessels. Natural gas (Pittsburgh), 12, 14-5.

Kliewer, J. E., Bell, C. A., \& Sosnovske, D. A. (1995). Investigation of the relationship between field performance and laboratory aging properties of asphalt mixtures. Engineering Properties of Asphalt Mixtures and the Relationship to Their Performance. ASTM International.

Lemke, Z., Sadek, H., Swiertz, D., Reichelt, S., \& Bahia, H. U. (2018). Effects of reheating procedure and oven type on performance testing results of asphalt mixtures. Transportation Research Record, 0361198118773886. 
Li, Y., \& Nazarian, S. (1995). Evaluation of aging of hot-mix asphalt using wave propagation techniques. Engineering Properties of Asphalt Mixtures and the Relationship to Their Performance. ASTM International.

Lu, X., Talon, Y., \& Redelius, P. (2008, May). Ageing of bituminous binders-laboratory tests and field data. Proceedings of the 4th Eurasphalt \& Eurobitume Congress, Copenhagen (pp. 21-23).

Ma, T., Huang, X. M., Mahmoud, E., \& Garibaldy, E. (2011). Effect of moisture on the aging behavior of asphalt binder. International Journal of Minerals, Metallurgy, and Materials, 18(4), 460. https://doi.org/10.1007/s12613-011-0463-4

Mollenhauer, K., \& Mouillet, V. (2011). Re-road-End of life strategies of asphalt pavements. European Commission DG Research.

Mollenhauer, K., Mouillet, V., Pierard, N., Tusar, M., \& Gabet, T. (2012, June). Laboratory aging of asphalt mixtures: Simulation of reclaimed asphalt and application as test method for durability. 5th Eurasphalt \& Eurobitume Congress (p. 8p).

Monismith, C. L., Hicks, R. G., Finn, F. N., Tayebali, A. A., Sousa, J. B., Harvey, J., ... \& Scholz, T. (1994). Accelerated performance-related tests for asphalt-aggregate mixes and their use in mix design and analysis systems (No. SHRP-A-417).

Morian, N., Hajj, E., Glover, C., \& Sebaaly, P. (2011). Oxidative aging of asphalt binders in hot-mix asphalt mixtures. Transportation Research Record: Journal of the Transportation Research Board, (2207), 107-116. https://doi.org/10.3141/2207-14

Mouillet, V., Farcas, F., Chailleux, E., \& Sauger, L. (2014). Evolution of bituminous mix behaviour submitted to UV rays in laboratory compared to field exposure. Materials and Structures, 47(8), 1287-1299. https://doi.org/10.1617/s11527-014-0258-y

Newcomb, D., Martin, A. E., Yin, F., Arambula, E., Park, E. S., Chowdhury, A., ... \& Jones, D. (2015). Short-term laboratory conditioning of asphalt mixtures (No. Project 09-52).

Nicholls, J. C., Carswell, I., Vanelstraete, A. N., Hirsch, V., \& Blab, R. (2007). Analysis of available data for validation of bitumen tests. na.

Ozer, H., Renshaw, G., Hasiba, K., \& Al-Qadi, I. L. (2016). Evaluation of the impacts of re-refined engine oil bottoms (REOB) on performance graded asphalt binders and asphalt mixtures. Illinois Center for Transportation/Illinois Department of Transportation.

Partl, M. N., Bahia, H. U., Canestrari, F., De la Roche, C., Di Benedetto, H., Piber, H., \& Sybilski, D. (Eds.). (2012). Advances in interlaboratory testing and evaluation of bituminous materials: Stateof-the-art report of the RILEM technical committee 206-ATB (Vol. 9). Springer Science \& Business Media.

Petersen, J. C. (2009). A review of the fundamentals of asphalt oxidation: Chemical, physicochemical, physical property, and durability relationships. Transportation Research E-Circular, (E-C140).

Rad, F. Y., Elwardany, M. D., Castorena, C., \& Kim, Y. R. (2018). Evaluation of chemical and rheological aging indices to track oxidative aging of asphalt mixtures. Transportation Research Record, 0361198118784138. 
Reed, J. (2010, December). Evaluation of the effects of aging on asphalt rubber pavements. Masters Abstracts International, 49,(3).

Rivera-Perez, J. J. (2017). Effect of specimen geometry and test configuration on the fracture process zone for asphalt materials (Master Thesis, University of Illinois at Urbana-Champaign).

Scholz, T. V. (1995). Durability of bituminous paving mixtures (Doctoral dissertation, University of Nottingham).

Sharew Yehualaeset, J. (2010). Development of a laboratory ageing method for bitumen in porous asphalt.

Sharma, B. K., Ma, J., Kunwar, B., Singhvi, P., Ozer, H., \& Rajagopalan, N. (2017). Modeling the performance properties of RAS and RAP blended asphalt mixes using chemical compositional information. Illinois Center for Transportation/Illinois Department of Transportation.

Tarbox, S., \& Daniel, J. (2012). Effects of long-term oven aging on reclaimed asphalt pavement mixtures. Transportation Research Record: Journal of the Transportation Research Board, (2294), 1-15. https://doi.org/10.3141/2294-01

Van den Bergh, W. (2011). The effect of ageing on the fatigue and healing properties of bituminous mortars.

Von Quintus, H. L., Hughes, C. S., \& Scherocman, J. A. (1992). NCHRP asphalt-aggregate mixture analysis system. Transportation Research Record, (1353).

Von Quintus, H. L., Scherocman, J., \& Hughes, C. (1989). Asphalt-aggregate mixtures analysis system: Philosophy of the concept. Asphalt Concrete Mix Design: Development of More Rational Approaches. ASTM International.

$\mathrm{Wu}, \mathrm{J}$. (2009). The influence of mineral aggregates and binder volumetrics on bitumen ageing. Nottingham: University of Nottingham.

Wu, S., Pang, L., Liu, G., \& Zhu, J. (2010). Laboratory study on ultraviolet radiation aging of bitumen. Journal of Materials in Civil Engineering, 22(8), 767-772. https://doi.org/10.1061/(ASCE)MT.19435533.0000010

Xiao, F., Newton, D., Putman, B., Punith, V. S., \& Amirkhanian, S. N. (2013). A long-term ultraviolet aging procedure on foamed WMA mixtures. Materials and structures, 46(12), 1987-2001. https://doi.org/10.1617/s11527-013-0031-7

Yin, F., Arámbula-Mercado, E., Epps Martin, A., Newcomb, D., \& Tran, N. (2017). Long-term ageing of asphalt mixtures. Road Materials and Pavement Design, 18(sup1), 2-27. https://doi.org/10.1080/14680629.2016.1266739

Zeng, W., Wu, S., Wen, J., \& Chen, Z. (2015). The temperature effects in aging index of asphalt during UV aging process. Construction and Building Materials, 93, 1125-1131.

https://doi.org/10.1016/j.conbuildmat.2015.05.022 


\section{APPENDIX A: JOB MIX FORMULAS OF PLANT-PRODUCED MIXES}

Table A.1. PM1

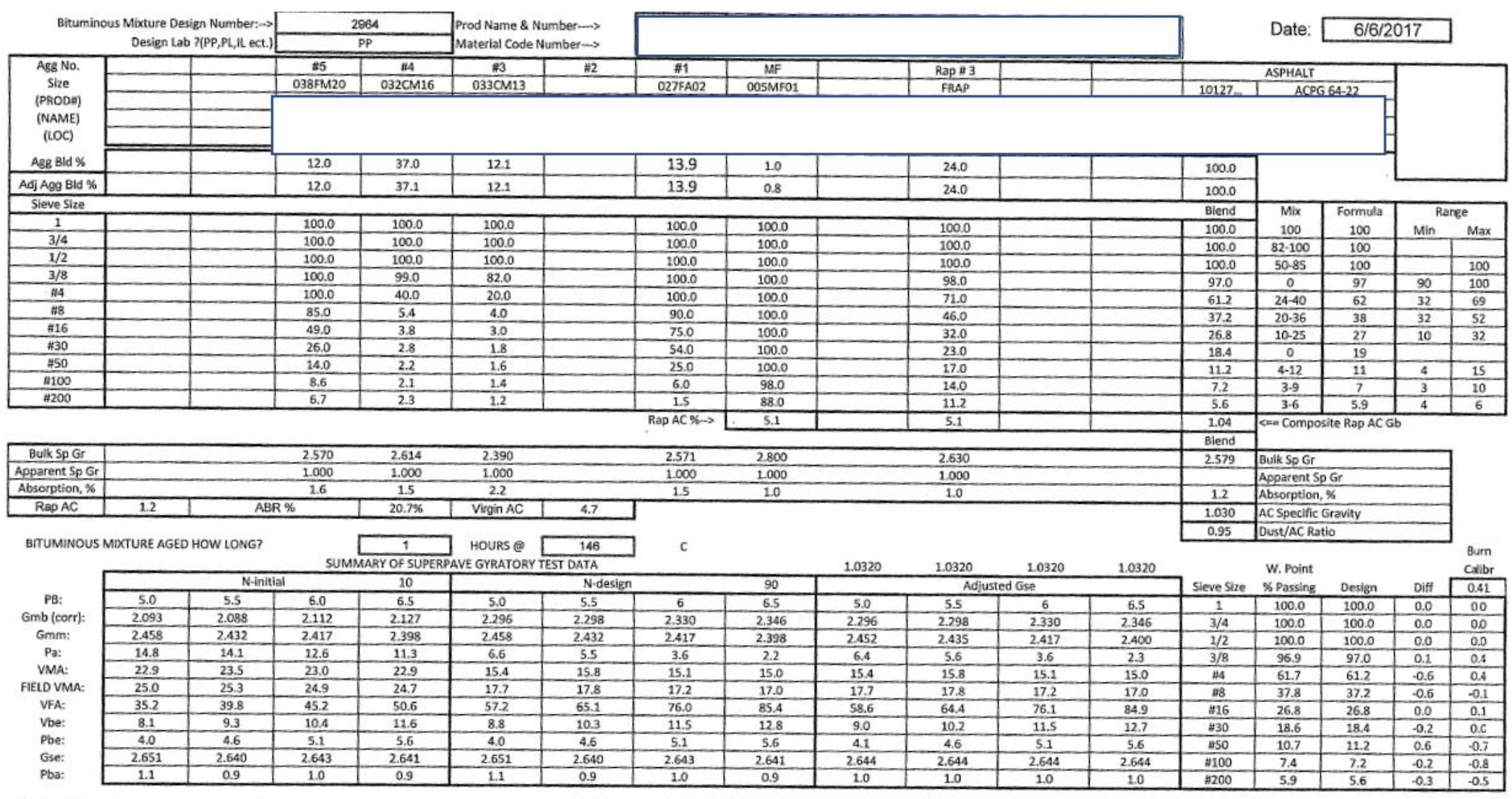

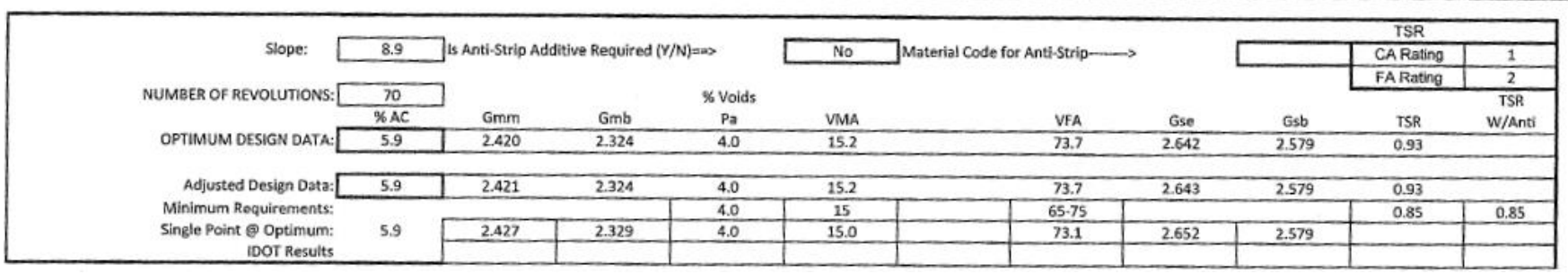


Table A.2. PM2

\begin{tabular}{|c|c|c|c|c|c|c|c|c|c|c|c|c|c|}
\hline \begin{tabular}{|r|} 
Plant Bin N \\
Size \\
Source ( PROO E) \\
(NAME) \\
(LOC)
\end{tabular} & $\frac{77}{022 \mathrm{CM} 1 \mathrm{y}}$ & $\frac{m}{\text { oxcmig }}$ & $\begin{array}{c}5 \\
022 \mathrm{CM} 16\end{array}$ & $\frac{14}{122 \mathrm{CM} 16}$ & $\frac{13}{028 F M 20}$ & $\frac{12}{12}$ & $\begin{array}{c}\text { "1 } \\
\text { O27FMO1 }\end{array}$ & $\begin{array}{l}\text { MF } \\
\text { MFMO1 }\end{array}$ & $\begin{array}{l}\text { FRAP :A } \\
\text { 017CN13 }\end{array}$ & $\begin{array}{l}\text { FRAP E3 } \\
\text { O17CM13 }\end{array}$ & RCY & RCY & \begin{tabular}{|c|} 
ASPHALT \\
10120
\end{tabular} \\
\hline \multirow[t]{5}{*}{ (ADD.INFO) } & 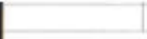 & & & & & & & & fisco & 157c- & & & Ses PG $70-22$ \\
\hline & Apgregatse Blend: & & & & & & & & $3.1^{\prime}$ & $s$. & 0.0 & $\begin{array}{c}0.0 \\
\text { Plan PG Grade }\end{array}$ & $\begin{array}{l}\text { *AS in RAP } \\
P G 70-22\end{array}$ \\
\hline & 0.0 & 0.0 & 45.8 & 11.2 & 23.0 & 0.0 & 8.0 & 0.9 & 0.0 & 11.1 & 0.0 & 0.0 & 100.0 \\
\hline & Mixture Bilend: & & & & & & & & & & & & totak: I \\
\hline & 0.0 & 0.0 & 43.0 & 10.5 & 21.6 & 00 & 7.5 & 0.8 & 0.0 & 11.0 & 0.0 & 0.0 & 100.0 \\
\hline
\end{tabular}

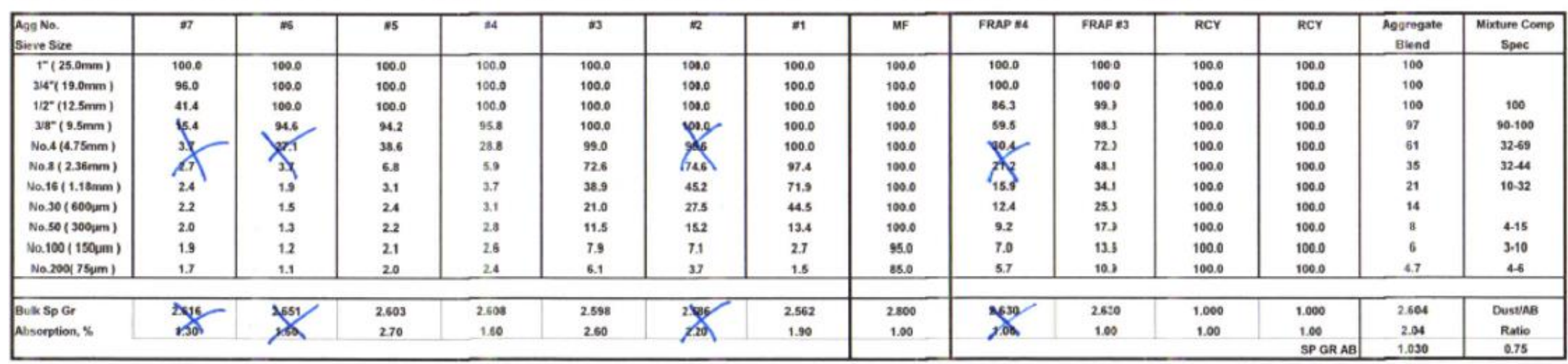

SUMMARY OF SUPERPAVE GYRATORY DESIGN DATA

\begin{tabular}{|c|c|c|c|c|c|c|c|c|c|}
\hline DATA for N-int. & 8 & & & & & & & & \\
\hline & AB, צMIX & Gmb & Gmm & Voids (Pa) & $\operatorname{vman}$ & VFA & vbe & Pbe & Pba \\
\hline $\operatorname{mix} 1$ & 5.2 & 2.075 & 2.489 & 166 & 20.5 & 320 & 7.82 & 2.88 & 1.39 \\
\hline $\operatorname{Mix} 2$ & 57 & 2000 & 2.479 & 15.1 & 24.7 & 348 & 8.60 & 4.26 & 1.59 \\
\hline $\operatorname{mix} 3$ & 6.2 & 2003 & 2456 & 148 & 24.6 & sas & 9.44 & 4.94 & 1.45 \\
\hline $\operatorname{Mix} 4$ & 6.7 & 2.103 & 2.437 & 13.7 & 24.7 & 44 & 10.96 & 5.37 & 1.43 \\
\hline
\end{tabular}

\begin{tabular}{|c|c|c|c|c|c|c|c|c|c|c|}
\hline DaTA for N-des. & 90 & & & & & & & & & \\
\hline & & amb & Grmm & Voids $(P a)$ & maa & VFA & vae & Poe & Gse & Pba \\
\hline $\operatorname{Mix} 1$ & 5.2 & 2.321 & 2.489 & 68 & 15.5 & 56.5 & 8.74 & 3.83 & 2.699 & 1.20 \\
\hline $\operatorname{Mix} 2$ & 5.7 & 2.396 & 2.479 & 5.8 & 15.4 & 62.6 & 9.66 & 4.26 & 2.709 & 1.80 \\
\hline $\operatorname{M} \times 3$ & 6.2 & 2.356 & 2.456 & 4.1 & 15.1 & 73.1 & 11.07 & 4.84 & 2703 & 1.05 \\
\hline MIX 4 & 6.7 & 2.366 & 2.437 & 29 & 15.2 & 80.9 & 12.34 & 8.37 & 2.702 & 1.00 \\
\hline
\end{tabular}

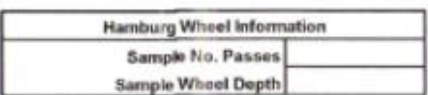

\begin{tabular}{|c|c|c|c|c|c|c|c|c|c|c|c|c|}
\hline \multicolumn{13}{|c|}{ OPTMUUM DESIGN DATA \& Ndes } \\
\hline GYRATIONS & $A B$ & Gmb & Gmm & XVOIDS (Pa) & VMA & VFA & Gse & Gsb & TSR & RCY AB & Virgin $A B$ & ABR \\
\hline
\end{tabular}


Table A.3. PM3

\begin{tabular}{|c|c|c|c|c|c|c|c|c|c|c|c|c|c|}
\hline \begin{tabular}{|l|} 
Plant Bin " \\
sine
\end{tabular} & $\frac{7}{222 \mathrm{CM} 11}$ & $\frac{16}{021 \mathrm{CM} 16}$ & $\begin{array}{c}05 \\
022 \mathrm{CM} 16\end{array}$ & $\frac{14}{1202015}$ & $\begin{array}{c}\text { M3 } \\
028 F M 20\end{array}$ & $\frac{12}{102}$ & O27F & $\begin{array}{l}\text { MF } \\
\text { MFEe01 }\end{array}$ & FRAP BA & FRAP & $\begin{array}{l}\text { FRAP 12 } \\
\text { O17TCM13 }\end{array}$ & $\begin{array}{l}\text { FRAP H1 } \\
\text { O17CN13 }\end{array}$ & $\begin{array}{c}\text { ASPHALT } \\
10129\end{array}$ \\
\hline \multicolumn{14}{|l|}{$\begin{array}{r}\text { Source (PROD B) } \\
\text { (NAME) } \\
\text { (LOC) }\end{array}$} \\
\hline & & & & & & & & & & & $/ 1570+0^{\circ}$ & 1570 . & ses PG $70-22$ \\
\hline & A & & & & & & & & 0.0 & 0.8 & 2.9 & 4.9 & KAB in RAP \\
\hline & 0.0 & 263 & 251 & 00 & 256 & 00 & 95 & 11 & 0.0 & 00 & 20 & Plan PQ Grade 3 & 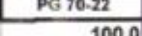 \\
\hline & Mixture Blend: & & & & & & & & & & 0.0 & 12.4 & 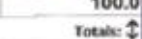 \\
\hline & 0.0 & 24.7 & 23.5 & 0.0 & 24.0 & 0.0 & 8.9 & 1.0 & 0.0 & 0.0 & 0.0 & 12.2 & 100.0 \\
\hline
\end{tabular}

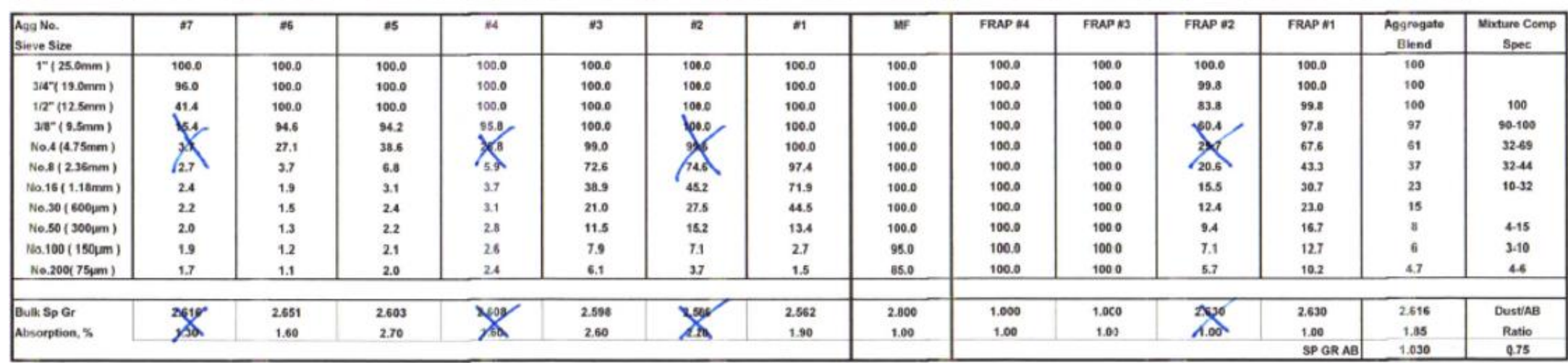

SUMMARY OF SUPERPAVE GYRATORY DESIGN DATA

\begin{tabular}{|c|c|c|c|c|c|c|c|c|c|}
\hline DATA for N-int & 8 & & & & & & & & \\
\hline & $A B, X M D X$ & Gmb & Gemm & Voids (Pa) & vMA & VFA & not & Pbo & Pta \\
\hline $\operatorname{vax} 1$ & 5.6 & 2.116 & 2.484 & 14.8 & 23.6 & 37.4 & 8.83 & 4.30 & 1.38 \\
\hline $\operatorname{sex} 2$ & 6.1 & 2.121 & 2.471 & 14.2 & 23.9 & 40.7 & 9.70 & 4.71 & 1.48 \\
\hline $\max 3$ & 6.6 & 2.137 & 2.453 & 12.9 & 237 & 457 & 10.83 & 5.22 & 1.48 \\
\hline $\max 4$ & 7.1 & 2.132 & 2436 & 12.5 & 24.3 & 48.6 & 11.80 & 5.70 & 1.51 \\
\hline
\end{tabular}

\begin{tabular}{|c|c|c|c|c|c|c|c|c|c|c|}
\hline DATA for N-des. & 90 & & & & & & & & & \\
\hline & & Gmb & Gmm & Voids (Pa) & vinea & VFA & vbe & Pbo & Gese & $\mathrm{Pba}$ \\
\hline $\operatorname{vax} 1$ & 5.6 & 2.387 & 2.494 & 5.1 & 15.0 & 658 & 2.94 & 4.20 & 2711 & 1.38 \\
\hline $\operatorname{six} 2$ & 6.1 & 2.363 & 2.471 & 4.4 & 15.2 & 71.2 & 10.81 & 4.71 & 2718 & 1.48 \\
\hline $\max 3$ & 6.6 & 2.381 & 2.453 & 2.9 & 15.0 & 80.4 & 12.07 & 5.22 & 2718 & 148 \\
\hline $\max 4$ & 71 & 2.389 & 2.436 & 19 & 152 & 873 & 13.22 & 5.70 & 2.720 & 151 \\
\hline
\end{tabular}

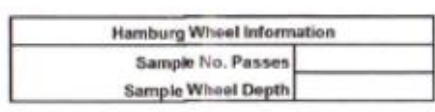

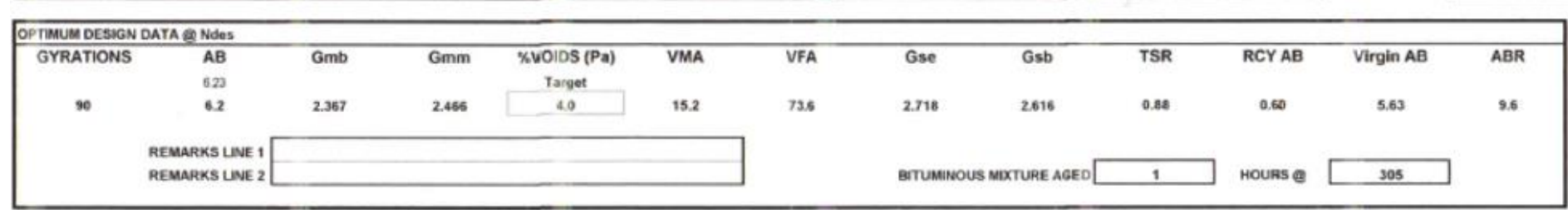


Table A.4. PM5

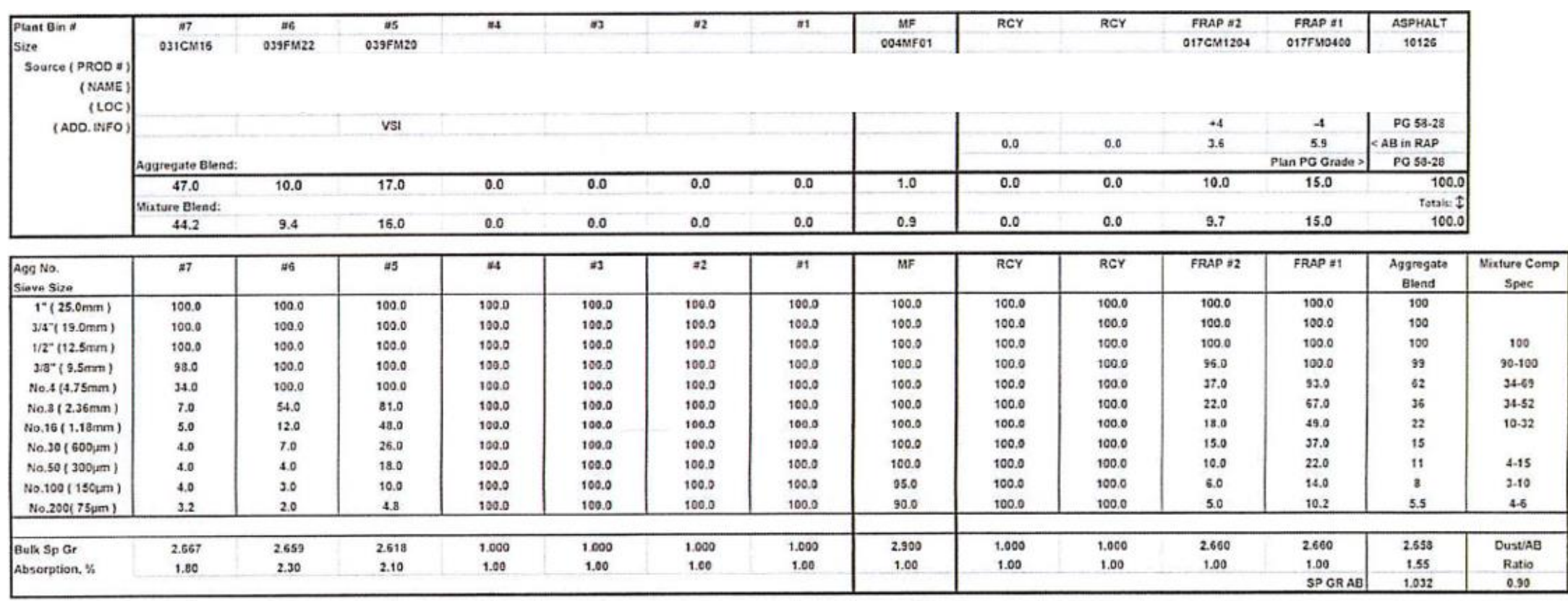

SUMMARY OF SUPERPAVE GYRATORY DESIGN DATA

\begin{tabular}{|c|c|c|c|c|c|c|c|c|c|}
\hline DATA for $N$-int. & 7 & & & & & & & & \\
\hline & $A B, \%=1 x$ & Gmb & Gmm & Voids (Pa) & VEA & VFA & vibe & Pbe & Pba \\
\hline $\operatorname{mx} 1$ & 5.5 & 2.124 & 2.517 & 15.6 & 24.5 & 36.3 & 8.87 & 4.31 & 1.26 \\
\hline $\operatorname{mix} 2$ & 6.0 & 2.133 & 2.492 & 14.4 & 24.6 & 41.4 & 10.17 & 4.92 & 1.15 \\
\hline $\max 3$ & 6.5 & 2.140 & 2475 & 12.5 & 24.7 & 452 & 11.20 & 5.40 & 1,18 \\
\hline $\mathrm{m} \times 4$ & 7.0 & 2.159 & 2453 & 12.0 & 24.5 & 51.0 & 12.47 & 5.96 & 1.12 \\
\hline
\end{tabular}

\begin{tabular}{|c|c|c|c|c|c|c|c|c|c|c|}
\hline DATA tor N-des. & 70 & & & & & & & & & \\
\hline & & Gmb & Gman & Voids (Pa) & VSAA & VFA & vbe & Pbe & Gse & Pbas \\
\hline $\max 1$ & 5.5 & 2.375 & 2.517 & 5.6 & 15.6 & 63.8 & 9.92 & 4.31 & 2.747 & 1.25 \\
\hline $\operatorname{Mix} 2$ & 6.0 & 2.389 & 2.492 & 4.4 & 15.5 & 72.4 & 11.39 & 4.92 & 2.739 & 1.15 \\
\hline$m \times 3$ & 6.5 & 2.401 & 2.475 & 30 & 15.5 & 20.8 & 12.55 & 5.40 & 2.741 & t.18 \\
\hline$M \times 4$ & 7.0 & 2.413 & 2.453 & 1.5 & 15.6 & 89.5 & 13.94 & 5.96 & 2.737 & t.12 \\
\hline
\end{tabular}

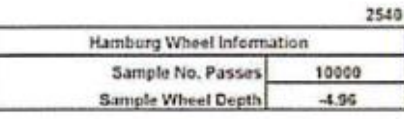

\begin{tabular}{|c|c|c|c|c|c|}
\hline GYRATIONS & $A B$ & Gmb & Gmm & $\%$ VOIDS (Pa) & VMA \\
\hline & 608 & & & Target & \\
\hline 70 & 6.1 & 2.390 & 2.490 & 40 & 15.5 \\
\hline
\end{tabular}

VFA

$+\frac{10}{10}$

(a)

RCYAB

\begin{tabular}{|c|c|}
\hline \multicolumn{2}{|c|}{ Hanburg Whoel Infomation } \\
\hline Sample No. Passers & 10000 \\
\hline Sample Wheel Depth] & +96 \\
\hline \multicolumn{2}{|c|}{300} \\
\hline \multicolumn{2}{|l|}{ TSR information } \\
\hline Condtrioned & 89.1 \\
\hline Uncondationed & 1053 \\
\hline TSR & 0.35 \\
\hline CA Strip Rating & 1 \\
\hline Fa Strip Rating & 1 \\
\hline Additive Prod $=$ & \\
\hline Additive Product Name & \\
\hline addative 5 & \\
\hline
\end{tabular}
REMARKS UNE 2 L.Fit $6300 \mathrm{gm}$

BITUMINOUS MIXTURE AGED 1 HOURS \& 146 
Table A.5. PM6

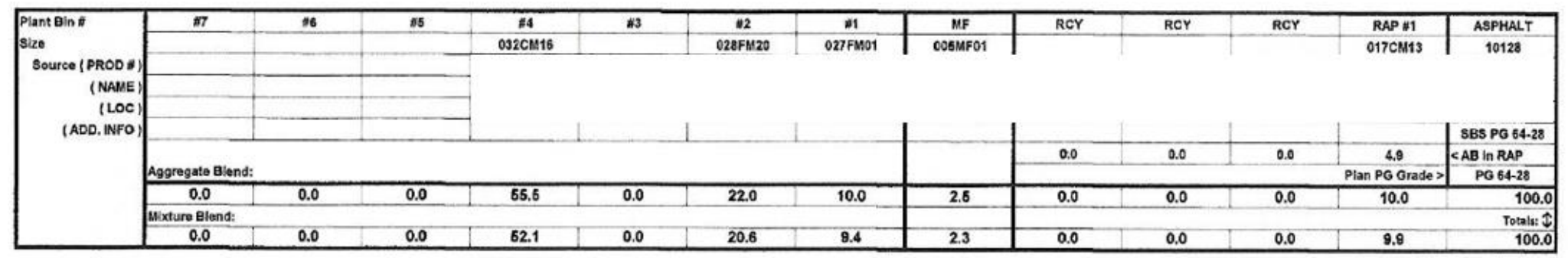

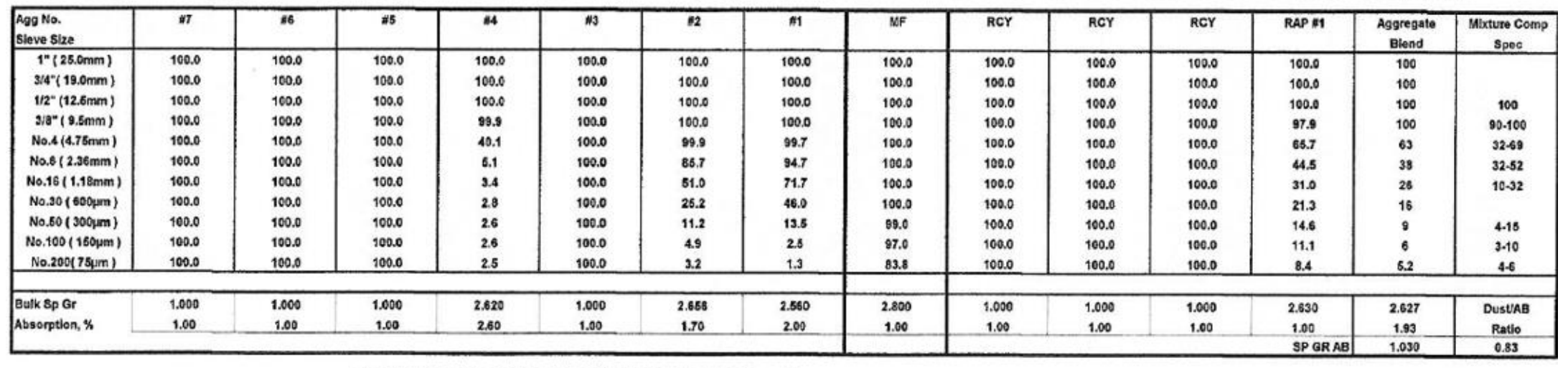

SUMMARY OF SUPERPAVE GYRATORY DESIGN DATA

\begin{tabular}{|c|c|c|c|c|c|c|c|c|c|}
\hline DATA for N-int. & 7 & & & & & & & & \\
\hline & $A B, \cos x$ & Gmb & Gmm & Volds $\left(\boldsymbol{P}_{\mathbf{a}}\right)$ & vaA & VFA & vbo & Pbe & Pba \\
\hline $\max 1$ & 6.2 & 2.168 & 2.472 & 12.3 & 22.6 & 45.6 & 10.29 & 4.89 & 1.40 \\
\hline $\max 2$ & 6.7 & 0.000 & 0.000 & 0.0 & 0.0 & 0.0 & 0.00 & "Drviot & \#Divio: \\
\hline $\operatorname{MEx} 3$ & 7.2 & 0.000 & 0.000 & 0.0 & 0.0 & 0.0 & 0.00 & MDNot & Howio! \\
\hline $\operatorname{MIX} 4$ & 7.7 & 0.000 & 0.000 & 0.0 & 0.0 & 0.0 & 0.00 & ADN/OR & Wonvior \\
\hline
\end{tabular}

\begin{tabular}{|c|c|c|c|c|c|c|c|c|c|c|}
\hline DATA for N-des. & 70 & & & & & & & & & \\
\hline & & Gmb & Gmm & Volds (Pa) & VMA & VFA & Vbe & Pbo & $G_{20}$ & Pba \\
\hline $\operatorname{Mix} 1$ & 6.2 & 2.372 & 2.472 & 4.1 & 18.3 & 73.6 & 11.26 & 4.89 & 2.724 & 140 \\
\hline $\operatorname{MIx} 2$ & 6.7 & 0.000 & 0.000 & 0.0 & 0.0 & 0.0 & 0.00 & aDNro: & 0.000 & ADNIOI \\
\hline $\operatorname{mix} 3$ & 7.2 & 0.000 & 0.000 & 0.0 & 0.0 & 0.0 & 0.00 & EDNNot & 0.000 & ODNTOI \\
\hline $\operatorname{Mx} 4$ & 7.7 & 0,000 & 0.000 & 0.0 & 0.0 & 0.0 & 0.00 & EON/OH & 0.000 & ADNiol \\
\hline
\end{tabular}

\begin{tabular}{|c|c|c|c|c|c|c|c|c|c|c|c|c|}
\hline \multicolumn{13}{|c|}{ OPTIMUM DESIGN DATA@ Ndes } \\
\hline \multirow[t]{2}{*}{70} & 6.2 & 2.326 & 2.423 & 4.9 & 17.0 & 76.4 & 2.861 & 2.627 & 1.01 & 0.48 & 5.73 & 7.7 \\
\hline & $\begin{array}{l}\text { REMARKS LINE } 1 \\
\text { REMARKS LNE } 2\end{array}$ & & & & & & вiтum & TURE AGED I & 1 & HOURs & 295 & \\
\hline
\end{tabular}


Table A.6. PM7

\begin{tabular}{|c|c|c|c|c|c|c|c|c|c|c|c|c|c|}
\hline $\begin{array}{l}\text { Plant Bin E } \\
\text { Sizto }\end{array}$ & $\frac{27}{022 \mathrm{CM}}$ & 26 & \#5 & $\$ 4$ & 23 & $\$ 2$ & $\$ 1$ & MF & FRAP 24 & FRAP $* 3$ & RAS $* 2$ & RCY & ASPHALT \\
\hline \multicolumn{14}{|l|}{$\left|\begin{array}{r}\text { Sizo } \\
\text { Source (PROD }) \text { ) } \\
\text { (NAME) } \\
\text { (LOC) }\end{array}\right|$} \\
\hline \multirow[t]{6}{*}{ (ADO. NFO) } & & & & & & & & & & & & & SBSPG 70-28 \\
\hline & \multirow{2}{*}{\multicolumn{7}{|c|}{ Aogregate Blend: }} & & 6.1 & 3.4 & 26.0 & 0.0 & $\angle A B$ in RAP \\
\hline & & & & & & & & & & & & Plan PG Grade > & PG 76-22 \\
\hline & 34.0 & 36.0 & 8.0 & 0.0 & 0.0 & 0.0 & 0.0 & 4.0 & 7.1 & 7.0 & 3.9 & 0.0 & 100.0 \\
\hline & Mixture Bliend: & דיו & & & & & & & & & & & Totals: $\downarrow$ \\
\hline & 31.8 & 33.7 & 7.5 & 0.0 & 0.0 & 0.0 & 0.0 & 3.7 & 7.1 & 6.8 & 4.9 & 0.0 & 100.0 \\
\hline
\end{tabular}

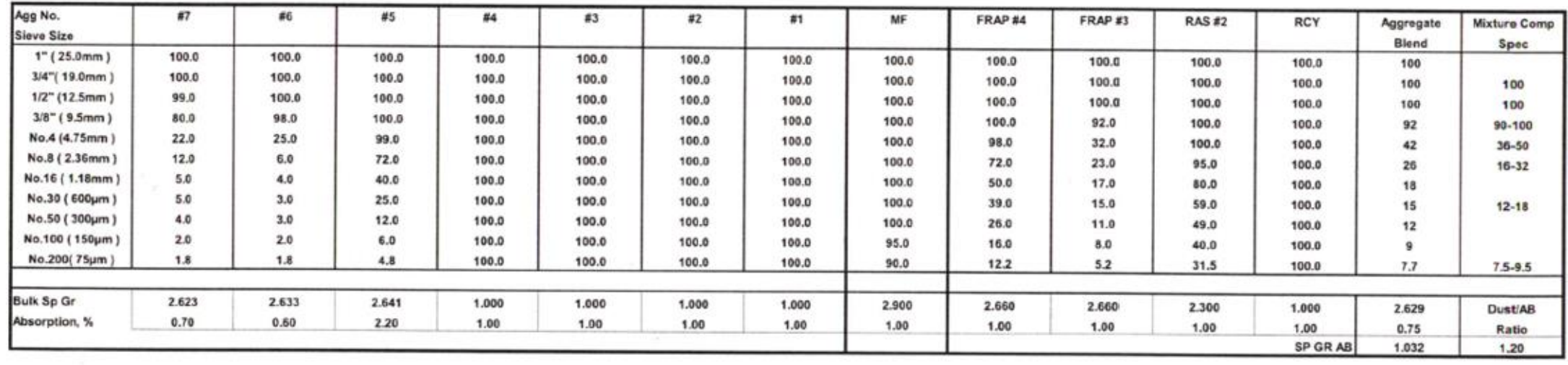

SUMMARY OF SUPERPAVE GYRATORY DESIGN DATA

\begin{tabular}{|c|c|c|c|c|c|c|c|c|c|}
\hline DATA for N-int. & 7 & & & & & & & & \\
\hline & $A B$, YMDX & Gmb & $\mathrm{Gmm}$ & Veids (Pa) & VMA & VFA & voo & Pbo & Pba \\
\hline $\operatorname{Mx} 1$ & 5.5 & 2.061 & 2.465 & 18.4 & 25.9 & 36.8 & 9.51 & 4.76 & 0.78 \\
\hline $\operatorname{Mix} 2$ & 6.0 & 2.076 & 2.447 & 15.2 & 25.8 & 41.2 & 10.60 & 5.27 & 0.78 \\
\hline $\operatorname{Mx} 3$ & 6.5 & 2.086 & 2.432 & 14.2 & 25.8 & 4.9 & 11.58 & 5.73 & 0.82 \\
\hline $\operatorname{Mx} 4$ & 7.0 & 2.091 & 2413 & 13.3 & 26.0 & 48.8 & 12.70 & 6.27 & 0.79 \\
\hline
\end{tabular}

\begin{tabular}{|c|c|c|c|c|c|c|c|c|c|c|}
\hline DATA for N-des. & 80 & & & & & & & & & \\
\hline & & Gmb & $\mathrm{Gmm}$ & Voids (Pa) & VMA & VFA & vbe & Pbe & Gse & Pba \\
\hline $\operatorname{MOx} 1$ & 5.5 & 2.322 & 2.465 & 5.8 & 16.5 & 64.9 & 10.71 & 4.76 & 2.662 & 0.78 \\
\hline $\operatorname{MDX} 2$ & 0.0 & 2.338 & 2.447 & 4.5 & 16.4 & 72.9 & 11.94 & 5.27 & 2.682 & 0.78 \\
\hline $\operatorname{MDx} 3$ & 6.5 & 2.351 & 2.432 & 3.3 & 16.4 & 79.7 & 13.05 & 5.73 & 2.685 & 0.82 \\
\hline $\operatorname{MDx} 4$ & 7.0 & 2.359 & 2.413 & 2.2 & 16.6 & 86.5 & 14.33 & 6.27 & 2.683 & 0.79 \\
\hline
\end{tabular}

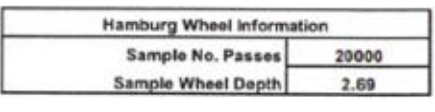

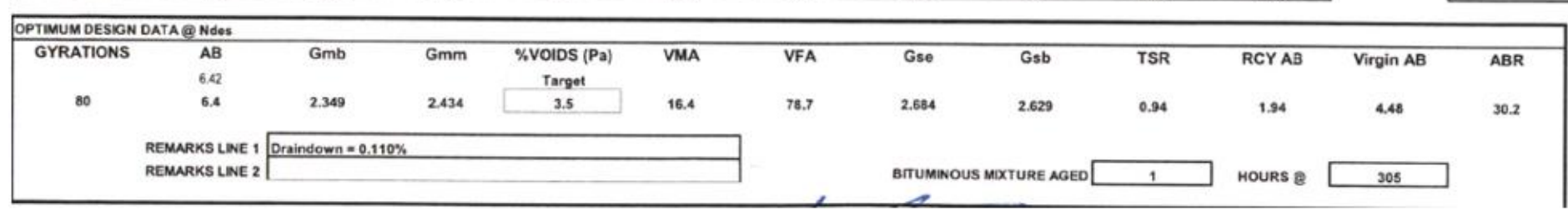


Table A.7. PM8

\begin{tabular}{|c|c|c|c|c|c|c|c|c|c|c|c|c|c|}
\hline \multirow{4}{*}{\begin{tabular}{|l} 
Plant Bin \# \\
Size \\
Source (PROD \#) \\
(NAME)
\end{tabular}} & $\# 7$ & $\# 6$ & \#5 & $\# 4$ & $\# 3$ & $\# 2$ & $\# 1$ & MF & RAP\#4 & FRAP\#3 & RAS \#2 & FRAP\#1 & ASPHALT \\
\hline & & & $032 \mathrm{CM} 16$ & & & 039FM20 & 037FM01 & 004MF01 & & & & $017 \mathrm{CM} 13$ & 10127 \\
\hline & & & & & & & & & & & & & \\
\hline & & & & & & & & & & & & & \\
\hline ( LOC & & & & & & & & & & & & & \\
\hline ( ADD. INFO & & & & & & & & & & & & Plant -1/2 & PG64-22 \\
\hline & & & & & & & & & 0.0 & 0.0 & 0.0 & 5.3 & $<A B$ in $\mathrm{RAP}$ \\
\hline & Aggregate Blenc & & & & & & & & & & & in PG Grade & PG64-22 \\
\hline & \begin{tabular}{|l|}
0.0 \\
\end{tabular} & 0.0 & 54.0 & 0.0 & 0.0 & 10.3 & 17.2 & 0.5 & 0.0 & 0.0 & 0.0 & 18.0 & 100.0 \\
\hline & Mixture Blend: & & & & & & & & & & & & Totals: $\uparrow$ \\
\hline & 0.0 & 0.0 & 50.8 & 0.0 & 0.0 & 9.7 & 16.2 & 0.5 & 0.0 & 0.0 & 0.0 & 17.9 & 100.0 \\
\hline
\end{tabular}

\begin{tabular}{|c|c|c|c|c|c|c|c|c|c|c|c|c|c|c|}
\hline \begin{tabular}{|l} 
Agg No. \\
Sieve Size \\
\end{tabular} & \#7 & $\# 6$ & $\# 5$ & $\# 4$ & $\# 3$ & $\# 2$ & $\# 1$ & MF & FRAP\#4 & FRAP \#3 & RAS \#2 & FRAP\#1 & $\begin{array}{c}\text { Aggregate } \\
\text { Blend }\end{array}$ & \begin{tabular}{|c|} 
Mixture Comp \\
Spec
\end{tabular} \\
\hline $1^{\prime \prime}(25.0 \mathrm{~mm})$ & 100.0 & 100.0 & 100.0 & 100.0 & 100.0 & 100.0 & 100.0 & 100.0 & 100.0 & 100.0 & 100.0 & 100.0 & 100 & \\
\hline $3 / 4 "(19.0 \mathrm{~mm})$ & 100.0 & 100.0 & 100.0 & 100.0 & 100.0 & 100.0 & 100.0 & 100.0 & 100.0 & 100.0 & 100.0 & 100.0 & 100 & \\
\hline $1 / 2 "(12.5 \mathrm{~mm})$ & 100.0 & 100.0 & 100.0 & 100.0 & 100.0 & 100.0 & 100.0 & 100.0 & 100.0 & 100.0 & 100.0 & 100.0 & 100 & 100 \\
\hline $3 / 8 "(9.5 \mathrm{~mm})$ & 100.0 & 94.0 & 94.0 & 100.0 & 100.0 & 100.0 & 100.0 & 100.0 & 100.0 & 100.0 & 100.0 & 100.0 & 97 & 90-100 \\
\hline No.4 $(4.75 \mathrm{~mm})$ & 100.0 & 29.6 & 35.0 & 95.0 & 100.0 & 95.0 & 96.0 & 100.0 & 100.0 & 100.0 & 100.0 & 81.0 & 60 & $32-69$ \\
\hline No.8 (2.36mm) & 100.0 & 5.8 & 7.0 & 69.0 & 100.0 & 69.0 & 77.0 & 100.0 & 100.0 & 100.0 & 100.0 & 56.0 & 35 & $32-52$ \\
\hline No. $16(1.18 \mathrm{~mm})$ & 100.0 & 3.0 & 5.0 & 45.0 & 100.0 & 45.0 & 55.0 & 100.0 & 100.0 & 100.0 & 100.0 & 41.0 & 25 & 10-32 \\
\hline 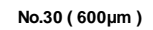 & 100.0 & 2.4 & 5.0 & 27.0 & 100.0 & 27.0 & 33.0 & 100.0 & 100.0 & 100.0 & 100.0 & 30.0 & 17 & \\
\hline No. $50(300 \mu \mathrm{m})$ & 100.0 & 2.2 & 4.0 & 13.0 & 100.0 & 13.0 & 10.0 & 100.0 & 100.0 & 100.0 & 100.0 & 19.0 & 9 & 4-15 \\
\hline No. $100(150 \mu \mathrm{m})$ & 100.0 & 2.0 & 4.0 & 6.0 & 100.0 & 6.0 & 2.0 & 100.0 & 100.0 & 100.0 & 100.0 & 13.0 & 6 & 3-10 \\
\hline \multirow{2}{*}{\multicolumn{15}{|c|}{ 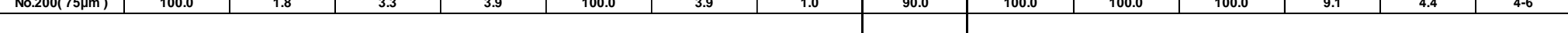 }} \\
\hline & & & & & & & & & & & & & & \\
\hline Bulk Sp Gr & 1.000 & 2.618 & 2.645 & 2.577 & 1.000 & 2.567 & 2.593 & 2.850 & 2.927 & 2.801 & 2.300 & 2.630 & 2.626 & Dust/AB \\
\hline Absorption, \% & 1.00 & 2.10 & 2.10 & 2.70 & 1.00 & 2.60 & 1.40 & 1.00 & 1.00 & 1.00 & 1.00 & 1.00 & 1.65 & Ratio \\
\hline & & & & & & & & & & & & SP GR AB & 1.035 & 0.74 \\
\hline
\end{tabular}

SUMMARY OF SUPERPAVE GYRATORY DESIGN DATA

\begin{tabular}{|cccccccccc|}
\hline DATA for N-int. & 6 & \multicolumn{10}{c}{ 6 } & AB, \%MIX & Gmb & Gmm & Voids (Pa) & VMA & VFA & Vbe & Pbe & Pba \\
\hline & 5.0 & 2.124 & 2.511 & 15.4 & 23.2 & 33.5 & 7.74 & 3.77 & 1.29 \\
MIX 1 & 5.5 & 2.146 & 2.493 & 13.9 & 22.8 & 38.9 & 8.83 & 4.26 & 1.31 \\
MIX 2 & 5.5 & 2.158 & 2.474 & 12.8 & 22.8 & 43.9 & 9.99 & 4.79 & 1.29 \\
MIX 3 & 6.0 & 2.169 & 2.457 & 11.7 & 22.8 & 48.5 & 11.07 & 5.28 & 1.31 \\
MIX 4 & 6.5 & 2.169 &
\end{tabular}

\begin{tabular}{|ccccccccccc|}
\hline DATA for N-des. & 50 & & & & & & & & & \\
\hline & & Gmb & Gmm & Voids (Pa) & VMA & VFA & Vbe & Pbe & Gse & Pba \\
MIX 1 & 5.0 & 2.349 & 2.511 & 6.5 & 15.0 & 57.1 & 8.56 & 3.77 & 2.715 & 1.29 \\
MIX 2 & 5.5 & 2.360 & 2.493 & 5.3 & 15.1 & 64.6 & 9.71 & 4.26 & 2.716 & 1.31 \\
MIX 3 & 6.0 & 2.374 & 2.474 & 4.0 & 15.0 & 73.1 & 10.99 & 4.79 & 2.715 & 1.29 \\
MIX 4 & 6.5 & 2.389 & 2.457 & 2.8 & 14.9 & 81.5 & 12.19 & 5.28 & 2.716 & 1.31 \\
\hline
\end{tabular}

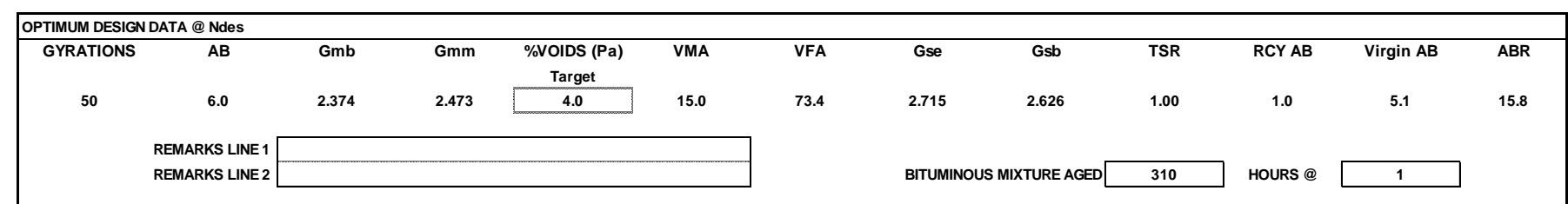


Table A.8. PM9

\begin{tabular}{|c|c|c|c|c|c|c|c|c|c|c|c|c|c|}
\hline \multirow{4}{*}{ 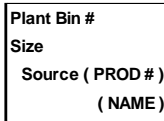 } & $\# 7$ & $\# 6$ & $\# 5$ & $\# 4$ & $\# 3$ & $\# 2$ & $\# 1$ & MF & RCY & RCY & RCY & RAP\#1 & ASPHALT \\
\hline & & & & $031 \mathrm{CM} 16$ & $022 \mathrm{CM} 16$ & 028FM20 & 027FA01 & 004FM01 & & & & $017 \mathrm{FM} 3800$ & 10131 \\
\hline & & & & & & & & & & & & & \\
\hline & & & & & & & & & & & & & \\
\hline (LOC) & & & & & & & & & & & & & \\
\hline ( ADD. INFO) & & & & & & & & & & & & & SBS PG 76-22 \\
\hline & & & & & & & & & 0.0 & 0.0 & 0.0 & 5.8 & $\angle A B$ in RAP \\
\hline & Aggregate Blend & & & & & & & & & & & an PG Grade = & PG76-22 \\
\hline & $\begin{array}{ll}0.0 \\
\end{array}$ & 0.0 & 0.0 & 25.0 & 40.0 & 15.0 & 9.0 & 1.0 & 0.0 & 0.0 & 0.0 & 10.0 & 100.0 \\
\hline & Mixture Blend: & & & & & & & & & & & & Totals: $\downarrow$ \\
\hline & 0.0 & 0.0 & 0.0 & 23.6 & 37.7 & 14.2 & 8.5 & 0.9 & 0.0 & 0.0 & 0.0 & 10.0 & 100.0 \\
\hline
\end{tabular}

\begin{tabular}{|c|c|c|c|c|c|c|c|c|c|c|c|c|c|c|}
\hline \begin{tabular}{|l|} 
Agg No. \\
Sieve Size \\
\end{tabular} & \#7 & $\# 6$ & $\# 5$ & $\# 4$ & $\# 3$ & $\# 2$ & $\# 1$ & MF & $\begin{array}{ll}\text { RCY } \\
\end{array}$ & RCY & RCY & RAP \#1 & $\begin{array}{c}\text { Aggregate } \\
\text { Blend }\end{array}$ & \begin{tabular}{|c|} 
Mixture Comp \\
Spec
\end{tabular} \\
\hline $1^{\prime \prime}(25.0 \mathrm{~mm})$ & 100.0 & 100.0 & 100.0 & 100.0 & 100.0 & 100.0 & 100.0 & 100.0 & 100.0 & 100.0 & 100.0 & 100.0 & 100 & \\
\hline $3 / 4 "(19.0 \mathrm{~mm})$ & 100.0 & 100.0 & 100.0 & 100.0 & 100.0 & 100.0 & 100.0 & 100.0 & 100.0 & 100.0 & 100.0 & 100.0 & 100 & \\
\hline $1 / 2 "(12.5 \mathrm{~mm})$ & 100.0 & 100.0 & 100.0 & 100.0 & 100.0 & 100.0 & 100.0 & 100.0 & 100.0 & 100.0 & 100.0 & 100.0 & 100 & 100 \\
\hline $3 / 8 "(9.5 \mathrm{~mm})$ & 100.0 & 100.0 & 100.0 & 95.0 & 98.0 & 100.0 & 100.0 & 100.0 & 100.0 & 100.0 & 100.0 & 100.0 & 98 & 90-100 \\
\hline No.4 $(4.75 \mathrm{~mm})$ & 100.0 & 100.0 & 100.0 & 37.0 & 45.0 & 100.0 & 85.0 & 100.0 & 100.0 & 100.0 & 100.0 & 88.0 & 60 & $32-69$ \\
\hline No.8 (2.36mm) & 100.0 & 100.0 & 100.0 & 9.0 & 7.0 & 88.0 & 74.0 & 100.0 & 100.0 & 100.0 & 100.0 & 63.0 & 32 & $32-52$ \\
\hline No. $16(1.18 \mathrm{~mm})$ & 100.0 & 100.0 & 100.0 & 4.0 & 3.5 & 60.0 & 54.0 & 100.0 & 100.0 & 100.0 & 100.0 & 44.0 & 22 & 10-32 \\
\hline 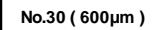 & 100.0 & 100.0 & 100.0 & 3.0 & 3.0 & 42.0 & 41.0 & 100.0 & 100.0 & 100.0 & 100.0 & 32.0 & 16 & \\
\hline No.50 ( $300 \mu \mathrm{m})$ & 100.0 & 100.0 & 100.0 & 2.0 & 2.0 & 29.0 & 13.0 & 99.0 & 100.0 & 100.0 & 100.0 & 19.0 & 10 & 4-15 \\
\hline No. $100(150 \mu \mathrm{m})$ & 100.0 & 100.0 & 100.0 & 1.5 & 1.6 & 17.0 & 2.0 & 96.0 & 100.0 & 100.0 & 100.0 & 13.0 & 6 & 3-10 \\
\hline \multirow{2}{*}{\multicolumn{15}{|c|}{ 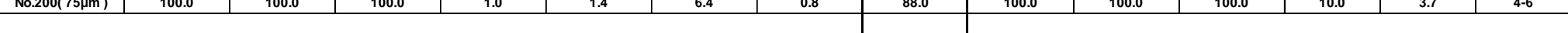 }} \\
\hline & & & & & & & & & & & & & & \\
\hline Bulk Sp Gr & 1.000 & 1.000 & 1.000 & 2.600 & 2.689 & 2.731 & 2.593 & 2.850 & 1.000 & 1.000 & 1.000 & 2.674 & 2.663 & Dust/AB \\
\hline Absorption, \% & 1.00 & 1.00 & 1.00 & 1.00 & 1.40 & 0.50 & 1.30 & 1.00 & 1.00 & 1.00 & 1.00 & 1.40 & 1.01 & Ratio \\
\hline & & & & & & & & & & & & SP GR AB & 1.033 & 0.66 \\
\hline
\end{tabular}

SUMMARY OF SUPERPAVE GYRATORY DESIGN DATA

\begin{tabular}{|cccccccccc|}
\hline DATA for N-int. & 7 & & & & & & & & \\
\hline & AB, \%MIX & Gmb & Gmm & Voids (Pa) & VMA & VFA & Vbe & Pbe & Pba \\
MIX 1 & 5.0 & 2.155 & 2.518 & 14.4 & 23.1 & 37.6 & 8.70 & 4.17 & 0.87 \\
MIX 2 & 5.5 & 2.150 & 2.505 & 14.2 & 23.7 & 40.2 & 9.51 & 4.57 & 0.98 \\
MIX 3 & 6.0 & 2.170 & 2.489 & 12.8 & 23.4 & 45.2 & 10.59 & 5.04 & 1.02 \\
MIX 4 & 6.5 & 2.167 & 2.472 & 12.3 & 23.9 & 48.4 & 11.58 & 5.52 & 1.05 \\
\hline
\end{tabular}

\begin{tabular}{|ccccccccccc|}
\hline DATA for N-des. & 70 & & & & & & & & & \\
\hline & & Gmb & Gmm & Voids (Pa) & VMA & VFA & Vbe & Pbe & Gse & Pba \\
MIX 1 & 5.0 & 2.386 & 2.518 & 5.2 & 14.9 & 64.8 & 9.63 & 4.17 & 2.724 & 0.87 \\
MIX 2 & 5.5 & 2.394 & 2.505 & 4.4 & 15.1 & 70.6 & 10.59 & 4.57 & 2.732 & 0.98 \\
MIX 3 & 6.0 & 2.410 & 2.489 & 3.2 & 14.9 & 78.8 & 11.76 & 5.04 & 2.735 & 1.02 \\
MIX 4 & 6.5 & 2.428 & 2.472 & 1.8 & 14.8 & 87.9 & 12.97 & 5.52 & 2.737 & 1.05 \\
\hline
\end{tabular}

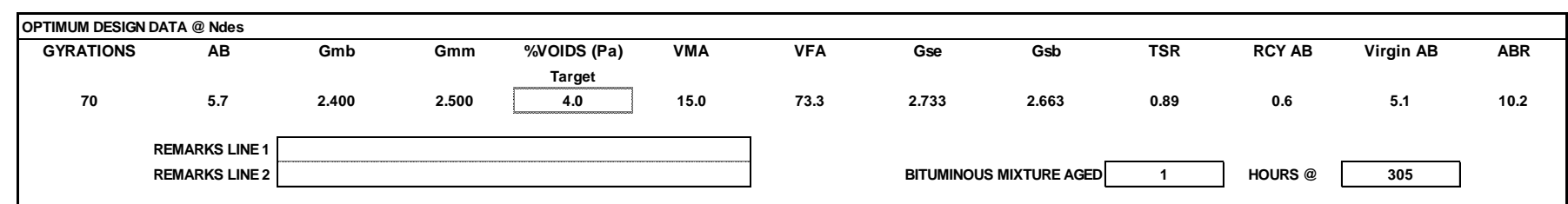


Table A.9. PM10

\begin{tabular}{|c|c|c|c|c|c|c|c|c|c|c|c|c|c|}
\hline \multirow{4}{*}{$\begin{array}{l}\text { Plant Bin \# } \\
\text { Size } \\
\text { Source ( PROD\#) ) } \\
\text { (NAME) }\end{array}$} & $\# 7$ & $\# 6$ & \#5 & $\# 4$ & \#3 & $\# 2$ & $\# 1$ & MF & RCY & RCY & RCY & FRAP\#1 & ASPHALT \\
\hline & & & 032CM16 & 031CM16 & 039FM22 & 039FM20 & 037 & 004MF01 & & & & $017 \mathrm{FM} 3800$ & 10131 \\
\hline & & & & & & & & & & & & & \\
\hline & & & & & & & & & & & & & \\
\hline ( LOC) & & & & & & & & & & & & & \\
\hline ( ADD. INFO) & & & & & & & & & & & & & SBS PG 76-22 \\
\hline & & & & & & & & & 0.0 & 0.0 & 0.0 & 6.1 & $\angle A B$ in $\mathrm{RAP}$ \\
\hline & Aggregate & & & & & & & & & & & lan PG Grade > & PG76-22 \\
\hline & $\begin{array}{l}0.0 \\
\end{array}$ & 0.0 & 26.0 & 26.0 & 11.0 & 18.0 & 7.0 & 2.0 & 0.0 & 0.0 & 0.0 & 10.0 & 100.0 \\
\hline & Mixture Ble & תח & nes & & & & & & & & & & Totals: 1 \\
\hline
\end{tabular}

\begin{tabular}{|c|c|c|c|c|c|c|c|c|c|c|c|c|c|c|}
\hline \begin{tabular}{|l|} 
Agg No. \\
Sieve Size
\end{tabular} & $\# 7$ & $\# 6$ & \#5 & $\# 4$ & $\# 3$ & $\# 2$ & $\# 1$ & MF & RCY & RCY & RCY & FAP \#1 & $\begin{array}{c}\text { Aggregate } \\
\text { Blend }\end{array}$ & $\begin{array}{c}\text { Mixture Comp } \\
\text { Spec }\end{array}$ \\
\hline $1^{\prime \prime}(25.0 \mathrm{~mm})$ & 100.0 & 100.0 & 100.0 & 100.0 & 100.0 & 100.0 & 100.0 & 100.0 & 100.0 & 100.0 & 100.0 & 100.0 & 100 & \\
\hline $3 / 4 "(19.0 \mathrm{~mm})$ & 100.0 & 100.0 & 100.0 & 100.0 & 100.0 & 100.0 & 100.0 & 100.0 & 100.0 & 100.0 & 100.0 & 100.0 & 100 & \\
\hline $1 / 2 "(12.5 \mathrm{~mm})$ & 100.0 & 100.0 & 100.0 & 100.0 & 100.0 & 100.0 & 100.0 & 100.0 & 100.0 & 100.0 & 100.0 & 100.0 & 100 & 100 \\
\hline $3 / 8 "(9.5 \mathrm{~mm})$ & 100.0 & 100.0 & 97.5 & 91.8 & 100.0 & 100.0 & 100.0 & 100.0 & 100.0 & 100.0 & 100.0 & 100.0 & 97 & $90-100$ \\
\hline No.4 $(4.75 \mathrm{~mm})$ & 100.0 & 100.0 & 31.1 & 23.5 & 94.0 & 97.3 & 99.8 & 100.0 & 100.0 & 100.0 & 100.0 & 87.8 & 60 & $32-69$ \\
\hline No. $8(2.36 \mathrm{~mm})$ & 100.0 & 100.0 & 10.2 & 3.5 & 43.7 & 77.8 & 84.4 & 100.0 & 100.0 & 100.0 & 100.0 & 60.6 & 36 & $32-52$ \\
\hline №.16 $(1.18 \mathrm{~mm})$ & 100.0 & 100.0 & 7.2 & 1.7 & 10.9 & 55.5 & 59.4 & 100.0 & 100.0 & 100.0 & 100.0 & 39.2 & 24 & $10-32$ \\
\hline No.30 ( $600 \mu \mathrm{m})$ & 100.0 & 100.0 & 6.3 & 1.2 & 3.4 & 32.4 & 39.7 & 100.0 & 100.0 & 100.0 & 100.0 & 33.0 & 16 & \\
\hline No. $50(300 \mu \mathrm{m})$ & 100.0 & 100.0 & 5.8 & 1.1 & 1.9 & 16.2 & 15.0 & 100.0 & 100.0 & 100.0 & 100.0 & 22.2 & 10 & 4-15 \\
\hline 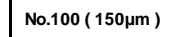 & 100.0 & 100.0 & 5.2 & 1.0 & 1.5 & 6.2 & 4.6 & 95.0 & 100.0 & 100.0 & 100.0 & 14.8 & 7 & 3-10 \\
\hline №. $200(75 \mu \mathrm{m})$ & 100.0 & 100.0 & 4.2 & 0.9 & 1.3 & 3.5 & 1.6 & 85.0 & 100.0 & 100.0 & 100.0 & 10.7 & 5.0 & $4-6$ \\
\hline \\
\hline Bulk Sp Gr & 1.000 & 1.000 & 2.658 & 2.617 & 2.576 & 2.564 & 2.584 & 2.850 & 1.000 & 1.000 & 1.000 & 2.657 & 2.619 & Dust/AB \\
\hline Absorption, \% & 1.00 & 1.00 & 1.90 & 2.10 & 2.60 & 2.60 & 1.80 & 1.00 & 1.00 & 1.00 & 1.00 & 1.00 & 1.88 & Ratio \\
\hline & & & & & & & & & & & & SP GR AB & 1.031 & 0.83 \\
\hline
\end{tabular}

SUMMARY OF SUPERPAVE GYRATORY DESIGN DATA

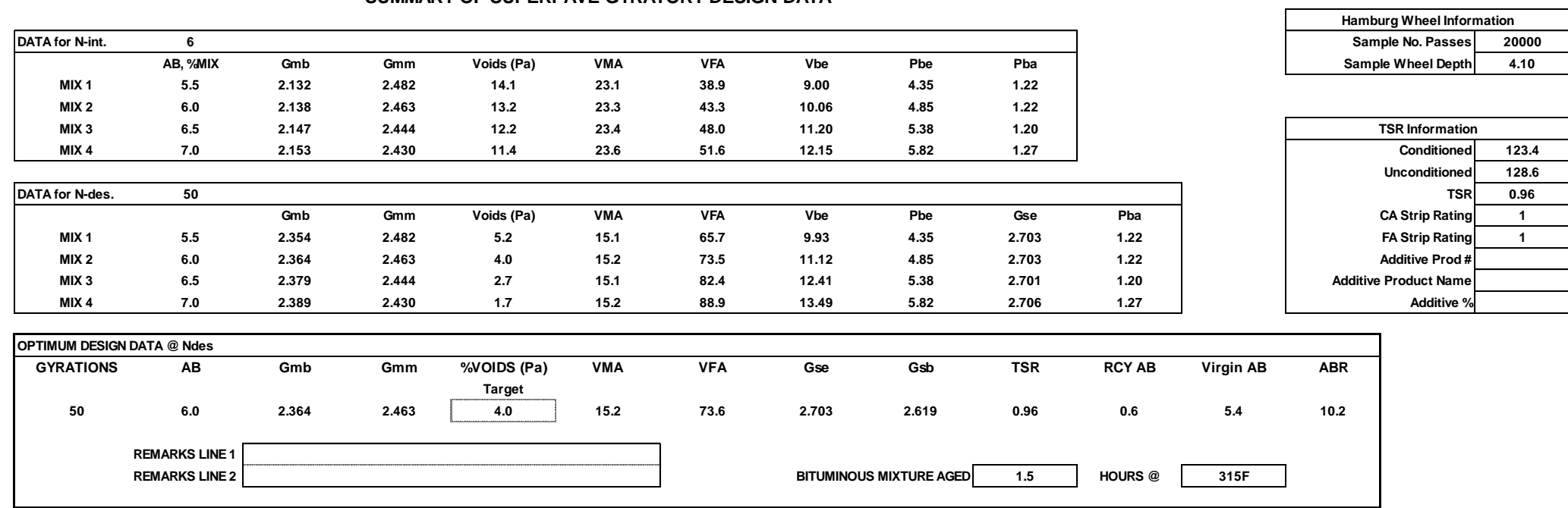


Table A.10. PM11

\begin{tabular}{|c|c|c|c|c|c|c|c|c|c|c|c|c|c|}
\hline \multirow{4}{*}{\begin{tabular}{|r|}
$\begin{array}{l}\text { Plant Bin\# } \\
\text { Size }\end{array}$ \\
Source ( PROD\#) \\
$\quad$ ( NAME)
\end{tabular}} & $\# 7$ & \#6 & $\# 5$ & $\# 4$ & $\# 3$ & $\# 2$ & $\# 1$ & MF & RCY & RCY & RCY & FAP\#1 & ASPHALT \\
\hline & & & $032 \mathrm{CM} 16$ & $031 \mathrm{CM} 16$ & 039FM22 & 039FM20 & 037FM01 & 004MF01 & & & & 017 FM 3800 & 10126 \\
\hline & & & & & & & & & & & & & \\
\hline & & & & & & & & & & & & & \\
\hline (LOC) & & & & & & & & & & & & & \\
\hline ( ADD. INFO) & & & & & & & & & & & & & PG58-28 \\
\hline & & & & & & & & & 0.0 & 0.0 & 0.0 & 6.1 & $<A B$ in $\mathrm{RAP}$ \\
\hline & Aggregate Blen & & & & & & & & & & & an PG Grade > & \begin{tabular}{|l|}
$P G 64-22$ \\
\end{tabular} \\
\hline & 0.0 & 0.0 & 24.0 & 24.0 & 10.0 & 10.0 & 7.0 & 1.0 & 0.0 & 0.0 & 0.0 & 24.0 & 100.0 \\
\hline & Mixture Blend: & & & & & & & & & & & & Totals: $\downarrow$ \\
\hline & 0.0 & 0.0 & 22.6 & 22.6 & 9.4 & 9.4 & 6.6 & 0.9 & 0.0 & 0.0 & 0.0 & 24.0 & 100.0 \\
\hline
\end{tabular}

\begin{tabular}{|c|c|c|c|c|c|c|c|c|c|c|c|c|c|c|}
\hline \begin{tabular}{|l} 
Agg No. \\
Sieve Size
\end{tabular} & \#7 & \#6 & \#5 & $\# 4$ & \#3 & \#2 & $\# 1$ & MF & RCY & RCY & RCY & FAP \#1 & $\begin{array}{c}\text { Aggregate } \\
\text { Blend }\end{array}$ & \begin{tabular}{|c|}
$\begin{array}{c}\text { Mixture Comp } \\
\text { Spec }\end{array}$ \\
\end{tabular} \\
\hline $1^{\prime \prime}(25.0 \mathrm{~mm})$ & 100.0 & 100.0 & 100.0 & 100.0 & 100.0 & 100.0 & 100.0 & 100.0 & 100.0 & 100.0 & 100.0 & 100.0 & 100 & \\
\hline 3/4"(19.0mm ) & 100.0 & 100.0 & 100.0 & 100.0 & 100.0 & 100.0 & 100.0 & 100.0 & 100.0 & 100.0 & 100.0 & 100.0 & 100 & \\
\hline $1 / 2 "(12.5 \mathrm{~mm})$ & 100.0 & 100.0 & 100.0 & 100.0 & 100.0 & 100.0 & 100.0 & 100.0 & 100.0 & 100.0 & 100.0 & 100.0 & 100 & 100 \\
\hline 3/8" (9.5mm ) & 100.0 & 100.0 & 97.5 & 91.8 & 100.0 & 100.0 & 100.0 & 100.0 & 100.0 & 100.0 & 100.0 & 100.0 & 97 & $90-100$ \\
\hline No.4 (4.75mm ) & 100.0 & 100.0 & 31.1 & 23.5 & 94.0 & 97.3 & 99.8 & 100.0 & 100.0 & 100.0 & 100.0 & 87.8 & 61 & $32-69$ \\
\hline No.8 $(2.36 \mathrm{~mm})$ & 100.0 & 100.0 & 10.2 & 3.5 & 43.7 & 77.8 & 84.4 & 100.0 & 100.0 & 100.0 & 100.0 & 60.6 & 37 & $32-52$ \\
\hline No. $16(1.18 \mathrm{~mm})$ & 100.0 & 100.0 & 7.2 & 1.7 & 10.9 & 55.5 & 59.4 & 100.0 & 100.0 & 100.0 & 100.0 & 39.2 & 23 & 10-32 \\
\hline No.30 ( $600 \mu \mathrm{m}$ ) & 100.0 & 100.0 & 6.3 & 1.2 & 3.4 & 32.4 & 39.7 & 100.0 & 100.0 & 100.0 & 100.0 & 33.0 & 17 & \\
\hline №.50 ( $300 \mu \mathrm{m}$ ) & 100.0 & 100.0 & 5.8 & 1.1 & 1.9 & 16.2 & 15.0 & 100.0 & 100.0 & 100.0 & 100.0 & 22.2 & 11 & 4-15 \\
\hline No. $100(150 \mu \mathrm{m})$ & 100.0 & 100.0 & 5.2 & 1.0 & 1.5 & 6.2 & 4.6 & 95.0 & 100.0 & 100.0 & 100.0 & 14.8 & 7 & 3-10 \\
\hline No. $200(75 \mu \mathrm{m})$ & 100.0 & 100.0 & 4.2 & 0.9 & 1.3 & 3.5 & 1.6 & 85.0 & 100.0 & 100.0 & 100.0 & 10.7 & 5.2 & 4-6 \\
\hline Bulk Sp Gr & 1.000 & 1.000 & 2.658 & 2.617 & 2.576 & 2.564 & 2.584 & 2.850 & 1.000 & 1.000 & 1.000 & 2.657 & 2.626 & Dust/AB \\
\hline Absorption, \% & 1.00 & 1.00 & 1.90 & 2.10 & 2.60 & 2.60 & 1.80 & 1.00 & 1.00 & 1.00 & 1.00 & 1.00 & 1.65 & Ratio \\
\hline & & & & & & & & & & & & SP GR AB & 1.031 & 0.87 \\
\hline
\end{tabular}

SUMMARY OF SUPERPAVE GYRATORY DESIGN DATA

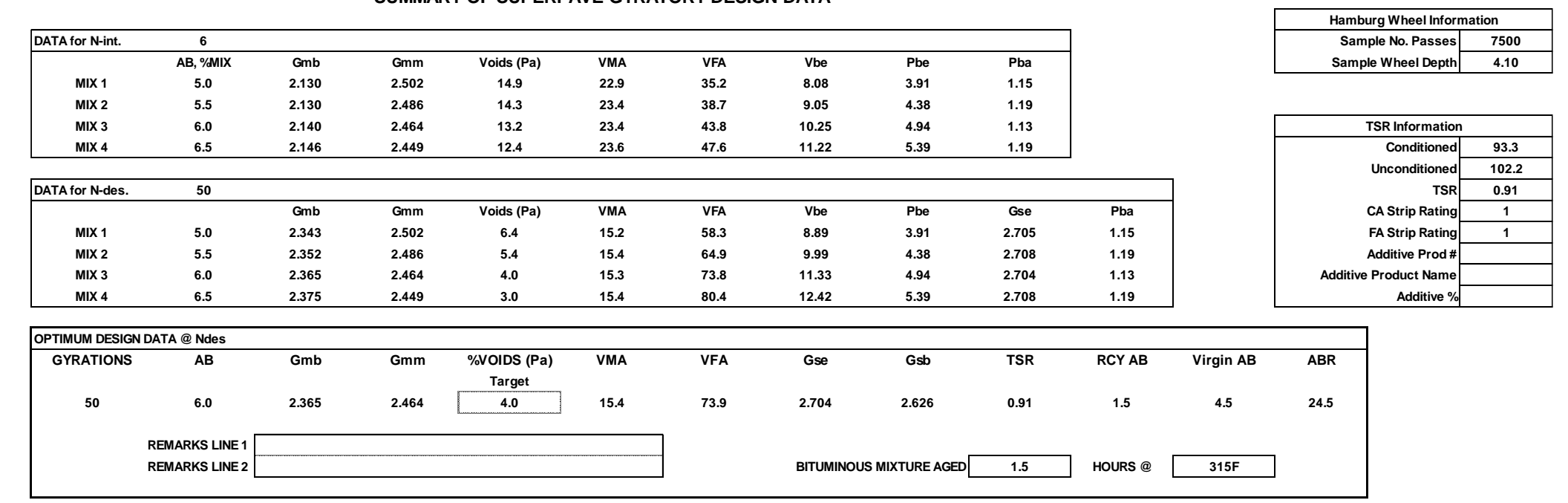


Table A.11. PM12

\begin{tabular}{|c|c|c|c|c|c|c|c|c|c|c|c|c|c|}
\hline \multirow{8}{*}{$\begin{array}{l}\text { Slao } \\
\text { Soureo (PROON) } \\
\text { (NANE) } \\
\text { (LOC) } \\
\text { (ADO. INFO) }\end{array}$} & at & 05 & as & 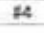 & E3 & 92 & si & MF & FRAD & FRAP $\approx 3$ & RASA2 & $\mathrm{RCY}$ & NPPNAT \\
\hline & $022 \mathrm{CM} 13$ & 032 CMis & O2aFNzo & & & & & 004MFOZ & О17F:0450 & $017 \mathrm{CM}: 204$ & 017\%kgs & & 10130 \\
\hline & & & & & & & & & & & & & \\
\hline & & & & & & & & & & & & & $\operatorname{SESPC} 70.20$ \\
\hline & Asgrogato Etenc: & & & & & & & & e.1 & 3.4 & 25.9 & $\frac{0.0}{P \tan P G \text { Grado }>}$ & $\begin{array}{c}\angle A B \text { in RNO } \\
P G 76-22\end{array}$ \\
\hline & 16.5 & 44.5 & 20.5 & 0.0 & 0.0 & 0.0 & 0.0 & 1.0 & 7.0 & 7.0 & 3.5 & $\frac{P \operatorname{san} P G G \text { rado }>}{0.0}$ & 100.0 \\
\hline & Moxture Bload: & & & & & & & & & & & & Touks 3 \\
\hline & 15.5 & 41.8 & 19.3 & 0.0 & 0.0 & 0.0 & 0.0 & 0.9 & 7.0 & 6.8 & 2.2 & 0.0 & 100.0 \\
\hline
\end{tabular}

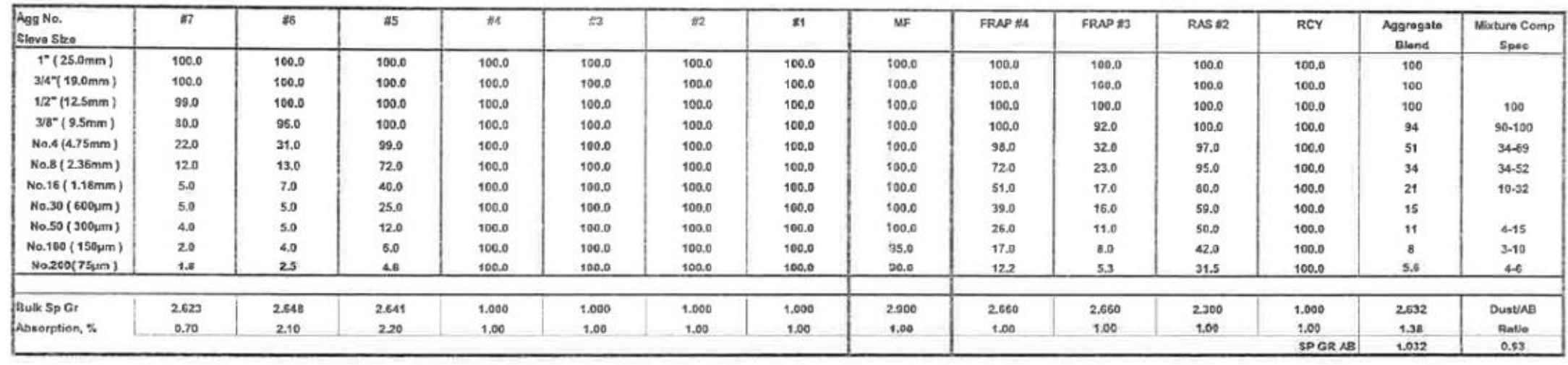

SUMMARY OF SUPERPAVE GYRATORY DESIGN DATA

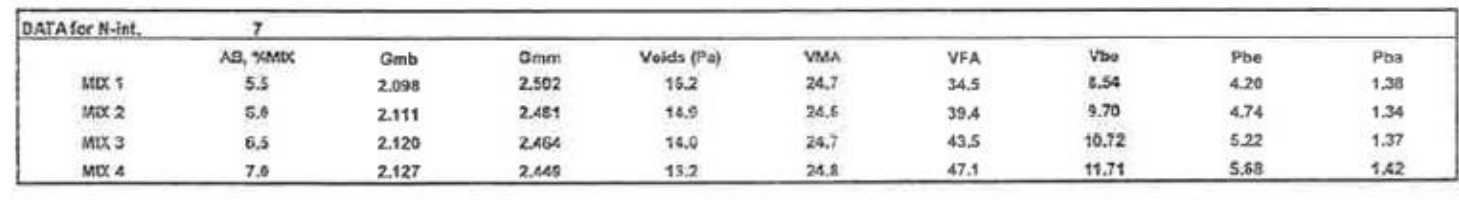

\begin{tabular}{|c|c|c|c|c|c|c|c|c|c|c|}
\hline Datafer $\mathrm{N}$-cos. & 70 & & & & & & & & & \\
\hline & & $\mathrm{Gmb}$ & Gmm & Volds $\left(\mathrm{Pa}_{\mathrm{a}}\right)$ & VMA & VFA & vas & PDo & Gso & Pba \\
\hline$M D \times 1$ & 5.5 & 2.365 & 2.502 & 5.5 & 15.1 & 63.7 & 9.63 & 4.20 & 2.728 & 1.38 \\
\hline$M \times 2$ & 6.0 & 2380 & 2.481 & 4.1 & 15.0 & 72.9 & 10.93 & 4.74 & 2.725 & 1.34 \\
\hline$M \times x_{3}$ & 6.5 & 2392 & 2464 & 2.9 & 15.0 & 80.5 & 12.10 & 5.22 & 2.727 & 1.37 \\
\hline$y \times 4$ & 7.0 & 2.403 & 2.449 & 1.9 & 15.1 & 37.5 & 13.23 & 5.68 & 2.733 & 1.42 \\
\hline
\end{tabular}

\begin{tabular}{|c|c|c|c|c|c|c|c|c|c|c|c|c|}
\hline \multicolumn{13}{|c|}{ OPTWAUM DESIGN DATA \&NEAOA } \\
\hline GYRATIONS & $A B$ & Gmb & Gmm & \%VOIDS (Pa) & VMEA & VEA & Goo & Gsb & TSR & RCYAB & Viesin $A B$ & $A B R$ \\
\hline \multirow[t]{2}{*}{70} & $\begin{array}{l}663 \\
6.0\end{array}$ & 2.2581 & 2.400 & $\begin{array}{c}\text { Targot } \\
4.0\end{array}$ & 15.0 & 73.3 & 2.725 & 2.632 & $0.9 n$ & .s1 & 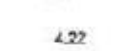 & 30.5 \\
\hline & $\begin{array}{l}\text { REMARKS UNE } 1 \\
\text { REMNRKS LINE } 2\end{array}$ & & & & & \multicolumn{3}{|c|}{ BTUMENOUS MOCTURE AGED [ } & 1 & HOURS $\oplus$ & 305 & \\
\hline
\end{tabular}


Table A.12. PM13

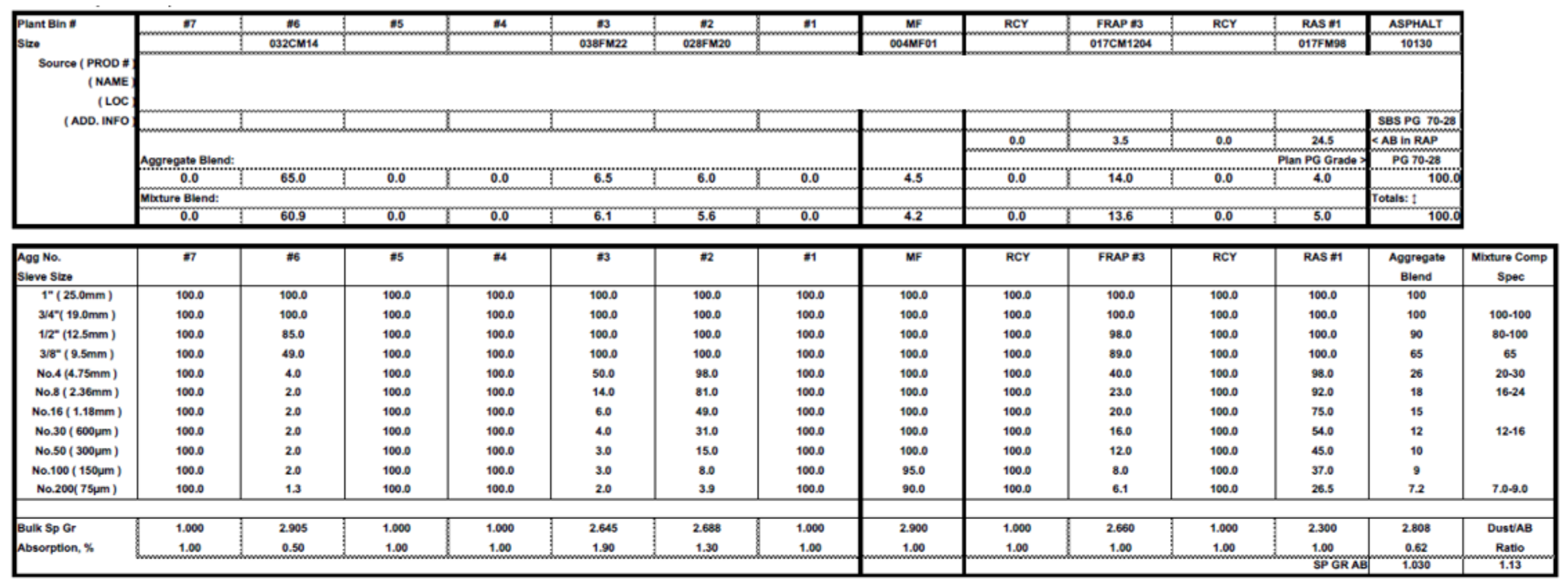

SUMMARY OF SUPERPAVE GYRATORY DESIGN DATA

\begin{tabular}{|c|c|c|c|c|c|c|c|c|c|}
\hline DATA for $\mathrm{N}$-int. & 7 & & & & & & & & \\
\hline & AB, ЖMIX & Gmb & $\mathrm{Gmm}$ & Volds (Pa) & VMA & VFA & Vbe & Pbe & Pba \\
\hline MIX 1 & 5.5 & 2.182 & 2.605 & 16.2 & 26.6 & 38.9 & 10.36 & 4.89 & 0.65 \\
\hline $\operatorname{MiX} 2$ & 6.0 & 2.206 & 2.583 & 14.6 & 26.2 & 44.2 & 11.57 & 5.40 & 0.64 \\
\hline MIX 3 & 6.5 & 2.230 & 2.562 & 13.0 & 25.8 & 49.7 & 12.80 & 5.91 & 0.63 \\
\hline MIX 4 & 7.0 & 2.232 & 2.545 & 12.3 & 26.1 & 52.8 & 13.78 & 6.36 & 0.69 \\
\hline
\end{tabular}

\begin{tabular}{|c|c|c|c|c|c|c|c|c|c|c|}
\hline \begin{tabular}{|l} 
DATA for N-des. \\
.
\end{tabular} & 80 & & & & & & & & & \\
\hline & & Gmb & Gmm & Volds $\left(\mathrm{Pa}_{\mathrm{a}}\right)$ & VMA & VFA & Vbo & $\mathrm{Pbe}_{0}$ & Gso & Pba \\
\hline MIX 1 & 5.5 & 2.442 & 2.605 & 6.3 & 17.8 & 64.9 & 11.59 & 4.89 & 2.859 & 0.65 \\
\hline MIX 2 & 6.0 & 2.465 & 2.583 & 4.6 & 17.5 & 73.9 & 12.92 & 5.40 & 2.858 & 0.64 \\
\hline $\operatorname{mix} 3$ & 6.5 & 2.486 & 2.562 & 3.0 & 17.2 & 82.8 & 14.26 & 5.91 & 2.857 & 0.63 \\
\hline $\operatorname{MIX} 4$ & 7.0 & 2.495 & 2.545 & 2.0 & 17.4 & 88.7 & 15.41 & 6.36 & 2.862 & 0.69 \\
\hline
\end{tabular}

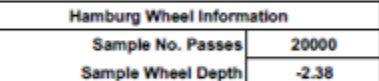

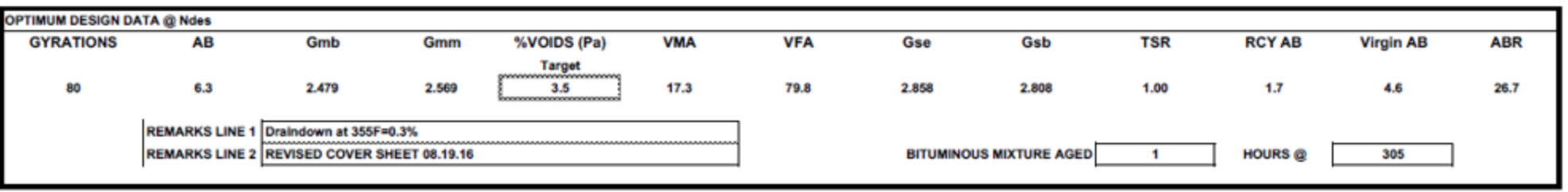




\section{APPENDIX B: I-FIT TEST RESULTS}

\section{Table B.1. Results for PM1 I-FIT Specimen Aging}

\begin{tabular}{|c|c|c|c|c|c|c|c|c|c|c|c|c|c|}
\hline \multicolumn{2}{|c|}{ PM1 } & \multicolumn{4}{|c|}{ Fracture Energy } & \multicolumn{4}{|c|}{ Flexibility Index } & \multicolumn{4}{|c|}{ Slope } \\
\hline Type & $\begin{array}{l}\text { Specimen } \\
\text { ID }\end{array}$ & $\begin{array}{c}\text { Energy } \\
\text { (LLD) (Gf) } \\
(\mathrm{J} / \mathrm{m} 2)\end{array}$ & Average & Std Dev & $\operatorname{CoV}(\%)$ & $\begin{array}{l}\text { Flexibility } \\
\text { Index }\end{array}$ & Average & Std Dev & $\operatorname{CoV}(\%)$ & Slope & Average & Std Dev & $\operatorname{CoV}(\%)$ \\
\hline \multirow{7}{*}{$75 \mathrm{C}, 1 \mathrm{~d}$} & $19-1$ & 1556.52 & \multirow{7}{*}{1502.91} & \multirow{7}{*}{64.13} & \multirow{7}{*}{4.27} & 1.67 & \multirow{7}{*}{1.45} & \multirow{7}{*}{0.63} & \multirow{7}{*}{42.98} & -9.33 & \multirow{7}{*}{-12.11} & \multirow{7}{*}{4.54} & \multirow{7}{*}{37.48} \\
\hline & $15-1$ & 1490.32 & & & & 0.93 & & & & -16.11 & & & \\
\hline & $3-1$ & 1562.08 & & & & 1.06 & & & & -14.68 & & & \\
\hline & $17-1$ & 1484.45 & & & & 1.75 & & & & -8.50 & & & \\
\hline & $12-2$ & 1361.61 & & & & 1.24 & & & & -10.99 & & & \\
\hline & $1-4$ & 1542.08 & & & & 0.79 & & & & -19.62 & & & \\
\hline & $18-2$ & 1523.32 & & & & 2.75 & & & & -5.54 & & & \\
\hline \multirow{8}{*}{$85 \mathrm{C}, 1 \mathrm{~d}$} & $6-4$ & 1586.08 & \multirow{8}{*}{1605.97} & \multirow{8}{*}{129.83} & & 1.90 & & & & -8.33 & & & \\
\hline & $19-4$ & 1710.72 & & & & 2.12 & & & & -8.07 & & & \\
\hline & $19-3$ & 1410.16 & & & & 1.79 & & & & -7.89 & & & \\
\hline & $18-4$ & 1669.15 & & & & 2.56 & & & & -6.52 & & & \\
\hline & $16-1$ & 1586.46 & & & 8.08 & 1.84 & 2.08 & 0.38 & 18.53 & -8.64 & -1.95 & 1.21 & 15.25 \\
\hline & $16-4$ & 1851.52 & & & & 2.29 & & & & -8.09 & & & \\
\hline & $18-3$ & 1564.69 & & & & 2.66 & & & & -5.89 & & & \\
\hline & $17-2$ & 1468.97 & & & & 1.45 & & & & -10.14 & & & \\
\hline & $16-2$ & 1350.42 & & & & 0.91 & & & & -14.77 & & & \\
\hline & $13-1$ & 1671.23 & & & & 1.43 & & & & -11.66 & & & \\
\hline $95 \mathrm{C} 1 \mathrm{~d}$ & $4-3$ & 1112.89 & 110100 & 17507 & 1240 & 0.41 & & & 2055 & -27.14 & 1401 & 509 & \\
\hline $95 \mathrm{C}, 1 \mathrm{~d}$ & $18-1$ & 1430.07 & 1404.29 & $1 / 5.21$ & 12.48 & 1.49 & 1.08 & 0.36 & 33.55 & -9.59 & -14.84 & 5.83 & 39.30 \\
\hline & $17-3$ & 1322.86 & & & & 1.21 & & & & -10.95 & & & \\
\hline & $13-3$ & 1538.29 & & & & 1.03 & & & & -14.94 & & & \\
\hline & $14-3$ & 1552.39 & & & & 1.48 & & & & -10.48 & & & \\
\hline & $8-3$ & 1632.30 & & & & 1.91 & & & & -8.53 & & & \\
\hline & $1-1$ & 1842.59 & & & & 1.73 & & & & -10.66 & & & \\
\hline $75 C, 3 d$ & $4-4$ & 1459.61 & 1598.53 & 119.79 & 7.49 & 1.22 & 1.56 & 0.23 & 15.03 & -11.99 & -10.41 & 1.06 & 10.21 \\
\hline & $5-3$ & 1482.43 & & & & 1.37 & & & & -10.81 & & & \\
\hline & $9-3$ & 1657.92 & & & & 1.78 & & & & -9.32 & & & \\
\hline & $13-2$ & 1562.48 & & & & 1.41 & & & & -11.09 & & & \\
\hline & $9-2$ & 1334.86 & & & & 0.09 & & & & -153.25 & & & \\
\hline & $10-4$ & 1677.70 & & & & 1.56 & & & & -10.77 & & & \\
\hline & $15-2$ & 1369.16 & & & & 0.50 & & & & -27.52 & & & \\
\hline $85 C, 3 d$ & $9-1$ & 1460.90 & 1464.18 & 211.08 & 14.42 & 0.76 & 0.75 & 0.45 & 60.63 & -19.22 & -39.39 & 46.95 & 119.2 \\
\hline & $15-3$ & 1091.25 & & & & 0.35 & & & & -31.63 & & & \\
\hline & $10-3$ & 1772.95 & & & & 0.97 & & & & -18.22 & & & \\
\hline & $6-3$ & 1542.45 & & & & 1.02 & & & & -15.12 & & & \\
\hline & $8-1$ & 1194.14 & & & & 0.06 & & & & -193.64 & & & \\
\hline & $14-2$ & 1220.06 & & & & 0.06 & & & & -188.51 & & & \\
\hline & $4-2$ & 878.90 & & & & 0.02 & & & & -386.11 & -22194 & & 5033 \\
\hline $95 c, 3 d$ & $1-3$ & 1248.07 & $10 / 5.09$ & 150.91 & 14.04 & 0.13 & 0.06 & 0.04 & 55.88 & -94.41 & -221.94 & $111 . / 0$ & 50.33 \\
\hline & $9-4$ & 999.74 & & & & 0.03 & & & & -355.76 & & & \\
\hline & $14-1$ & 909.65 & & & & 0.08 & & & & -113.21 & & & \\
\hline & P1TL & 1662.84 & & & & 3.44 & & & & -4.83 & & & \\
\hline & P1TR & 1581.03 & & & & 3.88 & & & & -4.08 & & & \\
\hline & P1BL & 1740.38 & & & & 4.58 & & & & -3.80 & & & \\
\hline HNACD & P1BR & 1742.17 & 181058 & 1120 & 701 & 4.26 & 110 & 065 & 1581 & -4.09 & 150 & 070 & $16-11$ \\
\hline UINAGED & P2TL & 1982.98 & 1810.58 & 143.20 & 1.91 & 4.93 & 4.10 & 0.65 & 15.84 & -4.02 & -4.52 & 0.13 & 10.11 \\
\hline & P2TR & 1947.86 & & & & 3.34 & & & & -5.84 & & & \\
\hline & P2BL & 1984.74 & & & & 4.99 & & & & -3.98 & & & \\
\hline & P2BR & 1842.61 & & & & 3.35 & & & & -5.50 & & & \\
\hline
\end{tabular}


Table B.2. Results for PM1 Loose Mixture Aging

\begin{tabular}{|c|c|c|c|c|c|c|c|c|c|c|c|c|c|}
\hline \multicolumn{2}{|c|}{ PM1 } & \multicolumn{4}{|c|}{ Fracture Energy } & \multicolumn{4}{|c|}{ Flexibility Index } & \multicolumn{4}{|c|}{ Slope } \\
\hline Type & $\begin{array}{l}\text { Specimen } \\
\text { ID }\end{array}$ & $\begin{array}{c}\text { Energy } \\
\text { (LLD) (Gf) } \\
(\mathrm{J} / \mathrm{m} 2)\end{array}$ & Average & Std Dev & $\operatorname{CoV}(\%)$ & $\begin{array}{l}\text { Flexibility } \\
\text { Index }\end{array}$ & Average & Std Dev & $\operatorname{CoV}(\%)$ & Slope & Average & Std Dev & $\operatorname{CoV}(\%)$ \\
\hline \multirow{8}{*}{$\begin{array}{l}75 \mathrm{C}, \\
16 \mathrm{hrs}\end{array}$} & P5-1 & 1477.05 & \multirow{8}{*}{1606.06} & \multirow{8}{*}{157.97} & \multirow{8}{*}{9.84} & 1.23 & \multirow{8}{*}{2.24} & \multirow{8}{*}{0.74} & \multirow{8}{*}{32.91} & -11.98 & \multirow{8}{*}{-7.97} & \multirow{8}{*}{2.51} & \multirow{8}{*}{31.47} \\
\hline & P5-2 & 1482.96 & & & & 2.57 & & & & -5.77 & & & \\
\hline & P5-3 & 1400.01 & & & & 1.18 & & & & -11.90 & & & \\
\hline & P5-4 & 1569.99 & & & & 2.50 & & & & -6.27 & & & \\
\hline & P6-1 & 1780.39 & & & & 3.03 & & & & -5.88 & & & \\
\hline & P6-2 & 1513.45 & & & & 1.78 & & & & -8.49 & & & \\
\hline & P6-3 & 1808.64 & & & & 3.32 & & & & -5.44 & & & \\
\hline & P6-4 & 1815.99 & & & & 2.27 & & & & -8.01 & & & \\
\hline \multirow{6}{*}{$\begin{array}{l}85 \mathrm{C}, \\
16 \mathrm{hrs}\end{array}$} & P3-2 & 1482.53 & \multirow{6}{*}{1531.42} & \multirow{6}{*}{80.76} & \multirow{6}{*}{5.27} & 1.10 & \multirow{6}{*}{1.56} & & & -13.45 & & & \\
\hline & P3-3 & 1694.35 & & & & 1.72 & & & & -9.85 & & & \\
\hline & P3-4 & 1445.67 & & & & 1.49 & & & & -9.69 & & & \\
\hline & P4-2 & 1534.53 & & & & 1.81 & & 0.23 & 14.75 & -8.47 & -10.05 & 1.59 & 15.77 \\
\hline & P4-3 & 1549.95 & & & & 1.68 & & & & -9.25 & & & \\
\hline & P4-4 & 1481.51 & & & & 1.54 & & & & -9.59 & & & \\
\hline & P11-1 & 1533.63 & & & & 0.33 & & & & -45.97 & & & \\
\hline & $\mathrm{P} 11-2$ & 1735.78 & & & & 1.76 & & & & -9.88 & & & \\
\hline & P11-3 & 1657.57 & & & & 1.48 & & & & -11.23 & & & \\
\hline & $\mathrm{P} 11-4$ & 1426.43 & 161595 & 13624 & 843 & 0.68 & 156 & 076 & 4825 & -20.99 & $-15 ? 0$ & & \\
\hline 1D75C & $\mathrm{P} 12-1$ & 1790.94 & 1615.95 & 136.24 & 8.43 & 2.67 & 1.56 & 0.16 & 48.35 & -6.70 & -15.20 & 12.36 & 81.28 \\
\hline & P12-2 & 1583.14 & & & & 1.47 & & & & -10.80 & & & \\
\hline & P12-3 & 1430.51 & & & & 1.57 & & & & -9.14 & & & \\
\hline & P12-4 & 1769.61 & & & & 2.56 & & & & -6.91 & & & \\
\hline & P9-1 & 1593.56 & & & & 1.51 & & & & -10.58 & & & \\
\hline & P9-2 & 1403.30 & & & & 1.01 & & & & -13.85 & & & \\
\hline & P9-3 & 1775.26 & & & & 1.99 & & & & -8.90 & & & \\
\hline $1085 C$ & P9-4 & 1554.77 & & & & 1.45 & & & & -10.72 & & & \\
\hline 1D85C & P10-1 & 1271.14 & 1502.15 & 147.81 & 9.84 & 0.64 & 1.30 & 0.37 & 28.76 & -19.93 & -12.38 & 3.15 & 25.44 \\
\hline & P10-2 & 1405.66 & & & & 1.14 & & & & -12.35 & & & \\
\hline & P10-3 & 1415.73 & & & & 1.22 & & & & -11.56 & & & \\
\hline & P10-4 & 1597.81 & & & & 1.43 & & & & -11.18 & & & \\
\hline & P7-1 & 1194.62 & & & & 0.54 & & & & -21.96 & & & \\
\hline & P7-3 & 1511.02 & & & & 0.70 & & & & -21.51 & & & \\
\hline & P8-1 & 1340.82 & & & & 1.52 & & & & -8.80 & & & \\
\hline 1D95C & P8-2 & 1442.90 & 1440.16 & 162.36 & 11.27 & 1.43 & 0.95 & 0.43 & 44.65 & -10.09 & -18.65 & 8.44 & 45.22 \\
\hline & P8-3 & 1423.83 & & & & 0.42 & & & & -33.79 & & & \\
\hline & P8-4 & 1727.74 & & & & 1.10 & & & & -15.77 & & & \\
\hline & P1TL & 1662.84 & & & & 3.44 & & & & -4.83 & & & \\
\hline & P1TR & 1581.03 & & & & 3.88 & & & & -4.08 & & & \\
\hline & P1BL & 1740.38 & & & & 4.58 & & & & -3.80 & & & \\
\hline INAGER & P1BR & 1742.17 & 181058 & 14320 & 701 & 4.26 & 410 & 065 & & -4.09 & & & \\
\hline UNAGED & P2TL & 1982.98 & 1810.58 & 143.20 & 7.91 & 4.93 & 4.10 & 0.65 & 15.84 & -4.02 & -4.52 & 0.73 & 16.11 \\
\hline & P2TR & 1947.86 & & & & 3.34 & & & & -5.84 & & & \\
\hline & P2BL & 1984.74 & & & & 4.99 & & & & -3.98 & & & \\
\hline & P2BR & 1842.61 & & & & 3.35 & & & & -5.50 & & & \\
\hline
\end{tabular}


Table B.3. Results for PM2 I-FIT Specimen Aging

\begin{tabular}{|c|c|c|c|c|c|c|c|}
\hline & & & Fractu & & & & Flexibility \\
\hline Type & Specimen ID & $\begin{array}{c}\text { Energy (LLD) } \\
\text { (Gf) }(\mathrm{J} / \mathrm{m} 2)\end{array}$ & Average & Std Dev & CoV (\%) & Flexibility Index & Average \\
\hline & $\begin{array}{l}36-4 \\
36-1\end{array}$ & $\begin{array}{l}2063.99 \\
3247.03\end{array}$ & & & & $\begin{array}{c}5.58 \\
1181\end{array}$ & \\
\hline & $\begin{array}{l}36-1 \\
34-2\end{array}$ & $\begin{array}{l}324 . .03 \\
2193.40\end{array}$ & & & & $\begin{array}{l}17.81 \\
5.42\end{array}$ & \\
\hline & $34-1$ & 2592.48 & 265848 & 43980 & 1654 & 8.82 & 51 \\
\hline $75 \mathrm{C}, 1 \mathrm{~d}$ & $31-4$ & 2834.92 & 2658.48 & 439.80 & 16.54 & 14.77 & 9.51 \\
\hline & $32-2$ & 2412.36 & & & & 7.78 & \\
\hline & $33-2$ & 3380.85 & & & & 14.21 & \\
\hline & $36-3$ & 2542.84 & & & & 7.68 & \\
\hline & $31-3$ & 2568.44 & & & & 8.21 & \\
\hline & $32-4$ & 3039.98 & & & & 0.67 & \\
\hline & $33-1$ & 2591.22 & & & & 8.12 & \\
\hline $85 \mathrm{C}, 1 \mathrm{~d}$ & $31-1$ & 2504.72 & 2577.88 & 223.13 & 8.66 & 9.24 & 7.85 \\
\hline $85 \mathrm{C}, 1 \mathrm{~d}$ & $33-3$ & 2596.00 & 2577.88 & 223.13 & 8.66 & 6.61 & 7.85 \\
\hline & $36-2$ & 2690.77 & & & & 7.73 & \\
\hline & $35-4$ & 2195.85 & & & & 5.50 & \\
\hline & $35-3$ & 2436.03 & & & & 6.73 & \\
\hline & $32-3$ & 2364.29 & & & & 6.08 & \\
\hline & $32-1$ & 2655.88 & & & & 7.18 & \\
\hline & $\frac{32-1}{33-4}$ & $\frac{2053.88}{2405.14}$ & & & & $\frac{1.18}{5.45}$ & \\
\hline $95 \mathrm{C}, 1 \mathrm{~d}$ & $35-2$ & 2714.82 & 2518.44 & 132.58 & 5.26 & 6.45 & 6.80 \\
\hline & $34-4$ & 2362.45 & & & & 6.97 & \\
\hline & $31-2$ & 2545.50 & & & & 6.61 & \\
\hline & $34-3$ & 2581.02 & & & & $\frac{0.01}{8.38}$ & \\
\hline & $2-2$ & 2955.67 & & & & $\begin{array}{l}0.00 \\
8.72\end{array}$ & \\
\hline & $12-1$ & 2794.13 & & & & 6.92 & \\
\hline $75 C, 3 d$ & $16-1$ & 2043.81 & 2570.92 & 355.65 & 13.83 & 4.90 & 6.89 \\
\hline $75 C, 3 d$ & $17-1$ & 2264.37 & 2570.92 & 355.65 & 13.83 & 5.60 & 6.89 \\
\hline & $20-1$ & 2397.61 & & & & 5.93 & \\
\hline & $20-4$ & 2969.91 & & & & 9.25 & \\
\hline & $2-1$ & 2531.49 & & & & 5.44 & \\
\hline & $3-1$ & 2355.76 & & & & 5.18 & \\
\hline $85 C, 3 d$ & $18-1$ & 2421.70 & 2435.52 & 62.79 & 2.58 & 5.43 & 5.19 \\
\hline & $21-2$ & 2433.12 & & & & 4.70 & \\
\hline & 14-1 & $\frac{2400.12}{2221.08}$ & & & & 3.63 & \\
\hline & $14-2$ & 2150.51 & & & & 3.21 & \\
\hline $95 \mathrm{C}, 3 \mathrm{~d}$ & $15-2$ & 1920.93 & 222159 & 200.22 & 901 & 2.15 & 3.19 \\
\hline $95 C, 3 d$ & $20-2$ & 2493.53 & 2221.59 & 200.22 & 9.01 & 4.25 & 3.19 \\
\hline & $20-3$ & 2089.96 & & & & 1.97 & \\
\hline & $22-1$ & 2453.55 & & & & 3.94 & \\
\hline & $1-2$ & 2145.94 & & & & 3.04 & \\
\hline & $8-3$ & 2589.50 & & & & 4.09 & \\
\hline & $9-3$ & 2553.84 & & & & 4.38 & \\
\hline $750 \cdot 5$ & $12-3$ & 2388.00 & 244583 & & & 7.61 & 503 \\
\hline $75 \mathrm{C}, 5 \mathrm{~d}$ & $16-2$ & 2267.16 & 2445.83 & 196.11 & 8.02 & 5.05 & 5.03 \\
\hline & $18-2$ & 2682.45 & & & & 6.30 & \\
\hline & $21-3$ & 2254.20 & & & & 3.87 & \\
\hline & $\frac{21-3}{21-4}$ & $\frac{254.20}{2685.54}$ & & & & $\begin{array}{l}3.87 \\
5.88\end{array}$ & \\
\hline & $1-1$ & 1923.29 & & & & 2.92 & \\
\hline & $3-2$ & 1922.76 & & & & 2.95 & \\
\hline & $4-1$ & 2282.30 & & & & 4.20 & \\
\hline $85 C, 5 d$ & $6-4$ & 2199.28 & 2086.72 & 206.18 & 9.88 & 3.02 & 3.29 \\
\hline & $9-4$ & 2414.39 & & & & 4.14 & \\
\hline & $11-1$ & 2070.63 & & & & 3.45 & \\
\hline & $11-2$ & 1794.42 & & & & 2.32 & \\
\hline & $1-4$ & 2347.48 & & & & 4.28 & \\
\hline & $6-2$ & 2062.47 & & & & 2.35 & \\
\hline & $10-1$ & 2230.65 & & & & 2.27 & \\
\hline & $13-1$ & 2509.51 & & & & 2.71 & \\
\hline $95 \mathrm{C}, 5 \mathrm{~d}$ & $13-3$ & 2007.67 & 2204.05 & 185.65 & 8.42 & 2.18 & 2.83 \\
\hline & $15-4$ & 2410.47 & & & & 3.26 & \\
\hline & $17-2$ & 2012.48 & & & & 2.56 & \\
\hline & $22-3$ & 2051.63 & & & & 3.06 & \\
\hline & $3-4$ & 2294.00 & & & & 4.59 & \\
\hline & $9-2$ & 2556.09 & & & & 4.77 & \\
\hline & $\frac{y-2}{22-4}$ & 2455.11 & & & & 4.80 & \\
\hline $75 C, 7 d$ & $12-2$ & 1963.76 & 2328.05 & 218.69 & 9.39 & 2.14 & 4.02 \\
\hline & $13-4$ & 2098.09 & & & & 2.80 & \\
\hline & $12-4$ & 2610.53 & & & & 4.55 & \\
\hline & $14-4$ & 2318.74 & & & & 4.46 & \\
\hline & $19-2$ & 2024.72 & & & & 2.51 & \\
\hline & $15-3$ & 1849.55 & & & & 2.00 & \\
\hline & $2-4$ & 2420.98 & & & & 4.97 & \\
\hline & $8-1$ & 2251.03 & & & & 3.46 & \\
\hline $85 \mathrm{C}, 7 \mathrm{~d}$ & $4-2$ & 1964.62 & 2100.84 & 163.61 & 7.79 & 1.75 & 2.84 \\
\hline & $11-3$ & 2122.79 & & & & 1.78 & \\
\hline & $4-3$ & 2105.24 & & & & 3.42 & \\
\hline & $5-2$ & 2067.79 & & & & 2.84 & \\
\hline & $7-4$ & 2197.94 & & & & $\begin{array}{l}2.04 \\
2.88 \\
\end{array}$ & \\
\hline & $\begin{array}{l}-4-1 \\
9-1\end{array}$ & $\frac{297.94}{2047.63}$ & & & & $\begin{array}{l}2.80 \\
1.80\end{array}$ & \\
\hline & $22-2$ & 1677.43 & & & & 1.79 & \\
\hline $95 \mathrm{C}, 7 \mathrm{~d}$ & $14-3$ & 1818.02 & 1948.23 & 188.75 & 9.69 & 1.20 & 1.83 \\
\hline & $21-1$ & 2154.02 & & & & 2.48 & \\
\hline & $15-1$ & 1999.12 & & & & 1.29 & \\
\hline & $19-1$ & 1743.46 & & & & 1.36 & \\
\hline & B2-P6-3 & 3042.51 & & & & 20.42 & \\
\hline & B2-P6-4 & 2658.64 & & & & 15.19 & \\
\hline & B2-P7-1 & 2643.90 & & & & 17.99 & \\
\hline UNAGED & B2-P7-2 & 2158.69 & 2612.72 & 265.45 & 10.16 & 11.42 & 16.32 \\
\hline & B2-P7-3 & 2703.44 & & & & 20.33 & \\
\hline & B2-P7-4 & 2469.14 & & & & 12.60 & \\
\hline
\end{tabular}


Table B.4. Results for PM2 Loose Mixture Aging

\begin{tabular}{|c|c|c|c|c|c|c|c|c|c|c|c|c|c|}
\hline & 12 & & Fractur & nergy & & & Flexibility & idex & & & & & \\
\hline Type & $\begin{array}{l}\text { Specimen } \\
\text { ID }\end{array}$ & $\begin{array}{c}\text { Energy } \\
\text { (LLD) (Gf) } \\
(\mathrm{J} / \mathrm{m} 2)\end{array}$ & Average & Std Dev & $\operatorname{CoV}(\%)$ & Flexibility Index & Average & Std Dev & $\mathrm{CoV}(\%)$ & Slope & Average & Std Dev & $\operatorname{CoV}(\%)$ \\
\hline & $\begin{array}{l}\text { P5-1 } \\
\text { P5-2 }\end{array}$ & $\begin{array}{l}2244.47 \\
2256.10\end{array}$ & & & & $\begin{array}{l}5.34 \\
5.13\end{array}$ & & & & $\begin{array}{l}-4.20 \\
-4.40\end{array}$ & & & \\
\hline & P5-3 & 2173.66 & & & & 4.24 & & & & $\begin{array}{l}-4.40 \\
-5.13\end{array}$ & & & \\
\hline & P5-4 & 2343.28 & & & & 5.25 & & & & -4.46 & & & \\
\hline $75 \mathrm{C}, 16 \mathrm{hrs}$ & P6-1 & 2393.27 & 2392.55 & 170.99 & 7.15 & 5.81 & 5.33 & 0.64 & 12.07 & -4.12 & -4.54 & 0.49 & 10.86 \\
\hline & P6-2 & 2732.62 & & & & 6.21 & & & & -4.40 & & & \\
\hline & P6-3 & 2448.90 & & & & 6.05 & & & & -4.05 & & & \\
\hline & P6-4 & 2548.10 & & & & 4.59 & & & & -5.55 & & & \\
\hline & P3-1 & 2436.60 & & & & 7.15 & & & & -3.41 & & & \\
\hline & P3-2 & 2344.06 & & & & 5.62 & & & & -4.17 & & & \\
\hline $85 \mathrm{C}, 16 \mathrm{hrs}$ & $\begin{array}{l}\text { P3-4 } \\
\text { P4-1 }\end{array}$ & $\frac{2566.32}{2433.44}$ & 2454.87 & 175.13 & 7.13 & $\frac{6.56}{8.48}$ & 6.99 & 109 & 1565 & $\frac{-3.91}{-2.87}$ & 350 & 053 & 1469 \\
\hline 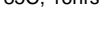 & P4-2 & 2226.32 & 2404.07 & 175.10 & 1.10 & 5.62 & 0.99 & 1.09 & 15.65 & $\begin{array}{l}-2.01 \\
-3.96\end{array}$ & -3.59 & 0.53 & 14.69 \\
\hline & P4-3 & 2813.56 & & & & 7.02 & & & & -4.01 & & & \\
\hline & P4-4 & 2363.77 & & & & 8.47 & & & & -2.79 & & & \\
\hline & $\mathrm{P} 1-2$ & 2261.17 & & & & 4.74 & & & & -4.77 & & & \\
\hline & $\mathrm{P} 1-3$ & 2234.83 & & & & 4.63 & & & & -4.83 & & & \\
\hline & $\mathrm{P} 1-4$ & 2046.07 & & & & 3.43 & & & & -5.97 & & & \\
\hline $95 \mathrm{C}, 16 \mathrm{hrs}$ & P2-1 & 2385.40 & 2315.72 & 139.39 & 6.02 & 5.60 & 4.87 & 0.89 & 18.35 & -4.26 & -4.90 & 0.81 & 16.61 \\
\hline & P2-2 & 2340.35 & & & & 6.21 & & & & -3.77 & & & \\
\hline & P2-3 & 2473.49 & & & & 4.00 & & & & -6.18 & & & \\
\hline & P2-4 & 2468.74 & & & & 5.46 & & & & -4.52 & & & \\
\hline & P11-1 & 2373.58 & & & & 6.67 & & & & -3.56 & & & \\
\hline & P11-2 & 2476.70 & & & & 7.00 & & & & -3.54 & & & \\
\hline & $\mathrm{P} 11-3$ & 2263.06 & & & & 5.49 & & & & -4.12 & & & \\
\hline $75 C, 1 d$ & P11-4 & 2640.32 & 2394.29 & 143.11 & 5.98 & 6.14 & 6.07 & 0.55 & 9.07 & -4.30 & -3.97 & 0.37 & 9.41 \\
\hline & P12-1 & 2173.96 & Letine & 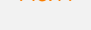 & (.) & 5.81 & 年 & (.) & (1) & -3.74 & & & \\
\hline & P12-3 & 2482.87 & & & & 5.36 & & & & -4.63 & & & \\
\hline & P12-4 & 2349.51 & & & & 6.02 & & & & -3.90 & & & \\
\hline & P9-1 & 2542.83 & & & & 6.45 & & & & -3.94 & & & \\
\hline & P9-2 & 2183.04 & & & & 5.50 & & & & -3.97 & & & \\
\hline $85 \mathrm{C}, 1 \mathrm{~d}$ & $\mathrm{P} 10-1$ & 2206.50 & 2291.80 & 126.16 & 5.50 & 4.10 & 5.14 & 0.74 & 14.40 & -5.38 & -4.53 & 0.50 & 10.98 \\
\hline & $\begin{array}{l}\mathrm{P} 10-2 \\
\mathrm{P} 10-3\end{array}$ & 2247.51 & दूरा.00 & 120.10 & 3.00 & 4.65 & 0.14 & 0.14 & 14.40 & -4.83 & & 0.50 & \\
\hline & $\begin{array}{l}\text { P10-3 } \\
\text { P10-4 }\end{array}$ & $\frac{2210.16}{2360.76}$ & & & & $\begin{array}{l}4.84 \\
5.29\end{array}$ & & & & $\begin{array}{l}-4.57 \\
-4.46\end{array}$ & & & \\
\hline & P7-1 & 2310.26 & & & & 4.91 & & & & -4.71 & & & \\
\hline & P7-2 & 2358.43 & & & & 4.80 & & & & -4.91 & & & \\
\hline & P7-3 & 2346.96 & & & & 4.97 & & & & -4.72 & & & \\
\hline & P7-4 & 2517.93 & & 215.29 & & 3.70 & 4.50 & & & -6.81 & & & \\
\hline $95 \mathrm{C}, 1 \mathrm{~d}$ & P8-1 & 2326.80 & $228 / .46$ & 215.29 & 9.41 & 4.54 & 4.50 & 0.49 & 10.91 & -5.13 & -5.13 & 0.69 & 13.46 \\
\hline & P8-2 & 1983.68 & & & & 3.75 & & & & -5.29 & & & \\
\hline & P8-3 & 1905.60 & & & & 4.36 & & & & -4.37 & & & \\
\hline & P8-4 & 2550.02 & & & & 4.97 & & & & -5.13 & & & \\
\hline & P17-1 & 2962.50 & & & & 9.23 & & & & -3.21 & & & \\
\hline & P17-2 & 2512.90 & & & & 11.07 & & & & -2.27 & & & \\
\hline & P17-3 & 2658.01 & & & & 6.37 & & & & -4.17 & & & \\
\hline $75 c \cdot 3 d$ & $\mathrm{P} 17-4$ & 2740.26 & 260122 & 21153 & 8.13 & 6.64 & 776 & 164 & 21.15 & -4.13 & 350 & 0.75 & 21.47 \\
\hline $75 C, 3 d$ & P18-1 & 2714.26 & 2601.22 & 211.53 & 8.13 & 6.60 & 7.16 & 1.64 & 21.15 & -4.11 & -3.50 & 0.15 & 21.41 \\
\hline & P18-2 & 2578.41 & & & & 5.86 & & & & -4.40 & & & \\
\hline & P18-3 & 2439.00 & & & & 8.13 & & & & -3.00 & & & \\
\hline & P18-4 & 2204.39 & & & & 8.16 & & & & -2.70 & & & \\
\hline & P15-1 & 1698.98 & & & & 2.51 & & & & -6.76 & & & \\
\hline & P15-2 & 1987.54 & & & & 3.06 & & & & -6.50 & & & \\
\hline & P15-3 & 2448.62 & & & & 3.75 & & & & -6.53 & & & \\
\hline $85 C \cdot 3 d$ & P15-4 & 1853.70 & 215974 & 270.24 & 1251 & 3.16 & 388 & 0.90 & 23.05 & -5.87 & -5.72 & 0.78 & 1360 \\
\hline $85 C, 3 d$ & P16-1 & 2487.58 & 2159.14 & 270.24 & 12.51 & 4.68 & 3.88 & 0.90 & 23.05 & -5.32 & $-5 . / 2$ & 0.18 & 13.60 \\
\hline & P16-2 & 2374.36 & & & & 5.43 & & & & -4.37 & & & \\
\hline & P16-3 & 2267.62 & & & & 4.34 & & & & -5.22 & & & \\
\hline & P16-4 & 2159.55 & & & & 4.14 & & & & $\begin{array}{l}-5.22 \\
-5.22\end{array}$ & & & \\
\hline & P13-1 & 1800.31 & & & & 1.89 & & & & -9.53 & & & \\
\hline & P13-2 & 1922.64 & & & & 3.70 & & & & -5.20 & & & \\
\hline & P14-1 & 2134.36 & & & 764 & 1.96 & 257 & & & -10.89 & & & \\
\hline $95 C, 3 d$ & P14-2 & 1747.27 & 1854.65 & 141.65 & 7.64 & 3.28 & $2.5 /$ & 0.70 & 27.30 & -5.32 & -1.18 & 2.18 & 28.00 \\
\hline & P14-3 & 1708.07 & & & & 2.57 & & & & -6.64 & & & \\
\hline & P14-4 & 1815.23 & & & & 2.00 & & & & -9.09 & & & \\
\hline & P23-1 & 2105.01 & & & & 4.09 & & & & -5.15 & & & \\
\hline & P23-2 & 2482.73 & & & & 5.01 & & & & -4.96 & & & \\
\hline & P23-3 & 2341.93 & & & & 4.61 & & & & -5.08 & & & \\
\hline & P23-4 & 2178.85 & & 16762 & 724 & 4.51 & 4.37 & 0.51 & & -4.83 & 5.35 & & 1015 \\
\hline $75 C, 7 d$ & P24-1 & 2522.34 & 2313.74 & 167.62 & 7.24 & 3.94 & 4.37 & 0.51 & 11.66 & -6.40 & -5.35 & 0.54 & 10.15 \\
\hline & P24-2 & 2050.45 & & & & 3.37 & & & & -6.09 & & & \\
\hline & P24-3 & 2397.09 & & & & 4.51 & & & & -5.32 & & & \\
\hline & P24-4 & 2431.52 & & & & 4.92 & & & & -4.94 & & & \\
\hline & P19-1 & & & & & 0.02 & & & & & & & \\
\hline & P19-2 & 953.80 & & & & 0.05 & & & & -205.00 & & & \\
\hline & P19-3 & 1308.22 & & & & 0.09 & & & & -151.98 & & & \\
\hline $95 \mathrm{C}, 7 \mathrm{~d}$ & P19-4 & 914.33 & 993.90 & 155.49 & 15.64 & 0.05 & 0.05 & 0.03 & 55.95 & -167.15 & -303.45 & 192.09 & 63.30 \\
\hline & P20-1 & 860.30 & & & & 0.01 & & & & -653.00 & & & \\
\hline & P20-3 & 1120.79 & & & & 0.04 & & & & -300.02 & & & \\
\hline & P20-4 & 973.74 & & & & 0.08 & & & & -118.50 & & & \\
\hline & B2-P6-3 & 3042.51 & & & & 20.42 & & & & -1.49 & & & \\
\hline & B2-P6-4 & 2658.64 & & & & 15.19 & & & & -1.75 & & & \\
\hline UNAGFD & B2-P7-1 & 2643.90 & 261272 & 265.45 & 1016 & 17.99 & 16.32 & 353 & & -1.47 & 165 & 023 & 1410 \\
\hline UNAGED & B2-P7-2 & 2158.69 & 2612.12 & 265.45 & 10.16 & 11.42 & 16.32 & 3.53 & 21.62 & -1.89 & -1.65 & 0.23 & 14.10 \\
\hline & B2-P7-3 & 2703.44 & & & & 20.33 & & & & -1.33 & & & \\
\hline & B2-P7-4 & 2469.14 & & & & 12.60 & & & & -1.96 & & & \\
\hline
\end{tabular}


Table B.5. Results for PM3 I-FIT Specimen Aging

\begin{tabular}{|c|c|c|c|c|c|c|c|c|c|c|c|c|c|}
\hline \multicolumn{2}{|c|}{ PM3 } & \multicolumn{4}{|c|}{ Fracture Energy } & \multicolumn{4}{|c|}{ Flexibility Index } & \multicolumn{4}{|c|}{ Slope } \\
\hline Type & Specimen ID & $\begin{array}{c}\text { Energy (LLD) } \\
\text { (Gf) (J/m2) }\end{array}$ & Average & Std Dev & $\operatorname{CoV}(\%)$ & $\begin{array}{l}\text { Flexibility } \\
\text { Index }\end{array}$ & Average & Std Dev & CoV (\%) & Slope & Average & Std Dev & CoV (\%) \\
\hline $75 \mathrm{C}, 1 \mathrm{~d}$ & $\begin{array}{r}34-1 \\
35-1 \\
33-4 \\
36-2 \\
31-3 \\
36-4 \\
\end{array}$ & $\begin{array}{l}2319.00 \\
2869.07 \\
2970.26 \\
2907.93 \\
2566.99 \\
2453.41 \\
\end{array}$ & 2681.11 & 247.11 & 9.22 & $\begin{array}{c}9.43 \\
9.35 \\
9.52 \\
10.61 \\
10.19 \\
9.29 \\
\end{array}$ & 9.73 & 0.49 & 5.07 & $\begin{array}{l}-2.46 \\
-3.07 \\
-3.12 \\
-2.74 \\
-2.52 \\
-2.64 \\
\end{array}$ & -2.76 & 0.25 & 9.22 \\
\hline 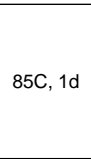 & $\begin{array}{r}34-2 \\
32-1 \\
32-2 \\
36-1 \\
34-3 \\
31-4 \\
32-3 \\
35-4 \\
\end{array}$ & $\begin{array}{l}3283.45 \\
2437.67 \\
3069.80 \\
2471.75 \\
3217.21 \\
2347.48 \\
2440.64 \\
2104.43 \\
\end{array}$ & 2671.55 & 419.13 & 15.69 & $\begin{array}{c}11.02 \\
5.59 \\
10.66 \\
10.30 \\
11.29 \\
7.38 \\
7.01 \\
8.52 \\
\end{array}$ & 8.97 & 2.00 & 22.35 & $\begin{array}{l}-2.98 \\
-4.36 \\
-2.88 \\
-2.40 \\
-2.85 \\
-3.18 \\
-3.48 \\
-2.47\end{array}$ & -3.08 & 0.59 & 19.05 \\
\hline $95 \mathrm{C}, 1 \mathrm{~d}$ & $\begin{array}{r}35-2 \\
33-3 \\
32-4 \\
36-3 \\
35-3 \\
34-4 \\
33-2 \\
31-1 \\
\end{array}$ & \begin{tabular}{|l|}
2400.21 \\
2508.07 \\
2959.33 \\
2345.47 \\
2853.41 \\
2697.12 \\
2236.03 \\
2663.13 \\
\end{tabular} & 2582.85 & 237.18 & 9.18 & $\begin{array}{l}7.74 \\
7.23 \\
9.83 \\
7.79 \\
8.81 \\
6.68 \\
7.63 \\
9.21 \\
\end{array}$ & 8.12 & 1.00 & 12.30 & $\begin{array}{l}-10 \\
-3.47 \\
-3.01 \\
-3.01 \\
-3.24 \\
-4.04 \\
-2.93 \\
-2.89 \\
\end{array}$ & -3.21 & 0.36 & 11.18 \\
\hline $75 C, 3 d$ & $\begin{array}{c}15-1 \\
3-1 \\
10-3 \\
16-2 \\
2-4 \\
15-2 \\
6-1 \\
2-2 \\
\end{array}$ & \begin{tabular}{|l|}
2530.37 \\
2585.44 \\
2195.91 \\
2257.92 \\
2419.24 \\
2607.01 \\
2475.63 \\
2661.67 \\
\end{tabular} & 2466.65 & 156.17 & 6.33 & $\begin{array}{l}6.71 \\
6.95 \\
5.75 \\
4.90 \\
5.54 \\
5.74 \\
5.15 \\
7.76 \\
\end{array}$ & 6.06 & 0.92 & 15.17 & $\begin{array}{l}-3.77 \\
-3.72 \\
-3.82 \\
-4.61 \\
-4.37 \\
-4.54 \\
-4.81 \\
-3.43 \\
\end{array}$ & -4.13 & 0.47 & 11.48 \\
\hline $85 C, 3 d$ & \begin{tabular}{|c|}
$19-6$ \\
$13-3$ \\
$7-2$ \\
$2-1$ \\
$4-1$ \\
$18-1$ \\
$4-4$ \\
\end{tabular} & $\begin{array}{l}2295.52 \\
2908.76 \\
2133.08 \\
1987.22 \\
2275.33 \\
2039.12 \\
2947.23 \\
\end{array}$ & 2369.47 & 368.41 & 15.55 & $\begin{array}{l}.10 \\
5.16 \\
8.05 \\
3.61 \\
4.06 \\
6.00 \\
4.86 \\
\end{array}$ & 5.24 & 1.69 & 32.24 & $\begin{array}{l}-0.40 \\
-4.45 \\
-3.25 \\
-5.27 \\
-5.51 \\
-5.61 \\
-3.40 \\
-6.06\end{array}$ & -4.79 & 1.03 & 21.53 \\
\hline $95 C, 3 d$ & $\begin{array}{c}19-5 \\
10-1 \\
7-3 \\
12-3 \\
3-2 \\
4-3 \\
1-2 \\
8-4 \\
\end{array}$ & $\begin{array}{r}2392.78 \\
2265.14 \\
2255.40 \\
1969.13 \\
1867.28 \\
2166.24 \\
2166.79 \\
2272.47 \\
\end{array}$ & 2169.40 & 161.43 & 7.44 & $\begin{array}{l}5.16 \\
4.57 \\
2.36 \\
3.59 \\
2.06 \\
2.87 \\
2.98 \\
4.65 \\
\end{array}$ & 3.53 & 1.08 & 30.49 & $\begin{array}{l}-4.64 \\
-4.96 \\
-9.57 \\
-5.48 \\
-9.07 \\
-7.56 \\
-7.27 \\
-4.89 \\
\end{array}$ & -6.68 & 1.84 & 27.48 \\
\hline $75 C, 5 d$ & \begin{tabular}{|c|}
$19-4$ \\
$10-2$ \\
$7-4$ \\
$6-2$ \\
$13-4$ \\
$14-2$ \\
$11-2$ \\
\end{tabular} & $\begin{array}{r}2444.41 \\
2454.46 \\
2249.40 \\
2246.19 \\
2207.30 \\
2217.09 \\
2450.59 \\
\end{array}$ & 2324.21 & 109.68 & 4.72 & $\begin{array}{l}4.05 \\
6.59 \\
5.93 \\
3.52 \\
3.74 \\
5.02 \\
5.57 \\
6.08 \\
\end{array}$ & 5.21 & 1.09 & 21.00 & $\begin{array}{l}-4.09 \\
-3.71 \\
-4.14 \\
-6.39 \\
-6.01 \\
-4.40 \\
-3.98 \\
-4.03\end{array}$ & -4.67 & 0.99 & 21.30 \\
\hline $95 \mathrm{C}, 5 \mathrm{~d}$ & $\begin{array}{c}9-1 \\
5-4 \\
1-3 \\
14-4 \\
10-4 \\
12-4 \\
8-1 \\
\end{array}$ & $\begin{array}{l}2352.47 \\
1929.49 \\
1893.79 \\
2108.31 \\
2225.91 \\
1907.81 \\
2281.47 \\
\end{array}$ & 2099.89 & 177.73 & 8.46 & $\begin{array}{l}.00 \\
4.78 \\
3.07 \\
1.90 \\
2.97 \\
3.86 \\
2.85 \\
5.27 \\
\end{array}$ & 3.53 & 1.09 & 30.96 & $\begin{array}{l}-4.05 \\
-4.92 \\
-6.28 \\
-9.97 \\
-7.11 \\
-5.76 \\
-6.70 \\
-4.33 \\
\end{array}$ & -6.44 & 1.70 & 26.40 \\
\hline $75 \mathrm{C}, 7 \mathrm{~d}$ & $\begin{array}{c}2-3 \\
3-4 \\
9-3 \\
11-3 \\
14-1 \\
14-3 \\
16-1 \\
19-1 \\
\end{array}$ & $\begin{array}{l}1823.74 \\
2167.37 \\
2286.35 \\
2863.30 \\
2439.38 \\
2365.21 \\
2635.73 \\
2321.28 \\
\end{array}$ & 2362.80 & 288.79 & 12.22 & $\begin{array}{l}3.49 \\
3.03 \\
7.42 \\
9.09 \\
5.46 \\
6.68 \\
5.35 \\
4.93 \\
\end{array}$ & 5.68 & 1.88 & 33.08 & $\begin{array}{l}-5.23 \\
-7.16 \\
-3.08 \\
-3.15 \\
-4.47 \\
-3.54 \\
-4.93 \\
-4.71\end{array}$ & -4.53 & 1.25 & 27.67 \\
\hline $85 C, 7 d$ & $\begin{array}{r}11-4 \\
13-2 \\
16-3 \\
16-4 \\
17-1 \\
18-2 \\
18-3 \\
19-2 \\
\end{array}$ & $\begin{array}{l}2593.09 \\
2073.73 \\
2113.01 \\
1823.40 \\
1915.24 \\
2263.64 \\
2341.96 \\
2237.08 \\
\end{array}$ & 2170.14 & 228.96 & 10.55 & $\begin{array}{l}4.90 \\
5.00 \\
2.20 \\
3.24 \\
2.47 \\
2.32 \\
3.59 \\
3.20 \\
4.22 \\
\end{array}$ & 3.28 & 0.91 & 27.85 & $\begin{array}{l}-5.19 \\
-9.44 \\
-6.52 \\
-7.37 \\
-8.25 \\
-6.31 \\
-7.31 \\
-5.30\end{array}$ & -6.96 & 1.35 & 19.41 \\
\hline $95 \mathrm{C}, 7 \mathrm{~d}$ & $\begin{array}{c}1-4 \\
3-3 \\
4-2 \\
11-1 \\
13-1 \\
15-4 \\
18-4 \\
19-3 \\
\end{array}$ & $\begin{array}{l}1754.53 \\
2083.72 \\
2062.71 \\
3021.07 \\
1695.50 \\
2215.66 \\
2148.99 \\
2409.56 \\
\end{array}$ & 2173.97 & 387.43 & 17.82 & $\begin{array}{l}1.84 \\
1.91 \\
1.93 \\
3.33 \\
1.96 \\
3.44 \\
3.18 \\
3.42 \\
\end{array}$ & 2.62 & 0.72 & 27.40 & $\begin{array}{l}-9.51 \\
-10.91 \\
-10.70 \\
-9.08 \\
-8.67 \\
-6.45 \\
-6.76 \\
-7.05\end{array}$ & -8.64 & 1.63 & 18.84 \\
\hline UNAGED & $\begin{array}{l}\text { B1-P3-1 } \\
\text { B1-P3-2 } \\
\text { B1-P3-4 } \\
\text { B1-P4-3 } \\
\end{array}$ & $\begin{array}{r}2723.57 \\
2719.72 \\
3238.43 \\
2479.66 \\
\end{array}$ & 2790.35 & 276.93 & 9.92 & $\begin{array}{l}1.07 \\
13.40 \\
16.61 \\
10.08 \\
\end{array}$ & 12.79 & 2.51 & 19.64 & $\begin{array}{l}-2.46 \\
-2.03 \\
-1.95 \\
-2.46 \\
\end{array}$ & -2.23 & 0.24 & 10.64 \\
\hline
\end{tabular}


Table B.6. Results for PM3 Loose Mixture Aging

\begin{tabular}{|c|c|c|c|c|c|c|c|c|c|c|c|c|c|}
\hline \multicolumn{2}{|c|}{ PM3 } & \multicolumn{4}{|c|}{ Fracture Energy } & \multicolumn{4}{|c|}{ Flexibility Index } & \multicolumn{4}{|c|}{ Slope } \\
\hline Type & Specimen ID & $\begin{array}{l}\text { Energy (LLD) } \\
\text { (Gf) (J/m2) }\end{array}$ & Average & Std Dev & $\operatorname{CoV}(\%)$ & $\begin{array}{l}\text { Flexibility } \\
\text { Index }\end{array}$ & Average & Std Dev & CoV (\%) & Slope & Average & Std Dev & $\operatorname{CoV}(\%)$ \\
\hline $75 \mathrm{C}, 16 \mathrm{hrs}$ & \begin{tabular}{|l|} 
P5-1 \\
P5-2 \\
P5-3 \\
P6-1 \\
P6-2 \\
P6-3 \\
P6-4 \\
\end{tabular} & $\begin{array}{l}2697.48 \\
2175.06 \\
2775.02 \\
2755.90 \\
2421.60 \\
2344.54 \\
2871.04 \\
\end{array}$ & 2577.23 & 242.59 & 9.41 & $\begin{array}{l}6.73 \\
4.93 \\
5.99 \\
5.68 \\
6.63 \\
6.46 \\
7.64 \\
\end{array}$ & 6.29 & 0.80 & 12.67 & $\begin{array}{l}-4.01 \\
-4.41 \\
-4.63 \\
-4.85 \\
-3.65 \\
-3.63 \\
-3.76 \\
\end{array}$ & -4.13 & 0.46 & 11.12 \\
\hline $85 \mathrm{C}, 16 \mathrm{hrs}$ & \begin{tabular}{|c|} 
P3-1 \\
P3-2 \\
P3-3 \\
P3-4 \\
P4-1 \\
P4-2 \\
P4-3 \\
P4-4 \\
\end{tabular} & $\begin{array}{l}2316.64 \\
2409.21 \\
2303.16 \\
2137.51 \\
2454.56 \\
2307.17 \\
2474.24 \\
2362.08 \\
\end{array}$ & 2345.57 & 100.10 & 4.27 & $\begin{array}{l}3.97 \\
5.68 \\
5.19 \\
4.96 \\
6.02 \\
4.84 \\
3.94 \\
4.41 \\
\end{array}$ & 4.87 & 0.71 & 14.47 & $\begin{array}{l}-5.84 \\
-4.24 \\
-4.44 \\
-4.31 \\
-4.08 \\
-4.77 \\
-6.28 \\
-5.36 \\
\end{array}$ & -4.92 & 0.77 & 15.57 \\
\hline $95 \mathrm{C}, 16 \mathrm{hrs}$ & $\begin{array}{l}\mathrm{P} 1-1 \\
\mathrm{P} 1-2 \\
\mathrm{P} 1-3 \\
\mathrm{P} 1-4 \\
\mathrm{P} 2-1 \\
\mathrm{P} 2-2 \\
\mathrm{P} 2-3 \\
\mathrm{P} 2-4 \\
\end{array}$ & $\begin{array}{l}2106.41 \\
2230.14 \\
2350.67 \\
2355.31 \\
1911.84 \\
2296.94 \\
2586.51 \\
2378.54 \\
\end{array}$ & 2277.05 & 187.98 & 8.26 & $\begin{array}{l}4.80 \\
2.80 \\
6.46 \\
5.90 \\
2.91 \\
4.06 \\
5.23 \\
4.05 \\
\end{array}$ & 4.52 & 1.24 & 27.34 & $\begin{array}{l}-4.39 \\
-7.97 \\
-3.64 \\
-3.99 \\
-6.57 \\
-5.66 \\
-4.95 \\
-5.88 \\
\end{array}$ & -5.38 & 1.35 & 25.08 \\
\hline $75 \mathrm{C}, 1 \mathrm{~d}$ & \begin{tabular}{|l|}
$\mathrm{P} 11-1$ \\
$\mathrm{P} 11-2$ \\
$\mathrm{P} 11-3$ \\
$\mathrm{P} 11-4$ \\
$\mathrm{P} 12-1$ \\
$\mathrm{P} 12-2$ \\
$\mathrm{P} 12-3$ \\
$\mathrm{P} 12-4$ \\
\end{tabular} & $\begin{array}{l}2447.13 \\
2720.00 \\
2207.70 \\
2597.78 \\
2622.55 \\
2354.17 \\
2391.35 \\
2565.66 \\
\end{array}$ & 2488.29 & 157.12 & 6.31 & $\begin{array}{l}8.50 \\
9.01 \\
7.10 \\
7.53 \\
6.52 \\
5.53 \\
6.04 \\
7.89 \\
\end{array}$ & 7.26 & 1.12 & 15.48 & $\begin{array}{l}-2.88 \\
-3.02 \\
-3.11 \\
-3.45 \\
-4.02 \\
-4.26 \\
-3.96 \\
-3.25 \\
\end{array}$ & -3.49 & 0.49 & 13.92 \\
\hline $85 \mathrm{C}, 1 \mathrm{~d}$ & $\begin{array}{c}\text { P9-1 } \\
\text { P9-2 } \\
\text { P9-3 } \\
\text { P9-4 } \\
\text { P10-1 } \\
\text { P10-2 } \\
\text { P10-3 } \\
\text { P10-4 } \\
\end{array}$ & $\begin{array}{l}2730.78 \\
2210.53 \\
2864.86 \\
2719.30 \\
2564.47 \\
2550.16 \\
2361.78 \\
2770.56 \\
\end{array}$ & 2596.56 & 206.96 & 7.97 & $\begin{array}{c}9.16 \\
3.88 \\
8.00 \\
10.18 \\
7.16 \\
6.22 \\
5.17 \\
6.43 \\
\end{array}$ & 7.03 & 1.93 & 27.51 & $\begin{array}{l}-2.98 \\
-5.70 \\
-3.58 \\
-2.67 \\
-3.58 \\
-4.10 \\
-4.57 \\
-4.31 \\
\end{array}$ & -3.94 & 0.90 & 22.81 \\
\hline $75 C, 3 d$ & $\begin{array}{r}\text { P17-1 } \\
\text { P17-2 } \\
\text { P17-3 } \\
\text { P17-4 } \\
\text { P18-1 } \\
\text { P18-2 } \\
\text { P18-3 } \\
\text { P18-4 }\end{array}$ & $\begin{array}{l}2357.20 \\
2079.69 \\
2507.50 \\
2689.00 \\
2511.38 \\
2494.26 \\
2202.71 \\
2526.54 \\
\end{array}$ & 2421.04 & 184.46 & 7.62 & $\begin{array}{l}6.32 \\
6.25 \\
5.74 \\
7.33 \\
6.07 \\
5.87 \\
4.94 \\
4.81 \\
\end{array}$ & 5.91 & 0.75 & 12.67 & $\begin{array}{l}-3.73 \\
-3.33 \\
-4.37 \\
-3.67 \\
-4.14 \\
-4.25 \\
-4.46 \\
-5.25 \\
\end{array}$ & -4.15 & 0.55 & 13.32 \\
\hline $85 C, 3 d$ & $\begin{array}{l}\text { P15-1 } \\
\text { P15-2 } \\
\text { P15-3 } \\
\text { P15-4 } \\
\text { P16-1 } \\
\text { P16-2 } \\
\text { P16-3 } \\
\text { P16-4 }\end{array}$ & $\begin{array}{l}2096.56 \\
2239.97 \\
2125.71 \\
1956.55 \\
2419.45 \\
2624.44 \\
2144.89 \\
2161.11 \\
2270\end{array}$ & 2221.09 & 195.70 & 8.81 & $\begin{array}{l}3.76 \\
3.67 \\
4.74 \\
4.42 \\
5.67 \\
5.02 \\
2.97 \\
4.19 \\
\end{array}$ & 4.30 & 0.79 & 18.46 & $\begin{array}{l}-5.57 \\
-6.11 \\
-4.48 \\
-4.43 \\
-4.27 \\
-5.23 \\
-7.21 \\
-5.16 \\
\end{array}$ & -5.31 & 0.93 & 17.49 \\
\hline $95 \mathrm{C}, 3 \mathrm{~d}$ & $\begin{array}{r}\text { P13-1 } \\
\text { P13-2 } \\
\text { P13-3 } \\
\text { P13-4 } \\
\text { P14-1 } \\
\text { P14-2 } \\
\text { P14-3 } \\
\text { P14-4 } \\
\end{array}$ & $\begin{array}{l}2279.74 \\
1604.14 \\
2479.14 \\
1813.18 \\
1702.39 \\
2306.95 \\
1993.35 \\
1648.24 \\
\end{array}$ & 1978.39 & 316.68 & 16.01 & $\begin{array}{l}2.58 \\
1.26 \\
2.63 \\
1.18 \\
1.84 \\
2.57 \\
1.50 \\
1.00 \\
\end{array}$ & 1.82 & 0.64 & 35.24 & $\begin{array}{c}-8.84 \\
-12.74 \\
-9.44 \\
-15.35 \\
-9.26 \\
-8.98 \\
-13.30 \\
-16.50 \\
\end{array}$ & -11.80 & 2.88 & 24.44 \\
\hline $75 \mathrm{C}, 7 \mathrm{~d}$ & \begin{tabular}{|l|} 
P23-1 \\
P23-2 \\
P23-3 \\
P23-4 \\
P24-1 \\
P24-2 \\
P24-3 \\
P24-4 \\
\end{tabular} & $\begin{array}{l}2467.55 \\
2278.59 \\
2147.62 \\
2308.99 \\
2195.87 \\
2250.22 \\
2421.44 \\
2621.68 \\
\end{array}$ & 2336.50 & 146.94 & 6.29 & $\begin{array}{l}6.14 \\
4.08 \\
4.56 \\
6.19 \\
4.21 \\
4.27 \\
5.99 \\
4.66 \\
\end{array}$ & 5.01 & 0.87 & 17.28 & $\begin{array}{l}-4.02 \\
-5.58 \\
-4.71 \\
-3.73 \\
-5.22 \\
-5.27 \\
-4.04 \\
-5.62 \\
\end{array}$ & -4.77 & 0.71 & 14.83 \\
\hline $85 C, 7 d$ & $\begin{array}{l}\text { P21-1 } \\
\text { P21-2 } \\
\text { P21-3 } \\
\text { P21-4 } \\
\text { P22-1 } \\
\text { P22-2 } \\
\text { P22-3 } \\
\text { P22-4 } \\
\end{array}$ & $\begin{array}{l}1921.25 \\
1678.83 \\
2030.61 \\
2006.41 \\
1787.04 \\
2097.40 \\
1791.78 \\
1684.84 \\
\end{array}$ & 1874.77 & 150.99 & 8.05 & $\begin{array}{l}1.93 \\
1.68 \\
2.48 \\
2.42 \\
1.27 \\
3.09 \\
1.36 \\
1.15 \\
\end{array}$ & 1.92 & 0.64 & 33.47 & $\begin{array}{c}-9.93 \\
-9.97 \\
-8.20 \\
-8.30 \\
-14.04 \\
-6.78 \\
-13.21 \\
-14.62 \\
\end{array}$ & -10.63 & 2.77 & 26.03 \\
\hline $95 \mathrm{C}, 7 \mathrm{~d}$ & \begin{tabular}{|l|} 
P19-1 \\
P19-2 \\
P19-3 \\
P20-1 \\
P20-2 \\
P20-3 \\
P20-4 \\
\end{tabular} & $\begin{array}{l}1552.14 \\
1462.92 \\
1516.30 \\
1528.14 \\
1427.74 \\
1649.77 \\
1162.93 \\
\end{array}$ & 1471.42 & 141.78 & 9.64 & $\begin{array}{l}1.02 \\
0.99 \\
0.94 \\
0.29 \\
1.24 \\
1.16 \\
0.18 \\
\end{array}$ & 0.83 & 0.39 & 46.87 & $\begin{array}{l}-15.22 \\
-14.73 \\
-16.15 \\
-53.07 \\
-11.51 \\
-14.28 \\
-63.85 \\
\end{array}$ & -26.97 & 20.16 & 74.76 \\
\hline UNAGED & $\begin{array}{l}\text { B1-P3-1 } \\
\text { B1-P3-2 } \\
\text { B1-P3-4 } \\
\text { B1-P4-3 }\end{array}$ & $\begin{array}{r}2723.57 \\
2719.72 \\
3238.43 \\
2479.66 \\
\end{array}$ & 2790.35 & 276.93 & 9.92 & $\begin{array}{l}11.07 \\
13.40 \\
16.61 \\
10.08 \\
\end{array}$ & 12.79 & 2.51 & 19.64 & $\begin{array}{r}-2.46 \\
-2.03 \\
-1.95 \\
-2.46 \\
\end{array}$ & -2.23 & 0.24 & 10.64 \\
\hline
\end{tabular}


Table B.7. Results for PM5 I-FIT Specimen Aging

\begin{tabular}{|c|c|c|c|c|c|c|c|c|c|c|c|c|c|}
\hline \multicolumn{2}{|c|}{ PM5 } & \multicolumn{4}{|c|}{ Fracture Energy } & \multicolumn{4}{|c|}{ Flexibility Index } & \multicolumn{4}{|c|}{ Slope } \\
\hline Type & $\begin{array}{l}\text { Specimen } \\
\text { ID }\end{array}$ & $\begin{array}{c}\text { Energy } \\
\text { (LLD) (Gf) } \\
\text { (J/m2) }\end{array}$ & Average & Std Dev & $\operatorname{CoV}(\%)$ & Flexibility Index & Average & Std Dev & $\operatorname{CoV}(\%)$ & Slope & Average & Std Dev & CoV (\%) \\
\hline \multirow{7}{*}{ Unaged } & $20-3$ & 1780.19 & \multirow{7}{*}{1823.56} & \multirow{7}{*}{108.90} & \multirow{7}{*}{5.97} & 9.47 & \multirow{7}{*}{12.48} & \multirow{7}{*}{2.10} & \multirow{7}{*}{16.84} & -1.88 & \multirow{7}{*}{-1.50} & & \\
\hline & $18-3$ & 1824.99 & & & & 9.61 & & & & -1.90 & & & \\
\hline & $19-2$ & 1702.58 & & & & 12.90 & & & & -1.32 & & & \\
\hline & $22-3$ & 1803.97 & & & & 15.42 & & & & -1.17 & & 0.26 & 17.41 \\
\hline & $21-2$ & 1702.09 & & & & 12.07 & & & & -1.41 & & & \\
\hline & $14-2$ & 1924.77 & & & & 14.36 & & & & -1.34 & & & \\
\hline & $15-5$ & 2026.34 & & & & 13.51 & & & & -1.50 & & & \\
\hline & $1-3$ & 1726.37 & & & & 12.07 & & & & -1.43 & & & \\
\hline & $3-2$ & 1747.48 & & & & 8.83 & & & & -1.98 & & & \\
\hline & $4-2$ & 2064.98 & 1890.21 & 126.20 & 6.68 & 9.56 & 9.56 & 236 & 24.67 & -2.16 & -2.11 & 0.55 & 26.22 \\
\hline $16 \mathrm{HRS}, 85 \mathrm{C}$ & $5-3$ & 2012.41 & 1890.21 & 126.20 & 6.68 & 7.29 & 9.56 & 2.36 & 24.67 & -2.76 & -2.11 & 0.55 & 26.22 \\
\hline & $6-3$ & 1857.42 & & & & 6.54 & & & & -2.84 & & & \\
\hline & $7-1$ & 1932.61 & & & & 13.06 & & & & -1.48 & & & \\
\hline & $1-2$ & 2303.13 & & & & 10.37 & & & & -2.22 & & & \\
\hline & $3-1$ & 2153.44 & & & & 10.45 & & & & -2.06 & & & \\
\hline $16 \mathrm{HRS}, 95 \mathrm{C}$ & $3-3$ & 2275.52 & 2173.23 & 139.19 & 6.40 & 9.25 & 10.08 & 0.79 & 7.84 & -2.46 & -2.16 & 0.17 & 7.72 \\
\hline & $4-3$ & 1914.41 & & & & 9.12 & & & & -2.10 & & & \\
\hline & $5-4$ & 2219.67 & & & & 11.21 & & & & -1.98 & & & \\
\hline & $1-1$ & 1283.24 & & & & 4.97 & & & & -2.58 & & & \\
\hline & $1-4$ & 2248.82 & & & & 7.01 & & & & -3.21 & & & \\
\hline 16HRS, & $2-4$ & 2114.89 & 196944 & 34058 & 1720 & 8.26 & 771 & 210 & 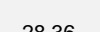 & -2.56 & 205 & 046 & 1721 \\
\hline $110 \mathrm{C}$ & $3-4$ & 2200.61 & 1969.44 & 340.58 & 17.29 & 11.46 & 7.71 & 2.19 & 28.36 & -1.92 & -2.65 & 0.46 & 17.24 \\
\hline & $4-1$ & 2174.28 & & & & 9.02 & & & & -2.41 & & & \\
\hline & $5-2$ & 1794.77 & & & & 5.56 & & & & -3.23 & & & \\
\hline & $1-1$ & 2105.75 & & & & 14.73 & & & & -1.43 & & & \\
\hline & $2-2$ & 2189.97 & & & & 18.25 & & & & -1.20 & & & \\
\hline & $5-4$ & 1944.85 & 10065 & 101 r & (0) & 10.51 & 1004 & 10 & 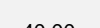 & -1.85 & & & \\
\hline 1D85C & $6-3$ & 1860.89 & 1936.54 & 181.04 & 9.35 & 8.31 & 10.84 & 4.34 & 40.00 & -2.24 & -2.01 & 0.57 & 28.16 \\
\hline & $8-3$ & 1890.96 & & & & 7.11 & & & & -2.66 & & & \\
\hline & $9-4$ & 1626.83 & & & & 6.12 & & & & -2.66 & & & \\
\hline & $2-1$ & 1687.48 & & & & 10.29 & & & & -1.64 & & & \\
\hline & $5-3$ & 1931.38 & & & & 6.33 & & & & -3.05 & & & \\
\hline 10950 & $7-1$ & 2012.77 & 191084 & 10809 & 566 & 7.45 & 852 & 167 & 1954 & -2.70 & 234 & 052 & 235 \\
\hline 1D95C & $10-1$ & 1989.30 & 1910.84 & 108.09 & 5.66 & 8.84 & 8.52 & 1.67 & 19.54 & -2.25 & -2.34 & 0.52 & 22.35 \\
\hline & $13-2$ & 1960.71 & & & & 7.26 & & & & -2.70 & & & \\
\hline & $13-3$ & 1883.38 & & & & 10.95 & & & & -1.72 & & & \\
\hline & $2-3$ & 1973.45 & & & & 11.75 & & & & -1.68 & & & \\
\hline & $4-3$ & 2339.75 & & & & 10.93 & & & & -2.14 & & & \\
\hline & $6-2$ & 1856.00 & & & & 6.65 & & & & -2.79 & & & \\
\hline $3 D 85 C$ & $7-2$ & 1855.54 & 1974.11 & 177.93 & 9.01 & 7.96 & 8.28 & 2.28 & 27.52 & -2.33 & -2.53 & 0.59 & 23.39 \\
\hline & $10-2$ & 1809.89 & & & & 6.78 & & & & -2.67 & & & \\
\hline & $12-2$ & 2010.00 & & & & 5.61 & & & & -3.58 & & & \\
\hline & $3-2$ & 1797.14 & & & & 4.21 & & & & -4.27 & & & \\
\hline 35050 & $5-2$ & 1746.78 & 176051 & 10212 & 580 & 4.32 & 424 & & 124 & -4.04 & 415 & & 5.48 \\
\hline 3D95C & $10-4$ & 1890.35 & 1760.51 & 102.12 & 5.80 & 4.26 & 4.24 & 0.05 & 1.24 & -4.44 & -4.15 & 0.23 & 5.48 \\
\hline & $11-3$ & 1607.78 & & & & 4.19 & & & & -3.84 & & & \\
\hline & $2-4$ & 1728.52 & & & & 6.55 & & & & -2.64 & & & \\
\hline & $3-1$ & 1779.54 & & & & 5.23 & & & & -3.40 & & & \\
\hline 5095 & $6-4$ & 1778.18 & 170407 & 160231 & 005 & 4.99 & 5,17 & 094 & 1725 & -3.56 & 327 & 0.57 & 1681 \\
\hline 5D85C & $9-2$ & 1609.68 & 1794.07 & 162.31 & 9.05 & 3.81 & 5.47 & 0.94 & 17.25 & -4.22 & -3.37 & 0.57 & 16.84 \\
\hline & $11-1$ & 1734.22 & & & & 6.52 & & & & -2.66 & & & \\
\hline & $12-4$ & 2134.25 & & & & 5.71 & & & & -3.74 & & & \\
\hline & $4-2$ & 1889.47 & & & & 4.28 & & & & -4.41 & & & \\
\hline & $6-1$ & 1697.33 & & & & 3.69 & & & & -4.60 & & & \\
\hline & $9-1$ & 1651.37 & & & & 2.13 & & & & -7.76 & & & \\
\hline 5D95C & $10-3$ & 1920.85 & 1827.72 & 131.32 & 7.18 & 3.10 & 3.44 & 0.72 & 20.91 & -6.20 & -5.57 & 1.25 & 22.46 \\
\hline & $11-4$ & 1778.45 & & & & 4.13 & & & & -4.31 & & & \\
\hline & $12-1$ & 2028.86 & & & & 3.30 & & & & -6.14 & & & \\
\hline
\end{tabular}


Table B.8. Results for PM5 Loose Mixture Aging

\begin{tabular}{|c|c|c|c|c|c|c|c|c|c|c|c|c|c|}
\hline \multicolumn{2}{|c|}{ PM5 } & \multicolumn{4}{|c|}{ Fracture Energy } & \multicolumn{4}{|c|}{ Flexibility Index } & \multicolumn{4}{|c|}{ Slope } \\
\hline Type & Specimen ID & $\begin{array}{c}\text { Energy (LLD) } \\
\text { (Gf) }(\mathrm{J} / \mathrm{m} 2)\end{array}$ & Average & Std Dev & $\operatorname{CoV}(\%)$ & $\begin{array}{l}\text { Flexibility } \\
\text { Index }\end{array}$ & Average & Std Dev & CoV (\%) & Slope & Average & Std Dev & $\operatorname{CoV}(\%)$ \\
\hline 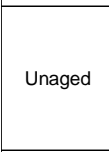 & $\begin{array}{c}20-3 \\
18-3 \\
19-2 \\
22-3 \\
21-2 \\
14-2 \\
15-5 \\
\end{array}$ & $\begin{array}{l}1780.19 \\
1824.99 \\
1702.58 \\
1803.97 \\
1702.09 \\
1924.77 \\
2026.34 \\
\end{array}$ & 1823.56 & 108.90 & 5.97 & $\begin{array}{c}9.47 \\
9.61 \\
12.90 \\
15.42 \\
12.07 \\
14.36 \\
13.51 \\
\end{array}$ & 12.48 & (n) & 16.84 & $\begin{array}{l}-1.88 \\
-1.90 \\
-1.32 \\
-1.17 \\
-1.41 \\
-1.34 \\
-1.50\end{array}$ & -1.50 & 0.26 & 17.41 \\
\hline 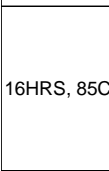 & $\begin{array}{c}\text { LP5 P1-1 } \\
\text { LP5 P1-2 } \\
\text { LP5 P1-3 } \\
\text { LP5 P1-4 } \\
\text { LP5 P2-1 } \\
\text { LP5 P2-2 } \\
\text { LP5 P2-3 } \\
\text { LP5 P2-4 }\end{array}$ & $\begin{array}{l}1899.37 \\
1877.93 \\
1749.54 \\
1759.42 \\
1979.93 \\
1819.26 \\
2138.83 \\
1714.97\end{array}$ & 1867.41 & 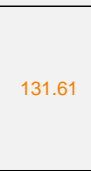 & 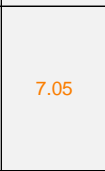 & $\begin{array}{c}5.79 \\
11.96 \\
9.36 \\
8.38 \\
15.71 \\
10.89 \\
18.93 \\
8.71 \\
\end{array}$ & 11.22 & 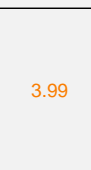 & 35.61 & $\begin{array}{l}-3.28 \\
-1.57 \\
-1.87 \\
-2.10 \\
-1.26 \\
-1.67 \\
-1.13 \\
-1.97\end{array}$ & -1.86 & 0.62 & 33.53 \\
\hline 16HRS, 95C & $\begin{array}{l}\text { LP5 P3-1 } \\
\text { LP5 P3-2 } \\
\text { LP5 P3-3 } \\
\text { LP5 P3-4 } \\
\text { LP5 P4-1 } \\
\text { LP5 P4-2 } \\
\text { LP5 P4-3 } \\
\text { LP5 P4-4 }\end{array}$ & $\begin{array}{l}2074.73 \\
1700.04 \\
1765.17 \\
2009.57 \\
1660.58 \\
2063.27 \\
1825.09 \\
2070.44 \\
\end{array}$ & 1896.11 & 165.54 & 8.73 & \begin{tabular}{|c|}
10.81 \\
5.59 \\
6.98 \\
5.68 \\
7.01 \\
13.06 \\
10.55 \\
13.80 \\
\end{tabular} & 9.18 & 3.08 & 33.49 & $\begin{array}{l}-1.92 \\
-3.04 \\
-2.53 \\
-3.54 \\
-2.37 \\
-1.58 \\
-1.73 \\
-1.50 \\
\end{array}$ & -2.28 & 0.69 & 30.14 \\
\hline $\begin{array}{c}\text { 16HRS, } \\
110 \mathrm{C}\end{array}$ & $\begin{array}{l}\text { LP5 P5-1 } \\
\text { LP5 P5-2 } \\
\text { LP5 P5-3 } \\
\text { LP5 P5-4 } \\
\text { LP5 P6-1 } \\
\text { LP5 P6-2 } \\
\text { LP5 P6-3 } \\
\text { LP5 P6-4 }\end{array}$ & $\begin{array}{l}1615.19 \\
1850.64 \\
1863.17 \\
1730.59 \\
1789.97 \\
1911.02 \\
1891.57 \\
1954.67 \\
\end{array}$ & 1825.85 & 103.03 & 5.64 & $\begin{array}{l}6.96 \\
6.98 \\
6.49 \\
8.40 \\
7.13 \\
7.03 \\
7.88 \\
7.82 \\
\end{array}$ & 7.34 & 0.59 & 8.03 & $\begin{array}{l}-2.32 \\
-2.65 \\
-2.87 \\
-2.06 \\
-2.51 \\
-2.72 \\
-2.40 \\
-2.50 \\
\end{array}$ & -2.50 & 0.24 & 9.41 \\
\hline $1 \mathrm{D} 85 \mathrm{C}$ & $\begin{array}{l}\text { LP5 P7-1 } \\
\text { LP5 P7-2 } \\
\text { LP5 P7-4 } \\
\text { LP5 P8-1 } \\
\text { LP5 P8-2 } \\
\text { LP5 P8-3 } \\
\text { LP5 P8-4 } \\
\end{array}$ & \begin{tabular}{|l|}
1918.93 \\
1844.01 \\
1688.89 \\
1894.68 \\
1910.97 \\
1664.89 \\
1766.07 \\
\end{tabular} & 1812.63 & 98.56 & 5.44 & $\begin{array}{l}7.50 \\
7.59 \\
7.75 \\
8.81 \\
7.03 \\
7.67 \\
6.61 \\
\end{array}$ & 7.57 & 0.63 & 8.36 & $\begin{array}{l}-2.56 \\
-2.43 \\
-2.18 \\
-2.15 \\
-2.72 \\
-2.17 \\
-2.67 \\
\end{array}$ & -2.41 & 0.23 & 9.47 \\
\hline $1 \mathrm{D} 95 \mathrm{C}$ & $\begin{array}{l}\text { LP5 P9-1 } \\
\text { LP5 P9-2 } \\
\text { LP5 P9-3 } \\
\text { LP5 P9-4 } \\
\text { LP5 P } 10-1 \\
\text { LP5 P10-2 } \\
\text { LP5 P10-3 } \\
\text { LP5 P10-4 }\end{array}$ & $\begin{array}{l}1687.06 \\
1665.90 \\
1967.52 \\
1637.90 \\
1783.97 \\
1902.47 \\
1821.19 \\
1793.26 \\
\end{array}$ & 1782.41 & 108.31 & 6.08 & $\begin{array}{l}6.25 \\
6.29 \\
9.46 \\
8.15 \\
6.46 \\
9.61 \\
6.80 \\
7.06 \\
\end{array}$ & 7.51 & 1.30 & 17.31 & $\begin{array}{l}-2.70 \\
-2.65 \\
-2.08 \\
-2.01 \\
-2.76 \\
-1.98 \\
-2.68 \\
-2.54 \\
\end{array}$ & -2.43 & 0.32 & 13.09 \\
\hline 1D110C & $\begin{array}{r}\text { LP5 P11-1 } \\
\text { LP5 P11-2 } \\
\text { LP5 P11-3 } \\
\text { LP5 P11-4 } \\
\text { LP5 P12-1 } \\
\text { LP5 P12-2 } \\
\text { LP5 P12-3 } \\
\text { LP5 P12-4 }\end{array}$ & $\begin{array}{l}1871.42 \\
1894.02 \\
2047.33 \\
1566.72 \\
1988.86 \\
1683.16 \\
1769.43 \\
2295.48\end{array}$ & 1889.55 & 212.46 & 11.24 & $\begin{array}{l}5.62 \\
5.22 \\
6.85 \\
4.21 \\
6.25 \\
4.95 \\
5.84 \\
6.71\end{array}$ & 5.71 & 0.84 & 14.77 & $\begin{array}{l}-3.33 \\
-3.63 \\
-2.99 \\
-3.72 \\
-3.18 \\
-3.40 \\
-3.03 \\
-3.42\end{array}$ & -3.34 & 0.25 & 7.36 \\
\hline $3 D 85 C$ & $\begin{array}{l}\text { LP5 P13-1 } \\
\text { LP5 P13-2 } \\
\text { LP5 P13-3 } \\
\text { LP5 P13-4 } \\
\text { LP5 P14-1 } \\
\text { LP5 P14-2 } \\
\text { LP5 P14-3 } \\
\text { LP5 P14-4 }\end{array}$ & $\begin{array}{l}1567.45 \\
2053.78 \\
1896.68 \\
1529.47 \\
1580.85 \\
1906.44 \\
1795.08 \\
2039.76\end{array}$ & 1796.19 & 199.30 & 11.10 & $\begin{array}{l}4.10 \\
7.81 \\
6.10 \\
4.10 \\
6.70 \\
6.86 \\
7.74 \\
10.62 \\
\end{array}$ & 6.75 & 1.99 & 29.41 & $\begin{array}{l}-3.82 \\
-2.63 \\
-3.11 \\
-3.73 \\
-2.36 \\
-2.78 \\
-2.32 \\
-1.92 \\
\end{array}$ & -2.83 & 0.63 & 22.39 \\
\hline $3 \mathrm{D} 95 \mathrm{C}$ & $\begin{array}{l}\text { LP5 P15-1 } \\
\text { LP5 P15-2 } \\
\text { LP5 P15-3 } \\
\text { LP5 P15-4 } \\
\text { LP5 P } 16-1 \\
\text { LP5 P16-2 } \\
\text { LP5 P16-3 } \\
\text { LP5 P16-4 }\end{array}$ & $\begin{array}{l}1821.60 \\
1689.01 \\
1550.74 \\
1571.05 \\
1784.39 \\
1696.63 \\
1741.02 \\
1736.94 \\
\end{array}$ & 1698.92 & 89.43 & 5.26 & $\begin{array}{l}3.70 \\
4.14 \\
2.86 \\
2.95 \\
3.14 \\
4.40 \\
3.78 \\
4.23 \\
\end{array}$ & 3.65 & 0.56 & 15.36 & $\begin{array}{l}-4.92 \\
-4.08 \\
-5.42 \\
-5.32 \\
-5.68 \\
-3.86 \\
-4.60 \\
-4.11 \\
\end{array}$ & -4.75 & 0.65 & 13.61 \\
\hline $5 \mathrm{D} 85 \mathrm{C}$ & $\begin{array}{l}\text { LP5 P17-1 } \\
\text { LP5 P17-2 } \\
\text { LP5 P17-3 } \\
\text { LP5 P18-1 } \\
\text { LP5 P18-2 } \\
\text { LP5 P18-3 } \\
\text { LP5 P18-4 }\end{array}$ & $\begin{array}{l}1876.92 \\
2032.15 \\
1877.97 \\
1810.95 \\
1878.95 \\
1637.52 \\
1725.29\end{array}$ & 1834.25 & 117.12 & 6.38 & $\begin{array}{l}4.36 \\
4.97 \\
6.10 \\
4.92 \\
5.40 \\
5.18 \\
5.32 \\
\end{array}$ & 5.18 & 0.49 & 9.48 & \begin{tabular}{r|}
-4.30 \\
-4.09 \\
-3.08 \\
-3.68 \\
-3.48 \\
-3.16 \\
-3.24
\end{tabular} & -3.58 & 0.44 & 12.24 \\
\hline $5 \mathrm{D} 95 \mathrm{C}$ & $\begin{array}{l}\text { LP5 P19-1 } \\
\text { LP5 P19-2 } \\
\text { LP5 P19-3 } \\
\text { LP5 P19-4 } \\
\text { LP5 P20-1 } \\
\text { LP5 P20-2 } \\
\text { LP5 P20-3 } \\
\text { LP5 P20-4 }\end{array}$ & $\begin{array}{l}1553.37 \\
1546.33 \\
1414.75 \\
1424.67 \\
1642.36 \\
1412.62 \\
1299.66 \\
1361.11 \\
\end{array}$ & 1456.86 & 106.29 & 7.30 & $\begin{array}{l}2.32 \\
3.00 \\
1.71 \\
1.90 \\
1.99 \\
2.47 \\
1.77 \\
2.10 \\
\end{array}$ & 2.16 & 0.40 & 18.49 & $\begin{array}{l}-6.70 \\
-5.16 \\
-8.25 \\
-7.51 \\
-8.24 \\
-5.71 \\
-7.34 \\
-6.48 \\
\end{array}$ & -6.92 & 1.05 & 15.18 \\
\hline
\end{tabular}


Table B.9. Results for PM6 I-FIT Specimen Aging

\begin{tabular}{|c|c|c|c|c|c|c|c|c|c|c|c|c|c|}
\hline \multicolumn{2}{|c|}{ PM6 } & \multicolumn{4}{|c|}{ Fracture Energy } & \multicolumn{4}{|c|}{ Flexibility Index } & \multicolumn{4}{|c|}{ Slope } \\
\hline Type & $\begin{array}{l}\text { Specimen } \\
\text { ID }\end{array}$ & $\begin{array}{c}\text { Energy } \\
\text { (LLD) (Gf) } \\
(\mathrm{J} / \mathrm{m} 2)\end{array}$ & Average & Std Dev & $\operatorname{CoV}(\%)$ & $\begin{array}{l}\text { Flexibility } \\
\text { Index }\end{array}$ & Average & Std Dev & $\operatorname{CoV}(\%)$ & Slope & Average & Std Dev & $\operatorname{CoV}(\%)$ \\
\hline \multirow{9}{*}{ Unaged } & & 1853.49 & \multirow{9}{*}{2057.80} & \multirow{9}{*}{192.94} & \multirow{9}{*}{9.38} & 6.99 & \multirow{9}{*}{7.37} & \multirow{9}{*}{1.50} & \multirow{9}{*}{20.42} & -2.65 & \multirow{9}{*}{-2.88} & \multirow{9}{*}{0.49} & \multirow{9}{*}{17.08} \\
\hline & & 2114.29 & & & & 8.74 & & & & -2.42 & & & \\
\hline & & 1930.85 & & & & 8.90 & & & & -2.17 & & & \\
\hline & & 2381.57 & & & & 9.41 & & & & -2.53 & & & \\
\hline & & 1951.06 & & & & 5.43 & & & & -3.59 & & & \\
\hline & & 2212.43 & & & & 8.19 & & & & -2.70 & & & \\
\hline & & 2307.33 & & & & 7.72 & & & & -2.99 & & & \\
\hline & & 1808.45 & & & & 5.56 & & & & -3.25 & & & \\
\hline & & 1960.71 & & & & 5.36 & & & & -3.66 & & & \\
\hline \multirow{6}{*}{$\begin{array}{c}\text { 16HRS, } \\
95 \mathrm{C}\end{array}$} & $4-1$ & 2012.54 & \multirow{6}{*}{2281.38} & \multirow{6}{*}{168.42} & \multirow{6}{*}{7.38} & 7.80 & \multirow{6}{*}{7.30} & & & -2.58 & & & \\
\hline & $5-2$ & 2235.12 & & & & 6.75 & & & & -3.31 & & & \\
\hline & $9-3$ & 2292.39 & & & & 8.40 & & & & -2.73 & & & \\
\hline & $10-4$ & 2587.90 & & & & 8.43 & & 0.95 & 13.09 & -3.07 & -3.18 & 0.43 & 13.44 \\
\hline & $11-2$ & 2251.00 & & & & 6.17 & & & & -3.65 & & & \\
\hline & 13-1 & 2309.33 & & & & 6.22 & & & & -3.71 & & & \\
\hline & $4-4$ & 2102.55 & & & & 6.94 & & & & -3.03 & & & \\
\hline & $6-1$ & 1932.61 & & & & 5.01 & & & & -3.86 & & & \\
\hline 16HRS, & $10-3$ & 1842.97 & & & & 3.75 & & & & -4.91 & & & \\
\hline $110 \mathrm{C}$ & $11-3$ & 2008.36 & 1951.21 & 142.42 & 7.30 & 5.41 & 4.93 & 1.19 & 24.16 & -3.71 & -4.13 & 0.74 & 17.96 \\
\hline & $12-1$ & 1710.23 & & & & 3.26 & & & & -5.24 & & & \\
\hline & $13-3$ & 2110.55 & & & & 5.21 & & & & -4.05 & & & \\
\hline & $8-4$ & 2239.93 & & & & 5.77 & & & & -3.88 & & & \\
\hline & $6-3$ & 2168.30 & & & & 7.06 & & & & -3.07 & & & \\
\hline & $1-4$ & 2063.08 & & & & 8.03 & & & & -2.57 & & & \\
\hline 1D95C & $2-3$ & 2052.78 & 2102.84 & 117.31 & 5.58 & 8.08 & 6.50 & 1.48 & 22.76 & -2.54 & -3.43 & 0.85 & 24.79 \\
\hline & $12-2$ & 1889.86 & & & & 3.78 & & & & -5.00 & & & \\
\hline & $11-4$ & 2203.09 & & & & 6.28 & & & & -3.51 & & & \\
\hline & $1-3$ & 1789.95 & & & & 5.26 & & & & -3.40 & & & \\
\hline & $7-3$ & 1813.71 & & & & 3.63 & & & & -4.99 & & & \\
\hline חم1101 & $9-1$ & 2062.73 & 101909 & 15551 & 797 & 4.23 & 134 & ? & 1655 & -4.88 & & & \\
\hline & $10-2$ & 1931.91 & 1949.99 & 155.51 & 7.91 & 4.07 & 4.34 & 0.12 & 16.55 & -4.75 & -4.59 & 0.63 & 13.69 \\
\hline & $11-1$ & 1867.06 & & & & 3.51 & & & & -5.32 & & & \\
\hline & 12.3 & 2234.59 & & & & 5.32 & & & & -4.20 & & & \\
\hline & $4-2$ & 1827.99 & & & & 3.66 & & & & -4.99 & & & \\
\hline & $5-1$ & 1759.86 & & & & 2.95 & & & & -5.97 & & & \\
\hline $3095 C$ & $13-4$ & 1938.88 & 1820 & 8930 & 400 & 3.64 & 369 & 063 & 1718 & -5.32 & 508 & 080 & $157 ?$ \\
\hline $3 D 95 C$ & $12-4$ & 1941.08 & 1822.47 & 89.30 & 4.90 & 4.17 & 3.69 & 0.63 & 17.18 & -4.66 & -5.08 & 0.80 & $15 . / 2$ \\
\hline & $1-2$ & 1718.55 & & & & 4.73 & & & & -3.63 & & & \\
\hline & $6-2$ & 1748.43 & & & & 2.96 & & & & -5.91 & & & \\
\hline & $9-4$ & 1925.01 & & & & 3.35 & & & & -5.75 & & & \\
\hline & $7-4$ & 1865.60 & & & & 3.64 & & & & -5.13 & & & \\
\hline & $10-1$ & 1636.78 & & & & 3.45 & & & & -4.74 & & & \\
\hline $5 D 85 C$ & $13-2$ & 1949.71 & 1850.68 & 106.09 & 5.73 & 4.04 & 3.65 & 0.23 & 6.24 & -4.83 & -5.09 & 0.31 & 7.23 \\
\hline & $5-3$ & 1917.78 & & & & 3.59 & & & & -5.34 & & & \\
\hline & $7-1$ & 1809.22 & & & & 3.82 & & & & -4.74 & & & \\
\hline
\end{tabular}


Table B.10. Results for PM6 Loose Mixture Aging

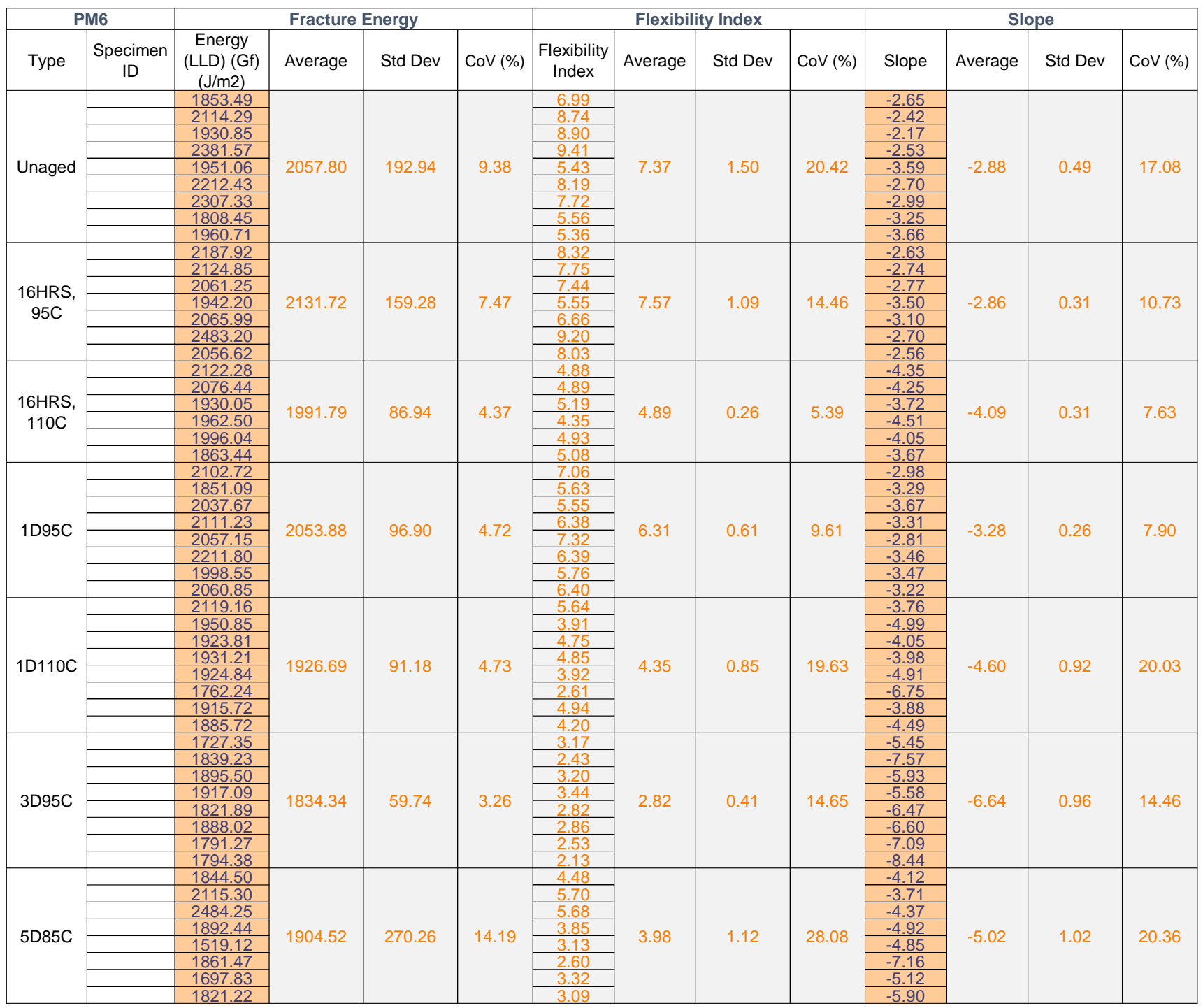


Table B.11. Results for PM7 I-FIT Specimen Aging

\begin{tabular}{|c|c|c|c|c|c|c|c|c|c|c|c|c|c|}
\hline \multicolumn{2}{|c|}{ PM7 } & \multicolumn{4}{|c|}{ Fracture Energy } & \multicolumn{4}{|c|}{ Flexibility Index } & \multicolumn{4}{|c|}{ Slope } \\
\hline Type & $\begin{array}{l}\text { Specimen } \\
\text { ID }\end{array}$ & $\begin{array}{c}\text { Energy } \\
\text { (LLD) (Gf) } \\
(\mathrm{J} / \mathrm{m} 2)\end{array}$ & Average & Std Dev & $\operatorname{CoV}(\%)$ & $\begin{array}{l}\text { Flexibility } \\
\text { Index }\end{array}$ & Average & Std Dev & CoV (\%) & Slope & Average & Std Dev & $\operatorname{CoV}(\%)$ \\
\hline \multirow{5}{*}{ Unaged } & $21-1$ & 2046.98 & \multirow{5}{*}{2017.80} & \multirow{5}{*}{59.37} & \multirow{5}{*}{2.94} & 7.78 & \multirow{5}{*}{7.40} & \multirow{5}{*}{0.46} & \multirow{5}{*}{6.18} & -2.63 & \multirow{5}{*}{-2.74} & \multirow{5}{*}{0.18} & \multirow{5}{*}{6.48} \\
\hline & $21-4$ & 1999.24 & & & & 6.78 & & & & -2.95 & & & \\
\hline & $23-4$ & 2037.51 & & & & 6.93 & & & & -2.94 & & & \\
\hline & $26-1$ & 1914.38 & & & & 7.63 & & & & -2.51 & & & \\
\hline & $26-4$ & 2090.90 & & & & 7.89 & & & & -2.65 & & & \\
\hline \multirow{7}{*}{$16 \mathrm{HRS}, 95 \mathrm{C}$} & $1-1$ & 2457.09 & \multirow{7}{*}{2240.07} & \multirow{7}{*}{201.79} & \multirow{7}{*}{9.01} & 9.91 & & & & -2.48 & & & \\
\hline & $2-1$ & 1962.90 & & & & 6.96 & & & & -2.82 & & & \\
\hline & $2-2$ & 2509.40 & & & & 9.03 & & & & -2.78 & & & \\
\hline & $3-2$ & 2416.75 & & & & 6.83 & 7.87 & 1.84 & 23.40 & -3.54 & -2.97 & 0.56 & 18.91 \\
\hline & $4-4$ & 2083.82 & & & & 6.02 & & & & -3.46 & & & \\
\hline & $8-2$ & 2067.90 & & & & 5.65 & & & & -3.66 & & & \\
\hline & $22-1$ & 2182.66 & & & & 10.70 & & & & -2.04 & & & \\
\hline & $6-1$ & 2560.62 & & & & 8.03 & & & & -3.19 & & & \\
\hline & $7-4$ & 1798.62 & & & & 5.62 & & & & -3.20 & & & \\
\hline & $8-1$ & 2435.54 & & & & 10.23 & & & & -2.38 & & & \\
\hline & $8-4$ & 2064.94 & & & & 6.37 & & & & -3.24 & & & \\
\hline & $20-2$ & 1961.33 & & & & 8.17 & & & & -2.40 & & & \\
\hline 16HRS, & $22-2$ & 2281.54 & 2209.16 & 226.78 & 10.27 & 6.27 & 7.57 & 1.52 & 20.13 & -3.64 & -2.99 & 0.43 & 14.23 \\
\hline & $23-3$ & 2358.69 & & & & 7.58 & & & & -3.11 & & & \\
\hline & $24-2$ & 2414.37 & & & & 9.58 & & & & -2.52 & & & \\
\hline & $25-3$ & 2019.69 & & & & 6.16 & & & & -3.28 & & & \\
\hline & $9-2$ & 2334.05 & & & & 9.19 & & & & -2.54 & & & \\
\hline & $11-1$ & 2071.33 & & & & 6.06 & & & & $\begin{array}{l}-3.42 \\
-3.42\end{array}$ & & & \\
\hline & $1-3$ & 2411.82 & & & & 9.76 & & & & -2.47 & & & \\
\hline & $4-2$ & 2421.98 & & & & 9.89 & & & & -2.45 & & & \\
\hline & $7-3$ & 2187.17 & & & & 7.39 & & & & -2.96 & & & \\
\hline & $9-1$ & 2414.89 & & & & 7.59 & & & & -3.18 & & & \\
\hline 1D95C & $21-2$ & 2130.53 & 2263.26 & 134.55 & 5.94 & 6.27 & 8.03 & 2.71 & 33.79 & -3.40 & -3.10 & 0.89 & 28.77 \\
\hline & $23-1$ & 2052.52 & & & & 4.08 & & & & -5.03 & & & \\
\hline & $25-1$ & 2333.23 & & & & 6.36 & & & & -3.67 & & & \\
\hline & $11-2$ & 2124.98 & & & & 6.88 & & & & -3.09 & & & \\
\hline & $12-4$ & 2292.24 & & & & 14.06 & & & & -1.63 & & & \\
\hline & $2-2$ & 2274.86 & & & & 10.63 & & & & -2.14 & & & \\
\hline & $5-1$ & 2437.77 & & & & 9.71 & & & & -2.51 & & & \\
\hline & $6-3$ & 1942.98 & & & & 4.69 & & & & -4.14 & & & \\
\hline & $9-4$ & 2479.93 & & & & 8.79 & & & & -2.82 & & & \\
\hline 1D110C & $20-3$ & 2175.42 & 2234.02 & 150.37 & 6.73 & 6.38 & 7.95 & 2.04 & 25.60 & -3.41 & -2.99 & 0.70 & 23.50 \\
\hline & $22-3$ & 2148.69 & & & & 6.55 & & & & -3.28 & & & \\
\hline & $26-2$ & 2212.10 & & & & 5.60 & & & & -3.95 & & & \\
\hline & $10-1$ & 2258.36 & & & & 9.22 & & & & -2.45 & & & \\
\hline & $11-4$ & 2176.11 & & & & 9.98 & & & & -2.18 & & & \\
\hline & $20-4$ & 2137.43 & & & & 6.23 & & & & -3.43 & & & \\
\hline & $21-3$ & 2168.26 & & & & 4.92 & & & & -4.41 & & & \\
\hline & $23-2$ & 2002.87 & & & & 3.54 & & & & -5.66 & & & \\
\hline 5D85C & $24-1$ & 2191.09 & 2162.80 & 102.99 & 4.76 & 4.53 & 4.81 & 0.82 & 17.16 & -4.84 & -4.61 & 0.67 & 14.42 \\
\hline & $25-2$ & 2126.56 & & & & 4.39 & & & & -4.84 & & & \\
\hline & $26-3$ & 2350.61 & & & & 5.24 & & & & -4.49 & & & \\
\hline
\end{tabular}


Table B.12. Results for PM8 I-FIT Specimen Aging

\begin{tabular}{|c|c|c|c|c|c|c|c|c|c|c|c|c|c|}
\hline \multicolumn{2}{|c|}{ PM8 } & \multicolumn{4}{|c|}{ Fracture Energy } & \multicolumn{4}{|c|}{ Flexibility Index } & \multicolumn{4}{|c|}{ Slope } \\
\hline Type & $\begin{array}{l}\text { Specimen } \\
\text { ID }\end{array}$ & $\begin{array}{c}\text { Energy } \\
\text { (LLD) (Gf) } \\
(\mathrm{J} / \mathrm{m} 2)\end{array}$ & Average & Std Dev & $\operatorname{CoV}(\%)$ & $\begin{array}{l}\text { Flexibility } \\
\text { Index }\end{array}$ & Average & Std Dev & $\operatorname{CoV}(\%)$ & Slope & Average & Std Dev & $\operatorname{CoV}(\%)$ \\
\hline \multirow{6}{*}{ Unaged } & $1-1$ & 2062.77 & \multirow{6}{*}{2055.77} & \multirow{6}{*}{169.93} & \multirow{6}{*}{8.27} & 20.84 & \multirow{6}{*}{15.02} & \multirow{6}{*}{4.00} & \multirow{6}{*}{26.60} & -0.99 & \multirow{6}{*}{-1.45} & \multirow{6}{*}{0.33} & \multirow{6}{*}{22.51} \\
\hline & $2-2$ & 1979.91 & & & & 14.45 & & & & -1.37 & & & \\
\hline & $5-1$ & 1789.82 & & & & 11.47 & & & & -1.56 & & & \\
\hline & $6-2$ & 1969.27 & & & & 10.05 & & & & -1.96 & & & \\
\hline & $12-3$ & 2282.15 & & & & 13.58 & & & & -1.68 & & & \\
\hline & $14-1$ & 2250.70 & & & & 19.74 & & & & -1.14 & & & \\
\hline \multirow{11}{*}{$16 \mathrm{HRS}, 95 \mathrm{C}$} & $1-4$ & 2020.13 & \multirow{6}{*}{2204.02} & \multirow{6}{*}{196.96} & \multirow{6}{*}{8.94} & 13.38 & \multirow{6}{*}{11.13} & \multirow{6}{*}{2.03} & \multirow{6}{*}{18.27} & -1.51 & \multirow{6}{*}{-2.04} & \multirow{6}{*}{0.37} & \multirow{6}{*}{18.29} \\
\hline & 4-4 & 2622.60 & & & & 13.45 & & & & -1.95 & & & \\
\hline & $10-3$ & 2100.95 & & & & 10.50 & & & & -2.00 & & & \\
\hline & $11-4$ & 2094.49 & & & & 9.35 & & & & -2.24 & & & \\
\hline & $12-4$ & 2172.20 & & & & 7.99 & & & & -2.72 & & & \\
\hline & $14-4$ & 2213.77 & & & & 12.10 & & & & -1.83 & & & \\
\hline & $3-2$ & 1836.87 & & & & 8.24 & & & & -2.23 & & & \\
\hline & $5-3$ & 2444.74 & & & & 8.89 & & & & -2.75 & & & \\
\hline & $9-4$ & 1922.14 & 2082.40 & 209.13 & 10.04 & 6.77 & 8.10 & 0.98 & 12.11 & -2.84 & -2.59 & 0.29 & 11.13 \\
\hline & $11-3$ & 2088.80 & & & & 7.23 & & & & -2.89 & & & \\
\hline & $12-2$ & 2119.47 & & & & 9.38 & & & & -2.26 & & & \\
\hline & $1-2$ & 2070.81 & & & & 10.84 & & & & -1.91 & & & \\
\hline & $2-1$ & 2094.31 & & & & 7.96 & & & & -2.63 & & & \\
\hline $1095 \mathrm{C}$ & $4-1$ & 2120.23 & 211824 & 11115 & 525 & 7.49 & 858 & 124 & 1450 & -2.83 & 251 & 030 & 1179 \\
\hline $1095 C$ & $6-1$ & 2250.68 & 2118.24 & 111.15 & 5.25 & 9.34 & 8.58 & 1.24 & 14.50 & -2.41 & -2.51 & 0.30 & $11 . / 9$ \\
\hline & $7-1$ & 2247.61 & & & & 8.68 & & & & -2.59 & & & \\
\hline & $14-3$ & 1925.81 & & & & 7.16 & & & & -2.69 & & & \\
\hline & $1-3$ & 1864.30 & & & & 6.71 & & & & -2.78 & & & \\
\hline & $3-3$ & 1708.65 & & & & 7.12 & & & & -2.40 & & & \\
\hline $1 \mathrm{D} 110 \mathrm{C}$ & $4-3$ & 1960.82 & 189320 & 16967 & 8.96 & 5.29 & 6.93 & 152 & 2188 & -3.71 & -284 & 0.55 & 1946 \\
\hline 1DI10C & 6-4 & 1786.65 & 1893.20 & $169.6 /$ & 8.96 & 5.18 & 6.93 & 1.52 & 21.88 & -3.45 & -2.84 & 0.55 & 19.46 \\
\hline & $10-1$ & 2231.58 & & & & 9.66 & & & & -2.31 & & & \\
\hline & $11-2$ & 1807.18 & & & & 7.63 & & & & -2.37 & & & \\
\hline & $4-2$ & 1966.47 & & & & 5.34 & & & & -3.68 & & & \\
\hline & $6-3$ & 1747.84 & & & & 3.63 & & & & -4.81 & & & \\
\hline & $7-4$ & 2147.40 & & & & 8.17 & & & & -2.63 & & & \\
\hline 5D85C & $10-2$ & 1862.54 & 1988.57 & 151.65 & 7.63 & 5.84 & 6.22 & 1.60 & 25.79 & -3.19 & -3.38 & 0.74 & 21.84 \\
\hline & $11-1$ & 2026.18 & & & & 6.10 & & & & -3.32 & & & \\
\hline & $12-1$ & 2181.00 & & & & 8.23 & & & & -2.65 & & & \\
\hline
\end{tabular}


Table B.13. Results for PM9 I-FIT Specimen Aging

\begin{tabular}{|c|c|c|c|c|c|c|c|c|c|c|c|c|c|}
\hline \multicolumn{2}{|c|}{ PM9 } & \multicolumn{4}{|c|}{ Fracture Energy } & \multicolumn{4}{|c|}{ Flexibility Index } & \multicolumn{4}{|c|}{ Slope } \\
\hline Type & $\begin{array}{l}\text { Specimen } \\
\text { ID }\end{array}$ & $\begin{array}{c}\text { Energy } \\
\text { (LLD) (Gf) } \\
(\mathrm{J} / \mathrm{m} 2)\end{array}$ & Average & Std Dev & $\operatorname{CoV}(\%)$ & Flexibility Index & Average & Std Dev & $\operatorname{CoV}(\%)$ & Slope & Average & Std Dev & $\operatorname{CoV}(\%)$ \\
\hline \multirow{6}{*}{ Unaged } & $2-1$ & 2332.85 & \multirow{6}{*}{2087.57} & \multirow{6}{*}{162.84} & \multirow{6}{*}{7.80} & 13.56 & \multirow{6}{*}{10.62} & \multirow{6}{*}{3.28} & \multirow{6}{*}{30.88} & -1.72 & \multirow{6}{*}{-2.17} & \multirow{6}{*}{0.67} & \multirow{6}{*}{30.77} \\
\hline & $3-1$ & 2029.92 & & & & 14.00 & & & & -1.45 & & & \\
\hline & $5-3$ & 1953.84 & & & & 6.98 & & & & -2.80 & & & \\
\hline & $7-4$ & 2124.91 & & & & 13.12 & & & & -1.62 & & & \\
\hline & $10-1$ & 2231.93 & & & & 10.43 & & & & -2.14 & & & \\
\hline & $12-2$ & 1851.99 & & & & 5.63 & & & & -3.29 & & & \\
\hline \multirow{5}{*}{$16 \mathrm{HRS}, 95 \mathrm{C}$} & $3-2$ & 2226.24 & \multirow{5}{*}{2082.92} & \multirow{5}{*}{82.89} & \multirow{5}{*}{3.98} & 9.64 & \multirow{5}{*}{8.24} & \multirow{5}{*}{0.89} & \multirow{5}{*}{10.82} & -2.31 & \multirow{5}{*}{-2.55} & \multirow{5}{*}{0.23} & \multirow{5}{*}{9.05} \\
\hline & $4-2$ & 1980.46 & & & & 8.61 & & & & -2.30 & & & \\
\hline & $6-4$ & 2106.43 & & & & 8.36 & & & & -2.52 & & & \\
\hline & $7-1$ & 2069.30 & & & & 7.55 & & & & -2.74 & & & \\
\hline & $11-4$ & 2032.18 & & & & 7.06 & & & & -2.88 & & & \\
\hline \multirow{6}{*}{$\begin{array}{c}16 \mathrm{HRS}, \\
110 \mathrm{C}\end{array}$} & $1-2$ & 1963.54 & & & & 6.38 & & & & -3.08 & & & \\
\hline & $4-3$ & 2360.69 & & & & 9.67 & & & & -2.44 & & & \\
\hline & $5-1$ & 1799.72 & 211477 & 208.90 & 988 & 5.49 & & 150 & 19.45 & -3.28 & -280 & 0.35 & 1253 \\
\hline & $6-1$ & 2015.81 & 2114.71 & 208.90 & 9.88 & 8.58 & 1.12 & 1.50 & 19.45 & -2.35 & -2.80 & 0.35 & 12.53 \\
\hline & $7-2$ & 2370.47 & & & & 9.08 & & & & -2.61 & & & \\
\hline & $9-1$ & 2178.39 & & & & 7.14 & & & & -3.05 & & & \\
\hline & $1-4$ & 2133.46 & & & & 8.71 & & & & -2.45 & & & \\
\hline & $2-4$ & 1890.47 & & & & 6.26 & & & & -3.02 & & & \\
\hline & $3-3$ & 2150.88 & 2123.81 & 111.18 & & 10.29 & & & & -2.09 & -2.54 & & 21.57 \\
\hline $1095 \mathrm{C}$ & $4-1$ & 2137.62 & 2123.81 & 111.18 & 5.24 & 6.79 & 8.90 & 2.59 & 29.06 & -3.15 & -2.54 & 0.55 & 21.57 \\
\hline & $9-3$ & 2246.80 & & & & 13.87 & & & & -1.62 & & & \\
\hline & $11-3$ & 2183.64 & & & & 7.48 & & & & -2.92 & & & \\
\hline & $1-3$ & 1995.48 & & & & 6.46 & & & & -3.09 & & & \\
\hline 1D110C & $2-2$ & 2070.98 & 215001 & 14776 & 6.87 & 6.53 & & & & -3.17 & & & \\
\hline $10110 \mathrm{C}$ & $4-4$ & 2144.43 & 2150.01 & $74 / .16$ & 6.87 & 6.83 & 6.61 & 0.14 & 2.10 & -3.14 & -3.26 & 0.21 & 6.53 \\
\hline & $12-1$ & 2389.13 & & & & 6.60 & & & & -3.62 & & & \\
\hline & $1-1$ & 2064.71 & & & & 5.24 & & & & -3.94 & & & \\
\hline & $6-3$ & 2314.35 & & & & 6.12 & & & & -3.78 & & & \\
\hline $5 \mathrm{D} 85 \mathrm{C}$ & $7-3$ & 1946.03 & 2084.86 & 126.04 & 6.05 & 5.26 & 5.72 & 0.41 & 7.09 & -3.70 & -3.65 & 0.23 & 6.40 \\
\hline & $8-3$ & 2001.68 & & & & 6.18 & & & & -3.24 & & & \\
\hline & $11-1$ & 2097.55 & & & & 5.81 & & & & -3.61 & & & \\
\hline
\end{tabular}


Table B.14. Results for PM10 I-FIT Specimen Aging

\begin{tabular}{|c|c|c|c|c|c|c|c|c|c|c|c|c|c|}
\hline \multicolumn{2}{|c|}{ PM10 } & \multicolumn{4}{|c|}{ Fracture Energy } & \multicolumn{4}{|c|}{ Flexibility Index } & \multicolumn{4}{|c|}{ Slope } \\
\hline Type & $\begin{array}{l}\text { Specimen } \\
\text { ID }\end{array}$ & $\begin{array}{c}\text { Energy } \\
\text { (LLD) (Gf) } \\
(\mathrm{J} / \mathrm{m} 2)\end{array}$ & Average & Std Dev & $\operatorname{CoV}(\%)$ & $\begin{array}{l}\text { Flexibility } \\
\text { Index }\end{array}$ & Average & Std Dev & $\operatorname{CoV}(\%)$ & Slope & Average & Std Dev & CoV $(\%)$ \\
\hline \multirow{5}{*}{ Unaged } & $4-3$ & 3089.37 & \multirow{5}{*}{2831.79} & \multirow{5}{*}{155.51} & \multirow{5}{*}{5.49} & 7.57 & \multirow{5}{*}{7.60} & \multirow{5}{*}{0.93} & \multirow{5}{*}{12.25} & -4.08 & \multirow{5}{*}{-3.78} & \multirow{5}{*}{0.51} & \multirow{5}{*}{13.55} \\
\hline & $5-2$ & 2633.89 & & & & 8.99 & & & & -2.93 & & & \\
\hline & $6-3$ & 2886.60 & & & & 7.87 & & & & -3.67 & & & \\
\hline & $8-2$ & 2828.59 & & & & 7.52 & & & & -3.76 & & & \\
\hline & $9-2$ & 2720.48 & & & & 6.07 & & & & -4.48 & & & \\
\hline \multirow{6}{*}{$16 \mathrm{HRS}, 95 \mathrm{C}$} & 1-4 & 2665.24 & \multirow{6}{*}{2552.70} & \multirow{6}{*}{172.16} & \multirow{6}{*}{6.74} & 4.49 & \multirow{6}{*}{4.56} & \multirow{6}{*}{0.96} & \multirow{6}{*}{21.01} & -5.93 & \multirow{6}{*}{-5.85} & \multirow{6}{*}{1.21} & \multirow{6}{*}{20.75} \\
\hline & $2-2$ & 2857.82 & & & & 5.41 & & & & -5.28 & & & \\
\hline & $4-4$ & 2533.53 & & & & 5.39 & & & & -4.70 & & & \\
\hline & $6-2$ & 2505.36 & & & & 3.82 & & & & -6.56 & & & \\
\hline & $9-3$ & 2314.67 & & & & 2.87 & & & & -8.07 & & & \\
\hline & $11-3$ & 2439.59 & & & & 5.39 & & & & -4.53 & & & \\
\hline & $1-1$ & 2208.99 & & & & 2.81 & & & & -7.86 & & & \\
\hline & $2-3$ & 2881.63 & & & & 5.77 & & & & -4.99 & & & \\
\hline & $3-3$ & 2695.41 & & & & 3.62 & & & & -7.45 & & & \\
\hline $110 \mathrm{C}$ & $6-4$ & 2326.43 & 2538.98 & 250.08 & 9.85 & 3.42 & 4.08 & 0.98 & 24.06 & -6.80 & -6.45 & 1.01 & 15.69 \\
\hline & $7-4$ & 2362.79 & & & & 3.98 & & & & -5.94 & & & \\
\hline & $8-4$ & 2758.63 & & & & 4.88 & & & & -5.65 & & & \\
\hline & $2-1$ & 2371.09 & & & & 3.35 & & & & -7.07 & & & \\
\hline & $3-1$ & 2472.49 & & & & 3.46 & & & & -7.15 & & & \\
\hline & $5-3$ & 2561.15 & & & 555 & 4.99 & & & 1842 & -5.13 & -6.14 & 0.88 & 1426 \\
\hline 1D95C & $7-1$ & 2187.24 & 2448.49 & 135.88 & 5.55 & 3.25 & 4.10 & 0.75 & 18.42 & -6.73 & -6.14 & 0.88 & 14.26 \\
\hline & 8-1 & 2587.41 & & & & 4.60 & & & & -5.63 & & & \\
\hline & $11-2$ & 2511.53 & & & & 4.92 & & & & -5.10 & & & \\
\hline & $1-2$ & 2908.22 & & & & 3.02 & & & & -9.62 & & & \\
\hline & $4-2$ & 2241.46 & & & & 2.59 & & & & -8.66 & & & \\
\hline 1D110C & $6-1$ & 2277.18 & 2431.67 & 246.19 & 10.12 & 3.07 & 3.11 & 0.37 & 12.03 & -7.41 & -7.92 & 1.10 & 13.86 \\
\hline & $8-3$ & 2425.79 & & & & 3.76 & & & & -6.46 & & & \\
\hline & $9-1$ & 2305.70 & & & & 3.09 & & & & -7.47 & & & \\
\hline & $1-3$ & 2234.65 & & & & 3.20 & & & & -6.99 & & & \\
\hline & $2-4$ & 2740.63 & & & & 4.41 & & & & -6.22 & & & \\
\hline & $3-4$ & 2296.83 & & & & 2.29 & & & & -10.02 & & & \\
\hline 5D85C & $9-4$ & 2355.24 & 2437.40 & 210.49 & 8.64 & 2.38 & 3.20 & 0.73 & 22.74 & -9.90 & -7.92 & 1.49 & 18.79 \\
\hline & $10-1$ & 2276.29 & & & & 3.27 & & & & -6.96 & & & \\
\hline & $12-1$ & 2720.78 & & & & 3.68 & & & & -7.40 & & & \\
\hline
\end{tabular}


Table B.15. Results for PM11 I-FIT Specimen Aging

\begin{tabular}{|c|c|c|c|c|c|c|c|c|c|c|c|c|c|}
\hline \multicolumn{2}{|c|}{ PM11 } & \multicolumn{4}{|c|}{ Fracture Energy } & \multicolumn{4}{|c|}{ Flexibility Index } & \multicolumn{4}{|c|}{ Slope } \\
\hline Type & $\begin{array}{l}\text { Specimen } \\
\text { ID }\end{array}$ & $\begin{array}{c}\text { Energy } \\
\text { (LLD) (Gf) } \\
(\mathrm{J} / \mathrm{m} 2)\end{array}$ & Average & Std Dev & $\operatorname{CoV}(\%)$ & $\begin{array}{l}\text { Flexibility } \\
\text { Index }\end{array}$ & Average & Std Dev & $\operatorname{CoV}(\%)$ & Slope & Average & Std Dev & CoV (\%) \\
\hline \multirow{6}{*}{ Unaged } & $1-4$ & 1985.00 & \multirow{6}{*}{1852.51} & \multirow{6}{*}{193.85} & \multirow{6}{*}{10.46} & 3.60 & \multirow{6}{*}{4.64} & \multirow{6}{*}{1.35} & \multirow{6}{*}{29.03} & -5.52 & \multirow{6}{*}{-4.22} & \multirow{6}{*}{0.88} & \multirow{6}{*}{20.75} \\
\hline & $2-1$ & 2181.85 & & & & 6.97 & & & & -3.13 & & & \\
\hline & $3-1$ & 1685.56 & & & & 3.36 & & & & -5.02 & & & \\
\hline & $4-2$ & 1622.66 & & & & 3.67 & & & & -4.42 & & & \\
\hline & $11-1$ & 1728.84 & & & & 4.31 & & & & -4.01 & & & \\
\hline & $12-3$ & 1911.17 & & & & 5.94 & & & & -3.22 & & & \\
\hline \multirow{8}{*}{$16 \mathrm{HRS}, 95 \mathrm{C}$} & $4-1$ & 2298.12 & \multirow{8}{*}{1998.41} & \multirow{8}{*}{165.42} & \multirow{8}{*}{8.28} & 3.72 & \multirow{8}{*}{3.38} & & & -6.18 & & & \\
\hline & $5-1$ & 1840.20 & & & & 2.78 & & & & -6.61 & & & \\
\hline & $11-4$ & 2132.60 & & & & 3.52 & & & & -6.06 & & & \\
\hline & $15-1$ & 2013.12 & & & & 3.27 & & & 13.16 & -6.16 & & & \\
\hline & $15-2$ & 1734.32 & & & & 3.16 & & 0.45 & 13.16 & -5.49 & -5.96 & 0.53 & 8.89 \\
\hline & $18-4$ & 2079.66 & & & & 4.34 & & & & -4.79 & & & \\
\hline & $19-3$ & 1901.58 & & & & 3.14 & & & & -6.06 & & & \\
\hline & $19-4$ & 1987.69 & & & & 3.15 & & & & -6.31 & & & \\
\hline & $1-1$ & 1817.31 & & & & 2.10 & & & & -8.67 & & & \\
\hline & $2-3$ & 1806.05 & & & & 2.30 & & & & -7.85 & & & \\
\hline & $7-3$ & 1749.62 & & & & 1.84 & & & & -9.53 & & & \\
\hline 16HRS, & $16-4$ & 1801.21 & 1782.22 & 132.62 & 7.44 & 1.87 & & & 26.94 & -9.62 & -7.42 & 1.66 & 22.34 \\
\hline $110 \mathrm{C}$ & $17-3$ & 1698.84 & $1 / 82.22$ & 132.62 & 1.44 & 2.89 & 2.54 & 0.69 & 26.94 & -5.88 & -1.42 & 1.60 & $2<.34$ \\
\hline & $18-2$ & 1987.93 & & & & 3.62 & & & & -5.49 & & & \\
\hline & $19-1$ & 1890.95 & & & & 3.59 & & & & -5.26 & & & \\
\hline & $20-3$ & 1505.85 & & & & 2.14 & & & & -7.03 & & & \\
\hline & $5-3$ & 1844.90 & & & & 2.75 & & & & -6.70 & & & \\
\hline & $12-1$ & 2117.51 & & & & 3.24 & & & & -6.54 & & & \\
\hline & $15-3$ & 1856.44 & & & & 3.59 & & & & -5.17 & & & \\
\hline $1 \mathrm{D} 95 \mathrm{C}$ & $16-1$ & 1924.12 & 1868.75 & 121.25 & 6.49 & 3.02 & 3.07 & 0.25 & 8.19 & -6.37 & -6.12 & 0.50 & 8.10 \\
\hline & $17-1$ & 1693.41 & & & & 2.98 & & & & -5.69 & & & \\
\hline & $18-3$ & 1789.23 & & & & 2.97 & & & & -6.03 & & & \\
\hline & $19-2$ & 1855.64 & & & & 2.93 & & & & -6.34 & & & \\
\hline & $1-2$ & 1854.63 & & & & 3.05 & & & & -6.08 & & & \\
\hline & $2-2$ & 1756.76 & & & & 2.36 & & & & -7.44 & & & \\
\hline & $7-4$ & 1778.80 & & & & 2.29 & & & & -7.76 & & & \\
\hline & $9-3$ & 1925.71 & & & & 3.12 & & & & -6.17 & & & \\
\hline 1D110C & $15-4$ & 1779.85 & 180285 & 74.48 & 4.13 & 2.69 & 255 & 0.51 & 19.86 & -6.61 & -735 & 1.44 & 1954 \\
\hline & $16-3$ & 1909.88 & & & 4.13 & 3.36 & 2.50 & 0.01 & & -5.69 & -1.30 & 1.44 & 19.04 \\
\hline & $17-2$ & 1767.48 & & & & 2.59 & & & & -6.82 & & & \\
\hline & $17-4$ & 1715.69 & & & & 2.32 & & & & -7.39 & & & \\
\hline & $20-2$ & 1847.28 & & & & 2.12 & & & & -8.72 & & & \\
\hline & $20-4$ & 1692.46 & & & & 1.57 & & & & -10.81 & & & \\
\hline & $1-3$ & 1939.74 & & & & 2.75 & & & & -7.06 & & & \\
\hline & $3-2$ & 1723.68 & & & & 2.05 & & & & -8.40 & & & \\
\hline & $4-4$ & 1736.00 & & & & 1.45 & & & & -11.98 & & & \\
\hline $5 \mathrm{D} 85 \mathrm{C}$ & 6-1 & 1746.44 & 1819.60 & 100.55 & 5.53 & 2.15 & 2.20 & 0.44 & 20.16 & -8.12 & -8.56 & 1.63 & 19.05 \\
\hline & $11-2$ & 1975.05 & & & & 2.74 & & & & -7.22 & & & \\
\hline & $12-4$ & 1796.71 & & & & 2.09 & & & & -8.58 & & & \\
\hline
\end{tabular}


Table B.16. Results for PM12 I-FIT Specimen Aging

\begin{tabular}{|c|c|c|c|c|c|c|c|c|c|c|c|c|c|}
\hline \multicolumn{2}{|c|}{ PM12 } & \multicolumn{4}{|c|}{ Fracture Energy } & \multicolumn{4}{|c|}{ Flexibility Index } & \multicolumn{4}{|c|}{ Slope } \\
\hline Type & $\begin{array}{l}\text { Specimen } \\
\text { ID }\end{array}$ & $\begin{array}{c}\text { Energy } \\
\text { (LLD) (Gf) } \\
(\mathrm{J} / \mathrm{m} 2)\end{array}$ & Average & Std Dev & $\operatorname{CoV}(\%)$ & $\begin{array}{l}\text { Flexibility } \\
\text { Index }\end{array}$ & Average & Std Dev & $\operatorname{CoV}(\%)$ & Slope & Average & Std Dev & $\operatorname{CoV}(\%)$ \\
\hline \multirow{5}{*}{ Unaged } & $22-3$ & 1961.11 & \multirow{5}{*}{1854.31} & \multirow{5}{*}{94.18} & \multirow{5}{*}{5.08} & 5.60 & \multirow{5}{*}{4.82} & \multirow{5}{*}{0.61} & \multirow{5}{*}{12.60} & -3.50 & \multirow{5}{*}{-3.91} & \multirow{5}{*}{0.52} & \multirow{5}{*}{13.37} \\
\hline & $23-3$ & 1936.73 & & & & 3.95 & & & & -4.90 & & & \\
\hline & $24-2$ & 1818.50 & & & & 5.12 & & & & -3.55 & & & \\
\hline & $25-4$ & 1696.67 & & & & 4.28 & & & & -3.96 & & & \\
\hline & $26-2$ & 1858.55 & & & & 5.13 & & & & -3.62 & & & \\
\hline \multirow{10}{*}{$16 \mathrm{HRS}, 95 \mathrm{C}$} & $6-4$ & 1783.33 & \multirow{10}{*}{2015.19} & \multirow{10}{*}{189.69} & \multirow{10}{*}{9.41} & 3.01 & & & & -5.92 & & & \\
\hline & $7-4$ & 1964.30 & & & & 4.71 & & & & -4.17 & & & \\
\hline & $8-3$ & 2414.62 & & & & 4.55 & & & & -5.31 & & & \\
\hline & $12-3$ & 1845.47 & & & & 5.18 & & & & -3.56 & & & \\
\hline & $20-4$ & 2153.03 & & & & 3.97 & 404 & & 1633 & -5.42 & -510 & 075 & 1464 \\
\hline & $23-1$ & 1900.52 & & & & 3.99 & 4.04 & 0.66 & 16.33 & -4.76 & -5.10 & 0.15 & 14.64 \\
\hline & $24-1$ & 2128.32 & & & & 3.52 & & & & -6.05 & & & \\
\hline & $26-4$ & 2032.97 & & & & 3.64 & & & & -5.58 & & & \\
\hline & $2-2$ & 2144.60 & & & & 4.52 & & & & -4.74 & & & \\
\hline & $4-3$ & 1784.69 & & & & 3.26 & & & & -5.47 & & & \\
\hline & $1-2$ & 2395.49 & & & & 4.72 & & & & -5.07 & & & \\
\hline & $4-1$ & 1793.62 & & & & 2.14 & & & & -8.39 & & & \\
\hline & $7-1$ & 1864.72 & & & & 3.53 & & & & -5.28 & & & \\
\hline & $8-2$ & 1755.47 & & & & 3.35 & & & & -5.24 & & & \\
\hline 16HRS, & $21-2$ & 1995.28 & 2014.13 & 246.15 & 12.22 & 5.03 & 3.89 & 1.01 & 25.87 & -3.97 & -5.45 & 1.24 & 22.73 \\
\hline & $24-4$ & 2459.70 & & & & 5.19 & & & & -4.74 & & & \\
\hline & $25-1$ & 1788.78 & & & & 3.29 & & & & -5.44 & & & \\
\hline & $12-1$ & 2112.27 & & & & 4.79 & & & & -4.41 & & & \\
\hline & $14-2$ & 1961.82 & & & & 3.00 & & & & -6.54 & & & \\
\hline & $1-4$ & 2145.95 & & & & 4.06 & & & & -5.28 & & & \\
\hline & $2-1$ & 2201.88 & & & & 5.05 & & & & -4.36 & & & \\
\hline & $6-2$ & 2092.22 & & & & 5.86 & & & & -3.57 & & & \\
\hline & $7-3$ & 2271.01 & & & & 4.92 & & & & -4.62 & & & \\
\hline $1 \mathrm{D} 95 \mathrm{C}$ & $20-2$ & 2029.42 & 2032.25 & 204.89 & 10.08 & 3.99 & 4.06 & 1.08 & 26.62 & -5.09 & -5.31 & 1.22 & 22.91 \\
\hline & $22-4$ & 1755.40 & & & & 2.18 & & & & -8.04 & & & \\
\hline & $25-2$ & 1742.29 & & & & 2.71 & & & & -6.43 & & & \\
\hline & $10-2$ & 2265.55 & & & & 4.19 & & & & -5.41 & & & \\
\hline & $12-2$ & 1786.57 & & & & 3.61 & & & & -4.95 & & & \\
\hline & $1-3$ & 1728.90 & & & & 2.62 & & & & -6.61 & & & \\
\hline & $3-1$ & 1805.50 & & & & 3.28 & & & & -5.50 & & & \\
\hline & $3-4$ & 1765.57 & & & & 3.74 & & & & -4.72 & & & \\
\hline 1D110C & $9-1$ & 2045.31 & 1926 ด & 13994 & 726 & 4.29 & $36 ?$ & 057 & 1568 & -4.77 & & & \\
\hline & $23-4$ & 2139.46 & 1926.90 & 139.94 & 1.26 & 3.77 & 3.62 & $0.5 /$ & 15.68 & -5.67 & -5.43 & 0.78 & 14.32 \\
\hline & $24-3$ & 2051.74 & & & & 3.11 & & & & -6.59 & & & \\
\hline & $26-3$ & 1911.17 & & & & 3.68 & & & & -5.19 & & & \\
\hline & $11-1$ & 1967.56 & & & & 4.46 & & & & -4.41 & & & \\
\hline & $8-1$ & 1721.79 & & & & 2.49 & & & & -6.92 & & & \\
\hline & $8-4$ & 1804.48 & & & & 2.65 & & & & -6.82 & & & \\
\hline $5 \mathrm{D} 85 \mathrm{C}$ & $11-2$ & 1661.89 & 1794.78 & 113.29 & 6.31 & 2.78 & 2.66 & 0.11 & 4.27 & -5.98 & -6.76 & 0.41 & 6.06 \\
\hline & $11-4$ & 1997.38 & & & & 2.78 & & & & -7.18 & & & \\
\hline & $12-4$ & 1788.36 & & & & 2.58 & & & & -6.92 & & & \\
\hline
\end{tabular}


Table B.17. Results for PM13 I-FIT Specimen Aging

\begin{tabular}{|c|c|c|c|c|c|c|c|c|c|c|c|c|c|}
\hline \multicolumn{2}{|c|}{ PM13 } & \multicolumn{4}{|c|}{ Fracture Energy } & \multicolumn{4}{|c|}{ Flexibility Index } & \multicolumn{4}{|c|}{ Slope } \\
\hline Type & $\begin{array}{c}\text { Specimen } \\
\text { ID }\end{array}$ & $\begin{array}{c}\text { Energy } \\
\text { (LLD) (Gf) } \\
\text { (J/m2) }\end{array}$ & Average & Std Dev & CoV (\%) & $\begin{array}{l}\text { Flexibility } \\
\text { Index }\end{array}$ & Average & Std Dev & $\operatorname{CoV}(\%)$ & Slope & Average & Std Dev & CoV (\%) \\
\hline 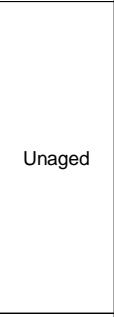 & \begin{tabular}{|c|}
$20-2$ \\
$44-4$ \\
$45-2$ \\
$53-4$ \\
$41-1$ \\
$42-1$ \\
$43-2$ \\
$44-3$ \\
$46-4$ \\
$48-1$ \\
$22-3$ \\
$23-1$ \\
$24-1$ \\
$24-2$ \\
$25-4$ \\
\end{tabular} & $\begin{array}{l}2176.75 \\
1959.97 \\
2210.44 \\
2347.32 \\
2055.38 \\
2580.49 \\
1855.51 \\
1784.39 \\
1794.38 \\
2131.16 \\
2534.50 \\
2143.15 \\
2165.15 \\
1811.88 \\
1663.98 \\
\end{array}$ & 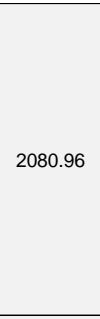 & 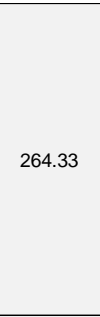 & 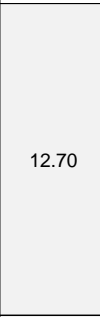 & $\begin{array}{c}10.42 \\
18.15 \\
10.68 \\
15.34 \\
12.38 \\
17.09 \\
10.37 \\
18.40 \\
5.37 \\
8.36 \\
11.63 \\
8.31 \\
7.90 \\
8.39 \\
6.63 \\
\end{array}$ & 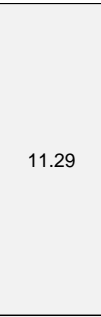 & 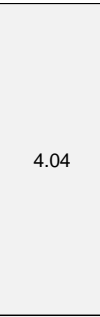 & 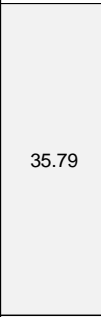 & \begin{tabular}{|l|}
-2.09 \\
-1.08 \\
-2.07 \\
-1.53 \\
-1.66 \\
-1.51 \\
-1.79 \\
-0.97 \\
-3.34 \\
-2.55 \\
-2.18 \\
-2.58 \\
-2.74 \\
-2.16 \\
-2.51 \\
\end{tabular} & -2.05 & 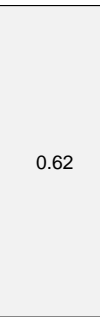 & 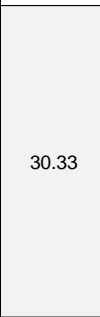 \\
\hline 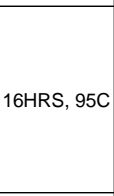 & \begin{tabular}{|c|}
$3-1$ \\
$5-4$ \\
$6-2$ \\
$8-1$ \\
$22-1$ \\
$24-4$ \\
$25-1$ \\
$9-1$ \\
$10-1$ \\
\end{tabular} & $\begin{array}{l}2004.94 \\
2124.99 \\
1632.67 \\
2260.56 \\
2084.24 \\
2173.32 \\
2383.16 \\
2322.18 \\
2133.36 \\
\end{array}$ & 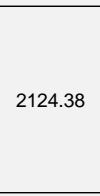 & . & 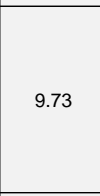 & $\begin{array}{c}7.68 \\
6.85 \\
6.69 \\
11.90 \\
12.33 \\
7.27 \\
10.32 \\
9.72 \\
7.41 \\
\end{array}$ & 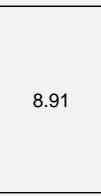 & 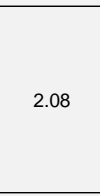 & . & $\begin{array}{l}-2.61 \\
-3.10 \\
-2.44 \\
-1.90 \\
-1.69 \\
-2.99 \\
-2.31 \\
-2.39 \\
-2.88 \\
\end{array}$ & -2.48 & 0.45 & 18.14 \\
\hline $\begin{array}{c}16 \mathrm{HRS}, \\
110 \mathrm{C}\end{array}$ & \begin{tabular}{|c|}
$3-4$ \\
$5-3$ \\
$6-1$ \\
$8-2$ \\
$20-4$ \\
$9-2$ \\
$9-3$ \\
\end{tabular} & $\begin{array}{l}2860.67 \\
2495.86 \\
2066.72 \\
2620.96 \\
2278.73 \\
2137.28 \\
2383.29 \\
\end{array}$ & 2406.22 & 257.75 & 10.71 & $\begin{array}{c}12.28 \\
13.56 \\
10.76 \\
9.67 \\
5.90 \\
9.37 \\
6.91 \\
\end{array}$ & 9.78 & 2.53 & 25.92 & $\begin{array}{l}-2.33 \\
-1.84 \\
-1.92 \\
-2.71 \\
-3.86 \\
-2.28 \\
-3.45 \\
\end{array}$ & -2.63 & 0.71 & 27.04 \\
\hline $1 \mathrm{D} 95 \mathrm{C}$ & $\begin{array}{c}5-1 \\
6-3 \\
7-1 \\
41-3 \\
43-1 \\
49-1 \\
50-4 \\
52-4 \\
54-3 \\
52-3 \\
7-3 \\
21-3 \\
24-3 \\
25-2 \\
25-3 \\
9-4 \\
10-4 \\
\end{array}$ & $\begin{array}{l}2476.60 \\
2629.83 \\
2564.52 \\
2531.48 \\
2480.64 \\
3224.61 \\
1889.32 \\
2315.12 \\
2044.50 \\
2064.31 \\
2658.93 \\
2367.42 \\
1548.33 \\
3086.81 \\
1808.50 \\
2135.07 \\
2366.06 \\
\end{array}$ & 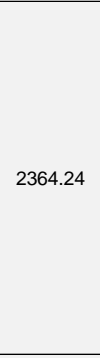 & 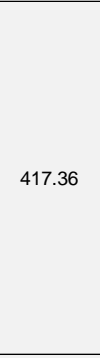 & 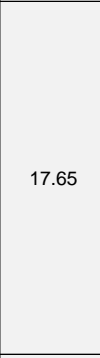 & $\begin{array}{c}10.77 \\
13.49 \\
11.55 \\
15.72 \\
14.59 \\
11.77 \\
4.86 \\
10.02 \\
5.78 \\
6.93 \\
15.92 \\
8.25 \\
6.10 \\
10.09 \\
7.97 \\
14.93 \\
11.49 \\
\end{array}$ & 10.60 & 更 & 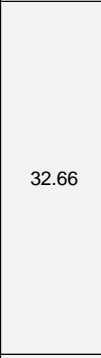 & $\begin{array}{l}-2.30 \\
-1.95 \\
-2.22 \\
-1.61 \\
-1.70 \\
-2.74 \\
-3.89 \\
-2.31 \\
-3.54 \\
-2.98 \\
-1.67 \\
-2.87 \\
-2.54 \\
-3.06 \\
-2.27 \\
-1.43 \\
-2.06 \\
\end{array}$ & -2.42 & 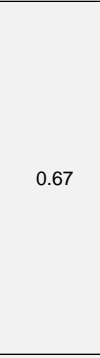 & 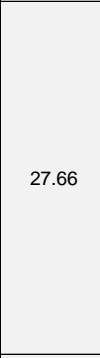 \\
\hline 1D110C & $\begin{array}{c}5-2 \\
47-2 \\
48-2 \\
50-3 \\
51-3 \\
54-2 \\
55-4 \\
6-4 \\
7-4 \\
10-2 \\
10-3 \\
\end{array}$ & $\begin{array}{l}2142.29 \\
2066.58 \\
1821.48 \\
2272.67 \\
2123.09 \\
2327.51 \\
2529.55 \\
2419.96 \\
2314.37 \\
2845.94 \\
2408.40 \\
\end{array}$ & 2297.44 & 255.51 & 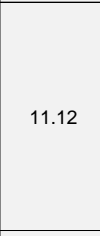 & $\begin{array}{c}10.82 \\
6.75 \\
5.05 \\
6.97 \\
5.85 \\
5.83 \\
9.84 \\
10.48 \\
10.06 \\
13.55 \\
11.69 \\
\end{array}$ & 8.81 & 2.69 & ( & \begin{tabular}{|l|}
-1.98 \\
-3.06 \\
-3.61 \\
-3.26 \\
-3.63 \\
-3.99 \\
-2.57 \\
-2.31 \\
-2.30 \\
-2.10 \\
-2.06 \\
\end{tabular} & -2.81 & 0.69 & 24.72 \\
\hline $5 \mathrm{D} 85 \mathrm{C}$ & $\begin{array}{l}11-1 \\
47-3 \\
48-3 \\
51-4 \\
52-2 \\
53-2 \\
54-4 \\
11-3 \\
11-4 \\
12-1 \\
12-2 \\
12-3 \\
\end{array}$ & $\begin{array}{l}2424.74 \\
1662.01 \\
2055.79 \\
2060.89 \\
1976.49 \\
2101.57 \\
1832.46 \\
1879.62 \\
2135.65 \\
1993.66 \\
1839.27 \\
2292.20 \\
\end{array}$ & 2021.20 & 199.82 & 9.89 & $\begin{array}{l}6.77 \\
3.29 \\
4.58 \\
5.37 \\
7.49 \\
8.18 \\
3.77 \\
6.71 \\
5.98 \\
5.09 \\
6.57 \\
8.13 \\
\end{array}$ & 5.99 & 1.54 & 25.70 & $\begin{array}{l}-3.58 \\
-5.05 \\
-4.49 \\
-3.84 \\
-2.64 \\
-2.57 \\
-4.86 \\
-2.80 \\
-3.57 \\
-3.92 \\
-2.80 \\
-2.82 \\
\end{array}$ & -3.58 & 0.84 & 23.53 \\
\hline 2D95C & $\begin{array}{l}42-3 \\
45-1 \\
46-1 \\
49-3 \\
51-1 \\
55-2 \\
53-3 \\
56-1 \\
\end{array}$ & $\begin{array}{l}2208.51 \\
3034.87 \\
2003.42 \\
2317.35 \\
2101.68 \\
1797.40 \\
1983.83 \\
1634.14\end{array}$ & 2135.15 & 396.00 & 18.55 & $\begin{array}{c}11.27 \\
14.45 \\
6.24 \\
5.62 \\
8.98 \\
5.60 \\
5.11 \\
4.21\end{array}$ & 7.69 & 3.35 & 43.56 & $\begin{array}{l}-1.96 \\
-2.10 \\
-3.21 \\
-4.12 \\
-2.34 \\
-3.21 \\
-3.88 \\
-3.88 \\
\end{array}$ & -3.09 & 0.80 & 26.01 \\
\hline $3 \mathrm{D} 95 \mathrm{C}$ & $\begin{array}{l}46-3 \\
47-1 \\
49-4 \\
50-2 \\
51-2 \\
53-1 \\
\end{array}$ & $\begin{array}{l}1765.11 \\
2020.53 \\
2165.78 \\
1935.10 \\
2106.82 \\
2004.60 \\
\end{array}$ & 1999.66 & 128.27 & 6.41 & $\begin{array}{l}5.83 \\
5.23 \\
4.41 \\
5.22 \\
4.02 \\
4.95 \\
\end{array}$ & 4.94 & 0.59 & 11.89 & $\begin{array}{l}-3.03 \\
-3.86 \\
-4.91 \\
-3.71 \\
-5.24 \\
-4.05 \\
\end{array}$ & -4.13 & 0.74 & 17.96 \\
\hline
\end{tabular}


Table B.18. Results for LM1 I-FIT Specimen Aging

\begin{tabular}{|c|c|c|c|c|c|c|c|c|c|c|}
\hline & M1 & & Fractur & hergy & & & Flexibi & Index & & \\
\hline Type & Specimen ID & $\begin{array}{c}\text { Energy (LLD) } \\
\text { (Gf) (J/m2) }\end{array}$ & Average & Std Dev & CoV (\%) & $\begin{array}{l}\text { Flexibility } \\
\text { Index }\end{array}$ & Average & Std Dev & CoV (\%) & Slope \\
\hline & $19-3$ & 1971.06 & & & & 7.98 & & & & -2.47 \\
\hline & $21-4$ & 1857.12 & & & & 8.97 & & & & -2.07 \\
\hline & $22-1$ & 1822.01 & & & & 6.58 & & & & -2.77 \\
\hline $75 \mathrm{C}, 1 \mathrm{~d}$ & $22-3$ & 2072.55 & 1962.60 & 127.25 & 6.48 & 11.26 & 810 & 1.44 & 1782 & -1.84 \\
\hline $15 \mathrm{c}, \mathrm{la}$ & $23-3$ & 1805.75 & 1962.60 & 127.25 & 6.48 & 6.79 & 8.10 & 1.44 & 17.82 & -2.66 \\
\hline & $\begin{array}{l}24-1 \\
25-1\end{array}$ & $\begin{array}{l}1913.70 \\
2085.19\end{array}$ & & & & 7.30 & & & & -2.62 \\
\hline & $\begin{array}{l}25-1 \\
26-1\end{array}$ & $\begin{array}{l}2085.19 \\
2173.45\end{array}$ & & & & $\begin{array}{l}7.19 \\
8.73\end{array}$ & & & & $\begin{array}{l}-2.90 \\
-2.49\end{array}$ \\
\hline & $20-2$ & 1835.41 & & & & $\begin{array}{l}0.15 \\
6.42\end{array}$ & & & & $\begin{array}{l}-2.45 \\
-2.86\end{array}$ \\
\hline & $\frac{2-2}{21-2}$ & 1980.21 & & & & 8.54 & & & & $\begin{array}{l}-2.00 \\
-2.32\end{array}$ \\
\hline & $21-3$ & 1920.63 & & & & 7.06 & & & & -2.72 \\
\hline $85 C, 1 d$ & $23-4$ & 2185.00 & 1958.07 & 119.70 & 6.11 & 8.03 & 7.14 & 0.80 & 11.15 & -2.72 \\
\hline & $24-2$ & 2066.94 & & & & 7.13 & & & & -2.90 \\
\hline & $25-2$ & 1844.25 & & & & 6.17 & & & & -2.99 \\
\hline & $25-3$ & 1874.06 & & & & 6.67 & & & & -2.81 \\
\hline & $19-4$ & 1938.41 & & & & $\frac{0.01}{4.02}$ & & & & -4.82 \\
\hline & $20-1$ & 1796.04 & & & & 4.83 & & & & -3.72 \\
\hline & $20-3$ & 2189.88 & & & & 7.37 & & & & -2.97 \\
\hline $95 \mathrm{C}, 1 \mathrm{~d}$ & $22-2$ & 2015.95 & 1971.46 & 169.21 & 8.58 & 6.65 & 5.96 & 1.09 & 18.31 & -3.03 \\
\hline 300,10 & $\frac{2<-2}{24-4}$ & 2213.93 & 1971.40 & 105.21 & 0.00 & 6.00 & 0.50 & 1.05 & 10.01 & $\begin{array}{l}-0.00 \\
-3.27\end{array}$ \\
\hline & $25-4$ & 1912.43 & & & & 5.76 & & & & -3.32 \\
\hline & $26-4$ & 1733.56 & & & & 6.30 & & & & -2.75 \\
\hline & $4-3$ & 2081.59 & & & & 6.80 & & & & -3.06 \\
\hline & $9-4$ & 2165.94 & & & & 7.01 & & & & -3.09 \\
\hline $75 \mathrm{c} .3 \mathrm{~d}$ & $1-4$ & 1806.49 & 199142 & 17074 & 857 & 5.87 & & 088 & 1251 & -3.08 \\
\hline $75 C, 3 d$ & $10-4$ & 1788.43 & 1991.42 & 170.74 & 8.57 & 6.32 & 7.04 & 0.88 & 12.51 & -2.83 \\
\hline & $12-4$ & 1889.73 & & & & 7.74 & & & & -2.44 \\
\hline & $2-4$ & 2216.32 & & & & 8.52 & & & & $\begin{array}{l}-2.44 \\
-2.60\end{array}$ \\
\hline & $1-1$ & 1844.55 & & & & 4.19 & & & & -4.40 \\
\hline & $\frac{1-1}{11-3}$ & 1672.98 & & & & 3.96 & & & & $\begin{array}{l}-4.40 \\
-4.22\end{array}$ \\
\hline & $4-2$ & 2028.70 & & & & $\begin{array}{l}0.90 \\
4.89\end{array}$ & & & & $\begin{array}{l}-4.22 \\
-4.15\end{array}$ \\
\hline $85 \mathrm{C} 3 \mathrm{~d}$ & $5-3$ & 1675.78 & 188212 & 20814 & 1106 & 4.12 & & 205 & 36.17 & -4.07 \\
\hline $85 C, 3 d$ & $6-3$ & 1631.77 & 1882.12 & 208.14 & 11.06 & 4.13 & 5.66 & 2.05 & 36.17 & -3.95 \\
\hline & $3-1$ & 2161.73 & & & & 9.69 & & & & -2.23 \\
\hline & $12-1$ & 2191.14 & & & & 8.27 & & & & -2.65 \\
\hline & $3-2$ & 1850.31 & & & & 6.05 & & & & -3.06 \\
\hline & $5-2$ & 1682.95 & & & & 3.05 & & & & -5.51 \\
\hline & $7-4$ & 1644.26 & & & & 3.69 & & & & -4.45 \\
\hline $95 C, 3 d$ & $12-2$ & 1364.34 & 1699.19 & 199.84 & 11.76 & 2.70 & 3.52 & 0.57 & 16.13 & -5.05 \\
\hline & $11-1$ & 1871.74 & & & & 3.84 & & & & -4.87 \\
\hline & $1-2$ & 1932.64 & & & & $\begin{array}{l}3.04 \\
4.29\end{array}$ & & & & $\begin{array}{l}-4.01 \\
-4.51\end{array}$ \\
\hline & $5-1$ & 2434.10 & & & & 5.42 & & & & -4.49 \\
\hline & $9-2$ & $\begin{array}{l}404.10 \\
1531.00\end{array}$ & & & & 2.70 & & & & $\begin{array}{l}-4.45 \\
-5.67\end{array}$ \\
\hline & $10-2$ & 2207.70 & & & & 4.77 & & & & $\begin{array}{l}-0.01 \\
-4.63\end{array}$ \\
\hline $75 C, 5 d$ & $16-2$ & 2452.88 & 2144.94 & 312.17 & 14.55 & 8.43 & 5.31 & 1.79 & 33.69 & -2.91 \\
\hline & $\frac{10-2}{17-2}$ & $\begin{array}{l}2452.00 \\
2232.31\end{array}$ & & & & $\begin{array}{l}0.45 \\
6.38\end{array}$ & & & & $\begin{array}{l}-2.91 \\
-3.50\end{array}$ \\
\hline & $18-4$ & 2011.62 & & & & 4.19 & & & & -4.80 \\
\hline & $11-2$ & 1752.20 & & & & 3.48 & & & & -5.04 \\
\hline & $13-3$ & 1803.33 & & & & 3.87 & & & & -4.66 \\
\hline & $14-2$ & 2271.56 & & & & 5.32 & & & & -4.27 \\
\hline $85 \mathrm{C}, 5 \mathrm{~d}$ & $\frac{14-2}{15-2}$ & $\begin{array}{l}221.56 \\
1924.70\end{array}$ & 1964.00 & 171.07 & 8.71 & $\begin{array}{l}5.32 \\
5.12\end{array}$ & 4.65 & 0.71 & 15.17 & $\begin{array}{l}-4.21 \\
-3.76\end{array}$ \\
\hline & $16-1$ & 2056.97 & & & & 5.02 & & & & -4.10 \\
\hline & $\frac{10-1}{17-4}$ & 1975.21 & & & & 5.09 & & & & $\begin{array}{l}-4.10 \\
-3.88\end{array}$ \\
\hline & $2-3$ & 1633.25 & & & & 2.84 & & & & $\begin{array}{l}-0.00 \\
-5.75\end{array}$ \\
\hline & $3-4$ & 1597.30 & & & & 2.46 & & & & -6.48 \\
\hline & $6-4$ & 1759.73 & & 110 & 651 & 2.90 & & & 1021 & -6.07 \\
\hline $95 \mathrm{C}, 5 \mathrm{~d}$ & 13-1 & 1533.45 & 1690.51 & 110.13 & 6.51 & 2.92 & 2.63 & 0.27 & 10.24 & $\begin{array}{l}-0.01 \\
-5.26\end{array}$ \\
\hline & $17-3$ & 1771.29 & & & & 2.19 & & & & -8.07 \\
\hline & $18-1$ & 1848.06 & & & & 2.48 & & & & $\begin{array}{l}-0.01 \\
-7.44\end{array}$ \\
\hline & $1-3$ & 1948.84 & & & & 4.96 & & & & -3.93 \\
\hline & $10-3$ & 1862.76 & & & & 4.51 & & & & -4.13 \\
\hline & $10=5$ & 2093.74 & & & & 5.67 & & & & $\begin{array}{l}-4.10 \\
-3.69\end{array}$ \\
\hline $75 \mathrm{C}, 7 \mathrm{~d}$ & $9-1$ & 1826.84 & 1837.85 & 199.60 & 10.86 & 3.32 & 4.22 & 0.94 & 22.39 & $\begin{array}{l}-5.05 \\
-5.50\end{array}$ \\
\hline & $8-4$ & $\begin{array}{l}1020.04 \\
1697.83\end{array}$ & & & & $\begin{array}{l}0.06 \\
3.89\end{array}$ & & & & -4.36 \\
\hline & $7-1$ & 1442.01 & & & & 2.64 & & & & -5.46 \\
\hline & $4-4$ & $\begin{array}{l}1442.01 \\
1992.96\end{array}$ & & & & 2.04 & & & & $\begin{array}{l}-5.40 \\
-4.40\end{array}$ \\
\hline & $8-1$ & 1711.88 & & & & $\begin{array}{l}4.00 \\
2.93\end{array}$ & & & & $\begin{array}{l}-4.40 \\
-5.85\end{array}$ \\
\hline & $7-3$ & 2020.77 & & & & 3.02 & & & & -6.69 \\
\hline & $2-1$ & 1214.77 & & & & $\frac{0.02}{2.15}$ & & & & $\begin{array}{l}-0.05 \\
-5.64\end{array}$ \\
\hline $85 C, 7 d$ & $8-1$ & $\begin{array}{l}1<4.121 \\
1912.08\end{array}$ & 1697.90 & 269.25 & 15.86 & 2.86 & 2.60 & 0.31 & 12.04 & $\begin{array}{l}-5.04 \\
-6.68 \\
\end{array}$ \\
\hline & $9-3$ & 1675.32 & & & & 2.49 & & & & -6.73 \\
\hline & $5-4$ & 1428.02 & & & & 2.27 & & & & $\begin{array}{r}-0.10 \\
-6.28\end{array}$ \\
\hline & $6-1$ & 1920.02 & & & & $\frac{2.27}{2.47}$ & & & & $\begin{array}{l}-0.20 \\
-7.77\end{array}$ \\
\hline & $3-3$ & 1569.90 & & & & 2.16 & & & & -7.26 \\
\hline & $10-1$ & 1685.62 & & & & 1.58 & & & & -10.65 \\
\hline & $4-1$ & 1471.14 & & & & 0.96 & & & & -15.26 \\
\hline $95 \mathrm{C}, 7 \mathrm{~d}$ & $2-2$ & 1473.60 & 1562.08 & 224.96 & 14.40 & 1.14 & 1.51 & 0.56 & 37.24 & $\begin{array}{l}-12.98 \\
-12.98\end{array}$ \\
\hline & $8-3$ & 2041.15 & & & & 2.51 & & & & -8.14 \\
\hline & $6-2$ & 1361.06 & & & & 1.27 & & & & -10.73 \\
\hline & $7-2$ & 1332.08 & & & & 0.97 & & & & 13.80 \\
\hline & $19-1$ & 1819.40 & & & & 10.28 & & & & -1.77 \\
\hline & $19-2$ & 1907.09 & & & & 10.90 & & & & -1.75 \\
\hline & $20-4$ & 2016.40 & & & & $\begin{array}{l}10.90 \\
11.39\end{array}$ & & & & $\begin{array}{l}-1.15 \\
-1.77\end{array}$ \\
\hline UNAGED & $21-1$ & 1754.35 & 1944.09 & 110.40 & 5.68 & 9.00 & 10.38 & 1.02 & 9.85 & $\begin{array}{l}-1.11 \\
-1.95\end{array}$ \\
\hline & $23-1$ & 2011.14 & & & & 9.23 & & & & -2.18 \\
\hline & $24-3$ & 2040.34 & & & & 9.90 & & & & -2.06 \\
\hline & $26-3$ & 2059.88 & & & & 11.98 & & & & -1.72 \\
\hline
\end{tabular}


Table B.19. Results for LM1 Loose Mixture Aging

\begin{tabular}{|c|c|c|c|c|c|c|c|c|c|c|}
\hline & M1 & & Fracture & nergy & & & Flexibil & y Index & & \\
\hline Type & $\begin{array}{l}\text { Specimen } \\
\text { ID }\end{array}$ & $\begin{array}{c}\text { Energy } \\
\text { (LLD) (Gf) } \\
(\mathrm{J} / \mathrm{m} 2)\end{array}$ & Average & Std Dev & $\operatorname{CoV}(\%)$ & $\begin{array}{c}\text { Flexibility } \\
\text { Index }\end{array}$ & Average & Std Dev & $\operatorname{CoV}(\%)$ & Slope \\
\hline & $\begin{array}{l}\text { P1-1 } \\
\text { P1-2 }\end{array}$ & $\begin{array}{l}1743.81 \\
2290.92\end{array}$ & & & & $\begin{array}{l}5.43 \\
8.39\end{array}$ & & & & $\begin{array}{l}-3.21 \\
-2.73\end{array}$ \\
\hline & $\mathrm{P} 1-3$ & 2062.07 & & & & 5.65 & & & & $\begin{array}{l}-2.13 \\
-3.65\end{array}$ \\
\hline & P1-4 & 2070.91 & & & & 8.89 & & & & -2.33 \\
\hline $75 \mathrm{C}, 1 \mathrm{~d}$ & P2-1 & 2221.36 & 2167.01 & 193.73 & 8.94 & 9.14 & 8.48 & 1.82 & 21.51 & -2.43 \\
\hline & P2-2 & 2386.87 & & & & 10.16 & & & & -2.35 \\
\hline & P2-3 & 2345.99 & & & & 9.46 & & & & -2.48 \\
\hline & P2-4 & 2214.13 & & & & 10.70 & & & & -2.07 \\
\hline & P3-1 & 2040.56 & & & & 6.63 & & & & -3.08 \\
\hline & P3-2 & 2050.07 & & & & 5.68 & & & & -3.61 \\
\hline & P3-3 & 1985.72 & & & & 5.07 & & & & -3.92 \\
\hline $85 C, 1 d$ & P3-4 & 1936.35 & 2205.82 & 274.16 & 12.43 & 5.96 & 5.99 & 0.51 & 8.57 & -3.25 \\
\hline & P4-1 & 2557.30 & & & & 6.68 & & & & -3.83 \\
\hline & P4-3 & 2684.98 & & & & 6.06 & & & & -4.43 \\
\hline & P4-4 & 2185.79 & & & & 5.88 & & & & -3.72 \\
\hline & P5-1 & 1909.55 & & & & 3.91 & & & & -4.89 \\
\hline & P5-2 & 2143.23 & & & & 4.31 & & & & -4.97 \\
\hline & P5-3 & 1715.98 & & & & 3.51 & & & & -4.89 \\
\hline & P5-4 & 1517.01 & & & & 3.17 & & & & -4.79 \\
\hline $95 \mathrm{C}, 1 \mathrm{~d}$ & P6-1 & 1886.71 & 1934.89 & 241.61 & 12.49 & 3.71 & 3.88 & 0.44 & 11.33 & -5.08 \\
\hline & P6-2 & 2299.36 & & & & 4.36 & & & & -5.27 \\
\hline & P6-3 & 2170.66 & & & & 4.49 & & & & -4.83 \\
\hline & P6-4 & 1836.59 & & & & 3.60 & & & & -5.10 \\
\hline & P7-1 & 2141.80 & & & & 4.66 & & & & -4.60 \\
\hline & P7-2 & 1851.48 & & & & 3.92 & & & & -4.72 \\
\hline & P7-3 & 1743.18 & & & & 4.48 & & & & -3.89 \\
\hline $75 C, 3 d$ & P7-4 & 1758.57 & 1873.91 & 126.98 & 6.78 & 4.43 & 4.03 & 0.54 & 13.41 & -3.97 \\
\hline & P8-1 & 1825.09 & & & & 4.21 & & & & -4.33 \\
\hline & P8-2 & 1841.23 & & & & 3.24 & & & & -5.69 \\
\hline & P8-4 & 1956.05 & & & & 3.25 & & & & -6.02 \\
\hline & P9-1 & 1737.01 & & & & 1.99 & & & & -8.74 \\
\hline & P9-2 & 1774.29 & & & & 2.85 & & & & -6.23 \\
\hline & P9-4 & 1490.20 & & & & 1.97 & & & & -7.57 \\
\hline $85 C, 3 d$ & P10-1 & 1769.60 & 1674.31 & 92.96 & 5.55 & 2.70 & 2.28 & 0.33 & 14.68 & -6.56 \\
\hline & P10-2 & 1630.90 & & 年 & & 2.24 & . & & & -7.28 \\
\hline & P10-3 & 1634.21 & & & & 1.96 & & & & -8.34 \\
\hline & $\mathrm{P} 10-4$ & 1683.96 & & & & 2.26 & & & & -7.45 \\
\hline & P11-1 & 1124.84 & & & & 0.32 & & & & -34.75 \\
\hline & P11-3 & 981.87 & & & & 0.17 & & & & -57.19 \\
\hline & P11-4 & 1003.65 & & & & 0.03 & & & & -365.49 \\
\hline $95 C, 3 d$ & P12-1 & 1178.12 & 1140.09 & 104.60 & 9.18 & 0.12 & 0.19 & 0.10 & 54.37 & -97.98 \\
\hline & P12-2 & 1256.42 & & & & 0.11 & & & & -116.47 \\
\hline & $\mathrm{P} 12-3$ & 1270.95 & & & & 0.27 & & & & -47.13 \\
\hline & $\mathrm{P} 12-4$ & 1164.78 & & & & 0.29 & & & & -40.16 \\
\hline & P13-BL & 1897.92 & & & & 4.15 & & & & -4.57 \\
\hline & P13-BR & 1788.85 & & & & 4.87 & & & & -3.67 \\
\hline & P13-TL & 1774.87 & & & & 3.18 & & & & -5.58 \\
\hline & P13-TR & 2319.43 & & & & 5.56 & & & & -4.17 \\
\hline $75 C, 5 d$ & P14-BL & 1841.47 & 1917.26 & 182.65 & 9.53 & 4.38 & 4.16 & 0.75 & 17.92 & -4.20 \\
\hline & P14-BR & 1994.39 & & & & 4.20 & & & & -4.75 \\
\hline & P14-TL & 2018.51 & & & & 3.47 & & & & -5.81 \\
\hline & P14-TR & 1702.64 & & & & 3.46 & & & & -4.92 \\
\hline & P15-BL & 1467.45 & & & & 1.16 & & & & -12.65 \\
\hline & P15-BR & 1541.24 & & & & 0.78 & & & & -19.65 \\
\hline & P15-TL & 1114.57 & & & & 0.20 & & & & -56.95 \\
\hline & P15-TR & 2586.21 & & 42870 & 2784 & 1.06 & & & 4731 & -24.42 \\
\hline $85 C, 5 d$ & P16-BL & 1637.85 & 1540.16 & 428.79 & 27.84 & 1.56 & 0.89 & 0.42 & 47.31 & -10.53 \\
\hline & P16-BR & 1463.75 & & & & 0.56 & & & & -26.14 \\
\hline & P16-TL & 1333.72 & & & & 1.28 & & & & -10.38 \\
\hline & P16-TR & 1176.49 & & & & 0.54 & & & & -21.83 \\
\hline & P19-1 & 1959.99 & & & & 4.22 & & & & -4.64 \\
\hline & P19-2 & 1881.25 & & & & 3.87 & & & & -4.86 \\
\hline & P19-3 & 2076.56 & & & & 3.78 & & & & -5.50 \\
\hline $75 C, 7 d$ & P19-4 & 1748.65 & 1882.86 & 110.49 & 5.87 & 3.53 & 3.88 & 0.26 & 6.67 & -4.96 \\
\hline & P20-1 & 1922.80 & & & & 3.88 & & & & -4.95 \\
\hline & P20-3 & 1854.89 & & & & 3.64 & & & & -5.10 \\
\hline & P20-4 & 1735.90 & & & & 4.28 & & & & -4.06 \\
\hline & P21-1 & 1420.92 & & & & 0.76 & & & & -18.75 \\
\hline & $\begin{array}{l}\text { P21-2 } \\
\text { P21-3 }\end{array}$ & 1510.73 & & & & $\begin{array}{l}1.65 \\
1.12\end{array}$ & & & & -9.13 \\
\hline $85 C, 7 d$ & $\begin{array}{l}\text { P21-3 } \\
\text { P21-4 }\end{array}$ & $\frac{1372.86}{1820.72}$ & 1208.49 & 478.75 & 39.62 & $\frac{1.12}{2.58}$ & 1.11 & 0.83 & 74.70 & $\begin{array}{c}-12.30 \\
-7.07\end{array}$ \\
\hline & P22-2 & 603.06 & & & & 0.52 & & & & 11.52 \\
\hline & P22-4 & 522.66 & & & & 0.02 & & & & -302.03 \\
\hline & P23-1 & 996.70 & & & & 0.30 & & & & -33.16 \\
\hline & P23-2 & 968.69 & & & & 0.07 & & & & -141.76 \\
\hline & P23-3 & 1205.13 & & & & 1.06 & & & & -11.38 \\
\hline & P23-4 & 1128.16 & & & & 0.24 & & & & -46.56 \\
\hline $95 \mathrm{C}, / \mathrm{d}$ & P24-1 & 1359.30 & $121 / .5 /$ & $1 / 7.88$ & 14.61 & 0.96 & 0.67 & 0.42 & 62.13 & -14.13 \\
\hline & P24-2 & 1348.68 & & & & 0.47 & & & & -28.82 \\
\hline & P24-3 & 1205.13 & & & & 1.06 & & & & -11.38 \\
\hline & P24-4 & 1528.74 & & & & 1.19 & & & & -12.84 \\
\hline & $19-1$ & 1819.40 & & & & 10.28 & & & & -1.77 \\
\hline & $19-2$ & 1907.09 & & & & 10.90 & & & & -1.75 \\
\hline & $20-4$ & 2016.40 & & & & 11.39 & & & & -1.77 \\
\hline UNAGED & $21-1$ & 1754.35 & 1944.09 & 110.40 & 5.68 & 9.00 & 10.38 & 1.02 & 9.85 & -1.95 \\
\hline & $23-1$ & 2011.14 & & & & 9.23 & & & & -2.18 \\
\hline & 24-3 & 2040.34 & & & & 9.90 & & & & $\begin{array}{l}-2.06 \\
170\end{array}$ \\
\hline & $26-3$ & 2059.88 & & & & 11.98 & & & & -1.72 \\
\hline
\end{tabular}


Table B.20. Results for LM2 I-FIT Specimen Aging

\begin{tabular}{|c|c|c|c|c|c|c|c|c|c|c|c|c|c|}
\hline \multicolumn{2}{|c|}{ LM2 } & \multicolumn{4}{|c|}{ Fracture Energy } & \multicolumn{4}{|c|}{ Flexibility Index } & \multicolumn{4}{|c|}{ Slope } \\
\hline Type & $\begin{array}{l}\text { Specimen } \\
\text { ID }\end{array}$ & $\begin{array}{c}\text { Energy } \\
\text { (LLD) (Gf) } \\
(\mathrm{J} / \mathrm{m} 2)\end{array}$ & Average & Std Dev & $\operatorname{CoV}(\%)$ & $\begin{array}{l}\text { Flexibility } \\
\text { Index }\end{array}$ & Average & Std Dev & $\operatorname{CoV}(\%)$ & Slope & Average & Std Dev & $\operatorname{CoV}(\%)$ \\
\hline \multirow{7}{*}{ Unaged } & $16-3$ & 2321.38 & \multirow{7}{*}{2295.52} & \multirow{7}{*}{173.23} & \multirow{7}{*}{7.55} & 16.94 & \multirow{7}{*}{15.55} & \multirow{7}{*}{1.80} & \multirow{7}{*}{11.59} & -1.37 & \multirow{7}{*}{-1.49} & \multirow{7}{*}{0.13} & \multirow{7}{*}{8.79} \\
\hline & $17-1$ & 2239.22 & & & & 13.09 & & & & -1.71 & & & \\
\hline & $17-4$ & 2086.98 & & & & 13.91 & & & & -1.50 & & & \\
\hline & $18-1$ & 2663.68 & & & & 18.63 & & & & -1.43 & & & \\
\hline & $18-3$ & 2285.44 & & & & 16.32 & & & & -1.40 & & & \\
\hline & $19-2$ & 2136.01 & & & & 15.82 & & & & -1.35 & & & \\
\hline & $19-3$ & 2335.95 & & & & 14.16 & & & & -1.65 & & & \\
\hline \multirow{6}{*}{$1 \mathrm{D} 95 \mathrm{C}$} & $1-1$ & 1976.58 & \multirow{6}{*}{2301.03} & \multirow{6}{*}{219.56} & \multirow{6}{*}{9.54} & 5.81 & \multirow{6}{*}{7.37} & \multirow{6}{*}{1.46} & \multirow{6}{*}{19.78} & -3.40 & & \multirow{6}{*}{0.58} & \\
\hline & $9-2$ & 2262.56 & & & & 7.72 & & & & -2.93 & & & \\
\hline & $10-1$ & 2241.88 & & & & 7.70 & & & & -2.91 & & & \\
\hline & $11-3$ & 2327.10 & & & & 8.59 & & & & -2.71 & -3.22 & & 18.10 \\
\hline & $14-2$ & 2722.54 & & & & 9.26 & & & & -2.94 & & & \\
\hline & $15-2$ & 2275.54 & & & & 5.13 & & & & -4.44 & & & \\
\hline & $4-1$ & 1901.15 & & & & 6.25 & & & & -3.04 & & & \\
\hline & $16-1$ & 2013.35 & & & & 4.36 & & & & -4.62 & & & \\
\hline & $16-2$ & 2143.10 & & & & 4.46 & & & & -4.81 & & & \\
\hline $2 \mathrm{D} 95 \mathrm{C}$ & $16-4$ & 2053.61 & 2076.65 & 112.13 & 5.40 & 6.02 & & & & -3.41 & & & \\
\hline 2095 & $18-2$ & 2313.05 & $20 / 0.05$ & 112.13 & 5.40 & 7.39 & 5.49 & 0.95 & 17.21 & -3.13 & -3.88 & 0.61 & 15.15 \\
\hline & $18-4$ & 2082.27 & & & & 5.19 & & & & -4.01 & & & \\
\hline & $19-1$ & 2009.54 & & & & 5.18 & & & & -3.88 & & & \\
\hline & $19-4$ & 2097.13 & & & & 5.07 & & & & -4.14 & & & \\
\hline & $4-4$ & 2482.27 & & & & 6.73 & & & & -3.69 & & & \\
\hline & $6-4$ & 2473.30 & & & & 5.50 & & & & -4.50 & & & \\
\hline $3 \mathrm{D} 95 \mathrm{C}$ & $8-2$ & 2148.21 & & & & 6.59 & & & & -3.26 & & & \\
\hline $3095 \mathrm{C}$ & $9-3$ & 2032.01 & 2232.28 & $17 / .95$ & 7.97 & 3.82 & 5.27 & 1.10 & 20.89 & -5.32 & -4.38 & 0.70 & 15.96 \\
\hline & $11-4$ & 2107.96 & & & & 4.55 & & & & -4.63 & & & \\
\hline & $13-1$ & 2149.90 & & & & 4.41 & & & & -4.87 & & & \\
\hline & $2-1$ & 1837.21 & & & & 3.04 & & & & -6.04 & & & \\
\hline & $9-4$ & 1845.89 & & & & 2.37 & & & & -7.79 & & & \\
\hline $5 \mathrm{D} 95 \mathrm{C}$ & $12-3$ & 1723.07 & 1784.29 & 9259 & 5.19 & 2.37 & 2.37 & 0.55 & 23.22 & -7.26 & -7.92 & 167 & 21.05 \\
\hline $5 D 95 C$ & $13-2$ & 1878.40 & $1 / 84.29$ & 92.59 & 5.19 & 3.02 & 2.31 & 0.55 & 23.22 & -6.21 & -1.92 & 1.67 & 21.05 \\
\hline & $14-1$ & 1607.20 & & & & 1.64 & & & & -9.82 & & & \\
\hline & $15-1$ & 1813.99 & & & & 1.74 & & & & -10.40 & & & \\
\hline & $1-2$ & 2155.57 & & & & 4.93 & & & & -4.37 & & & \\
\hline & $2-3$ & 1934.42 & & & & 3.77 & & & & -5.13 & & & \\
\hline & $6-3$ & 1977.41 & & & & 3.99 & & & & -4.96 & & & \\
\hline $5085 C$ & $8-1$ & 2424.80 & 2163.96 & 169.69 & 1.84 & 6.20 & 5.12 & 1.11 & 21.68 & -3.91 & -4.35 & 0.61 & 13.91 \\
\hline & $10-2$ & 2202.28 & & & & 4.98 & & & & -4.42 & & & \\
\hline & $12-4$ & 2289.26 & & & & 6.87 & & & & -3.33 & & & \\
\hline
\end{tabular}


Table B.21. Results for LM3 I-FIT Specimen Aging

\begin{tabular}{|c|c|c|c|c|c|c|c|c|c|c|c|c|c|}
\hline \multicolumn{2}{|c|}{ LM3 } & \multicolumn{4}{|c|}{ Fracture Energy } & \multicolumn{4}{|c|}{ Flexibility Index } & \multicolumn{4}{|c|}{ Slope } \\
\hline Type & $\begin{array}{l}\text { Specimen } \\
\text { ID }\end{array}$ & $\begin{array}{c}\text { Energy } \\
\text { (LLD) (Gf) } \\
(\mathrm{J} / \mathrm{m} 2)\end{array}$ & Average & Std Dev & $\operatorname{CoV}(\%)$ & $\begin{array}{l}\text { Flexibility } \\
\text { Index }\end{array}$ & Average & Std Dev & $\operatorname{CoV}(\%)$ & Slope & Average & Std Dev & $\operatorname{CoV}(\%)$ \\
\hline \multirow{6}{*}{ Unaged } & $4-2$ & 2278.14 & \multirow{6}{*}{2179.59} & \multirow{6}{*}{88.48} & \multirow{6}{*}{4.06} & 17.00 & \multirow{6}{*}{19.35} & \multirow{6}{*}{4.03} & \multirow{6}{*}{20.83} & -1.34 & \multirow{6}{*}{-1.17} & \multirow{6}{*}{0.20} & \multirow{6}{*}{16.95} \\
\hline & $6-2$ & 2043.11 & & & & 16.34 & & & & -1.25 & & & \\
\hline & $9-2$ & 2187.35 & & & & 16.83 & & & & -1.30 & & & \\
\hline & $10-2$ & 2149.29 & & & & 24.15 & & & & -0.89 & & & \\
\hline & $13-1$ & 2298.76 & & & & 25.83 & & & & -0.89 & & & \\
\hline & $16-2$ & 2120.86 & & & & 15.95 & & & & -1.33 & & & \\
\hline \multirow{6}{*}{$1 \mathrm{D} 95 \mathrm{C}$} & $1-4$ & 2490.44 & \multirow{6}{*}{2031.78} & \multirow{6}{*}{229.70} & \multirow{6}{*}{11.31} & 15.37 & \multirow{6}{*}{11.61} & \multirow{6}{*}{2.81} & \multirow{6}{*}{24.25} & -1.62 & & \multirow{6}{*}{0.37} & \multirow{6}{*}{20.03} \\
\hline & $5-2$ & 1930.07 & & & & 8.14 & & & & -2.37 & & & \\
\hline & $9-3$ & 1868.11 & & & & 13.94 & & & & -1.34 & & & \\
\hline & $13-3$ & 2088.47 & & & & 10.77 & & & & -1.94 & -1.83 & & \\
\hline & $15-3$ & 1775.25 & & & & 8.11 & & & & -2.19 & & & \\
\hline & $16-1$ & 2038.35 & & & & 13.32 & & & & -1.53 & & & \\
\hline \multirow{6}{*}{$2 \mathrm{D} 95 \mathrm{C}$} & $4-1$ & 1469.16 & & & & 8.30 & & & & -1.77 & & & \\
\hline & $6-4$ & 2058.86 & & & & 8.65 & & & & -2.38 & & & \\
\hline & $7-3$ & 2127.96 & 187761 & 24277 & 1293 & 9.85 & 8.40 & 1.34 & 16.01 & -2.16 & -2.27 & 0.30 & 13.19 \\
\hline & $8-2$ & 2101.40 & 1817.61 & 242.11 & 12.93 & 10.10 & 8.40 & 1.34 & 10.01 & -2.08 & -2.21 & 0.30 & 13.19 \\
\hline & $11-4$ & 1827.29 & & & & 7.08 & & & & -2.58 & & & \\
\hline & $12-3$ & 1681.00 & & & & 6.39 & & & & -2.63 & & & \\
\hline & $1-1$ & 2392.40 & & & & 10.22 & & & & -2.34 & & & \\
\hline & $5-3$ & 1982.69 & & & & 6.93 & & & & -2.86 & & & \\
\hline $3095 \mathrm{C}$ & $8-1$ & 1999.68 & & & & 6.31 & & & & -3.17 & & & \\
\hline $3095 \mathrm{C}$ & $10-1$ & 1890.30 & 2102.20 & 184.67 & 8.78 & 8.63 & 8.90 & 1.77 & 19.94 & -2.19 & -2.44 & 0.43 & 17.60 \\
\hline & $11-3$ & 2316.15 & & & & 11.08 & & & & -2.09 & & & \\
\hline & $12-1$ & 2031.95 & & & & 10.21 & & & & -1.99 & & & \\
\hline & $2-4$ & 1964.26 & & & & 5.85 & & & & -3.36 & & & \\
\hline & $7-2$ & 1842.92 & & & & 6.25 & & & & -2.95 & & & \\
\hline & $9-4$ & 2075.87 & & 133.54 & 6.63 & 7.16 & & & & -2.90 & & & 17.31 \\
\hline $5095 C$ & $11-2$ & 2199.86 & 2013.24 & 133.54 & 6.63 & 4.73 & 5.93 & 0.88 & 14.16 & -4.65 & -3.41 & 0.60 & 17.31 \\
\hline & $13-2$ & 1863.36 & & & & 4.93 & & & & -3.78 & & & \\
\hline & $15-1$ & 2133.17 & & & & 6.67 & & & & -3.20 & & & \\
\hline & $1-3$ & 1991.61 & & & & 7.40 & & & & -2.69 & & & \\
\hline & $2-3$ & 1750.22 & & & & 6.43 & & & & -2.72 & & & \\
\hline $5 D 85 C$ & 5-1 & 1852.87 & 2049.39 & 242.06 & 11.81 & 7.24 & 7.26 & 0.52 & 7.12 & -2.56 & -2.82 & 0.22 & 7.65 \\
\hline & $14-3$ & 2261.57 & & & & 7.16 & & & & -3.16 & & & \\
\hline & $16-4$ & 2390.70 & & & & 8.05 & & & & -2.97 & & & \\
\hline
\end{tabular}


Table B.22. Results for LM4 I-FIT Specimen Aging

\begin{tabular}{|c|c|c|c|c|c|c|c|c|c|c|c|c|c|}
\hline \multicolumn{2}{|c|}{ LM4 } & \multicolumn{4}{|c|}{ Fracture Energy } & \multicolumn{4}{|c|}{ Flexibility Index } & \multicolumn{4}{|c|}{ Slope } \\
\hline Type & $\begin{array}{c}\text { Specimen } \\
\text { ID }\end{array}$ & $\begin{array}{c}\text { Energy } \\
\text { (LLD) (Gf) } \\
(\mathrm{J} / \mathrm{m} 2)\end{array}$ & Average & Std Dev & CoV (\%) & $\begin{array}{l}\text { Flexibility } \\
\text { Index }\end{array}$ & Average & Std Dev & $\operatorname{CoV}(\%)$ & Slope & Average & Std Dev & $\operatorname{CoV}(\%)$ \\
\hline \multirow{6}{*}{ Unaged } & $2-3$ & 1914.75 & \multirow{6}{*}{1960.75} & \multirow{6}{*}{140.39} & \multirow{6}{*}{7.16} & 13.21 & \multirow{6}{*}{17.18} & \multirow{6}{*}{2.40} & \multirow{6}{*}{13.99} & -1.45 & \multirow{6}{*}{-1.17} & \multirow{6}{*}{0.18} & \multirow{6}{*}{15.72} \\
\hline & 3-4 & 2028.13 & & & & 20.08 & & & & -1.01 & & & \\
\hline & $7-2$ & 1767.43 & & & & 19.42 & & & & -0.91 & & & \\
\hline & $9-3$ & 2221.69 & & & & 17.92 & & & & -1.24 & & & \\
\hline & $11-3$ & 1949.19 & & & & 14.99 & & & & -1.30 & & & \\
\hline & $13-1$ & 1883.31 & & & & 17.44 & & & & -1.08 & & & \\
\hline \multirow{7}{*}{ 1D95C } & $2-1$ & 1998.45 & \multirow{7}{*}{1952.88} & \multirow{7}{*}{73.13} & \multirow{7}{*}{3.74} & 8.96 & \multirow{7}{*}{10.99} & \multirow{7}{*}{1.31} & \multirow{7}{*}{11.96} & -2.23 & \multirow{7}{*}{-1.80} & \multirow{7}{*}{0.23} & \\
\hline & $2-4$ & 1870.36 & & & & 11.20 & & & & -1.67 & & & \\
\hline & $6-3$ & 1862.06 & & & & 10.07 & & & & -1.85 & & & \\
\hline & $8-2$ & 2067.91 & & & & 10.14 & & & & -2.04 & & & 12.98 \\
\hline & $9-4$ & 1910.81 & & & & 11.37 & & & & -1.68 & & & \\
\hline & $10-1$ & 1936.71 & & & & 11.88 & & & & -1.63 & & & \\
\hline & $13-3$ & 2023.85 & & & & 13.31 & & & & -1.52 & & & \\
\hline & $1-4$ & 1802.69 & & & & 6.70 & & & & -2.69 & & & \\
\hline & 3-3 & 1962.77 & & & & 8.08 & & & & -2.43 & & & \\
\hline $2 \mathrm{D} 95 \mathrm{C}$ & $4-3$ & 2029.33 & 193371 & 13250 & 685 & 10.25 & 782 & 149 & 1912 & -1.98 & -253 & 032 & \\
\hline 20950 & $4-4$ & 2151.26 & 1933.11 & 132.50 & 6.85 & 9.15 & 1.82 & 1.49 & 19.12 & -2.35 & -2.53 & 0.32 & 12.58 \\
\hline & $14-3$ & 1762.73 & & & & 6.10 & & & & -2.89 & & & \\
\hline & $15-1$ & 1893.45 & & & & 6.62 & & & & -2.86 & & & \\
\hline & $1-3$ & 1823.61 & & & & 5.84 & & & & -3.12 & & & \\
\hline & $8-3$ & 1769.71 & & & & 6.39 & & & & -2.77 & & & \\
\hline & $9-2$ & 2310.96 & & & & 7.94 & & & & -2.91 & & & \\
\hline $3095 \mathrm{C}$ & $10-4$ & 2234.30 & 1985.16 & 243.11 & 12.25 & 8.76 & 6.89 & 1.29 & 18.10 & -2.55 & -2.92 & 0.26 & $8.8 /$ \\
\hline & $12-3$ & 1669.06 & & & & 4.97 & & & & -3.36 & & & \\
\hline & $15-2$ & 2103.33 & & & & 7.46 & & & & -2.82 & & & \\
\hline & $6-1$ & 1609.89 & & & & 3.70 & & & & -4.35 & & & \\
\hline & $7-4$ & 1659.33 & & & & 3.54 & & & & -4.69 & & & \\
\hline $5 \mathrm{D} 95 \mathrm{C}$ & $8-4$ & 1771.96 & 176468 & 14930 & 846 & 5.52 & 438 & 0.72 & 1633 & -3.21 & -4.09 & 0.47 & 1140 \\
\hline 50950 & $12-2$ & 1958.73 & 1704.08 & 149.30 & 8.40 & 5.04 & 4.38 & 0.12 & 10.33 & -3.89 & $-4.0 y$ & 0.47 & 11.40 \\
\hline & $13-2$ & 1621.95 & & & & 3.99 & & & & -4.07 & & & \\
\hline & $16-2$ & 1966.19 & & & & 4.53 & & & & -4.34 & & & \\
\hline & $2-2$ & 1795.94 & & & & 5.00 & & & & -3.59 & & & \\
\hline & $7-1$ & 1599.90 & & & & 4.89 & & & & -3.27 & & & \\
\hline & $8-1$ & 2085.73 & & & & 7.12 & & & & -2.93 & & & \\
\hline $5085 \mathrm{C}$ & $9-1$ & 1367.12 & 1879.00 & 359.00 & 19.11 & 4.67 & 0.21 & 1.68 & 26.84 & -2.93 & -3.06 & 0.30 & 9.85 \\
\hline & $11-2$ & 2496.46 & & & & 9.46 & & & & -2.64 & & & \\
\hline & $16-1$ & 1928.87 & & & & 6.49 & & & & -2.97 & & & \\
\hline
\end{tabular}


Table B.23. Results for LM5 I-FIT Specimen Aging

\begin{tabular}{|c|c|c|c|c|c|c|c|c|c|c|c|c|c|}
\hline \multicolumn{2}{|c|}{ LM5 } & \multicolumn{4}{|c|}{ Fracture Energy } & \multicolumn{4}{|c|}{ Flexibility Index } & \multicolumn{4}{|c|}{ Slope } \\
\hline Type & $\begin{array}{l}\text { Specimen } \\
\text { ID }\end{array}$ & $\begin{array}{c}\text { Energy } \\
\text { (LLD) (Gf) } \\
(\mathrm{J} / \mathrm{m} 2)\end{array}$ & Average & Std Dev & $\operatorname{CoV}(\%)$ & $\begin{array}{l}\text { Flexibility } \\
\text { Index }\end{array}$ & Average & Std Dev & $\operatorname{CoV}(\%)$ & Slope & Average & Std Dev & $\operatorname{CoV}(\%)$ \\
\hline \multirow{5}{*}{ Unaged } & $2-1$ & 2131.25 & \multirow{5}{*}{2291.24} & \multirow{5}{*}{273.77} & \multirow{5}{*}{11.95} & 27.32 & \multirow{5}{*}{22.46} & \multirow{5}{*}{3.09} & \multirow{5}{*}{13.75} & -0.78 & \multirow{5}{*}{-1.04} & \multirow{5}{*}{0.17} & \multirow{5}{*}{16.76} \\
\hline & $3-3$ & 2792.05 & & & & 21.48 & & & & -1.30 & & & \\
\hline & $5-2$ & 2053.46 & & & & 18.01 & & & & -1.14 & & & \\
\hline & $6-1$ & 2104.63 & & & & 21.48 & & & & -0.98 & & & \\
\hline & $6-4$ & 2374.79 & & & & 23.99 & & & & -0.99 & & & \\
\hline \multirow{6}{*}{$1 \mathrm{D} 95 \mathrm{C}$} & $2-4$ & 1875.39 & \multirow{6}{*}{2289.19} & \multirow{6}{*}{311.83} & \multirow{6}{*}{13.62} & 8.26 & \multirow{6}{*}{15.30} & \multirow{6}{*}{4.29} & \multirow{6}{*}{28.03} & -2.27 & \multirow{6}{*}{-1.60} & \multirow{6}{*}{0.41} & \multirow{6}{*}{25.26} \\
\hline & $3-1$ & 2901.20 & & & & 19.74 & & & & -1.47 & & & \\
\hline & $5-3$ & 2357.30 & & & & 14.12 & & & & -1.67 & & & \\
\hline & $6-3$ & 2134.32 & & & & 19.95 & & & & -1.07 & & & \\
\hline & $7-1$ & 2196.07 & & & & 17.85 & & & & -1.23 & & & \\
\hline & $12-1$ & 2270.87 & & & & 11.89 & & & & -1.91 & & & \\
\hline \multirow{6}{*}{$2 \mathrm{D} 95 \mathrm{C}$} & $1-1$ & 2190.68 & \multirow{6}{*}{2169.32} & \multirow{6}{*}{171.40} & & 11.35 & & & & -1.93 & & & \\
\hline & $3-2$ & 2257.03 & & & & 7.92 & & & & -2.85 & & & \\
\hline & $5-1$ & 1884.79 & & & 790 & 13.27 & 1016 & 180 & 1774 & -1.42 & -2 २? & 0.49 & 22,27 \\
\hline & $6-2$ & 2012.57 & & & 1.90 & 10.11 & 10.16 & 1.80 & 17.74 & -1.99 & -2.22 & 0.49 & 22.21 \\
\hline & $7-4$ & 2396.30 & & & & 9.98 & & & & -2.40 & & & \\
\hline & $10-3$ & 2274.53 & & & & 8.33 & & & & -2.73 & & & \\
\hline & $1-3$ & 2202.62 & & & & 6.97 & & & & -3.16 & & & \\
\hline & $4-3$ & 2004.30 & & & & 6.96 & & & & -2.88 & & & \\
\hline & $7-3$ & 2072.31 & & & & 8.46 & & & & -2.45 & & & \\
\hline $3 D 95 C$ & $9-2$ & 2041.16 & 2084.50 & 102.37 & 4.91 & 7.09 & 7.59 & 0.81 & 10.10 & -2.88 & -2.11 & 0.24 & 8.82 \\
\hline & $9-3$ & 1951.35 & & & & 7.10 & & & & -2.75 & & & \\
\hline & $10-2$ & 2235.27 & & & & 8.98 & & & & -2.49 & & & \\
\hline & $3-4$ & 2613.87 & & & & 10.17 & & & & -2.57 & & & \\
\hline & $4-1$ & 2094.12 & & & & 6.00 & & & & -3.49 & & & \\
\hline & $5-4$ & 1953.07 & & & & 7.23 & & & & -2.70 & & & \\
\hline $5085 C$ & $9-1$ & 2035.29 & 2138.12 & 217.28 & 10.16 & 10.83 & 8.08 & 1.85 & 22.90 & -1.88 & -2.76 & 0.54 & 19.55 \\
\hline & $10-1$ & 2054.90 & & & & 8.03 & & & & -2.56 & & & \\
\hline & $11-2$ & 2081.07 & & & & 6.19 & & & & -3.36 & & & \\
\hline
\end{tabular}

Table B.24. Results for LM6 I-FIT Specimen Aging

\begin{tabular}{|c|c|c|c|c|c|c|c|c|c|c|c|c|c|}
\hline \multicolumn{2}{|c|}{ LM6 } & \multicolumn{4}{|c|}{ Fracture Energy } & \multicolumn{4}{|c|}{ Flexibility Index } & \multicolumn{4}{|c|}{ Slope } \\
\hline Type & $\begin{array}{l}\text { Specimen } \\
\text { ID }\end{array}$ & $\begin{array}{c}\text { Energy } \\
\text { (LLD) (Gf) } \\
(\mathrm{J} / \mathrm{m} 2)\end{array}$ & Average & Std Dev & $\operatorname{CoV}(\%)$ & $\begin{array}{l}\text { Flexibility } \\
\text { Index }\end{array}$ & Average & Std Dev & $\operatorname{CoV}(\%)$ & Slope & Average & Std Dev & $\operatorname{CoV}(\%)$ \\
\hline \multirow{6}{*}{ Unaged } & $1-4$ & 2271.86 & \multirow{6}{*}{2186.71} & \multirow{6}{*}{90.78} & \multirow{6}{*}{4.15} & 14.20 & \multirow{6}{*}{17.74} & \multirow{6}{*}{3.94} & \multirow{6}{*}{22.21} & -1.60 & \multirow{6}{*}{-1.29} & \multirow{6}{*}{0.26} & \multirow{6}{*}{19.99} \\
\hline & $6-4$ & 2080.09 & & & & 18.25 & & & & -1.14 & & & \\
\hline & $7-3$ & 2061.64 & & & & 12.89 & & & & -1.60 & & & \\
\hline & $9-4$ & 2219.63 & & & & 24.39 & & & & -0.91 & & & \\
\hline & $11-3$ & 2181.71 & & & & 15.92 & & & & -1.37 & & & \\
\hline & $12-4$ & 2305.34 & & & & 20.77 & & & & -1.11 & & & \\
\hline \multirow{6}{*}{$1 \mathrm{D} 95 \mathrm{C}$} & $1-1$ & 2010.71 & \multirow{6}{*}{2095.62} & \multirow{6}{*}{114.82} & \multirow{6}{*}{5.48} & 8.31 & \multirow{6}{*}{9.70} & \multirow{6}{*}{1.68} & \multirow{6}{*}{17.36} & -2.42 & \multirow{6}{*}{-2.20} & \multirow{6}{*}{0.25} & \multirow{6}{*}{11.46} \\
\hline & $2-1$ & 2118.56 & & & & 8.51 & & & & -2.49 & & & \\
\hline & $9-3$ & 2110.69 & & & & 9.91 & & & & -2.13 & & & \\
\hline & $10-2$ & 1902.14 & & & & 8.03 & & & & -2.37 & & & \\
\hline & $11-1$ & 2166.72 & & & & 10.57 & & & & -2.05 & & & \\
\hline & $11-2$ & 2264.89 & & & & 12.87 & & & & -1.76 & & & \\
\hline \multirow{6}{*}{$3 \mathrm{D} 95 \mathrm{C}$} & $1-2$ & 1905.17 & \multirow{6}{*}{2040.89} & \multirow{6}{*}{183.25} & \multirow{6}{*}{8.98} & 4.46 & \multirow{6}{*}{6.69} & \multirow{6}{*}{2.11} & & -4.27 & & & \\
\hline & $6-2$ & 2097.61 & & & & 6.79 & & & & -3.09 & & & \\
\hline & $7-2$ & 2060.30 & & & & 7.75 & & & & -2.66 & & & \\
\hline & $9-2$ & 2336.62 & & & & 10.20 & & & 31.52 & -2.29 & -3.30 & 0.82 & 24.84 \\
\hline & $10-1$ & 1743.75 & & & & 3.85 & & & & -4.53 & & & \\
\hline & $11-4$ & 2101.86 & & & & 7.08 & & & & -2.97 & & & \\
\hline & $6-1$ & 2113.56 & & & & 6.58 & & & & -3.21 & & & \\
\hline & $7-1$ & 2310.90 & & & & 6.30 & & & & -3.67 & & & \\
\hline $5 D 85 C$ & $9-1$ & 2297.06 & 2158.97 & 127.23 & 5.89 & 7.07 & 6.76 & 0.58 & 8.59 & -3.25 & -3.21 & 0.30 & 9.47 \\
\hline & $10-4$ & 2094.99 & & & & 7.73 & & & & -2.71 & & & \\
\hline & $12-3$ & 1978.32 & & & & 6.12 & & & & -3.23 & & & \\
\hline
\end{tabular}


Table B.25. Results for LM7 I-FIT Specimen Aging

\begin{tabular}{|c|c|c|c|c|c|c|c|c|c|c|c|c|c|}
\hline \multicolumn{2}{|c|}{ LM7 } & \multicolumn{4}{|c|}{ Fracture Energy } & \multicolumn{4}{|c|}{ Flexibility Index } & \multicolumn{4}{|c|}{ Slope } \\
\hline Type & $\begin{array}{l}\text { Specimen } \\
\text { ID }\end{array}$ & $\begin{array}{c}\text { Energy } \\
\text { (LLD) (Gf) } \\
(\mathrm{J} / \mathrm{m} 2)\end{array}$ & Average & Std Dev & $\operatorname{CoV}(\%)$ & $\begin{array}{l}\text { Flexibility } \\
\text { Index }\end{array}$ & Average & Std Dev & CoV (\%) & Slope & Average & Std Dev & $\operatorname{CoV}(\%)$ \\
\hline \multirow{6}{*}{ Unaged } & $3-4$ & 1839.74 & \multirow{6}{*}{1974.13} & \multirow{6}{*}{147.76} & \multirow{6}{*}{7.48} & 19.78 & \multirow{6}{*}{20.83} & \multirow{6}{*}{5.40} & \multirow{6}{*}{25.91} & -0.93 & \multirow{6}{*}{-1.00} & \multirow{6}{*}{0.24} & \multirow{6}{*}{23.58} \\
\hline & $4-1$ & 1993.52 & & & & 14.88 & & & & -1.34 & & & \\
\hline & $5-1$ & 2004.67 & & & & 16.85 & & & & -1.19 & & & \\
\hline & $6-1$ & 2058.64 & & & & 18.22 & & & & -1.13 & & & \\
\hline & $10-1$ & 1745.53 & & & & 24.24 & & & & -0.72 & & & \\
\hline & $11-3$ & 2202.65 & & & & 31.02 & & & & -0.71 & & & \\
\hline \multirow{6}{*}{$1 \mathrm{D} 95 \mathrm{C}$} & $1-1$ & 1868.29 & \multirow{6}{*}{1917.72} & \multirow{6}{*}{91.08} & \multirow{6}{*}{4.75} & 9.07 & \multirow{6}{*}{9.91} & \multirow{6}{*}{1.14} & \multirow{6}{*}{11.48} & -2.06 & \multirow{6}{*}{-1.95} & \multirow{6}{*}{0.15} & \multirow{6}{*}{7.76} \\
\hline & $2-4$ & 1917.68 & & & & 8.92 & & & & -2.15 & & & \\
\hline & $3-3$ & 1828.05 & & & & 9.37 & & & & -1.95 & & & \\
\hline & $6-2$ & 2029.37 & & & & 12.23 & & & & -1.66 & & & \\
\hline & $10-3$ & 2046.46 & & & & 10.39 & & & & -1.97 & & & \\
\hline & $11-1$ & 1816.47 & & & & 9.46 & & & & -1.92 & & & \\
\hline \multirow{6}{*}{$3 D 95 C$} & $1-3$ & 1776.77 & \multirow{6}{*}{1905.68} & \multirow{6}{*}{86.29} & \multirow{6}{*}{4.53} & 5.73 & \multirow{6}{*}{6.10} & & & -3.10 & & & \\
\hline & $2-1$ & 1832.14 & & & & 4.89 & & & & -3.75 & & & \\
\hline & $3-2$ & 2050.34 & & & & 8.33 & & & & -2.46 & & & \\
\hline & $4-3$ & 1944.00 & & & & 5.45 & & 1.32 & 21.62 & -3.57 & -3.25 & 0.57 & 17.65 \\
\hline & 6-3 & 1909.64 & & & & 4.79 & & & & -3.99 & & & \\
\hline & $11-2$ & 1921.16 & & & & 7.39 & & & & -2.60 & & & \\
\hline & $1-4$ & 2145.78 & & & & 6.66 & & & & -3.22 & & & \\
\hline & $3-1$ & 1729.22 & & & & 5.42 & & & & -3.19 & & & \\
\hline & $4-4$ & 2172.97 & 108294 & 15938 & 803 & 5.50 & & & 1266 & -3.95 & 338 & 014 & 1205 \\
\hline $5 D 85 C$ & $5-3$ & 1902.20 & 1983.94 & 159.38 & 8.03 & 4.86 & 5.96 & 0.15 & 12.66 & -3.91 & -3.38 & 0.44 & 13.05 \\
\hline & $6-4$ & 2073.71 & & & & 6.28 & & & & -3.30 & & & \\
\hline & $11-4$ & 1879.73 & & & & 7.01 & & & & -2.68 & & & \\
\hline
\end{tabular}




\section{APPENDIX C: R27-175 MIXES FIELD CORES I-FIT RESULTS}

\section{Table C.1. Results for PM1 Field Cores}

\begin{tabular}{|c|c|c|c|c|c|c|c|c|c|c|c|c|}
\hline \multirow{2}{*}{$\begin{array}{l}\text { M1 Cores } \\
\text { Specimen } \\
\text { ID }\end{array}$} & \multicolumn{2}{|c|}{ Fracture Energy } & \multicolumn{2}{|c|}{ Flexibility Index } & \multicolumn{2}{|c|}{ Slope } & \multicolumn{2}{|c|}{ Air Voids } & \multicolumn{4}{|c|}{ Corrected Flexibility Index } \\
\hline & $\begin{array}{c}\text { Energy } \\
\text { (LLD) (Gf) } \\
(\mathrm{J} / \mathrm{m} 2)\end{array}$ & Average & $\begin{array}{l}\text { Flexibility } \\
\text { Index }\end{array}$ & Average & Slope & Average & Air Voids & Average & $\begin{array}{l}\text { Flexibility } \\
\text { Index }\end{array}$ & Average & Std Dev & CoV $(\%)$ \\
\hline A1-1 & 1902.63 & \multirow{8}{*}{2093.84} & 5.66 & \multirow{8}{*}{6.21} & -3.36 & \multirow{8}{*}{-3.44} & 5.2 & \multirow{8}{*}{5.31} & 7.50 & \multirow{8}{*}{8.05} & \multirow{8}{*}{1.74} & \multirow{8}{*}{21.64} \\
\hline A1-2 & 2190.55 & & 6.35 & & -3.45 & & 5.5 & & 8.01 & & & \\
\hline A2-1 & 2263.61 & & 7.86 & & -2.88 & & 5.2 & & 10.45 & & & \\
\hline $\mathrm{A} 2-2$ & 1938.72 & & 4.98 & & -3.89 & & 5.4 & & 6.39 & & & \\
\hline A3-1 & 1991.51 & & 6.17 & & -3.23 & & 5.3 & & 8.05 & & & \\
\hline A3-2 & 1675.73 & & 4.02 & & -4.17 & & 5.3 & & 5.25 & & & \\
\hline A4-1 & 2741.90 & & 8.44 & & -3.25 & & 5.4 & & 10.81 & & & \\
\hline A4-2 & 2046.05 & & 6.24 & & -3.28 & & 5.4 & & 7.94 & & & \\
\hline B1-1 & 1959.18 & \multirow{8}{*}{2092.32} & 5.68 & \multirow{8}{*}{6.30} & -3.45 & \multirow{8}{*}{-3.38} & 6.1 & \multirow{8}{*}{5.83} & 6.42 & \multirow{8}{*}{7.49} & \multirow{8}{*}{1.24} & \multirow{8}{*}{16.62} \\
\hline B1-2 & 2237.61 & & 6.08 & & -3.68 & & 5.8 & & 7.23 & & & \\
\hline B2-1 & 1897.61 & & 5.10 & & -3.72 & & 6.0 & & 5.90 & & & \\
\hline B2-2 & 2251.14 & & 7.17 & & -3.14 & & 5.8 & & 8.55 & & & \\
\hline B3-1 & 2165.62 & & 8.02 & & -2.70 & & 5.8 & & 9.56 & & & \\
\hline B3-2 & 2023.68 & & 6.81 & & -2.97 & & 5.8 & & 8.18 & & & \\
\hline B4-1 & 2164.27 & & 6.52 & & -3.32 & & 5.6 & & 8.10 & & & \\
\hline B4-2 & 2039.46 & & 5.00 & & -4.08 & & 5.8 & & 5.94 & & & \\
\hline
\end{tabular}

Table C.2. Results for PM2 Field Cores

\begin{tabular}{|c|c|c|c|c|c|c|c|c|c|c|c|c|c|c|}
\hline \multirow{2}{*}{\begin{tabular}{|l} 
Mix2 Cores \\
$\begin{array}{c}\text { Specimen } \\
\text { ID }\end{array}$
\end{tabular}} & \multicolumn{2}{|c|}{ Fracture Energy } & \multicolumn{2}{|c|}{ Flexibility Index } & \multicolumn{2}{|c|}{ Slope } & \multicolumn{2}{|c|}{ Air voids } & \multicolumn{2}{|c|}{ Dimensions } & \multicolumn{4}{|c|}{ Corrected Flexibility Index } \\
\hline & $\begin{array}{c}\text { Energy } \\
\text { (LLD) (Gf) } \\
(\mathrm{J} / \mathrm{m} 2)\end{array}$ & Average & $\begin{array}{l}\text { Flexibility } \\
\text { Index }\end{array}$ & Average & Slope & Average & Air Voids & Average & $\begin{array}{l}\text { Avg. } \\
\text { Ligament } \\
(\mathrm{mm})\end{array}$ & $\begin{array}{l}\text { Avg. } \\
\text { Thickness } \\
(\mathrm{mm})\end{array}$ & $\begin{array}{l}\text { Flexibility } \\
\text { Index }\end{array}$ & Average & Std Dev & $\operatorname{CoV}(\%)$ \\
\hline M2 A1-1 & 3025.55 & \multirow{7}{*}{3008.96} & 16.27 & \multirow{7}{*}{13.49} & -1.86 & \multirow{7}{*}{-2.29} & 4.2 & \multirow{7}{*}{4.09} & 58.8 & 36.0 & 18.85 & \multirow{7}{*}{16.31} & \multirow{7}{*}{3.08} & \multirow{7}{*}{18.90} \\
\hline M2 A1-2 & 3369.60 & & 16.85 & & -2.00 & & 4.0 & & 59.1 & 35.6 & 20.55 & & & \\
\hline M2 A2-1 & 2821.01 & & 10.69 & & -2.64 & & 4.0 & & 57.9 & 36.4 & 13.29 & & & \\
\hline M2 A2-2 & 2781.51 & & 12.15 & & -2.29 & & 4.1 & & 58.9 & 35.9 & 14.52 & & & \\
\hline M2 A3-2 & 2959.11 & & 10.64 & & -2.78 & & 4.2 & & 58.9 & 37.7 & 12.86 & & & \\
\hline M2 A4-1 & 2785.43 & & 11.70 & & -2.38 & & 4.2 & & 57.8 & 37.1 & 14.14 & & & \\
\hline M2 A4-2 & 3320.51 & & 16.12 & & -2.06 & & 4.0 & & 59.1 & 36.5 & 19.94 & & & \\
\hline M2 B1-1 & 2307.50 & \multirow{8}{*}{2721.71} & 13.49 & \multirow{8}{*}{13.31} & -1.71 & \multirow{8}{*}{-2.08} & 5.1 & \multirow{8}{*}{4.73} & 59.3 & 34.1 & 12.48 & \multirow{8}{*}{13.66} & \multirow{8}{*}{2.64} & \multirow{8}{*}{19.29} \\
\hline M2 B1-2 & 2779.35 & & 12.46 & & -2.23 & & 4.8 & & 58.9 & 34.8 & 12.39 & & & \\
\hline M2 B2-1 & 2783.00 & & 14.35 & & -1.94 & & 4.4 & & 57.7 & 36.9 & 16.22 & & & \\
\hline M2 B2-2 & 2988.05 & & 14.79 & & -2.02 & & 4.8 & & 58.4 & 37.3 & 15.83 & & & \\
\hline M2 B3-1 & 2653.88 & & 11.20 & & -2.37 & & 4.7 & & 57.6 & 35.7 & 11.73 & & & \\
\hline M2 B3-2 & 3032.43 & & 17.53 & & -1.73 & & 4.6 & & 58.7 & 35.4 & 18.53 & & & \\
\hline M2 B4-1 & 2746.46 & & 12.15 & & -2.26 & & 4.9 & & 59.1 & 34.1 & 11.48 & & & \\
\hline M2 B4-2 & 2482.99 & & 10.52 & & -2.36 & & 4.6 & & 59.1 & 34.0 & 10.65 & & & \\
\hline
\end{tabular}


Table C.3. Results for PM3 Field Cores

\begin{tabular}{|c|c|c|c|c|c|c|c|c|c|c|c|c|c|c|}
\hline \multirow{2}{*}{\begin{tabular}{|l} 
M3 Cores \\
$\begin{array}{c}\text { Specimen } \\
\text { ID }\end{array}$
\end{tabular}} & \multicolumn{2}{|c|}{ Fracture Energy } & \multicolumn{2}{|c|}{ Flexibility Index } & \multicolumn{2}{|c|}{ Slope } & \multicolumn{2}{|c|}{ Air voids } & \multicolumn{2}{|c|}{ Dimensions } & \multicolumn{4}{|c|}{ Corrected Flexibility Index } \\
\hline & $\begin{array}{c}\text { Energy } \\
\text { (LLD) (Gf) } \\
(\mathrm{J} / \mathrm{m} 2)\end{array}$ & Average & $\begin{array}{l}\text { Flexibility } \\
\text { Index }\end{array}$ & Average & Slope & Average & Air Voids & Average & $\begin{array}{l}\text { Avg. } \\
\text { Ligament } \\
(\mathrm{mm})\end{array}$ & $\begin{array}{l}\text { Avg. } \\
\text { Thickness } \\
\text { (mm) }\end{array}$ & $\begin{array}{l}\text { Flexibility } \\
\text { Index }\end{array}$ & Average & Std Dev & $\operatorname{CoV}(\%)$ \\
\hline M3 A1-1 & 2189.73 & \multirow{8}{*}{2312.23} & 9.56 & \multirow{8}{*}{12.10} & -2.29 & \multirow{8}{*}{-1.96} & 5.5 & \multirow{8}{*}{5.20} & 58.3 & 33.9 & 8.14 & \multirow{8}{*}{10.29} & \multirow{8}{*}{2.31} & \multirow{8}{*}{22.42} \\
\hline M3 A1-2 & 2096.43 & & 11.84 & & -1.77 & & 5.9 & & 58.2 & 32.6 & 9.14 & & & \\
\hline M3 A2-1 & 2308.61 & & 10.45 & & -2.21 & & 5.0 & & 59.5 & 32.3 & 9.19 & & & \\
\hline M3 A2-2 & 2278.68 & & 11.63 & & -1.96 & & 4.9 & & 58.2 & 30.7 & 10.03 & & & \\
\hline M3 A3-1 & 2564.19 & & 17.33 & & -1.48 & & 4.9 & & 57.8 & 32.2 & 15.72 & & & \\
\hline M3 A3-2 & 2436.76 & & 10.41 & & -2.34 & & 5.1 & & 59.2 & 31.0 & 8.64 & & & \\
\hline M3 A4-1 & 2218.03 & & 11.26 & & -1.97 & & 5.1 & & 58.5 & 31.3 & 9.57 & & & \\
\hline M3 A4-2 & 2405.42 & & 14.32 & & -1.68 & & 5.3 & & 58.6 & 31.9 & 11.89 & & & \\
\hline M3 B1-1 & 2580.76 & \multirow{8}{*}{2597.76} & 15.55 & \multirow{8}{*}{14.74} & -1.66 & \multirow{8}{*}{-1.79} & 5.1 & \multirow{8}{*}{5.27} & 58.5 & 32.4 & 13.47 & \multirow{8}{*}{12.61} & \multirow{8}{*}{1.76} & \multirow{8}{*}{13.99} \\
\hline M3 B1-2 & 2680.51 & & 16.25 & & -1.65 & & 5.4 & & 58.7 & 31.2 & 13.01 & & & \\
\hline M3 B2-1 & 2486.59 & & 15.07 & & -1.65 & & 5.3 & & 58.6 & 32.6 & 12.73 & & & \\
\hline M3 B2-2 & 2523.84 & & 14.93 & & -1.69 & & 5.5 & & 58.7 & 31.4 & 11.74 & & & \\
\hline M3 B3-1 & 2910.48 & & 14.70 & & -1.98 & & 5.3 & & 58.6 & 33.8 & 12.84 & & & \\
\hline M3 B3-2 & 2454.69 & & 12.72 & & -1.93 & & 5.1 & & 58.6 & 35.3 & 12.15 & & & \\
\hline M3 B4-1 & 2856.45 & & 17.97 & & -1.59 & & 5.2 & & 58.8 & 33.2 & 15.87 & & & \\
\hline M3 B4-2 & 2288.79 & & 10.75 & & -2.13 & & 5.3 & & 58.9 & 32.9 & 9.10 & & & \\
\hline
\end{tabular}

Table C.4. Results for PM5 Field Cores

\begin{tabular}{|c|c|c|c|c|c|c|c|c|c|c|c|c|c|c|}
\hline \multirow{2}{*}{\begin{tabular}{|c} 
M5 Cores \\
Specimen \\
ID
\end{tabular}} & \multicolumn{2}{|c|}{ Fracture Energy } & \multicolumn{2}{|c|}{ Flexibility Index } & \multicolumn{2}{|c|}{ Slope } & \multicolumn{2}{|c|}{ Air voids } & \multicolumn{2}{|c|}{ Dimensions } & \multicolumn{4}{|c|}{ Corrected Flexibility Index } \\
\hline & $\begin{array}{c}\text { Energy } \\
\text { (LLD) (Gf) } \\
(\mathrm{J} / \mathrm{m} 2)\end{array}$ & Average & $\begin{array}{l}\text { Flexibility } \\
\text { Index }\end{array}$ & Average & Slope & Average & Air Voids & Average & $\begin{array}{l}\text { Avg. } \\
\text { Ligament } \\
(\mathrm{mm})\end{array}$ & $\begin{array}{l}\text { Avg. } \\
\text { Thickness } \\
(\mathrm{mm})\end{array}$ & $\begin{array}{l}\text { Flexibility } \\
\text { Index }\end{array}$ & Average & Std Dev & $\operatorname{CoV}(\%)$ \\
\hline M5 A1-1 & 1957.66 & \multirow{8}{*}{2063.10} & 18.30 & \multirow{8}{*}{21.40} & -1.07 & \multirow{8}{*}{-0.99} & 6.5 & \multirow{8}{*}{6.82} & 58.7 & 49.6 & 19.35 & \multirow{8}{*}{21.32} & \multirow{8}{*}{3.33} & \multirow{8}{*}{15.61} \\
\hline M5 A1-2 & 2046.91 & & 17.65 & & -1.16 & & 6.5 & & 58.1 & 51.5 & 19.43 & & & \\
\hline M5 A2-1 & 2050.61 & & 28.09 & & -0.73 & & 6.6 & & 58.3 & 47.2 & 27.87 & & & \\
\hline M5 A2-2 & 2181.60 & & 18.03 & & -1.21 & & 6.5 & & 58.7 & 46.9 & 18.20 & & & \\
\hline M5 A3-1 & 2153.80 & & 21.12 & & -1.02 & & 6.6 & & 58.9 & 49.1 & 21.85 & & & \\
\hline M5 A3-2 & 2191.80 & & 26.09 & & -0.84 & & 6.8 & & 56.9 & 47.7 & 25.51 & & & \\
\hline M5 A4-1 & 1932.47 & & 22.21 & & -0.87 & & 7.7 & & 56.4 & 49.2 & 20.08 & & & \\
\hline M5 A4-2 & 1989.98 & & 19.70 & & -1.01 & & 7.3 & & 57.8 & 48.4 & 18.29 & & & \\
\hline M5 B1-1 & 2135.82 & \multirow{8}{*}{2020.13} & 19.59 & \multirow{8}{*}{17.43} & -1.09 & \multirow{8}{*}{-1.21} & 5.4 & \multirow{8}{*}{5.58} & 58.6 & 48.0 & 23.95 & \multirow{8}{*}{20.78} & \multirow{8}{*}{4.40} & \multirow{8}{*}{21.16} \\
\hline M5 B1-2 & 1881.03 & & 11.76 & & -1.60 & & 5.3 & & 59.0 & 47.9 & 14.49 & & & \\
\hline M5 B2-1 & 2116.61 & & 19.97 & & -1.06 & & 5.5 & & 58.0 & 47.1 & 23.66 & & & \\
\hline M5 B2-2 & 1992.87 & & 12.38 & & -1.61 & & 5.3 & & 58.8 & 47.3 & 15.24 & & & \\
\hline M5 B3-1 & 1739.71 & & 13.81 & & -1.26 & & 5.8 & & 58.6 & 49.8 & 16.30 & & & \\
\hline M5 B3-2 & 2209.52 & & 18.88 & & -1.17 & & 5.4 & & 58.9 & 51.4 & 24.83 & & & \\
\hline M5 B4-1 & 2103.76 & & 19.48 & & -1.08 & & 6.0 & & 56.2 & 48.1 & 21.58 & & & \\
\hline M5 B4-2 & 1981.75 & & 23.59 & & -0.84 & & 5.9 & & 55.6 & 47.4 & 26.18 & & & \\
\hline
\end{tabular}

Table C.5. Results for PM6 Field Cores

\begin{tabular}{|c|c|c|c|c|c|c|c|c|c|c|c|c|c|c|}
\hline \multirow{2}{*}{$\begin{array}{l}\text { M6 Cores } \\
\text { Specimen } \\
\text { ID }\end{array}$} & \multicolumn{2}{|c|}{ Fracture Energy } & \multicolumn{2}{|c|}{ Flexibility Index } & \multicolumn{2}{|c|}{ Slope } & \multicolumn{2}{|c|}{ Air voids } & \multicolumn{2}{|c|}{ Dimensions } & \multicolumn{4}{|c|}{ Corrected Flexibility Index } \\
\hline & $\begin{array}{c}\text { Energy } \\
\text { (LLD) (Gf) } \\
(\mathrm{J} / \mathrm{m} 2)\end{array}$ & Average & $\begin{array}{l}\text { Flexibility } \\
\text { Index }\end{array}$ & Average & Slope & Average & Air Voids & Average & $\begin{array}{c}\text { Avg. } \\
\text { Ligament } \\
(\mathrm{mm})\end{array}$ & $\begin{array}{l}\text { Avg. } \\
\text { Thickness } \\
(\mathrm{mm})\end{array}$ & $\begin{array}{l}\text { Flexibility } \\
\text { Index }\end{array}$ & Average & Std Dev & $\operatorname{CoV}(\%)$ \\
\hline M6 A1-1 & 2131.69 & \multirow{6}{*}{2131.63} & 20.11 & \multirow{6}{*}{20.67} & -1.06 & \multirow{6}{*}{-1.06} & 4.8 & \multirow{6}{*}{5.10} & 58.3 & 34.5 & 19.81 & \multirow{6}{*}{19.18} & \multirow{6}{*}{3.99} & \multirow{6}{*}{20.83} \\
\hline M6 A1-2 & 2141.96 & & 18.15 & & -1.18 & & 4.7 & & 58.8 & 35.6 & 18.79 & & & \\
\hline M6 A3-1 & 2469.44 & & 30.12 & & -0.82 & & 5.1 & & 59.1 & 33.7 & 27.06 & & & \\
\hline M6 A3-2 & 2022.84 & & 19.64 & & -1.03 & & 4.9 & & 59.1 & 32.7 & 18.07 & & & \\
\hline M6 A4-1 & 1929.50 & & 19.89 & & -0.97 & & 5.5 & & 57.7 & 35.1 & 17.62 & & & \\
\hline M6 A4-2 & 2094.35 & & 16.11 & & -1.30 & & 5.6 & & 58.8 & 34.9 & 13.75 & & & \\
\hline M6 B1-1 & 1870.99 & \multirow{8}{*}{2045.52} & 17.65 & \multirow{8}{*}{16.84} & -1.06 & \multirow{8}{*}{-1.26} & 4.7 & \multirow{8}{*}{4.74} & 59.1 & 34.1 & 17.56 & \multirow{8}{*}{17.56} & \multirow{8}{*}{3.66} & \multirow{8}{*}{20.87} \\
\hline M6 B1-2 & 2405.90 & & 23.59 & & -1.02 & & 4.4 & & 58.8 & 34.1 & 24.73 & & & \\
\hline M6 B2-1 & 1856.35 & & 12.54 & & -1.48 & & 4.7 & & 57.7 & 37.9 & 13.72 & & & \\
\hline M6 B2-2 & 1957.51 & & 13.98 & & -1.40 & & 4.7 & & 57.8 & 35.1 & 14.31 & & & \\
\hline M6 B3-1 & 1935.52 & & 17.60 & & -1.10 & & 5.0 & & 59.2 & 37.9 & 18.26 & & & \\
\hline M6 B3-2 & 2014.99 & & 13.71 & & -1.47 & & 4.6 & & 58.6 & 38.0 & 15.37 & & & \\
\hline M6 B4-1 & 2233.29 & & 21.47 & & -1.04 & & 4.8 & & 58.1 & 35.4 & 21.75 & & & \\
\hline M6 B4-2 & 2089.58 & & 14.21 & & -1.47 & & 5.0 & & 58.5 & 37.9 & 14.78 & & & \\
\hline
\end{tabular}


Table C.6. Results for PM7 Field Cores

\begin{tabular}{|c|c|c|c|c|c|c|c|c|c|c|c|c|c|c|}
\hline \multirow{2}{*}{\begin{tabular}{|l} 
M7 cores \\
$\begin{array}{c}\text { Specimen } \\
\text { ID }\end{array}$
\end{tabular}} & \multicolumn{2}{|c|}{ Fracture Energy } & \multicolumn{2}{|c|}{ Flexibility Index } & \multicolumn{2}{|c|}{ Slope } & \multicolumn{2}{|c|}{ Air voids } & \multicolumn{2}{|c|}{ Dimensions } & \multicolumn{4}{|c|}{ Corrected Flexibility Index } \\
\hline & $\begin{array}{c}\text { Energy } \\
\text { (LLD) (Gf) } \\
(\mathrm{J} / \mathrm{m} 2)\end{array}$ & Average & $\begin{array}{l}\text { Flexibility } \\
\text { Index }\end{array}$ & Average & Slope & Average & Air Voids & Average & $\begin{array}{l}\text { Avg. } \\
\text { Ligament } \\
(\mathrm{mm})\end{array}$ & $\begin{array}{l}\text { Avg. } \\
\text { Thickness } \\
(\mathrm{mm})\end{array}$ & $\begin{array}{l}\text { Flexibility } \\
\text { Index }\end{array}$ & Average & Std Dev & $\operatorname{CoV}(\%)$ \\
\hline M7 1-1 & 2493.51 & \multirow{8}{*}{2546.82} & 26.81 & \multirow{8}{*}{24.99} & -0.93 & \multirow{8}{*}{-1.05} & 4.4 & \multirow{8}{*}{4.75} & 59.2 & 34.1 & 28.08 & \multirow{8}{*}{25.77} & \multirow{8}{*}{4.93} & \multirow{8}{*}{19.15} \\
\hline M7 1-2 & 2418.52 & & 21.99 & & -1.10 & & 4.9 & & 58.9 & 33.4 & 20.62 & & & \\
\hline M7 2-1 & 2354.27 & & 29.80 & & -0.79 & & 5.0 & & 58.1 & 35.6 & 28.86 & & & \\
\hline M7 2-2 & 2796.83 & & 33.70 & & -0.83 & & 4.8 & & 58.2 & 36.3 & 34.60 & & & \\
\hline M7 3-1 & 2875.29 & & 24.58 & & -1.17 & & 4.6 & & 54.9 & 36.0 & 26.21 & & & \\
\hline M7 3-2 & 2757.60 & & 26.02 & & -1.06 & & 4.8 & & 58.6 & 35.4 & 26.13 & & & \\
\hline M7 4-1 & 1960.76 & & 15.44 & & -1.27 & & 4.7 & & 58.4 & 38.2 & 17.22 & & & \\
\hline M7 4-2 & 2717.80 & & 21.57 & & -1.26 & & 4.7 & & 58.8 & 39.0 & 24.43 & & & \\
\hline M7 5-1 & 2305.25 & \multirow{8}{*}{2513.01} & 23.77 & \multirow{8}{*}{21.61} & -0.97 & \multirow{8}{*}{-1.18} & 4.8 & \multirow{8}{*}{4.59} & 58.6 & 32.3 & 21.95 & \multirow{8}{*}{22.57} & \multirow{8}{*}{3.62} & \multirow{8}{*}{16.02} \\
\hline M7 5-2 & 3044.93 & & 26.48 & & -1.15 & & 4.5 & & 58.8 & 33.4 & 26.68 & & & \\
\hline M7 6-1 & 2135.29 & & 15.94 & & -1.34 & & 4.8 & & 58.4 & 35.8 & 16.10 & & & \\
\hline M7 6-2 & 2402.67 & & 20.36 & & -1.18 & & 4.7 & & 58.8 & 36.3 & 21.48 & & & \\
\hline M7 7-1 & 2714.31 & & 26.87 & & -1.01 & & 4.4 & & 58.2 & 34.7 & 28.77 & & & \\
\hline M7 7-2 & 2456.54 & & 21.00 & & -1.17 & & 4.5 & & 59.2 & 34.4 & 21.84 & & & \\
\hline M7 8-1 & 2649.26 & & 18.66 & & -1.42 & & 4.6 & & 58.7 & 36.7 & 20.42 & & & \\
\hline M7 8-2 & 2395.85 & & 19.80 & & -1.21 & & 4.4 & & 58.9 & 37.9 & 23.30 & & & \\
\hline
\end{tabular}

Table C.7. Results for PM12 Field Cores

\begin{tabular}{|c|c|c|c|c|c|c|c|c|c|c|c|c|c|c|}
\hline \multirow{2}{*}{$\begin{array}{l}\text { M12 Cores } \\
\text { Specimen } \\
\text { ID }\end{array}$} & \multicolumn{2}{|c|}{ Fracture Energy } & \multicolumn{2}{|c|}{ Flexibility Index } & \multicolumn{2}{|c|}{ Slope } & \multicolumn{2}{|c|}{ Air voids } & \multicolumn{2}{|c|}{ Dimensions } & \multicolumn{4}{|c|}{ Corrected Flexibility Index } \\
\hline & $\begin{array}{c}\text { Energy } \\
\text { (LLD) (Gf) } \\
(\mathrm{J} / \mathrm{m} 2)\end{array}$ & Average & $\begin{array}{l}\text { Flexibility } \\
\text { Index }\end{array}$ & Average & Slope & Average & Air Voids & Average & $\begin{array}{l}\text { Avg. } \\
\text { Ligament } \\
(\mathrm{mm})\end{array}$ & $\begin{array}{l}\text { Avg. } \\
\text { Thickness } \\
(\mathrm{mm})\end{array}$ & $\begin{array}{l}\text { Flexibility } \\
\text { Index }\end{array}$ & Average & Std Dev & $\operatorname{CoV}(\%)$ \\
\hline M12 1-1 & 2897.50 & \multirow{6}{*}{2271.03} & 23.18 & \multirow{6}{*}{16.21} & -1.25 & \multirow{6}{*}{-1.43} & 5.9 & \multirow{6}{*}{6.59} & 58.7 & 49.5 & 26.86 & \multirow{6}{*}{17.00} & \multirow{6}{*}{4.56} & \multirow{6}{*}{26.82} \\
\hline M12 1-2 & 2161.56 & & 13.77 & & -1.57 & & 6.5 & & 59.2 & 49.6 & 14.54 & & & \\
\hline M12 2-1 & 2054.20 & & 12.53 & & -1.64 & & 6.4 & & 59.0 & 48.3 & 13.22 & & & \\
\hline M12 3-2 & 2182.31 & & 17.46 & & -1.25 & & 7.2 & & 59.7 & 50.5 & 17.10 & & & \\
\hline M12 4-1 & 2379.48 & & 14.60 & & -1.63 & & 6.6 & & 59.4 & 48.4 & 14.91 & & & \\
\hline $\mathrm{M} 124-2$ & 1951.12 & & 15.73 & & -1.24 & & 6.9 & & 59.3 & 48.2 & 15.33 & & & \\
\hline M12 5-1 & 1985.60 & \multirow{8}{*}{2331.22} & 9.59 & \multirow{8}{*}{15.63} & -2.07 & \multirow{8}{*}{-1.56} & 6.4 & \multirow{8}{*}{6.44} & 59.5 & 49.6 & 10.40 & \multirow{8}{*}{16.75} & \multirow{8}{*}{3.76} & \multirow{8}{*}{22.45} \\
\hline M12 5-2 & 2459.62 & & 11.71 & & -2.10 & & 6.6 & & 59.0 & 49.7 & 12.24 & & & \\
\hline M12 6-1 & 2376.69 & & 14.76 & & -1.61 & & 5.9 & & 59.1 & 48.9 & 16.82 & & & \\
\hline M12 6-2 & 2337.57 & & 19.48 & & -1.20 & & 6.0 & & 58.7 & 49.2 & 22.03 & & & \\
\hline M12 7-1 & 2356.01 & & 14.82 & & -1.59 & & 6.6 & & 59.0 & 49.5 & 15.55 & & & \\
\hline M12 7-2 & 2328.43 & & 16.28 & & -1.43 & & 6.6 & & 58.7 & 48.9 & 16.74 & & & \\
\hline M12 8-1 & 2277.03 & & 18.66 & & -1.22 & & 6.7 & & 59.8 & 50.2 & 19.55 & & & \\
\hline M12 8-2 & 2528.81 & & 19.76 & & -1.28 & & 6.7 & & 59.4 & 50.0 & 20.67 & & & \\
\hline
\end{tabular}

Table C.8. Results for PM13 Field Cores

\begin{tabular}{|c|c|c|c|c|c|c|c|c|c|c|c|c|c|c|}
\hline \multirow{2}{*}{\begin{tabular}{|c} 
M13 Cores \\
$\begin{array}{c}\text { Specimen } \\
\text { ID }\end{array}$
\end{tabular}} & \multicolumn{2}{|c|}{ Fracture Energy } & \multicolumn{2}{|c|}{ Flexibility Index } & \multicolumn{2}{|c|}{ Slope } & \multicolumn{2}{|c|}{ Air voids } & \multicolumn{2}{|c|}{ Dimensions } & \multicolumn{4}{|c|}{ Corrected Flexibility Index } \\
\hline & $\begin{array}{c}\text { Energy } \\
\text { (LLD) (Gf) } \\
(\mathrm{J} / \mathrm{m} 2)\end{array}$ & Average & $\begin{array}{l}\text { Flexibility } \\
\text { Index }\end{array}$ & Average & Slope & Average & Air Voids & Average & $\begin{array}{l}\text { Avg. } \\
\text { Ligament } \\
(\mathrm{mm})\end{array}$ & $\begin{array}{l}\text { Avg. } \\
\text { Thickness } \\
(\mathrm{mm})\end{array}$ & $\begin{array}{l}\text { Flexibility } \\
\text { Index }\end{array}$ & Average & Std Dev & $\operatorname{CoV}(\%)$ \\
\hline M13 1-1 & 2283.76 & \multirow{6}{*}{2303.43} & 24.56 & \multirow{6}{*}{29.87} & -0.93 & \multirow{6}{*}{-0.78} & 4.2 & \multirow{6}{*}{4.30} & 58.8 & 35.8 & 28.47 & \multirow{6}{*}{33.77} & \multirow{6}{*}{4.35} & \multirow{6}{*}{12.88} \\
\hline M13 1-2 & 2551.60 & & 31.50 & & -0.81 & & 4.5 & & 59.1 & 34.9 & 33.28 & & & \\
\hline M13 2-1 & 2633.89 & & 32.52 & & -0.81 & & 4.5 & & 58.3 & 37.1 & 36.90 & & & \\
\hline M13 2-2 & 2699.51 & & 30.68 & & -0.88 & & 3.8 & & 59.2 & 36.5 & 39.85 & & & \\
\hline M13 4-1 & 1544.89 & & 30.29 & & -0.51 & & 5.0 & & 58.9 & 33.7 & 28.03 & & & \\
\hline M13 4-2 & 2106.90 & & 29.67 & & -0.71 & & 3.8 & & 58.7 & 34.4 & 36.08 & & & \\
\hline M13 5-1 & 2261.42 & \multirow{5}{*}{2193.47} & 33.26 & \multirow{5}{*}{25.10} & -0.68 & \multirow{5}{*}{-0.90} & 5.0 & \multirow{5}{*}{4.98} & 59.0 & 34.8 & 31.54 & \multirow{5}{*}{24.29} & \multirow{5}{*}{5.34} & \multirow{5}{*}{21.99} \\
\hline M13 5-2 & 1722.91 & & 18.53 & & -0.93 & & 4.6 & & 59.4 & 34.3 & 18.65 & & & \\
\hline M13 6-1 & 2561.11 & & 23.94 & & -1.07 & & 4.9 & & 58.8 & 36.0 & 24.30 & & & \\
\hline M13 6-2 & 2076.71 & & 28.06 & & -0.74 & & 4.7 & & 59.2 & 35.4 & 28.81 & & & \\
\hline M13 8-1 & 2345.22 & & 21.72 & & -1.08 & & 5.7 & & 59.2 & 34.2 & 18.14 & & & \\
\hline
\end{tabular}


Table C.9. Six Months Field Cores Results for PM1

\begin{tabular}{|c|c|c|c|c|c|c|c|c|c|c|c|c|c|c|}
\hline \multirow{2}{*}{$\begin{array}{l}\text { M1 6months } \\
\text { Specimen ID }\end{array}$} & \multicolumn{2}{|c|}{ Fracture Energy } & \multicolumn{2}{|c|}{ Flexibility Index } & \multicolumn{2}{|c|}{ Slope } & \multicolumn{2}{|c|}{ Air voids } & \multicolumn{2}{|c|}{ Dimensions } & \multicolumn{4}{|c|}{ Corrected Flexibility Index } \\
\hline & $\begin{array}{c}\text { Energy } \\
\text { (LLD) (Gf) } \\
(\mathrm{J} / \mathrm{m} 2)\end{array}$ & Average & $\begin{array}{l}\text { Flexibility } \\
\text { Index }\end{array}$ & Average & Slope & Average & Air Voids & Average & $\begin{array}{l}\text { Avg. } \\
\text { Ligament } \\
(\mathrm{mm})\end{array}$ & $\begin{array}{c}\text { Avg. } \\
\text { Thickness } \\
(\mathrm{mm})\end{array}$ & $\begin{array}{l}\text { Flexibility } \\
\text { Index }\end{array}$ & Average & Std Dev & $\operatorname{CoV}(\%)$ \\
\hline M1-6 C1-1 & 2151.00 & \multirow{8}{*}{1911.06} & 5.30 & \multirow{8}{*}{3.70} & -4.06 & \multirow{8}{*}{-5.33} & 5.9 & \multirow{8}{*}{5.75} & 56.8 & 50.4 & 6.25 & \multirow{8}{*}{4.42} & \multirow{8}{*}{0.86} & \multirow{8}{*}{19.38} \\
\hline M1-6 C1-2 & 1679.67 & & 3.54 & & -4.74 & & 5.6 & & 55.8 & 50.1 & 4.39 & & & \\
\hline M1-6 C2-1 & 2129.90 & & 3.11 & & -6.85 & & 5.6 & & 59.5 & 49.5 & 3.80 & & & \\
\hline M1-6 C2-2 & 1819.85 & & 2.61 & & -6.96 & & 5.5 & & 55.8 & 49.9 & 3.25 & & & \\
\hline M1-6 C3-1 & 1761.67 & & 3.88 & & -4.54 & & 6.0 & & 59.2 & 49.4 & 4.39 & & & \\
\hline M1-6 C3-2 & 1849.30 & & 4.14 & & -4.47 & & 5.6 & & 56.8 & 49.4 & 4.99 & & & \\
\hline M1-6 C4-1 & 1575.47 & & 3.22 & & -4.90 & & 6.0 & & 59.2 & 50.5 & 3.76 & & & \\
\hline M1-6 C4-2 & 2321.58 & & 3.78 & & -6.14 & & 5.8 & & 57.6 & 50.1 & 4.53 & & & \\
\hline M1-6 D1-1 & 1372.47 & \multirow{8}{*}{1505.31} & 2.72 & \multirow{8}{*}{2.62} & -5.05 & \multirow{8}{*}{-6.15} & 5.3 & \multirow{8}{*}{5.25} & 56.8 & 49.3 & 3.45 & \multirow{8}{*}{3.47} & \multirow{8}{*}{1.00} & \multirow{8}{*}{28.72} \\
\hline M1-6 D1-2 & 1467.74 & & 2.45 & & -5.99 & & 5.4 & & 56.7 & 49.2 & 3.10 & & & \\
\hline M1-6 D2-1 & 1754.46 & & 3.93 & & -4.46 & & 5.1 & & 59.2 & 51.0 & 5.37 & & & \\
\hline M1-6 D2-2 & 1243.84 & & 2.91 & & -4.28 & & 5.3 & & 59.0 & 50.5 & 3.84 & & & \\
\hline M1-6 D3-1 & 1364.83 & & 1.93 & & -7.09 & & 5.3 & & 58.6 & 51.4 & 2.56 & & & \\
\hline M1-6 D3-2 & 1435.82 & & 2.83 & & -5.08 & & 5.2 & & 54.3 & 51.0 & 3.83 & & & \\
\hline M1-6 D4-1 & 1314.58 & & 1.33 & & -9.87 & & 5.3 & & 54.1 & 50.6 & 1.75 & & & \\
\hline M1-6 D4-2 & 2088.76 & & 2.84 & & -7.36 & & 5.1 & & 59.1 & 50.8 & 3.84 & & & \\
\hline
\end{tabular}

Table C.10. Six Months Field Cores Results for PM2

\begin{tabular}{|c|c|c|c|c|c|c|c|c|c|c|c|c|c|c|}
\hline \multirow{2}{*}{$\begin{array}{l}\text { M2 6months } \\
\text { Specimen ID }\end{array}$} & \multicolumn{2}{|c|}{ Fracture Energy } & \multicolumn{2}{|c|}{ Flexibility Index } & \multicolumn{2}{|c|}{ Slope } & \multicolumn{2}{|c|}{ Air voids } & \multicolumn{2}{|c|}{ Dimensions } & \multicolumn{4}{|c|}{ Corrected Flexibility Index } \\
\hline & $\begin{array}{c}\text { Energy } \\
\text { (LLD) (Gf) } \\
(\mathrm{J} / \mathrm{m} 2)\end{array}$ & Average & $\begin{array}{l}\text { Flexibility } \\
\text { Index }\end{array}$ & Average & Slope & Average & Air Voids & Average & $\begin{array}{l}\text { Avg. } \\
\text { Ligament } \\
(\mathrm{mm})\end{array}$ & $\begin{array}{l}\text { Avg. } \\
\text { Thickness } \\
(\mathrm{mm})\end{array}$ & $\begin{array}{l}\text { Flexibility } \\
\text { Index }\end{array}$ & Average & Std Dev & CoV (\%) \\
\hline M2 FC-6 A2-1 & 2867.20 & \multirow{8}{*}{2603.67} & 16.77 & \multirow{8}{*}{12.93} & -1.71 & \multirow{8}{*}{-2.19} & 3.8 & \multirow{8}{*}{4.10} & 59.5 & 36.3 & 21.53 & \multirow{8}{*}{15.90} & \multirow{8}{*}{6.23} & \multirow{8}{*}{39.20} \\
\hline M2 FC-6 A2-2 & 2628.02 & & 10.35 & & -2.54 & & 3.9 & & 57.1 & 35.5 & 12.82 & & & \\
\hline M2 FC-6 A4-1 & 1981.08 & & 6.29 & & -3.15 & & 4.1 & & 59.4 & 36.8 & 7.70 & & & \\
\hline M2 FC-6 A4-2 & 2652.88 & & 15.51 & & -1.71 & & 3.7 & & 59.3 & 37.3 & 21.20 & & & \\
\hline M2 FC-6 B2-1 & 3275.10 & & 21.27 & & -1.54 & & 4.0 & & 59.0 & 36.9 & 26.52 & & & \\
\hline M2 FC-6 B2-2 & 2901.57 & & 14.65 & & -1.98 & & 4.5 & & 59.5 & 36.8 & 16.49 & & & \\
\hline M2 FC-6 B4-1 & 2108.48 & & 8.71 & & -2.42 & & 4.6 & & 59.4 & 36.1 & 9.43 & & & \\
\hline M2 FC-6 B4-2 & 2415.05 & & 9.86 & & -2.45 & & 4.3 & & 58.6 & 37.3 & 11.51 & & & \\
\hline
\end{tabular}

Table C.11. Six Months Field Cores Results for PM3

\begin{tabular}{|c|c|c|c|c|c|c|c|c|c|c|c|c|c|c|}
\hline \multirow{2}{*}{$\begin{array}{l}\text { M3 6months } \\
\text { Specimen ID }\end{array}$} & \multicolumn{2}{|c|}{ Fracture Energy } & \multicolumn{2}{|c|}{ Flexibility Index } & \multicolumn{2}{|c|}{ Slope } & \multicolumn{2}{|c|}{ Air voids } & \multicolumn{2}{|c|}{ Dimensions } & \multicolumn{4}{|c|}{ Corrected Flexibility Index } \\
\hline & $\begin{array}{c}\text { Energy } \\
\text { (LLD) (Gf) } \\
(\mathrm{J} / \mathrm{m} 2)\end{array}$ & Average & $\begin{array}{l}\text { Flexibility } \\
\text { Index }\end{array}$ & Average & Slope & Average & Air Voids & Average & $\begin{array}{c}\text { Avg. } \\
\text { Ligament } \\
(\mathrm{mm})\end{array}$ & $\begin{array}{l}\text { Avg. } \\
\text { Thickness } \\
(\mathrm{mm})\end{array}$ & $\begin{array}{l}\text { Flexibility } \\
\text { Index }\end{array}$ & Average & Std Dev & $\operatorname{CoV}(\%)$ \\
\hline M3 FC-6 A2-1 & 2290.51 & \multirow{8}{*}{2327.72} & 20.27 & \multirow{8}{*}{17.09} & -1.13 & \multirow{8}{*}{-1.44} & 5.2 & \multirow{8}{*}{5.08} & 59.2 & 32.0 & 17.11 & \multirow{8}{*}{15.28} & \multirow{8}{*}{5.19} & \multirow{8}{*}{33.95} \\
\hline M3 FC-6 A2-2 & 1975.20 & & 10.29 & & -1.92 & & 5.0 & & 59.0 & 31.6 & 8.98 & & & \\
\hline M3 FC-6 A4-1 & 2160.02 & & 17.42 & & -1.24 & & 5.2 & & 58.0 & 32.0 & 14.79 & & & \\
\hline M3 FC-6 A4-2 & 1847.17 & & 15.39 & & -1.20 & & 5.9 & & 59.5 & 33.0 & 11.97 & & & \\
\hline M3 FC-6 B2-1 & 2552.40 & & 14.67 & & -1.74 & & 5.0 & & 59.0 & 33.1 & 13.43 & & & \\
\hline M3 FC-6 B2-2 & 2437.04 & & 13.24 & & -1.84 & & 4.7 & & 58.7 & 32.5 & 12.41 & & & \\
\hline M3 FC-6 B4-1 & 2341.80 & & 16.97 & & -1.38 & & 4.9 & & 59.4 & 33.8 & 16.06 & & & \\
\hline M3 FC-6 B4-2 & 3017.62 & & 28.47 & & -1.06 & & 4.8 & & 58.9 & 34.0 & 27.47 & & & \\
\hline
\end{tabular}


Table C.12. Six Months Field Cores Results for PM5

\begin{tabular}{|c|c|c|c|c|c|c|c|c|c|c|c|c|c|c|}
\hline \multirow{2}{*}{$\begin{array}{l}\text { M5 6months } \\
\text { Specimen ID }\end{array}$} & \multicolumn{2}{|c|}{ Fracture Energy } & \multicolumn{2}{|c|}{ Flexibility Index } & \multicolumn{2}{|c|}{ Slope } & \multicolumn{2}{|c|}{ Air voids } & \multicolumn{2}{|c|}{ Dimensions } & \multicolumn{4}{|c|}{ Corrected Flexibility Index } \\
\hline & $\begin{array}{c}\text { Energy } \\
\text { (LLD) (Gf) } \\
(\mathrm{J} / \mathrm{m} 2)\end{array}$ & Average & $\begin{array}{l}\text { Flexibility } \\
\text { Index }\end{array}$ & Average & Slope & Average & Air Voids & Average & $\begin{array}{l}\text { Avg. } \\
\text { Ligament } \\
(\mathrm{mm})\end{array}$ & $\begin{array}{l}\text { Avg. } \\
\text { Thickness } \\
(\mathrm{mm})\end{array}$ & $\begin{array}{l}\text { Flexibility } \\
\text { Index }\end{array}$ & Average & Std Dev & $\operatorname{CoV}(\%)$ \\
\hline $2 A-1$ & 2128.45 & \multirow{8}{*}{2284.65} & 18.84 & \multirow{8}{*}{23.57} & -1.13 & \multirow{8}{*}{-1.02} & 4.2 & \multirow{8}{*}{4.57} & 60.2 & 34.1 & 20.73 & \multirow{8}{*}{24.32} & \multirow{8}{*}{6.23} & \multirow{8}{*}{25.63} \\
\hline $2 A-2$ & 2351.63 & & 29.40 & & -0.80 & & 4.6 & & 57.6 & 33.7 & 29.19 & & & \\
\hline 2B-1 & 2166.97 & & 18.36 & & -1.18 & & 4.6 & & 59.0 & 36.0 & 19.68 & & & \\
\hline $2 B-2$ & 2304.47 & & 28.81 & & -0.80 & & 4.3 & & 57.6 & 37.6 & 34.02 & & & \\
\hline $4 \mathrm{~A}-1$ & 2540.61 & & 28.87 & & -0.88 & & 4.6 & & 59.5 & 32.7 & 27.83 & & & \\
\hline $4 A-2$ & 2529.97 & & 30.12 & & -0.84 & & 4.7 & & 58.2 & 33.8 & 29.70 & & & \\
\hline 4B-1 & 2098.10 & & 17.20 & & -1.22 & & 4.5 & & 59.2 & 33.7 & 17.66 & & & \\
\hline $4 B-2$ & 2157.01 & & 16.98 & & -1.27 & & 5.1 & & 58.1 & 34.2 & 15.77 & & & \\
\hline
\end{tabular}

Table C.13. Six Months Field Cores Results for PM6

\begin{tabular}{|c|c|c|c|c|c|c|c|c|c|c|c|c|c|c|}
\hline \multirow{2}{*}{$\begin{array}{l}\text { M6 6months } \\
\text { Specimen ID }\end{array}$} & \multicolumn{2}{|c|}{ Fracture Energy } & \multicolumn{2}{|c|}{ Flexibility Index } & \multicolumn{2}{|c|}{ Slope } & \multicolumn{2}{|c|}{ Air voids } & \multicolumn{2}{|c|}{ Dimensions } & \multicolumn{4}{|c|}{ Corrected Flexibility Index } \\
\hline & $\begin{array}{c}\text { Energy } \\
\text { (LLD) (Gf) } \\
(\mathrm{J} / \mathrm{m} 2)\end{array}$ & Average & $\begin{array}{l}\text { Flexibility } \\
\text { Index }\end{array}$ & Average & Slope & Average & Air Voids & Average & $\begin{array}{c}\text { Avg. } \\
\text { Ligament } \\
(\mathrm{mm})\end{array}$ & $\begin{array}{l}\text { Avg. } \\
\text { Thickness } \\
(\mathrm{mm})\end{array}$ & $\begin{array}{l}\text { Flexibility } \\
\text { Index }\end{array}$ & Average & Std Dev & $\operatorname{CoV}(\%)$ \\
\hline $2 A-1$ & 1948.86 & \multirow{8}{*}{1638.39} & 34.19 & \multirow{8}{*}{22.23} & -0.57 & \multirow{8}{*}{-0.78} & 6.7 & \multirow{8}{*}{6.05} & 60.2 & 34.1 & 24.37 & \multirow{8}{*}{17.77} & \multirow{8}{*}{4.97} & \multirow{8}{*}{27.98} \\
\hline $2 A-2$ & 1692.25 & & 19.68 & & -0.86 & & 5.7 & & 57.6 & 33.7 & 16.17 & & & \\
\hline 2B-1 & 1191.06 & & 19.53 & & -0.61 & & 7.5 & & 59.0 & 36.0 & 13.22 & & & \\
\hline 2B-2 & 1168.67 & & 21.64 & & -0.54 & & 8.0 & & 57.6 & 37.6 & 14.35 & & & \\
\hline $4 \mathrm{~A}-1$ & 1785.05 & & 15.26 & & -1.17 & & 5.2 & & 59.5 & 32.7 & 13.24 & & & \\
\hline $4 \mathrm{~A}-2$ & 1549.19 & & 14.34 & & -1.08 & & 5.0 & & 58.2 & 33.8 & 13.28 & & & \\
\hline 4B-1 & 1933.84 & & 24.48 & & -0.79 & & 5.2 & & 59.2 & 33.7 & 21.98 & & & \\
\hline $4 B-2$ & 1838.16 & & 28.72 & & -0.64 & & 5.3 & & 58.1 & 34.2 & 25.58 & & & \\
\hline
\end{tabular}

\section{Table C.14. Six Months Field Cores Results for PM7}

\begin{tabular}{|c|c|c|c|c|c|c|c|c|c|c|c|c|c|c|}
\hline \multirow{2}{*}{$\begin{array}{l}\text { M7 6months } \\
\text { Specimen ID }\end{array}$} & \multicolumn{2}{|c|}{ Fracture Energy } & \multicolumn{2}{|c|}{ Flexibility Index } & \multicolumn{2}{|c|}{ Slope } & \multicolumn{2}{|c|}{ Air voids } & \multicolumn{2}{|c|}{ Dimensions } & \multicolumn{4}{|c|}{ Corrected Flexibility Index } \\
\hline & $\begin{array}{c}\text { Energy } \\
(\mathrm{LLD})(\mathrm{Gf}) \\
(\mathrm{J} / \mathrm{m} 2)\end{array}$ & Average & $\begin{array}{l}\text { Flexibility } \\
\text { Index }\end{array}$ & Average & Slope & Average & Air Voids & Average & $\begin{array}{c}\text { Avg. } \\
\text { Ligament } \\
(\mathrm{mm})\end{array}$ & $\begin{array}{l}\text { Avg. } \\
\text { Thickness } \\
(\mathrm{mm})\end{array}$ & $\begin{array}{l}\text { Flexibility } \\
\text { Index }\end{array}$ & Average & Std Dev & $\operatorname{CoV}(\%)$ \\
\hline $1 \mathrm{~A}-1$ & 2638.50 & \multirow{8}{*}{2483.31} & 28.37 & \multirow{8}{*}{29.05} & -0.93 & \multirow{8}{*}{-0.87} & 6.7 & \multirow{8}{*}{6.05} & 60.2 & 34.1 & 20.22 & \multirow{8}{*}{23.53} & \multirow{8}{*}{4.53} & \multirow{8}{*}{19.27} \\
\hline $1 \mathrm{~A}-2$ & 2335.36 & & 28.83 & & -0.81 & & 5.7 & & 57.6 & 33.7 & 23.70 & & & \\
\hline 1B-1 & 2488.10 & & 27.04 & & -0.92 & & 7.5 & & 59.0 & 36.0 & 18.31 & & & \\
\hline $1 \mathrm{~B}-2$ & 2598.20 & & 27.94 & & -0.93 & & 8.0 & & 57.6 & 37.6 & 18.52 & & & \\
\hline $4 A-1$ & 2618.24 & & 36.88 & & -0.71 & & 5.2 & & 59.5 & 32.7 & 32.01 & & & \\
\hline $4 A-2$ & 2335.03 & & 30.72 & & -0.76 & & 5.0 & & 58.2 & 33.8 & 28.44 & & & \\
\hline $4 \mathrm{~B}-1$ & 2880.55 & & 24.41 & & -1.18 & & 5.2 & & 59.2 & 33.7 & 21.92 & & & \\
\hline 4B-2 & 1972.46 & & 28.18 & & -0.70 & & 5.3 & & 58.1 & 34.2 & 25.09 & & & \\
\hline
\end{tabular}

\section{Table C.15. Six Months Field Cores Results for PM12}

\begin{tabular}{|c|c|c|c|c|c|c|c|c|c|c|c|c|c|c|}
\hline \multirow{2}{*}{$\begin{array}{l}\text { M12 6months } \\
\text { Specimen ID }\end{array}$} & \multicolumn{2}{|c|}{ Fracture Energy } & \multicolumn{2}{|c|}{ Flexibility Index } & \multicolumn{2}{|c|}{ Slope } & \multicolumn{2}{|c|}{ Air voids } & \multicolumn{2}{|c|}{ Dimensions } & \multicolumn{4}{|c|}{ Corrected Flexibility Index } \\
\hline & $\begin{array}{l}\text { Energy } \\
\text { (LLD) (Gf) } \\
(\mathrm{J} / \mathrm{m} 2)\end{array}$ & Average & $\begin{array}{l}\text { Flexibility } \\
\text { Index }\end{array}$ & Average & Slope & Average & Air Voids & Average & $\begin{array}{c}\text { Avg. } \\
\text { Ligament } \\
(\mathrm{mm})\end{array}$ & $\begin{array}{l}\text { Avg. } \\
\text { Thickness } \\
(\mathrm{mm})\end{array}$ & $\begin{array}{l}\text { Flexibility } \\
\text { Index }\end{array}$ & Average & Std Dev & $\operatorname{CoV}(\%)$ \\
\hline $2 A-1$ & 2698.12 & \multirow{8}{*}{2515.58} & 15.97 & \multirow{8}{*}{14.04} & -1.69 & \multirow{8}{*}{-1.83} & 6.7 & \multirow{8}{*}{6.05} & 60.2 & 34.1 & 11.38 & \multirow{8}{*}{11.28} & \multirow{8}{*}{2.43} & \multirow{8}{*}{21.57} \\
\hline $2 A-2$ & 2767.78 & & 19.36 & & -1.43 & & 5.7 & & 57.6 & 33.7 & 15.91 & & & \\
\hline $2 B-1$ & 1933.07 & & 10.28 & & -1.88 & & 7.5 & & 59.0 & 36.0 & 6.96 & & & \\
\hline $2 \mathrm{~B}-2$ & 2930.97 & & 16.19 & & -1.81 & & 8.0 & & 57.6 & 37.6 & 10.74 & & & \\
\hline $4 A-1$ & 2552.84 & & 12.27 & & -2.08 & & 5.2 & & 59.5 & 32.7 & 10.65 & & & \\
\hline $4 A-2$ & 2281.94 & & 12.82 & & -1.78 & & 5.0 & & 58.2 & 33.8 & 11.87 & & & \\
\hline $4 \mathrm{~B}-1$ & 2468.10 & & 10.68 & & -2.31 & & 5.2 & & 59.2 & 33.7 & 9.59 & & & \\
\hline $4 \mathrm{~B}-2$ & 2491.82 & & 14.74 & & -1.69 & & 5.3 & & 58.1 & 34.2 & 13.13 & & & \\
\hline
\end{tabular}


Table C.16. Six Months Field Cores Results for PM13

\begin{tabular}{|c|c|c|c|c|c|c|c|c|c|c|c|c|c|c|}
\hline \multirow{2}{*}{$\begin{array}{l}\text { M13 6months } \\
\text { Specimen ID }\end{array}$} & \multicolumn{2}{|c|}{ Fracture Energy } & \multicolumn{2}{|c|}{ Flexibility Index } & \multicolumn{2}{|c|}{ Slope } & \multicolumn{2}{|c|}{ Air voids } & \multicolumn{2}{|c|}{ Dimensions } & \multicolumn{4}{|c|}{ Corrected Flexibility Index } \\
\hline & $\begin{array}{c}\text { Energy } \\
\text { (LLD) (Gf) } \\
(\mathrm{J} / \mathrm{m} 2)\end{array}$ & Average & $\begin{array}{l}\text { Flexibility } \\
\text { Index }\end{array}$ & Average & Slope & Average & Air Voids & Average & $\begin{array}{l}\text { Avg. } \\
\text { Ligament } \\
(\mathrm{mm})\end{array}$ & $\begin{array}{l}\text { Avg. } \\
\text { Thickness } \\
(\mathrm{mm})\end{array}$ & $\begin{array}{l}\text { Flexibility } \\
\text { Index }\end{array}$ & Average & Std Dev & $\operatorname{CoV}(\%)$ \\
\hline $1 \mathrm{~A}-1$ & 1976.24 & \multirow{8}{*}{2099.06} & 23.81 & \multirow{8}{*}{27.43} & -0.83 & \multirow{8}{*}{-0.80} & 6.7 & \multirow{8}{*}{6.05} & 60.2 & 34.1 & 16.97 & \multirow{8}{*}{22.57} & \multirow{8}{*}{7.40} & \multirow{8}{*}{32.77} \\
\hline $1 \mathrm{~A}-2$ & 1717.49 & & 22.60 & & -0.76 & & 5.7 & & 57.6 & 33.7 & 18.57 & & & \\
\hline $1 \mathrm{~B}-1$ & 2008.24 & & 24.79 & & -0.81 & & 7.5 & & 59.0 & 36.0 & 16.79 & & & \\
\hline $1 \mathrm{~B}-2$ & 2244.08 & & 20.97 & & -1.07 & & 8.0 & & 57.6 & 37.6 & 13.90 & & & \\
\hline $2 A-1$ & 2298.39 & & 22.76 & & -1.01 & & 5.2 & & 59.5 & 32.7 & 19.75 & & & \\
\hline $2 A-2$ & 2204.83 & & 37.37 & & -0.59 & & 5.0 & & 58.2 & 33.8 & 34.60 & & & \\
\hline 3B-1 & 2189.20 & & 29.99 & & -0.73 & & 5.2 & & 59.2 & 33.7 & 26.93 & & & \\
\hline $3 B-2$ & 2154.01 & & 37.14 & & -0.58 & & 5.3 & & 58.1 & 34.2 & 33.07 & & & \\
\hline
\end{tabular}

Table C.17. Twelve Months Field Cores Results for PM1

\begin{tabular}{|c|c|c|c|c|c|c|c|c|c|c|c|c|c|c|}
\hline \multirow{2}{*}{$\begin{array}{l}\text { M1 12months } \\
\text { Specimen ID }\end{array}$} & \multicolumn{2}{|c|}{ Fracture Energy } & \multicolumn{2}{|c|}{ Flexibility Index } & \multicolumn{2}{|c|}{ Slope } & \multicolumn{2}{|c|}{ Air voids } & \multicolumn{2}{|c|}{ Dimensions } & \multicolumn{4}{|c|}{ Corrected Flexibility Index } \\
\hline & $\begin{array}{c}\text { Energy } \\
\text { (LLD) (Gf) } \\
(\mathrm{J} / \mathrm{m} 2)\end{array}$ & Average & $\begin{array}{l}\text { Flexibility } \\
\text { Index }\end{array}$ & Average & Slope & Average & Air Voids & Average & $\begin{array}{l}\text { Avg. } \\
\text { Ligament } \\
(\mathrm{mm})\end{array}$ & \begin{tabular}{|c|} 
Avg. \\
Thicknes \\
$\mathrm{s}(\mathrm{mm})$
\end{tabular} & $\begin{array}{l}\text { Flexibility } \\
\text { Index }\end{array}$ & Average & Std Dev & $\operatorname{CoV}(\%)$ \\
\hline $1 \mathrm{~A}-1$ & 1310.81 & \multirow{8}{*}{1364.58} & 2.38 & \multirow{8}{*}{3.00} & -5.50 & \multirow{8}{*}{-4.77} & 5.9 & \multirow{8}{*}{5.66} & 58.5 & 49.7 & 2.78 & \multirow{8}{*}{3.67} & \multirow{8}{*}{0.96} & \multirow{8}{*}{26.17} \\
\hline $1 \mathrm{~A}-2$ & 1131.66 & & 2.66 & & -4.26 & & 5.7 & & 59.2 & 50.3 & 3.25 & & & \\
\hline 1B-1 & 1586.64 & & 4.56 & & -3.48 & & 5.9 & & 58.5 & 49.9 & 5.31 & & & \\
\hline 1B-2 & 1153.16 & & 2.03 & & -5.67 & & 5.9 & & 59.1 & 50.5 & 2.40 & & & \\
\hline $3 A-1$ & 1526.34 & & 3.35 & & -4.55 & & 5.4 & & 59.2 & 49.7 & 4.28 & & & \\
\hline $3 A-2$ & 1448.33 & & 3.10 & & -4.67 & & 5.5 & & 58.7 & 49.7 & 3.87 & & & \\
\hline 3B-1 & 1475.64 & & 2.24 & & -6.59 & & 5.5 & & 58.4 & 50.3 & 2.82 & & & \\
\hline $3 B-2$ & 1284.07 & & 3.70 & & -3.47 & & 5.5 & & 58.2 & 50.0 & 4.65 & & & \\
\hline
\end{tabular}

Table C.18. Twelve Months Field Cores Results for PM2

\begin{tabular}{|c|c|c|c|c|c|c|c|c|c|c|c|c|c|c|}
\hline \multirow{2}{*}{$\begin{array}{l}\text { M2 12months } \\
\text { Specimen ID }\end{array}$} & \multicolumn{2}{|c|}{ Fracture Energy } & \multicolumn{2}{|c|}{ Flexibility Index } & \multicolumn{2}{|c|}{ Slope } & \multicolumn{2}{|c|}{ Air voids } & \multicolumn{2}{|c|}{ Dimensions } & \multicolumn{4}{|c|}{ Corrected Flexibility Index } \\
\hline & $\begin{array}{c}\text { Energy } \\
\text { (LLD) (Gf) } \\
(\mathrm{J} / \mathrm{m} 2)\end{array}$ & Average & $\begin{array}{l}\text { Flexibility } \\
\text { Index }\end{array}$ & Average & Slope & Average & Air Voids & Average & $\begin{array}{c}\text { Avg. } \\
\text { Ligament } \\
(\mathrm{mm})\end{array}$ & $\begin{array}{l}\text { Avg. } \\
\text { Thickness } \\
(\mathrm{mm})\end{array}$ & $\begin{array}{l}\text { Flexibility } \\
\text { Index }\end{array}$ & Average & Std Dev & $\operatorname{CoV}(\%)$ \\
\hline $1 \mathrm{~A}-1$ & 2793.40 & \multirow{8}{*}{2423.71} & 15.78 & \multirow{8}{*}{11.96} & -1.77 & \multirow{8}{*}{-2.08} & 3.6 & \multirow{8}{*}{4.03} & 59.1 & 31.8 & 18.84 & \multirow{8}{*}{14.20} & \multirow{8}{*}{2.85} & \multirow{8}{*}{20.06} \\
\hline $1 \mathrm{~A}-2$ & 2316.43 & & 12.06 & & -1.92 & & 4.0 & & 59.1 & 32.7 & 13.47 & & & \\
\hline 1B-1 & 2448.28 & & 13.38 & & -1.83 & & 4.3 & & 58.4 & 37.1 & 15.56 & & & \\
\hline 1B-2 & 2459.13 & & 10.16 & & -2.42 & & 4.3 & & 59.0 & 37.6 & 12.17 & & & \\
\hline $3 A-1$ & 2602.82 & & 13.84 & & -1.88 & & 3.6 & & 58.9 & 34.2 & 17.97 & & & \\
\hline $3 A-2$ & 2176.26 & & 8.57 & & -2.54 & & 3.7 & & 59.4 & 34.4 & 10.70 & & & \\
\hline 3B-1 & 2217.75 & & 9.56 & & -2.32 & & 4.4 & & 59.0 & 37.5 & 10.98 & & & \\
\hline $3 B-2$ & 2375.57 & & 12.31 & & -1.93 & & 4.3 & & 59.0 & 36.0 & 13.93 & & & \\
\hline
\end{tabular}

Table C.19. Twelve Months Field Cores Results for PM3

\begin{tabular}{|c|c|c|c|c|c|c|c|c|c|c|c|c|c|c|}
\hline \multirow{2}{*}{$\begin{array}{l}\text { M3 12months } \\
\text { Specimen ID }\end{array}$} & \multicolumn{2}{|c|}{ Fracture Energy } & \multicolumn{2}{|c|}{ Flexibility Index } & \multicolumn{2}{|c|}{ Slope } & \multicolumn{2}{|c|}{ Air voids } & \multicolumn{2}{|c|}{ Dimensions } & \multicolumn{4}{|c|}{ Corrected Flexibility Index } \\
\hline & $\begin{array}{c}\text { Energy } \\
(\text { LLD) (Gf) } \\
(\mathrm{J} / \mathrm{m} 2)\end{array}$ & Average & $\begin{array}{l}\text { Flexibility } \\
\text { Index }\end{array}$ & Average & Slope & Average & Air Voids & Average & $\begin{array}{l}\text { Avg. } \\
\text { Ligament } \\
(\mathrm{mm})\end{array}$ & $\begin{array}{l}\text { Avg. } \\
\text { Thickness } \\
(\mathrm{mm})\end{array}$ & $\begin{array}{l}\text { Flexibility } \\
\text { Index }\end{array}$ & Average & Std Dev & $\operatorname{CoV}(\%)$ \\
\hline $1 \mathrm{~A}-1$ & 1508.90 & \multirow{8}{*}{1889.20} & 5.49 & \multirow{8}{*}{11.19} & -2.75 & \multirow{8}{*}{-1.96} & 4.9 & \multirow{8}{*}{4.81} & 59.0 & 33.2 & 5.05 & \multirow{8}{*}{8.92} & \multirow{8}{*}{2.87} & \multirow{8}{*}{32.16} \\
\hline $1 \mathrm{~A}-2$ & 2127.61 & & 12.89 & & -1.65 & & 4.8 & & 59.2 & 34.9 & 12.90 & & & \\
\hline 1B-1 & 1394.60 & & 9.49 & & -1.47 & & 4.7 & & 58.9 & 34.1 & 9.41 & & & \\
\hline 1B-2 & 1663.24 & & 5.70 & & -2.92 & & 4.9 & & 59.1 & 33.0 & 5.26 & & & \\
\hline $3 A-1$ & 2076.70 & & 10.82 & & -1.92 & & 4.6 & & 59.2 & 31.8 & 10.28 & & & \\
\hline $3 A-2$ & 2109.21 & & 12.12 & & -1.74 & & 4.7 & & 58.8 & 34.2 & 11.99 & & & \\
\hline 3B-1 & 1741.77 & & 7.85 & & -2.22 & & 5.1 & & 58.8 & 36.0 & 7.55 & & & \\
\hline $3 B-2$ & 2491.55 & & 25.17 & & -0.99 & & 4.8 & & 59.3 & 34.3 & & & & \\
\hline
\end{tabular}




\section{APPENDIX D: SHELF AGING I-FIT RESULTS}

Table D.1. Six Months Shelf Aging for PM2

\begin{tabular}{|c|c|c|c|c|c|c|c|c|c|c|c|c|c|}
\hline \multicolumn{2}{|c|}{ SH6 PM2 } & \multicolumn{4}{|c|}{ Fracture Energy } & \multicolumn{4}{|c|}{ Flexibility Index } & \multicolumn{4}{|c|}{ Slope } \\
\hline Aging Condition & $\begin{array}{c}\text { Specimen } \\
\text { ID }\end{array}$ & $\begin{array}{l}\text { Energy } \\
\text { (LLD) (Gf) } \\
\text { (J/m2) }\end{array}$ & Average & Std Dev & $\operatorname{CoV}(\%)$ & $\begin{array}{l}\text { Flexibility } \\
\text { Index }\end{array}$ & Average & Std Dev & $\operatorname{CoV}(\%)$ & Slope & Average & Std Dev & $\operatorname{CoV}(\%)$ \\
\hline \multirow{6}{*}{ Unaged } & $1-4$ & 2205.22 & \multirow{6}{*}{2480.17} & \multirow{6}{*}{188.61} & \multirow{6}{*}{7.60} & 5.51 & \multirow{6}{*}{7.64} & \multirow{6}{*}{2.03} & \multirow{6}{*}{26.65} & -4.00 & \multirow{6}{*}{-3.42} & \multirow{6}{*}{0.66} & \multirow{6}{*}{19.39} \\
\hline & $2-3$ & 2711.68 & & & & 10.16 & & & & -2.67 & & & \\
\hline & $3-2$ & 2328.14 & & & & 5.49 & & & & -4.24 & & & \\
\hline & $5-3$ & 2674.11 & & & & 10.53 & & & & -2.54 & & & \\
\hline & $6-2$ & 2372.47 & & & & 7.48 & & & & -3.17 & & & \\
\hline & $6-4$ & 2589.37 & & & & 6.64 & & & & -3.90 & & & \\
\hline \multirow{7}{*}{$24 \mathrm{hrs} / 95 \mathrm{C}$} & $1-2$ & 2509.53 & \multirow{7}{*}{2512.74} & \multirow{7}{*}{270.46} & \multirow{7}{*}{10.76} & 6.26 & \multirow{7}{*}{4.53} & \multirow{7}{*}{1.17} & \multirow{7}{*}{25.90} & -4.01 & \multirow{7}{*}{-5.59} & \multirow{7}{*}{1.00} & \multirow{7}{*}{17.96} \\
\hline & $1-3$ & 2481.79 & & & & 3.94 & & & & -6.30 & & & \\
\hline & $2-4$ & 2428.73 & & & & 4.25 & & & & -5.71 & & & \\
\hline & $3-1$ & 2790.47 & & & & 5.89 & & & & -4.74 & & & \\
\hline & $4-1$ & 2273.64 & & & & 4.00 & & & & -5.69 & & & \\
\hline & $5-1$ & 2124.42 & & & & 2.87 & & & & -7.39 & & & \\
\hline & $6-1$ & 2980.61 & & & & & & & & -5.31 & & & \\
\hline \multirow{5}{*}{5 days $/ 85 \mathrm{C}$} & $1-1$ & 1965.61 & \multirow{5}{*}{2125.38} & \multirow{5}{*}{197.57} & \multirow{5}{*}{9.30} & 1.80 & \multirow{5}{*}{2.50} & \multirow{5}{*}{0.51} & \multirow{5}{*}{20.49} & -10.94 & \multirow{5}{*}{-8.79} & & \\
\hline & $2-1$ & 1882.09 & & & & 1.93 & & & & -9.75 & & & \\
\hline & $2-2$ & 2206.32 & & & & 2.50 & & & & -8.81 & & & \\
\hline & $5-2$ & 2183.19 & & & & 2.79 & & & & -7.82 & & 1.51 & 17.19 \\
\hline & $\begin{array}{l}5-4 \\
6-3\end{array}$ & $\begin{array}{l}2028.78 \\
2486.31\end{array}$ & & & & $\begin{array}{l}3.30 \\
2.68\end{array}$ & & & & $\begin{array}{l}-6.15 \\
-9.29\end{array}$ & & & \\
\hline
\end{tabular}

Table D.2. Six Months Shelf Aging for PM3

\begin{tabular}{|c|c|c|c|c|c|c|c|c|c|c|c|c|c|}
\hline \multicolumn{2}{|c|}{ SH6 PM3 } & \multicolumn{4}{|c|}{ Fracture Energy } & \multicolumn{4}{|c|}{ Flexibility Index } & \multicolumn{4}{|c|}{ Slope } \\
\hline Aging Condition & $\begin{array}{c}\text { Specimen } \\
\text { ID }\end{array}$ & $\begin{array}{c}\text { Energy } \\
(\mathrm{LLD})(\mathrm{Gf}) \\
(\mathrm{J} / \mathrm{m} 2)\end{array}$ & Average & Std Dev & CoV (\%) & $\begin{array}{l}\text { Flexibility } \\
\text { Index }\end{array}$ & Average & Std Dev & $\operatorname{CoV}(\%)$ & Slope & Average & Std Dev & $\operatorname{CoV}(\%)$ \\
\hline \multirow{5}{*}{ Unaged } & $21-1$ & 3423.51 & \multirow{5}{*}{2588.40} & \multirow{5}{*}{445.51} & \multirow{5}{*}{17.21} & 18.71 & \multirow{5}{*}{11.07} & \multirow{5}{*}{3.85} & \multirow{5}{*}{34.75} & -1.83 & \multirow{5}{*}{-2.45} & \multirow{5}{*}{0.39} & \multirow{5}{*}{16.05} \\
\hline & $22-1$ & 2526.67 & & & & 8.62 & & & & -2.93 & & & \\
\hline & $23-1$ & 2564.37 & & & & 9.46 & & & & -2.71 & & & \\
\hline & $28-3$ & 2283.92 & & & & 8.75 & & & & -2.61 & & & \\
\hline & $29-1$ & 2143.55 & & & & 9.79 & & & & -2.19 & & & \\
\hline \multirow{6}{*}{$16 \mathrm{hrs} / 95 \mathrm{C}$} & $20-3$ & 2435.71 & \multirow{6}{*}{2522.19} & \multirow{6}{*}{148.88} & \multirow{6}{*}{5.90} & 6.25 & \multirow{6}{*}{6.18} & \multirow{6}{*}{0.82} & \multirow{6}{*}{13.32} & -3.90 & \multirow{6}{*}{-4.15} & \multirow{6}{*}{0.54} & \multirow{6}{*}{12.99} \\
\hline & $22-2$ & 2598.51 & & & & 6.26 & & & & -4.15 & & & \\
\hline & $25-3$ & 2489.11 & & & & 7.39 & & & & -3.37 & & & \\
\hline & $26-1$ & 2469.26 & & & & 6.33 & & & & -3.90 & & & \\
\hline & $28-1$ & 2806.02 & & & & 6.26 & & & & -4.48 & & & \\
\hline & $30-2$ & 2334.50 & & & & 4.58 & & & & -5.10 & & & \\
\hline \multirow{6}{*}{$16 \mathrm{hrs} / 110 \mathrm{C}$} & $20-4$ & 2908.82 & & & & 6.96 & & & & -4.18 & & & \\
\hline & $24-1$ & 2642.58 & & & & 8.93 & & & & -2.96 & & & \\
\hline & $25-4$ & 2406.63 & & & & 3.91 & & & & -6.16 & & & \\
\hline & $26-4$ & 2618.96 & 2717.17 & 178.40 & 6.57 & 5.87 & 6.99 & 1.74 & 24.95 & -4.46 & -4.15 & 1.04 & 25.06 \\
\hline & $27-1$ & 2858.27 & & & & 7.37 & & & & -3.88 & & & \\
\hline & $30-1$ & 2867.78 & & & & 8.88 & & & & -3.23 & & & \\
\hline & $22-3$ & 2392.88 & & & & 3.83 & & & & -6.25 & & & \\
\hline & $25-1$ & 2907.23 & & & & 5.76 & & & & -5.05 & & & \\
\hline & $26-2$ & 2387.64 & & & & 3.74 & & & & -6.39 & & & \\
\hline 24hrs/95C & $28-4$ & 3005.73 & 2704.38 & 238.17 & 8.81 & 8.87 & 5.44 & 1.70 & 31.34 & -3.39 & -5.28 & 0.98 & 18.64 \\
\hline & $29-2$ & 2725.57 & & & & 5.22 & & & & -5.22 & & & \\
\hline & $30-3$ & 2807.23 & & & & 5.23 & & & & -5.37 & & & \\
\hline & $21-4$ & 2706.03 & & & & 6.80 & & & & -3.98 & & & \\
\hline & $25-2$ & 2376.37 & & & & 4.24 & & & & -5.60 & & & \\
\hline $24 \mathrm{hrs} / 110 \mathrm{C}$ & $27-4$ & 2794.09 & 2648.17 & 191.46 & 7.23 & 7.35 & 6.02 & 1.11 & 18.36 & -3.80 & -4.53 & 0.58 & 12.74 \\
\hline & $29-3$ & 2926.39 & & & & 6.38 & & & & -4.59 & & & \\
\hline & $30-4$ & 2438.37 & & & & 5.35 & & & & -4.56 & & & \\
\hline & $21-2$ & 2222.82 & & & & 3.26 & & & & -6.81 & & & \\
\hline & $21-3$ & 2412.25 & & & & 3.26 & & & & -7.39 & & & \\
\hline 5days/85C & $22-4$ & 2424.76 & 2216.98 & 249.26 & 11.24 & 3.65 & 3.08 & 0.56 & 18.32 & -6.65 & -7.33 & 0.71 & 9.63 \\
\hline & $29-4$ & 1808.09 & & & & 2.14 & & & & -8.45 & & & \\
\hline
\end{tabular}


Table D.3. Six Months Shelf Aging for PM5

\begin{tabular}{|c|c|c|c|c|c|c|c|c|c|c|c|c|c|}
\hline Aging Condition & Specimen ID & $\begin{array}{c}\text { Energy (LLD) } \\
\text { (Gf) (J/m2) }\end{array}$ & Average & Std Dev & $\operatorname{CoV}(\%)$ & $\begin{array}{l}\text { Flexibility } \\
\text { Index }\end{array}$ & Average & Std Dev & $\operatorname{CoV}(\%)$ & Slope & Average & Std Dev & $\operatorname{CoV}(\%)$ \\
\hline \multirow{12}{*}{ Unaged } & $1-1$ & 1764.81 & \multirow{12}{*}{1837.05} & \multirow{12}{*}{142.19} & \multirow{12}{*}{7.74} & 13.58 & \multirow{12}{*}{11.96} & \multirow{12}{*}{2.52} & \multirow{12}{*}{21.10} & -1.30 & \multirow{12}{*}{-1.59} & \multirow{12}{*}{0.27} & \multirow{12}{*}{17.09} \\
\hline & $1-2$ & 1763.29 & & & & 11.30 & & & & -1.56 & & & \\
\hline & $1-3$ & 2123.63 & & & & 18.47 & & & & -1.15 & & & \\
\hline & $1-4$ & 1743.97 & & & & 13.21 & & & & -1.32 & & & \\
\hline & $2-1$ & 1879.97 & & & & 10.05 & & & & -1.87 & & & \\
\hline & $2-2$ & 1786.46 & & & & 9.71 & & & & -1.84 & & & \\
\hline & $2-3$ & 2085.69 & & & & 12.13 & & & & -1.72 & & & \\
\hline & $2-4$ & 1854.10 & & & & 8.91 & & & & -2.08 & & & \\
\hline & P1-1 & 1751.15 & & & & 11.67 & & & & -1.50 & & & \\
\hline & $\mathrm{P} 1-2$ & 1911.53 & & & & 14.16 & & & & -1.35 & & & \\
\hline & P1-3 & 1593.84 & & & & 10.63 & & & & -1.50 & & & \\
\hline & P3-3 & 1786.19 & & & & 9.76 & & & & -1.83 & & & \\
\hline \multirow{6}{*}{$1 \mathrm{D} / 95 \mathrm{C}$} & $1-3$ & 1671.36 & \multirow{6}{*}{1824.94} & \multirow{6}{*}{103.41} & \multirow{6}{*}{5.67} & 6.17 & \multirow{6}{*}{7.17} & \multirow{6}{*}{1.05} & \multirow{6}{*}{14.62} & -2.71 & \multirow{6}{*}{-2.58} & \multirow{6}{*}{0.40} & \multirow{6}{*}{15.60} \\
\hline & $2-2$ & 1882.88 & & & & 7.98 & & & & -2.36 & & & \\
\hline & $2-3$ & 1781.81 & & & & 6.36 & & & & -2.80 & & & \\
\hline & $3-2$ & 1887.53 & & & & 7.90 & & & & -2.39 & & & \\
\hline & $5-2$ & 1743.57 & & & & 5.93 & & & & -2.94 & & & \\
\hline & $6-3$ & 1982.50 & & & & 8.66 & & & & -2.29 & & & \\
\hline \multirow{4}{*}{$3 D / 95 C$} & $3-3$ & 1535.06 & \multirow{4}{*}{1690.49} & \multirow{4}{*}{99.78} & \multirow{4}{*}{5.90} & 3.38 & \multirow{4}{*}{4.19} & \multirow{4}{*}{0.64} & \multirow{4}{*}{15.37} & -4.54 & \multirow{4}{*}{-4.10} & & \\
\hline & $7-2$ & 1767.71 & & & & 4.13 & & & & -4.28 & & & \\
\hline & $8-2$ & 1671.96 & & & & 4.06 & & & & -4.12 & & 0.40 & 9.83 \\
\hline & $8-4$ & 1787.24 & & & & 5.18 & & & & -3.45 & & & \\
\hline & $1-4$ & 2174.16 & & & & 7.96 & & & & -2.73 & & & \\
\hline & $2-1$ & 1848.19 & & & & 4.85 & & & & -3.81 & & & \\
\hline & 3-1 & 1648.15 & & & & 4.33 & & & & -3.81 & & & \\
\hline $5 D / 85 C$ & $3-4$ & 1978.95 & $18 / 0.88$ & $16 / .80$ & 8.91 & 6.34 & 5.90 & 1.18 & 20.03 & -3.12 & -3.25 & 0.43 & 13.12 \\
\hline & 4-1 & 1814.20 & & & & 6.39 & & & & -2.84 & & & \\
\hline & $5-1$ & 1761.60 & & & & 5.56 & & & & -3.17 & & & \\
\hline
\end{tabular}

Table D.4. Six Months Shelf Aging for PM6

\begin{tabular}{|c|c|c|c|c|c|c|c|c|c|c|c|c|c|}
\hline \multicolumn{2}{|c|}{ SH6 PM6 } & \multicolumn{4}{|c|}{ Fracture Energy } & \multicolumn{4}{|c|}{ Flexibility Index } & \multicolumn{4}{|c|}{ Slope } \\
\hline Aging Condition & Specimen ID & $\begin{array}{c}\text { Energy } \\
\text { (LLD) (Gf) } \\
(\mathrm{J} / \mathrm{m} 2)\end{array}$ & Average & Std Dev & $\operatorname{CoV}(\%)$ & $\begin{array}{l}\text { Flexibility } \\
\text { Index }\end{array}$ & Average & Std Dev & $\operatorname{CoV}(\%)$ & Slope & Average & Std Dev & $\operatorname{CoV}(\%)$ \\
\hline \multirow{6}{*}{ Unaged } & $1-1$ & 2205.81 & \multirow{6}{*}{2157.06} & \multirow{6}{*}{176.11} & \multirow{6}{*}{8.16} & 11.86 & \multirow{6}{*}{12.69} & \multirow{6}{*}{3.55} & \multirow{6}{*}{27.97} & -1.86 & \multirow{6}{*}{-1.79} & \multirow{6}{*}{0.33} & \multirow{6}{*}{18.57} \\
\hline & $2-1$ & 1923.90 & & & & 8.19 & & & & -2.35 & & & \\
\hline & $3-1$ & 2086.39 & & & & 10.81 & & & & -1.93 & & & \\
\hline & $4-1$ & 2459.17 & & & & 19.67 & & & & -1.25 & & & \\
\hline & $5-1$ & 2007.11 & & & & 11.74 & & & & -1.71 & & & \\
\hline & $6-2$ & 2259.97 & & & & 13.86 & & & & -1.63 & & & \\
\hline \multirow{6}{*}{$1 \mathrm{D} / 95 \mathrm{C}$} & $2-3$ & 2076.56 & \multirow{6}{*}{1975.71} & \multirow{6}{*}{232.69} & \multirow{6}{*}{11.78} & 5.67 & \multirow{6}{*}{6.28} & \multirow{6}{*}{1.20} & \multirow{6}{*}{19.18} & -3.66 & \multirow{6}{*}{-3.20} & \multirow{6}{*}{0.33} & \multirow{6}{*}{10.33} \\
\hline & $2-4$ & 1544.00 & & & & 4.49 & & & & -3.44 & & & \\
\hline & $5-4$ & 2150.80 & & & & 7.76 & & & & -2.77 & & & \\
\hline & $6-1$ & 2210.22 & & & & 7.89 & & & & -2.80 & & & \\
\hline & $7-2$ & 2077.01 & & & & 6.13 & & & & -3.39 & & & \\
\hline & $7-4$ & 1795.65 & & & & 5.74 & & & & -3.13 & & & \\
\hline \multirow{6}{*}{$5 \mathrm{D} / 85 \mathrm{C}$} & $1-2$ & 2000.24 & \multirow{6}{*}{1911.59} & \multirow{6}{*}{180.95} & \multirow{6}{*}{9.47} & 4.81 & \multirow{6}{*}{3.79} & \multirow{6}{*}{1.31} & \multirow{6}{*}{34.66} & -4.16 & \multirow{6}{*}{-5.54} & & \\
\hline & $2-2$ & 2130.68 & & & & 6.00 & & & & -3.55 & & & \\
\hline & $3-2$ & 1800.35 & & & & 2.22 & & & & -8.10 & & 154 & 2775 \\
\hline & $5-3$ & 1584.50 & & & & 2.84 & & & & -5.57 & & 1.54 & 27.75 \\
\hline & $6-3$ & 2056.58 & & & & 4.05 & & & & -5.08 & & & \\
\hline & $7-1$ & 1897.17 & & & & 2.79 & & & & -6.79 & & & \\
\hline
\end{tabular}


Table D.5. Six Months Shelf Aging for PM8

\begin{tabular}{|c|c|c|c|c|c|c|c|c|c|c|c|c|c|}
\hline \multicolumn{2}{|c|}{ SH6 PM8 } & \multicolumn{4}{|c|}{ Fracture Energy } & \multicolumn{4}{|c|}{ Flexibility Index } & \multicolumn{4}{|c|}{ Slope } \\
\hline Aging Condition & Specimen ID & $\begin{array}{c}\text { Energy } \\
\text { (LLD) (Gf) } \\
(\mathrm{J} / \mathrm{m} 2)\end{array}$ & Average & Std Dev & $\operatorname{CoV}(\%)$ & $\begin{array}{l}\text { Flexibility } \\
\text { Index }\end{array}$ & Average & Std Dev & $\operatorname{CoV}(\%)$ & Slope & Average & Std Dev & $\operatorname{CoV}(\%)$ \\
\hline \multirow{6}{*}{ Unaged } & $1-1$ & 1979.14 & \multirow{6}{*}{2008.04} & \multirow{6}{*}{140.41} & \multirow{6}{*}{6.99} & 10.64 & \multirow{6}{*}{10.60} & \multirow{6}{*}{1.51} & \multirow{6}{*}{14.26} & -1.86 & \multirow{6}{*}{-1.93} & \multirow{6}{*}{0.30} & \multirow{6}{*}{15.41} \\
\hline & $2-3$ & 1956.15 & & & & 11.64 & & & & -1.68 & & & \\
\hline & $3-3$ & 2152.00 & & & & 11.21 & & & & -1.92 & & & \\
\hline & $4-3$ & 1852.84 & & & & 11.09 & & & & -1.67 & & & \\
\hline & $5-3$ & 2235.32 & & & & 11.70 & & & & -1.91 & & & \\
\hline & $7-2$ & 1872.80 & & & & 7.32 & & & & -2.56 & & & \\
\hline \multirow{6}{*}{$1 \mathrm{D} / 95 \mathrm{C}$} & $1-3$ & 1701.52 & \multirow{6}{*}{1985.21} & \multirow{6}{*}{147.64} & \multirow{6}{*}{7.44} & 5.27 & \multirow{6}{*}{7.09} & \multirow{6}{*}{1.17} & \multirow{6}{*}{16.52} & -3.23 & \multirow{6}{*}{-2.85} & \multirow{6}{*}{0.31} & \multirow{6}{*}{10.97} \\
\hline & $3-2$ & 1917.81 & & & & 7.26 & & & & -2.64 & & & \\
\hline & $4-1$ & 2144.85 & & & & 9.05 & & & & -2.37 & & & \\
\hline & $5-4$ & 2117.17 & & & & 7.15 & & & & -2.96 & & & \\
\hline & $7-4$ & 2038.36 & & & & 7.58 & & & & -2.69 & & & \\
\hline & $8-2$ & 1991.54 & & & & 6.20 & & & & -3.21 & & & \\
\hline \multirow{6}{*}{$3 D / 95 C$} & $1-4$ & 2018.71 & \multirow{6}{*}{2012.12} & \multirow{6}{*}{218.70} & \multirow{6}{*}{10.87} & 6.99 & \multirow{6}{*}{5.65} & & & -2.89 & & & \\
\hline & $4-2$ & 2331.75 & & & & 6.64 & & & & -3.51 & & & \\
\hline & $5-1$ & 1802.65 & & & & 4.11 & & 103 & 1821 & -4.39 & 264 & 047 & 1203 \\
\hline & $6-1$ & 1877.83 & & & & 4.74 & & 1.03 & 18.21 & -3.96 & -3.64 & 0.47 & 12.93 \\
\hline & $6-3$ & 2268.57 & & & & 6.13 & & & & -3.70 & & & \\
\hline & $8-1$ & 1773.18 & & & & 5.28 & & & & -3.36 & & & \\
\hline & $1-2$ & 2019.80 & & & & 5.06 & & & & -3.99 & & & \\
\hline & $2-4$ & 2347.65 & & & & 7.85 & & & & -2.99 & & & \\
\hline $50 / 850$ & $4-4$ & 1936.43 & & & & 5.39 & & & & -3.59 & & & \\
\hline $5 \mathrm{D} / 85 \mathrm{C}$ & $6-2$ & 1792.22 & 2010.34 & 173.28 & 8.62 & 5.30 & 5.45 & 1.14 & 21.00 & -3.38 & -3.79 & 0.52 & 13.85 \\
\hline & $7-3$ & 2059.61 & & & & 4.90 & & & & -4.20 & & & \\
\hline & $8-3$ & 1906.30 & & & & 4.18 & & & & -4.56 & & & \\
\hline
\end{tabular}

Table D.6. Six Months Shelf Aging for PM9

\begin{tabular}{|c|c|c|c|c|c|c|c|c|c|c|c|c|c|}
\hline \multicolumn{2}{|c|}{ SH PM9 } & \multicolumn{4}{|c|}{ Fracture Energy } & \multicolumn{4}{|c|}{ Flexibility Index } & \multicolumn{4}{|c|}{ Slope } \\
\hline Aging Condition & Specimen ID & $\begin{array}{l}\text { Energy } \\
\text { (LLD) (Gf) } \\
(\mathrm{J} / \mathrm{m} 2)\end{array}$ & Average & Std Dev & $\operatorname{CoV}(\%)$ & $\begin{array}{l}\text { Flexibility } \\
\text { Index }\end{array}$ & Average & Std Dev & $\operatorname{CoV}(\%)$ & Slope & Average & Std Dev & $\operatorname{CoV}(\%)$ \\
\hline \multirow{7}{*}{ Unaged } & $1-4$ & 2003.84 & \multirow{7}{*}{2057.14} & \multirow{7}{*}{192.68} & \multirow{7}{*}{9.37} & 12.93 & \multirow{7}{*}{9.89} & \multirow{7}{*}{2.81} & \multirow{7}{*}{28.46} & -1.55 & \multirow{7}{*}{-2.22} & \multirow{7}{*}{0.51} & \multirow{7}{*}{22.83} \\
\hline & $2-2$ & 2373.88 & & & & 13.72 & & & & -1.73 & & & \\
\hline & $3-2$ & 1982.15 & & & & 9.22 & & & & -2.15 & & & \\
\hline & $4-3$ & 1885.78 & & & & 6.33 & & & & -2.98 & & & \\
\hline & $5-3$ & 2336.16 & & & & 12.17 & & & & -1.92 & & & \\
\hline & $6-4$ & 1906.68 & & & & 8.22 & & & & -2.32 & & & \\
\hline & $8-1$ & 1911.46 & & & & 6.66 & & & & -2.87 & & & \\
\hline \multirow{6}{*}{$1 \mathrm{D} / 95 \mathrm{C}$} & $1-3$ & 2122.37 & \multirow{6}{*}{1933.64} & \multirow{6}{*}{130.91} & \multirow{6}{*}{6.77} & 10.15 & \multirow{6}{*}{6.96} & \multirow{6}{*}{1.64} & \multirow{6}{*}{23.53} & -2.09 & \multirow{6}{*}{-2.89} & \multirow{6}{*}{0.49} & \multirow{6}{*}{16.97} \\
\hline & $2-4$ & 1813.02 & & & & 7.19 & & & & -2.52 & & & \\
\hline & $3-1$ & 2003.04 & & & & 6.52 & & & & -3.07 & & & \\
\hline & $4-4$ & 1988.29 & & & & 7.08 & & & & -2.81 & & & \\
\hline & $6-1$ & 1722.67 & & & & 4.80 & & & & -3.59 & & & \\
\hline & $7-4$ & 1952.42 & & & & 5.99 & & & & -3.26 & & & \\
\hline \multirow{6}{*}{$3 \mathrm{D} / 95 \mathrm{C}$} & $1-1$ & 2277.40 & \multirow{6}{*}{1976.24} & \multirow{6}{*}{194.86} & \multirow{6}{*}{9.86} & 5.67 & \multirow{6}{*}{4.53} & & & -4.02 & & & \\
\hline & $2-1$ & 2083.43 & & & & 4.96 & & & & -4.20 & & & \\
\hline & $3-4$ & 2105.52 & & & & 5.41 & & & & -3.89 & & & \\
\hline & $4-2$ & 1770.37 & & & & 3.65 & & 0.81 & 19.14 & -4.85 & -4.45 & 0.44 & 9.91 \\
\hline & $5-2$ & $\begin{array}{l}1886.20 \\
1734.51\end{array}$ & & & & $\frac{4.07}{3.41}$ & & & & $\begin{array}{r}-4.64 \\
-509\end{array}$ & & & \\
\hline & $6-3$ & 1734.51 & & & & 3.41 & & & & -5.09 & & & \\
\hline & $1-2$ & 2022.96 & & & & 5.47 & & & & -3.70 & & & \\
\hline & $2-3$ & 1943.28 & & & & 4.10 & & & & -4.74 & & & \\
\hline $5 \mathrm{D} / 85 \mathrm{C}$ & 3-3 & 1940.15 & 194581 & 99.77 & 5.13 & 4.47 & 4.92 & 0.72 & 14.73 & -4.34 & -4.02 & 0.48 & 12.01 \\
\hline & 4-1 & 2038.98 & & & & 6.25 & & & & -3.26 & & & \\
\hline & $5-1$ & 1738.43 & & & & 4.55 & & & & -3.82 & & & \\
\hline
\end{tabular}


Table D.7. Six Months Shelf Aging for PM10

\begin{tabular}{|c|c|c|c|c|c|c|c|c|c|c|c|c|c|}
\hline \multicolumn{2}{|c|}{ SH6 PM10 } & \multicolumn{4}{|c|}{ Fracture Energy } & \multicolumn{4}{|c|}{ Flexibility Index } & \multicolumn{4}{|c|}{ Slope } \\
\hline Aging Condition & Specimen ID & $\begin{array}{c}\text { Energy } \\
\text { (LLD) (Gf) } \\
(\mathrm{J} / \mathrm{m} 2)\end{array}$ & Average & Std Dev & $\operatorname{CoV}(\%)$ & $\begin{array}{l}\text { Flexibility } \\
\text { Index }\end{array}$ & Average & Std Dev & $\operatorname{CoV}(\%)$ & Slope & Average & Std Dev & $\operatorname{CoV}(\%)$ \\
\hline \multirow{9}{*}{ Unaged } & $1-3$ & 2441.35 & \multirow{9}{*}{2599.54} & \multirow{9}{*}{146.82} & \multirow{9}{*}{5.65} & 5.37 & \multirow{9}{*}{6.39} & \multirow{9}{*}{1.75} & \multirow{9}{*}{27.34} & -4.55 & \multirow{9}{*}{-4.37} & \multirow{9}{*}{1.21} & \multirow{9}{*}{27.56} \\
\hline & $2-4$ & 2507.95 & & & & 6.62 & & & & -3.79 & & & \\
\hline & $3-2$ & 2603.65 & & & & 7.84 & & & & -3.32 & & & \\
\hline & $4-3$ & 2485.84 & & & & 7.82 & & & & -3.18 & & & \\
\hline & $5-1$ & 2946.22 & & & & 9.66 & & & & -3.05 & & & \\
\hline & $6-1$ & 2622.02 & & & & 6.46 & & & & -4.06 & & & \\
\hline & $7-3$ & 2462.60 & & & & 3.54 & & & & -6.96 & & & \\
\hline & $8-4$ & 2655.51 & & & & 4.80 & & & & -5.53 & & & \\
\hline & $9-1$ & 2670.73 & & & & 5.44 & & & & -4.91 & & & \\
\hline \multirow{8}{*}{$1 \mathrm{D} / 95 \mathrm{C}$} & $1-4$ & 2410.59 & \multirow{8}{*}{2522.33} & \multirow{8}{*}{143.21} & \multirow{8}{*}{5.68} & 4.69 & \multirow{8}{*}{4.38} & \multirow{8}{*}{0.78} & \multirow{8}{*}{17.93} & -5.14 & \multirow{8}{*}{-5.90} & \multirow{8}{*}{0.82} & \multirow{8}{*}{13.81} \\
\hline & $3-1$ & 2583.44 & & & & 3.99 & & & & -6.48 & & & \\
\hline & $4-2$ & 2709.36 & & & & 5.89 & & & & -4.60 & & & \\
\hline & $5-2$ & 2512.67 & & & & 4.36 & & & & -5.76 & & & \\
\hline & $6-3$ & 2545.92 & & & & 4.09 & & & & -6.22 & & & \\
\hline & $7-1$ & 2538.49 & & & & 4.06 & & & & -6.26 & & & \\
\hline & $8-2$ & 2217.60 & & & & 3.00 & & & & -7.38 & & & \\
\hline & $9-3$ & 2660.57 & & & & 4.95 & & & & -5.38 & & & \\
\hline \multirow{8}{*}{$3 \mathrm{D} / 95 \mathrm{C}$} & $1-2$ & 2366.63 & & & & 3.49 & & & & -6.79 & & & \\
\hline & $2-3$ & 2273.04 & & & & 3.95 & & & & -5.76 & & & \\
\hline & $3-3$ & 2505.84 & & & & 2.99 & & & & -8.37 & & & \\
\hline & $4-4$ & 2342.49 & $23316 ?$ & 17421 & 747 & 3.54 & 299 & & & -6.62 & & & \\
\hline & $5-3$ & 2025.83 & 2331.62 & $1 / 4.21$ & 7.47 & 2.47 & 2.99 & 0.63 & 21.18 & -8.21 & -8.15 & 1.72 & 21.07 \\
\hline & $6-2$ & 2433.50 & & & & 2.67 & & & & -9.10 & & & \\
\hline & $7-4$ & 2579.64 & & & & 2.97 & & & & -8.68 & & & \\
\hline & $9-2$ & 2126.02 & & & & 1.82 & & & & -11.68 & & & \\
\hline & $2-1$ & 2341.05 & & & & 2.86 & & & & -8.18 & & & \\
\hline & $3-4$ & 2612.89 & & & & 4.00 & & & & -6.54 & & & \\
\hline & $4-1$ & 2626.08 & & & & 4.28 & & & & -6.13 & & & \\
\hline & $5-4$ & 2488.74 & & & & 3.86 & & & & -6.44 & & & \\
\hline $5 D / 85 C$ & $6-4$ & 2386.22 & $24 / 6.44$ & 103.64 & 4.18 & 3.69 & 3.46 & 0.64 & 18.62 & -6.46 & -7.45 & 1.70 & 22.11 \\
\hline & $7-2$ & 2347.21 & & & & 3.66 & & & & -6.42 & & & \\
\hline & $8-1$ & 2505.21 & & & & 3.17 & & & & -7.91 & & & \\
\hline & $9-4$ & 2504.12 & & & & 2.17 & & & & -11.53 & & & \\
\hline
\end{tabular}


Table D.8. Six Months Shelf Aging for PM11

\begin{tabular}{|c|c|c|c|c|c|c|c|c|c|c|c|c|c|}
\hline \multicolumn{2}{|c|}{ SH6 PM11 } & \multicolumn{4}{|c|}{ Fracture Energy } & \multicolumn{4}{|c|}{ Flexibility Index } & \multicolumn{4}{|c|}{ Slope } \\
\hline Aging Condition & Specimen ID & $\begin{array}{c}\text { Energy } \\
\text { (LLD) (Gf) } \\
(\mathrm{J} / \mathrm{m} 2)\end{array}$ & Average & Std Dev & $\operatorname{CoV}(\%)$ & $\begin{array}{l}\text { Flexibility } \\
\text { Index }\end{array}$ & Average & Std Dev & $\operatorname{CoV}(\%)$ & Slope & Average & Std Dev & $\operatorname{CoV}(\%)$ \\
\hline \multirow{6}{*}{ Unaged } & $1-1$ & 1684.82 & \multirow{6}{*}{1826.26} & \multirow{6}{*}{130.51} & \multirow{6}{*}{7.15} & 5.40 & \multirow{6}{*}{4.78} & \multirow{6}{*}{1.06} & \multirow{6}{*}{22.29} & -3.12 & \multirow{6}{*}{-3.99} & \multirow{6}{*}{0.80} & \multirow{6}{*}{20.12} \\
\hline & $3-3$ & 2074.62 & & & & 6.38 & & & & -3.25 & & & \\
\hline & $5-1$ & 1858.95 & & & & 5.37 & & & & -3.46 & & & \\
\hline & $7-1$ & 1860.38 & & & & 4.40 & & & & -4.23 & & & \\
\hline & $7-4$ & 1699.49 & & & & 3.15 & & & & -5.39 & & & \\
\hline & $8-3$ & 1779.32 & & & & 3.95 & & & & -4.51 & & & \\
\hline \multirow{7}{*}{ 1D/95C } & $1-2$ & 1869.49 & \multirow{7}{*}{1754.06} & \multirow{7}{*}{64.94} & \multirow{7}{*}{3.70} & 4.29 & \multirow{7}{*}{3.57} & \multirow{7}{*}{0.72} & \multirow{7}{*}{20.26} & -4.36 & \multirow{7}{*}{-5.17} & \multirow{7}{*}{1.31} & \multirow{7}{*}{25.29} \\
\hline & $2-3$ & 1783.91 & & & & 4.04 & & & & -4.42 & & & \\
\hline & $3-1$ & 1708.14 & & & & 4.07 & & & & -4.20 & & & \\
\hline & $3-2$ & 1717.72 & & & & 3.85 & & & & -4.46 & & & \\
\hline & $4-3$ & 1737.10 & & & & 3.11 & & & & -5.59 & & & \\
\hline & $8-1$ & 1803.58 & & & & 3.61 & & & & -5.00 & & & \\
\hline & $8-2$ & 1658.46 & & & & 2.02 & & & & -8.19 & & & \\
\hline \multirow{7}{*}{$3 D / 95 C$} & $1-3$ & 1595.26 & \multirow{7}{*}{1973.94} & \multirow{7}{*}{606.85} & \multirow{7}{*}{30.74} & 1.95 & & & & -8.17 & & & \\
\hline & $2-2$ & 1754.46 & & & & 2.96 & & & & -5.93 & & & \\
\hline & $2-4$ & 1849.29 & & & & 2.66 & & & & -6.95 & & & \\
\hline & $3-4$ & 1604.23 & & & & 2.62 & 2.59 & 0.66 & 25.63 & -6.12 & -7.69 & 1.26 & 16.41 \\
\hline & $4-2$ & 1886.71 & & & & 1.99 & & & & -9.47 & & & \\
\hline & $7-3$ & 3438.61 & & & & 3.94 & & & & -8.72 & & & \\
\hline & $8-4$ & 1689.02 & & & & 2.00 & & & & -8.44 & & & \\
\hline & $1-4$ & 1769.10 & & & & 2.39 & & & & -7.41 & & & \\
\hline & $2-1$ & 1519.23 & & & & 2.36 & & & & -6.44 & & & \\
\hline $5 \mathrm{D} / 85 \mathrm{C}$ & $4-1$ & 1772.13 & 1634.49 & 131.44 & 8.04 & 2.73 & 2.15 & 0.40 & 18.55 & -6.48 & -7.80 & 1.14 & 14.60 \\
\hline & $5-2$ & 1717.51 & & & & 2.02 & & & & -8.52 & -1.00 & & \\
\hline & $6-3$ & 1420.65 & & & & 1.48 & & & & -9.60 & & & \\
\hline & $7-2$ & 1608.32 & & & & 1.93 & & & & -8.32 & & & \\
\hline
\end{tabular}

Table D.9. Six Months Shelf Aging for PM12

\begin{tabular}{|c|c|c|c|c|c|c|c|c|c|c|c|c|c|}
\hline \multicolumn{2}{|c|}{ SH6 PM12 } & \multicolumn{4}{|c|}{ Fracture Energy } & \multicolumn{4}{|c|}{ Flexibility Index } & \multicolumn{4}{|c|}{ Slope } \\
\hline Aging Condition & Specimen ID & $\begin{array}{c}\text { Energy } \\
\text { (LLD) (Gf) } \\
(\mathrm{J} / \mathrm{m} 2)\end{array}$ & Average & Std Dev & $\operatorname{CoV}(\%)$ & $\begin{array}{l}\text { Flexibility } \\
\text { Index }\end{array}$ & Average & Std Dev & $\operatorname{CoV}(\%)$ & Slope & Average & Std Dev & $\operatorname{CoV}(\%)$ \\
\hline \multirow{7}{*}{ Unaged } & $1-4$ & 1685.28 & \multirow{7}{*}{1895.83} & \multirow{7}{*}{176.49} & \multirow{7}{*}{9.31} & 3.75 & \multirow{7}{*}{4.91} & \multirow{7}{*}{0.76} & \multirow{7}{*}{15.46} & -4.50 & \multirow{7}{*}{-3.93} & \multirow{7}{*}{0.53} & \multirow{7}{*}{13.59} \\
\hline & $2-3$ & 1846.89 & & & & 3.95 & & & & -4.67 & & & \\
\hline & $2-4$ & 2170.61 & & & & 5.44 & & & & -3.99 & & & \\
\hline & $3-1$ & 2115.88 & & & & 5.41 & & & & -3.91 & & & \\
\hline & $3-2$ & 1681.71 & & & & 5.39 & & & & -3.12 & & & \\
\hline & $4-3$ & 1910.79 & & & & 5.86 & & & & -3.26 & & & \\
\hline & $8-1$ & 1859.62 & & & & 4.59 & & & & -4.05 & & & \\
\hline \multirow{6}{*}{ 1D/95C } & $3-4$ & 2245.51 & \multirow{6}{*}{1933.36} & \multirow{6}{*}{202.84} & \multirow{6}{*}{10.49} & 4.18 & \multirow{6}{*}{4.12} & \multirow{6}{*}{1.50} & \multirow{6}{*}{36.36} & -5.37 & \multirow{6}{*}{-5.26} & \multirow{6}{*}{1.69} & \multirow{6}{*}{32.14} \\
\hline & $5-3$ & 2017.84 & & & & 6.91 & & & & -2.92 & & & \\
\hline & $6-1$ & 1972.39 & & & & 3.75 & & & & -5.26 & & & \\
\hline & $6-3$ & 1950.66 & & & & 4.44 & & & & -4.39 & & & \\
\hline & $7-2$ & 1569.28 & & & & 1.83 & & & & -8.56 & & & \\
\hline & $8-4$ & 1844.47 & & & & 3.63 & & & & -5.08 & & & \\
\hline \multirow{6}{*}{$3 \mathrm{D} / 95 \mathrm{C}$} & $2-1$ & 1627.25 & \multirow{6}{*}{1905.35} & \multirow{6}{*}{217.90} & \multirow{6}{*}{11.44} & 2.73 & \multirow{6}{*}{3.12} & & & -5.96 & & & \\
\hline & $4-1$ & 1977.59 & & & & 2.99 & & & & -6.61 & & & \\
\hline & $5-1$ & 2278.62 & & & & 4.24 & & & & -5.37 & & & \\
\hline & $6-4$ & 1996.92 & & & & 3.82 & & 0.68 & 21.94 & -5.23 & -6.27 & 0.82 & 13.13 \\
\hline & $7-3$ & 1673.23 & & & & 2.40 & & & & -6.96 & & & \\
\hline & $8-2$ & 1878.46 & & & & 2.51 & & & & -7.49 & & & \\
\hline & $3-3$ & 2137.28 & & & & 4.87 & & & & -4.39 & & & \\
\hline & $4-2$ & 1934.08 & & & & 3.05 & & & & -6.34 & & & \\
\hline & $5-2$ & 1840.01 & & & & 2.73 & & & & -6.75 & & & \\
\hline 5D/85C & $6-2$ & 1773.24 & 1909.51 & 144.89 & 7.59 & 2.43 & 3.15 & 0.87 & 27.63 & -7.31 & -6.36 & 1.05 & 16.56 \\
\hline & $7-4$ & 1729.70 & & & & 2.29 & & & & -7.54 & & & \\
\hline & $8-3$ & 2042.75 & & & & 3.52 & & & & -5.80 & & & \\
\hline
\end{tabular}


Table D.10. Six Months Shelf Aging for PM13

\begin{tabular}{|c|c|c|c|c|c|c|c|c|c|c|c|c|c|}
\hline \multicolumn{2}{|c|}{ SH6 PM13 } & \multicolumn{4}{|c|}{ Fracture Energy } & \multicolumn{4}{|c|}{ Flexibility Index } & \multicolumn{4}{|c|}{ Slope } \\
\hline Aging Condition & Specimen ID & $\begin{array}{c}\text { Energy } \\
\text { (LLD) (Gf) } \\
\text { (J/m2) }\end{array}$ & Average & Std Dev & $\operatorname{CoV}(\%)$ & $\begin{array}{l}\text { Flexibility } \\
\text { Index }\end{array}$ & Average & Std Dev & CoV (\%) & Slope & Average & Std Dev & CoV (\%) \\
\hline \multirow{6}{*}{$3 D / 95 C$} & $1-4$ & 2018.71 & \multirow{6}{*}{2012.12} & \multirow{6}{*}{218.70} & \multirow{6}{*}{10.87} & 6.99 & \multirow{6}{*}{5.65} & \multirow{6}{*}{1.03} & \multirow{6}{*}{18.21} & -2.89 & \multirow{6}{*}{-3.64} & \multirow{6}{*}{0.47} & \multirow{6}{*}{12.93} \\
\hline & $4-2$ & 2331.75 & & & & 6.64 & & & & -3.51 & & & \\
\hline & $5-1$ & 1802.65 & & & & 4.11 & & & & -4.39 & & & \\
\hline & $6-1$ & 1877.83 & & & & 4.74 & & & & -3.96 & & & \\
\hline & $6-3$ & 2268.57 & & & & 6.13 & & & & -3.70 & & & \\
\hline & $8-1$ & 1773.18 & & & & 5.28 & & & & -3.36 & & & \\
\hline \multirow{6}{*}{$5 \mathrm{D} / 85 \mathrm{C}$} & $1-2$ & 2019.80 & \multirow{6}{*}{2010.34} & \multirow{6}{*}{173.28} & \multirow{6}{*}{8.62} & 5.06 & \multirow{6}{*}{5.45} & \multirow{6}{*}{1.14} & \multirow{6}{*}{21.00} & -3.99 & \multirow{6}{*}{-3.79} & \multirow{6}{*}{0.52} & \multirow{6}{*}{13.85} \\
\hline & $2-4$ & 2347.65 & & & & 7.85 & & & & -2.99 & & & \\
\hline & $4-4$ & 1936.43 & & & & 5.39 & & & & -3.59 & & & \\
\hline & $6-2$ & 1792.22 & & & & 5.30 & & & & -3.38 & & & \\
\hline & $7-3$ & 2059.61 & & & & 4.90 & & & & -4.20 & & & \\
\hline & $8-3$ & 1906.30 & & & & 4.18 & & & & -4.56 & & & \\
\hline
\end{tabular}

Table D.11. Twelve Months Shelf Aging for PM1

\begin{tabular}{|c|c|c|c|c|c|c|c|c|c|c|c|c|c|}
\hline \multicolumn{2}{|c|}{ SH12 PM1 } & \multicolumn{4}{|c|}{ Fracture Energy } & \multicolumn{4}{|c|}{ Flexibility Index } & \multicolumn{4}{|c|}{ Slope } \\
\hline Aging Condition & Specimen ID & $\begin{array}{c}\text { Energy } \\
\text { (LLD) (Gf) } \\
\text { (J/m2) }\end{array}$ & Average & Std Dev & $\operatorname{CoV}(\%)$ & $\begin{array}{l}\text { Flexibility } \\
\text { Index }\end{array}$ & Average & Std Dev & $\operatorname{CoV}(\%)$ & Slope & Average & Std Dev & $\operatorname{CoV}(\%)$ \\
\hline \multirow{6}{*}{ Unaged } & $1-2$ & 1422.24 & \multirow{6}{*}{1415.14} & \multirow{6}{*}{96.85} & \multirow{6}{*}{6.84} & 1.70 & \multirow{6}{*}{1.32} & \multirow{6}{*}{0.27} & \multirow{6}{*}{20.25} & -8.38 & \multirow{6}{*}{-11.15} & \multirow{6}{*}{2.32} & \multirow{6}{*}{20.84} \\
\hline & $1-4$ & 1319.03 & & & & 1.00 & & & & -13.21 & & & \\
\hline & $2-3$ & 1502.93 & & & & 1.24 & & & & -12.14 & & & \\
\hline & $2-4$ & 1256.28 & & & & 1.68 & & & & -7.47 & & & \\
\hline & $3-3$ & 1520.07 & & & & 1.15 & & & & -13.17 & & & \\
\hline & $3-4$ & 1470.31 & & & & 1.18 & & & & -12.51 & & & \\
\hline \multirow{6}{*}{$1 \mathrm{D} / 95 \mathrm{C}$} & $1-1$ & 1145.29 & \multirow{6}{*}{1306.14} & \multirow{6}{*}{176.73} & \multirow{6}{*}{13.53} & 0.17 & \multirow{6}{*}{0.53} & \multirow{6}{*}{0.45} & \multirow{6}{*}{84.47} & -68.58 & \multirow{6}{*}{-66.11} & \multirow{6}{*}{57.51} & \multirow{6}{*}{87.00} \\
\hline & $1-3$ & 1096.15 & & & & 0.10 & & & & -114.11 & & & \\
\hline & $2-1$ & 1566.68 & & & & 0.97 & & & & -16.17 & & & \\
\hline & $2-2$ & 1320.11 & & & & 0.68 & & & & -19.47 & & & \\
\hline & $3-1$ & 1204.76 & & & & 0.07 & & & & -165.99 & & & \\
\hline & $3-2$ & 1503.83 & & & & 1.22 & & & & -12.33 & & & \\
\hline
\end{tabular}

Table D.12. Twelve Months Shelf Aging for PM2

\begin{tabular}{|c|c|c|c|c|c|c|c|c|c|c|c|c|c|}
\hline \multicolumn{2}{|c|}{ SH12 PM2 } & \multicolumn{4}{|c|}{ Fracture Energy } & \multicolumn{4}{|c|}{ Flexibility Index } & \multicolumn{4}{|c|}{ Slope } \\
\hline Aging Condition & Specimen ID & $\begin{array}{c}\text { Energy } \\
\text { (LLD) (Gf) } \\
(\mathrm{J} / \mathrm{m} 2)\end{array}$ & Average & Std Dev & $\operatorname{CoV}(\%)$ & $\begin{array}{l}\text { Flexibility } \\
\text { Index }\end{array}$ & Average & Std Dev & $\operatorname{CoV}(\%)$ & Slope & Average & Std Dev & $\operatorname{CoV}(\%)$ \\
\hline \multirow{6}{*}{ Unaged } & $1-3$ & 2521.98 & \multirow{6}{*}{2556.86} & \multirow{6}{*}{142.62} & \multirow{6}{*}{5.58} & 7.14 & \multirow{6}{*}{7.61} & \multirow{6}{*}{1.98} & \multirow{6}{*}{26.00} & -3.53 & \multirow{6}{*}{-3.52} & \multirow{6}{*}{0.60} & \multirow{6}{*}{17.14} \\
\hline & $2-1$ & 2733.67 & & & & 8.28 & & & & -3.30 & & & \\
\hline & $3-4$ & 2460.42 & & & & 6.67 & & & & -3.69 & & & \\
\hline & $4-4$ & 2354.41 & & & & 5.44 & & & & -4.33 & & & \\
\hline & $5-1$ & 2519.23 & & & & 6.51 & & & & -3.87 & & & \\
\hline & $6-4$ & 2751.42 & & & & 11.61 & & & & -2.37 & & & \\
\hline \multirow{6}{*}{$1 \mathrm{D} / 95 \mathrm{C}$} & $1-2$ & 2808.67 & \multirow{6}{*}{2374.33} & \multirow{6}{*}{208.54} & \multirow{6}{*}{8.78} & 6.52 & \multirow{6}{*}{4.89} & \multirow{6}{*}{0.93} & \multirow{6}{*}{18.96} & -4.31 & \multirow{6}{*}{-4.97} & \multirow{6}{*}{0.64} & \multirow{6}{*}{12.89} \\
\hline & $2-4$ & 2296.29 & & & & 5.38 & & & & -4.27 & & & \\
\hline & $3-3$ & 2445.44 & & & & 5.08 & & & & -4.81 & & & \\
\hline & 4-1 & 2243.87 & & & & 3.72 & & & & -6.04 & & & \\
\hline & $5-4$ & 2222.05 & & & & 4.00 & & & & -5.55 & & & \\
\hline & $6-3$ & 2229.68 & & & & 4.64 & & & & -4.81 & & & \\
\hline \multirow{6}{*}{$3 \mathrm{D} / 95 \mathrm{C}$} & $1-4$ & 2604.76 & \multirow{6}{*}{2159.09} & \multirow{6}{*}{344.80} & \multirow{6}{*}{15.97} & 4.64 & \multirow{6}{*}{3.45} & & & -5.61 & & & \\
\hline & $2-3$ & 1479.49 & & & & 2.99 & & & & -4.95 & & & \\
\hline & 3-1 & 2343.94 & & & & 4.09 & & 0.82 & 23.73 & -5.73 & -6.53 & 163 & 2501 \\
\hline & $4-2$ & 2068.97 & & & & 2.08 & & 0.02 & 23.13 & -9.96 & -0.53 & 1.03 & 20.01 \\
\hline & $5-2$ & 2207.75 & & & & 3.24 & & & & -6.82 & & & \\
\hline & $6-2$ & 2249.61 & & & & 3.68 & & & & -6.11 & & & \\
\hline & $1-1$ & 2025.28 & & & & 2.93 & & & & -6.91 & & & \\
\hline & $2-2$ & 2174.98 & & & & 3.31 & & & & -6.58 & & & \\
\hline $5 \mathrm{D} / 85 \mathrm{C}$ & $3-2$ & 1930.67 & 2177.69 & 375.42 & 17.24 & 2.51 & & & & -7.69 & & & 5.51 \\
\hline $5 D / 85 C$ & $4-3$ & 1908.78 & 2171.69 & $3 / 5.42$ & 17.24 & 2.90 & 3.17 & 0.62 & 19.60 & -6.58 & -6.91 & 0.38 & 5.51 \\
\hline & $5-3$ & 2994.84 & & & & 4.46 & & & & -6.71 & & & \\
\hline & $6-1$ & 2031.58 & & & & 2.92 & & & & -6.96 & & & \\
\hline
\end{tabular}


Table D.13. Twelve Months Shelf Aging for PM3

\begin{tabular}{|c|c|c|c|c|c|c|c|c|c|c|c|c|c|}
\hline \multicolumn{2}{|c|}{ SH12 PM3 } & \multicolumn{4}{|c|}{ Fracture Energy } & \multicolumn{4}{|c|}{ Flexibility Index } & \multicolumn{4}{|c|}{ Slope } \\
\hline Aging Condition & Specimen ID & $\begin{array}{c}\text { Energy } \\
\text { (LLD) (Gf) } \\
(\mathrm{J} / \mathrm{m} 2)\end{array}$ & Average & Std Dev & $\operatorname{CoV}(\%)$ & $\begin{array}{l}\text { Flexibility } \\
\text { Index }\end{array}$ & Average & Std Dev & $\operatorname{CoV}(\%)$ & Slope & Average & Std Dev & $\operatorname{CoV}(\%)$ \\
\hline \multirow{7}{*}{ Unaged } & $1-4$ & 2758.84 & \multirow{7}{*}{2738.14} & \multirow{7}{*}{124.93} & \multirow{7}{*}{4.56} & 8.51 & \multirow{7}{*}{9.34} & \multirow{7}{*}{1.32} & \multirow{7}{*}{14.17} & -3.24 & \multirow{7}{*}{-2.98} & \multirow{7}{*}{0.39} & \multirow{7}{*}{12.99} \\
\hline & $2-1$ & 2744.61 & & & & 10.76 & & & & -2.55 & & & \\
\hline & $3-3$ & 2990.88 & & & & 9.81 & & & & -3.05 & & & \\
\hline & $4-2$ & 2790.86 & & & & 10.73 & & & & -2.60 & & & \\
\hline & $4-4$ & 2663.24 & & & & 10.40 & & & & -2.56 & & & \\
\hline & $5-1$ & 2568.59 & & & & 7.78 & & & & -3.30 & & & \\
\hline & $6-3$ & 2649.96 & & & & 7.38 & & & & -3.59 & & & \\
\hline \multirow{7}{*}{$1 \mathrm{D} / 95 \mathrm{C}$} & $1-2$ & 2583.38 & \multirow{7}{*}{2500.15} & \multirow{7}{*}{186.51} & \multirow{7}{*}{7.46} & 6.76 & \multirow{7}{*}{5.51} & \multirow{7}{*}{0.84} & \multirow{7}{*}{15.25} & -3.82 & \multirow{7}{*}{-4.63} & \multirow{7}{*}{0.67} & \multirow{7}{*}{14.50} \\
\hline & $2-2$ & 2881.50 & & & & 5.80 & & & & -4.97 & & & \\
\hline & $3-1$ & 2435.60 & & & & 6.44 & & & & -3.78 & & & \\
\hline & $4-3$ & 2244.73 & & & & 5.06 & & & & -4.44 & & & \\
\hline & $5-2$ & 2387.03 & & & & 5.08 & & & & -4.70 & & & \\
\hline & $6-1$ & 2549.40 & & & & 5.29 & & & & -4.82 & & & \\
\hline & $7-4$ & 2419.39 & & & & 4.11 & & & & -5.89 & & & \\
\hline \multirow{7}{*}{$3 \mathrm{D} / 95 \mathrm{C}$} & $1-3$ & 2588.85 & \multirow{7}{*}{2374.54} & \multirow{7}{*}{263.54} & & 4.38 & & & & -5.91 & & & \\
\hline & $2-3$ & 2299.52 & & & & 4.56 & & & & -5.04 & & & \\
\hline & $3-2$ & 1798.27 & & & & 2.89 & & & & -6.23 & & & \\
\hline & $4-1$ & 2328.49 & & & 11.10 & 4.20 & 4.45 & 0.90 & 20.14 & -5.54 & -5.46 & 0.66 & 12.16 \\
\hline & $5-3$ & 2546.93 & & & & 5.84 & & & & -4.36 & & & \\
\hline & $6-2$ & 2423.90 & & & & 3.89 & & & & -6.23 & & & \\
\hline & $7-1$ & 2635.81 & & & & 5.37 & & & & -4.91 & & & \\
\hline & $1-1$ & 2381.84 & & & & 3.43 & & & & -6.94 & & & \\
\hline & $2-4$ & 2682.52 & & & & 5.21 & & & & -5.15 & & & \\
\hline & $3-4$ & 2106.70 & & & & 3.19 & & & & -6.61 & & & \\
\hline $5 \mathrm{D} / 85 \mathrm{C}$ & $5-4$ & 2647.08 & 2450.28 & 193.49 & 7.90 & 5.79 & 4.42 & 0.97 & 21.98 & -4.57 & -5.74 & 0.90 & 15.70 \\
\hline & $6-4$ & 2581.58 & & & & 4.83 & & & & -5.35 & & & \\
\hline & $7-2$ & 2472.16 & & & & 5.06 & & & & -4.89 & & & \\
\hline & $7-3$ & 2280.09 & & & & 3.41 & & & & -6.69 & & & \\
\hline
\end{tabular}




\section{APPENDIX E: R27-161 MIXES FIELD CORE I-FIT RESULTS}

Table E.1. Field Core Results for R27-161 Project

\begin{tabular}{|c|c|c|c|c|c|c|c|c|c|c|c|c|c|}
\hline \multicolumn{2}{|c|}{ R27-161 Cores } & \multicolumn{4}{|c|}{ Fracture Energy } & \multicolumn{4}{|c|}{ Slope } & \multicolumn{4}{|c|}{ Flexibility Index } \\
\hline Mix & Specimen & $\begin{array}{c}\text { Energy (LLD) } \\
\text { (Gf) }(\mathrm{J} / \mathrm{m} 2)\end{array}$ & Average & Std Dev & $\operatorname{CoV}(\%)$ & Slope & Average & Std Dev & $\operatorname{CoV}(\%)$ & $\mathrm{FI}$ & Average & Std Dev & $\operatorname{CoV}(\%)$ \\
\hline \multirow{4}{*}{$140 Y 02$} & B1 & 1844.1 & \multirow{4}{*}{1802.5} & \multirow{4}{*}{123.0} & \multirow{4}{*}{6.8} & -2.65 & \multirow{4}{*}{-2.69} & \multirow{4}{*}{0.28} & \multirow{4}{*}{10.31} & 7.0 & \multirow{4}{*}{6.8} & \multirow{4}{*}{0.87} & \multirow{4}{*}{12.88} \\
\hline & B2 & 1975.0 & & & & -2.73 & & & & 7.2 & & & \\
\hline & T1 & 1640.0 & & & & -3.08 & & & & 5.3 & & & \\
\hline & T2 & 1751.0 & & & & -2.30 & & & & 7.6 & & & \\
\hline \multirow{4}{*}{$156 Y 03$} & B1 & 2207.9 & \multirow{4}{*}{2086.8} & \multirow{4}{*}{111.8} & \multirow{4}{*}{5.4} & -3.52 & \multirow{4}{*}{-4.50} & \multirow{4}{*}{0.59} & \multirow{4}{*}{13.23} & 6.3 & \multirow{4}{*}{4.8} & \multirow{4}{*}{0.93} & \multirow{4}{*}{19.34} \\
\hline & B2 & 2014.5 & & & & -4.75 & & & & 4.3 & & & \\
\hline & $\mathrm{T} 1$ & 2182.6 & & & & -4.59 & & & & 4.8 & & & \\
\hline & T2 & 1942.3 & & & & -5.12 & & & & 3.8 & & & \\
\hline \multirow{4}{*}{$157 Y 03$} & B1 & 1709.1 & \multirow{4}{*}{1911.6} & \multirow{4}{*}{141.8} & \multirow{4}{*}{7.4} & -6.01 & \multirow{4}{*}{-5.46} & & & 2.8 & & & \\
\hline & B2 & 1985.0 & & & & -5.15 & & 34 & 619 & 3.9 & 35 & 045 & 1278 \\
\hline & $\mathrm{T} 1$ & 2089.6 & & & & -5.22 & & 0.34 & 6.19 & 4.0 & 3.5 & 0.45 & 12.78 \\
\hline & T2 & 1862.5 & & & & -5.44 & & & & 3.4 & & & \\
\hline & B1 & 2025.6 & & & & -2.23 & & & & 9.1 & & & \\
\hline $150 \mathrm{Y} 2$ & B2 & 2045.6 & 21383 & 2820 & 120 & -2.03 & & & & 10.1 & & & \\
\hline $159 y 02$ & $\mathrm{~T} 1$ & 1869.7 & 2138.3 & 282.0 & 13.2 & -2.72 & -2.16 & 0.38 & 17.54 & 6.9 & 10.4 & 3.21 & 30.87 \\
\hline & T2 & 2612.2 & & & & -1.67 & & & & 15.6 & & & \\
\hline & B1 & 1846.0 & & & & -1.52 & & & & 12.1 & & & \\
\hline & B2 & 1628.0 & & & & -2.28 & & & & 7.2 & & & \\
\hline $159 Y 04$ & $\mathrm{~T} 1$ & 1671.7 & 1747.1 & 98.5 & 5.6 & -2.12 & -1.75 & 0.48 & 27.64 & 7.9 & 11.1 & 4.04 & 36.30 \\
\hline & T2 & 1842.7 & & & & -1.07 & & & & 17.3 & & & \\
\hline & B1 & 1929.1 & & & & -1.82 & & & & 10.6 & & & \\
\hline $177 Y \bigcirc 4$ & B2 & 1874.5 & 10153 & 081 & 51 & -1.78 & 180 & 0.15 & 829 & 10.5 & 107 & 039 & 362 \\
\hline $17 / 704$ & $\mathrm{~T} 1$ & 2063.6 & 1915.3 & 98.1 & 5.1 & -2.01 & -1.80 & 0.15 & 8.29 & 10.2 & 10.7 & 0.39 & 3.02 \\
\hline & T2 & 1794.2 & & & & -1.59 & & & & 11.3 & & & \\
\hline
\end{tabular}

Table E.2. One Year Field Core Results for R27-161 Project

\begin{tabular}{|c|c|c|c|c|c|c|c|c|c|c|c|c|c|}
\hline \multicolumn{2}{|c|}{1 Year R27-161 } & \multirow{2}{*}{\begin{tabular}{|l} 
Thickness \\
Thickness
\end{tabular}} & \multirow{2}{*}{$\begin{array}{c}\text { Air void } \\
\text { AV }\end{array}$} & \multicolumn{2}{|c|}{ Fracture Energy } & \multicolumn{2}{|c|}{ Slope } & \multicolumn{3}{|c|}{ Flexibility Index } & \multicolumn{3}{|c|}{ Corrected FI } \\
\hline Mix & Specimen & & & $\begin{array}{c}\text { Energy (LLD) } \\
\text { (Gf) }(\mathrm{J} / \mathrm{m} 2)\end{array}$ & $\operatorname{CoV}(\%)$ & Slope & $\operatorname{CoV}(\%)$ & $\mathrm{FI}$ & FI Avg. & $\operatorname{CoV}(\%)$ & FI Corr & $\begin{array}{c}\text { FI Corr. } \\
\text { Avg }\end{array}$ & $\operatorname{CoV}(\%)$ \\
\hline \multirow{4}{*}{ 140Y02 } & EB21-1 & 32.98 & $7.06 \%$ & 2081.9 & \multirow{4}{*}{10.23} & -1.17 & \multirow{4}{*}{14.54} & 17.8 & \multirow{4}{*}{19.0} & \multirow{4}{*}{23.58} & 11.6 & \multirow{4}{*}{12.6} & \multirow{4}{*}{2521} \\
\hline & EB21-2 & 32.99 & $7.91 \%$ & 2181.0 & & -1.05 & & 20.8 & & & 12.3 & & \\
\hline & EB27-1 & 32.7 & $6.39 \%$ & 2269.5 & & -0.95 & & 23.9 & & & 17.0 & & \\
\hline & EB27-2 & 33.12 & $6.52 \%$ & 1781.0 & & -1.33 & & 13.4 & & & 9.5 & & \\
\hline \multirow{5}{*}{$156 \mathrm{Y} 03$} & $6-1$ & 30.49 & $7.34 \%$ & 2244.1 & \multirow{5}{*}{14.91} & -2.56 & \multirow{5}{*}{19.20} & 8.8 & \multirow{5}{*}{8.0} & \multirow{5}{*}{14.82} & 5.1 & \multirow{5}{*}{4.7} & \multirow{5}{*}{15.55} \\
\hline & $6-2$ & 30.2 & $7.26 \%$ & 1780.1 & & -2.81 & & 6.3 & & & 3.7 & & \\
\hline & $3-1$ & 32.45 & $7.80 \%$ & 1633.8 & & -2.09 & & 7.8 & & & 4.6 & & \\
\hline & $3-2$ & 31.98 & $7.80 \%$ & 1683.9 & & -221 & & 7.6 & & & 4.4 & & \\
\hline & $7-1$ & 32.14 & $7.60 \%$ & 1586.1 & & -1.68 & & 9.4 & & & 5.6 & & \\
\hline \multirow{4}{*}{$157 Y 03$} & $25-1$ & 30.63 & $4.92 \%$ & 2047.9 & \multirow{4}{*}{11.58} & -1.92 & \multirow{4}{*}{33.39} & 10.7 & & & 9.1 & & \\
\hline & $25--2$ & 30.63 & $4.51 \%$ & 2527.2 & & -1.43 & & 17.7 & & & 16.4 & & \\
\hline & $29-1$ & 30 & $4.66 \%$ & 1975.8 & & -3.09 & & 6.4 & 71.3 & 41.29 & 5.6 & 10.3 & 43.33 \\
\hline & $29--2$ & 30.47 & $4.25 \%$ & 2082.7 & & -1.97 & & 10.6 & & & 10.3 & & \\
\hline & WB5--1 & 32.46 & $8.47 \%$ & 2245.6 & & -1.62 & & 13.9 & & & 7.6 & & \\
\hline & WB5--2 & 31.97 & $8.38 \%$ & 2003.0 & & -2.05 & & 9.8 & & & 5.3 & & \\
\hline $159 Y 02$ & Wb12-1 & 31.17 & $8.12 \%$ & 2005.5 & 16.11 & -2.02 & 14.87 & 9.9 & 12.9 & 30.63 & 5.4 & 7.0 & 29.99 \\
\hline & Wb12-2 & 31.31 & $8.29 \%$ & 2775.6 & & -1.53 & & 18.1 & & & 9.7 & & \\
\hline & $18-1$ & 33.07 & $5.97 \%$ & 1813.5 & & -0.52 & & 34.9 & & & 26.8 & & \\
\hline & $18--2$ & 32.19 & $6.12 \%$ & 1615.7 & & -0.54 & & 29.9 & & & 21.8 & & \\
\hline 159Y04 & $30-1$ & 36.15 & $4.87 \%$ & 1660.1 & 8.83 & -0.64 & 17.65 & 25.9 & 36.5 & 25.10 & 26.3 & 34.0 & 37.46 \\
\hline & $28--1$ & 30.05 & $4.30 \%$ & 1937.9 & & -0.42 & & 46.1 & & & 43.8 & & \\
\hline & $28--2$ & 30.48 & $3.69 \%$ & 1968.0 & & -0.43 & & 45.8 & & & 51.1 & & \\
\hline & $6-1$ & 30.69 & $2.69 \%$ & 1660.2 & & -0.97 & & 17.1 & & & 26.1 & & \\
\hline & $6-2$ & 30.51 & $2.71 \%$ & 1837.0 & & -1.11 & & 16.5 & & & 25.0 & & \\
\hline $177 Y 04$ & $4-1$ & 34.12 & $3.08 \%$ & 1753.1 & 735 & -1.09 & 7.17 & 16.1 & 170 & 716 & 24.0 & 241 & 1057 \\
\hline $177 \mathrm{ru}$ & $4-2$ & 34 & $3.11 \%$ & 1767.1 & 1.50 & -0.97 & 1.11 & 18.2 & 11.0 & 1.10 & 26.8 & 24.1 & 10.51 \\
\hline & $16-1$ & 36.85 & $4.10 \%$ & 2054.4 & & -1.10 & & 18.7 & & & 22.8 & & \\
\hline & $16--2$ & 36.41 & $3.88 \%$ & 1788.6 & & -1.15 & & 15.6 & & & 19.8 & & \\
\hline & SB3-1 & 31.62 & $1.82 \%$ & 2417.6 & & -1.44 & & 16.8 & & & 38.6 & & \\
\hline & SB3--2 & 30.79 & $2.15 \%$ & 2636.7 & & -1.44 & & 18.3 & & & 35.0 & & \\
\hline $306 \mathrm{M} 30$ & NB16-1 & 30.89 & $1.91 \%$ & 2673.6 & 425 & -1.14 & 917 & 23.5 & 189 & 1233 & 50.2 & 388 & 1765 \\
\hline 30 blVIS0 & NB16--2 & 31.52 & $1.72 \%$ & 2632.9 & 4.25 & -1.49 & 9.11 & 17.7 & 18.9 & 12.33 & 43.0 & 38.8 & 17.65 \\
\hline & NB1-1 & 30.45 & $2.40 \%$ & 2549.5 & & -1.38 & & 18.5 & & & 31.3 & & \\
\hline & NB1-2 & 30.85 & $2.23 \%$ & 2734.4 & & -1.45 & & 18.9 & & & 34.8 & & \\
\hline
\end{tabular}


Table E.3. Two Year Field Core Results for R27-161 Project

\begin{tabular}{|c|c|c|c|c|c|c|c|c|c|c|c|c|c|}
\hline \multicolumn{2}{|c|}{2 Year R27-161 } & \multirow{2}{*}{\begin{tabular}{|l} 
Thickness \\
Thickness
\end{tabular}} & \multirow{2}{*}{$\begin{array}{c}\text { Air void } \\
\text { AV }\end{array}$} & \multicolumn{2}{|c|}{ Fracture Energy } & \multicolumn{2}{|c|}{ Slope } & \multicolumn{3}{|c|}{ Flexibility Index } & \multicolumn{3}{|c|}{ Corrected FI } \\
\hline Mix & Specimen & & & $\begin{array}{c}\text { Energy } \\
\text { (LLD) (Gf) } \\
(\mathrm{J} / \mathrm{m} 2)\end{array}$ & $\operatorname{CoV}(\%)$ & Slope & $\operatorname{CoV}(\%)$ & $\mathrm{FI}$ & FI Avg. & $\operatorname{CoV}(\%)$ & FI Corr & $\begin{array}{c}\text { FI Corr. } \\
\text { Avg }\end{array}$ & $\operatorname{CoV}(\%)$ \\
\hline \multirow{4}{*}{ 140Y02 } & $5--1$ & 30.75 & $4.30 \%$ & 2308.47 & \multirow{4}{*}{6.51} & -0.83 & \multirow{4}{*}{19.25} & 27.75 & \multirow{4}{*}{19.89} & \multirow{4}{*}{26.88} & 27.0 & \multirow{4}{*}{20.48} & \multirow{4}{*}{22.99} \\
\hline & $5--2$ & 30.57 & $3.84 \%$ & 1986.40 & & -1.08 & & 18.39 & & & 19.8 & & \\
\hline & $12--1$ & 32.10 & $3.98 \%$ & 2059.16 & & -1.17 & & 17.58 & & & 19.2 & & \\
\hline & $12--2$ & 32.60 & $4.43 \%$ & 2118.11 & & -1.34 & & 15.83 & & & 15.8 & & \\
\hline \multirow{4}{*}{ 156Y03 } & $2--1$ & 28.05 & $3.90 \%$ & 2072.52 & \multirow{4}{*}{7.91} & -2.77 & \multirow{4}{*}{13.19} & 7.49 & \multirow{4}{*}{8.14} & \multirow{4}{*}{20.02} & 7.3 & \multirow{4}{*}{7.70} & \multirow{4}{*}{15.23} \\
\hline & $2--2$ & 28.52 & $4.09 \%$ & 2186.14 & & -3.23 & & 6.77 & & & 6.4 & & \\
\hline & $10--1$ & 30.30 & $4.05 \%$ & 2360.56 & & -3.03 & & 7.80 & & & 7.9 & & \\
\hline & $10--2$ & 30.40 & $4.74 \%$ & 2477.29 & & -2.36 & & 10.50 & & & 9.2 & & \\
\hline \multirow{8}{*}{ 157Y03 } & $19--1$ & 30.38 & $3.71 \%$ & 1773.16 & \multirow{8}{*}{20.84} & -2.41 & \multirow{8}{*}{21.64} & 7.35 & \multirow{8}{*}{6.64} & \multirow{8}{*}{22.74} & 8.1 & \multirow{8}{*}{7.35} & \\
\hline & $19--2$ & 30.66 & $3.81 \%$ & 2428.78 & & -1.79 & & & & & & & \\
\hline & $22--1$ & 30.85 & $4.50 \%$ & 2031.93 & & -2.46 & & 8.25 & & & 7.7 & & \\
\hline & $22--2$ & 30.66 & $4.63 \%$ & 1929.90 & & -2.98 & & 6.47 & & & 5.9 & & \\
\hline & $25--1$ & 30.93 & $4.29 \%$ & 1426.01 & & -3.62 & & 3.94 & & & 3.9 & & 38.41 \\
\hline & $25--2$ & 30.73 & $5.09 \%$ & 1289.31 & & -2.33 & & 5.52 & & & 4.6 & & \\
\hline & $28--1$ & 30.16 & $2.72 \%$ & 2153.01 & & -2.68 & & 8.02 & & & 11.9 & & \\
\hline & $28--2$ & 30.12 & $2.98 \%$ & 2272.49 & & -3.29 & & 6.91 & & & 9.4 & & \\
\hline & $24--1$ & 27.14 & $8.28 \%$ & 1770.86 & & -1.27 & & & & & & & \\
\hline & $24--2$ & 26.96 & $8.27 \%$ & 1451.63 & & -2.03 & & 7.16 & & & 3.3 & & \\
\hline & $32--1$ & 29.82 & $7.44 \%$ & 1295.57 & & -2.28 & & 5.69 & & & 3.2 & & \\
\hline $159 y 0 ?$ & $32--2$ & 30.55 & $7.68 \%$ & 1275.70 & 1218 & -1.76 & 1879 & 7.27 & 672 & 1325 & 4.1 & 333 & 1315 \\
\hline $159 Y 0 \angle$ & $20--1$ & 24.62 & $8.26 \%$ & 1299.68 & 12.10 & -2.00 & 10.19 & 6.50 & 0.12 & 13.20 & 2.8 & 3.30 & 13.10 \\
\hline & $20--2$ & 24.67 & $7.65 \%$ & 1442.02 & & -1.79 & & 8.07 & & & 3.7 & & \\
\hline & $22--1$ & 28.05 & $7.43 \%$ & 1319.67 & & -2.37 & & 5.57 & & & 3.0 & & \\
\hline & $22--2$ & 28.65 & $8.24 \%$ & 1586.28 & & -2.35 & & 6.76 & & & 3.3 & & \\
\hline & $3--1$ & 30.73 & $4.38 \%$ & 1917.00 & & -0.57 & & 33.51 & & & 32.1 & & \\
\hline $159 Y 04$ & $3--2$ & 30.98 & $4.53 \%$ & 2435.59 & 1209 & -0.40 & & & & & & 3312 & 441 \\
\hline $159 Y 04$ & $15--1$ & 30.77 & $4.83 \%$ & 2076.24 & 12.09 & -0.52 & 15.96 & 40.00 & 35.33 & 11.51 & 34.8 & 33.12 & 4.41 \\
\hline & $15--2$ & 30.59 & $4.17 \%$ & 1889.03 & & -0.58 & & 32.46 & & & 32.5 & & \\
\hline
\end{tabular}


Table E.4. Three Year Field Core Results for R27-161 Project

\begin{tabular}{|c|c|c|c|c|c|c|c|c|c|c|c|c|c|}
\hline \multicolumn{2}{|c|}{3 Year R27-161 } & \multirow{2}{*}{\begin{tabular}{|l|} 
Thickness \\
Thickness
\end{tabular}} & \multirow{2}{*}{$\begin{array}{r}\text { Air void } \\
\text { AV }\end{array}$} & \multicolumn{2}{|c|}{ Fracture Energy } & \multicolumn{2}{|c|}{ Slope } & \multicolumn{3}{|c|}{ Flexibility Index } & \multicolumn{3}{|c|}{ Corrected FI } \\
\hline Mix & Specimen & & & $\begin{array}{c}\text { Energy } \\
\text { (LLD) (Gf) } \\
(\mathrm{J} / \mathrm{m} 2)\end{array}$ & $\operatorname{CoV}(\%)$ & Slope & $\operatorname{CoV}(\%)$ & $\mathrm{FI}$ & FI Avg. & $\operatorname{CoV}(\%)$ & FI Corr & $\begin{array}{c}\text { FI Corr. } \\
\text { Avg }\end{array}$ & $\operatorname{CoV}(\%)$ \\
\hline \multirow{6}{*}{ 140Y02 } & 140Y02-12-1 & 31.83 & $4.32 \%$ & 2091.78 & \multirow{6}{*}{15.20} & -1.70 & \multirow{6}{*}{12.45} & 12.34 & \multirow{6}{*}{12.95} & \multirow{6}{*}{15.33} & 12.3 & \multirow{6}{*}{12.76} & \multirow{6}{*}{13.77} \\
\hline & 140Y02-12-2 & 31.95 & $4.42 \%$ & 2282.27 & & -1.62 & & 14.09 & & & 13.9 & & \\
\hline & 140Y02-13-1 & 31.58 & $4.46 \%$ & 2651.08 & & -1.63 & & 16.30 & & & 15.7 & & \\
\hline & 140Y02-13-2 & 31.22 & $4.26 \%$ & 1766.83 & & -1.61 & & 10.99 & & & 10.9 & & \\
\hline & 140Y02-8-1 & 30.57 & $4.27 \%$ & 1851.71 & & -1.46 & & 12.73 & & & 12.3 & & \\
\hline & 140Y02-8-2 & 30.74 & $4.12 \%$ & 2334.02 & & -2.08 & & 11.25 & & & 11.4 & & \\
\hline \multirow{4}{*}{$156 Y 03$} & 156Y03-3-1 & 33.08 & $5.22 \%$ & 1421.59 & \multirow{4}{*}{19.93} & -2.62 & \multirow{4}{*}{16.92} & 5.43 & \multirow{4}{*}{6.19} & \multirow{4}{*}{15.11} & 4.7 & \multirow{4}{*}{5.53} & \multirow{4}{*}{19.62} \\
\hline & 156Y03-3-2 & 32.61 & $5.33 \%$ & 1154.57 & & -2.10 & & 5.49 & & & 4.6 & & \\
\hline & 156Y03-9-1 & 34.13 & $5.01 \%$ & 1536.73 & & -2.07 & & 7.42 & & & 6.9 & & \\
\hline & 156Y03-9-2 & 33.85 & $5.12 \%$ & 1874.61 & & -2.91 & & 6.43 & & & 5.8 & & \\
\hline \multirow{5}{*}{ 157Y03 } & 157Y03-21-1 & 34.87 & $5.09 \%$ & 1278.98 & \multirow{5}{*}{14.65} & -3.78 & \multirow{5}{*}{10.61} & 3.38 & & & 3.2 & & \\
\hline & 157Y03-21-2 & 34.74 & $5.01 \%$ & 1305.07 & & -3.15 & & 4.15 & & & 3.9 & & \\
\hline & 157Y03-28-1 & 33.59 & $5.45 \%$ & 941.79 & & -3.35 & & 2.81 & 3.26 & 19.88 & 2.4 & 2.97 & 25.30 \\
\hline & 157Y03-25-1 & 32.37 & $5.33 \%$ & 986.52 & & -4.00 & & 2.47 & & & 2.1 & & \\
\hline & 157Y03-23-1 & 33.99 & $4.91 \%$ & 1118.09 & & -3.22 & & 3.47 & & & 3.3 & & \\
\hline & 159Y02-28-1 & 29.22 & $6.57 \%$ & 1880.41 & & -2.68 & & 7.02 & & & 4.3 & & \\
\hline & 159Y02-28-2 & 29.10 & $6.04 \%$ & 1465.58 & & -2.21 & & 6.63 & & & 4.4 & & \\
\hline 159Y02 & 159Y02-31-1 & 28.41 & $6.41 \%$ & 1764.62 & 18.07 & -3.94 & 23.89 & 4.48 & 5.85 & 21.58 & 2.8 & 3.74 & 22.34 \\
\hline & 159Y02-31-2 & 29.90 & $6.44 \%$ & 1755.65 & & -3.92 & & 4.48 & & & 2.9 & & \\
\hline & 159Y02-32-2 & 30.01 & $6.53 \%$ & 2380.92 & & -3.57 & & 6.67 & & & 4.3 & & \\
\hline & 159Y04-8-1 & 30.20 & $4.01 \%$ & 2047.62 & & -1.06 & & 19.35 & & & 19.7 & & \\
\hline & 159Y04-8-2 & 29.71 & $3.93 \%$ & 1759.53 & & -1.07 & & 16.41 & & & 16.8 & & \\
\hline 159 Y04 & 159Y04-11-1 & 29.06 & $4.87 \%$ & 2025.67 & 6.92 & -0.94 & 10.26 & 21.48 & 19.81 & 12.80 & 17.6 & 18.04 & 6.76 \\
\hline & 159Y04-11-2 & 29.49 & $4.93 \%$ & 1891.27 & & -0.86 & & 21.99 & & & 18.0 & & \\
\hline & 177Y04-25-1 & 33.40 & $2.77 \%$ & 1946.35 & & -1.83 & & 10.61 & & & 17.2 & & \\
\hline & 177Y04-25-2 & 33.12 & $2.67 \%$ & 1969.83 & & -1.77 & & 11.11 & & & 18.5 & & \\
\hline 177 Y04 & 177Y04-26-1 & 32.64 & $2.14 \%$ & 1908.63 & 2.58 & -1.71 & 11.71 & 11.16 & 11.56 & 10.66 & 22.6 & 20.58 & 15.89 \\
\hline & 177Y04-26-2 & 32.64 & $2.42 \%$ & 1856.47 & & -1.39 & & 13.38 & & & 24.0 & & \\
\hline & $306 \mathrm{M} 30-12-1$ & 32.78 & $2.28 \%$ & 2950.26 & & -2.02 & & 14.59 & & & 28.0 & & \\
\hline & $306 \mathrm{M} 30-12-2$ & 33.04 & $2.46 \%$ & 2550.57 & & -1.56 & & 16.37 & & & 29.3 & & \\
\hline & 306М30-14-1 & 33.13 & $1.81 \%$ & 2981.27 & & -1.72 & & 17.36 & & & 42.1 & & \\
\hline $306 \mathrm{M} 30$ & $306 \mathrm{M} 30-14-2$ & 33.24 & $1.39 \%$ & 3590.03 & 12.13 & -1.60 & 9.63 & 22.42 & 18.33 & 15.64 & 70.6 & 42.68 & 36.16 \\
\hline & 306M30-13-1 & 33.99 & $2.34 \%$ & 3457.75 & & -1.67 & & 20.68 & & & 40.2 & & \\
\hline & $306 \mathrm{M} 30-13-2$ & 33.92 & $1.81 \%$ & 3070.42 & & -1.66 & & 18.55 & & & 45.9 & & \\
\hline
\end{tabular}


Table E.5. Four Year Field Core Results for Project R27-161

\begin{tabular}{|c|c|c|c|c|c|c|c|c|c|c|c|c|c|}
\hline \multicolumn{2}{|c|}{4 Year R27-161 } & \multirow{2}{*}{\begin{tabular}{|l|} 
Thickness \\
Thickness
\end{tabular}} & \multirow{2}{*}{$\begin{array}{r}\text { Air void } \\
\text { AV }\end{array}$} & \multicolumn{3}{|c|}{ Flexibility Index } & \multicolumn{3}{|c|}{ Corrected FI } & \multicolumn{2}{|c|}{ Slope } & \multicolumn{2}{|c|}{ Fracture Energy } \\
\hline Mix & Specimen & & & $\mathrm{FI}$ & FI Avg. & $\operatorname{CoV}(\%)$ & FI Corr & $\begin{array}{c}\text { FI Corr. } \\
\text { Avg }\end{array}$ & $\operatorname{CoV}(\%)$ & Slope & $\operatorname{CoV}(\%)$ & $\begin{array}{c}\text { Energy } \\
(\mathrm{LLD})(\mathrm{Gf}) \\
(\mathrm{J} / \mathrm{m} 2)\end{array}$ & $\operatorname{CoV}(\%)$ \\
\hline \multirow{8}{*}{ 140Y02 } & $3-1$ & 33.2 & 5.1 & 6.3 & \multirow{8}{*}{7.5} & \multirow{8}{*}{11.75} & 5.6 & \multirow{8}{*}{6.2} & \multirow{8}{*}{13.89} & -2.94 & \multirow{8}{*}{18.2} & 1848.99 & \multirow{8}{*}{12.02} \\
\hline & $3-2$ & 34.2 & 5.0 & 8.1 & & & 7.5 & & & -1.83 & & 1479.60 & \\
\hline & $4-1$ & 30.5 & 4.8 & 7.4 & & & 6.4 & & & -2.30 & & 1703.83 & \\
\hline & $4-2$ & 30.7 & 4.9 & 8.1 & & & 6.9 & & & -2.03 & & 1634.81 & \\
\hline & $7-1$ & 29.0 & 5.3 & 6.2 & & & 4.7 & & & -1.99 & & 1234.96 & \\
\hline & $7-2$ & 28.7 & 5.5 & 8.4 & & & 6.0 & & & -1.93 & & 1617.54 & \\
\hline & $8-1$ & 31.7 & 5.1 & 7.0 & & & 6.0 & & & -2.31 & & 1626.75 & \\
\hline & $8-2$ & 31.0 & 5.5 & 8.3 & & & 6.4 & & & -1.71 & & 1416.08 & \\
\hline & $9-1$ & 38.3 & 5.2 & 0.7 & & & 0.7 & & & -18.43 & & 1335.60 & \\
\hline & $9-2$ & 37.5 & 5.0 & 3.1 & & & 3.2 & & & -6.29 & & 1926.91 & \\
\hline & $11-1$ & 41.2 & 5.1 & 0.9 & & & 1.0 & & & -19.18 & & 1662.15 & \\
\hline 156Y03 & $12-1$ & 40.8 & 5.7 & 2.4 & 1.4 & 66.56 & 2.4 & 1.4 & 68.10 & -6.91 & 49.8 & 1676.17 & 17.06 \\
\hline & $12-2$ & 40.1 & 5.5 & 0.8 & & & 0.8 & & & -24.07 & & 1921.08 & \\
\hline & 14-1 & 39.3 & 6.0 & 0.7 & & & 0.7 & & & -22.14 & & 1622.42 & \\
\hline & $14-2$ & 39.9 & 5.9 & 1.3 & & & 1.2 & & & -8.89 & & 1187.80 & \\
\hline & 3-1 & 42.5 & 4.2 & 2.1 & & & 2.9 & & & -5.19 & & 1100.04 & \\
\hline & $3-2$ & 42.0 & 4.5 & 1.2 & & & 1.5 & & & -8.35 & & 1007.68 & \\
\hline & $5-1$ & 41.9 & 4.7 & 2.3 & & & 2.8 & & & -5.79 & & 1303.92 & \\
\hline & $5-2$ & 42.0 & 4.5 & 2.6 & & & 3.3 & & & -5.34 & & 1402.58 & \\
\hline $15 / Y 03$ & $7-1$ & 39.2 & 4.6 & 3.3 & 2.1 & 36.41 & 3.8 & 2.7 & 32.05 & -5.73 & 18.7 & 1880.52 & 32.39 \\
\hline & $7-2$ & 39.3 & 4.2 & 2.6 & & & 3.3 & & & -5.06 & & 1321.47 & \\
\hline & $8-1$ & 42.5 & 4.0 & 1.7 & & & 2.4 & & & -6.34 & & 1064.44 & \\
\hline & $8-2$ & 42.2 & 4.2 & 1.0 & & & 1.4 & & & -5.08 & & 520.01 & \\
\hline & $9-1$ & 29.6 & 4.9 & 1.3 & & & 1.1 & & & -13.13 & & 1749.63 & \\
\hline & $9-2$ & 29.7 & 5.1 & 2.6 & & & 2.1 & & & -6.03 & & 1592.14 & \\
\hline & $10-1$ & 28.8 & 5.0 & 3.5 & & & 2.8 & & & -4.40 & & 1557.54 & \\
\hline & $10-2$ & 28.1 & 5.0 & 2.0 & & & 1.6 & & & -5.60 & & 1133.89 & \\
\hline $159 Y 02$ & $14-1$ & 26.9 & 5.5 & 2.6 & 3.1 & 74.45 & 1.8 & 2.3 & 70.05 & -5.09 & 52.6 & 1312.06 & 34.21 \\
\hline & $14-2$ & 26.3 & 5.0 & 2.5 & & & 1.8 & & & -4.46 & & 1132.54 & \\
\hline & $15-1$ & 27.5 & 5.4 & 8.7 & & & 6.1 & & & -2.95 & & 2565.17 & \\
\hline & $15-2$ & 26.8 & 5.1 & 1.8 & & & 1.3 & & & -5.20 & & 927.73 & \\
\hline & $1-1$ & 32.0 & 4.0 & 15.9 & & & 17.4 & & & -1.10 & & 1745.75 & \\
\hline & $1-2$ & 37.0 & 3.6 & 16.8 & & & 23.5 & & & -1.11 & & 1865.39 & \\
\hline & $2-1$ & 34.0 & 3.5 & 19.4 & & & 25.2 & & & -1.05 & & 2042.05 & \\
\hline & $2-2$ & 33.9 & 3.8 & 13.8 & & & 16.5 & & & -1.27 & & 1746.92 & \\
\hline $159 Y 04$ & $5-1$ & 33.2 & 3.9 & 20.0 & 17.3 & 14.07 & 23.2 & 20.9 & 16.50 & -0.99 & 9.5 & 1980.90 & 6.49 \\
\hline & $5-2$ & 33.2 & 3.8 & 18.6 & & & 21.8 & & & -1.06 & & 1966.36 & \\
\hline & $7-1$ & 33.2 & 3.9 & 14.5 & & & 16.8 & & & -1.28 & & 1859.13 & \\
\hline & $7-2$ & 33.6 & 3.9 & 19.7 & & & 22.9 & & & -1.05 & & 2063.34 & \\
\hline & $9-1$ & 40.3 & 2.9 & 9.4 & & & 17.6 & & & -1.94 & & 1828.99 & \\
\hline & $9-2$ & 42.0 & 2.9 & 5.9 & & & 11.5 & & & -3.19 & & 1891.23 & \\
\hline & $10-1$ & 37.2 & 3.3 & 8.5 & & & 13.0 & & & -2.27 & & 1935.28 & \\
\hline & $10-2$ & 37.1 & 3.1 & 10.3 & 92 & 2053 & 16.3 & 149 & 1691 & -1.95 & 210 & 2003.26 & 460 \\
\hline $1 / 7 Y 04$ & $11-1$ & 41.2 & 3.3 & 7.1 & 9.2 & 20.53 & 11.9 & 14.9 & 16.91 & -2.48 & 21.9 & 1772.36 & 4.60 \\
\hline & $11-2$ & 42.5 & 3.2 & 10.2 & & & 18.1 & & & -1.98 & & 2023.67 & \\
\hline & $16-1$ & 33.5 & 3.4 & 11.6 & & & 15.3 & & & -1.72 & & 1991.50 & \\
\hline & $16-2$ & 37.7 & 3.4 & 10.5 & & & 15.6 & & & -1.86 & & 1952.60 & \\
\hline & $1-1$ & 39.0 & 2.2 & 9.8 & & & 23.2 & & & -2.60 & & 2542.96 & \\
\hline & $1-2$ & 39.0 & 1.9 & 7.1 & & & 19.7 & & & -3.13 & & 2219.25 & \\
\hline & 4-1 & 36.2 & 1.9 & 11.6 & & & 29.3 & & & -2.13 & & 2470.73 & \\
\hline & $4-2$ & 35.8 & 2.1 & 11.5 & & & 26.2 & & & -1.96 & & 2251.70 & \\
\hline 306M30 & $6-1$ & 33.1 & 2.4 & 14.9 & 11.4 & 24.59 & 27.7 & 24.7 & 17.72 & -1.65 & 22.5 & 2457.98 & 5.89 \\
\hline & $6-2$ & 35.1 & 2.5 & 15.7 & & & 29.5 & & & -1.63 & & 2561.67 & \\
\hline & $8-1$ & 34.2 & 2.5 & 9.7 & & & 17.4 & & & -2.31 & & 2247.21 & \\
\hline & $8-2$ & 34.7 & 2.0 & 11.0 & & & 24.9 & & & -2.27 & & 2488.93 & \\
\hline
\end{tabular}




\section{APPENDIX F: COMPARISON BETWEEN AASHTO R30 AND PROPOSED PROTOCOL}

The current standard of asphalt concrete $(A C)$ conditioning for long-term aging is specified by AASHTO R30. In the AASHTO R30 procedure, all long-term aging is conducted on the as-compacted gyratory pills, which are then cut into prepared semi-circular test specimens. In this study, long-term aging was conducted on fully prepared, semi-circular test specimens. To compare the effects on the $\mathrm{Fl}$ of the two specimen types when long-term aged, a study using the R30 procedure was conducted to compare with specimens already tested in this study using the proposed protocol. The tests were conducted on lab-produced, lab-compacted (LPLC) mixes LM1 through LM5.

\section{F.1 SPECIMEN PREPARATION}

Asphalt concrete raw materials (binder and aggregates) were first heated to the required mixing temperature, and then prepared as a loose AC mix using a mechanical stirrer. Next, the batched samples were subjected to a two-hour conditioning cycle so that the material could reach its required compaction temperature. After the conditioning cycle, the materials were compacted to $180 \mathrm{~mm}$ pills.

In accordance with AASHTO R30, pills were extruded from the compaction molds after cooling for two hours and then they were cooled at room temperature for 16 hours. The compacted pills were placed in a forced-draft oven for five days ( 120 hours) at a temperature of $185^{\circ} \mathrm{F}\left(85^{\circ} \mathrm{C}\right)$. After the long-term conditioning, the oven was turned off and doors were opened to allow the aged pills to cool to room temperature without touching them.

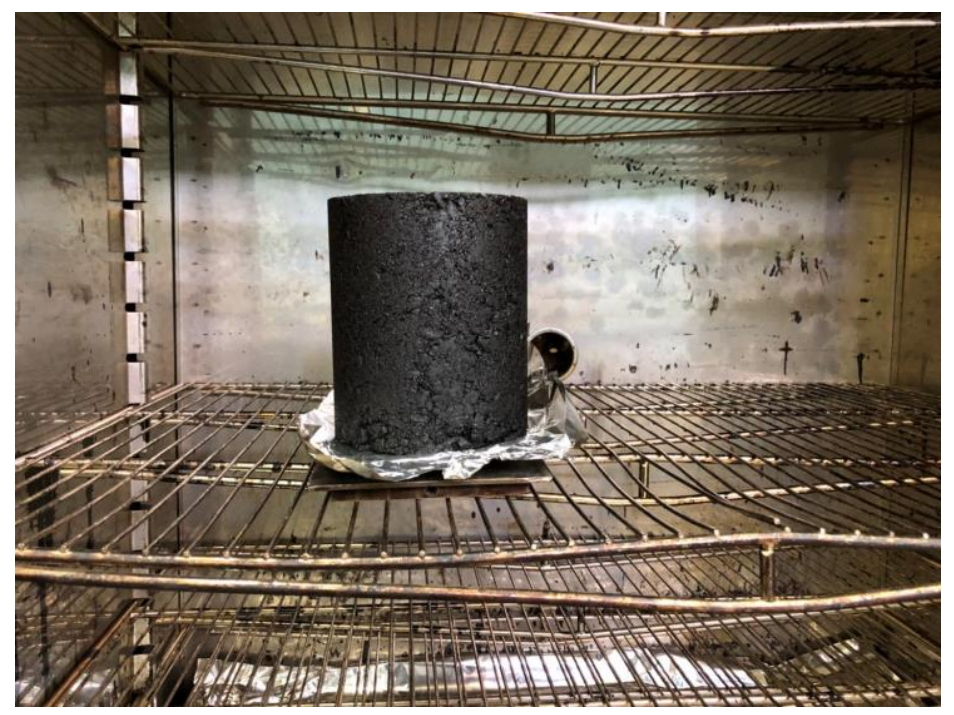

Figure F.1. Compacted $180 \mathrm{~mm}$ pills aging in a forced-draft oven.

No distortion was observed after aging. I-FIT test specimens were later prepared in accordance with AASHTO TP124 discussed in Chapter 3. 


\section{F.2 RESULTS AND DISCUSSION}

All calculated FI results were corrected using $7 \%$ air voids using equation (5). It should be noted that the correction factor developed in Barry (2016) evaluated short-term conditioned specimens. Further research would be required to validate that this correction factor calculation remains constant irrespective of conditioning level. Figure F.2 compares mean FI for AASHTO R30 and proposed 3D/95C methods aged specimens.

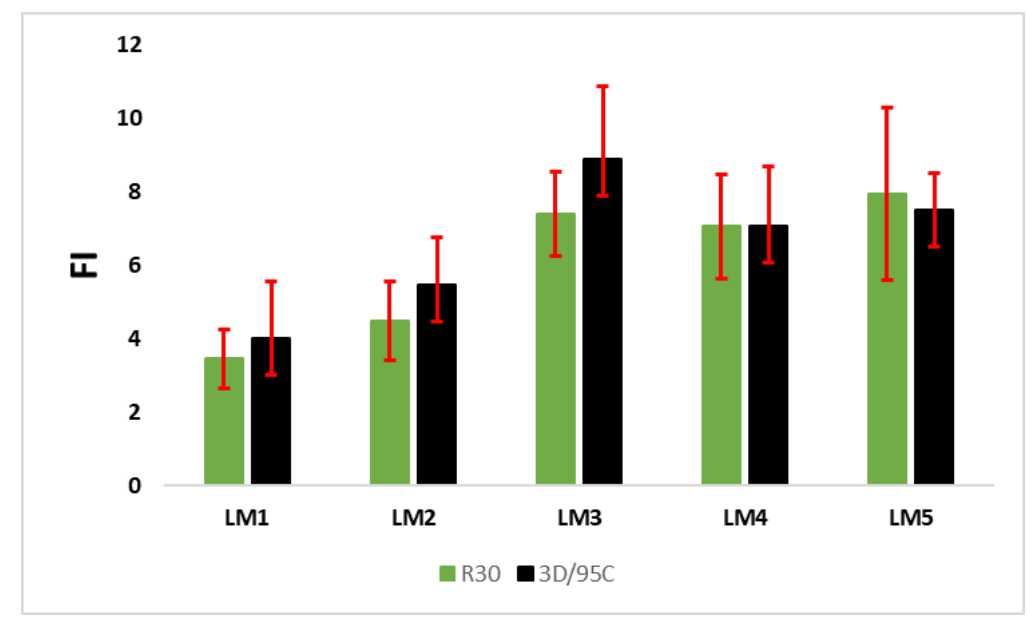

Figure F.2. Comparison of FI for AASHTO R30 and proposed protocol using aged specimens.

To statistically analyze if there is a significant difference between the effects of these two aging methods, an independent two-sample $t$ test was conducted. The significance level of 0.05 was selected.

The two-sample $t$ test requires the data to be normally distributed. The Shapiro-Wilk test was conducted and Table F.1 shows the resulting p-values.

Table F.1. Shapiro-Wilk Test Results

\begin{tabular}{c|ccccc}
\hline Method\Mix ID & LM1 & LM2 & LM3 & LM4 & LM5 \\
\hline AASHTO R30 & 0.2558 & 0.5030 & 0.7055 & 0.6454 & 0.1716 \\
3D/95C & 0.3019 & 0.2583 & 0.2067 & 0.4892 & 0.1255 \\
\hline
\end{tabular}

All p-values are larger than the significance level of 0.05 , thus, it is safe to conclude that normality assumptions are not violated. An independent two-sample $t$ test also requires that the two groups should have equal variance. Fisher's F test was conducted and Table F.2 presents the test results; $p$ values. 
Table F.2. Fisher's F Test Results

\begin{tabular}{c|ccccc}
\hline Mix ID & LM1 & LM2 & LM3 & LM4 & LM5 \\
& & & & & \\
\hline p-value & 0.1140 & 0.5900 & 0.1816 & 0.7204 & 0.0949 \\
& & & & & \\
\hline
\end{tabular}

Since all $p$-values are larger than significance level of 0.05 , the assumptions of equal variances are not violated. Hence, an independent two-sample $t$ test can be safely conducted with all assumptions met. Table F.3 illustrates the resulted p-values.

Table F.3. Independent Two-Sample T Test Results

\begin{tabular}{c|ccccc}
\hline Mix ID & LM1 & LM2 & LM3 & LM4 & LM5 \\
\hline p-value & 0.4051 & 0.9113 & 0.2763 & 0.6761 & 0.4708 \\
\hline
\end{tabular}

All p-values are larger than significance level of 0.05, which indicates that for all tested mixes; mean $\mathrm{FI}$ of AASHTO R30 and 3D/95C methods aged specimens are not statistically different.

\section{F.3 SUMMARY}

As stated before, this project proposes aging a fully prepared I-FIT specimen at $203^{\circ} \mathrm{F}\left(95^{\circ} \mathrm{C}\right)$ for three days using a forced-draft oven as the long-term aging protocol for AC surface mixtures. AASHTO R30, which is the current practice to simulate long-term aging, requires aging compacted pills at $185^{\circ} \mathrm{F}$ $\left(85^{\circ} \mathrm{C}\right)$ for five days. Results presented above show that these two methods have statistically similar effects on aged specimen $\mathrm{Fl}$. Thus, an extrapolation can be made that the proposed long-term aging protocol is able to simulate $5-10$ years of field aging.

However, as illustrated in Chapter 2, the AASHTO R30 method results in a significant aging gradient through out the specimen, which is not ideal for use in fracture tests like I-FIT. 
F.4 RAW DATA

Table F.4. AASHTO R30 Raw Data

\begin{tabular}{|c|c|c|c|c|c|c|c|c|c|c|c|c|}
\hline \multicolumn{2}{|c|}{ AASHTO R30 } & \multirow{2}{*}{$\begin{array}{r}\text { Air void } \\
\text { AV }\end{array}$} & \multicolumn{3}{|c|}{ Flexibility Index } & \multicolumn{3}{|c|}{ Corrected FI } & \multicolumn{2}{|c|}{ Slope } & \multicolumn{2}{|c|}{ Fracture Energy } \\
\hline Mix & Specimen & & $\mathrm{FI}$ & FI Avg. & $\operatorname{CoV}(\%)$ & FI Corr & $\begin{array}{c}\text { FI Corr. } \\
\text { Avg }\end{array}$ & $\operatorname{CoV}(\%)$ & Slope & CoV $(\%)$ & $\begin{array}{c}\text { Energy } \\
\text { (LLD) (Gf) } \\
\text { (J/m2) }\end{array}$ & CoV (\%) \\
\hline \multirow{8}{*}{ LM1 } & $1-1$ & 6.9 & 4.5 & \multirow{8}{*}{3.2} & \multirow{8}{*}{27.14} & 4.5 & \multirow{8}{*}{3.5} & \multirow{8}{*}{23.03} & -4.88 & \multirow{8}{*}{18.4} & 2192.75 & \multirow{8}{*}{9.61} \\
\hline & $1-2$ & 6.5 & 3.3 & & & 3.6 & & & -5.61 & & 1871.74 & \\
\hline & $1-3$ & 6.6 & 3.9 & & & 4.2 & & & -5.03 & & 1970.80 & \\
\hline & $1-4$ & 6.2 & 2.2 & & & 2.4 & & & -7.25 & & 1588.48 & \\
\hline & $2-1$ & 6.2 & 2.5 & & & 2.8 & & & -7.16 & & 1819.98 & \\
\hline & $2-2$ & 5.8 & 2.4 & & & 2.9 & & & -7.59 & & 1851.45 & \\
\hline & $2-3$ & 6.1 & 2.6 & & & 2.9 & & & -6.86 & & 1760.89 & \\
\hline & $2-4$ & 6.4 & 4.0 & & & 4.3 & & & -5.09 & & 2022.75 & \\
\hline \multirow{7}{*}{ LM2 } & $1-1$ & 6.5 & 4.1 & \multirow{7}{*}{4.1} & \multirow{7}{*}{23.05} & 4.3 & \multirow{7}{*}{4.5} & \multirow{7}{*}{23.57} & -4.85 & \multirow{7}{*}{16.7} & 1980.20 & \multirow{7}{*}{11.61} \\
\hline & $1-2$ & 6.4 & 2.7 & & & 2.9 & & & -6.80 & & 1801.68 & \\
\hline & $1-3$ & 6.4 & 3.7 & & & 4.0 & & & -5.12 & & 1889.79 & \\
\hline & $2-1$ & 6.3 & 5.1 & & & 5.7 & & & -4.69 & & 2400.41 & \\
\hline & $2-2$ & 6.1 & 4.5 & & & 5.2 & & & -5.25 & & 2384.79 & \\
\hline & $2-3$ & 6.4 & 5.2 & & & 5.7 & & & -4.06 & & 2123.81 & \\
\hline & $2-4$ & 6.7 & 4.6 & & & 4.9 & & & -4.67 & & 2167.83 & \\
\hline \multirow{8}{*}{ LM3 } & $1-1$ & 6.4 & 6.4 & \multirow{8}{*}{6.9} & \multirow{8}{*}{14.08} & 7.0 & \multirow{8}{*}{7.4} & & -3.04 & & 1957.59 & \\
\hline & $1-2$ & 6.6 & 5.0 & & & 5.3 & & & -3.58 & & 1798.79 & \\
\hline & $1-3$ & 6.8 & 6.3 & & & 6.4 & & & -3.35 & & 2107.48 & \\
\hline & $1-4$ & 6.5 & 7.6 & & & 8.1 & & & -3.12 & & 2367.78 & \\
\hline & $2-1$ & 6.3 & 8.0 & & & 8.8 & & 15.46 & -2.84 & 8.6 & 2284.55 & 8.91 \\
\hline & $2-2$ & 6.4 & 7.6 & & & 8.3 & & & -2.98 & & 2271.53 & \\
\hline & $2-3$ & 6.3 & 6.9 & & & 7.6 & & & -3.01 & & 2073.76 & \\
\hline & $2-4$ & 6.6 & 7.4 & & & 7.8 & & & -2.78 & & 2046.89 & \\
\hline & $1-1$ & 6.4 & 7.3 & & & 7.9 & & & -2.75 & & 1999.17 & \\
\hline & $1-2$ & 6.4 & 8.5 & & & 9.3 & & & -2.35 & & 1993.76 & \\
\hline & $1-3$ & 6.6 & 6.1 & & & 6.4 & & & -2.97 & & 1801.54 & \\
\hline & $1-4$ & 6.2 & 4.9 & & & 5.4 & & & -4.01 & & 1947.42 & \\
\hline LM4 & $2-1$ & 6.6 & 5.9 & 6.4 & 18.73 & 6.2 & 7.0 & 20.12 & -3.23 & 15.3 & 1914.22 & 9.20 \\
\hline & $2-2$ & 6.1 & 6.4 & & & 7.3 & & & -3.20 & & 2052.00 & \\
\hline & $2-3$ & 6.7 & 5.1 & & & 5.4 & & & -3.34 & & 1717.26 & \\
\hline & $2-4$ & 6.0 & 7.3 & & & 8.4 & & & -3.19 & & 2324.44 & \\
\hline & $1-1$ & 6.8 & 7.6 & & & 7.7 & & & -2.98 & & 2250.51 & \\
\hline & $1-2$ & 6.4 & 5.7 & & & 6.2 & & & -3.26 & & 1867.04 & \\
\hline LMS & $1-3$ & 7.2 & 6.6 & 7.7 & 29.07 & 6.5 & 7.9 & 29.61 & -2.93 & 17.2 & 1946.24 & 10.54 \\
\hline & $1-4$ & 6.7 & 10.9 & & & 11.3 & & & -2.13 & & 2313.59 & \\
\hline
\end{tabular}




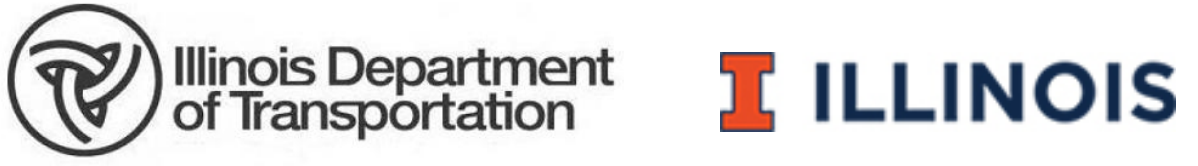

